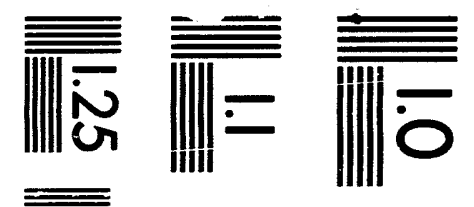

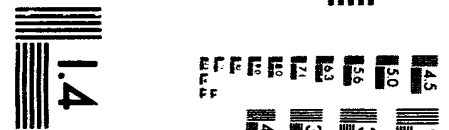

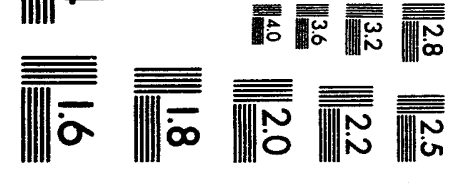



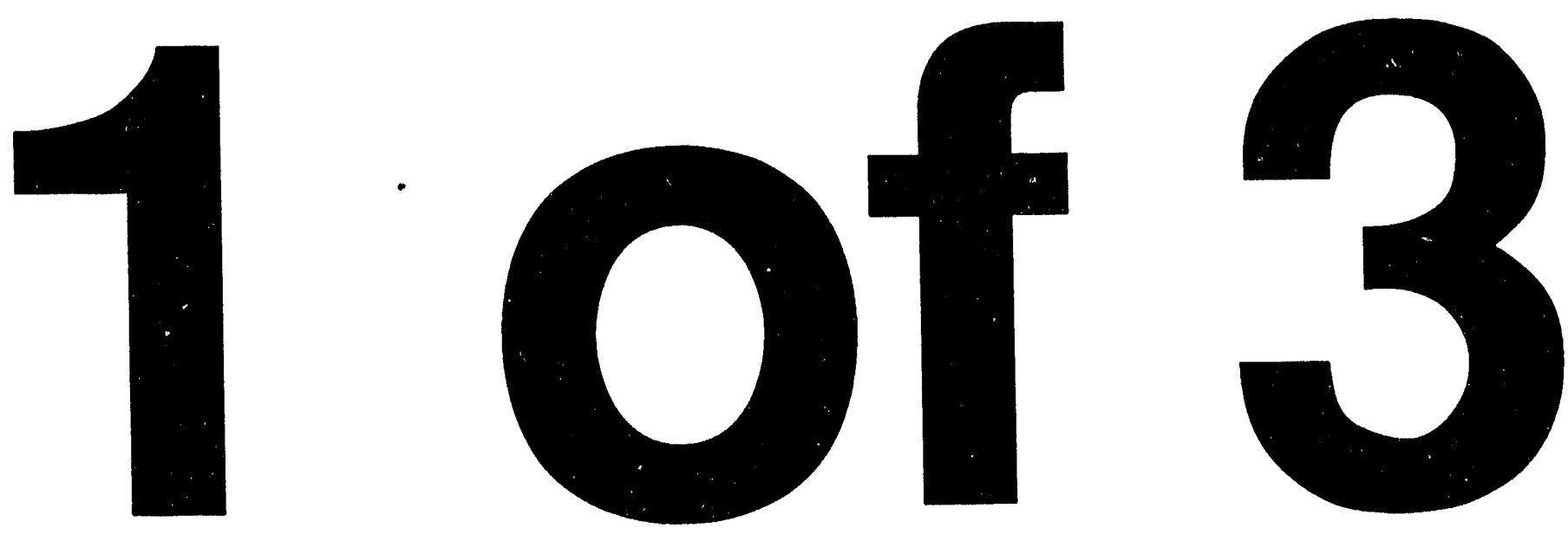
NUREG/CR-4832

UCID-21245

Vol. 8

RX

\title{
Analysis of the LaSalle Unit 2 Nuclear Power Plant: Risk Methods Integration and Evaluation Program (RMIEP)
}

\author{
Seismic Analysis
}

Manuscript Complcted: June 1993

Date Published: November 1993

Prepared by

J. E. Wells, D. A. Lappa, D. L. Bernreuter, J. C. Chen, T. Y. Chuang*, Lawrence Livermore National Laboratory

J. J. Johnson, R. D. Campbell, P. S. Hashimoto, O. R. Maslenikov, L. W. Tiong, M. K. Ravindra,

R. H. Kincaid, EQE Incorporated

R. H. Sues, C. S.Putcha, NTS Engineering

Lawrence Livermore National Laboratory

7000 East Avenue

Livermore, CA 94550

Subcontractors:

$\mathrm{EQE}$, International

Lakeshore Towers

18101 Von Karman Avenue, Suite 400

Irvine, CA 92715

NTS Engineering

6695 East Pacific Coast Highway

Long Beach, CA 90803

\section{DISCLAIMER}

\section{Prepared for}

Division of Safety Issue Resolution

Office of Nuclear Regulatory Research

U.S. Nuclear Regulatory Commission

Washington, DC 20555-0001

NRC FIN A1386

This report was prepared as an account of work sponsored by an agency of the United States Government. Neither the United States Government nor any agency thereof, nor any of their employees, makes any warranty, express or implied, or assumes any legal liability or responsibility for the accuracy, completeness, or usefulness of any information, apparatus, product, or process disclosed, or represents that its use would not infringe privately owned rights. Reference herein to any specific commercial product, process, or service by trade name, trademark, manufacturer, or otherwise does not necessarily constitute or imply its endorsement, recommendation, or favoring by the United States Government or any agency thereof. The views and opinions of authors expressed herein do not necessarily state or reflect those of the United States Government or any agency thereof.

*Consultant 


\title{
Seismic Analysis of the LaSalle Unit 2 Nuclear Power Plant--Risk Methodology Integration Evaluation Program (RMIEP)*
}

\begin{abstract}
Lawrence Livermore National Laboratory (LLNL) developed a detailed seismic probabilistic risk assessment (PRA) method for nuclear power plants under the Seismic Safety Margins Research Program (SSMRP) sponsored by the U.S. Nuclear Regulatory Commission (USNRC). Subsequent to the development of this detailed seismic PRA, a simplified methodology was developed to reduce the costs while still providing adequate results. This simplified seismic risk methodology was der onstrated by its application to a pressurized water reactor (PWR) plant - the Zion Nuclear Power Plant. In order to further demonstrate its applicability, and, if possible, to provide a basis for comparing the seismic risk of PWRs and boiling water reactors (BWRs), this simplified seismic risk assessment methodology was also applied to a BWR plant - the LaSalle County Station nuclear power plant. This report describes the methodology used and the results obtained for the LaSalle BWR plant.
\end{abstract}

The methodology applied to this analysis made use of SMACS and SEISIM computer codes to calculate structure and component responses, and failure frequencies. The process started with development of seismic hazard curves and a set of seismic time histories for two of the six earthquake levels of interest. These time histories were used as input to SMACS for computing some median responses for plant structures while taking into account soil-structure interaction effects. The responses obtained from this limited SMACS analysis were then used to scale design calculations to obtain median structural responses for all six earthquake levels. The variabilities and correlations of seismic responses were based on those values documented in the report for the simplified seismic risk assessment methodology. Core damage frequencies due to earthquakes were calculated using SEISIM code and the structural responses, plant fragilities, and logic models (event and fault trees) of the plant systems.

Using the RMIEP developed event and fault trees, our analysis resulted in a seismically ind'sced core damage frequency point estimate of 6.0E-7/yr for LaSalle. It was found that 89 percent $c^{\circ}$ " ihe risk fell in the first three (of six) earthquake levels with 57 percent of the risk in earthquake if 9 i (lowest acceleration). This result, combined with the component importance analysis, indic ine in system failures were dominated by random events. The dominant components include generator failures (failure to swing, failure to start, failure to run after started), and the cond nsarte storage tank.

* Although published in 1993, the majority of the analytical effort associated with this simplified seismig was performed in the period 1982 to 1986 . Therefore, because of the advancements in the state-of-the-an .: seismic PRAs, there may be differences in the procedures reported herein and what is commonly accepted practici today. 


\section{Table of Contents}

Page

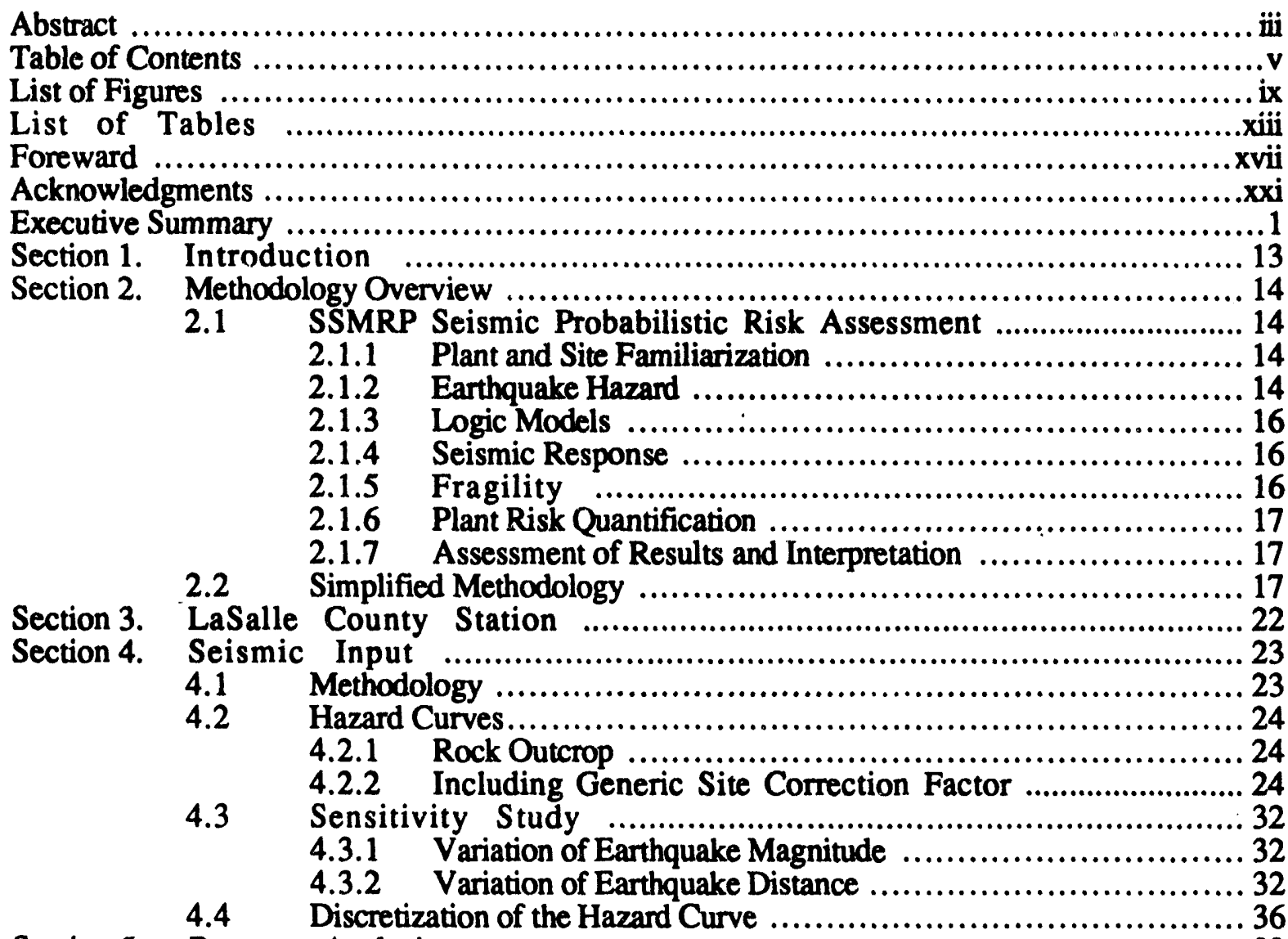

Section 5. Response Analysis ........................................................ 39

5.1 Seismic Response Requirements for a Seismic PRA and Overview ..... 39

5.2 Description of Site and Structures ................................. 41

$5.3 \quad$ SSI and Structure Models ........................................................45

5.3.1 Free-Field Ground Motion and Soil Model ....................... 46

5.3.2 SSI Parameters ....................................................... 47

5.3.3 Structure Models ........................................................ 56

5.4 Probabilistic Responses ....................................................... 99

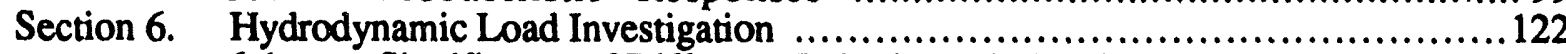

6.1 Significance of Different Hydrodynamic Loads ......................122

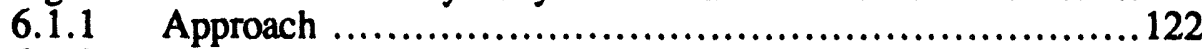

6.1.2 Description of Hydrodynamic Loads ............................124

6.1.3 Hydrodynamic Loads Screening ................................128

6.1.4 Evaluation of Median and Variability of SRV Loading .........132

6.1.5 Summary .................................................133

6.2 Load Combination Approach ………………….........................133

6.2.1 Introduction ...........................................133

6.2.2 Load Combination Methodology .............................138 
Table of Contents (cont.)

Page

6.3 Probabilistic Load Combination Case Studies .......................... 151

6.3.1 Introduction .............................................. 151

6.3.2 1 SSE Case Study ............................................ 153

6.3.3 3 SSE Case Study .......................................... 160

6.3.4 Recommended Response Combination Rule ....................171

Section 7. Structural Fragilities ....................................................... 175

$7.1 \quad$ Method of Fragility Evaluation ..........................................175

7.2 Development of Structural Capacities ...............................176

7.2.1 Structural Element Ultimate Strengths ......................176

$7.3 \quad$ S.2.2 Structure Inelastic Energy Absorption ........................ 177

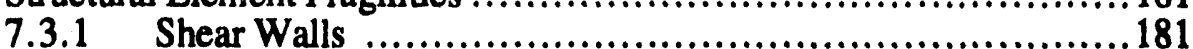

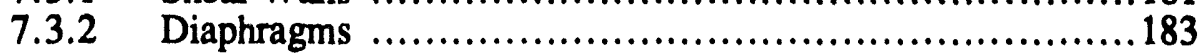

7.3.3 Primary Containment and Internal Structures ..................192

7.4 Condensate Storage Tank .............................................. 196

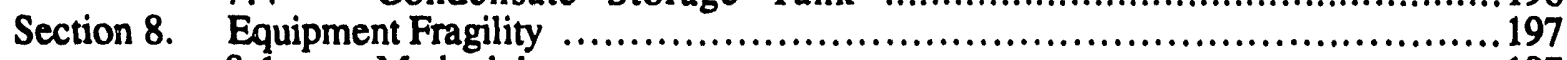

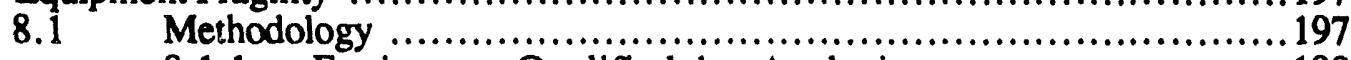

8.1.1 Equipment Qualified by Analysis ................................ 198

8.1.2 Equipment Qualified by Test ...................................200

8.1.3 Hydrodynamic Loads .....................................202

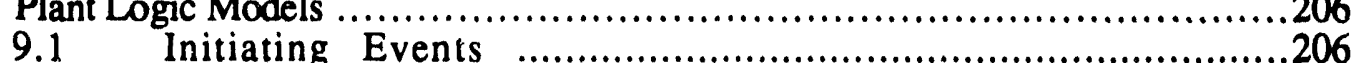

$9.1 .1 \quad$ Reactor Vessel Rupture .......................................206

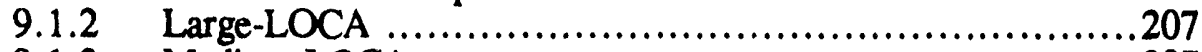

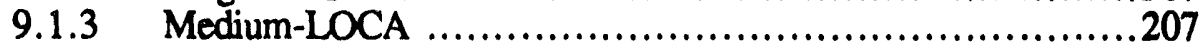

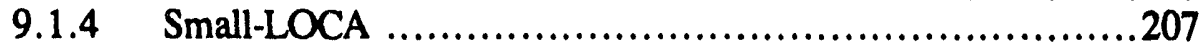

9.1.5 Transient ....................................................207

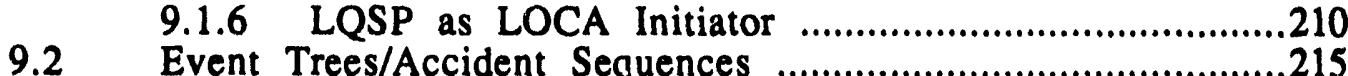

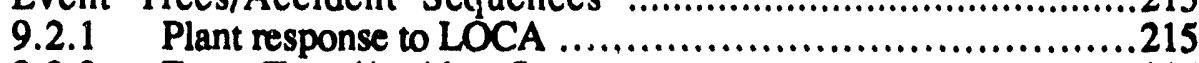

9.2.2 Event Trees/Accident Sequences ............................217

9.3 Fault Tree/Safety Systems ...........................................222

9.3.1 System descriptions .......................................222

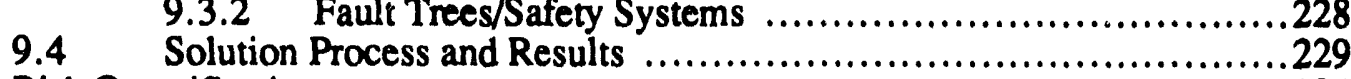

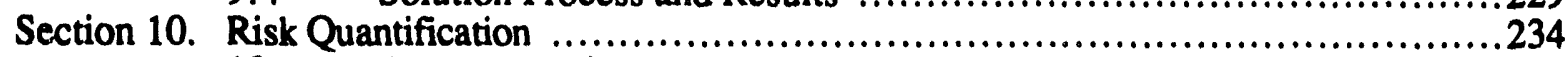

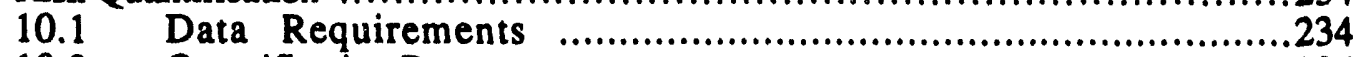

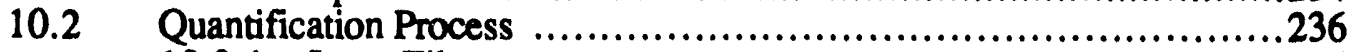

10.2.1 Input Files ................................................236

10.2.2 SEISIM Computational Procedure Summary ..................236

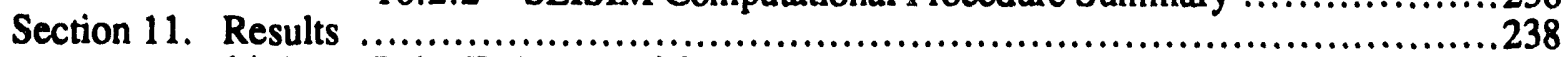

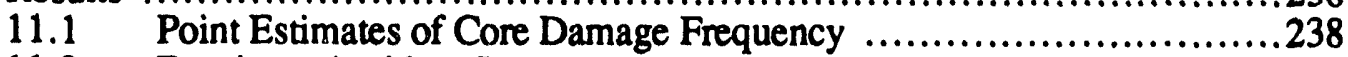

11.2 Dominant Accident Sequences ........................................2.239

11.3 Dominant Component Failures ..........................................239 
Section 12. Conclusions ...........................................................247

12.1 Comparison of BWR Seismic Risk to PWR Seismic Risk ................247

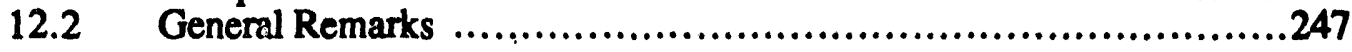

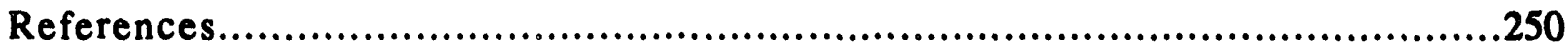

Appendix A. Use of Altemate Event and Fault Trees ......................................A-1

A.1 Event Trees/Accident Sequences Used in Additional Analysis .............A-1

A.2 Fault Trees/Safety Systems Used in Additional Analysis ...................A-2

A.3 Solution Process and Results Used in Additional Analysis ..............A-2

A.3.1 Solution Process .................................................

A.3.2 Point Estimate of Core Damage Frequency ...................A-9

A.3.3 Dominant Accident Sequences ..............................A-9

A.4 Comparison of RMIEP and Additional Analyses Results ................. A-15 


\section{List of Figures}

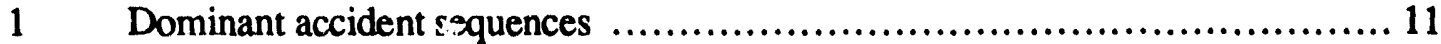

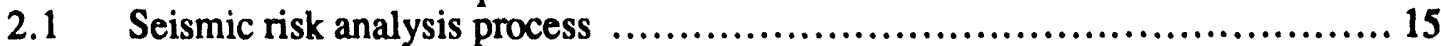

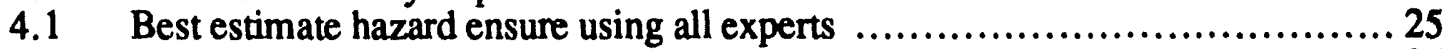

4.2 Best estimate hazard curve from each seismicity expert ............................... 26

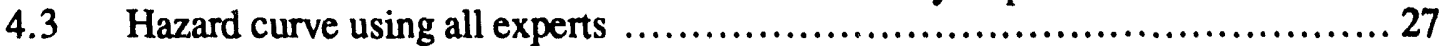

4.4 Arithmetic and geornetric mean hazard curves using all experts $\ldots \ldots \ldots \ldots \ldots \ldots \ldots . \ldots 28$

4.5 Best estimate hazard curve including Site correction using all experts ................................................................ 29

4.6 Best estimate hazard curve including site correction from

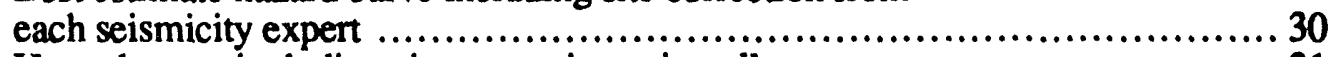

4.7 Hazard curve including site correction using all experts ........................ 31

4.8 Arithmetic and geometric mean hazard curves including site correction using all experts 33

4.9 Comparison of best estimate hazard curves between soil and hypothetical rock outcrop at the LaSalle site ................................... 34

4.10 Hazard curves for cases when only earthquakes of limited magnitude ranges are considered .35

4.11 Hazard curves for cases when only earthquakes in limited distance ranges are considered

5.1 Stratigraphic column with geophysical data summary ........................... 42

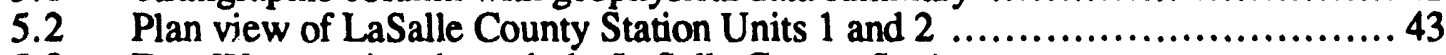

5.3 East-West section through the LaSalle County Station building complex

5.4 Schematic representation of the elements of the substructure approach to SSI analysis ....

5.5 Shear modulus degradation curves........................................... 50

5.6 Damping ratios vs strain................................................. 51

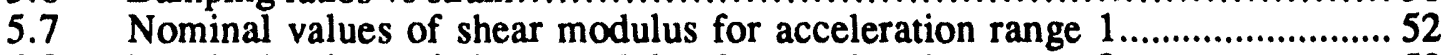

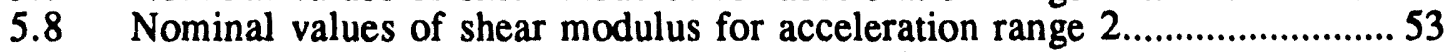

5.9 Mean free-field ground response spectra on soil free surface, el. 710', acceleration range 1 .................................. 54

5.10 Mean free-field ground response spectra on soil free surface, el. $710^{\prime}$, acceleration range 2 ..................................... 55

5.11 Foundation discretization for the LaSalle County Station structure complex impedance calculations .................................... 57

5.12 Representation LaSalle Foundation impedances .............................. 58

a) Horizontal translation, C-direction (\#/ft.).

b) Horizontal translation--rocking coupling (\#/rad).

c) Rocking about Y-axis (ft. \#/rad).

d) Vertical translation.

e) Torsion (ft. \#/rad).

5.13 Schematic of the LaSalle County Station Complex horizontal structure mode 


\section{List of Figures (cont.)}

5.14 Locations of mass points of horizontal model 63

5.15 Schematic of spring elements representing shear walls and shear wall groups.

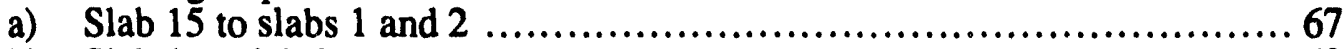

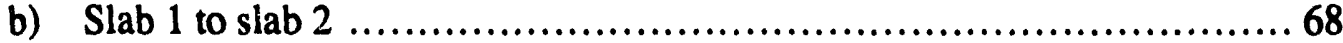

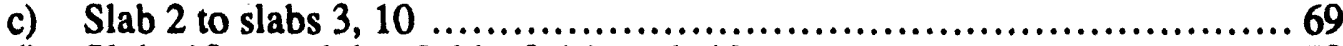

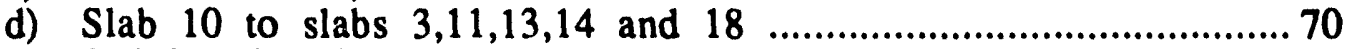

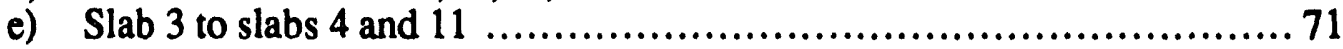

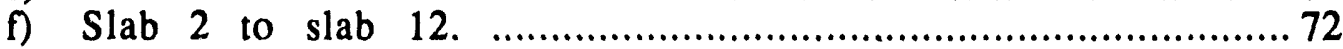

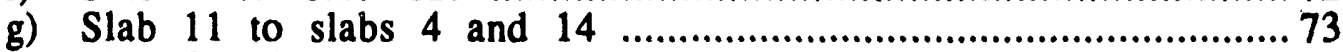

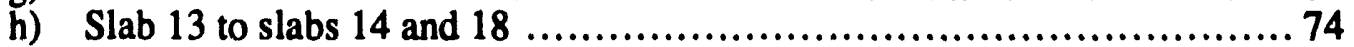

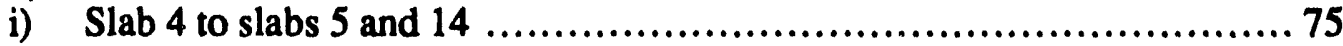

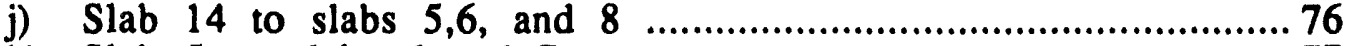

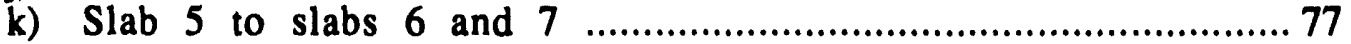

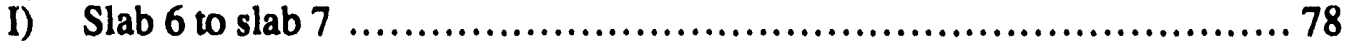

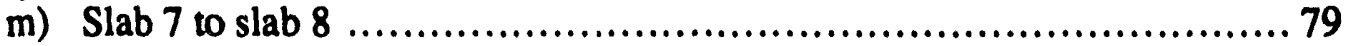

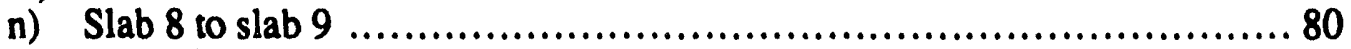

5.16 Vertical seismic model of reactor and auxiliary buildings ........................... 93

5.17 Vertical seismic model of turbine, heater bay and radwaste buildings ...........95

5.18 Vertical seismic model of RPV, sacrificial shield, and reactor pedestal ..........97 97

5.19 Horizontal in-structure response spectra, median acceleration ranges 1 and 2 , and design spectra.

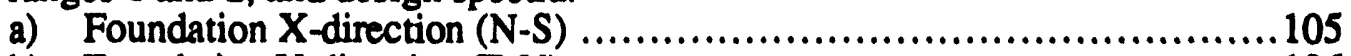

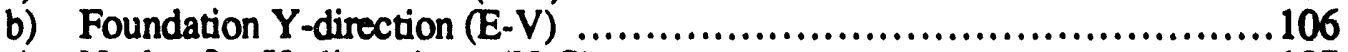

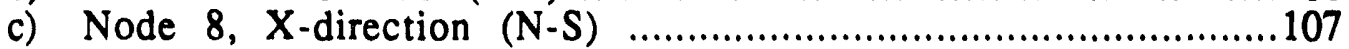

d) Node 8, Y-direction (E-W) ............................................... 108

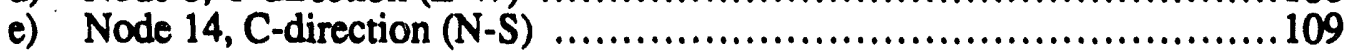

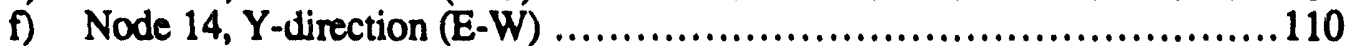

g) Node 34, X-direction (N-S) ............................................111

h) Node 34, Y-direction (E-W) ......................................112

5.20 Vertical in-structure response spectra, median acceleration ranges 1 and 2 , and design spectra.

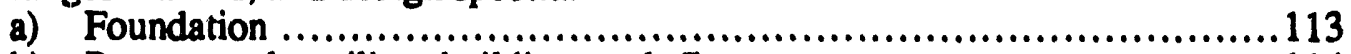

b) Reactor and auxiliary building, node 7 ................................114

c) Reactor and auxiliary building, node 22 .....................................115

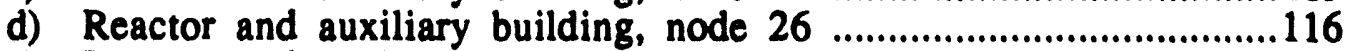

e) Reactor and auxiliary building, node 33 .................................117

6.1 Load event tree..............................................................123

6.2 Example hydrodynamic event conditional probability curves.................... 125

6.3 LaSalle County Station reactor and containment building model ...................131

6.4 Schematic of the LaSalle County Station complex horizontal

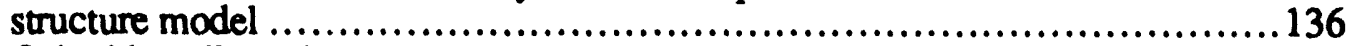

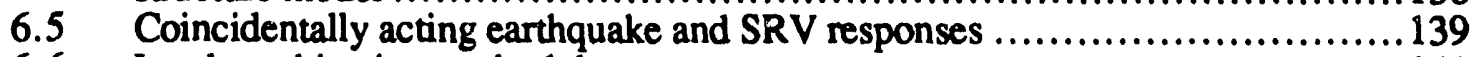

6.6 Load combination methodology ............................................... 141

6.7 Calculation of probability density and upcrossing rate functions.................143 


\section{List of Figures (cont.)}

6.8 Variation of upcrossing rate (of level $0.25 \mathrm{~g}$ ) with time 144

6.9 Variation of upcrossing rate (at time $=4 \mathrm{sec}$.) with acceleration level

6.10 Distribution of the SRV time log random variable, $\theta$

6.11 Time-averaged upcrossing rates for earthquake range 1 , SRV, and combined response (oscillator frequency $=2 \mathrm{~Hz}$, damping $=5 \%$ )

6.12 Time-averaged upcrossing rates for earthquake range 1 , SRV, and combined response (oscillator frequency $=4 \mathrm{~Hz}$, damping $=5 \%$ )

6.13 Time-averaged upcrossing rates for earthquake range 1 , SRV, and combined response (oscillator frequency $=25 \mathrm{~Hz}$, damping $=5 \%$ )

6.14 Time-averaged upcrossing rates for earthquake range 2 , SRV, and combined response (oscillator frequency $=2 \mathrm{~Hz}$, damping $=5 \%$ )

6.15 Time-averaged upcrossing rates for earthquake range 2 , SRV, and combined response (oscillator frequency $=2.8 \mathrm{~Hz}$, damping $=5 \%$ )

6.16 Time-averaged upcrossing rates for earthquake range 2 , SRV, and combined response (oscillator frequency $=3 \mathrm{~Hz}$, damping $=5 \%$ )

6.17 Time-averaged upcrossing rates for earthquake range 2 , SRV, and combined response (oscillator frequency $=4 \mathrm{~Hz}$, damping $=5 \%$ )

6.18 Variation of upcrossing rate of $0.20 \mathrm{~g}$ with time (earthquake range $2, \mathrm{f}=2.8 \mathrm{~Hz}$ ) 169

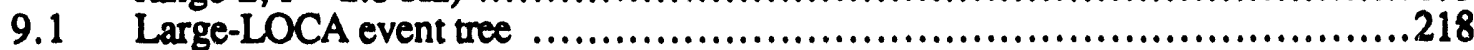
173

9.2 Medium-LOCA event tree ......................................................219

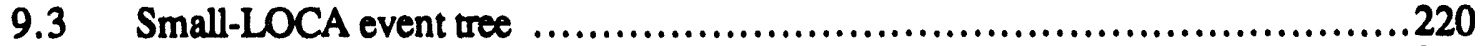

9.4 Loss-of-offsite power event tree ...........................................221

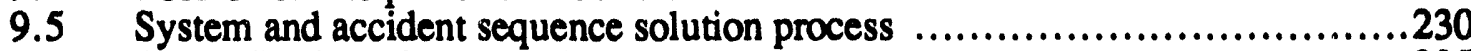

10.1 Quantification of seismic risk ...............................................235

A.1 Large-LOCA event tree (used in additional analysis) .................................

A.2 Medium-LOCA event tree (used in additional analysis) ...........................

A.3 Small-LOCA event tree (used in additional analysis) .................................5

A.4 Loss-of-offsite power event tree (used in additional analysis) ..................A-6 


\section{List of Tables}

Page

1 Seismic occurrence data for the LaSalle site .....................................9

2 Unconditional accident sequence probabilities ................................. 10

$2.1 \quad$ Response random and modeling uncertainties ................................ 20

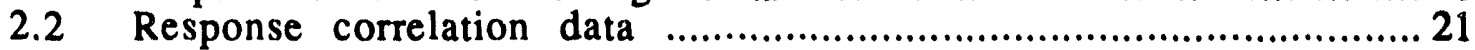

4.1 Seismic occurrence data for the LaSalle site ................................... 28

5.1 LaSalle soil characteristics ............................................... 49

5.2 Description of mass points in horizontal seismic model ....................... 64

5.3 Identification of shear walls and shear wall groups, horizon-

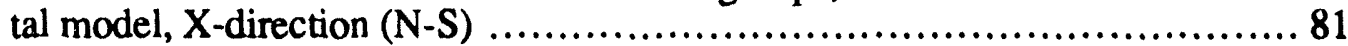

5.4 Identification of shear walls and shear wall groups, horizon-

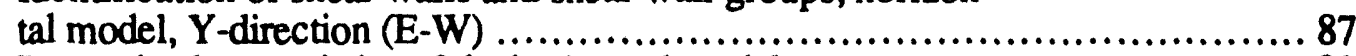

5.5 Dynamic characteristics of the horizontal model .............................. 92

5.6 Dynamic characteristics of the vertical model of the reactor

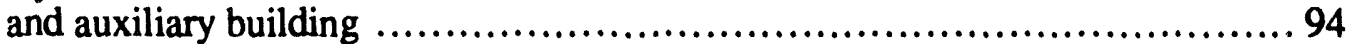

5.7 Dynamic characteristics of the vertical model of the turbine, heater bay and radwaste buildings ........................................... 96

5.8 Dynamic characteristics of the vertical model of the RPV, sacrificial shield and reactor pedestal ....................................... 98

5.9 In-structure forces and moments for acceleration ranges 1

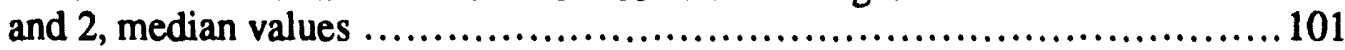

5.10 Equipment responses for all earthquake levels ..............................120

6.1 Horizontal accelerations for SRV-asymmetric and seismic

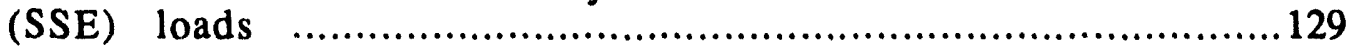

6.2 Vertical accelerations for SRV-all and seismic (SSE) loads .......................130

6.3 Horizontal and vertical accelerations for condensationoscillation loads

6.4 Median horizontal SRV spectral accelerations (SRV- . asymmetric)

6.5 Median horizontal SRV spectral accelerations at discrete frequencies

6.6 Upcrossing rates for earthquake range $1, \mathrm{SRV}$, and combined response (oscillator frequency $=2 \mathrm{~Hz}$, damping $=5 \%$ ) $\ldots \ldots \ldots \ldots \ldots \ldots \ldots \ldots \ldots \ldots$

6.7 Upcrossing rates for earthquake range $1, \mathrm{SRV}$, and combined

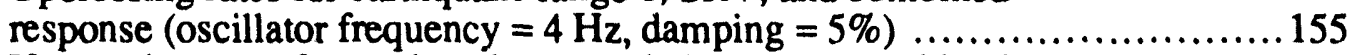

6.8 Upcrossing rates for earthquake range $1, \mathrm{SRV}$, and combined response (oscillator frequency $=25 \mathrm{~Hz}$, damping $=5 \%$ ) ..........................156

6.9 Comparison of SRSS and absolute sum combination With probabilistic combination (earthquake $=$ range 1 )

6.10 Upcrossing rates for earthquake range 2, SRV, and combined

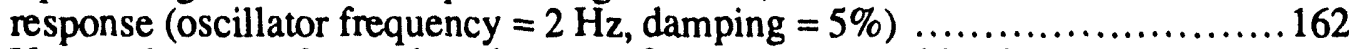

6.11 Upcrossing rates for earthquake range $2, S R V$, and combined response (oscillator frequency $=2.8 \mathrm{~Hz}$, damping $=5 \%$ ) 


\section{List of Tables (cont.)}

6.12 Upcrossing rates for earthquake range 2, SRV, and combined response (oscillator frequency $=3 \mathrm{~Hz}$, damping $=5 \%$ )

6.13 Upcrossing rates for earthquake range $2, \mathrm{SRV}$, and combined response (oscillator frequency $=4 \mathrm{~Hz}$, damping $=5 \%$ )

6.14 Comparison of SRSS and absolute sum combination with

probabilistic combination (earthquake $=$ range 2 )
6.15........ Sensitivity of combined upcrossing rate to time lag variable distribution

7.1 Median material strengths and variabilities

7.2 Summary of shear wall fragilities

7.3 Comparison of shear wall equivalent elastic capacities with elastic loads acceleration range 1

Comparison of shear wall equivalent elastic capacities with

7.4 Comparison of shear wall equivalent e
elastic loads acceleration range 2

7.5 Summary of diaphragm fragilities

7.6 Comparison of diaphragm equivalent elastic capacities with

elastic loads acceleration range 1

7.7 Comparison of diaphragm equivalent elastic capacities with elastic loads acceleration range 2

7.8 Summary of primary containment fragilities

7.9 Comparison of primary containment equivalent elastic capacities with elastic loads acceleration range 1

7.10 Comparison of primary containment equivalent elastic capacities with elastic loads acceleration range 2

Earthquake component combination factors

8.1

LaSalle county station equipment fragilities

8.2

Generic seismic equipment fragilities

fren

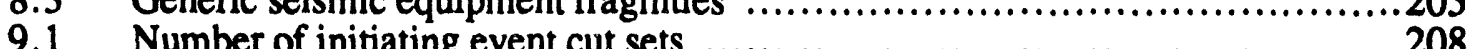

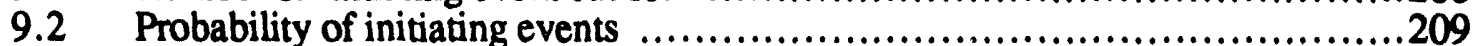

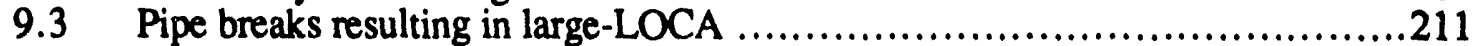

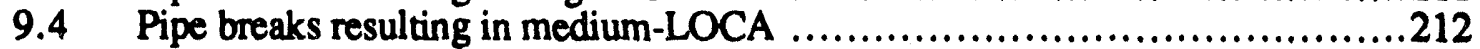

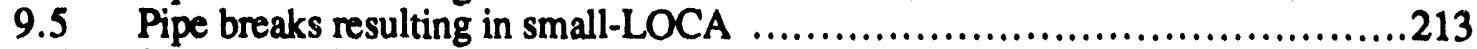

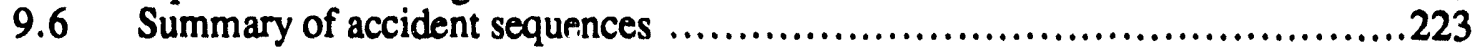

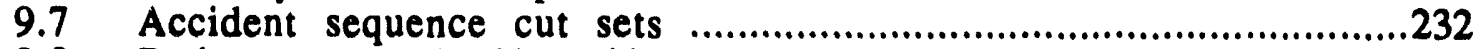

9.8 Basic events contained in accident sequence cut sets ..........................233

10.1 SEISIM input files ............................................................237

11.1 Unconditional accident sequence frequencies...................................240

11.2 Accident sequence probabilities conditional or earthquake probability

11.3 Accident sequence probabilities conditional on Initiator and earthquake probability

11.4 Core damage frequency contribution by initiating events $\ldots \ldots \ldots \ldots \ldots \ldots \ldots \ldots \ldots . .244$

A.1 Summary of accident sequences (additional analysis) .............................

A.2 Number of gates and basic events in safety system fault 


\section{List of Tables (cont.)}

A.3 Number of safety system cut sets after culling (additional analysis)

A.4 Culled accident sequence cut set numbers (additional analysis)

A.5 Unconditional accident sequence frequencies (additional analysis) A-11

A.6 Accident sequence probabilities conditional on earthquake probability (additional analysis)

A.7 Accident sequence probabilities conditional on initiator and earthquake probability (additional analysis) 


\section{FOREWORD}

\section{LaSalle Unit 2 Level III Probabilistic Risk Assessment}

In recent years, applications of Probabilistic Risk Assessment (PRA) to nuclear power plants have experienced increasing acceptance and use, particularly in addressing regulatory issues. Although progress on the PRA front has been impressive, the usage of PRA methods and insights to address increasingly broader regulatory issues has resulted in the need for continued improvement in and expansion of PRA methods to support the needi of the Nuclear Regulatory Commission (NRC).

Before any new PRA methods can be considered suitable for routine use in the regulatory arena, they need to be integrated into the overall framework of a PRA, appropriate interfaces defined, and the utility of the methods evaluated. The LaSalle Unit 2 Level III PRA, described in this and associated reports, integrates new methods and new applications of previous methods into a PRA framework that provides for this integration and evaluation. It helps lay the bases for both the routine use or the methods and the preparation of procedures that will provide guidance for future PRAs used in addressing regulatory issues. These new methods, once integrated into the framework of a PRA and evaluated, lead to a more complete PRA analysis, a better understanding of the uncertainties in PRA resuits, and broader insights into the importance of plant design and operational characteristics to public risk.

In order to satisfy the needs described above, the LaSalle Unit 2, Level III PRA addresses the following broad objectives:

1) To develop and apply methods to integrate internal, exterinal, and dependent failure risk methods to achieve greater efficiency, consistency, and completeness in the conduct of risk assessments;

2) To evaluate PRA technology developments and formulate improved PRA procedures;

3) To identify, evaluate, and effectively display the uncertainties in PRA risk predictions that stem from limitations in plant modeling, PRA methods, data, or physical processes that occur during the evolution of a severe accident;

4) To conduct a PRA on a BWR 5, Mark II nuclear power plant, ascertain the plant's dominant accident sequences, evaluate the core and containment response to accidents, calculate the consequences of the accidents, and assess overall risk; and finally;

5) To formulate the results in such a manner as to allow the PRA to be easily updated and to allow testing of future improvements in methodology, data, and the treatment of phenomena.

The LaSalle Unit 2 PRA was performed for the NRC by Sandia National Laboratories (SNL) with substantial help from Commonwealth Edison (CECo) and its contractors. Because of the size and scope of the PRA, various related programs were set up to conduct different aspects of the analysis. Additior.ally, existing programs had tasks added to perform some analyses for the LaSalle PRA. The responsibility for overall direction of the PRA was assigned to the Risk Methods Integration and Evaluation Program (RMIEP). RMIEP was specifically responsible for all aspects of the Level I analysis (i.e., the core damage analysis). The Phenomenology and Risk Uncertainty Evaluation Program (PRUEP) was responsible for the Level II/III analysis (i.e., 
accident progression, source term, consequence analyses, and risk integration). Other programs provided support in various areas or performed some of the subanalyses. These programs include the Seismic Safety Margins Research Program (SSMRP) at Lawrence Livermore National Laboratory (LLNL), which performed the seismic analysis; the Integrated Dependent Failure Analysis Program, which developed methods and analyzed data for dependent failure modeling; the MELCOR Program, which modified the MELCOR code in response to the PRA's modeling needs; the Fire Research Program, which performed the fire analysis; the PRA Methods Development Program, which developed some of the new methods used in the PRA; and the Data Programs, which provided new and updated data for BWR plants similar to LaSalle. CECo provided plant design and operational information and reviewed many of the analysis results.

The LaSalle PRA was begun before the NUREG-1150 analysis and the LaSalle program has supplied the NUREG-1150 program with simplified location analysis methods for integrated analysis of external events, insights on possible subtle interactions that come from the very detailed system models used in the LaSalle PRA, core vulnerable sequence resolution methods, methods for handling and propagating statistical uncertainties in an integrated way through the entire analysis, and BWR thermal hydraulic models which were adapted for the Peach Bottom and Grand Gulf analyses.

The Level I results of the LaSalle Unit 2 PRA are presented in "Analysis of the LaSalle Unit 2 Nuclear Power Plant: Risk Methods Integration and Evaluation Program (RMIEP)," NUREG/CR4832, SAND87-7157, ten volumes. The reports are organized as follows:

NUREG/CR-4832 - Volume 1: Summary Report.

NUREG/CR-4832 - Volume 2: Integrated Quantification and Uncertainty Analysis.

NUREG/CR-4832 - Volume 3: Internal Events Accident Sequence Quantification.

NUREG/CR-4832 - Volume 4: Initiating Events and Accident Sequence Delineation

NUREG/CR-4832 - Volume 5: Parameter Estimation Analysis and Human Reliability Screening Analysis.

NUREG/CR-4832 - Volume 6: System Descriptions and Fault Tree Definition.

NUREG/CR-4832 - Volume 7: External Event Scoping Quantification.

NUREG/CR-4832 - Volume 8: Seismic Analysis.

NUREG/CR-4832 - Volume 9: Internal Fire Analysis.

NUREG/CR-4832 - Volume 10: Internal Flcod Analysis.

The Level II/III results of the LaSalle Unit 2 PRA are presented in: "Integrated Risk Assessment For the LaSalle Unit 2 Nuclear Power Plant: Phenomenology and Risk Uncertainty Evaluation Program (PRUEP)," NUREG/CR5305, SAND90-2765, 3 volumes. The reports are organized as follows:

NUREG/CR-5305 - Volume 1: Main Report 


\section{NUREG/CR-5305 - Volume 2: Appendices A-G \\ NUREG/CR-5305 - Volume 3: MELCOR Code Calculations}

Important associated reports have been issued by the RMIEP Methods Development Program in: NUREG/CR-4834, Recovery Actions in PRA for the Risk Methods Integration and Evaluation Program (RMIEP); NUREG/CR-4835, Comparison and Application of Quantitative Human Reliability Analysis Methods for the Risk Methods Ir'egration and Evaluation Program (RMIEP); NUREG/CR-4836, Approaches to Uncertainty Analysis in Probabilistic Risk Assessment; NUREG/CR-4837, A Screening Approach to Dependent Failure Analysis; NUREG/CR-4838, Microcomputer Applications and Modifications to the Modular Fault Trees; and NUREG/CR4840, Procedures for the External Event Core Damage Frequency Analysis for NUREG-1150.

Some of the computer codes, expert judgment elicitations, and other supporting information used in this analysis are documented in associated reports, including: NUREG/CR-4586, User's Guide for a Personal-Computer Based Nuclear Power Plant Fire Data Base; NUREG/CR-4598, A User's Guide for the Top Event Matrix Analysis Code (TEMAC); NUREG/CR-5032, Modeling Time to Recovery and Initiating Event Frequency for Loss of Off-Site Power Incidents at Nuclear Power Plants; NUREG/CR-5088, Fire Risk Scoping Study: Investigation of Nuclear Power Plant Fire Risk, Including Previously Unaddressed Issues; NUREG/CR-5174, A Reference Manual for the Event Progression Analysis Code (EVNTRE); NUREG/CR-5253, PARTITION: A Program for Defining the Source Term/Consequence Analysis Interface in the NUREG-1150 Probabilistic Risk Assessments, User's Guide; NUREG/CR5262, PRAMIS: Probabilistic Risk Assessment Model Integration System, User's Guide; NUREG/CR-5331, MELCOR Analysis for Accident Progression Issues; NUREG/CR-5346, Assessment of the XXSOR Codes; NUREG/CR-5380, A User's Manual for the Postprocessing Program PSTEVNT; NUREG/CR-5444, Incorporation of Uncertainties into Reactor Accident Source Term Estimates; and NUREG/CR-5445, User's Guide for the RELTRAC Reactor Accident Source Term Model. In addition the reader is directed to the NUREG-1150 technical support reports in NUREG/CR-4550 and 4551.

Arthur C. Payne, Jr.

Principal Investigator

Phenomenology and Risk Uncertainty Evaluation Program and

Risk Methods Integration and Evaluation Program Division

6412, Reactor Systems Safety Analysis

Sandia National Laboratories

Albuquerque, New Mexico 87185 


\section{Acknowledgments}

This report is the final product of the LaSalle Seismic Risk Analysis Project. A team approach accomplished the objectives of the project in an efficient manner and provided the best technical product possible using the analysis assumptions. The team consisted of a core of LLNL personnel and selected consultants.

We would like to thank G. E. Cummings, former Program Manager of the Nuclear Systems Safety Program at LLNL for his helpful, critical review of this report. I would also like to thank Howard Lambert, a consultant to LLNL, for his review of the event and fault trees used and for assisting in the development of the initiating events.

The manuscript of this report was prepared and edited by Chris Peaslee and Jan Brosius. Their diligence, patience, and dedication are deeply appreciated. Finally, we wish to thank the NRC Project Manager, R. M. Kenneally, for his support and guidance. 


\section{Executive Summary}

\section{Background}

Lawrence Livermore National Laboratory (LLNL) developed a detailed seismic probabilistic risk assessment (PRA) method for nuclear power plants under the Seismic Safety Margins Research Program (SSMRP) sponsored by the U.S. Nuclear Regulatory Commission (USNRC.). Subsequent to the development of this detailed seismic PRA, a simplified methodology was developed to reduce the costs while still providing adequate results. This simplified seismic risk assessment methodology was demonstrated by its application to a pressurized water reactor (PWR) plant - the Zion Nuclear Power Plant. In order to further demonstrate its applicability, and, if possible, to provide a basis for comparing the seismic risk of PWRs and boiling water reactors (BWRs), this simplified seismic risk assessment methodology was also applied to a BWR plant the LaSalle County Station nuclear power plant. This report describes the methodology used and the results obtained for the LaSalle BWR plant.

\section{Methodology Overview}

Seismic risk analysis can be considered in five steps: seismic hazard characterization (seismic hazard curve, frequency characteristics of the motion); seismic response of structures and components; structure and component failure descriptions; plant logic models (fault trees and event trees); and probabilistic risk quantification.

Key elements of the LaSalle simplified seismic risk analysis are to:

1. Develop the seismic hazard at the LaSalle site including the effect of local site conditions.

2. Compare best estimate seismic response of structures, components, and piping systems with design values for the purposes of specifying medien responses in the seismic risk calculations.

3. Develop building and component fragilities for important structures and components.

4. Investigate the effects of hydrodynamic loads on seismic risk.

5. Develop the systems models (e.g., event and fault trees).

6. Estimate the seismically induced core damage frequency. 


\section{LaSalle County Station}

LaSalle County Station is located in the agricultural area of Brookfield Township, LaSalle County, Illinois. It is approximately 55 direct-line miles southwest of Chicago and 20 miles west of the Dresden Power Station. The station utilizes two single-cycle forced-circulation boiling water reactors, each rated at $3293 \mathrm{MWt}$ and designed for $3434 \mathrm{MWt}$. The gross electric output of each unit is $1122 \mathrm{MWe}$; the net output is $1078 \mathrm{MWe}$ from each General Electric (GE) turbine-generator. The nuclear steam supply system (NSSS) supplier was GE (Nuclear Energy Division). LaSalle Unit-2 was analyzed for this study.

The containment design employs the BWR Mark 11 concept of over-under pressure suppression with multiple downcomers connecting the reactor drywell to the water-filled pressure suppression chamber. The primary containment is a steel-lined, post-tensioned, concrete enclosure, housing the reactor and the suppression pool. This primary containment is entirely enclosed in the reinforced concrete reactor building which is the secondary containment structure The power generation complex includes several contiguous buildings: two reactor buildings, an auxiliary building (housing the control room), the turbine building, diesel-generator buildings, the radwaste building, the service building, and the off-gas building.

\section{Seismic Input}

The methodology used to develop the hazard curves (the probability of exceeding any given level of peak ground acceleration, PGA) is mostly based on the methodology and data given in the NRC sponsored Eastern United States Seismic Hazard Characterization study. Descriptions of the zonation, seismicity, and choice of ground motion model are based on the opinions of eleven seismicity experts and five ground motion experts. These expert opinions were used to supplement the available data. One of the important features of the methodology is to identity and assess the uncertainty in all the parameters of the analysis, and propagate that uncertainty using a Monte Carlo simulation approach. The parameters used in the simulations are described by probability distributions. The zonation maps and the ground motion models have discrete probability distributions. All other variables have continuous probability distributions.

The one major departure from the methodology described in the Eastern United States Seismic Hazard Characterization study was in the way local site conditions were taken into account. In the Eastern United States Seismic Hazard Characterization study, three approaches are used to incorporate the local site effect. The first approach is to make no correction for site type. The second approach is to use only a simple soil or rock classification for a site and apply a simple constant correction factor for each site category. The third approach is to apply a set of generic site correction factors to the category of a site. The generic site correction factors were obtained with 1-D wave propagation analysis. Eight site categories were developed based on the available site parameters of eastern U.S. power plant sites. The ground motion panel experts then provided weights for the three approaches. A fourth approach, similar to the third approach but limited only to the range of soil conditions at the site under analysis, was discussed in Ref. 2.1. This fourth approach was used for the LaSalle site and is similar to the approach used to develop the time histories for the Zion site. ${ }^{[1.3]}$ 
In this study, the time histories of motion used in the structural analysis for the LaSalle structures were developed in the following manner. First, hazard curves were developed at a hypothetical rock outcrop at the LaSalle site using the approach discussed above and based on the models given in the Easiern United States Seismic Hazard Characterization study. Secondly, strictly speaking, a set of event-specific spectra should have been developed for the LaSalle site. However, in keeping with the simplified methods approach, we used the two sets of rock outcrop time histories developed for the Zion site based on the argument that the LaSalle site is reasonably close to the Zion site; thus, the distribution of earthquakes contributing to the hazard would be similar for the two sites. Finally, to correct for the local soil conditions, these rock outcrop time histories were then propagated through the LaSalle soil column to get the time histories used in the SSI analysis. It should be noted that these time histories have the appropriate durations for distribution of earthquakes occurring near the LaSalle site. The earthquake durations vary from 5 to 18 seconds.

Monte Carlo numerical simulation techniques were applied to account for uncertainty. A total of 2750 simulations were used. Each simulation generates one hazard curve. The site specific values for the LaSalle site generally show a deamplification at the $0.6 \mathrm{~g}$ PGA rock outcrop level. This is because the soil column at the LaSalle site is very soft and exhibits a large nonlinear effect at the higher PGA levels leading to a deamplification of the computed PGA.

\section{Seismic Response of Structures and Components}

For each level of earthquake described by the seismic hazard curve, three aspects of seismic response are necessary to perform the seismic risk analysis: median level (or best estimate) response, variability of response, and correlation of responses. Seismic responses are required for all structures and components contained in the plant logic models. These responses, together with fragilities, allow for the calculation of seismically induced failure probabilities. The three aspects of seismic response are discussed below.

- Median level Response. The median level response given an earthquake occurrence is used. In general, this median level response differs from the design values because, in the latter case, design analysis procedures, parameter selection, and qualification procedures are conservatively biased.

- Variability of Response. Variability in seismic response resulting from variations in the earthquake excitation, the physical properties of the soil structure/piping system, and our ability to model them must be acknowledged and included in the seismic risk analysis to permit calculation of the component failure probability and core damage frequency.

- Correlation of Response. Tendency for pairs of responses to have simultaneously high or low values results from two sources--the level of the earthquake and the dynamic characteristics of the system. The level of the earthquake affects correlation since a large earthquake (large peak acceleration) may cause all responses to be large, whereas a small earthquake tends to produce small responses. The second source of correlation is due to system response itself. For example, floors within a structure may all experience high values of response simultaneously due to the dynamic characteristics of the structure itself. 
Three approaches to developing median level responses are possible: recalculation using bestestimate methods and parameters such as was done in the SSMRP; scaling of design responses to account for conservatisms introduced in their development; and a combination of the two preceding approaches, i.e., a limited amount of recalculation of the response using best-estimate methods and parameters, and the use of scale factors applied to the design responses. The latter approach was applied in the LaSalle seismic risk assessment.

The basic strategy for developing median level responses was to perform selected probabilistic response analyses of the LaSalle structures for two ranges of earthquakes--a lower level earthquake and a higher level earthquake; henceforth, called acceleration range 1 and 2, respectively. Two acceleration levels were considered in order to permit interpolation for other earthquakes of different peak accelerations. Results of the analyses gave probability distributions for two types of response: in-structure forces and moments to be used in the fragility evaluation of the structures themselves and in-structure response spectra at equipment and component locations for their fragility evaluation. Each element of the probabilistic response analysis is discussed below.

- Seismic Hazard. The seismic hazard for the LaSalle site is specified on a rock outcrop due to the presence of a shallow soil layer (approximately $170 \mathrm{ft}$.) overlying the stiff bedrock. Local site amplification was taken into account explicitly as described below. Specifying the seismic hazard for the purpose of the seismic risk assessment entailed specifying the hazard curve (i.e., the probability of occurrence of an earthquake of given peak ground acceleration) and the frequency characteristics of the motion (i.e., an ensemble of acceleration time histories on the rock outcrop).

- Local Site Amplification. Using equivalent linear viscoelastic soil properties developed as a function of earthquake excitation level and the assumption of vertically propagating waves, earthquake motions on the soil surface were developed for response prediction. This representation of local site amplification is a source of modeling uncertainty.

- Soil-Structure Interaction (SSI) Parameters. The soil configuration and low-strain soil properties were established based on the boring logs and soil response for the LaSalle site. Nominal soil properties as a function of excitation level were estimated from a series of SHAKE analyses using rock outcrop motion as input and material property variations vs. strain relations developed by the geotechnical engineer for the outlay. SSI parameters (foundation impedances and scattering matrices) were developed for the important LaSalle buildings and the CLASSI programs. The LaSalle structure analyzed in detail is a single complex structure which contains the reactor building, the auxiliary building, the turbine building, the off-gas filter building, the service building, and the diesel generator buildings. An average embedment of approximately $51 \mathrm{ft}$. was considered when developing the SSI parameters.

- Structure Model. Structure models developed by the utility and used in the design seismic analysis were used in the probabilistic response analyses. Fixedbase eigensystems for the horizontal and vertical models were developed by the utility for use in the analyses. The SMACS methodology of the SSMRP was used here. SSI is treated by the substructure approach. 
- Response Analyses. SMACS analyses were performed on the LaSalle structure complex including the effects of SSI. SMACS links together seismic input, SSI, structure response, and piping system and component response. All aspects except piping system responses, which were calibrated by using design values, were calculated. Component response was determined from in-structure response spectra. Variability is treated in SMACS. For the LaSalle SMACS analyses, variability due to inherent randomness only was treated. Forty-eight in-structure response spectra were calculated for equipment and component failure assessment. One hundred forty five structure forces and moments were calculated for structure failure assessment.

Median responses were calculated directly for the two acceleration ranges or scaled from the design results based on scale factors developed here or in previous studies. Variability and correlation of responses were assigned based on previous studies and recorded data.

Median in-structure response spectra provided the response input for the probabilistic failure calculations of components. In some instances, the LaSalle SSE design response spectra were overplotted with these in-structure spectra for comparison purposes. One difficulty in interpreting the comparisons of median response spectra of acceleration range 1 and 2 and the design spectra is a lack of correlation of the design free-field ground motion and that of acceleration range 1 and/or 2. The LaSalle seismic design criteria specify the design ground motion at foundation level ( 44 feet below the surface) and no free surface response spectra are available. The LaSalle seismic hazard for this study was defined on a hypothetical rock outcrop and local site amplification was treated explicitly. Hence, no direct comparison of free-field ground motion can easily be made. The median peak ground acceleration (PGA) of acceleration ranges 1 and 2 are approximately $0.2 \mathrm{~g}$ and $0.35 \mathrm{~g}$, respectively. Neither of these correspond to the design PGA of $0.2 \mathrm{~g}$ at foundation level. Frequency content of the free surface motion for this study and the design are likely to be quite different. Recognizing the difficulty in comparing the results, one still observes that the design spectra are generally conservative compared to median response spectra for acceleration range 1 . In some frequency ranges, notably above $2 \mathrm{~Hz}$, they are very conservative. Comparing the design spectra with those of acceleration range 2, the latter often exceed the former at low frequencies, but the design spectra are generally very conservative in the high frequency range.

\section{Structure and Component Fragilities}

The development of structure fragilities proceeded as follows. A review of the seismic design analysis results and development of a preliminary set of structure element capacities initiated the task. Simultaneously, a preliminary SMACS analysis was performed for a single earthquake simulation at near the SSE level to provide a basis of comparison with the design results. Having reviewed the design analysis results and structure model, changes in the structure model which better captured the expected behavior of the structure were recommended and incorporated into the SMACS analysis. Additional preliminary SMACS analyses were performed, loads generated, and an assessment of the model modification made.

The initial model changes led to limited load redistribution and motivated a second set of model changes which were incorporated into the SMACS model and again evaluated. The result was the best-estimate structure model. The one hundred forty five structure forces and moments at two excitation levels were obtained from the SMACS analyses and used in the fragility development. 
A comparison of the capacities of the structural elements with the SMACS generated median loads showed very large ratios for acceleration ranges 1 and 2 which make it highly unlikely that structural failures will occur for any excitation level considered in the hazard curve, i.e., structural element failures have low probabilities of occurrence.

Component fragilities were developed for major LaSalle components identified as important in terms of systems behavior and risk. Examples are items such as: reactor pressure vessel, reactor internals, recirculation pump, diesel generators, pumps (HPCS, RCIC, RHR, SW), batteries, switchgear, SBLC pump and tank, and suction strainers (HPCS, RCIC, LPCS, RHR). LaSalle specific design reports and equipment qualification data were used as the principal basis for fragility assessment.

Median level responses were used in the fragility assessment as generated from the SMACS analyses. A comparison of median response for acceleration ranges 1 and 2 with the capacities of components considered shows large margins. Thus the failure probabilities of these components due to seismic events is quite small.

\section{Hydrodynamic Loads.}

In a seismic risk analysis of a BWR, it is necessary to consider the combined effects of seismic loads and one or more hydrodynamic loads since seismic events can potentially induce hydrodynamic events (Safety Relief Valve (SRV) discharge, pool swell, condensate oscillation, and chugging). For LaSalle, we evaluated which of these loads were likely to occur simultaneously with an earthquake and developed an approach for combination of the seismic and hydrodynamic responses. The approach was applied to the combination of seismic and SRV discharge responses. Based on the results of several case studies, simple combination rules for obtaining the median and the logarithmic standard deviation of the combined responses from the median and logarithmic standard deviation of the individual responses were developed. The seismic risk analysis is performed for the combined seismic and SRV events.

The need for combining hydrodynamic load responses with seismic response depends on the relative magnitudes of the individual responses, the time-phasing of the loads, and the conditional probability of the hydrodynamic load being induced by earthquakes. Based on the screening criteria developed for this study, only certain SRV had responses and, possibly, condensation oscillation load responses need be combined with seismic responses.

The SRV loading was considered in detail. Our efforts focused on the effect of the hydrodynamic loads on equipment function rather than stress related failures of ductile components and structures. It was judged that the high frequency nature on the loads would have negligible effect on stress to failure of ductile components. Hence, we focused on equipment response as characterized by in-structure response spectra. Two sets of information formed the basis for estimating median SRV responses: the in-structure design response spectra and the results of limited in-plant tests. From the in-plant test results, in-structure design response spectra were reduced to an estimated median response level. The logarithmic standard deviation of the SRV loading was estimated to be $\mathbf{0 . 3}$.

The median combined response (seismic and SRV) may be obtained approximately by the SRSS 
The median combined response (seismic and SRV) may be obtained approximately by the SRSS combination of the median maximum responses of the individual values. This rule was evaluated for a number of cases using the time history response due to seismic and that due to SRV. The logarithmic standard deviation of the combined response is expressed as a function of the median and logarithmic standard deviations of the individual maximum responses. These rules were used to combine the seismic responses for each acceleration level of the seismic hazard curve with the SRV responses for the seismic risk analysis. Qualitatively, the effect of SRV loading on the combined seismic and SRV response was small for the components of interest.

\section{Plant Logic Models}

The accident sequences in the LaSalle analysis were identified using event trees. These event trees were developed by the Risk Methodology Integration Evaluation Program (RMIEP). These event trees included the large, medium, and small loss-of-coolant accidents (LOCAs) and loss-of-offsite power (LOSP). The LOSP was assumed to occur as a result of an earthquake in the range being studied.

The initiating event definitions can be considered part of the plant logic model. Because of the potential for seismically relateci failures, the probability of an initiating event occurring during a seismic event is increased over that of an event containing only random failures. All the initiating events defined by the event trees were studied to determine how they were affected by the seismic event. These pipe and component failures were then used to define initiating event minimal cut sets that were necessary for the quantification process.

Fault tree analysis was used to define the failure paths in the accident sequences. These fault trees, as with the event trees, were developed by the RMIEP and modified by LLNL for seismic initiators. The RMIEP developed fault trees for all the major safety systems at LaSalle and included the support systems, such as electric power and component cooling water. Consequently, the trees are quite large, involving thousands of gates and events. The trees became even larger upon modification for seismic events. To make the problem manageable, the fault trees were probabilistically culled to obtain the minimal cut sets.

The system fault tree minimal cut sets were combined according to the event tree logic to give Boolean expressions for each accident sequence. Each accident sequence was culled so that it contained only the most probable cut sets. Again this was done to make the solution and quantification processes manageable.

\section{Seismic Risk Quantification}

The quantification of seismically induced failure probabilities and core damage frequencies are accomplished using the computer program SEISIM. SEISIM computes the failure probabilities taking into account the dependence between basic events. SEISIM does this by computing the multi-normal integral whose integrand is specified by the means, standard deviations, and correlations of responses and fragilities. 


\section{Results}

The earthquake level definitions and their associated frequencies, based on the LaSalle hazard curve, are given in Table 1. Our analysis resulted in a seismically induced core damage frequency point estimate of $6.0 \mathrm{E}-7 / \mathrm{yr}$ for LaSalle (see Table 2). It was found that 89 percent of the risk fell in the first three (of six) earthquake levels with 57 percent of the risk in earthquake level 1 (lowest acceleration). We believe that this dominance by the lower level earthquakes can be traced to our assumption of a loss-of-offsite power during an earthquake. Note that this result is different from the Zion analysis in that the bulk of risk fell into the middle earthquake levels at Zion (earthquake levels 2,3 , and 4 ). It should be further noted that the Zion analysis contained only seismically induced failures (except maintenance and human error) while this analysis of LaSalle contains both random failures and seismically induced failures. This result, combined with the component importance analysis, showed that system failures were dominated by random events. Table 2 shows that 90 percent of the core damage frequency is due to only three accident sequences: LOSP-Trans-1, LOSP-Trans-3, and LOSP-Trans-4. These accident sequences are highlighted in Fig. 1. The most dominant sequence, LOSP-Trans-3, contributes 42 percent to the total core damage frequency.

The dominant components included diesel generator failures (failure to swing, failure to start, failure to run after started) and failure of the condensate storage tank (CST). In this analysis, the assumption was made that failure of the CST would cause failure of both the HPCS and RCIC pumps because of loss of suction.

\section{Additional Analysis}

During the study a second set of event trees and fault trees was generated. These trees were generated independently of the RMIEP event and fault trees. The fault trees in the second set were less detailed in that fewer systems were modeled and the systems modeled were modeled in less detail than the RMIEP fault trees. Two sets of trees were generated so that a comparison might be made between them to indicate whether or not highly detailed fault trees are warranted for a simplified seismic risk analysis. The second analysis resulted in a seismically induced core damage frequency point estimate of $8.4 \mathrm{E}-6 / \mathrm{yr}$. Ninety five percent of the risk fell in the lower three earthquake levels with 70 percent of the risk in earthquake level 1 (lowest acceleration).

These results differ from the other analysis mainly because of modeling differences in the event trees and fault trees. Both analysis did, however, indicate that LOSP accident sequences dominated, random events dominated, and failure of the diesel generators was a large contributor to the overall risk.

With respect to the event trees, there were a couple of important assumptions made that affected the results. In the additional analysis, it was assumed that RHR was required to prevent core damage. For example, the additional analysis Large-LOCA-1 sequence is defined as success of the Reactor Protection System (RPS), success of the Vapor Suppression (VS) system, success of the High Pressure Core Spray (HPCS) System, and failure of the Residual Heat Removal (RHR) System. Because of the failure of RHR, this sequence is assumed to cause core damage. This same sequence is not considered to lead to core damage in the RMIEP analysis. 
Table 1. Seismic occurrence data for the LaSalle site.

\begin{tabular}{ccc}
$\begin{array}{c}\text { Earthquake } \\
\text { Level }\end{array}$ & $\begin{array}{c}\text { Rock Out-Crop } \\
\text { Acceleration Range }\end{array}$ & $\begin{array}{c}\text { Frequency } \\
\text { Per Year }\end{array}$ \\
\hline 1 & $.18-.27$ & $1.1 \mathrm{E}-4$ \\
2 & $.27-.36$ & $2.9 \mathrm{E}-5$ \\
3 & $.36-.46$ & $1.1 \mathrm{E}-5$ \\
4 & $.46-.58$ & $4.7 \mathrm{E}-6$ \\
5 & $.58-.73$ & $2.1 \mathrm{E}-6$ \\
6 & $>.73$ & $1.0 \mathrm{E}-6$
\end{tabular}


Table 2. Unconditional accident sequence probabilities.

Earthquake Level

\section{Accident}

Sequence

Level 1 Level 2 Level $3 \quad$ Level $4 \quad$ Level 5 Level 6 Total

\begin{tabular}{|c|c|c|c|c|c|c|c|}
\hline $\begin{array}{l}\text { Large-LOCA-1 } \\
\text { Large-LOCA-2 } \\
\text { Largc-LOCA-3 } \\
\text { Subtotal }\end{array}$ & $\begin{array}{l}1.8 \mathrm{E}-11 \\
0.0 \mathrm{E}+0 \\
0.0 \mathrm{E}+0 \\
1.8 \mathrm{E}-11\end{array}$ & $\begin{array}{l}8.4 \mathrm{E}-12 \\
0.0 \mathrm{E}+0 \\
0.0 \mathrm{E}+0 \\
8.4 \mathrm{E}-12\end{array}$ & $\begin{array}{l}4.9 \mathrm{E}-12 \\
0.0 \mathrm{E}+0 \\
0.0 \mathrm{E}+0 \\
4.9 \mathrm{E}-12\end{array}$ & $\begin{array}{l}3.0 \mathrm{E}-12 \\
0.0 \mathrm{E}+0 \\
0.0 \mathrm{E}+0 \\
3.0 \mathrm{E}-12\end{array}$ & $\begin{array}{l}1.8 \mathrm{E}-12 \\
0.0 \mathrm{E}+0 \\
0.0 \mathrm{E}+0 \\
1.8 \mathrm{E}-12\end{array}$ & $\begin{array}{l}1.1 \mathrm{E}-12 \\
0.0 \mathrm{E}+0 \\
0.0 \mathrm{E}+0 \\
1.1 \mathrm{E}-12\end{array}$ & $\begin{array}{l}3.8 \mathrm{E}-11 \\
0.0 \mathrm{E}+0 \\
0.0 \mathrm{E}+0 \\
3.8 \mathrm{E}-11\end{array}$ \\
\hline $\begin{array}{l}\text { Medium-LOCA-1 } \\
\text { Medium-LOCA-2 } \\
\text { Medium-LOCA-3 } \\
\text { Medium-LOCA-4 } \\
\text { Subtotal }\end{array}$ & $\begin{array}{l}6.9 \mathrm{E}-10 \\
7.8 \mathrm{E}-10 \\
0.0 \mathrm{E}+0 \\
0.0 \mathrm{E}+0 \\
1.5 \mathrm{E}-9\end{array}$ & $\begin{array}{l}3.1 \mathrm{E}-10 \\
2.2 \mathrm{E}-10 \\
0.0 \mathrm{E}+0 \\
0.0 \mathrm{E}+0 \\
5.3 \mathrm{E}-10\end{array}$ & $\begin{array}{l}1.8 \mathrm{E}-10 \\
9.2 \mathrm{E}-11 \\
0.0 \mathrm{E}+0 \\
0.0 \mathrm{E}+0 \\
2.7 \mathrm{E}-10\end{array}$ & $\begin{array}{l}1.1 \mathrm{E}-10 \\
4.4 \mathrm{E}-11 \\
0.0 \mathrm{E}+0 \\
0.0 \mathrm{E}+0 \\
1.5 \mathrm{E}-10\end{array}$ & $\begin{array}{l}6.9 \mathrm{E}-11 \\
2.2 \mathrm{E}-11 \\
0.0 \mathrm{E}+0 \\
0.0 \mathrm{E}+0 \\
9.1 \mathrm{E}-11\end{array}$ & $\begin{array}{l}4.3 \mathrm{E}-11 \\
1.2 \mathrm{E}-11 \\
0.0 \mathrm{E}+0 \\
0.0 \mathrm{E}+0 \\
4.5 \mathrm{E}-11\end{array}$ & $\begin{array}{l}1.4 \mathrm{E}-9 \\
1.2 \mathrm{E}-9 \\
0.0 \mathrm{E}+0 \\
0.0 \mathrm{E}+0 \\
2.6 \mathrm{E}-9\end{array}$ \\
\hline $\begin{array}{c}\text { Small-LOCA-1 } \\
\text { Small-LOCA-2 } \\
\text { Small-LOCA-3 } \\
\text { Small-LOCA-4 } \\
\text { Small-LOCA-5 } \\
\text { Small-LOCA-6 } \\
\text { Subtotal }\end{array}$ & $\begin{array}{l}4.6 \mathrm{E}-9 \\
0.0 \mathrm{E}+0 \\
1.5 \mathrm{E}-8 \\
1.7 \mathrm{E}-8 \\
0.0 \mathrm{E}+0 \\
0.0 \mathrm{E}+0 \\
3.7 \mathrm{E}-8\end{array}$ & $\begin{array}{l}1.9 \mathrm{E}-9 \\
0.0 \mathrm{E}+0 \\
6.9 \mathrm{E}-9 \\
4.9 \mathrm{E}-9 \\
0.0 \mathrm{E}+0 \\
0.0 \mathrm{E}+0 \\
1.4 \mathrm{E}-8\end{array}$ & $\begin{array}{l}9.6 \mathrm{E}-10 \\
0.0 \mathrm{E}+0 \\
4.0 \mathrm{E}-9 \\
2.0 \mathrm{E}-9 \\
0.0 \mathrm{E}+0 \\
0.0 \mathrm{E}+0 \\
7.0 \mathrm{E}-9\end{array}$ & $\begin{array}{l}4.9 \mathrm{E}-10 \\
0.0 \mathrm{E}+0 \\
2.5 \mathrm{E}-9 \\
9.7 \mathrm{E}-10 \\
0.0 \mathrm{E}+0 \\
0.0 \mathrm{E}+0 \\
4.0 \mathrm{E}-9\end{array}$ & $\begin{array}{l}2.3 \mathrm{E}-10 \\
0.0 \mathrm{E}+0 \\
1.5 \mathrm{E}-9 \\
4.9 \mathrm{E}-10 \\
0.0 \mathrm{E}+0 \\
0.0 \mathrm{E}+0 \\
2.2 \mathrm{E}-9\end{array}$ & $\begin{array}{l}9.0 \mathrm{E}-11 \\
0.0 \mathrm{E}+0 \\
9.4 \mathrm{E}-10 \\
2.6 \mathrm{E}-10 \\
0.0 \mathrm{E}+0 \\
0.0 \mathrm{E}+0 \\
1.3 \mathrm{E}-9\end{array}$ & $\begin{array}{l}8.2 \mathrm{E}-9 \\
0.0 \mathrm{E}+0 \\
3.1 \mathrm{E}-8 \\
2.6 \mathrm{E}-8 \\
0.0 \mathrm{E}+0 \\
0.0 \mathrm{E}+0 \\
6.5 \mathrm{E}-8\end{array}$ \\
\hline $\begin{array}{c}\text { LOSP-Trans-1 } \\
\text { LOSP-Trans-2 } \\
\text { LOSP-Trans-3 } \\
\text { LOSP-Trans-4 } \\
\text { LOSP-Trans-5 } \\
\text { LOSP-Trans-6 } \\
\text { LOSP-Trans-7 } \\
\text { Subtotal }\end{array}$ & $\begin{array}{l}3.7 \mathrm{E}-8 \\
0.0 \mathrm{E}+0 \\
1.2 \mathrm{E}-7 \\
1.4 \mathrm{E}-7 \\
0.0 \mathrm{E}+0 \\
0.0 \mathrm{E}+0 \\
0.0 \mathrm{E}+0 \\
3.0 \mathrm{E}-7\end{array}$ & $\begin{array}{l}1.5 \mathrm{E}-8 \\
0.0 \mathrm{E}+0 \\
5.6 \mathrm{E}-8 \\
4.0 \mathrm{E}-8 \\
0.0 \mathrm{E}+0 \\
0.0 \mathrm{E}+0 \\
0.0 \mathrm{E}+0 \\
1.1 \mathrm{E}-7\end{array}$ & $\begin{array}{l}7.8 \mathrm{E}-9 \\
0.0 \mathrm{E}+0 \\
3.3 \mathrm{E}-8 \\
1.6 \mathrm{E}-8 \\
0.0 \mathrm{E}+0 \\
0.0 \mathrm{E}+0 \\
0.0 \mathrm{E}+0 \\
5.7 \mathrm{E}-8\end{array}$ & $\begin{array}{l}4.0 \mathrm{E}-9 \\
0.0 \mathrm{E}+0 \\
2.0 \mathrm{E}-8 \\
7.9 \mathrm{E}-9 \\
0.0 \mathrm{E}+0 \\
0.0 \mathrm{E}+0 \\
0.0 \mathrm{E}+0 \\
3.2 \mathrm{E}-8\end{array}$ & $\begin{array}{l}1.8 \mathrm{E}-9 \\
0.0 \mathrm{E}+0 \\
1.2 \mathrm{E}-8 \\
3.9 \mathrm{E}-9 \\
0.0 \mathrm{E}+0 \\
0.0 \mathrm{E}+0 \\
0.0 \mathrm{E}+0 \\
1.8 \mathrm{E}-8\end{array}$ & $\begin{array}{l}7.3 \mathrm{E}-10 \\
0.0 \mathrm{E}+0 \\
7.7 \mathrm{E}-9 \\
2.1 \mathrm{E}-9 \\
0.0 \mathrm{E}+0 \\
0.0 \mathrm{E}+0 \\
0.0 \mathrm{E}+0 \\
1.1 \mathrm{E}-8\end{array}$ & $\begin{array}{l}6.7 \mathrm{E}-8 \\
0.0 \mathrm{E}+0 \\
2.5 \mathrm{E}-7 \\
2.1 \mathrm{E}-7 \\
0.0 \mathrm{E}+0 \\
0.0 \mathrm{E}+0 \\
0.0 \mathrm{E}+0 \\
5.3 \mathrm{E}-7\end{array}$ \\
\hline GrandTotal & $3.4 \mathrm{E}-7$ & $1.3 \mathrm{E}-7$ & $6.4 \mathrm{E}-8$ & $3.6 \mathrm{E}-8$ & $2.0 \mathrm{E}-8$ & $1.2 \mathrm{E}-8$ & $6.0 \mathrm{E}-7$ \\
\hline
\end{tabular}

Note: Estimates listed as $0.0 \mathrm{E}+0$ actually represent insignifcant, but finite, values. 


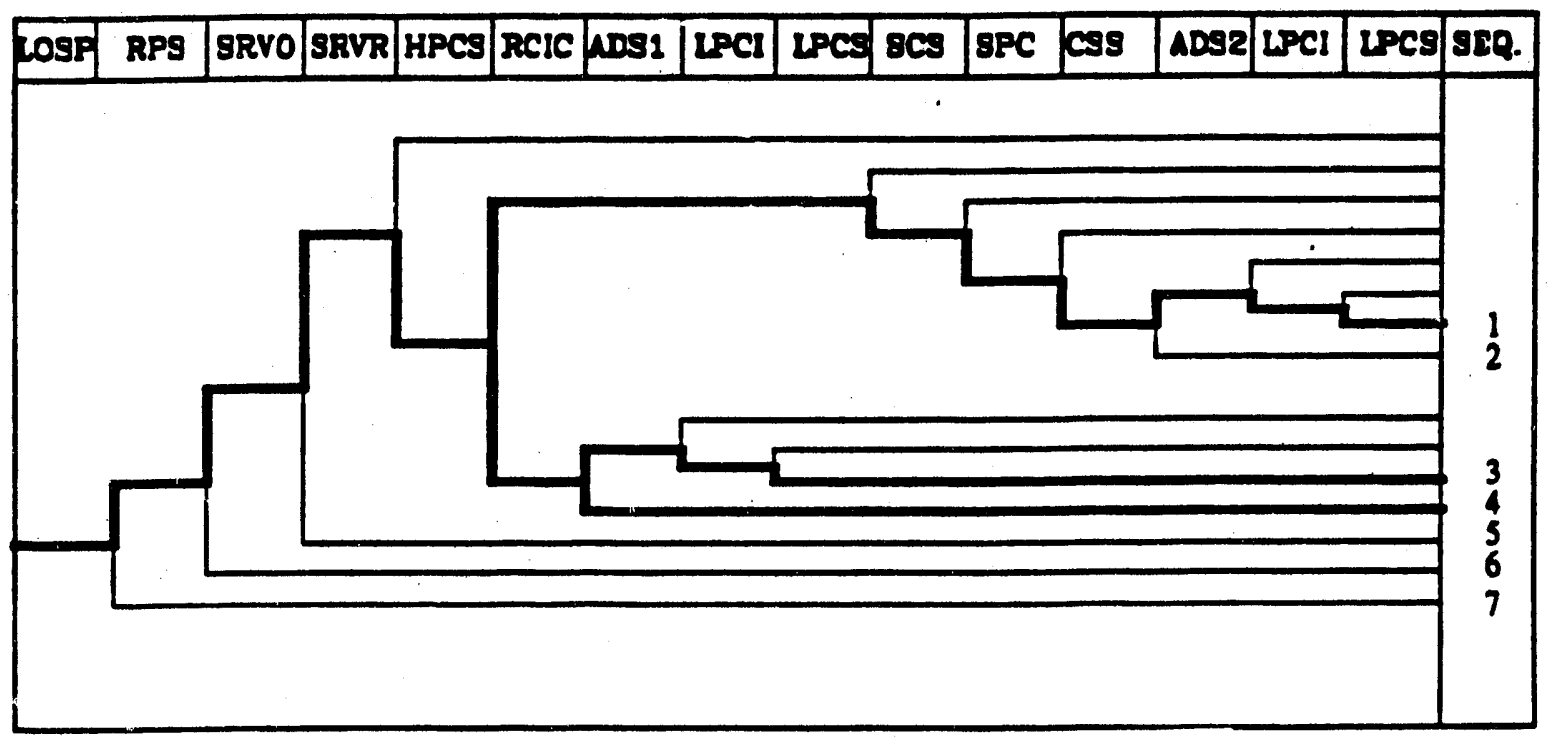

Figure 1. Dominant accident sequences. 
Another difference between the two sets of event trees was in the use of Automatic Depressurization System (ADS). The RMIEP event trees, Small-LOCA and LOSP-Trans, allow for the ADS to be used in the event Suppression Pool Cooling (SPC) and the Containment Spray System (CSS) fail. The second set of event trees do not consider this.

In addition to the event tree differences, there was one fault tree difference that proved to be significant. In the RMEIP analysis, the assumption was made that failure of the CST would cause failure of both the HPCS and RCIC pumps because of loss of suction. This assumption was not made in the other analysis.

Given these differences, it was found that the difference in event tree logic had the biggest effect on the final results rather the degree of detail in the two analyses.

\section{Conclusions}

This study had several goals. These included performing a seismic risk analysis of a BWR, exercise the simplified methodology developed by the SSMRP, and comparing the seismic risks of a BWR and PWR.

As result of our study there are some conclusions which can be drawn.

- The combination of structure and foundation configuration with the LaSalle soil profile led to considerably lower seismic response as compared to Zion.

- Hydrodynamic loads, which may be experienced in BWRs during an earthquake, are not significant at LaSalle.

- Based on the LaSalle and Zion analyses alone, it is difficult to make general statements regarding the relative seismic risk of BWRs vs PWRs.

- The only seismic initiating event of significance at LaSalle is the LOSP, which is assumed to occur. The LOCAs examined were dominated by the random failure of the SRVs to reclose following the LOSP transient.

- Our assumption of LOSP proved to be important. The high probability of random failure of the diesel generators leads to a high probability of station blackout following loss-of-offsite power.

- The only seismically-induced component failure of significance in the analysis is the failure of the condensate storage tank. Random failures dominated. 


\section{Section 1. Introduction}

Under the Seismic Safety Margins Research Program (SSMRP) sponsored by the U.S. Nuclear Regulatory Commission (USNRC), the Lawrence Livermore National Laboratory (LLNL) developed a detailed seismic probabilistic risk assessment (PRA) method for nuclear power plants ${ }^{[1,1]}$. This seismic PRA method consists of five major components: (1) determine seismic hazard, (2) construct plant logic, (3) estimate plant fragility, (4) calculate seismic response, and (5) quantify plant risk. Subsequent to the development of this detailed seismic PRA, a simplified methodology was developed ${ }^{1.2]}$ to reduce the costs while still providing adequate results. The simplification was concentrated on reducing tine effort that is required to determine seismic hazard and to calculate seismic response for structures, systems, and components (SSC) important to safety. This simplified seismic risk assessment methodology was demonstrated by its application to a pressurized water reactor (PWR) plant - the Zion Nuclear Power Plant ${ }^{[1.3]}$. In order to further demonstrate its applicability, and, if possible, to provide a basis for comparing the seismic risk of PWRs and boiling water reactors (BWRs), this simplified seismic risk assessment methodology was also applied to a BWR plant - the LaSalle County Station nuclear power plant. This report describes the methodology used and the results obtained for the LaSalle BWR plant.

An overview of the entire risk assessment process is presented in Sec. 2. Section 3 contains a description of the LaSalle nuclear power plant. Section 4 describes the seismic input requirements to include a description of the methodology used, the hazard curves, and the sensitivity study.

The calculation of seismic responses of buildings, piping, and components is presented in Sec. 5. These calculations were performed using the SMACS computer code. The structures analyzed will be described, SSI and structure models discussed, and the responses given.

The hydrodynamic loads will be discussed in Sec. 6. The approach, description of the hydrodynamic loads, and the resulting responses will be discussed. The statistically based failure relations (fragilities) for the structure are discussed in Sec. 7 and for the equipment in Sec. 8. Each of the major structured elements (shear walls, diagrams, primary containment, and internal structures) will be discussed. The equipment fragility methodology and results will also be discussed.

Section 9 describes the initiating events, event trees, fault trees, and solution process. Section 10 describes the risk quantification process. In this section we will discuss both the data requirements and the quantification process using the SEISIM computer code.

Section 11 of the report presents all the numerical results. We will also be discussing the important accident sequences and components for the analysis. In Sec. 12 we will provide our conclusions. This discussion will include comparison of BWR seismic risk to PWR seismic risk.

The accident sequences used in the analysis will be combined with the internal events analysis accident sequences being developed by the NRC sponsored Risk Methodology Integration Evaluation Program (RMIEP). ${ }^{[1.4]}$ This will allow RMIEP to provide NRC with a more complete analysis. This seismic analysis will also be included in both RMIEP's sensitivity and uncertainty analyses. 


\section{Section 2. Methodology Overview}

\subsection{SSMRP Seismic Probabilistic Risk Assessment}

Our seismic risk analysis can be considered a seven-step process. These seven steps include:

1. Plant and site familiarization

2. Earthquake hazard determination

3. Plant logic modeling

4. Seismic response determination

5. Fragility determination

6. Plant risk quantification

7. Assessment of results and interpretation

Figure 2.1 illustrates the flow of this methodology. What is not shown clearly is the interface between the systems analyst and the fragility analyst and also between the systems analysts and the subsystem, structural, and seismic hazard analysts. Much interaction takes place between these analysts as an interactive process. This interactive process adds to the efficiency in utilizing all of the resources available to the project.

\subsubsection{Plant and Site Familiarization}

Conducting a detailed seismic risk assessment requires integrating diverse sources of information and analysis. The first task is to become familiar with the plant and site. This will lay down the framework for the hazard, structure and subsystem, and plant logic models to be constructed.

The data and drawings of the reactor systems, necessary to construct plant logic models and to become familiar with the plant, should be available through either the plant owner or the internal event PRA team. The other data, which is unique to plant seismic analysis, should be available through the plant owner/operator. It is necessary to walk-through each plant to identify unique plant features and potential weak points of structures, equipment, components, and piping systems; e.g., weak ceilings, clearance between building, etc., to see that no such features are overlooked in the analysis. Site conditions are a feature unique to each seismic risk assessment and must be examined closely.

\subsubsection{Earthquake Hazard}

The purpose of this step is to develop a seismic hazard curve and time histories. The hazard curve is used to quantify the risk and the time histories are used to help benchmark the seismic response of structures and piping systems. 


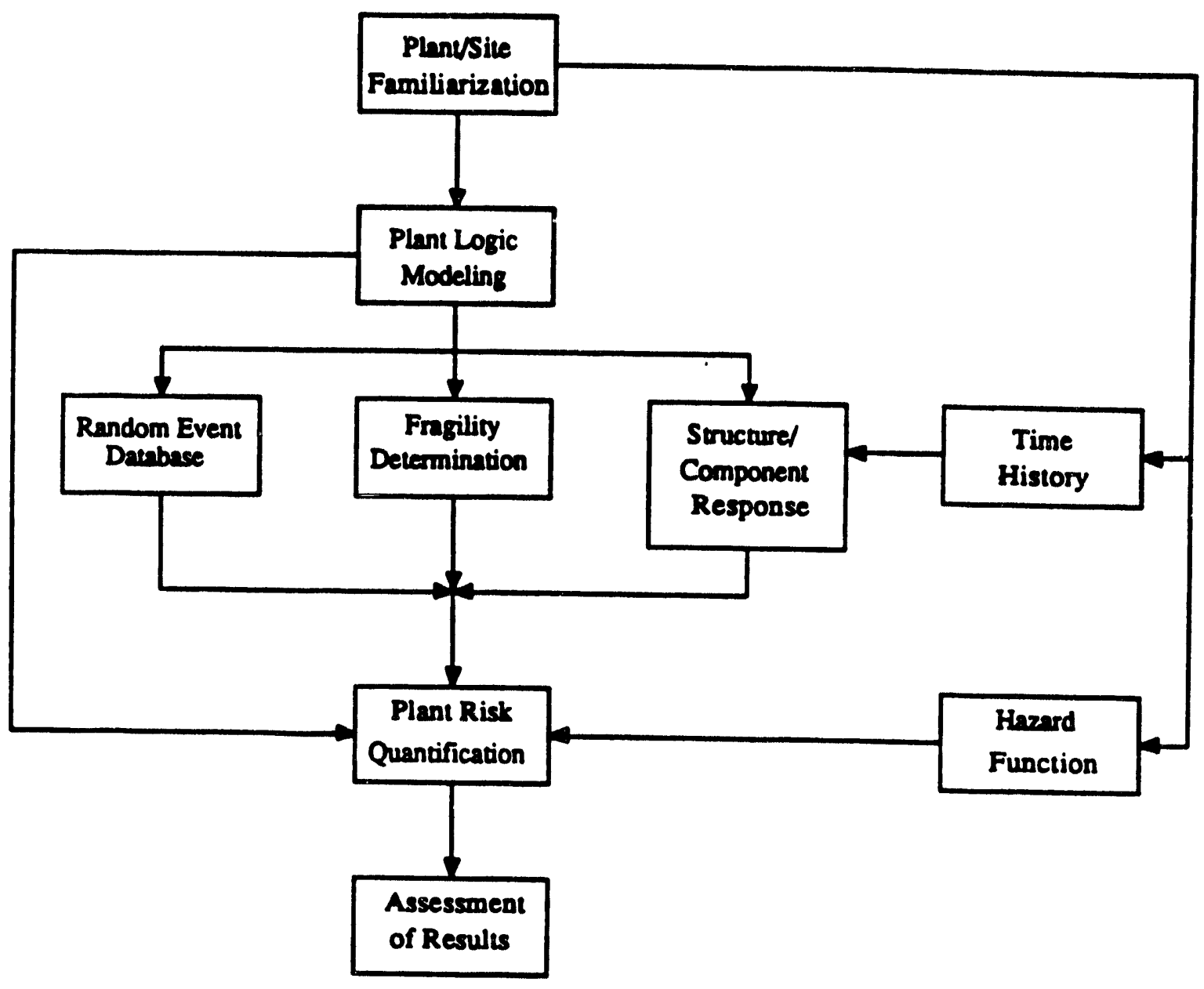

Figure 2.1. Seismic risk analysis process. 


\subsubsection{Plant Logic Models}

The seismically-induced initiating events are generally classified as RVR (Reactor Vessel Rupture or any break that results in a loss-of-coolant at a rate that exceeds mitigation capability), LOCA (loss-of-coolant accident), or transient. If an initiating event occurs, safety systems are required to operate in order to either prevent a core melt or to reduce the severity of its consequences. Event trees are used to identify the possible paths that a reactor could follow during a shutdown. These paths usually involve an accident and a subsequent failure of one or more safety systems and are referred to as accident sequences. A nuclear power plant is a collection of systems. Each system consists of numerous items of equipment.

During the course of a severe earthquake or abnormal conditions, failure of one or more items of equipment could prevent the system from performing its safety function. A risk assessment identifies and quantifies the component or equipment contributing to the failure of the system. The technique used to determine the failure modes for the safety systems is a system fault tree.

Construction of a fault tree begins by identifying the immediate causes of system failure. Then each of these causes is examined for more fund imental causes, until one has constructed a downward branching tree, at the bottom of which are failures not further reducible, i.e., failures of mechanical or electrical components due to all causes, such as structural failure or human error. These lowestorder failures on the fault tree are called basic events.

The fault trees used contain two types of basic events. The first type is the basic event in which failure probabilities are obtained by using response/fragility curves. These basic events are, typically, failures of hardware components. The second type is the random basic event. These consist of failures. such as human errors and other events. These failure probabilities are not related to the level of seismic excitation. Random basic events are treated as independent.

\subsubsection{Seismic Response}

In the plant familiarization task, structures housing the safety systems and equipment, and safety system piping were identified and reviewed. In the hazard determination task, the earthquake time histories were generated. This seismic response task generates probabilistic seismic responses for all structures, equipment, and subsystems identified in the plant logic models.

For each level of earthquake described by the seismic hazard curve, three aspects of seismic response are necessary to perform the seismic risk analysis: best-estimate response, variability of response, and correlation of responses. These seismic responses are required for all structures and components contained in the plant logic models (fault trees and event trees).

\subsubsection{Fragility}

The failure (fragility) of structures or structural elements may occur in one of several possible modes. If the structure provides a pressure boundary, then a failure mode is loss of pressure boundary integrity. Structures whose main purpose is to support subsystems and components fail 
when they no longer provide adequate support. Secondary subsystems and components fail when structural elements collapse. Component failure is defined as either loss of pressure boundary integrity or loss of operability. In all cases, failure is characterized by a cumulative distribution function which describes the probability that failure has occurred given a value of loading. The loading is described by local response quantities such as spectral acceleration, local peak acceleration, or an internal force resultant such as moment, depending on the component and failure mode under consideration, rather than being related directly to the free-field peak acceleration.

\subsubsection{Plant Risk Quantification}

To calculate the accident sequence and core damage frequencies, several items are integrated in this plant seismic safety evaluation. These items are the fault tree and event tree model, hazard curves, seismic response of structures and subsystems, fragilities, and data for random basic event failure probability.

Boolean equations are used to specify the logical failure relationships between structural, piping, and equipment failures within the reactor systems. The common-cause failure of component groups introduced by an earthquake is explicitly included in the computation of the probability of such component failure groups. This unique feature sets the SSMRP methodology apart from other existing seismic risk assessment methods.

The scope of the safety evaluation can consist of, but is not limited to, point value estimates, importance measures analysis, sensitivity studies, uncertainty analysis, and case studies.

\subsubsection{Assessment of Results and Interpretation}

The final task for a seismic PRA is to interpret and document the results produced in the preceding task. For primary objectives, the report should include the frequencies of core damage, and dominant accident sequences contributing to the release. Dominant hardware components and human errors should be documented in the report. Case studies can be used to gain engineering insight to the physical meaning and importance of different assumptions.

\subsection{Simplified Methodology}

As mentioned in the introduction (Section 1), the work presented in this report is a demonstration in the application of the simplified seismic PRA methodology[1.2] to a BWR plant. In this subsection, we will highlight some of the features of this sunplified methodology and indicate how and why that differs from the more detailed method described in Subsection 2.1.

Plant and Site Familiarization. There is no significant differences between the detailed and simplified approaches in the plant and site familiarization area.

Earthquake Hazard. In the detailed analysis, the seismic hazard is characterized in terms of 
seismic hazard curves, ground response spectra, and earthquake time histories. At each acceleration range of interest, typically 30 , but as many as 90 sets of three components of realistic time histories of motion, are specified at the surface of the soil.

In the simplified analysis, the seismic hazard curves are mostly based on the methodology and data given in the NRC sponsored studies of sites in the Eastern United States ${ }^{[2.1,4.1]}$ and comparable industry studies. Western sites would require a site-specific hazard characterization as part of the risk assessment.

The simplified methodology is fundamentally different from the detailed in that the simplified methodology requires spectra but not time histories. Some time histories may be developed as part of the simplified seismic $r^{*} k$ assessment for the purpose of benchmarking the seismic response of structures or piping systems, but this is optional. For the LaSalle study, two sets of rock outcrop time histories were used (one set for each of two earthquake levels) to benchmark the seismic analysis results obtained in design calculations.

Plant Logis: Models. There are no significant differences between the SSMRP detailed and simplified approaches in the development of plant logic models.

Seismic Response. For each level of earthquake described by the seismic hazard curve, three aspects of seismic response are necessary to perform the seismic risk analysis: median or bestestimate response, variability of response, and correlation of responses. In the detailed methodology, the seismic responses are calculated for each level of earthquake and for all three aspects of the responses. To reduce the amount of work, our simplified analysis of LaSalle BWR plant utilizes the simplified methodology documented in Ref. 1.2 and the existing design calculations for the plant as follows:

(1) A limited amount of structural response recalculations was performed using bestestimate methods and parameters. Using the calculated responses, scale factors were obtained so median responses can be scaled from the existing design calculations for LaSalle structures. The recalculations were performed for only two ranges of earthquakes (a lower level earthquake and a higher level earthquake) instead of all earthquake levels. These two levels permitted us to interpolate responses for other earthquake levels.

(2) Differing from the detailed approach, the variabilities and the correlations of seismic response were not based on our calculations described in Item (1) but were taken from the report for simplified method[1.2]. Table 2.1 shows the variabilities of structural responses in terms of random and modeling uncertainty values. The simplified method report specifies correlations of response for four different earthquake levels. We used that information to develop a correlation matrix that was weighted over the earthquake range. The response correlation data are given in Table 2.2.

The seismic response calculation method described in Items (1) and (2) provides a simple yet sufficiently accurate means of obtaining needed seismic response information for LaSalle plant. We recommend that the same approach be applied to the seismic risk assessment of other plants. Please note that there are variabilities in the responses associated with this simplified approach of obtaining response information. However, the variabilities are small compared to the values presented in Table 2.1. In short, Table 2.1 needs not be modified further to reflect the additional 
uncertainties associated with the simplified seismic response calculation method described in Items (1) and (2).

Conservatisms and nonconservatisms are usually introduced in the analysis using various analytical methods (such as SSI analysis using CLASSI and time history method of subsystem seismic analysis using SAP that are incorporated in SMACS code) because of conservative of nonconservative parameters used. It is almost always on the conservative side in the design calculations of a nuclear power plant structure. To yield meaningful PRA results, conservatisms and nonconservatisms need to be identified, quantified, and considered in estimating median response. For the simplified LaSalle and Zion PRAs, median (or best-estimate) responses were obtained by estimating response factors and applying these response factors to the calculated responses. Detailed descriptions of this methodology can be found in Ref. 1.2.

Fragility. There are no significant differences between the SSMRP detailed and the simplified PRA for LaSalle and Zion plants in the fragility area. Fragilities have to be developed for each risk assessment based on the information available at the time. There was a considerable amount of effort in the nuclear power industry to qualify equipment in older nuclear power plants using earthquake experience data 2.2$]$. Since the commercial facilities from which the data were obtained did not have a seismic analysis, the estimates contain a great deal of uncertainty. We have not evaluated the feasibility of using the experience data to improve fragility estimates for the equipment in LaSalle and Zion plants.

Plant Risk Quantification. There are no significant differences between the SSMRP detailed and simplified approaches in this area. Point estimates are useful for sensitivity studies but should be used with caution as an estimator of the mean. The mean should be estimated through uncertainty analysis using recommended values for the modeling uncertainty. This will require a number of SEISIM runs.

Assessment of Results and Interpretation. There are no significant differences between the SSMRP detailed and simplified approaches in the analysis results and interpretation area. 
Table 2.1. Response random and modeling uncertainties.

Random
Uncertainty $\quad \begin{gathered}\text { Modeling } \\ \text { Uncertainty }\end{gathered}$

1. Structure

.28

.27

2. Piping moment

.40

.57

3. Valve acceleration

.34

.43

4. Equipment mounted on building with fundamental frequencies nos near soil structure frequencies

5. Equipment mounted on buildings with fundamental frequencies near soil structure frequencies 
Table 2.2. Response correlation data.

\begin{tabular}{|c|c|c|c|c|}
\hline & $\begin{array}{l}\text { Structure/ } \\
\text { Equipment }\end{array}$ & $\begin{array}{l}\text { Piping } \\
\text { Moment }\end{array}$ & $\begin{array}{l}\text { Valve } \\
\text { Acceleration }\end{array}$ & Electrical \\
\hline $\begin{array}{l}\text { Structures/Equipment } \\
\text { Piping Movement } \\
\text { Valve Acceleration } \\
\text { Electrical }\end{array}$ & 0.91 & $\begin{array}{l}0.10 \\
0.29\end{array}$ & $\begin{array}{l}0.26 \\
0.14 \\
0.76\end{array}$ & $\begin{array}{l}0.40 \\
0.04 \\
0.24 \\
0.99\end{array}$ \\
\hline
\end{tabular}




\section{Section 3. LaSalle County Station}

The plant being analyzed, the LaSalle County Station, ${ }^{(3.1)}$ is located in the agricultural area of Brookfield Township, LaSalle County, Illinois. It is approximately 55 direct-line miles southwest of Chicago and 20 miles west of Dresden power station. The station utilizes two single-cycle forced-circulation boiling water reactors, each rated at $3293 \mathrm{MWt}$ and designed for $3434 \mathrm{MWt}$. The gross electric output of each unit is $1122 \mathrm{MWe}$; the net output is $1078 \mathrm{MWe}$ from each General Electric (GE) turbine-generator. The nuclear steam supply system (NSSS) supplier was GE (Nuclear Energy Division).

The containment design employs the BWR Mark II concept of over-under pressure suppression with multiple downcomers connecting the reactor drywell to the water-filled pressure suppression chamber. The primary containment is a steel-lined, post-tensioned, concrete enclosure, housing the reactor and the suppression pool. This primary containment is entirely enclosed in the reinforced concrete reactor building which is the secondary containment structure. The power generation complex includes several contiguous buildings-two reactor buildings, an auxiliary building (housing the control room), the turbine building, diesel-generator buildings, the radwaste building, the service building, and the off-gas building. 


\section{Section 4. Seismic Input}

To perform a seismic risk assessment at the LaSalle Nuclear Power Station, sets of hazard curves and time histories are required. In this section, we briefly discuss the methodology used to develop the seismic hazard curves for the quantification of risk and acceleration times histories for seismic response analysis. Acceleration time histories were only developed for $0.2 \mathrm{~g}$ and $0.6 \mathrm{~g}$ levels. Hazard curves were developed for two general cases: one for rock outcropping condition at the LaSalle site and another which included the local site effect (using the generic site amplifications appropriate for the LaSalle site). We also extended our analysis to investigate the effect of the variation of magnitude and distance of earthquakes on the hazard curves. Acceleration time histories required for structural response calculations were based on 1-D wave propagation theory and equivalent linear method. The site specific soil properties, including their uncertainty, were used to model the LaSalle site to generate the design acceleration time histories for each level. The uncertainty in soil properties were selected according the a Latin hypercube sampling procedure.

\subsection{Methodology}

The methodology used to develop the hazard curves (the probability of exceeding any given level of peak ground acceleration, PGA) is developed under the EUS Seismic Hazard Characterization project (SHC) sponsored by the USNRC (4.1), and is based on the methodology and data files given in Bernreuter et al.(2.1) The description of the zonation, seismicity and choice of ground motion model are based on expert opinions. We used data from a group of seismicity, experts and ground motion experts. These expert opinions were used to supplement the available data. One of the important features of the developed methodology is to identify and assess the uncertainty in all the parameters of the analysis, and propagate that uncertainty using a Monte Carlo simulation approach. The parameters used in simulations are described by a probability distribution. The zonation maps and the ground motion models have discrete probability distributions. All other variables parameters have continuous probability distributions.

One major departure from the methodology described in Ref. 2.1 was the way site conditions were accounted for. In that study three types of approaches are used to incorporate the local site effect. The first approach is to make no corrections for site type. The second approach is to use only a simple soil or rock classification for a site and apply a simple constant correction factor for each site category. The third approach is to apply a set of generic site correction factors to the category of a site. The generic site correction factors were obtained with 1-D wave propagation analysis. Eight site categories were developed based on the available site parameters of eastern United State power plant sites. The Ground Motion Panel experts then provided weights for the three approaches. A fourth approach, similar to the third approach but limited only to the range of soil conditions at the site under analysis, was discussed in Ref. 2.1. This fourth approach was used for the LaSalle site and is similar to the approach used to develop the time histories for the Zion site. ${ }^{[1.3]}$

The time histories used in the structural analysis for the LaSalle structures were developed in the following manner. We followed the approach used for the Zion site to handle local site effects. Hazard curves were developed at a hypothetical rock outcrop at the LaSalle site using the approach discussed above and based on the models given in Ref. 2.1. Strictly speaking, a set of event specific spectra should have been developed for the LaSalle site. However, in keeping with the 
simplified methods approach we used the two sets of rock outcrop time histories developed for the Zion site based on the fact that the LaSalle site is reasonably close to the Zion site, thus the distribution of earthquakes contributing to the hazard would be similar for the two sites. These rock outcrop time histories are then propagated through the LaSalle soil column to get the time histories used in the SSI analysis.

\subsection{Hazard Curves}

\subsubsection{Rock outcrop}

The hazard curves developed for the LaSalle site are for a hypothetical rock outcrop at the site such that the local soil effect is removed. The hazard curves were developed using five ground motion experts and eleven seismicity experts as discussed in Ref. 2.1. Figure 4.1 shows the best estimate of hazard curve using all experts. Best estimate was based on overall of 55 calculations that the seismic and ground motion parameters were set by each expert. For each seismicity expert, the best estimates of the seismic hazard, combined over five ground motion models, were computed and the results are shown in Fig. 4.2. Significant uncertainty is observed among the eleven seismicity experts. Monte Carlo numerical simulation techniques were applied to account for uncertainty. In the calculation, fifty simulations per ground motion expert were used for each seismicity expert. Thus, a total of 2750 calculation were performed for each acceleration level. Figure 4.3 shows the 15 th, 50 th and 85 th percentiles hazard curves. Figure 4.4 shows the arithmetic mean and geometric mean hazard curves using all experts and simulation. It is noted that the values computed from geometric mean are close to the median values shown in Fig. 4.3.

\subsubsection{Including Generic Site Correction Factor}

For comparison purposes, hazard curves were developed at the soil surface using the geneiic approach outlined in Ref. 2.1. Figure 4.5 shows the best estimate hazard curve, which was computed based on the best estimate parameters defined by each expert. Figure 4.6 shows the best estimate of each seismicity expert combined over five ground motion experts. Figure 4.7 shows the 15 th, 50 th and 85 th percentile hazard curves based on 2750 simulations.

As stated in Section 4.1, the seismic hazard curves used in this PRA were developed under the NRC sponsored EUS Seismic Hazard Characterization Project (SHC). It is important to point out that the seismic hazard curves used in the LaSalle PRA are the preliminary results of the SHC Project. Preliminary results were used due to scheduling consideration. The final curves for LaSalle plant are documented in Ref. 4.1 along with 68 other EUS sites.

To show the difference between the final and the preliminary LaSalle seismic hazard curves, the final 15th, 50th, and 85th percentile seismic hazard curves are plotted over the preliminary curves in Figure 4.7. It is clear that the final LaSalle constant percentile seismic hazard curves (CPHC) generally fall below the corresponding preliminary curves except at high peak ground acceleration values. The exceedance is rather small and is limited to high percentile hazard curves. The impact of the differences on the core damage frequency is discussed in Section 11. 


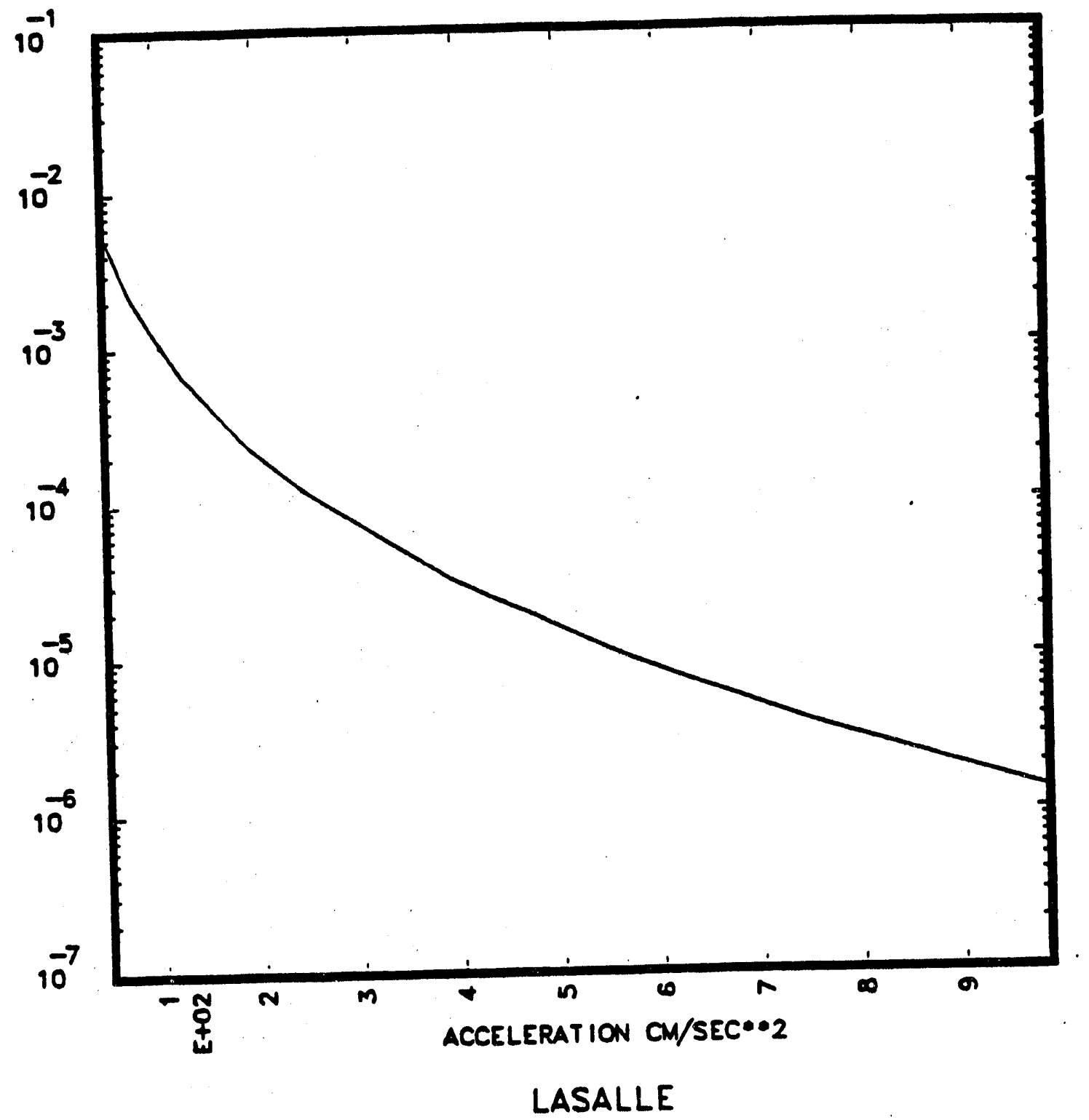

Figure 4.1. Best estimate hazard ensure using all experts. 


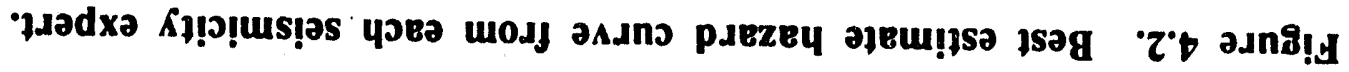

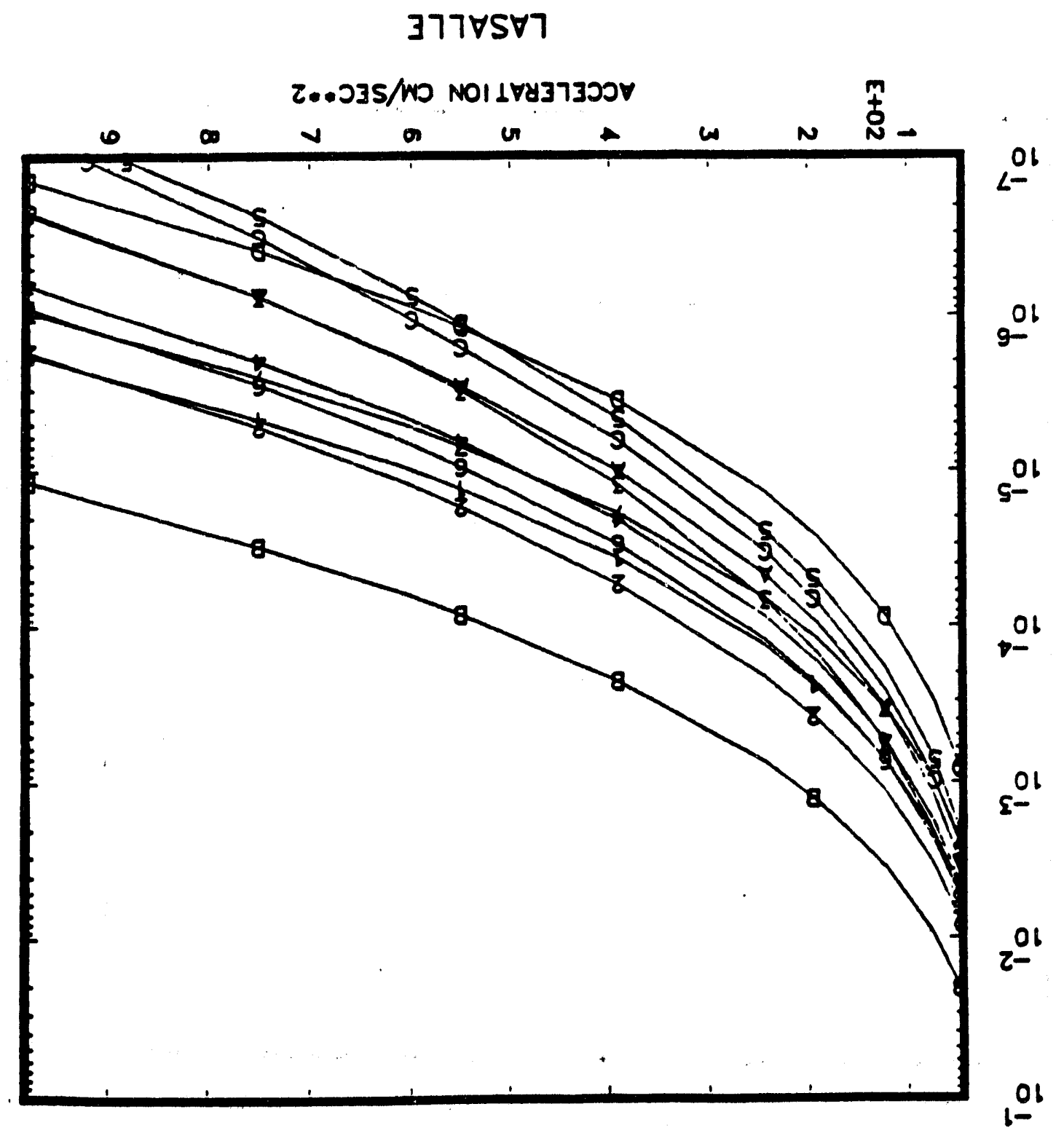




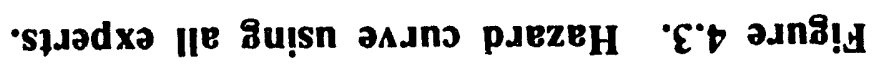

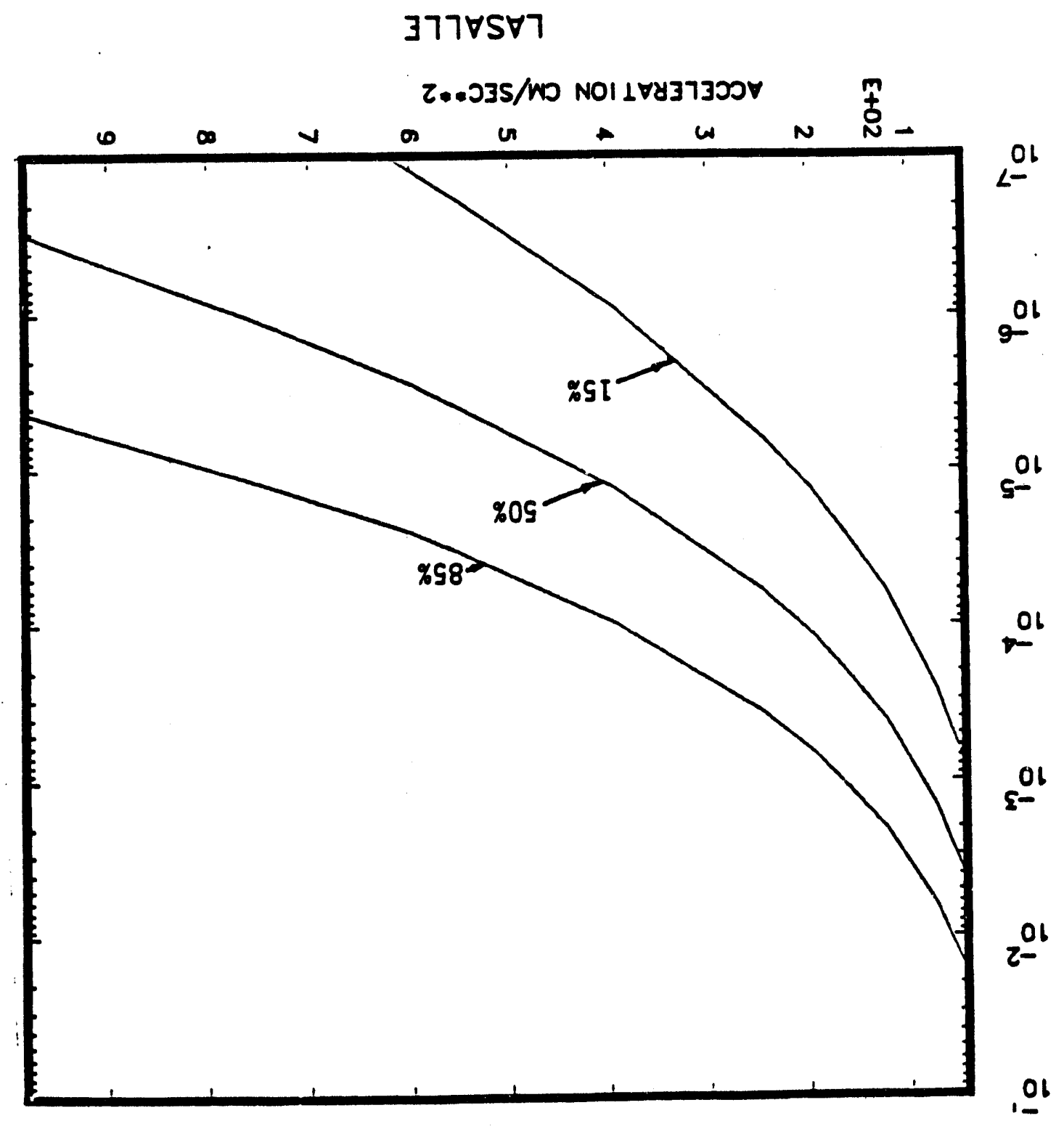




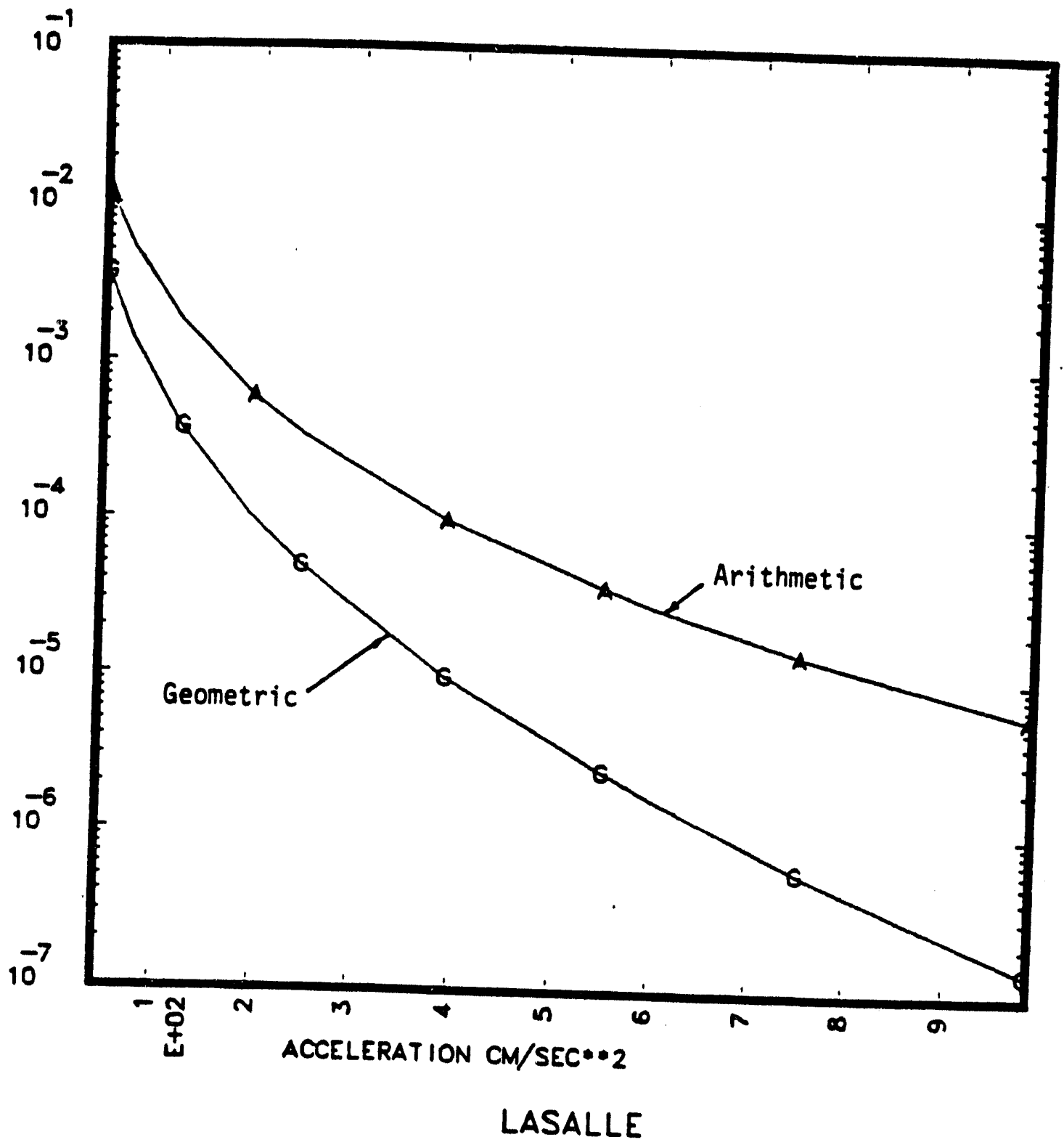

Figure 4.4. Arithmetic and geometric mean hazard curves using all experts. 


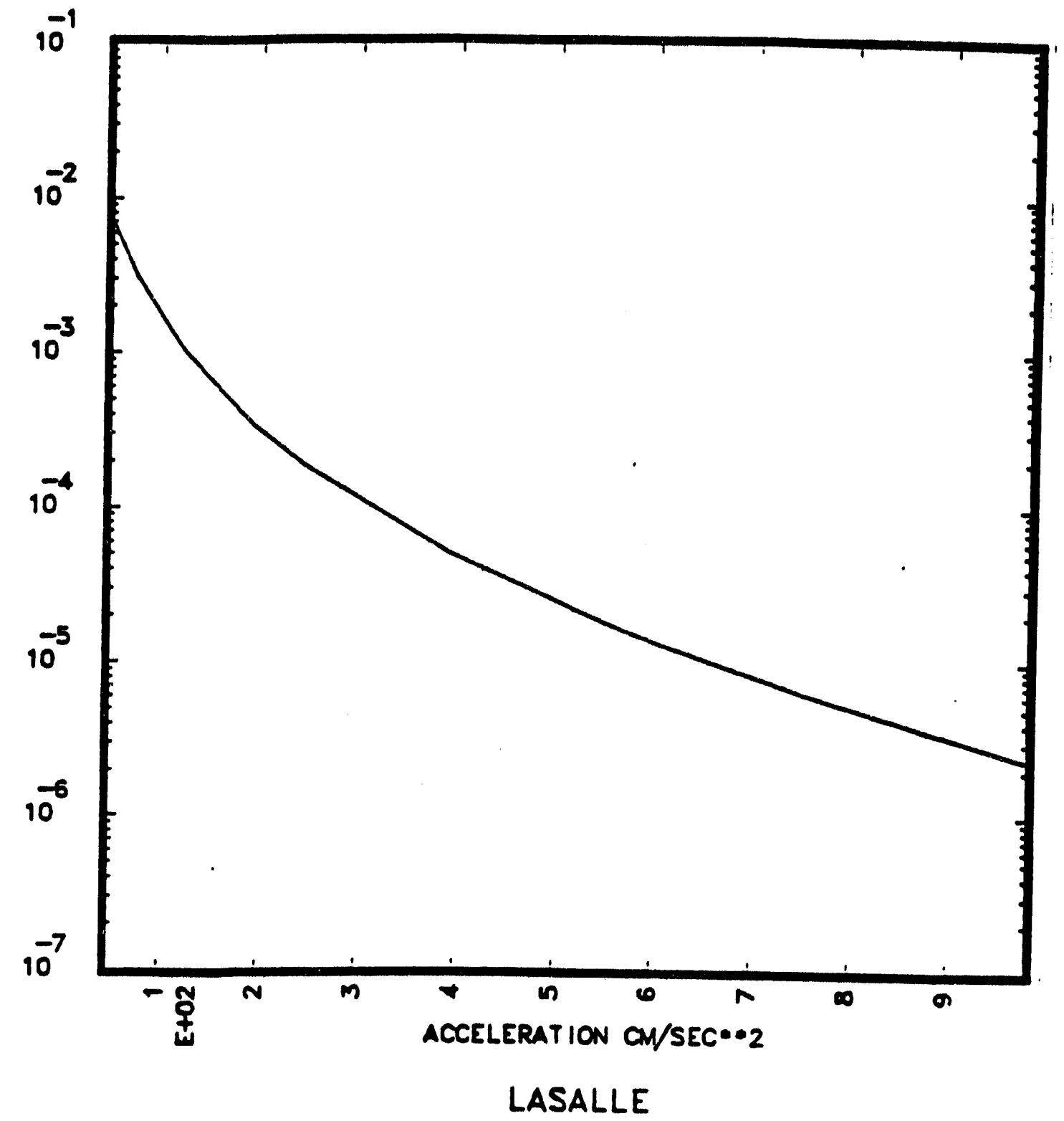

Figure 4.5. Best estimate hazard curve including site correction using all experts. 


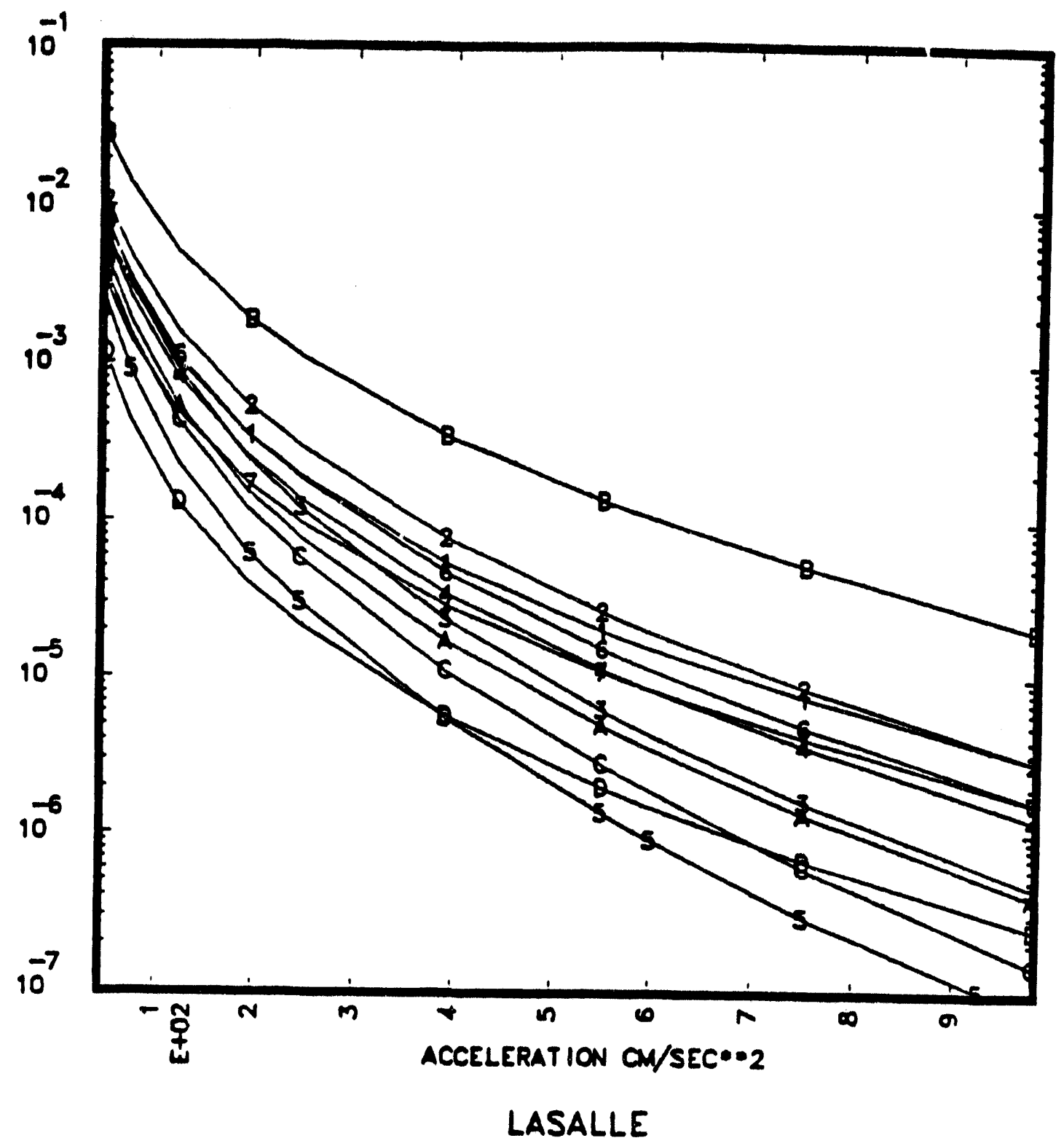

Figure 4.6. Best estimate hazard curve including site correction from each seismicity expert. 


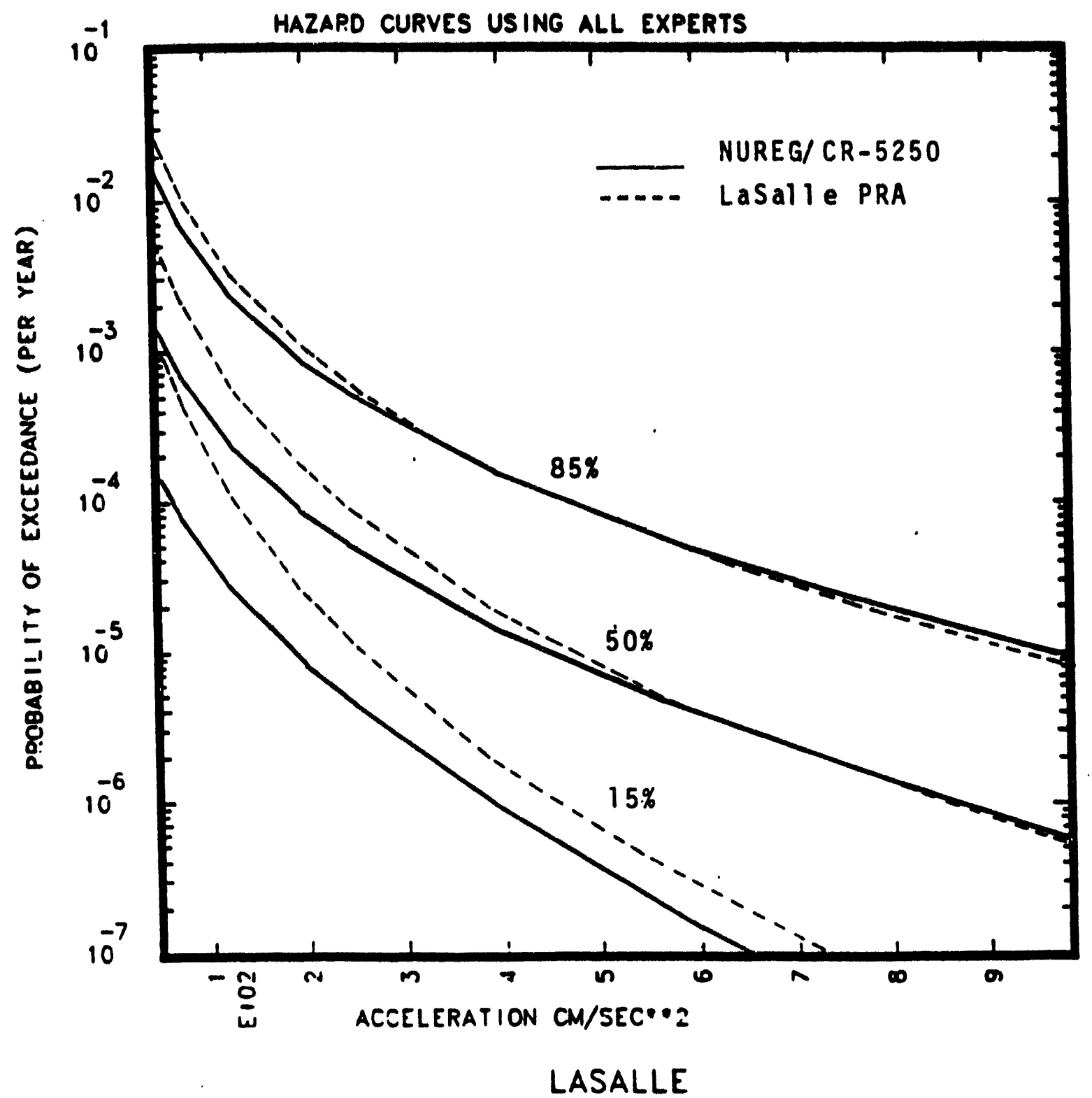

Figure 4.7. Hazard curve including site correction using all experts. 
Figure 4.8 shows the arithmetic and geometric mean of 2750 simulations. The comparison of the best estimate hazard curves between soil and hypothetical rock outcrop at the LaSalle site is shown in Fig. 4.9. Also shown in Fig. 4.9 are the range of the actual computed values of the POA at the top of the soil column at the LaSalle site at the $0.2 \mathrm{~g}$ and $0.6 \mathrm{~g}$ rock outcrop PGA levels. The site specific values for the LaSalle site are seen to be significantly lower than the generic case particularly at the $0.6 \mathrm{~g}$ PGA rock outcrop level. This is because the soil column at the LaSalle site is much softer and exhibits a larger nonlinear effect at the higher PGA levels than was the case for the soil column of the median site used to represent the generic case.

\subsubsection{Seismic Hazard Study by EPRI}

Subsequent to the completion of NRC sponsored work on seismic hazard characterization of EUS sites by LLNL [4.1], Electric Power Research Institute (EPRI) conducted a separate seismic hazard characterization effort of the EUS sites. Experience to date indicates that application of the methodologies included in these two studies can yield significantly different mean results. In 1992 NRC funded LLNL to update the 1989 results by re-elicting the Seismicity Experts to carefully examine the uncertainty bounds they provided. In addition the Ground Motion Experts also were re-elicted and allowed to update their input. This process is still ongoing, but preliminary results have brought the LLNL and EPRI results much closer together. In addition a joint effort has been funded by NRC/DOE/EPRI to examine their issues and make recommendations as to how to update the results from the various studies. Because this effort is still ongoing, updated results have not been used, and the hazard curves used in this report are based on the earlier 1989 results with generic site corrections and also on site-specific soil corrections based on data available from the FSAR for the LaSalle site. The impact on the LaSalle core damage frequencies due to differences in seismic hazard methodologies was not assessed in this report because the DOE Seismic Working Group has not reached a final position at the time this report was published.

\subsection{Sensitivity Study}

We developed the hazard curves with the contribution to the hazard from earthquakes in various magnitude ranges and for various distance ranges in order to improve the specification of the fragility curves.

\subsubsection{Variation of Earthquake Magnitude}

To study the sensitivity of the hazard curve to the change in earthquake magnitude, we defined four ranges of earthquake magnitude (MR): (1) very small--from 3.75 to $4.4 ;$ (2) small--from 4.5 to 5.4 ; (3) medium--from 5.5 to 6.4 ; and (4) large--from 6.5 to 8.5 . We only developed the best estimate of the hazard curve using parameters given by all ground motion and seismicity experts. Figure 4.10 shows the variation of hazard curves resulting from changes in earthquake magnitude. The largest contribution to the hazard is from earthquakes in the 5.5 to 6.4 range. Very small earthquakes and large earthquakes do not contribute significantly to the hazard except at very low

PGA levels. At the SSE level the hazard is equally divided between medium earthquakes (5.5-6.4) and small earthquakes (4.5-5.4).

\subsubsection{Variation of Earthquake Distance}

To Investigate the effect of earthquake distance from the LaSalle site on the hazard curve, we selected three distance ranges for this study. Three distance ranges are: from 0 to $25 \mathrm{~km}$, from 25 


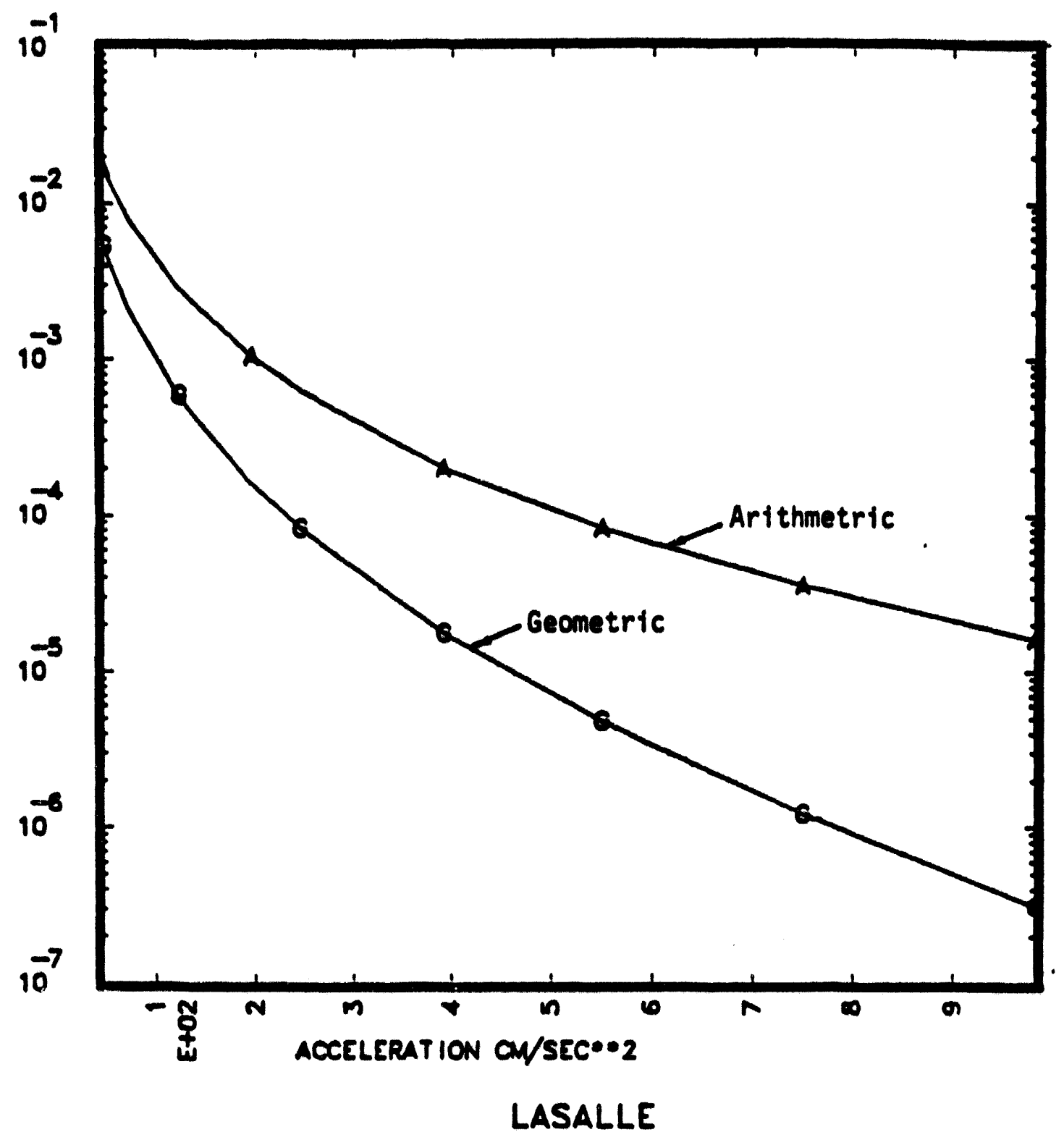

Figure 4.8. Arithmetic and geometric mean hazard curves including site correction using all experts. 


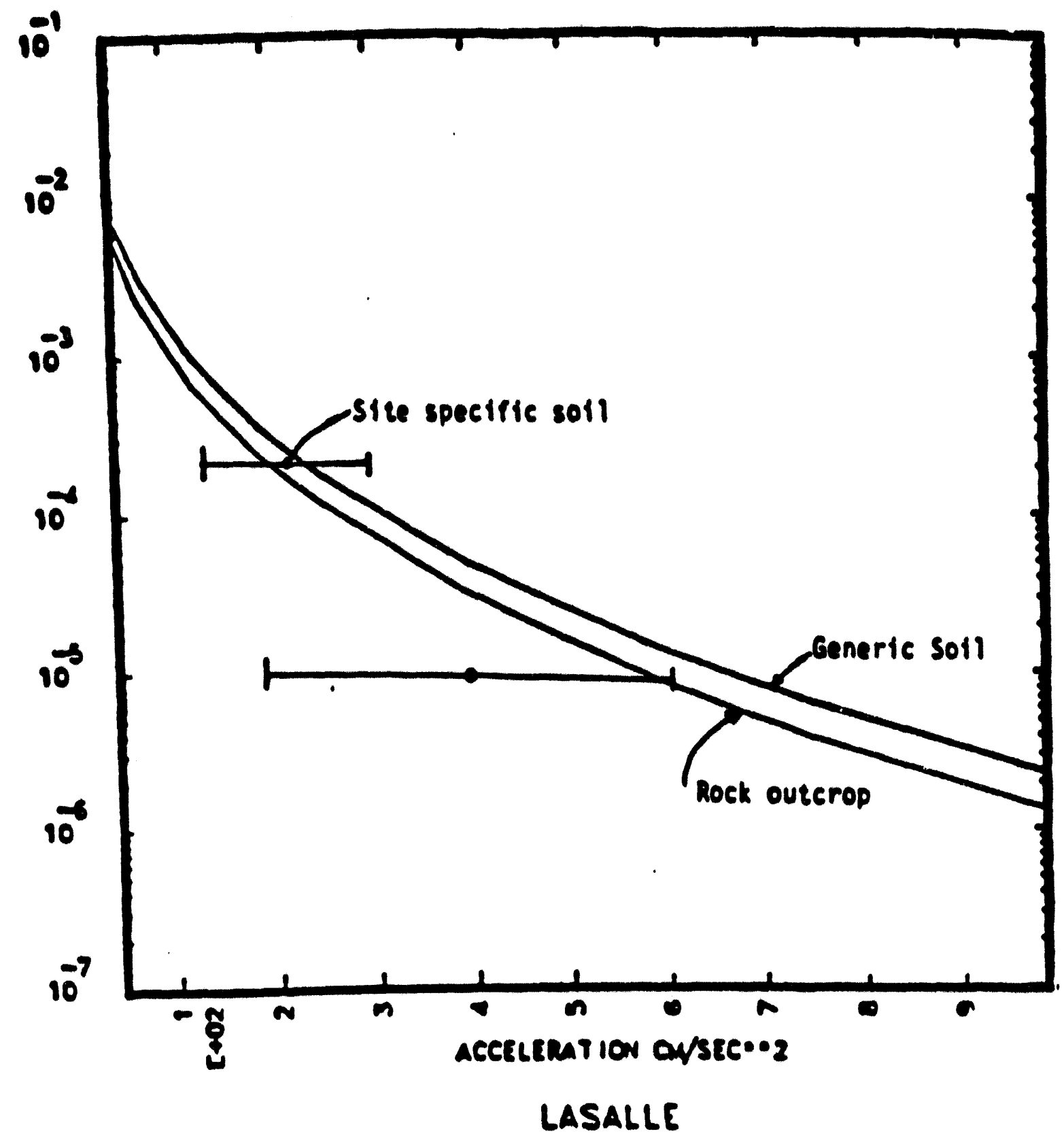

Figure 4.9. Comparison of best estimate hazard curves between soil and hypothetical rock outcrop at the LaSalle site 


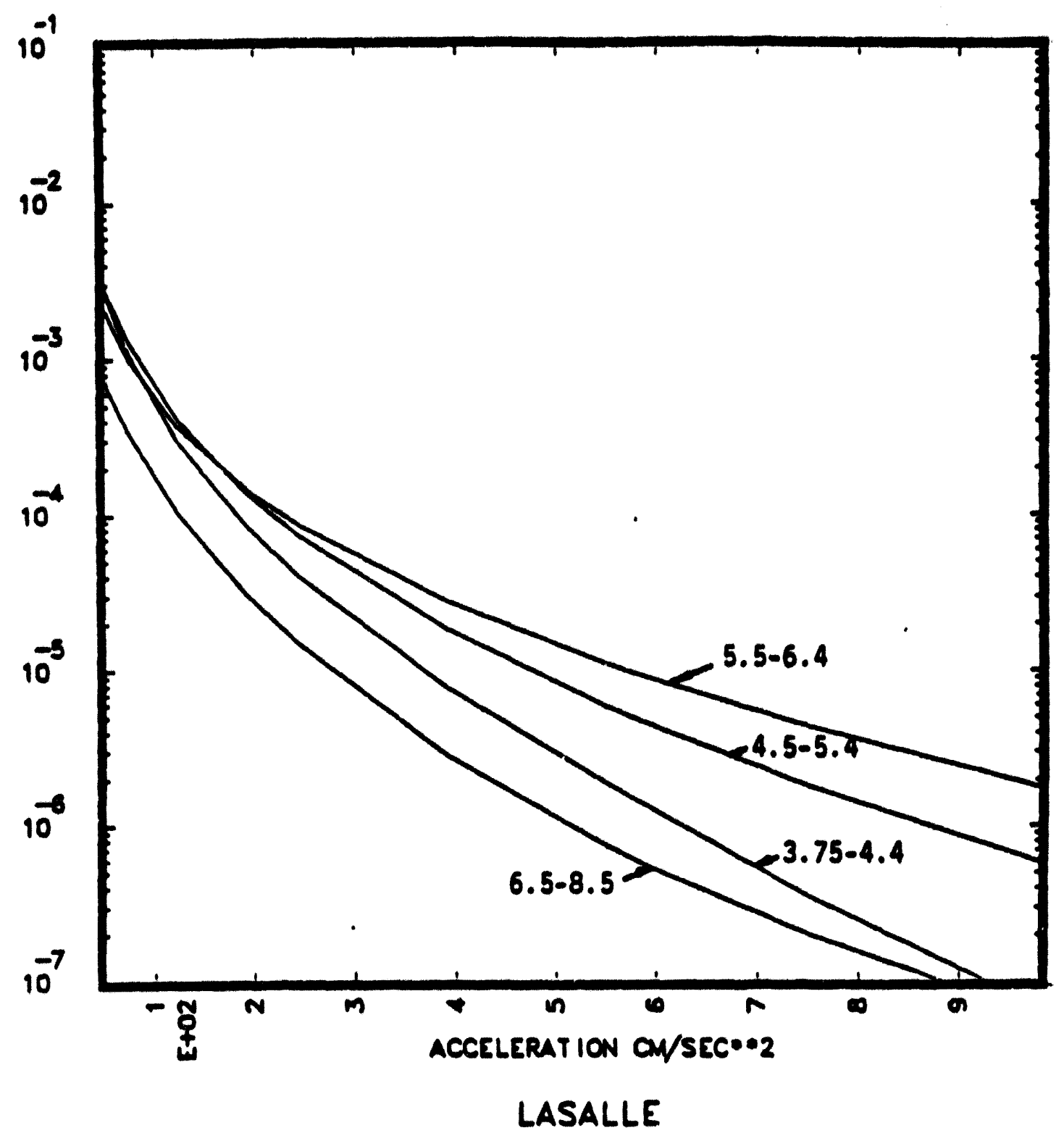

Figure 4.10. Hazard curves for cases when only earthquakes of limited magnitude ranges are considered. 
to $150 \mathrm{~km}$, and from 150 to $500 \mathrm{~km}$. The comparison of hazard curves resulting from the change of these distance Is shown In Fig. 4.11. We see from Fig. 4.11, earthquakes within $25 \mathrm{~km}$ of the LaSalle zone are the most significant contributors to the seismic hazard at the LaSalle site. Below an acceleration of $0.2 \mathrm{~g}$ the contribution from earthquakes having distances between 25 to $150 \mathrm{~km}$ are also important. distant earthquakes (more than $150 \mathrm{~km}$ from the LaSalle site) contribute very little to the seismic hazard at the LaSalle site.

\subsection{Discretization of the Hazard Curve}

The hazard curve was discretized so as to allow for the convolution of the systems analysis results with the seismic hazard curve. In our analyses, the seismic hazard curve was discretized, for calculational purposes, into six intervals of peak horizontal acceleration on the rock outcrop .18$.27, .27-.36, .36-.46, .46-.58, .58-.73$, and $>.73 \mathrm{~g}$. Discretization permits excitation-sensitive parameters to be incorporated into the calculations, and serves as an approximate numerical integration in computing the seismic risk over the entire range of hypothesized earthquake levels. The frequencies of occurrence of earthquakes having peak rock outcrop accelerations in each of the discretized ranges of accelerations on the hazard curve are given in Table 4.1. 


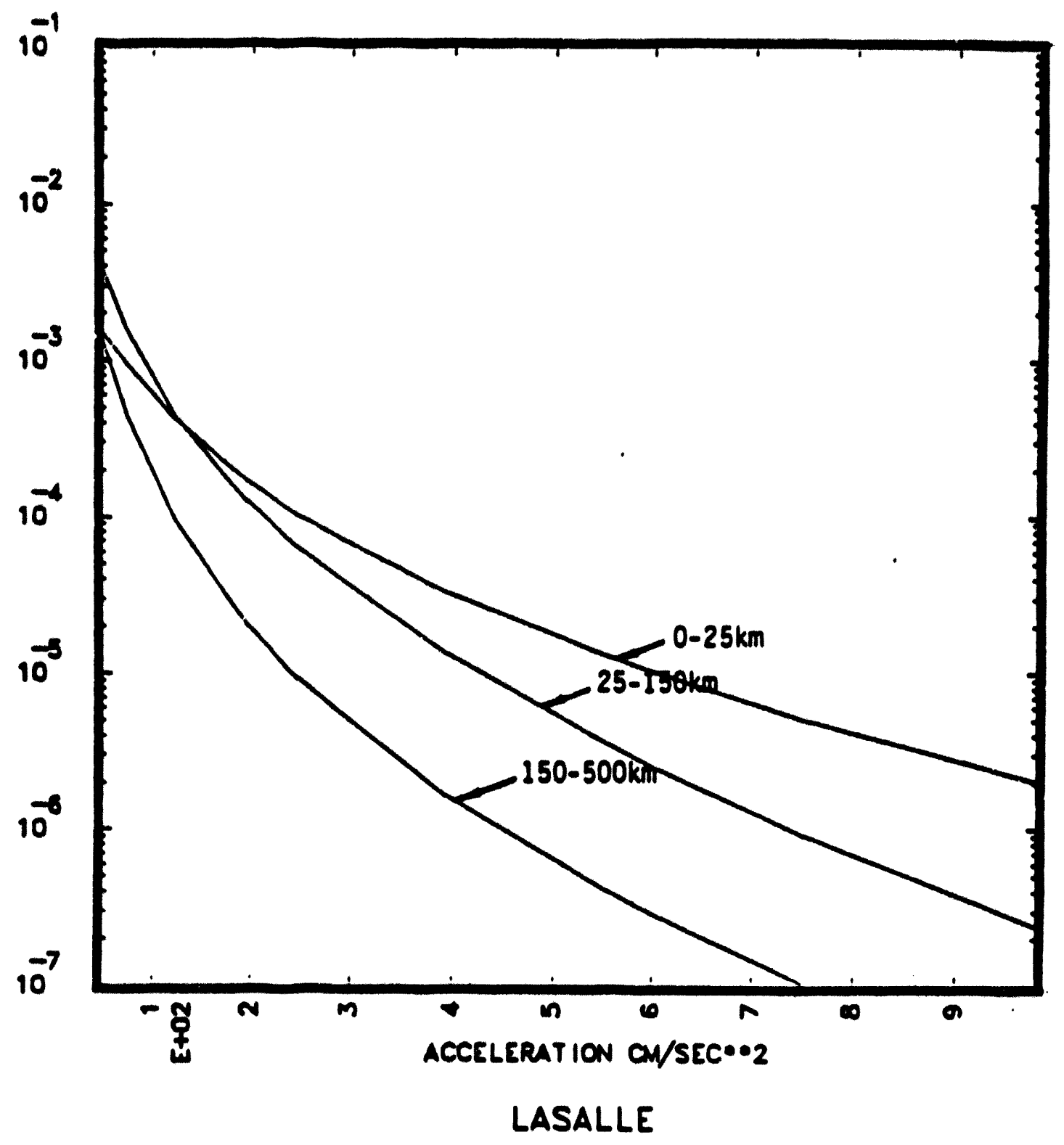

Figure 4.11. Hazard curves for cases when only earthquakes in limited distance ranges are considered. 
Table 4.1. Seismic occurrence data for the LaSalle site.

\begin{tabular}{ccc}
$\begin{array}{c}\text { Earthquake } \\
\text { Level }\end{array}$ & $\begin{array}{c}\text { Rock Out-crop } \\
\text { Acceleration Range }\end{array}$ & $\begin{array}{c}\text { Frequency } \\
\text { Per Year }\end{array}$ \\
\hline 1 & $.18-.27$ & $1.1 \mathrm{E}-4$ \\
2 & $.27-.36$ & $2.9 \mathrm{E}-5$ \\
3 & $.36-.46$ & $1.1 \mathrm{E}-5$ \\
4 & $.46-.58$ & $4.7 \mathrm{E}-6$ \\
5 & $.58-.73$ & $2.1 \mathrm{E}-6$ \\
6 & $>.73$ & $1.0 \mathrm{E}-6$
\end{tabular}




\section{Section 5. Response Analysis}

\subsection{Seismic Response Requirements for a Seismic PRA and Overview}

For each level of earthquake described by the seismic hazard curve, three aspects of seismic response are necessary to perform the seismic risk analysis: median level (or best estimate) response, variability of response, and correlation of responses. Seismic responses are required for all structures and components contained in the plant logic models. The three aspects of seismic response are discussed:

Median Level Response. The median level response, given an earthquake occurrence, is needed. In general, this median level response differs from the design values because, in the latter case, design analysis procedures, parameter selection, and qualification procedures are conservatively biased.

Variability of Response. Variability in seismic response resulting from variations in the earthquake excitation, physical properties of the soil structure/piping system, and our ability to model them must be acknowledged and included in the seismic risk analysis to permit calculation of probability of component failure and core damage frequency.

Correlation of Responses. The tendency for pairs of responses to have simultaneously high or low values results from two sources--the level of the earthquake and the dynamic characteristics of the system. The level of the earthquake affects correlation since a large earthquake (large peak acceleration) may cause all responses to be large, whereas, a small earthquake produces the opposite effect. The second source of correlation is due to system response itself. For example, floors within a structure may all experience high values of response simultaneously due to the dynamic characteristics of the structure itself.

Three approaches to developing median level responses are possible: recalculation using best estimate methodology and parameters such as was done in the SSMRP; use design responses scaled to account for conservatisms introduced in their development; and a combination of the two, i.e., a limited amount of recalculation of response using best estimate methodology and parameters is performed and scale factors developed to be applied to design responses. The latter case is being applied to the LaSalle County Station seismic risk analysis.

The basic strategy for developing median level responses was to perform selected probabilistic response analyses of the LaSalle County Station structures for two ranges of earthquakes--a lower level earthquake and a higher level earthquake. Results of the analyses gave probability distributions on two types of response--in-structure forces and moments to be used in the fragility evaluation of the structures themselves; and in-structure response spectra at equipment and component locations for their fragility evaluation. Two acceleration levels were considered to permit interpolation for other earthquakes of different peak accelerations. Each element of the probabilistic response analyses is discussed: 
- Seismic Hazard. Specifying the seismic hazard for the purpose of the seismic risk analysis entails specifying hazard curves (probability of exceeding any given level of peak ground acceleration including magnitude and distance characteristics), and the frequency characteristics of the motion. As described in Sec. 4, hazard curves were developed at a hypothetical rock outcrop at the LaSalle site. The frequency characteristics of the motion were ensembles of acceleration time histories (three components per earthquake simulation) again specified on a hypothetical rock outcrop. These two elements of the seismic hazard were specified on a hypothetical rock outcrop to permit explicit treatment of local site amplification as described below. The LaSalle site Is composed of approximately $170 \mathrm{ft}$. of soft soil overlying the bedrock and it is expected that this soft soil layer will have an important effect on the free-field ground motion.

- Local Site Amplification. Using equivalent linear viscoelastic soil properties developed as a function of earthquake excitation level and the assumption of vertically propagating waves, earthquake motions on the soil surface were developed for response prediction. This representation of local site amplification is a source of modeling uncertainty.

- Soil-Structure Interaction (SSI) Parameters. The soil configuration and low strain soil properties were established based on the boring logs and soil reports for the LaSalle site. ${ }^{5.11}$ Nominal soil properties as a function of excitation level were estimated from a series of SHAKE analyses using rock outcrop motion as input and the strain-dependent dynamic soil characteristics of the LaSalle site. SSI parameters (foundation impedances and scattering matrices) were developed for the important LaSalle buildings using the CLASSI programs and the nominal soil properties calculated using SHAKE. The LaSalle structure, analyzed in detail, is a single complex which contains the reactor building, the auxiliary building, the turbine building, off-gas filter building, and diesel generator buildings. An average embedment of approximately $51 \mathrm{ft}$. was treated in developing the SSI parameters. Section 5.3 describes the SSI information.

- Structure Model. Structure models developed by Sargent \& Lundy for the utility and used in the design seismic analysis formed the basis for the probabilistic response analyses. Fixed-base eigensystems for the horizontal and vertical models were developed by Sargent \& Lundy for the utility for use in the analyses. Preliminary analyses were performed and the results were reviewed from the perspective of structural fragility. Changes to the structure model were proposed to better capture its expected behavior. The structure was re-analyzed. These initial model changes led to limited load redistribution and motivated a second set of model changes which were incorporated and again evaluated. The result was the best estimate structure model. In all the analyses, SSI was included and treated by the substructure approach. Section 5.3 discusses the structure model.

- Response Analyses. SMACS ${ }^{[5.1]}$ analyses were performed on the LaSalle structure complex including the effects of SSI. SMACS links together seismic input, SSI, structure and subsystem models to generate response of structures, piping systems and components. All aspects except piping system response were 
treated here--component response was determined from in-structure response spectra. Variability is treated in SMACS. Ensembles of three component acceleration time histories define earthquake variability. Variability in SSI and structure response is treated by varying a discrete number of input parameters of the soil and structures (soil shear modulus, soil material damping, structure frequency, and structure damping). SMACS performs repeated deterministic analyses, each analysis simulating an earthquake occurrence. By performing many such analyses and by varying the values of the input parameters, uncertainty in deterministic analyses is taken into account. The discrete input parameters were assigned variability described by $\log$ normal distributions and sampled for each earthquake simulation. The Latin hypercube experimental design was used. For the LaSalle SMACS analyses, random variation in the parameters was considered. Thirty earthquake simulations were treated at each of the two excitation levels. Sections 5.3 and 5.4 describe aspects of the process and the results. Forty-eight in-structure response spectra were calculated for equipment and component fragility assessment. One hundred-and-forty-five structure forces and moments were calculated for the structure element fragility assessment.

\subsection{Description of Site and Structures}

As noted in Sec. 3, LaSalle County Station is located in the agricultural area of Brookfield Township, LaSalle County, Illinois. It is approximately $\mathbf{5 5}$ direct-line miles southwest of Chicago and 20 miles west of Dresden power station. The plant is on flat terrain about 220 feet above the Illinois River channel which traverses north central Illinois some 3-1/2 miles to the north of the site.

The site soil conditions are characterized by approximately 170 feet of predominantly Wisconsinan glacial drift which overlies the bedrock surface. Figure 5.1 is a stratigraphic column showing the subsurface geology.

The power generation complex includes several contiguous buildings--two reactor buildings, an auxiliary building (housing the control room), the turbine building, diesel-generator buildings, the radwaste building, the service building, and the off-gas building. Other buildings such as the gatehouse, warehouses, etc., are also located in the general plant area. A lake screen house on the intake flume is located about 800 feet east of the main building complex. The plant layout of Fig. 5.2 shows the location of the main buildings.

The major vertical and lateral load resisting elements of the complex are reinforced concrete shear walls. The upper stories of the reactor and turbine buildings are braced steel frames. The complex is founded on an integral basemat with thicknesses that vary from 7 feet beneath the reactor building, to 6 ' -4 " beneath the auxiliary-turbine-heater bay buildings, to 5 feet beneath the radwaste building. The basemat profile features a step at the reactor-auxiliary building intersection. The turbine foundations are structurally isolated from the rest of the complex. Figure 5.3 shows a section through the complex. The principal buildings of interest are the reactor, auxiliary and turbine buildings and are described generally below.

The reactor building extends from foundation (top of foundation elevation of 673'-4") to a roof-top elevation of 894'-6". It is of reinforced concrete construction up to the operating floor at elevation $843^{\prime}-6^{\prime \prime}$. The roof over the operating floor is supported on braced steel columns. Two reactor units 


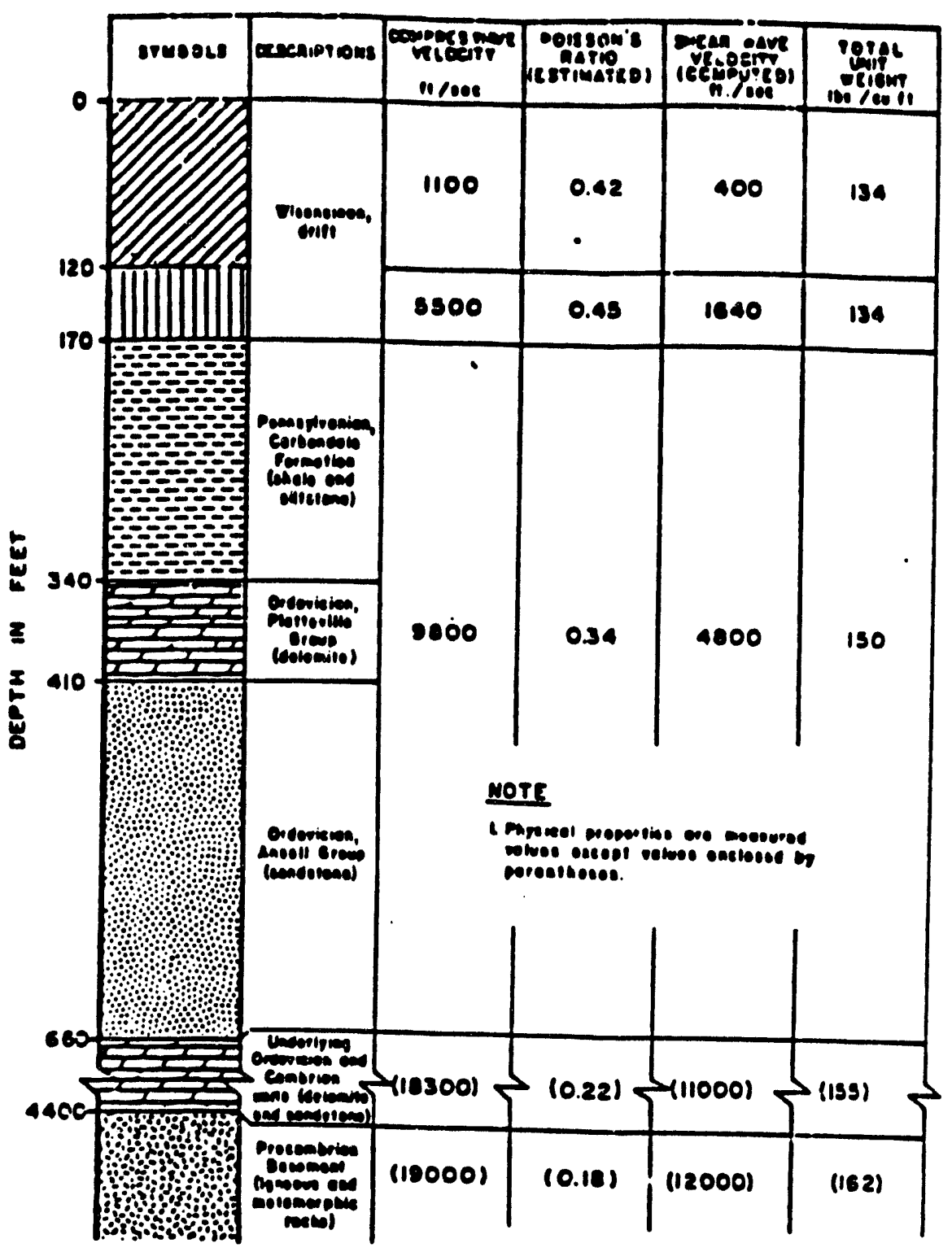

Figure 5.1. Stratigraphic column with geophysical data summary. 


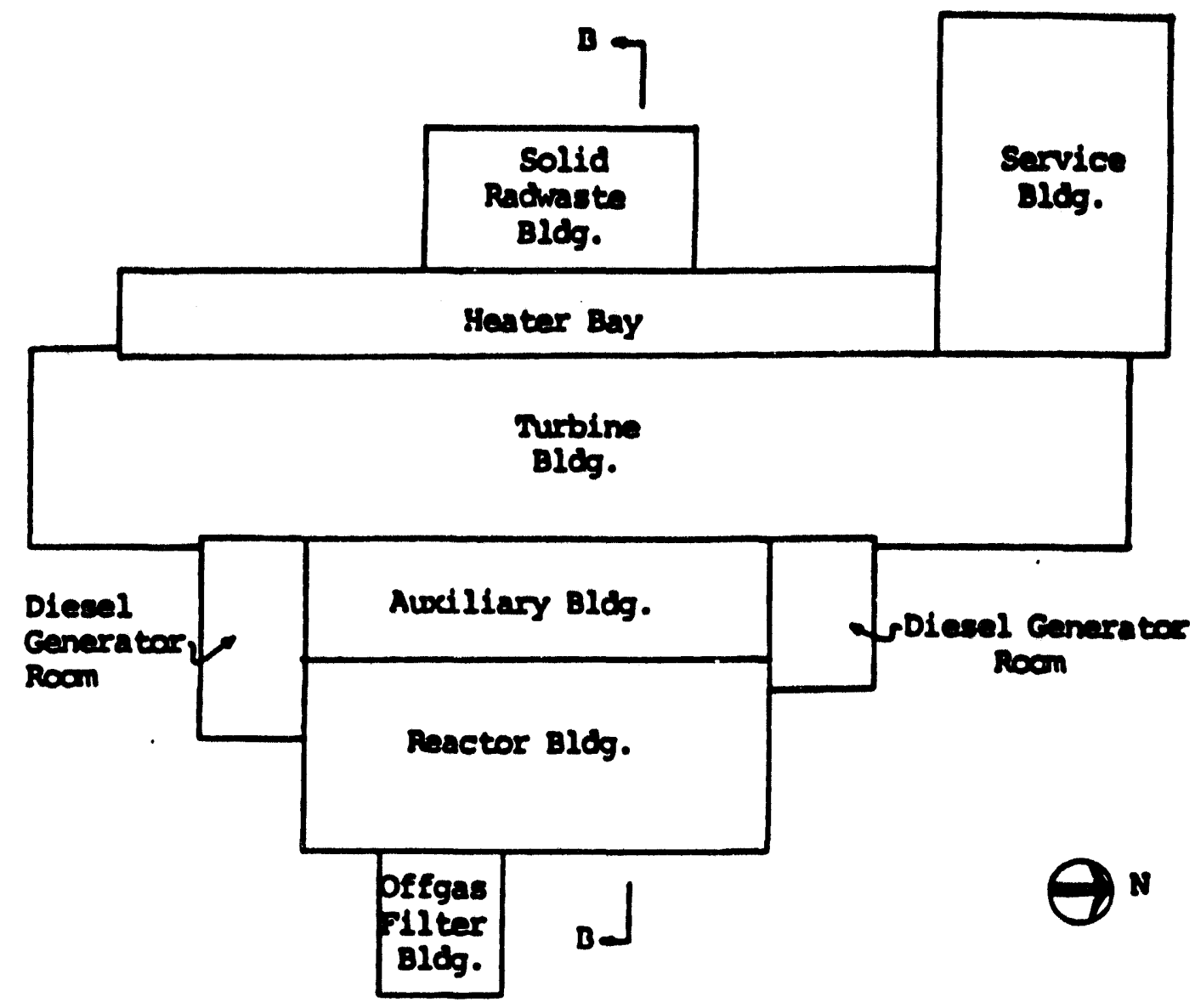

Figure 5.2. Plan view of LaSalle County Station Units 1 and 2. 


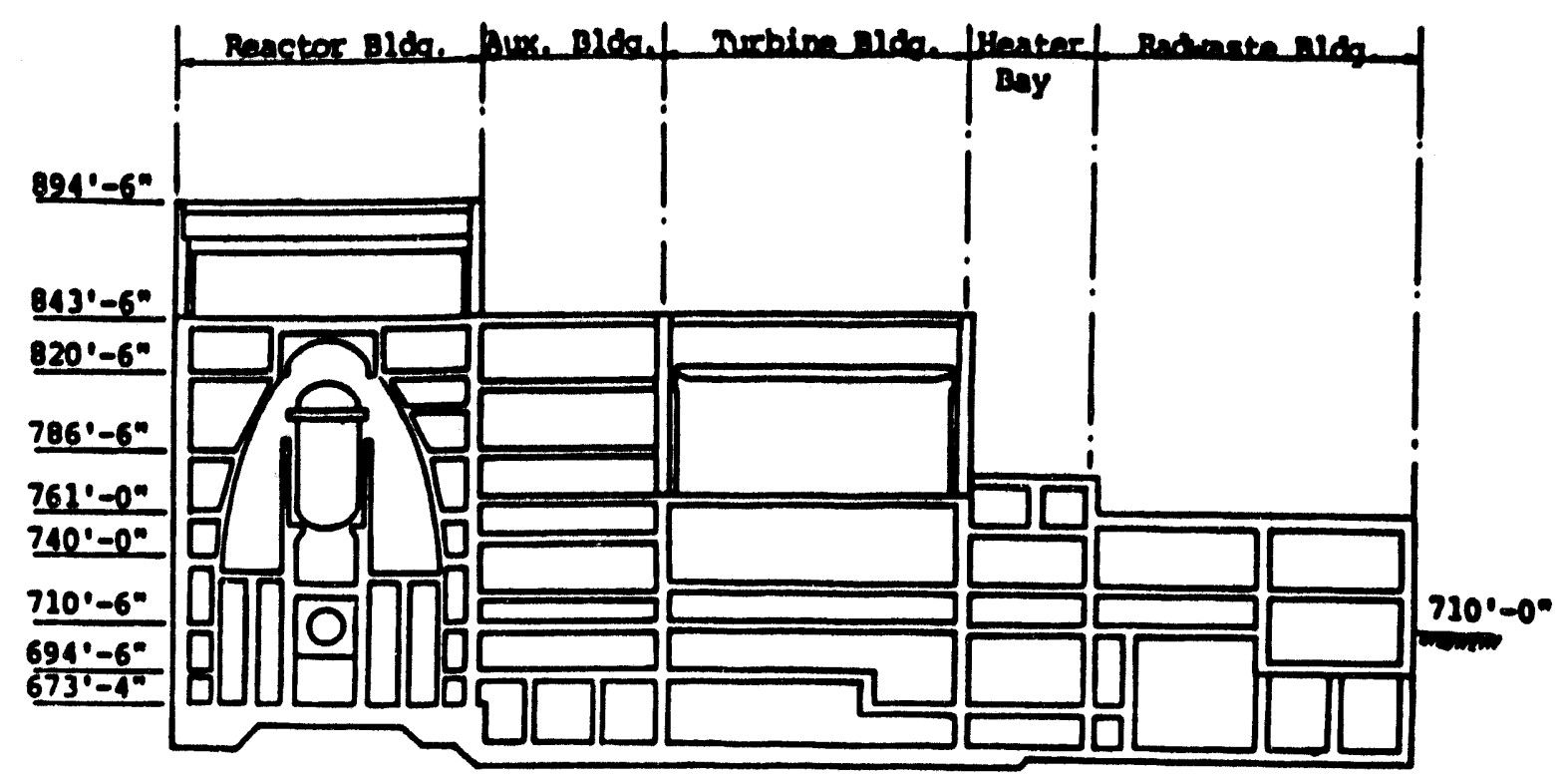

Figure 5.3. East-West section through the LaSalle County Station building complex. 
are housed within the reactor building. The primary containment is a steel-lined, post-tensioned concrete enclosure housing the reactor and the suppression pool. The containment walls also support several levels of floor slabs. The top of each containment is capped by a steel dome head. The reactor pressure vessel residing within the primary containment rests upon a cylindrical reinforced concrete pedestal which in tum is founded on the basemat. The top of the reactor vessel is supported laterally by a stabilizer truss. The refueling bellows also connects the reactor to the containment walls.

The auxiliary building located between the reactor and the turbine buildings is of reinforced concrete construction. It houses the control room, the HVAC equipment, the station vent stack, and much of the station electrical switchgear.

The turbine building is of reinforced concrete construction from basemat to elevation 710'. The floors above this elevation are supported on a steel structure. This building houses the power conversion equipment and feedwater cleanup equipment.

\subsection{SSI and Structure Models}

Soil-structure interaction (SSI) analysis methods can be grouped into continuum mechanics approach and finite element approach. These two approaches are best represented by two widely used computer codes: CLASSI in the continuum mechanics approach and SASSI ${ }^{[5.4]}$ in the finite element approach.

CLASSI was originally developed by Luco and Wong ${ }^{[5.5)}$ and was implemented in SMACS ${ }^{[5.1]}$ code by LLNL to perform both SSI, structural response, and subsystem response analyses in one computer run. CLASSI was used extensively in the nuclear power industry since 1979. SASSI was developed by Professor Lysmer of the University of California, Berkeley, and became available in the mid-1980s. There is no significant differences in the technical basis for these two computer codes because both codes are based on three-dimensional linear elastic wave propagation theory and use equivalent linear soil properties.

Compared to SASSI, CLASSI has the advantage of computational efficiency because of its use of closed-form solutions; in particular, when the basemat can be idealized as a circular rigid disk. The computational efficiency is important in our PRA of LaSalle and Zion plants because many SSI analyses need be performed in a Latin Hypercube experimental design. In the past few years, SASSI is gaining popularity over CLASSI because of its versatility inherent in a finite element method, its capability of handling embeddment effects, and the significant advances in computational speed of computers. It is versatile because it can handle foundation of any shape (including embeddment) and the soil properties are allowed to vary in both vertical and horizontal directions. CLASSI, on the other hand, has several restrictions: (1) surface foundation, (2) flat and rigid foundation, and (3) soil property varying in vertical direction only. CLASSI/ASD, ${ }^{[5.6]}$ a version of CLASSI by ASD International, has embeddment capability. However, it is not clear if this capability is properly validated. A comparison of SASSI and CLASSI/ASD results by the General Electric in the design of Advanced Boiling Water Reactor (ABWR) standard plant indicates that CLASSI/ASD may over-estimate responses by more than 100 percent (Fig. 3G.2-22(c), Amendment 4, Ref. 5.6) compared to SASSI. 
CLASSI was used in the PRAs for LaSalle and Zion plants simply because SASSI was not available when these analyses were performed. We have not assessed the effects if SASSI, instead of CLASSI, were incorporated in SMACS. The embeddment was considered approximately in LaSalle and Zion PRA. The accuracy of this approximation could not be evaluated at the time these PRAs were performed. The accuracy can be verified now because the availability of SASSI code. However, veritifcation was not performed because of the amount of effort involved. Assuming small embeddment effect, the seismic responses, we believe, would not be significantly affected if SASSI were used in the PRAs for LaSalle and Zion plants because restrictions (2) and (3) of CLASSI code usually have small effects on safety related structures of nuclear power plants, which generally have very thick basemat and heavy shear walls.

The CLASSI family of programs uses substructure approach to SSI analysis. The substructure approach divides the problem into a series of simpler problems, solves each independently, then superposes the results. The key elements of the approach are: specification of the free-field ground motion, determination of the foundation input motion, determination of the foundation impedances, calculation of the dynamic characteristics of the structures, and analysis of the coupled soilstructure system. The process culminates in a prediction of the response of the soil-structure system; i.e., SSI and detailed structural response are determined simultaneously. The set of continuum linear analysis (CLA) programs calculates the foundation input motion and impedances, then the SSIN program analyzes the coupled soil-structure system. SSIN forms the core of the SSI and major structure response calculations in SMACS. The steps of the substructure approach are depicted schematically in Fig. 5.4. Each aspect is described in the following subsections.

\subsubsection{Free-Field Ground Motion and Soil Model}

Specification of the free-field ground motion entails specifying the control point, the frequency characteristics of the co.ttrol motion (typically, time histories or response spectra), and the spatial variation of the motion. For SMACS, the free-field ground motion is specified by three components of acceleration time histories on the free surface of the soil. The three components correspond to two horizontal and the vertical directions. For each of the two acceleration ranges analyzed here, an ensemble of thirty earthquakes was defined. Local site amplification was treated explicitly for the LaSalle site, as introduced previously. Hence, the basic process was as follows:

- Define the seismic hazard on a hypothetical rock outcrop at the LaSalle site. Discretize the hazard curve into intervals for seismic response analysis and for the probabilistic failure and release calculations. For each interval of interest, generate acceleration time histories which represent the free-field ground motion.

- Perform preliminary analyses, using representative rock outcrop acceleration time histories, to estimate nominal values of equivalent linear soil properties to be used in the local site amplification and SSI analyses. LaSalle specific data, such as soil layering, low-strain and strain independent material properties, and relationships for shear modulus degradation and increases in material damping with strain, are used. The results are nominal soil properties for each of the acceleration ranges to be analyzed.

- Perform a series of free-field one-dimensional wave propagation analyses starting with rock outcrop acceleration time histories and generating acceleration time 
histories on the free surface of the soil for the SSI analyses. Vertically propagating shear anil dilatational waves are assumed. Soil properties (soil shear modulus and material damping) are varied according to the Latin hypercube experimental design used in the SMACS analyses. This maintains consistency between the SSI analyses and the free-field ground motion. Variability in the free-field surface motions result from variability in the rock outcrop motions and in the soil properties.

Properties of the soil profile are shown in Table 5.1 and Figs. 5.5 and 5.6. Nominal values of the equivalent linear soil properties were generated for two acceleration ranges centered at $0.2 \mathrm{~g}$ and $0.6 \mathrm{~g}$ on the rock outcrop. Figures 5.7 and 5.8 show nominal soil shear moduli for the two acceleration ranges. The dashed line overplotted in the figures represents the properties used for the calculation of the SSI parameters (scattering and impedance matrices). Table 5.1 summarizes the soil properties used for the two ranges. Note, the soil conditions for the two ranges are extremely soft, i.e., the nominal shear modulus (and shear wave velocities) are very low. This is due to the shear modulus degradation curves determined by Sargent \& Lundy for the utility and reported in Ref. 3.1. These properties were determined by laboratory and field tests performed by the utility. These extremely soft properties are a source of modeling uncertainty and may warrant further consideration.

The results of the site amplification studies were two ensembles of earthquake motions on the soil free surface--one ensemble for each of the acceleration ranges. Each ensemble contained the freefield motions for thirty earthquake simulations. Each simulation was represented by acceleration time histories--two horizontal and the vertical components. The mean spectra of the free surface motions are shown in Figs. 5.9 and 5.10.

\subsubsection{SSI Parameters}

To perform SSI analysis, the foundation scattering and Impedance matrices must be developed to characterize the kinematic and inertial behavior of the foundation-soil system. Applying the scattering matrix to the free-field surface ground motion yields the foundation Input motion. The foundation input motion differs from the free-field ground motion in all cases, except for surface foundations subjected to vertically incident waves. First, the free-field motion varies with soil depth. Second, the soil-foundation interface scatters waves because points on the foundation are constrained to move according to its geometry and stiffness. The foundation input motion $U^{*}(\omega)$ is related to the free-field ground motion by means of a transformation defined by a scattering matrix [S( $\omega)]$, which is complex-valued and frequency dependent:

$$
U^{*}(\omega)=[S(\omega)] f(\omega)
$$

The vector $f(\omega)$ is the complex Fourier transform of the free-field ground motion, which contains its complete description. The impedance matrix describes the harmonic force-displacement characteristics of the soil. It depends on soil configuration and material behavior, the frequency of the excitation, and the geometry of the foundation. The LaSalle analyses assumed rigid foundations for which the force-displacement characteristics are uniquely defined by a $6 \times 6$ matrix relating a 


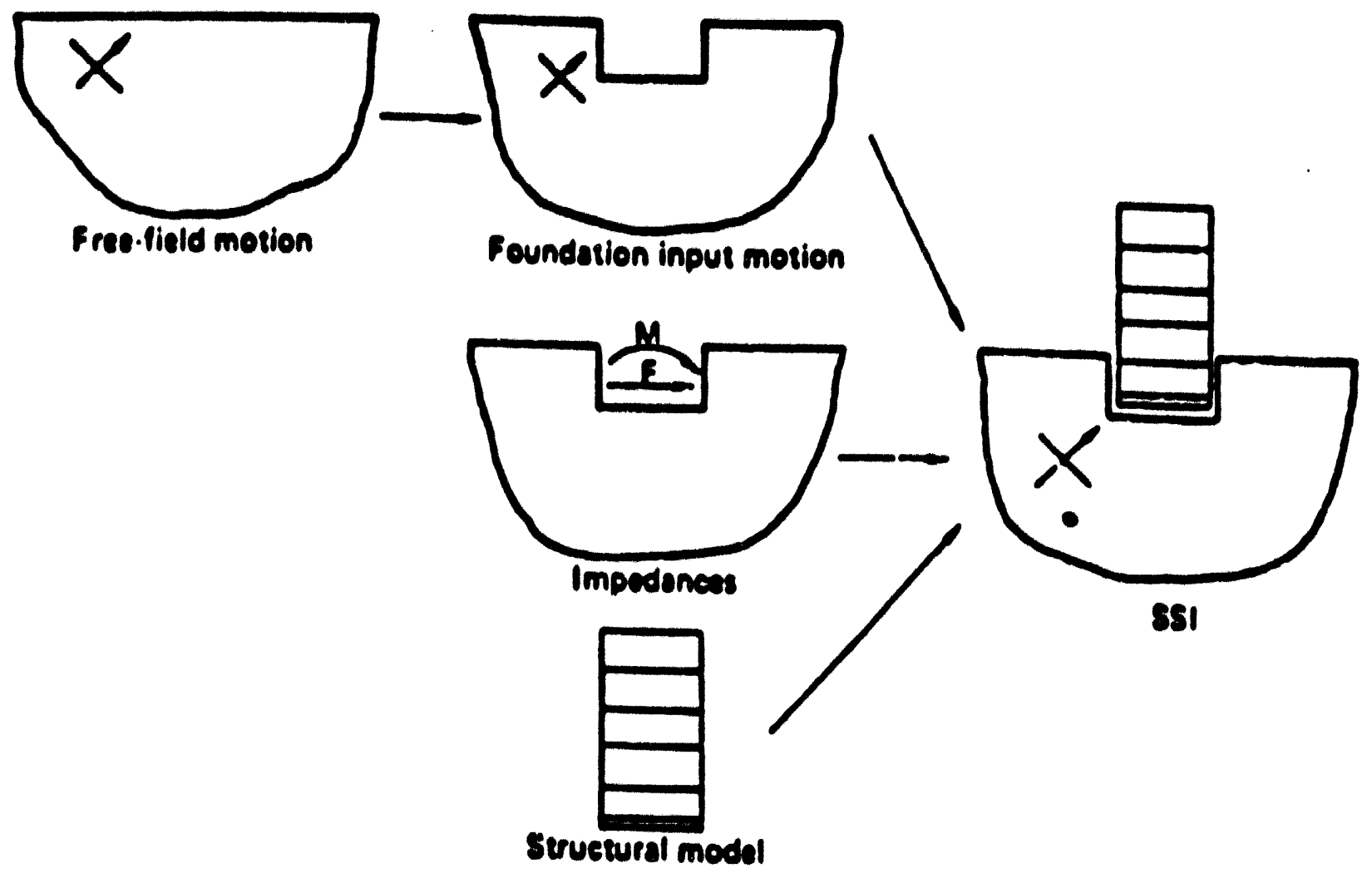

Figure 5.4. Schematic representation of the elements of the substructure approach to SSI analysis. 
(a) Low Strain and Strain Independent Parameters

\begin{tabular}{|c|c|c|c|c|}
\hline & 1 & 2 & Layer Number & 4 \\
\hline $\begin{array}{l}\text { Thickness (ft) } \\
\text { Density (pcf) } \\
\text { Poisson's Ratio }\end{array}$ & $\begin{array}{l}90 \\
134 \\
0.42\end{array}$ & $\begin{array}{c}30 \\
134 \\
0.42\end{array}$ & $\begin{array}{c}30 \\
134 \\
0.45\end{array}$ & $\begin{array}{l}20 \\
134 \\
0.45\end{array}$ \\
\hline
\end{tabular}

(b) Nominal Values of Soil Properties for Acceleration Range 1

Layer

Number

1
2
3
4
5
Shear Modulus (KSF)

$$
644
$$

1400

2450

3360

65,000
Shear Wave

Yelocity (fog)

393

582

767

898

3737

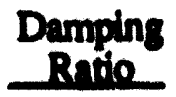

.126

.107

.102

.100

.010

(c) Nominal Values of Soil Properties for Acceleration Range 2

$$
\begin{gathered}
\text { Layer } \\
\text { Number }
\end{gathered}
$$

1
2
3
4
5

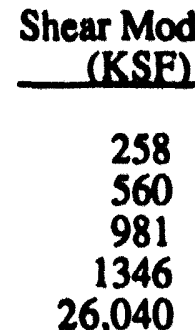

Shear wave

Velocity (fos)

$$
249
$$

368

485

568

2370

\section{Damping Rario}

.176

.150

.142

.140

.010

Data is presented for the layer discretization used in calculating foundation impedances and scattering. Data at a finer interval is shown in Figs. 2.7 and 2.8 for shear modulus. 


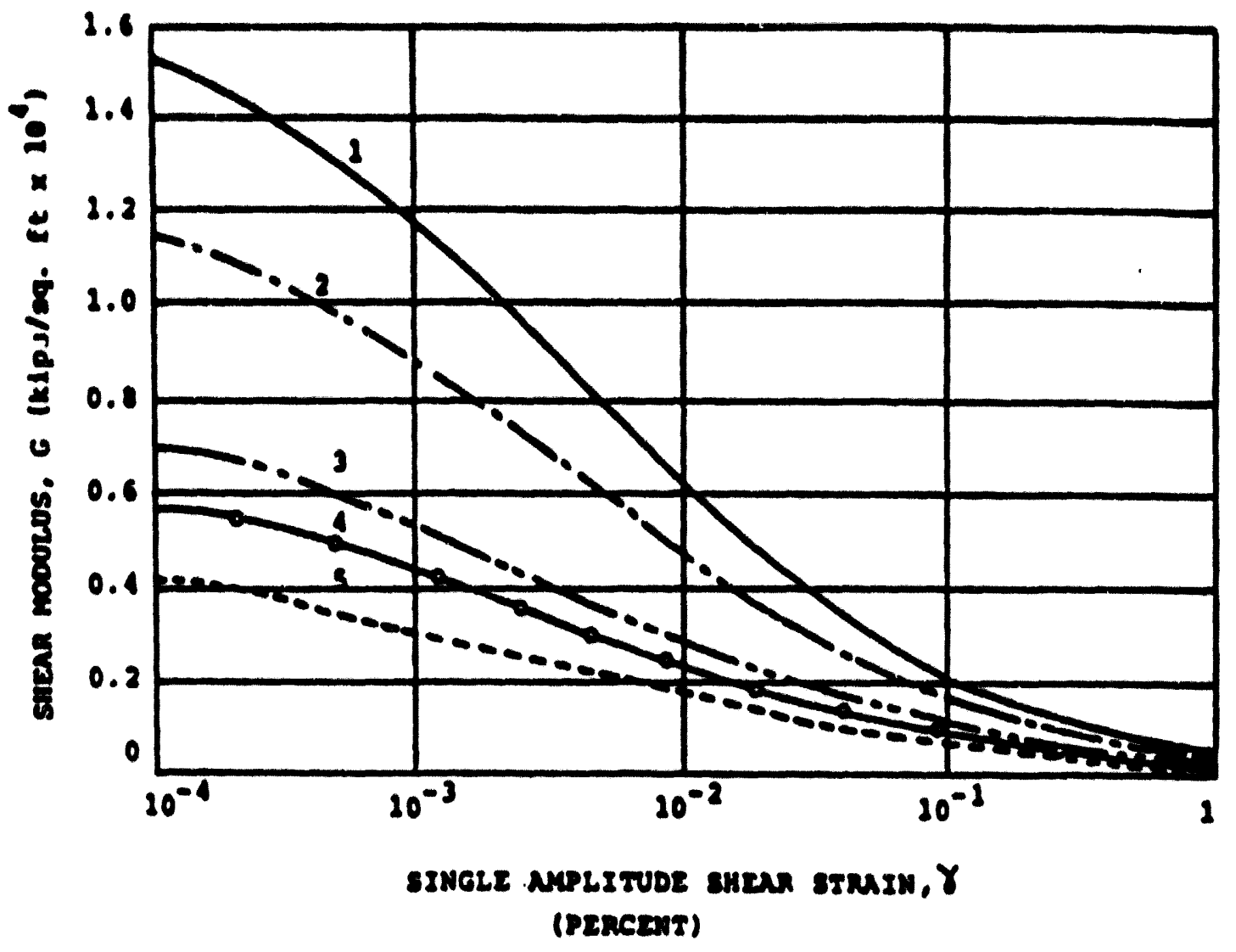

\begin{tabular}{|c|c|c|c|}
\hline Lexd & & and 1 & $\begin{array}{l}\text { Properticnaldty recter } \\
\text { y.r.tanv }\end{array}$ \\
\hline & 21. $350^{\circ} 510^{\circ}$ & 2 & 3.722 \\
\hline & 2d. $590^{\circ}-300^{\circ}$ & 2 & 2.033 \\
\hline 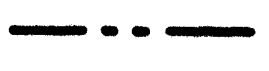 & 2. $\left(2 x^{\circ}-5200^{\circ}\right.$ & 3 & 2.722 \\
\hline$-\infty-\infty$ & $\begin{array}{l}\text { 21. } 710^{\circ}-690^{\circ} \\
\text { E1. } 690^{\circ}-620^{\circ}\end{array}$ & $\begin{array}{l}4 \\
5\end{array}$ & $\begin{array}{l}1.306 \\
1.000\end{array}$ \\
\hline
\end{tabular}

Figure 5.5. Shear modulus degradation curves. 


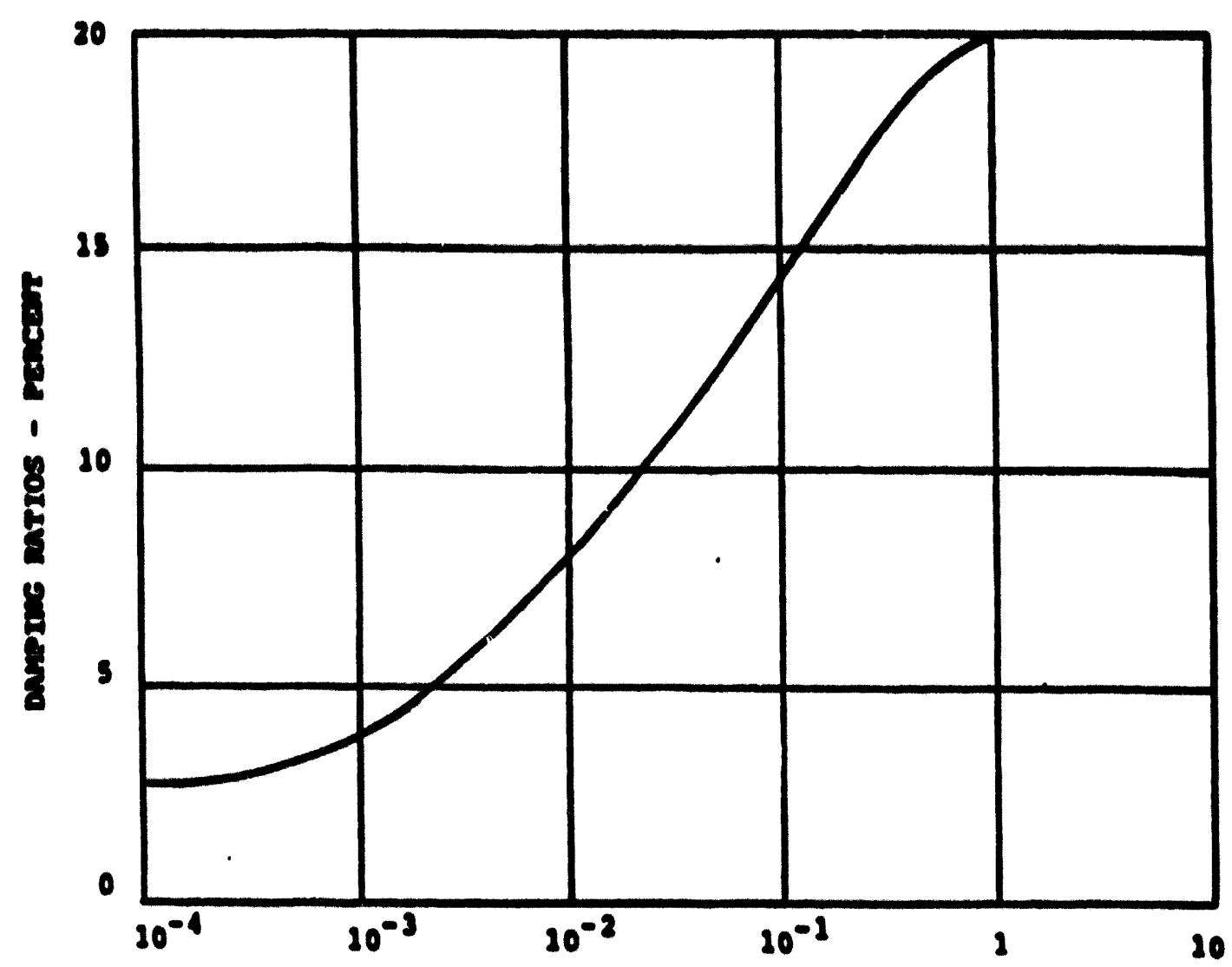

SIMOLE AMPLISUDE IMEAN STRAIn, $Y$ (Dencent)

Figure 5.6. Damping ratios vs strain.

-51 - 


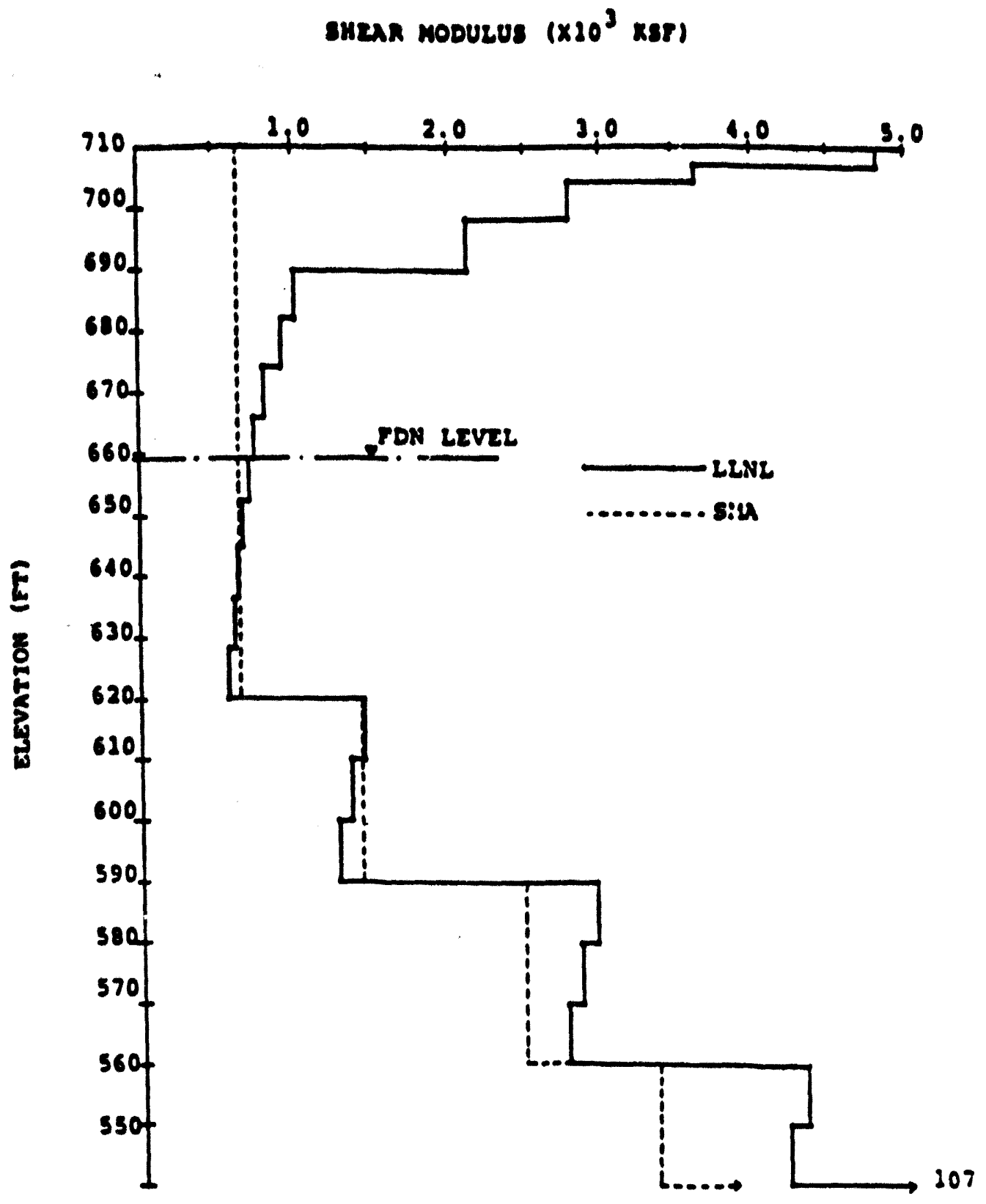

Figure 5.7. Nominal values of shear modulus for acceleration range 1. 


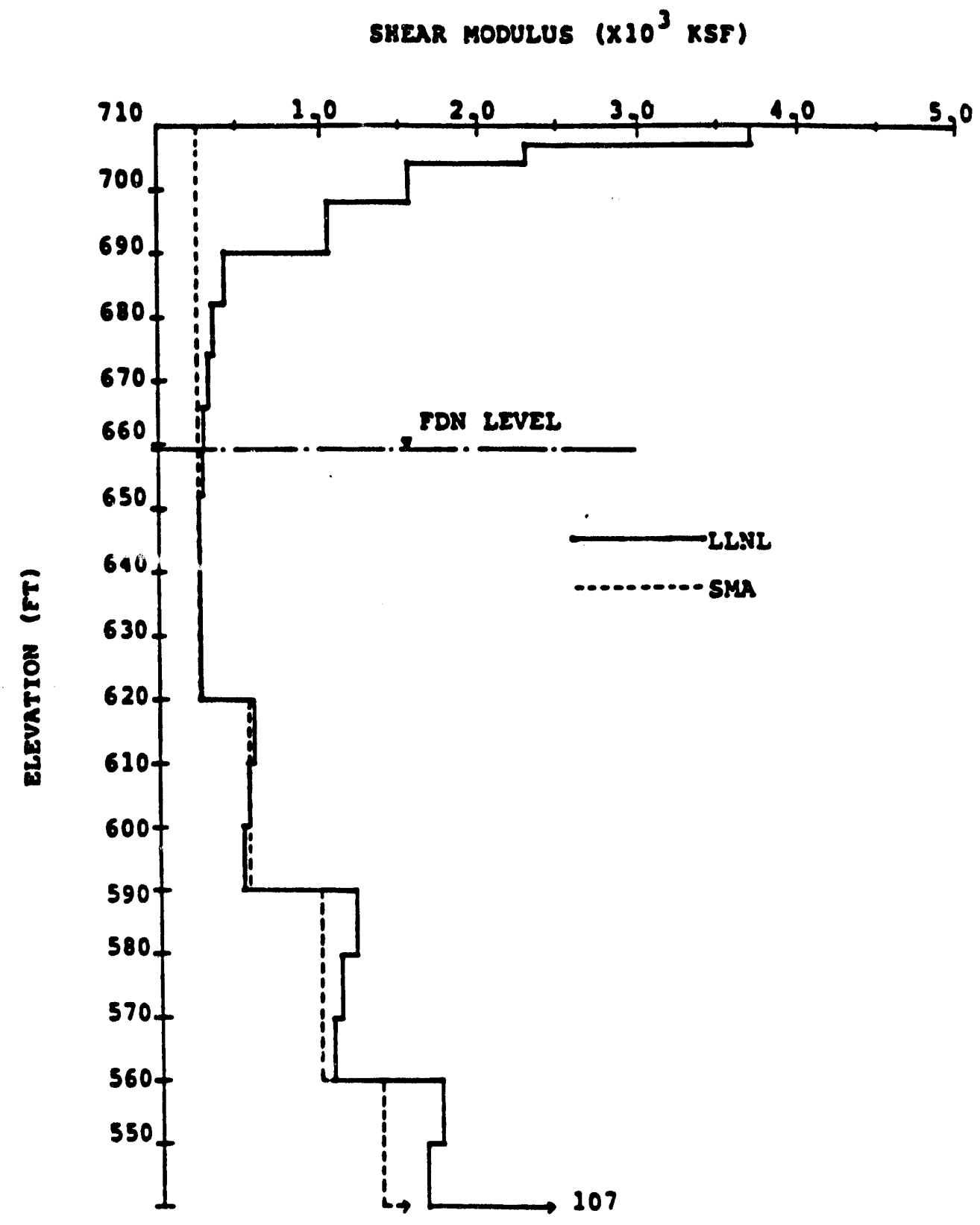

Figure 5.8. Nominal values of shear modulus for acceleration range 2. 

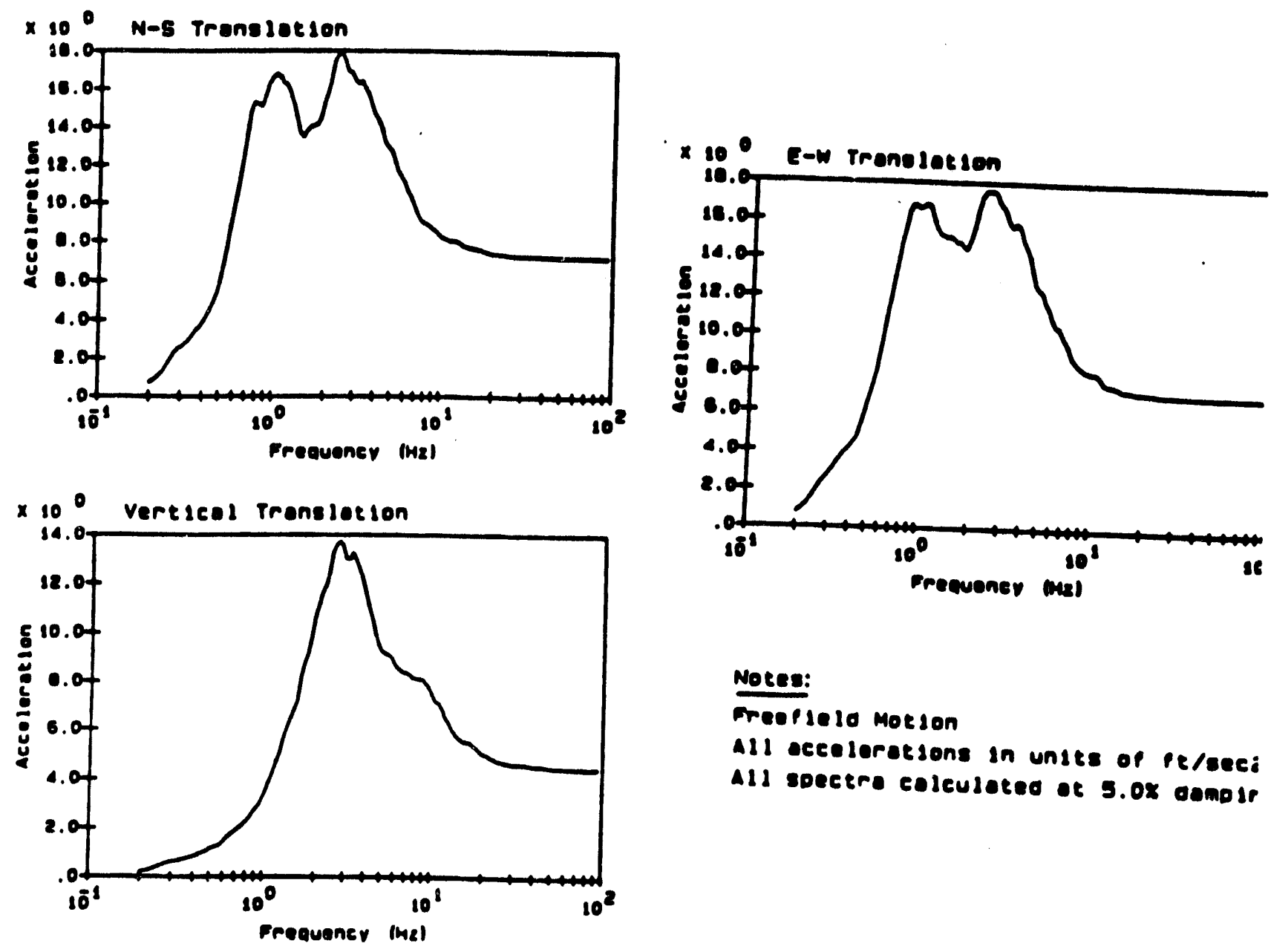

Notes:

Mreepiela Motion

All ocesiorotions in undts of ptece: all spectro colculoted ot $5.0 x$ oomplr

Figure 5.9. Mean free-field ground response spectra on soil free surface, el. $710^{\prime}$, acceleration range 1. 

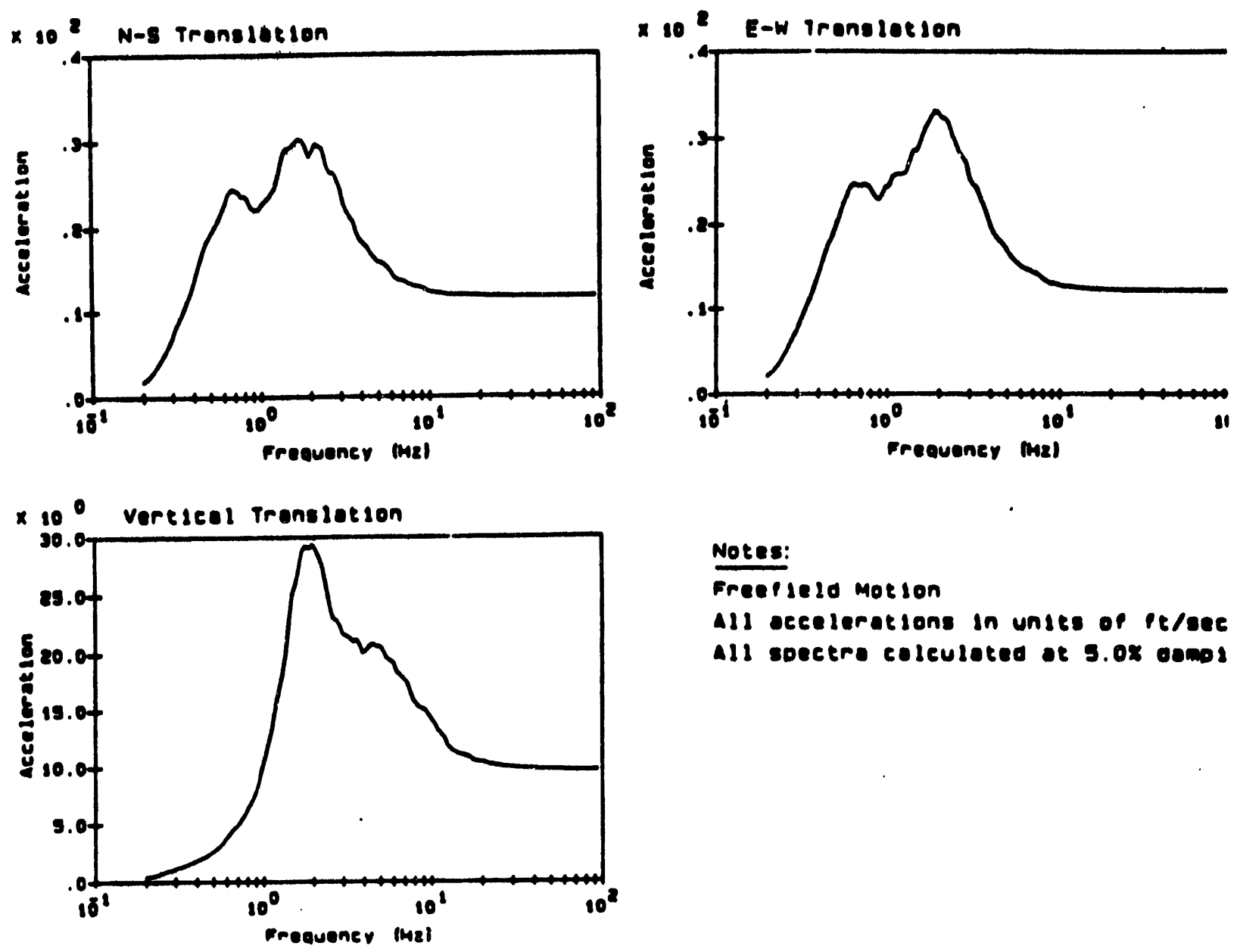

Notes:

Freetield motion

All eccelerations in units of ptoec All soectro celculeted of $\mathrm{g.0x}$ oums

Figure 5.10. Mean free-field ground response spectra on soil free surface, el. 710', acceleration range 2. 
resultant set of forces and moments to the six rigid-body degrees-of-freedom. In general, for linear elastic or viscoelastic material and a uniform or horizontally stratified soil deposit, each element of the impedance matrix $\left[K_{s}(\omega)\right]$ is complex-valued and frequency dependent. Each complex element of the matrix, can be thought of as a pair of functions: the real part approximating the stiffness of the soil, and the imaginary part the damping. For a rigid foundation, the impedance matrix is a $6 \mathrm{x}$ 6 which relates a resultant set of forces and moments to the six rigid-body degrees-of-freedom.

The procedure used to calculate embedded foundation impedances and scattering elements follows that described in Ref. 5.2 and applied to the Zion AFT complex. The procedure is discussed in three calculational steps. The terms equivalent radius and average embedment depth are used. These are the dimensions of a circular cylinder selected to yield cross-sectional area and excavated volume equal to that of the actual foundation. In the case of LaSalle, the equivalent radius was approximately $252 \mathrm{ft}$. and average embedment depth was $51 \mathrm{ft}$.

- In the first step, impedances for a flat foundation having the shape of the actual LaSalle foundation were calculated. The CLASSI programs denoted CLAN were used. The foundation is placed on the surface of a truncated soil column where the top portion of soil, i.e., to the average embedment depth, has been removed. The foundation model is shown in Fig. 5.11. The foundation reference point has been chosen so as to minimize coupling between horizontal translation and torsion and between vertical translation and rocking.

- The second step Involves the calculation of impedances and scattering elements for an embedded cylinder having the equivalent radius and embedment depth previously discussed. Vertically propagating shear and dilatational waves were assumed. The CLASSI programs denoted CLAE were used.

- In step three, impedances for a circular disk resting on the truncated soil column were calculated.

The method used to account for embedment, at the same time retaining the effects of the nonsymmetric LaSalle foundation shape, is to apply a correction to the impedances calculated in step one. The correction terms are obtained by subtracting the impedances for the circular disk (step three) from that of the embedded cylinder (step two). Selected foundation impedances corrected for embedment are shown in Fig. 5.12. In addition, the scattering matrix for the embedded foundation is used to model kinematic interaction.

The above steps were performed using nominal soil properties for acceleration range 1 . For acceleration range 2 , the foundation impedances and scattering were approximated by scaling those developed for range 1 by a constant factor to reflect a uniform reduction in nominal soil properties at the higher acceleration range.

\subsubsection{Structure Models}

The dynamic characteristics of the structures to be analyzed are described by their fixed-base eigensystem and modal damping factors. Typically, structural data for SMACS is calculated using the finite element program SAP4 in conjunction with the post processor INSSIN. The analyses 


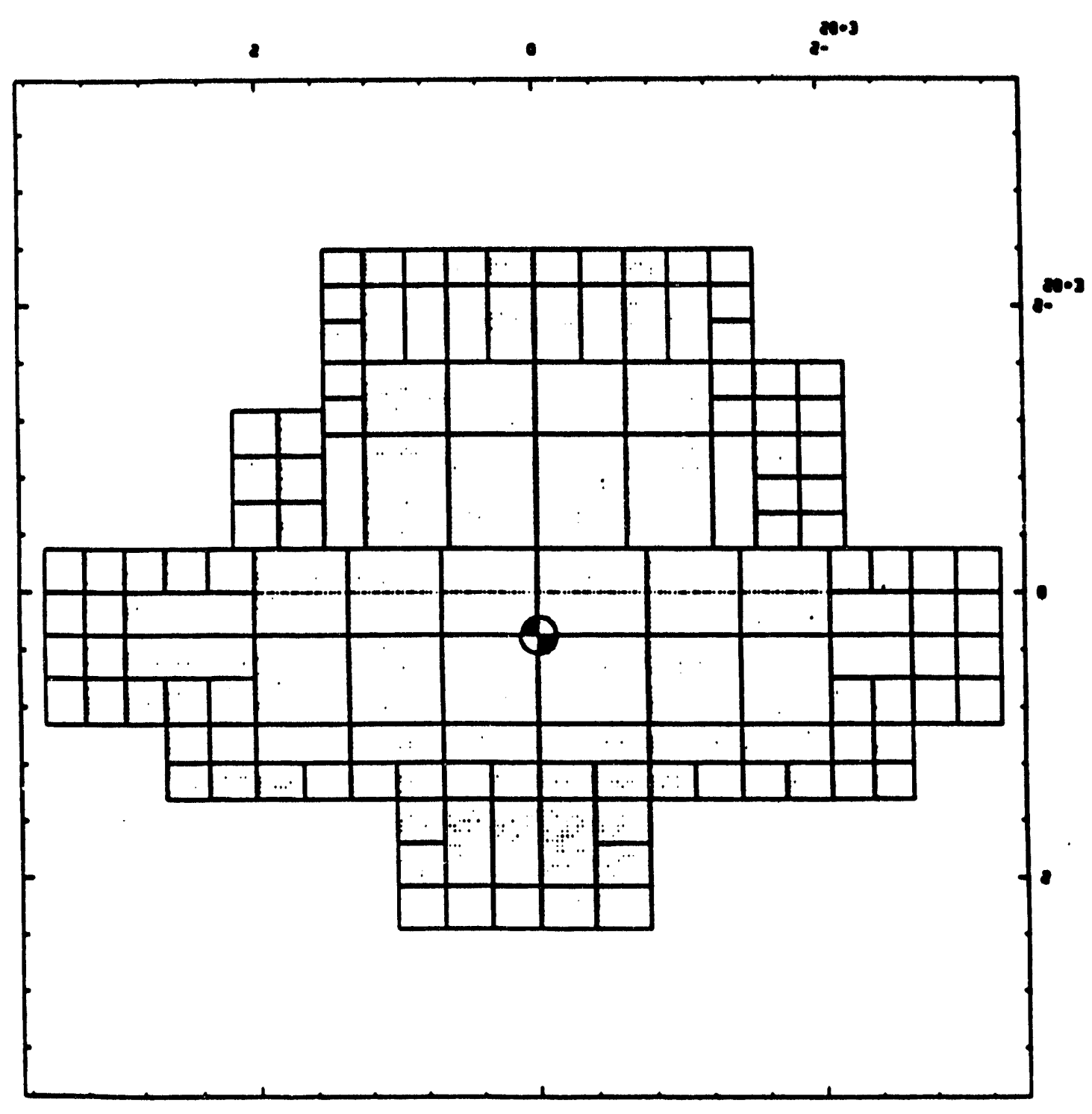

Figure 5.11. Foundation discretization for the LaSalle County Station structure complex impedance calculations. 

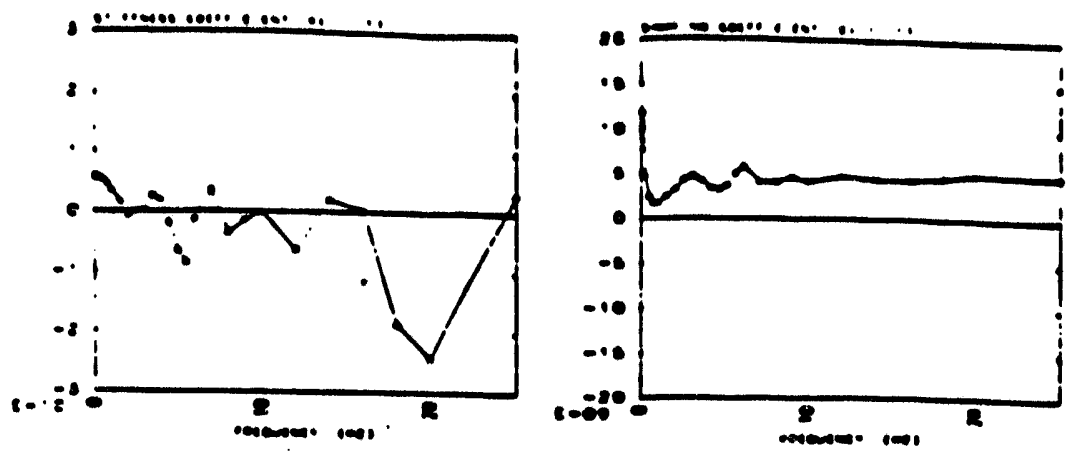

a) Morizontal rranslation, $x$-Dirwetion (1/8t)
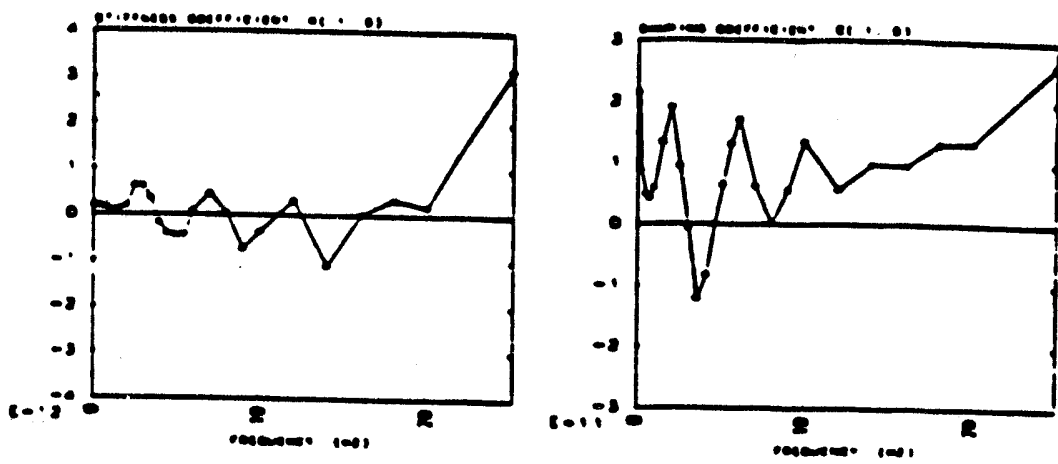

b) Borizantal traselation - Rocking Coplling (1/rad)
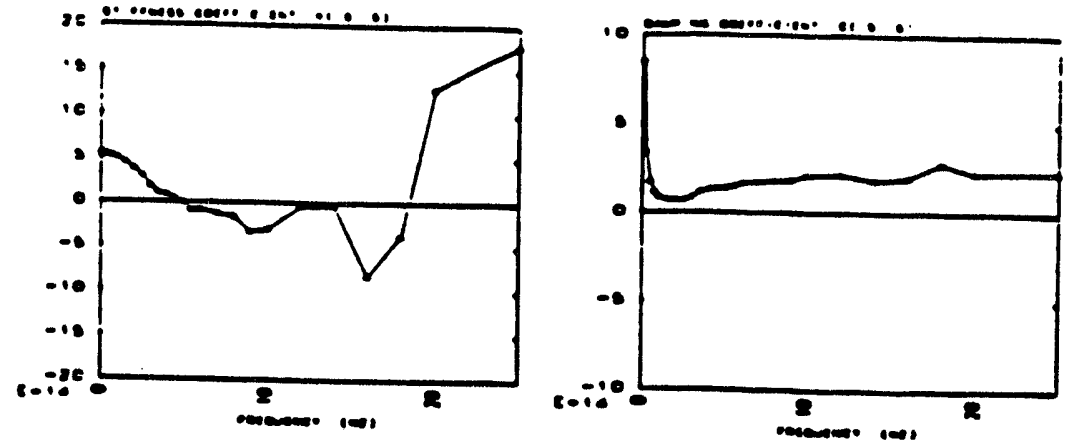

C) Rocking Abourt Y-Ruds (ft. 1./rad)

Figure 5.12. Representation LaSalle Foundation Impedances: a) Horizontal translation, C-direction (\#/ft.); b) Horizontal translation--rocking coupling (\#/rad); c) Rocking about Y-axis (ft. \#/rad); d) Vertical translation; and e) Torsion (ft.\#/rad). 

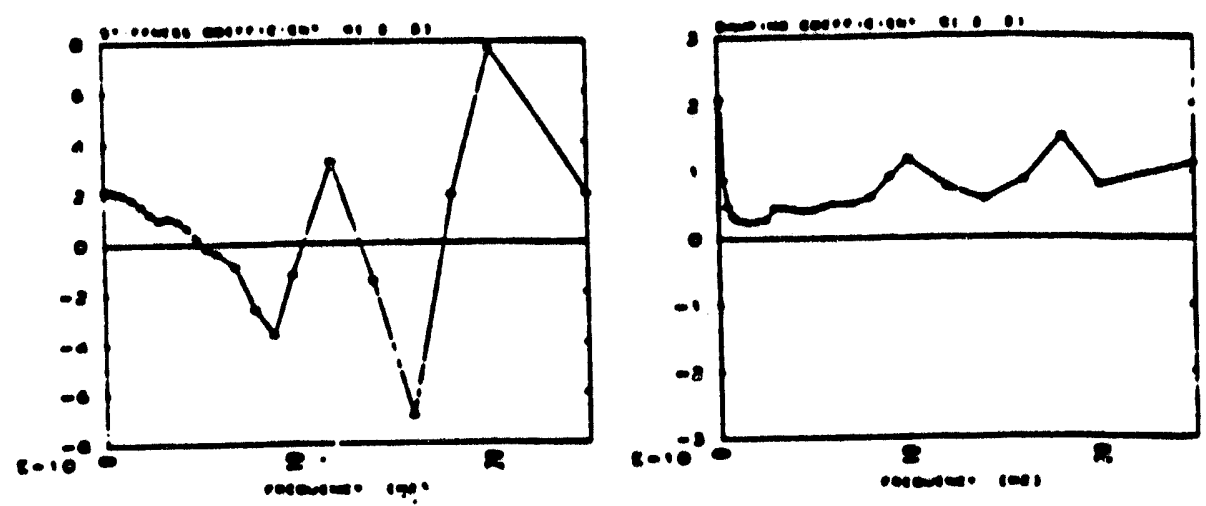

d) Vertiend iranalation (I/ft)
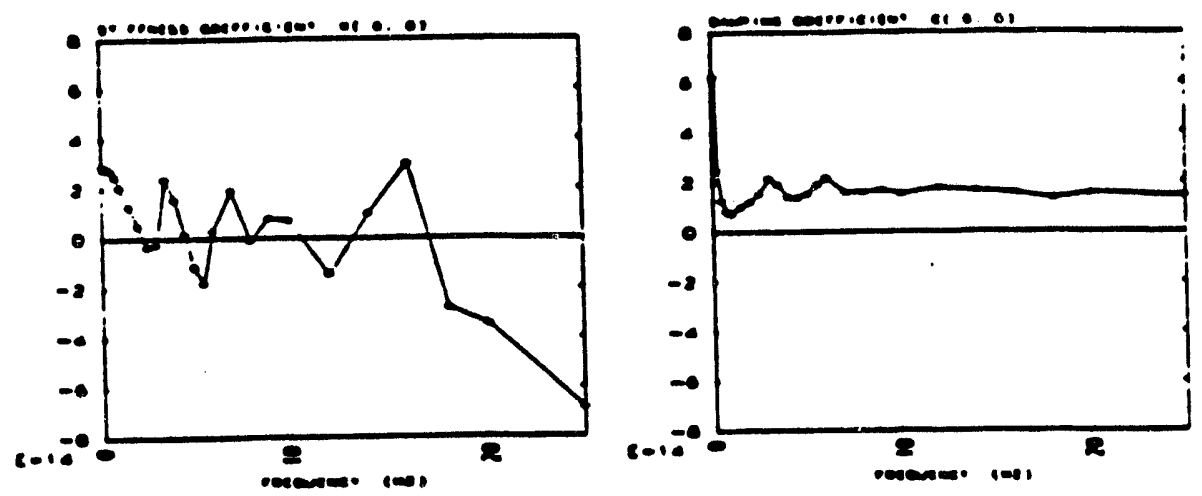

e) Torrion (ft. 1/rad)

Figure 5.12. Representation LaSalle Foundation Impedances. (cont.) 
performed here used, as a basis, the structure models developed by Sargent and Lundy. A horizontal model and three vertical models were developed by Sargent and Lundy for the LaSalle County Station. The dynamic data of these models was received from the $\mathrm{A} / \mathrm{E}$ and the models were reconstructed in SAP4 format. This permitted ease of use in SMACS and permitted modifications to be made to the horizontal model in order to obtain better estimates of local response. The reconstructed models were verified by comparing structural frequencies and modal participation factors with the original models. In addition, in the case of the horizontal model, the mass normalized modal coordinates for several major modes were compared and found to be in close agreement. The following sections describe in detail each of the four models--the descriptions are those of Sargent and Lundy ${ }^{(5.3)}$ and are presented here for completeness.

Horizontal Seismic Model. The horizontal model includes the reactor, auxiliary, turbine, heater-bay and radwaste buildings. Elements within the reactor building, such as the containment, sacrificial shield, reactor pedestal and reactor pressure vessel (RPV), are explicitly modeled.

A lumped mass-spring representation is used for the shear wall-slab structural system found in the buildings. The floor slabs, deemed rigid in-plane, are permitted three degrees-of-freedom--two inplane translations and a torsional rotation about a normal to the plane. The slab mass, including equipment and tributary walls, is lumped at the mass centroid. A mass polar moment of inertia was calculated about a normal through the centroid to represent torsional inertia. The shear walls are assumed to have negligible out-of-plane stiffness. The in-plane stiffness is calculated considering both flexural and shear effects induced by end translations without rotation. In order to capture the torsional stiffness of the structure, the shear wall spring elements are placed at an appropriate distance from the floor masses. In the original model the spring elements are slaved to floor masses thereby modeling a rigid floor diaphragm. However, in some instances, floor slabs feature large openings resulting in flexible strips of slab. Such a situation occurred at slabs $5,6,7$, and 10. Appropriate modifications were made to the horizontal model to account for floor slab flexibility, relieving stresses in shear walls located in their vicinity and, hence, more realistically predicting shear wall forces. Another set of modifications consisting of revised wall spring stiffnesses as described in Tables 8 and 9 of Ref. 5.3 were, also, incorporated.

Figure 5.13 shows a schematic of the horizontal model. Solid lines represent groups of shear walls. Dashed lines are beam members for structures in the reactor containment portion of the complex. Nodal points with masses assigned are shown as solid dots. Figure 5.14 shows the plan view location of the slab nodes with respect to the building outline. The origin of global coordinates chosen for the model is also shown. Table 5.2 lists the elevation of all mass points based on the MSL reference datum, and the X-and Y-coordinates of the points. It can be seen from the table that node points on the containment, sacrificial shield, RPV, and pedestal are collinear with either node 35 or node 61 . This is due to these concentric structures sharing a common axis.

Figures 5.15 reproduced from S\&L's exhibits 16 through 29 show the shear walls included in the model. Each wall or group of walls is uniquely identified by a seven digit code number. Leading zeros are implied in any number which has less than seven digits. The numbers are decoded as shown below, starting with the most significant digits:

1st two digits: Slab number to which the lower end of the wall spring is attached

2nd two digits: Slab number to which the upper end of the all spring is attached 
Last 3 digits: $\quad$ Spring number. An odd number denotes an $X$-spring representing a shear wall oriented in the N-S direction. An even number denotes a Yspring oriented in the E-W direction.

The wall spring groups are segregated into groups representing shear wall systems that can be regarded as structurally continuous through several floors of the complex. The groups and component walls are listed in Tables 5.3 and 5.4, for X- and Y-springs respectively. These tables provide the cross-reference for results such as shear and moments presented in later sections.

Table 5.5 presents selected dynamic characteristics of the modified horizontal model.

Vertical Model of the Reactor and Auxiliary Buildings. The vertical model of the reactor and auxiliary buildings is shown schemstically in Fig. 5.16. All node points have a vertical degreeof-freedom. The extreme right vertical column represents the containment walls. Other columns represent the walls of the reactor and auxiliary buildings. Horizontal members spanning between columns represent the reactor building slabs and the fuel pool walls. The cantilever members with masses lumped at their ends simulate slabs in the auxiliary building and the reactor containment.

Node points are established at the elevations of major floor slabs. The tributary masses of the shear walls are lumped at the nearest nodes. Two thirds of each slab mass is lumped with the wall masses at the slab elevation. The remaining one third is assigned to the single degree-of-freedom oscillator at midspan of the horizontal members.

The stiffness of the vertical members are derived from summation of the axial stiffness of all tributary walls at each level. For the containment, the effective axial area used for calculating stiffness is that of the annular cross-section. Where the radius changes over the height of a vertical member, the average area is used.

The single degree-of-freedom oscillators representing the fundamental modes are determined from standard formulas. Table 5.6 summarizes the dynamic characteristics of the model.

Vertical Model of the Turbine, Heater Bay and Radwaste Buidings. Figure 5.17 is a schematic of the vertical model representing the turbine, heater bay, and radwaste buildings. The two vertical columns model the turbine building walls. Mass points on the column represent tributary wall masses as well as tributary slab and equipment masses. The wall stiffness at each level is derived from a summation of the axial stiffnesses of individual walls at that level. Horizontal members interconnecting the columns and supprting lumped masses at midspan simulate the vertical response of floor slabs. One third of the floor mass together with any attached equipment are assumed to participate in the fundamental slab mode. The fundamental frequency is determined from standard formulas. Where higher modes are considered important, the effective slab mass is distributed equally between several of these single degree-of-freedom oscillators and their frequencies individually tuned by adjusting stiffness parameters. The cantilevered oscillators represent the heater bay and radwaste slabs of interest. Table 5.7 lists the dynamic characteristics of this model.

Vertical Model of RPV, Sacrificial Shield, and Reactor Pedestal. Figure 5.18 illustrates the vertical model for the RPV, sacrifical shield and the reactor pedestal. The floor slabs are relatively rigid and no amplification effect is expected. Hence, the total floor masses are lumped with the wall masses. The dynamic properties are listed in Table 5.8. 


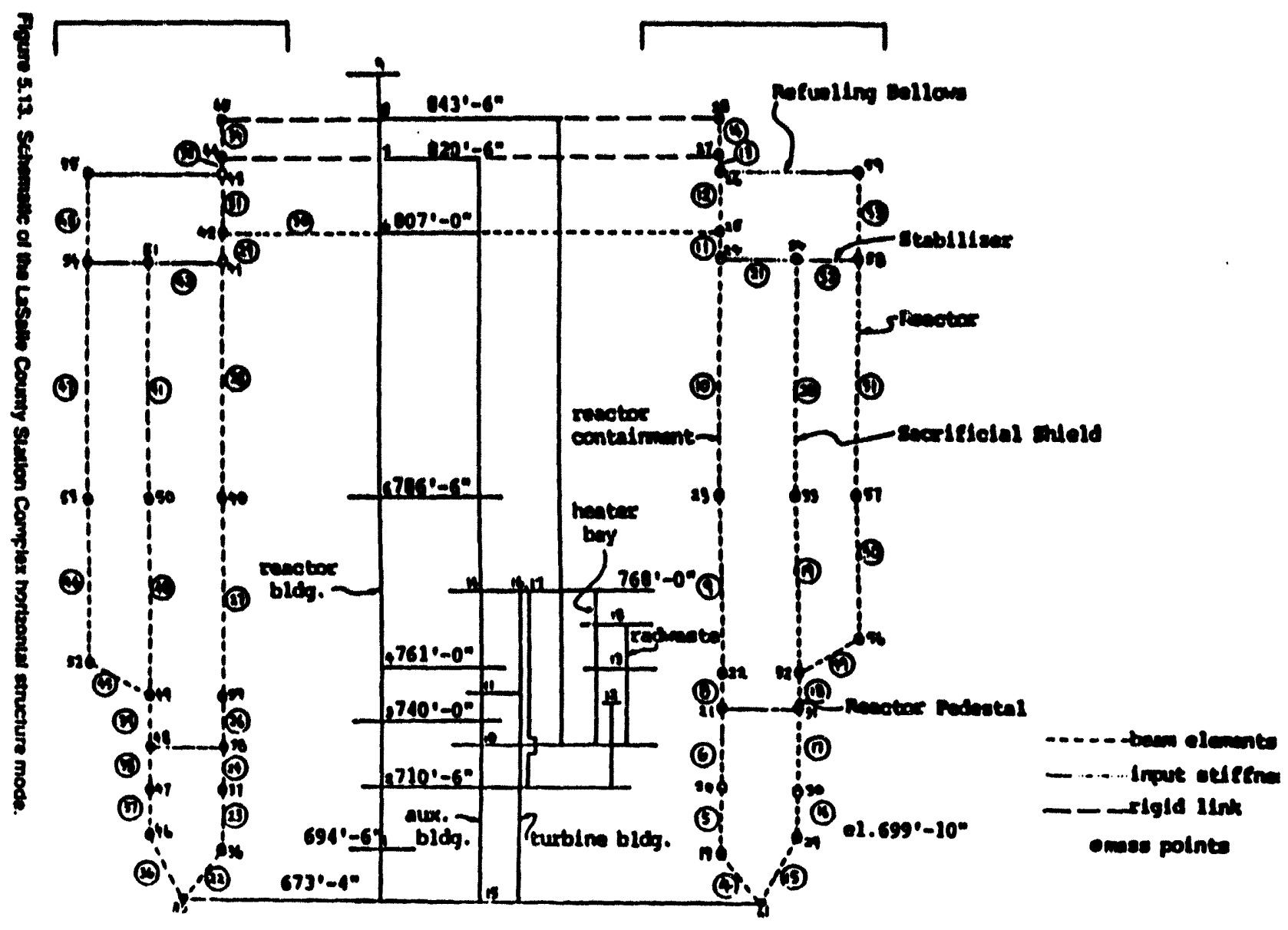

Figure 5.13. Schematic of the LaSalle County Station Complex horizontal structure mode. 


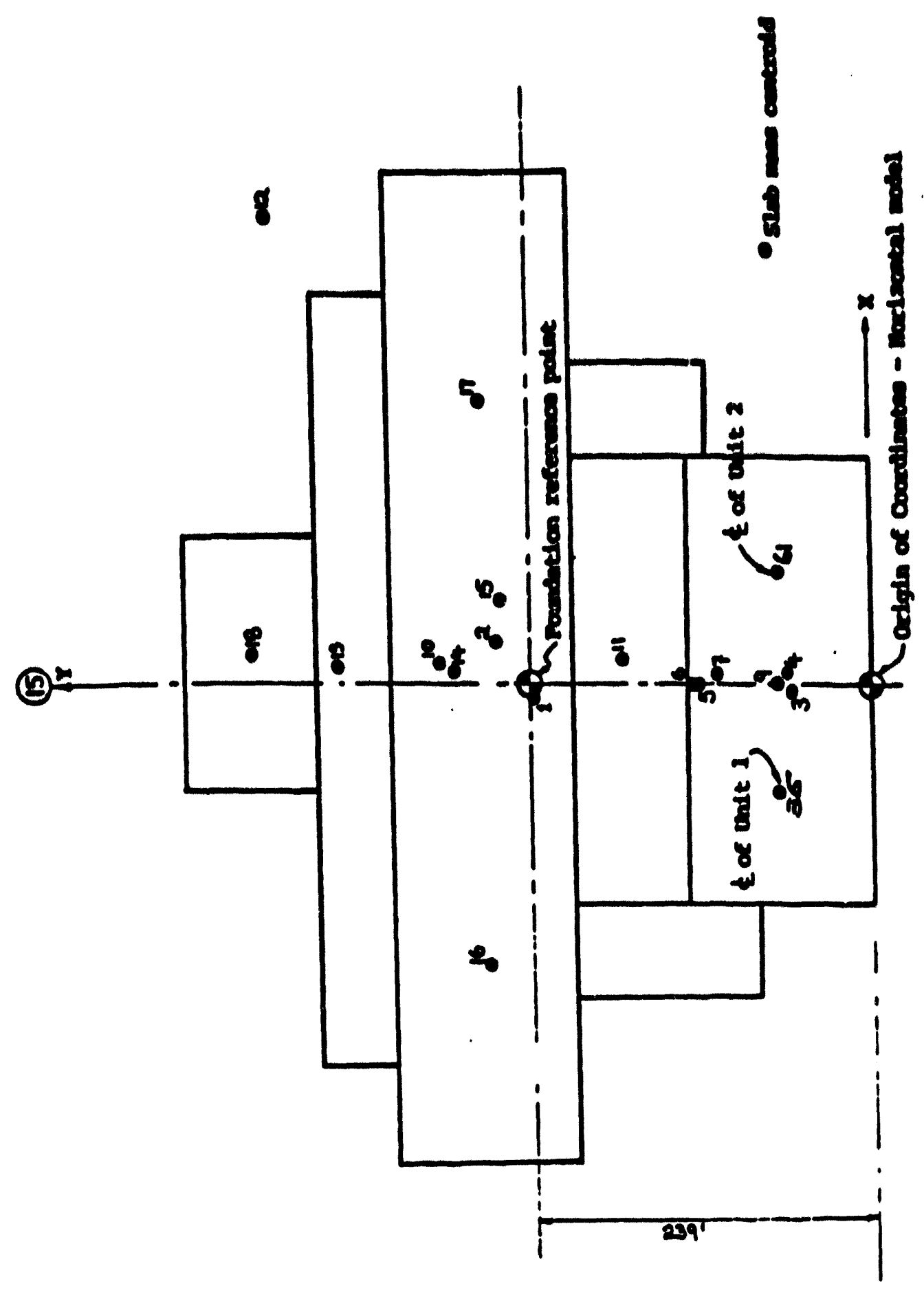

Figure 5.14. Locations of mass points of horizontal model. 
Table 5.2. Description of mass points in horizontal seismic model.

\begin{tabular}{|c|c|c|c|c|}
\hline $\begin{array}{l}\text { Mass } \\
\text { Boins }\end{array}$ & Dascription & Elevation & $\underset{x}{\text { Coordine }}$ & Point \\
\hline 1 & $\begin{array}{l}\text { Reactor, Auxiliary, Turbine } \\
\text { Bldg., Heater Bay Floor }\end{array}$ & $694^{\prime}-6^{\prime \prime}$ & -6.40 & 238.30 \\
\hline 2 & $\begin{array}{l}\text { Reactor, Auxiliary, Turbine } \\
\text { Blds.. Heater Bay Floor }\end{array}$ & $710^{\prime} \cdot 6^{\prime \prime}$ & 27.80 & 261.56 \\
\hline 3 & Reactor Building Floor & $740^{\circ}-0^{\prime \prime}$ & .6 .29 & 54.85 \\
\hline 4 & Reactor Building Floor & $761^{\prime}-0^{\prime \prime}$ & 3.85 & 57.59 \\
\hline 5 & Reaclor, Auxiliary Bldg. Floor & $786^{\prime}-6^{\prime \prime}$ & -0.92 & 119.99 \\
\hline 6 & Reactor Building Floor & $807-0^{n}$ & -1.55 & 124.13 \\
\hline 7 & Reactor, Auxiliary Bldg. & $820^{\prime} \cdot 6^{\prime \prime}$ & 2.28 & 107.45 \\
\hline 8 & Reaclor Building Floor & $843^{\prime}-6^{n}$ & 0.95 & 103.11 \\
\hline 9 & Rcactor Building Roof & $894^{\prime}-0^{n}$ & 0.00 & 64.00 \\
\hline 10 & $\begin{array}{l}\text { Auxiliary, Turbinc, Heater } \\
\text { Bay, Radwaste Bldg. Floor }\end{array}$ & $731^{\prime} 0^{\prime \prime}$ & 12.68 & 299.22 \\
\hline 11 & Auxiliary Building Floor & $749^{\prime}-0^{\prime \prime}$ & .3 .73 & 172.75 \\
\hline 12 & Radwaste Building Floor & $743^{\prime} \cdot 0^{n}$ & 317.00 & 413.44 \\
\hline 13 & Healer Bay Floor & $754^{\prime}-0^{\prime \prime}$ & 9.40 & 370.97 \\
\hline 14 & Auxiliary, Turbine Bldg. Floor & $768^{\prime}-0^{\prime \prime}$ & 5.25 & 290.80 \\
\hline 15 & $\begin{array}{l}\text { Reactor, Auxiliary, Turbine } \\
\text { Bldg., Heatcr Bay, Radwaste } \\
\text { Bldg. Base Slab }\end{array}$ & $673^{\circ} 4^{\prime \prime}$ & 55.38 & 259.96 \\
\hline
\end{tabular}


Table 5.2. Description of mass points in horizontal seismic model. (cont.)

Mass

Poins

16

17

18

21

25

27

28

31

32

34

35

36

37

38

39

40

42

44
Deecrimion

Turbine Bldg. Operating Floor

Turbine Bldg. Operating Floor

Radwaste Building Roof

Containment

Containment

Containment

Containment

Reactor Suppon Pedestal

Top of Pedestal

Top of Biological Shield

Containment

Containment

Containment

Containment

Containment

Containment

Containment

Containment
Elovation

768'-0"

$768^{\prime}-0^{\prime \prime}$

761'-8"

$735^{\prime}-0^{\prime \prime}$

807'-0"

$820^{\prime}-0^{\prime \prime}$

$843^{\prime} \cdot 6^{\prime \prime}$

735'-0"

$775^{\prime} \cdot 1 \cdot 1 / 4^{\prime \prime}$

803'-0-5/8"

$673^{\prime}-4^{\prime \prime}$

$694^{\prime} \cdot 6^{\prime \prime}$

$710^{\prime} \cdot 6^{\prime \prime}$

735'-0"

$755^{\prime} \cdot 1-1 / 4^{\prime \prime}$

$786^{\prime}-6^{\prime \prime}$

$807^{\prime} \cdot 0^{\prime \prime}$

$820^{\prime}-6^{n}$
Coordinates of Mass Points (F)

$\mathbf{x}$

.193 .09

190.16

13.40

76.00

76.00

76.00

76.00

76.00

76.00

76.00

76.00

.76 .00

$-76.00$

.76 .00

.76 .00

.76 .00

.76 .00

.76 .00
270.46

270.46

429.33

64.50

64.50

64.50

64.50

64.50

64.50

64.50

64.50

64.50

64.50

64.50

64.50

64.50

64.50

64.50 
Table 5.2. Description of mass points in horizontal seismic model. (cont.)

Mass

Point

Darcription

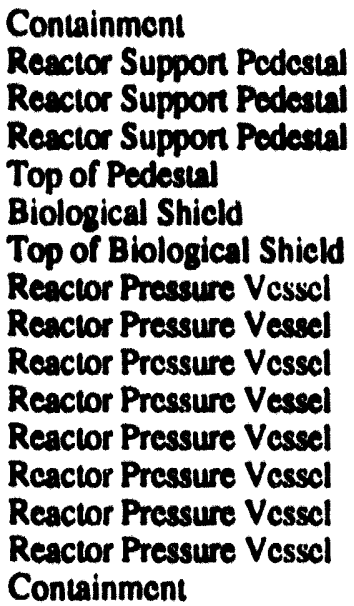

Elcyation

$843^{\prime}-6^{\prime \prime}$

$699^{\prime} \cdot 10^{n}$

$710^{\circ} \cdot 6^{n}$

$735^{\prime}-0^{n}$

$755^{\prime}-1 \cdot 1 / 4^{\prime \prime}$

$786^{\prime} \cdot 6^{\prime \prime}$

$803^{\prime} \cdot 5 / 8^{\prime \prime}$

$760^{\prime}-13 / 16^{\prime \prime}$

$789^{\prime} \cdot 13 / 32^{\prime \prime}$

$803^{\prime} \cdot 5 / 8^{\prime \prime}$

$818^{\prime} \cdot 5 / 8^{\prime \prime}$

$760^{\prime}-13 / 16^{\prime \prime}$

$789^{\prime}-13 / 32^{\prime \prime}$

$803^{\prime} \cdot 5 / 8^{\prime \prime}$

$818^{\prime}-5 / 8^{\prime \prime}$

$673^{\prime}-4^{n}$

\section{Coordinates of Mase Points (Fi)}

\begin{tabular}{c}
$X$ \\
\hline \\
.76 .00 \\
.76 .00 \\
.76 .00 \\
.76 .00 \\
.76 .00 \\
.76 .00 \\
.76 .00 \\
.76 .00 \\
.76 .00 \\
.7600 \\
.76 .00 \\
76.00 \\
76.00 \\
7600 \\
76.00 \\
76.00
\end{tabular}

64.50

64.50

64.50

64.50

64.50

64.50

64.50

64.50

64.50

64.50

64.50

64.50

64.50

64.50

64.50

64.50 


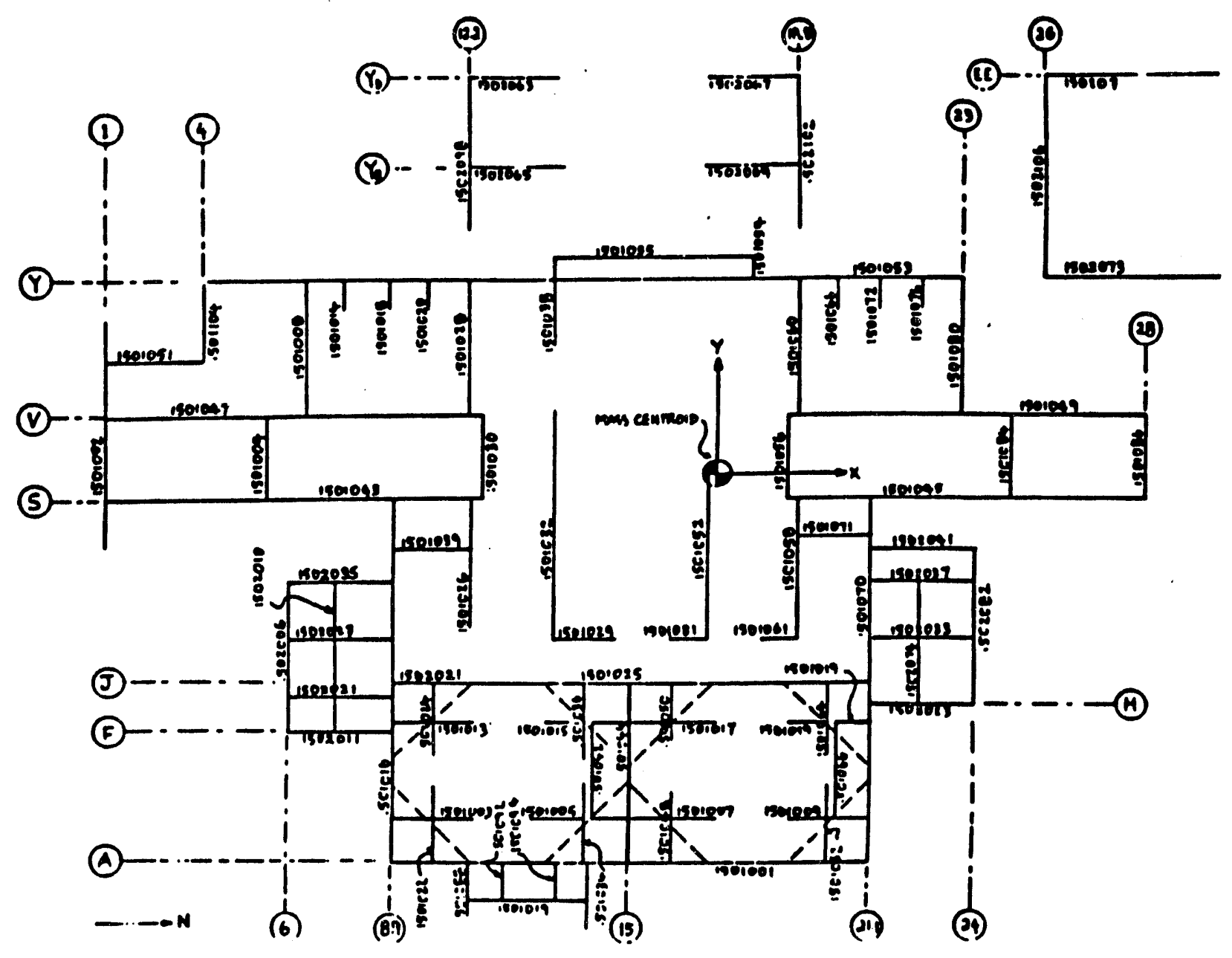

Figure 5.15. Schematic of spring elements representing shear walls and shear wall groups: a) Slab 15 to slabs 1 and 2. 


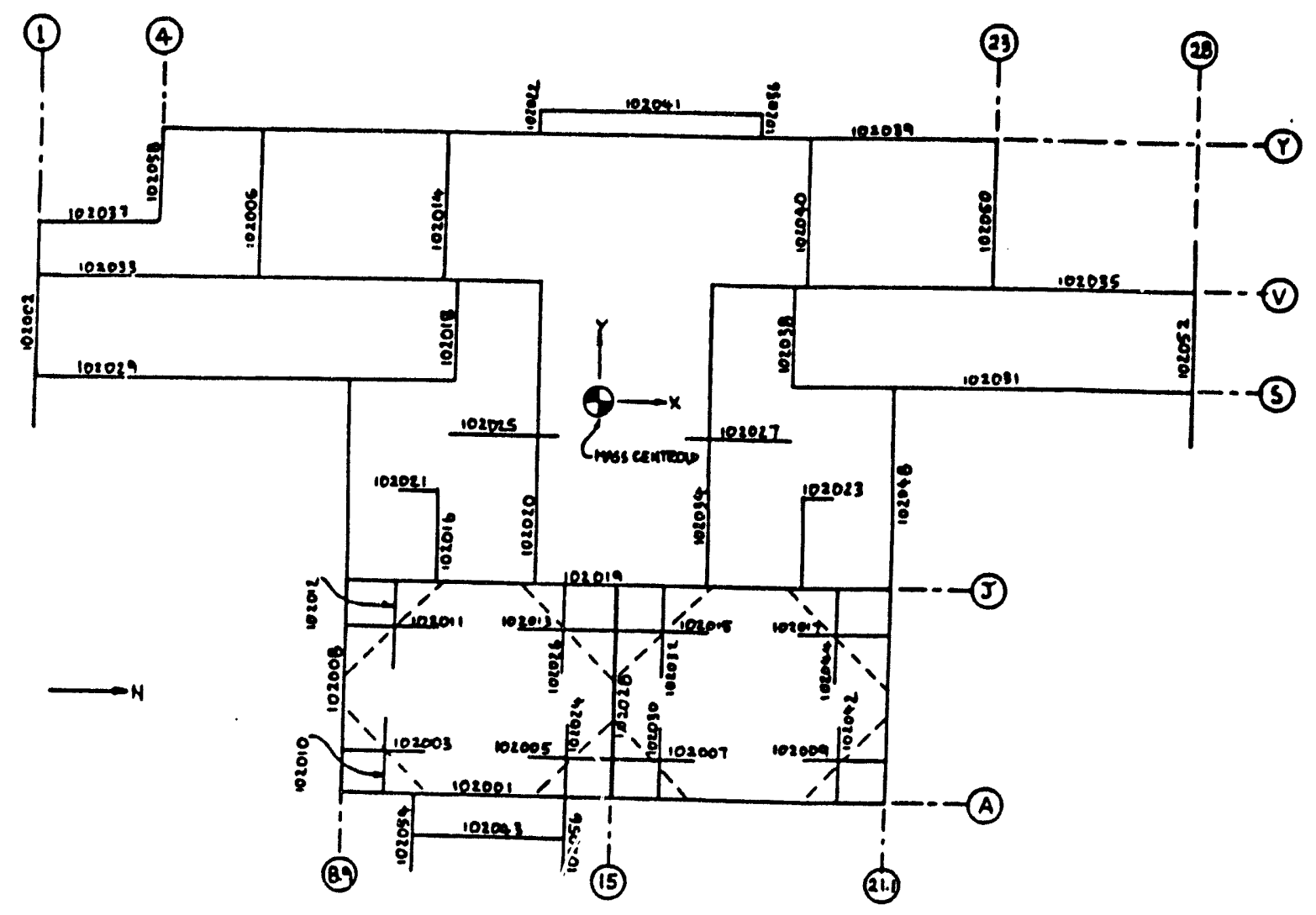

Figure 5.15. Schematic of spring elements representing shear walls and shear wall groups: b) Slab 1 to slab 2. 


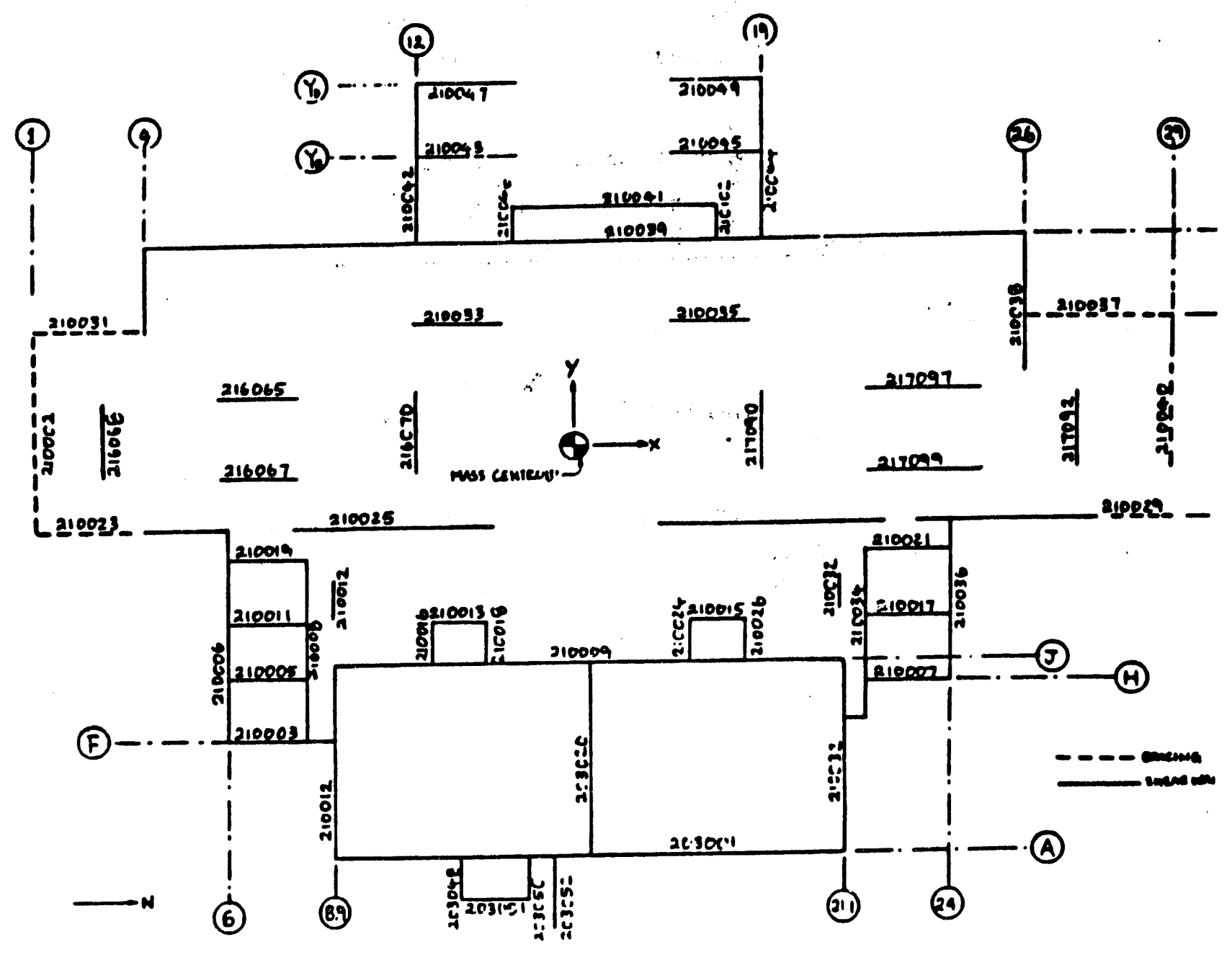

Figure 5.15. Schematic of spring elements representing shear walls and shear wall groups: c) Slab 2 to slabs $3,10$. 


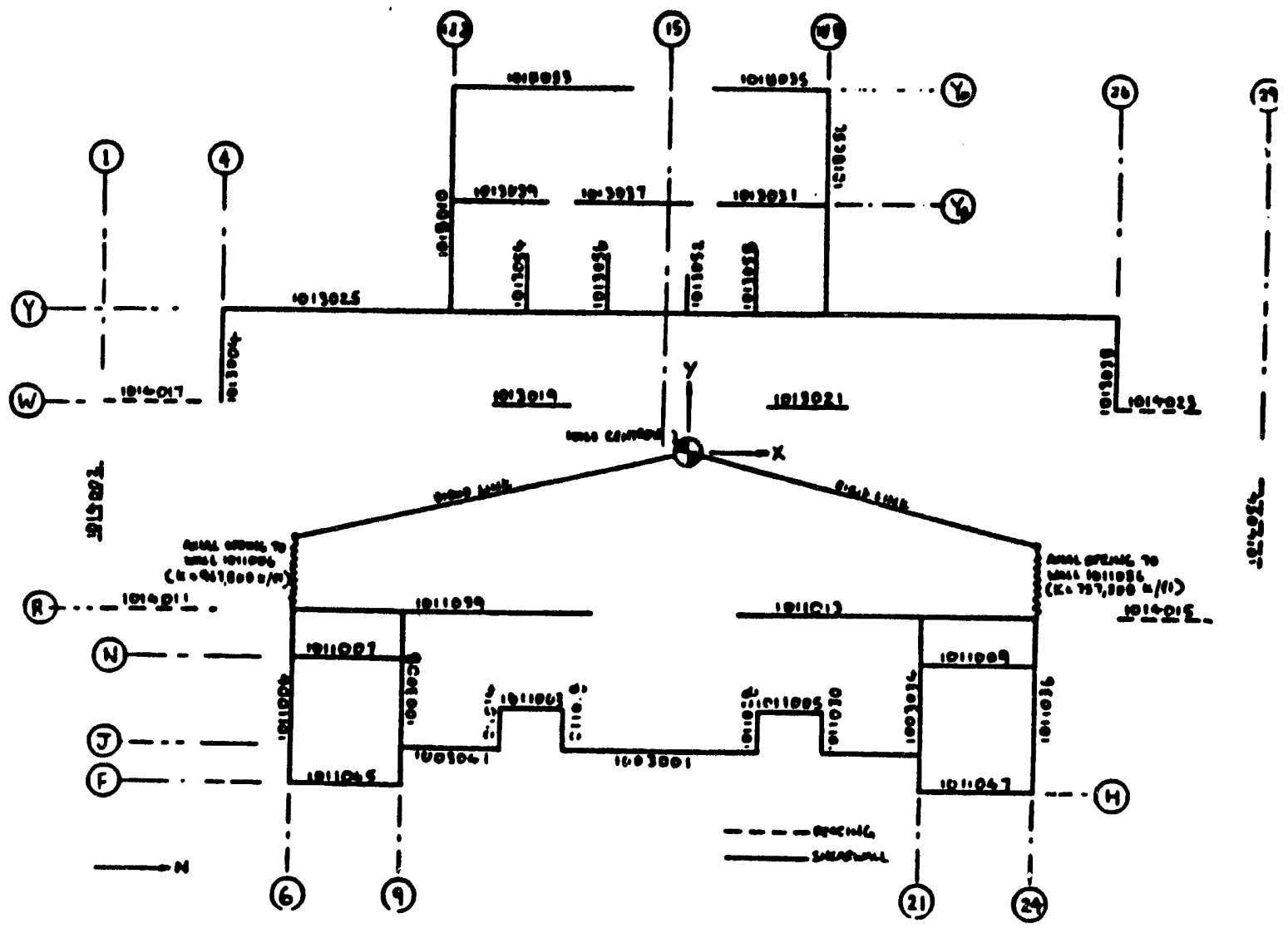

Figure 5.15. Schematic of spring elements representing shear walls and shear wall groups: d) Slab 10 to slabs $3,11,13,14$ and 18 . 


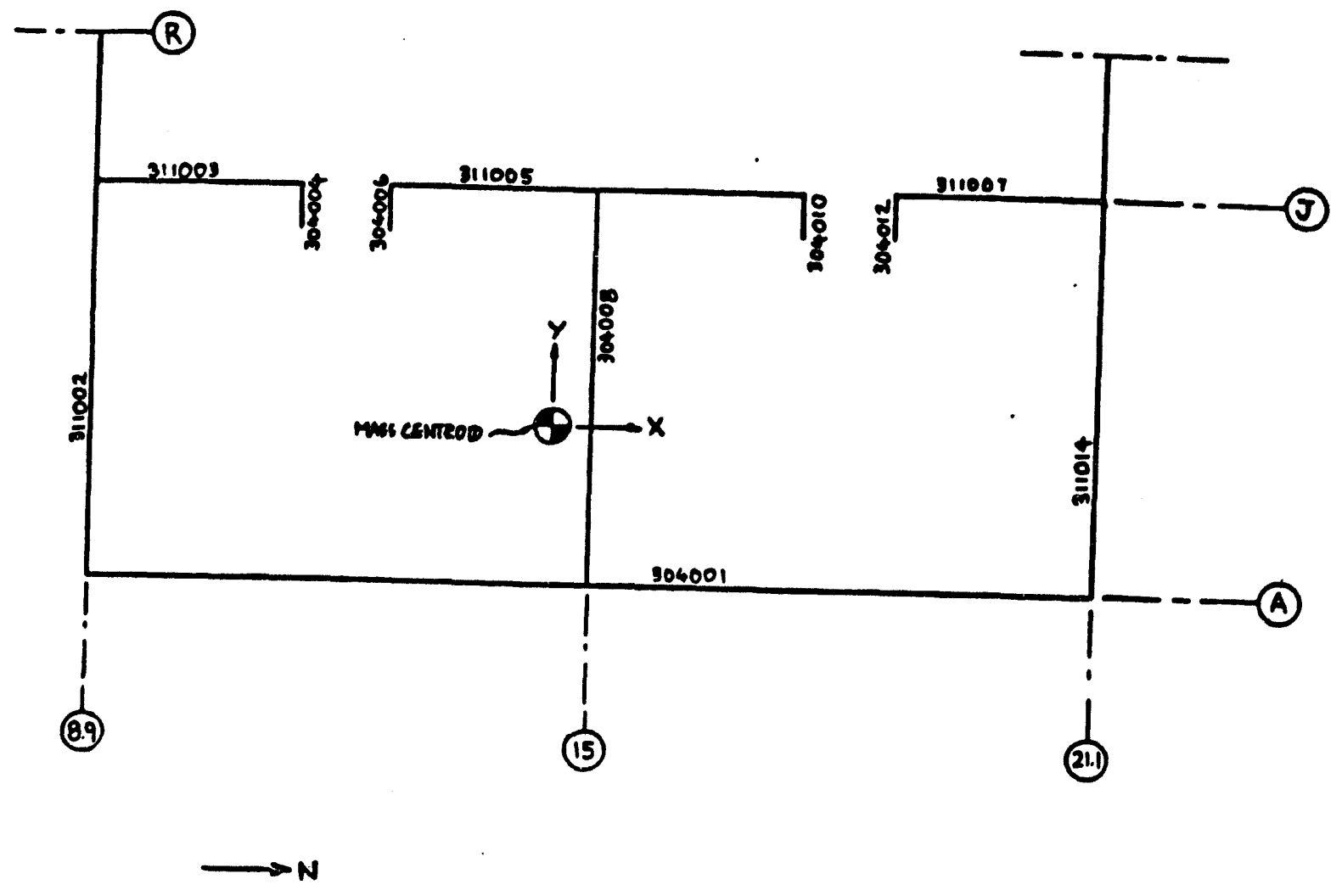

Figure 5.15. Schematic of spring elements representing shear walls and shear wall groups: e) Slab 3 to slabs 4 and 11. 


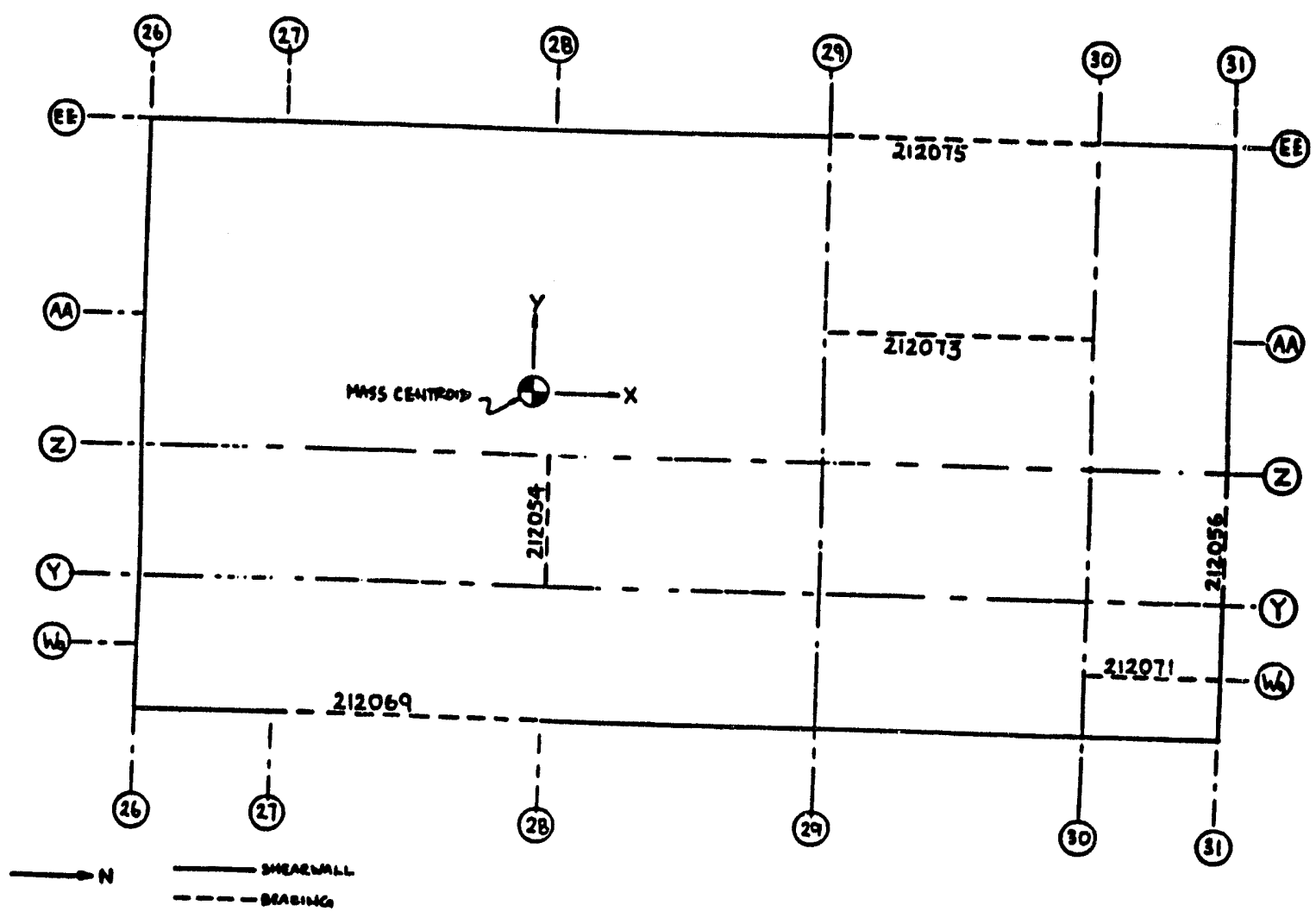

Figure 5.15. Schematic of spring elements representing shear walls and shear wall groups: f) Slab 2 to slab 12 . 


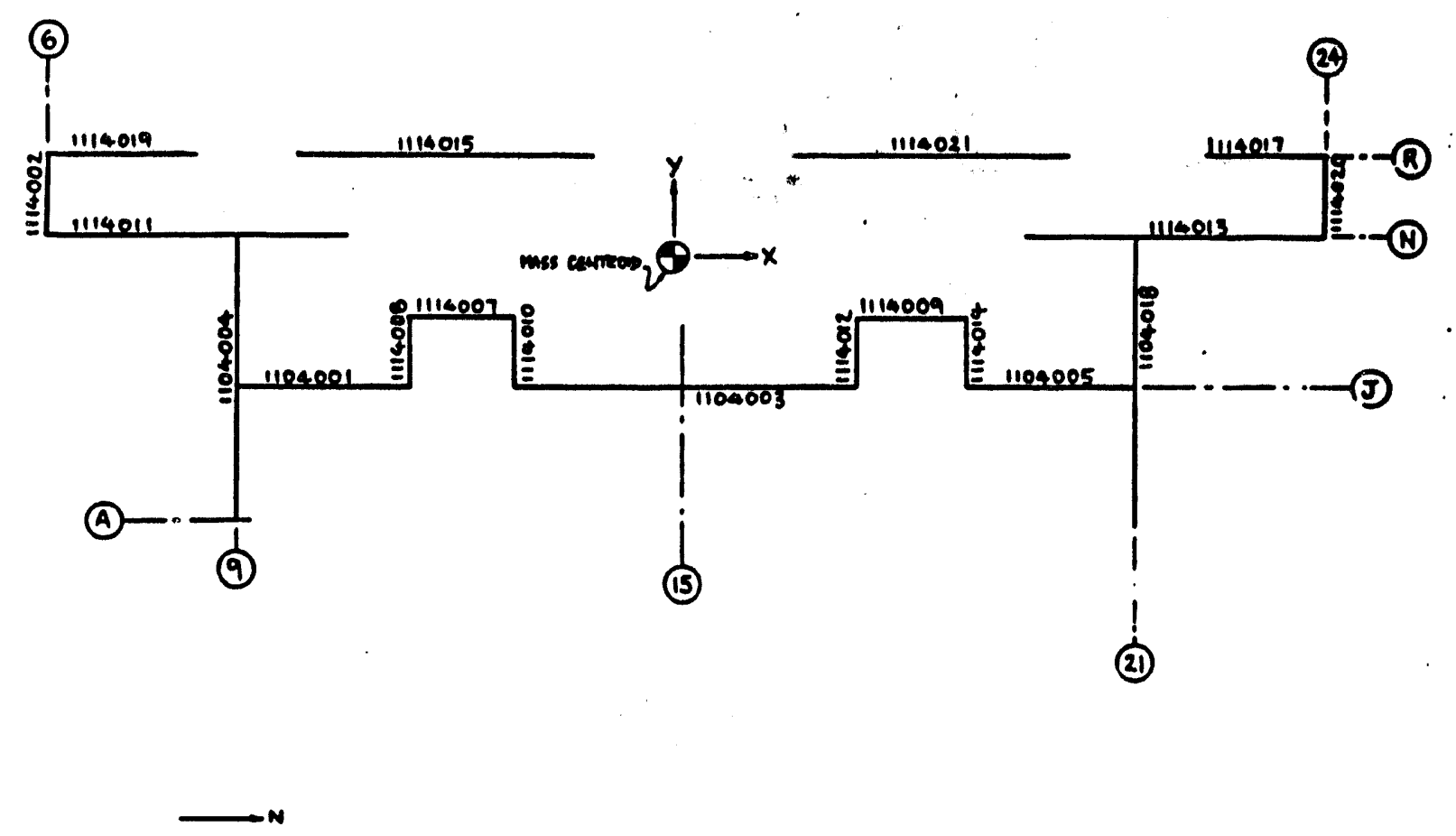

Figure 5.15. Schematic of spring elements representing shear walls and shear wall groups: g) Slab 11 to slabs 4 and 14 . 


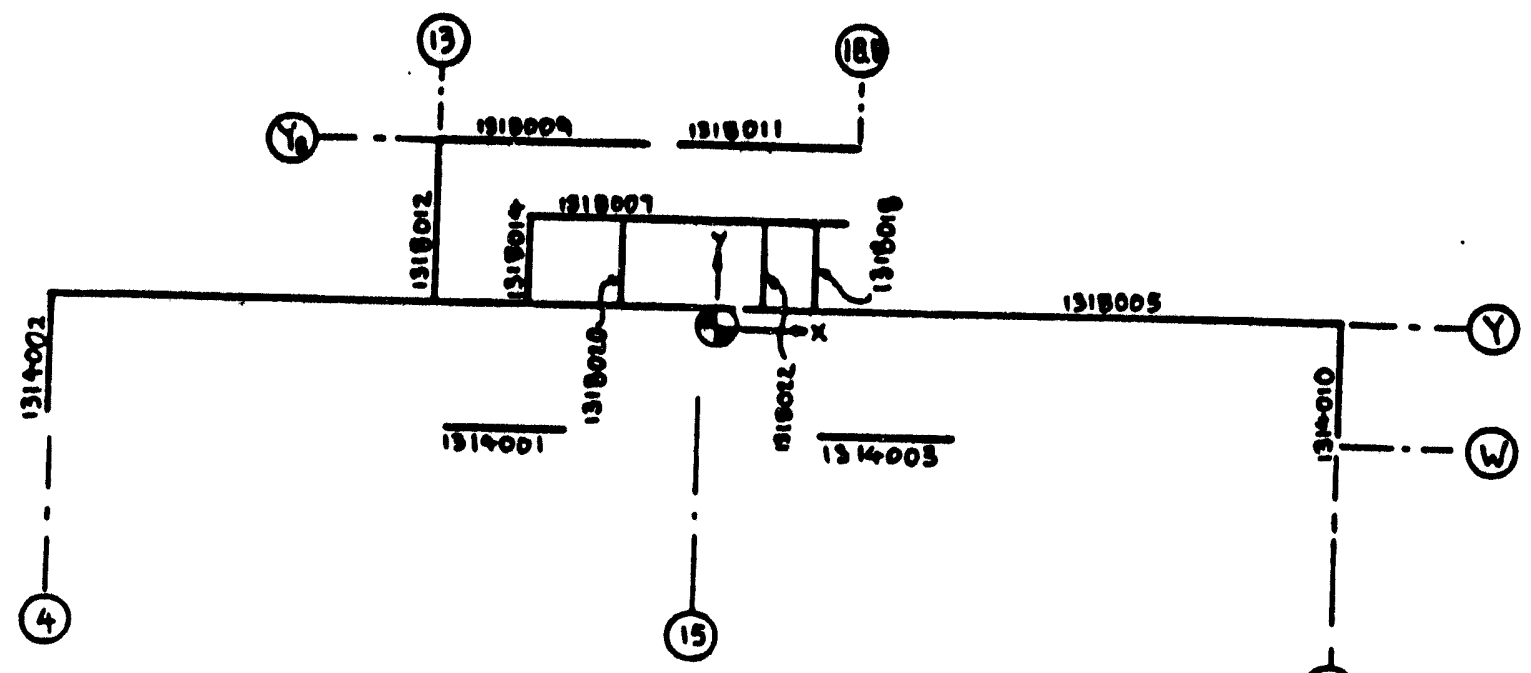

(26)

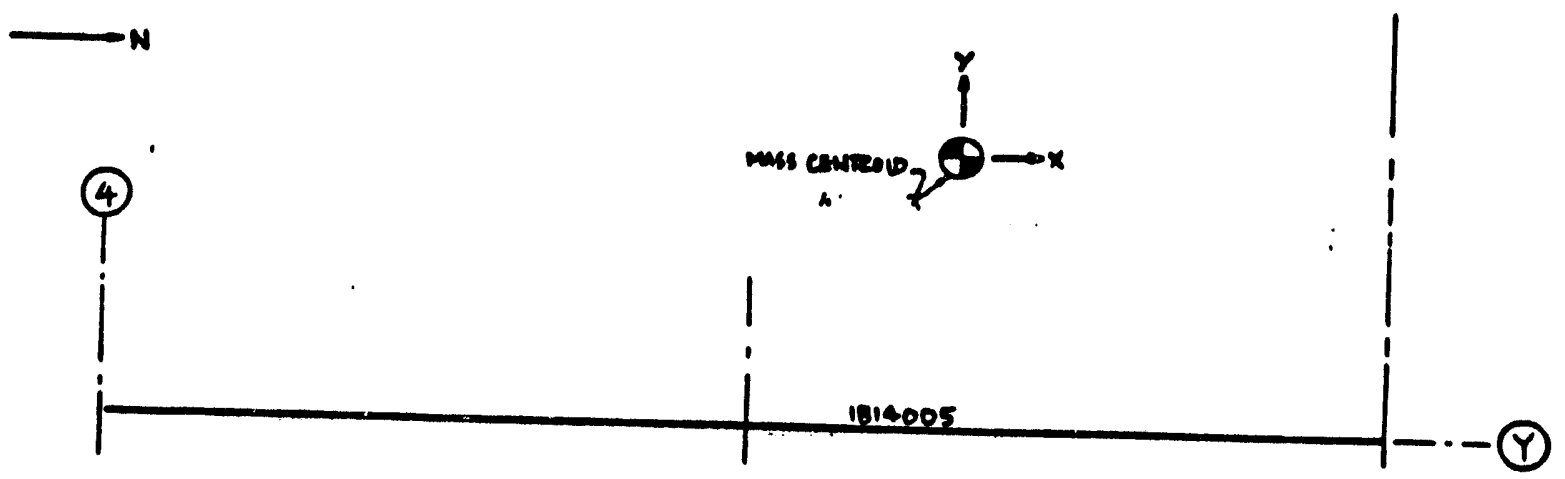

Figure 5.15. Schematic of spring elements representing shear walls and shear wall groups: h) Slab 13 to slabs 14 and 18. 
•t pue s sqejs ol $\bullet$ qeis (! :sdnod8

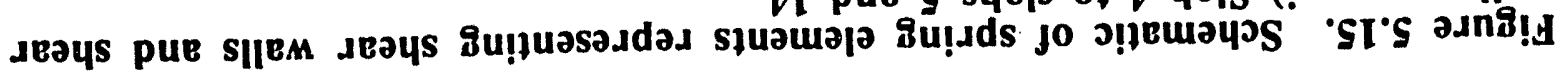
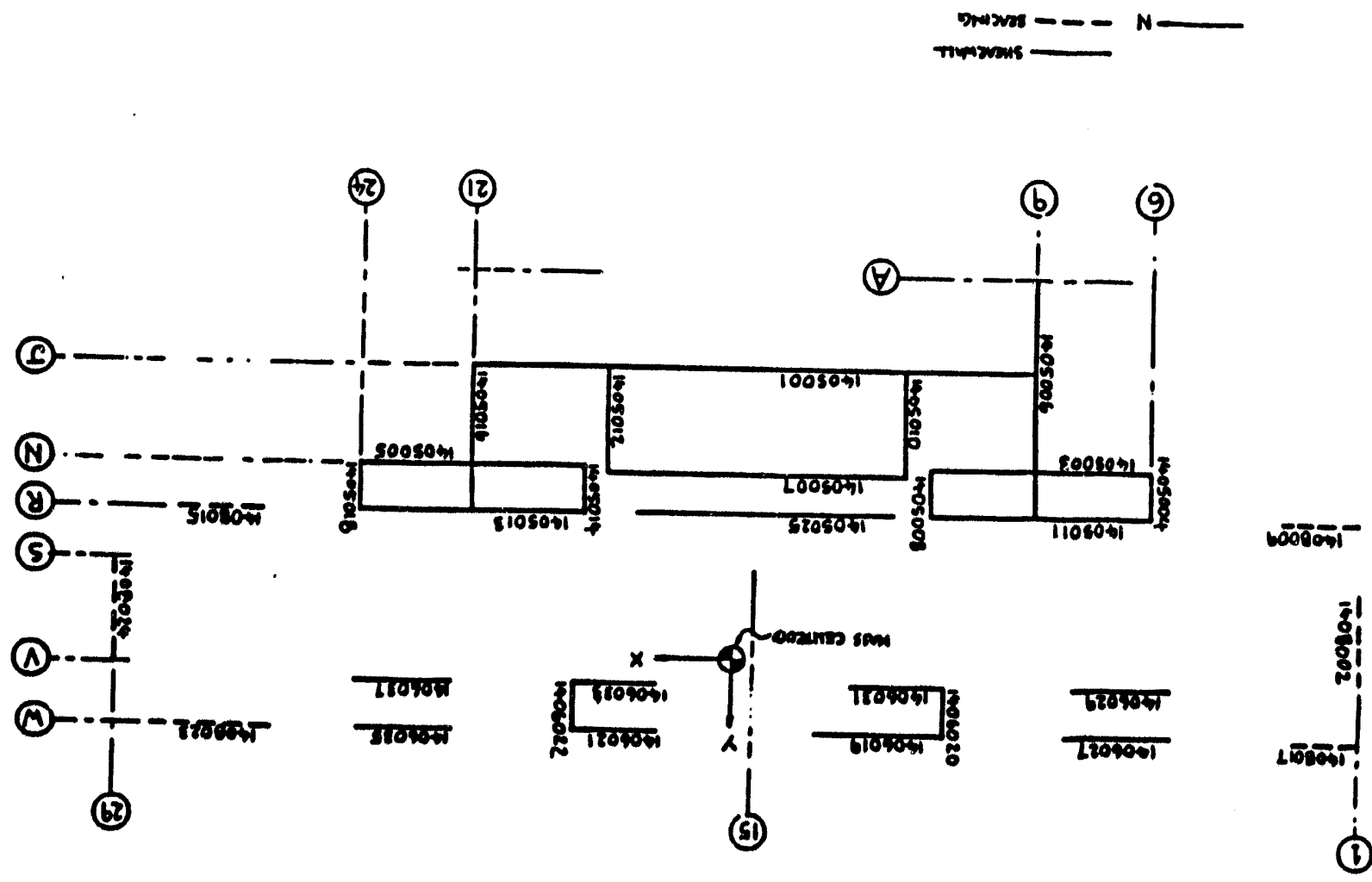


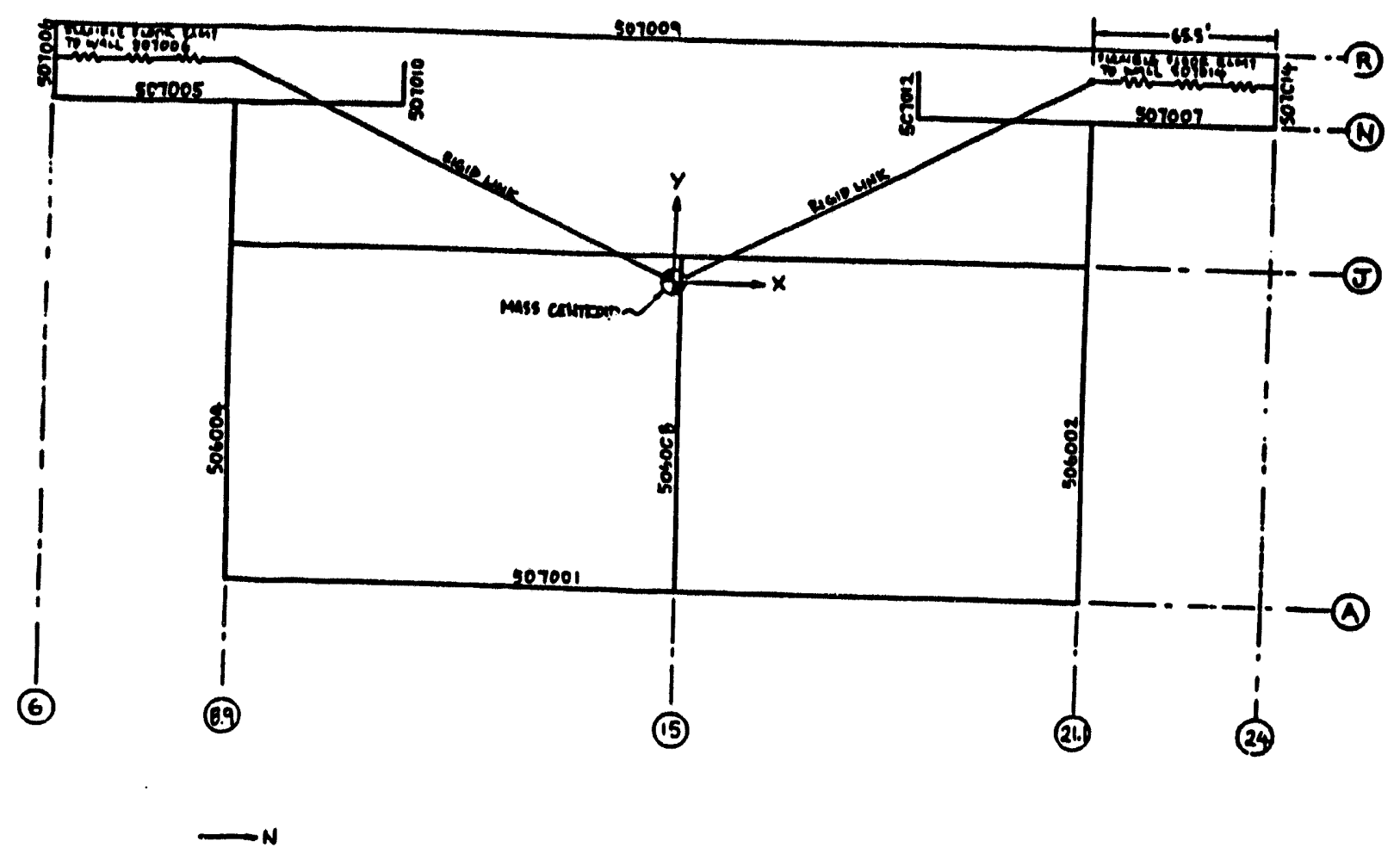

Figure 5.15. Schematic of spring elements representing shear walls and shear wall groups: j) Slab 14 to slabs 5, 6 and 8. 


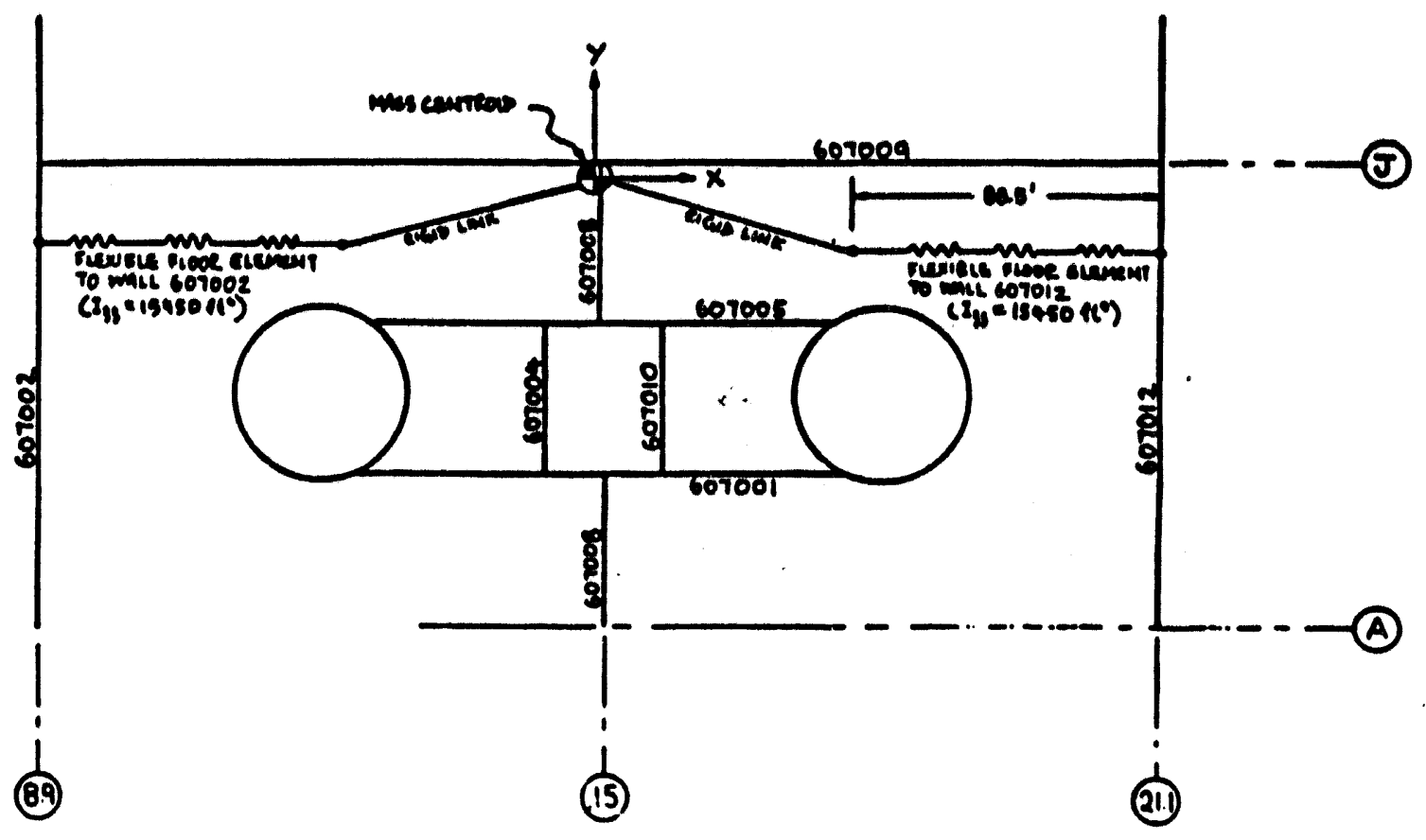

Figure 5.15. Schematic of spring elements representing shear walls and shear wall groups:L k) Slab 5 to slabs 6 and 7.

$-77-$ 


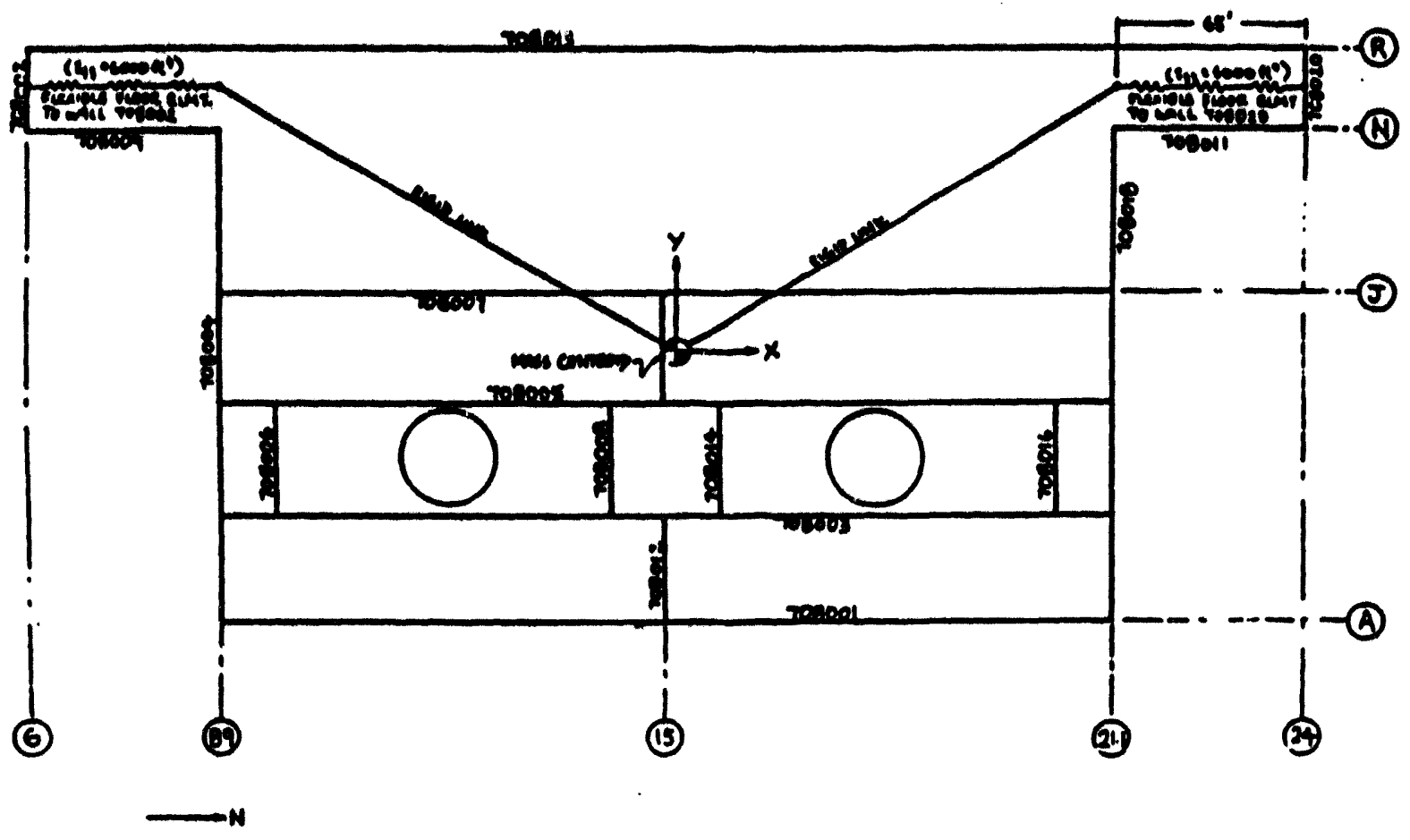

Figure 5.15. Schematic of spring elements representing shear walls and shear wall groups: I) Slab 6 to slab 7. 


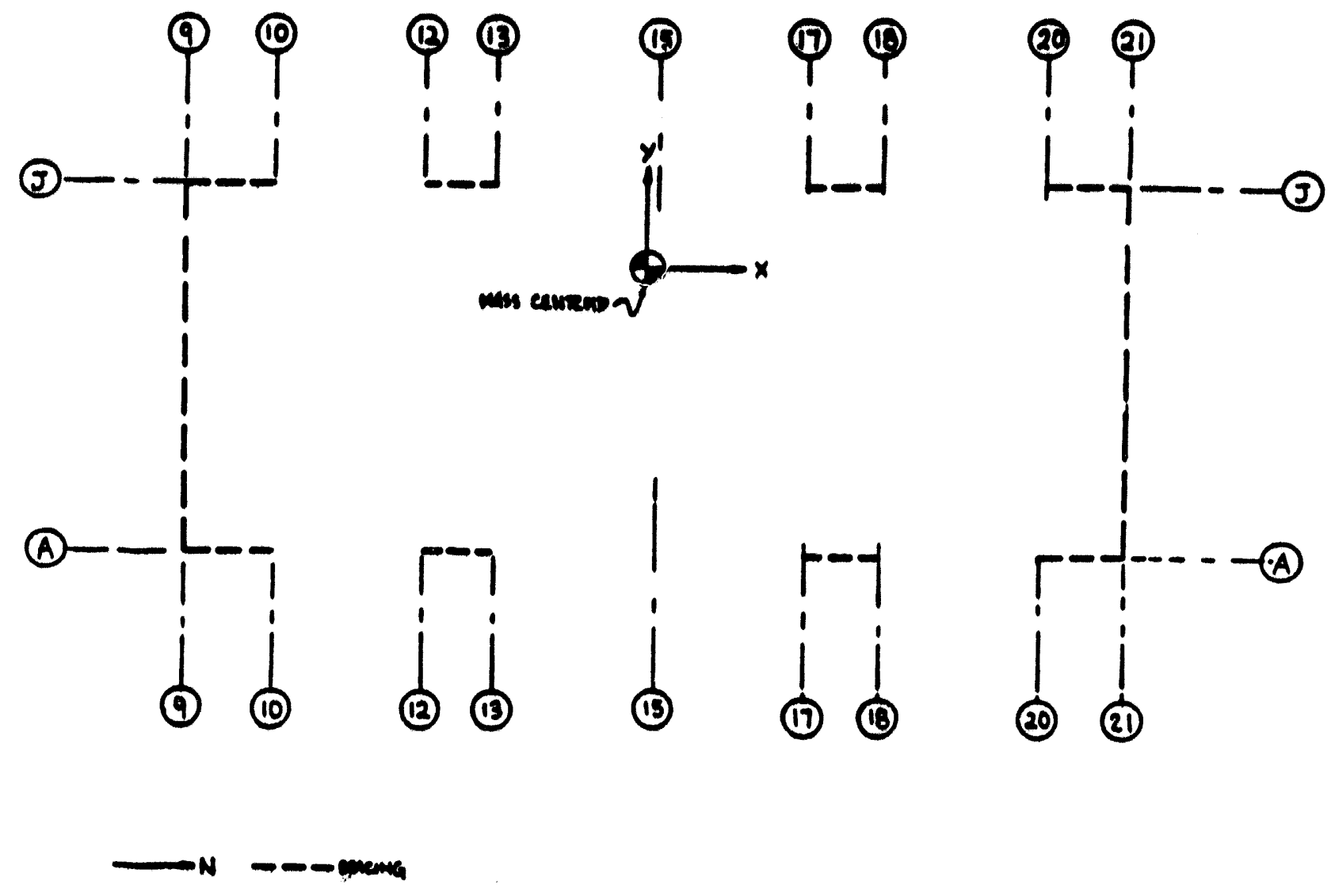

Figure 5.15. Schematic of spring elements representing shear walls and shear wall groups: $m$ ) Slab 7 to slab 8. 


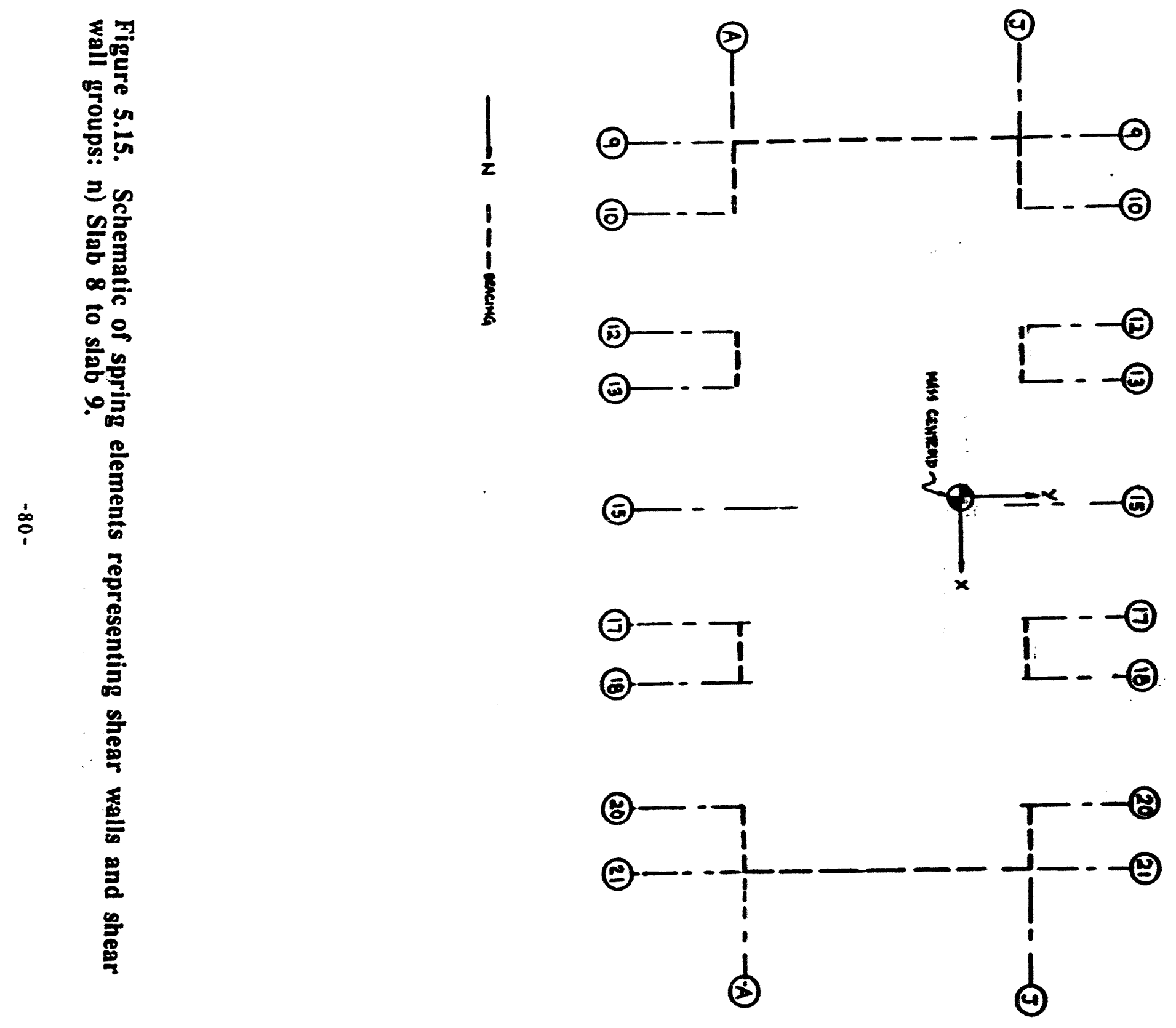




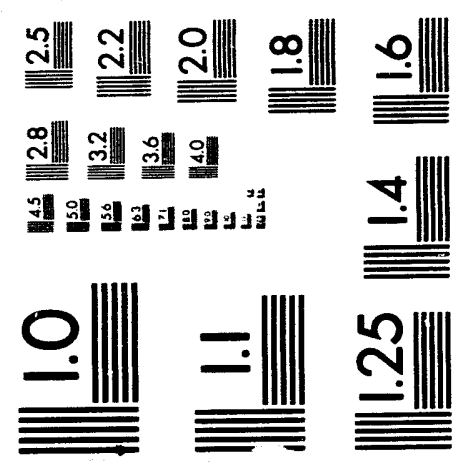



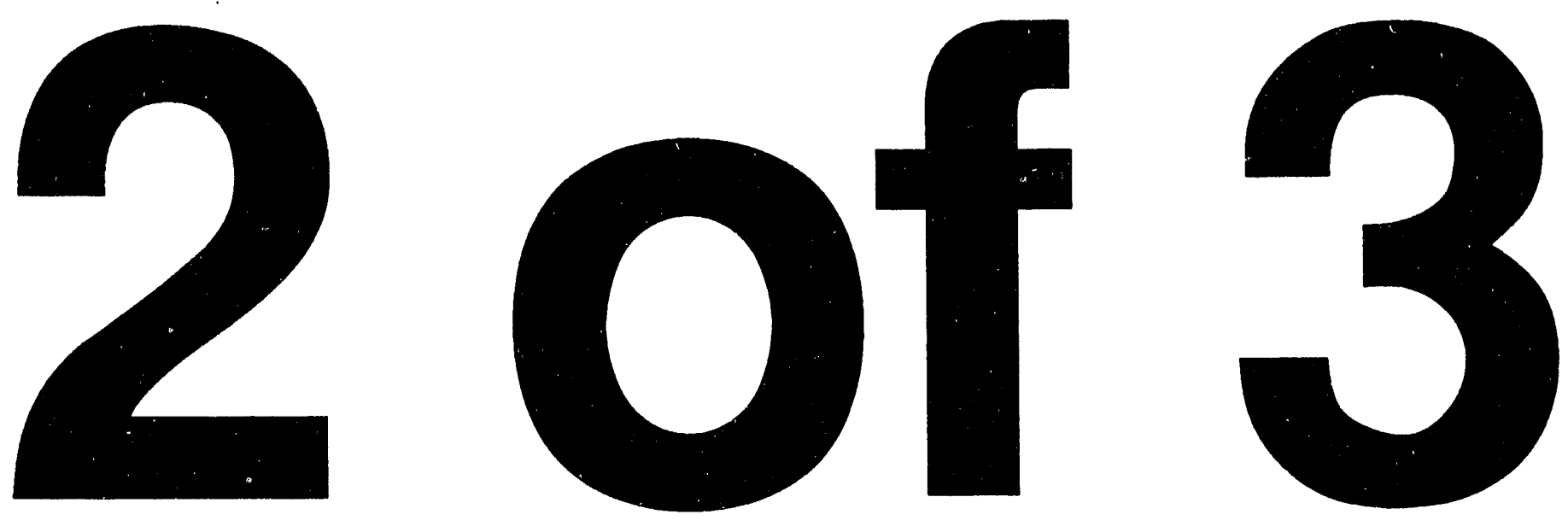
Table 5.3. Identification of shear walls and shear wall groups, horizontal model, X-direction (N-S).

\begin{tabular}{|c|c|c|c|}
\hline $\begin{array}{c}\text { Group } \\
\text { No. }\end{array}$ & $\begin{array}{l}\text { Slab } \\
\text { No. }\end{array}$ & $\begin{array}{l}\text { Spring } \\
\text { Above }\end{array}$ & $\begin{array}{l}\text { Spring } \\
\text { Below }\end{array}$ \\
\hline 1 & $\begin{array}{r}8 \\
7 \\
5 \\
4 \\
3 \\
2 \\
1 \\
15\end{array}$ & $\begin{array}{r}708001 \\
507001 \\
405001 \\
304001 \\
203001 \\
102001 \\
1501001\end{array}$ & $\begin{array}{r}708001 \\
507001 \\
405001 \\
304001 \\
203001 \\
102001 \\
1501001\end{array}$ \\
\hline 2 & $\begin{array}{r}8 \\
7 \\
6 \\
5 \\
14 \\
4 \\
11 \\
3 \\
10 \\
2 \\
1 \\
15\end{array}$ & $\begin{array}{r}708007 \\
607009 \\
506003 \\
1405001 \\
414003 \\
1104003 \\
311005 \\
1003001 \\
210009 \\
102019 \\
1501025\end{array}$ & $\begin{array}{r}708007 \\
607009 \\
506003 \\
1405001 \\
414003 \\
1104003 \\
311005 \\
1003001 \\
210009 \\
102019 \\
1501025\end{array}$ \\
\hline 3 & $\begin{array}{r}2 \\
1 \\
15\end{array}$ & $\begin{array}{r}102029 \\
1501043\end{array}$ & $\begin{array}{r}102029 \\
1501043\end{array}$ \\
\hline 4 & $\begin{array}{r}2 \\
1 \\
15\end{array}$ & $\begin{array}{r}102031 \\
1501045\end{array}$ & $\begin{array}{r}102031 \\
1501045\end{array}$ \\
\hline 5 & $\begin{array}{r}2 \\
1 \\
15\end{array}$ & $\begin{array}{r}102033 \\
1501047\end{array}$ & $\begin{array}{r}102033 \\
1501047\end{array}$ \\
\hline 6 & $\begin{array}{r}2 \\
1 \\
15\end{array}$ & $\begin{array}{r}102035 \\
1501049\end{array}$ & $\begin{array}{r}102035 \\
1501049\end{array}$ \\
\hline 7 & $\begin{array}{r}14 \\
18 \\
13 \\
10 \\
2\end{array}$ & $\begin{array}{r}1814005 \\
1318005 \\
1013025 \\
210039\end{array}$ & $\begin{array}{r}1814005 \\
1318005 \\
1013025 \\
210039 \\
102039\end{array}$ \\
\hline
\end{tabular}


Table 5.3. Identification of shear walls and shear wall groups, horizontal model, X-direction (N-S). (cont.)

\begin{tabular}{|c|c|c|c|}
\hline & $\begin{array}{r}1 \\
15\end{array}$ & $\begin{array}{r}102039 \\
1501053\end{array}$ & 1501053 \\
\hline 8 & $\begin{array}{r}10 \\
2 \\
1 \\
15\end{array}$ & $\begin{array}{r}210041 \\
102041 \\
1501055\end{array}$ & $\begin{array}{r}210041 \\
102041 \\
1501055\end{array}$ \\
\hline 9 & $\begin{array}{r}2 \\
15\end{array}$ & 1502057 & 1502057 \\
\hline 10 & $\begin{array}{r}3 \\
2 \\
1 \\
15\end{array}$ & $\begin{array}{r}203051 \\
102043 \\
1501059\end{array}$ & $\begin{array}{r}203051 \\
102043 \\
1501059\end{array}$ \\
\hline 11 & $\begin{array}{r}18 \\
13 \\
10 \\
2 \\
15\end{array}$ & $\begin{array}{r}1318009 \\
1013039 \\
210043 \\
1502065\end{array}$ & $\begin{array}{r}1318009 \\
1013039 \\
210043 \\
1502065\end{array}$ \\
\hline 12 & $\begin{array}{r}18 \\
13 \\
10 \\
2 \\
15\end{array}$ & $\begin{array}{r}1318011 \\
1013031 \\
210045 \\
1502069\end{array}$ & $\begin{array}{r}1318011 \\
1013031 \\
210045 \\
1502069\end{array}$ \\
\hline 13 & $\begin{array}{r}18 \\
10 \\
2 \\
15\end{array}$ & $\begin{array}{r}1018033 \\
210047 \\
1502063\end{array}$ & $\begin{array}{r}1018033 \\
210047 \\
1502063\end{array}$ \\
\hline 14 & $\begin{array}{r}18 \\
10 \\
2 \\
15\end{array}$ & $\begin{array}{r}1018035 \\
210049 \\
1502067\end{array}$ & $\begin{array}{r}1018035 \\
210049 \\
1502067\end{array}$ \\
\hline 15 & $\begin{array}{r}2 \\
1 \\
15\end{array}$ & $\begin{array}{r}102005 \\
1501005\end{array}$ & $\begin{array}{r}102005 \\
1501005\end{array}$ \\
\hline 16 & $\begin{array}{r}2 \\
1 \\
15\end{array}$ & $\begin{array}{r}102007 \\
1501007\end{array}$ & $\begin{array}{r}102007 \\
1501007\end{array}$ \\
\hline
\end{tabular}


Table 5.3. Identification of shear walls and shear wall groups, horizontal model, X-direction (N-S). (cont.)

\begin{tabular}{|c|c|c|c|}
\hline 17 & $\begin{array}{r}2 \\
1 \\
15\end{array}$ & $\begin{array}{r}102013 \\
1501015\end{array}$ & $\begin{array}{r}102013 \\
1501015\end{array}$ \\
\hline 18 & $\begin{array}{r}2 \\
1 \\
15\end{array}$ & $\begin{array}{r}102015 \\
1501017\end{array}$ & $\begin{array}{r}102015 \\
1501017\end{array}$ \\
\hline 19 & $\begin{array}{r}2 \\
1 \\
15\end{array}$ & $\begin{array}{r}102003 \\
1501003\end{array}$ & $\begin{array}{r}102003 \\
1501003\end{array}$ \\
\hline 20 & $\begin{array}{r}2 \\
1 \\
15\end{array}$ & $\begin{array}{r}102009 \\
1501009\end{array}$ & $\begin{array}{r}102009 \\
1501009\end{array}$ \\
\hline 21 & $\begin{array}{r}2 \\
1 \\
15\end{array}$ & $\begin{array}{r}102011 \\
1501013\end{array}$ & $\begin{array}{r}102011 \\
1501013\end{array}$ \\
\hline 22 & $\begin{array}{r}2 \\
1 \\
15\end{array}$ & $\begin{array}{r}102017 \\
1501019\end{array}$ & $\begin{array}{r}102017 \\
1501019\end{array}$ \\
\hline 23 & $\begin{array}{r}11 \\
10 \\
2 \\
15\end{array}$ & $\begin{array}{r}1011045 \\
210003 \\
1502011\end{array}$ & $\begin{array}{r}1011045 \\
210003 \\
1502011\end{array}$ \\
\hline 24 & $\begin{array}{r}11 \\
10 \\
2 \\
15\end{array}$ & $\begin{array}{r}1011047 \\
210007 \\
1502023\end{array}$ & $\begin{array}{r}1011047 \\
210007 \\
1502023\end{array}$ \\
\hline 25 & $\begin{array}{r}10 \\
2 \\
15\end{array}$ & $\begin{array}{r}210017 \\
1502033\end{array}$ & $\begin{array}{r}210017 \\
1502033\end{array}$ \\
\hline 26 & $\begin{array}{r}10 \\
2 \\
15\end{array}$ & $\begin{array}{r}210021 \\
1502037\end{array}$ & $\begin{array}{r}210021 \\
1502037\end{array}$ \\
\hline 27 & $\begin{array}{r}2 \\
1 \\
15\end{array}$ & $\begin{array}{r}102037 \\
1501051\end{array}$ & $\begin{array}{r}102037 \\
1501051\end{array}$ \\
\hline
\end{tabular}


Table 5.3. Identification of shear walls and shear wall groups, horizontal model, X-direction (N-S). (cont.)

\begin{tabular}{|c|c|c|c|}
\hline 28 & $\begin{array}{r}8 \\
7 \\
5 \\
14 \\
11 \\
10\end{array}$ & $\begin{array}{r}708009 \\
507005 \\
1405003 \\
1114011 \\
1011007\end{array}$ & $\begin{array}{r}708009 \\
507005 \\
1405003 \\
1114011 \\
1011007\end{array}$ \\
\hline 29 & $\begin{array}{r}8 \\
7 \\
5 \\
14 \\
11 \\
10\end{array}$ & $\begin{array}{r}708011 \\
507007 \\
1405005 \\
1114013 \\
1011009\end{array}$ & $\begin{array}{r}708011 \\
507007 \\
1405005 \\
1114013 \\
1011009\end{array}$ \\
\hline 30 & $\begin{array}{l}13 \\
10\end{array}$ & 1013037 & 1013037 \\
\hline 31 & $\begin{array}{l}18 \\
13\end{array}$ & 1318007 & 1318007 \\
\hline 32 & $\begin{array}{r}8 \\
7 \\
5 \\
14 \\
11 \\
10 \\
2 \\
1\end{array}$ & $\begin{array}{r}708013 \\
507009 \\
1405013 \\
1114021 \\
1011013 \\
210027 \\
102025\end{array}$ & $\begin{array}{r}708013 \\
507009 \\
1405013 \\
1114021 \\
1011013 \\
210027 \\
102025\end{array}$ \\
\hline 33 & $\begin{array}{r}11 \\
10 \\
2\end{array}$ & $\begin{array}{r}1011003 \\
210013\end{array}$ & $\begin{array}{r}1011003 \\
210013\end{array}$ \\
\hline 34 & $\begin{array}{r}11 \\
10 \\
2\end{array}$ & $\begin{array}{r}1011005 \\
210015\end{array}$ & $\begin{array}{r}1011005 \\
210015\end{array}$ \\
\hline 35 & $\begin{array}{r}8 \\
7 \\
5 \\
14 \\
11 \\
10 \\
2 \\
1\end{array}$ & $\begin{array}{r}708013 \\
5070(09 \\
1405011 \\
1114015 \\
1011039 \\
210025 \\
102025\end{array}$ & $\begin{array}{r}708013 \\
507009 \\
1405011 \\
1114015 \\
1011039 \\
210025 \\
102025\end{array}$ \\
\hline
\end{tabular}


Table 5.3. Identification of shear walls and shear wall groups, horizontal model, X-direction (N-S). (cont.)

\begin{tabular}{|c|c|c|c|}
\hline 36 & $\begin{array}{r}6 \\
14 \\
13 \\
10 \\
2\end{array}$ & $\begin{array}{r}1406019 \\
1314001 \\
1013019 \\
210033\end{array}$ & $\begin{array}{r}1406019 \\
1314001 \\
1013019 \\
210033\end{array}$ \\
\hline 37 & $\begin{array}{r}6 \\
14 \\
13 \\
10 \\
2\end{array}$ & $\begin{array}{r}1406021 \\
1314003 \\
1013021 \\
210035\end{array}$ & $\begin{array}{r}1406021 \\
1314003 \\
1013021 \\
210035\end{array}$ \\
\hline 38 & $\begin{array}{r}16 \\
2\end{array}$ & 216067 & 216067 \\
\hline 39 & $\begin{array}{r}17 \\
2\end{array}$ & 217097 & 217097 \\
\hline 40 & $\begin{array}{l}8 \\
7 \\
6\end{array}$ & $\begin{array}{l}708005 \\
607005\end{array}$ & $\begin{array}{l}708005 \\
607005\end{array}$ \\
\hline 41 & $\begin{array}{l}8 \\
7 \\
6\end{array}$ & $\begin{array}{l}708003 \\
607001\end{array}$ & $\begin{array}{l}708003 \\
607001\end{array}$ \\
\hline 42 & $\begin{array}{r}5 \\
14\end{array}$ & 1405025 & 1405025 \\
\hline 43 & $\begin{array}{r}6 \\
14\end{array}$ & 1406029 & 1406029 \\
\hline 44 & $\begin{array}{r}6 \\
14\end{array}$ & 1406031 & 1406031 \\
\hline 45 & $\begin{array}{r}6 \\
14\end{array}$ & 1406033 & 1406033 \\
\hline 46 & $\begin{array}{r}5 \\
14 \\
11 \\
10 \\
2\end{array}$ & $\begin{array}{r}1405013 \\
1114017 \\
1011013 \\
210027\end{array}$ & $\begin{array}{r}1405013 \\
1114017 \\
1011013 \\
210027\end{array}$ \\
\hline 47 & $\begin{array}{r}6 \\
14\end{array}$ & 1406037 & 1406037 \\
\hline
\end{tabular}


Table 5.3. Identification of shear walls and shear wall groups, horizontal model, $\mathrm{X}$-direction (N-S). (cont.)

\begin{tabular}{rrrr}
\hline 48 & 10 & & 210005 \\
& 2 & 210005 & 1502021 \\
& 15 & 1502021 & \\
49 & 2 & & 1502073 \\
& 15 & 1502073 & 1502075
\end{tabular}


Table 5.4. Identification of shear walls and shear wall groups, horizontal model, Y-direction

\begin{tabular}{|c|c|c|c|}
\hline $\begin{array}{c}\text { Group } \\
\text { No. }\end{array}$ & $\begin{array}{l}\text { Slab } \\
\text { No. }\end{array}$ & $\begin{array}{l}\text { Spring } \\
\text { Above }\end{array}$ & $\begin{array}{l}\text { Spring } \\
\text { Below }\end{array}$ \\
\hline 1 & $\begin{array}{r}2 \\
1 \\
15\end{array}$ & $\begin{array}{r}102002 \\
1501002\end{array}$ & $\begin{array}{r}102002 \\
1501002\end{array}$ \\
\hline 2 & $\begin{array}{r}2 \\
1 \\
15\end{array}$ & $\begin{array}{r}102016 \\
1501026\end{array}$ & $\begin{array}{r}102016 \\
1501026\end{array}$ \\
\hline 3 & $\begin{array}{r}2 \\
1 \\
15\end{array}$ & $\begin{array}{r}102014 \\
1501028\end{array}$ & $\begin{array}{r}102014 \\
1501028\end{array}$ \\
\hline 4 & $\begin{array}{r}18 \\
13 \\
10 \\
2 \\
1 \\
15\end{array}$ & $\begin{array}{r}1318018 \\
1013058 \\
210102 \\
102036 \\
1501054\end{array}$ & $\begin{array}{r}1318018 \\
1013058 \\
210102 \\
102036 \\
1501054\end{array}$ \\
\hline 5 & $\begin{array}{r}2 \\
1 \\
15\end{array}$ & $\begin{array}{r}102046 \\
1501058\end{array}$ & $\begin{array}{r}102046 \\
1501058\end{array}$ \\
\hline 6 & $\begin{array}{r}2 \\
15\end{array}$ & 1502010 & 1502010 \\
\hline 7 & $\begin{array}{r}8 \\
7 \\
6 \\
5 \\
14 \\
4 \\
11 \\
3 \\
10 \\
2 \\
1 \\
15\end{array}$ & $\begin{array}{r}708004 \\
607002 \\
506004 \\
1405006 \\
414002 \\
1104004 \\
311002 \\
1003008 \\
210012 \\
102008 \\
1501016\end{array}$ & $\begin{array}{r}708004 \\
607002 \\
506004 \\
1405006 \\
414002 \\
1104004 \\
311002 \\
1003008 \\
210012 \\
102008 \\
1501016\end{array}$ \\
\hline 8 & $\begin{array}{l}8 \\
7 \\
6 \\
5\end{array}$ & $\begin{array}{l}708018 \\
607012 \\
506002\end{array}$ & $\begin{array}{r}708018 \\
607012 \\
506002 \\
1405016\end{array}$ \\
\hline
\end{tabular}


Table 5.4. Identification of shear walls and shear wall groups, horizontal model, Y-direction

\begin{tabular}{|c|c|c|c|}
\hline & $\begin{array}{r}14 \\
4 \\
11 \\
3 \\
10 \\
2 \\
1 \\
15\end{array}$ & $\begin{array}{r}1405016 \\
414006 \\
1104018 \\
311014 \\
1003034 \\
210032 \\
102048 \\
1501070\end{array}$ & $\begin{array}{r}414006 \\
1104018 \\
311014 \\
1003034 \\
210032 \\
102048 \\
1501070\end{array}$ \\
\hline 9 & $\begin{array}{r}2 \\
1 \\
15\end{array}$ & $\begin{array}{r}102010 \\
1501022\end{array}$ & $\begin{array}{r}102010 \\
1501022\end{array}$ \\
\hline 10 & $\begin{array}{r}2 \\
1 \\
15\end{array}$ & $\begin{array}{r}102020 \\
1501032\end{array}$ & $\begin{array}{r}102020 \\
1501032\end{array}$ \\
\hline 11 & $\begin{array}{r}2 \\
1 \\
15\end{array}$ & $\begin{array}{r}102034 \\
1501052\end{array}$ & $\begin{array}{r}102034 \\
1501052\end{array}$ \\
\hline 12 & $\begin{array}{r}2 \\
1 \\
15\end{array}$ & $\begin{array}{r}102018 \\
1501030\end{array}$ & $\begin{array}{r}102018 \\
1501030\end{array}$ \\
\hline 13 & $\begin{array}{r}14 \\
13 \\
10 \\
2 \\
1 \\
15\end{array}$ & $\begin{array}{r}1314002 \\
1013004 \\
210004 \\
102058 \\
1501104\end{array}$ & $\begin{array}{r}1314002 \\
1013004 \\
210004 \\
102058 \\
1501104\end{array}$ \\
\hline 14 & $\begin{array}{r}8 \\
7 \\
6 \\
5 \\
4 \\
3 \\
2 \\
1 \\
15\end{array}$ & $\begin{array}{r}708012 \\
607008 \\
506008 \\
405004 \\
304008 \\
203020 \\
102028 \\
1501044\end{array}$ & $\begin{array}{r}708012 \\
607008 \\
506008 \\
405004 \\
304008 \\
203020 \\
102028 \\
1501044\end{array}$ \\
\hline 15 & $\begin{array}{r}2 \\
1 \\
15\end{array}$ & $\begin{array}{r}102006 \\
1501008\end{array}$ & $\begin{array}{r}102006 \\
1501008\end{array}$ \\
\hline
\end{tabular}


Table 5.4. Identification of shear walls and shear wall groups, horizontal model, Y-direction

\begin{tabular}{|c|c|c|c|}
\hline 16 & $\begin{array}{r}18 \\
13 \\
10 \\
2 \\
1 \\
15\end{array}$ & $\begin{array}{r}1318014 \\
1013054 \\
210046 \\
102022 \\
1501038\end{array}$ & $\begin{array}{r}1318014 \\
1013054 \\
210046 \\
102022 \\
1501038\end{array}$ \\
\hline 17 & $\begin{array}{r}18 \\
10 \\
2 \\
15\end{array}$ & $\begin{array}{r}1018010 \\
210042 \\
1502098\end{array}$ & $\begin{array}{r}1018010 \\
210042 \\
1502098\end{array}$ \\
\hline 18 & $\begin{array}{r}18 \\
10 \\
2 \\
15\end{array}$ & $\begin{array}{r}1018050 \\
210044 \\
1502102\end{array}$ & $\begin{array}{r}1018050 \\
210044 \\
1502102\end{array}$ \\
\hline 19 & $\begin{array}{r}2 \\
1 \\
15\end{array}$ & $\begin{array}{r}102040 \\
1501060\end{array}$ & $\begin{array}{r}102040 \\
1501060\end{array}$ \\
\hline 20 & $\begin{array}{r}2 \\
1 \\
15\end{array}$ & $\begin{array}{r}102050 \\
1501080\end{array}$ & $\begin{array}{r}102050 \\
1501080\end{array}$ \\
\hline 21 & $\begin{array}{l}18 \\
13 \\
10\end{array}$ & $\begin{array}{l}1318020 \\
1013052\end{array}$ & $\begin{array}{l}1318022 \\
1013052\end{array}$ \\
\hline 22 & $\begin{array}{l}18 \\
13 \\
10\end{array}$ & $\begin{array}{l}1318020 \\
1013056\end{array}$ & $\begin{array}{l}1318020 \\
1013056\end{array}$ \\
\hline 23 & $\begin{array}{r}2 \\
1 \\
15\end{array}$ & $\begin{array}{r}102052 \\
1501086\end{array}$ & $\begin{array}{r}102052 \\
1501086\end{array}$ \\
\hline 24 & $\begin{array}{r}10 \\
2 \\
15\end{array}$ & $\begin{array}{r}210036 \\
1502082\end{array}$ & $\begin{array}{r}210036 \\
1502082\end{array}$ \\
\hline 25 & $\begin{array}{r}14 \\
11 \\
10 \\
2\end{array}$ & $\begin{array}{r}1114008 \\
1011014 \\
210016\end{array}$ & $\begin{array}{r}1114008 \\
1011014 \\
210016\end{array}$ \\
\hline
\end{tabular}


Table 5.4. Identification of shear walls and shear wall groups, horizontal model, Y-direction

\begin{tabular}{|c|c|c|c|}
\hline 26 & $\begin{array}{r}2 \\
1 \\
15\end{array}$ & $\begin{array}{r}102054 \\
1501088\end{array}$ & $\begin{array}{r}102054 \\
1501088\end{array}$ \\
\hline 27 & $\begin{array}{r}8 \\
7 \\
5 \\
14 \\
11 \\
10 \\
2 \\
15\end{array}$ & $\begin{array}{r}708002 \\
507006 \\
1405004 \\
1114002 \\
1011006 \\
210006 \\
1502006\end{array}$ & $\begin{array}{r}708002 \\
507006 \\
1405004 \\
1114002 \\
1011006 \\
210006 \\
1502006\end{array}$ \\
\hline 28 & $\begin{array}{r}8 \\
7 \\
5 \\
14 \\
11 \\
10 \\
2 \\
15\end{array}$ & $\begin{array}{r}708(020 \\
507014 \\
1405018 \\
1114020 \\
1011036 \\
210036 \\
1502082\end{array}$ & $\begin{array}{r}708020 \\
507014 \\
1405018 \\
1114020 \\
1011036 \\
210036 \\
1502082\end{array}$ \\
\hline 29 & $\begin{array}{r}7 \\
5 \\
14\end{array}$ & $\begin{array}{r}507010 \\
1405008\end{array}$ & $\begin{array}{r}507010 \\
1405008\end{array}$ \\
\hline 30 & $\begin{array}{r}7 \\
5 \\
14\end{array}$ & $\begin{array}{r}507012 \\
1405014\end{array}$ & $\begin{array}{r}507012 \\
1405014\end{array}$ \\
\hline 31 & $\begin{array}{r}6 \\
14\end{array}$ & 1406020 & 1406020 \\
\hline 32 & $\begin{array}{r}5 \\
14\end{array}$ & 1405010 & 1405010 \\
\hline 33 & $\begin{array}{l}8 \\
7 \\
6\end{array}$ & $\begin{array}{l}708008 \\
607004\end{array}$ & $\begin{array}{l}708008 \\
607004\end{array}$ \\
\hline 34 & $\begin{array}{l}8 \\
7 \\
6\end{array}$ & $\begin{array}{l}708014 \\
607010\end{array}$ & $\begin{array}{l}708014 \\
607010\end{array}$ \\
\hline 35 & $\begin{array}{l}8 \\
7\end{array}$ & 708006 & 708006 \\
\hline
\end{tabular}


Table 5.4. Identification of shear walls and shear wall groups, horizontal model, Y-direction

\begin{tabular}{|c|c|c|c|}
\hline 36 & $\begin{array}{r}14 \\
11 \\
10 \\
2\end{array}$ & $\begin{array}{r}1114014 \\
1011030 \\
210026\end{array}$ & $\begin{array}{r}1114014 \\
1011030 \\
210026\end{array}$ \\
\hline 37 & $\begin{array}{r}14 \\
13 \\
10 \\
2\end{array}$ & $\begin{array}{r}1314010 \\
1013038 \\
210038\end{array}$ & $\begin{array}{r}1314010 \\
1013038 \\
210038\end{array}$ \\
\hline 38 & $\begin{array}{r}16 \\
2\end{array}$ & 216068 & 216068 \\
\hline 39 & $\begin{array}{r}17 \\
2\end{array}$ & 217090 & 217090 \\
\hline 40 & $\begin{array}{r}2 \\
15\end{array}$ & 1502106 & 1502106 \\
\hline 41 & $\begin{array}{r}2 \\
15\end{array}$ & 1502108 & 1502108 \\
\hline
\end{tabular}


Table 5.5. Dynamic characteristics of the horizontal model.

\section{Mass Participation (pct)}

Mode

Frequency (Hz. )

X-Trans

Y-Trans

1

1.3

1.7

3.0

3.7

3.7

4.1

4.2

4.9

5.3

5.3

5.7

5.8

5.9

6.4

6.4

7.1

7.3

7.4

8.4

11.3

11.9

12.1

13.0

12.4

13.4

13.8

14.2

16.4

16.9

17.3

17.7

18.5

18.5

18.6

19.1

19.6

22.1

22.4

22.6

$40 \quad 24.2$

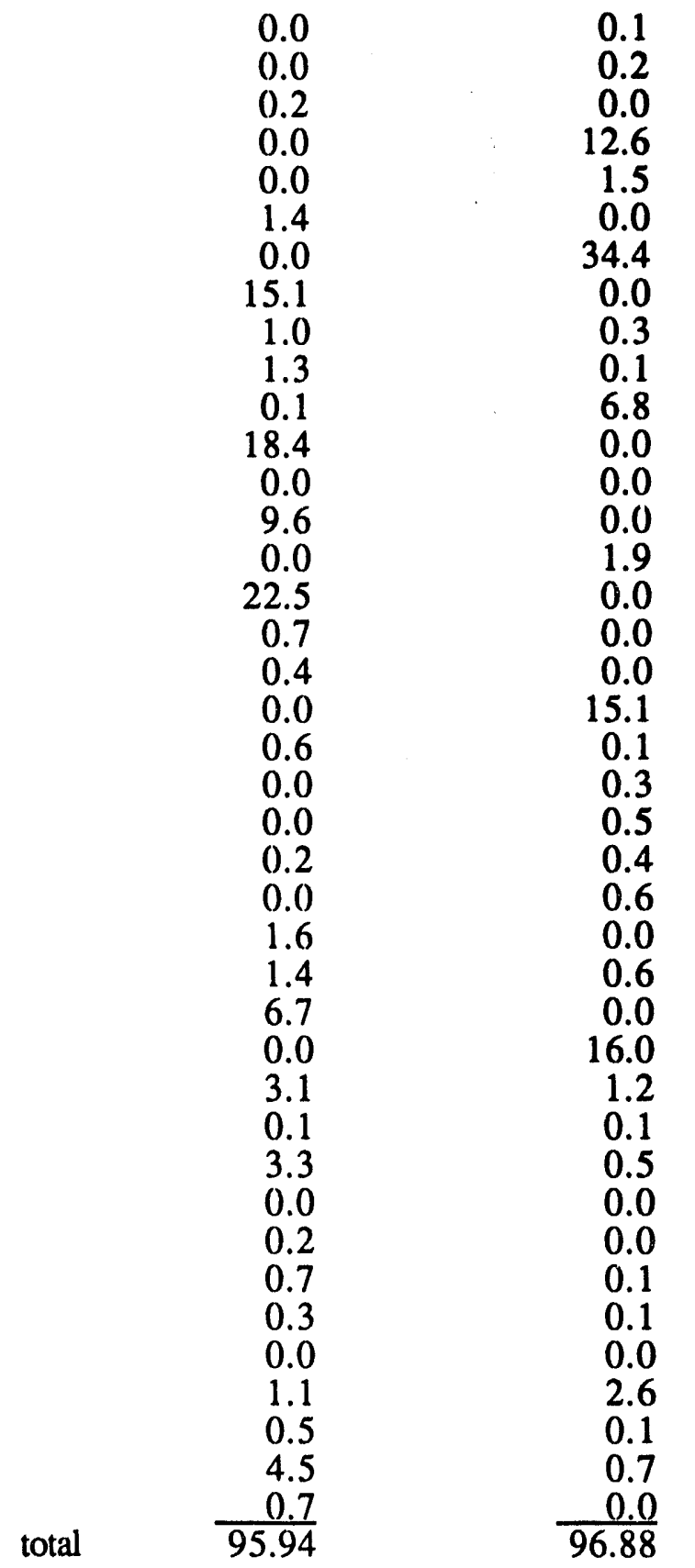




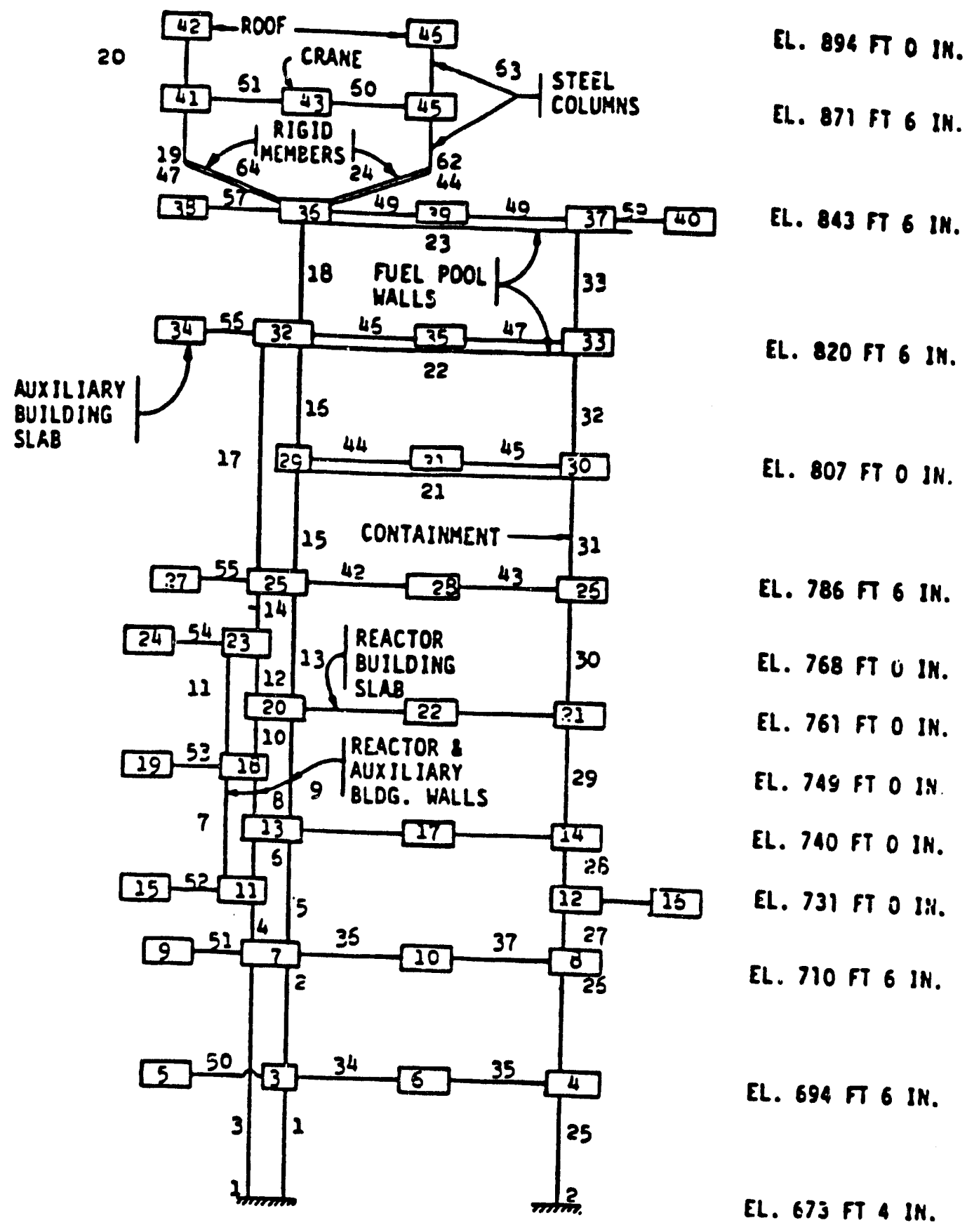

Figure 5.16. Vertical seismic model of reactor and auxiliary buildings. 
Table 5.6. Dynamic characteristics of the vertical model of the reactor and auxiliary building.

Mass Participation (pct)

Mode

1

2

3

4

5

6

8

9

10

11

12

13

14

15

16

17

18

19

20

21

22

23

24

25

26

27

28

29

30

31

32

33

34

35

36

37

38

39

40
Frequency (Hz.)

3.8

5.0

9.0

11.1

13.0

13.8

14.8

14.9

14.9

15.0

15.0

15.0

15.0

15.0

15.0

15.0

15.0

15.0

15.0

15.0

15.0

16.6

16.8

18.0

18.0

18.5

21.0

21.0

21.3

21.5

21.6

24.0

24.0

37.4

38.3

52.7

52.7

58.2

61.6

79.1
Z-Trans

0.1

0.2

0.8

72.7

0.2

1.2

2.2

0.5

0.1

0.1

0.0

0.1

0.0

0.0

0.0

0.0

0.0

0.0

0.0

0.0

0.0

4.0

0.2

0.0

0.0

2.0

0.0

0.0

0.8

0.0

0.6

0.0

0.3

7.3

0.5

0.0

0.0

4.2

0.6

total $\quad \frac{0.2}{98.8}$ 


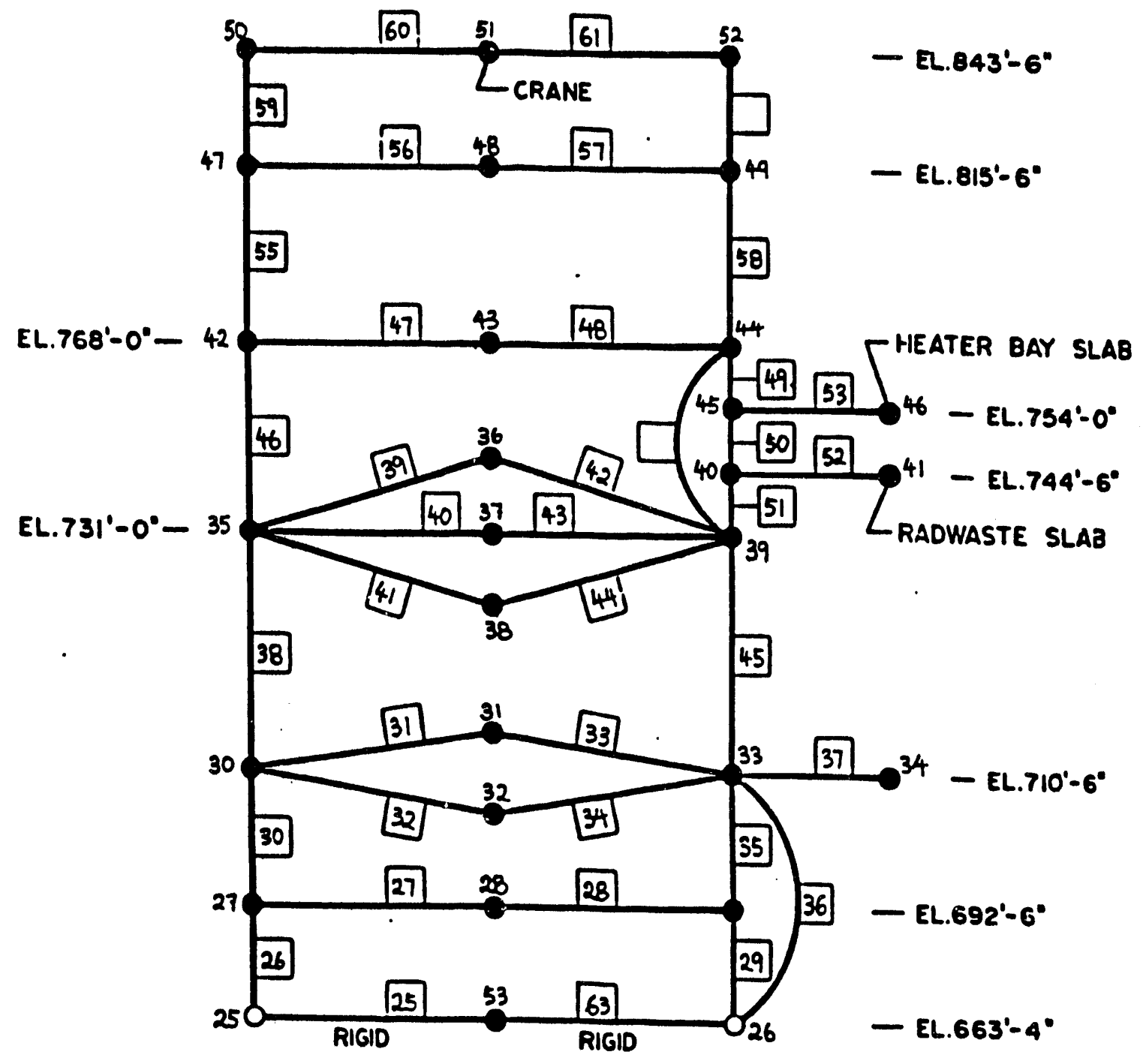

Figure 5.17. Vertical seismic model of turbine, heater bay and radwaste buildings. 
Table 5.7. Dynamic characteristics of the vertical model of the turbine, heater bay and radwaste buildings.

\begin{tabular}{|c|c|c|}
\hline \multicolumn{3}{|c|}{ Mass Participation (pct) } \\
\hline Mode & Frequency $(\mathrm{Hz})$. & Z-Trans \\
\hline $\begin{array}{c}1 \\
2 \\
3 \\
4 \\
5 \\
6 \\
7 \\
8 \\
9 \\
10 \\
11 \\
12 \\
13 \\
14 \\
15 \\
16 \\
17 \\
18 \\
19 \\
20 \\
21 \\
22 \\
23 \\
24 \\
25 \\
26\end{array}$ & $\begin{array}{r}6.0 \\
11.3 \\
17.8 \\
19.5 \\
21.0 \\
22.4 \\
23.2 \\
23.6 \\
26.8 \\
30.8 \\
32.8 \\
33.2 \\
33.2 \\
34.2 \\
36.7 \\
40.2 \\
41.5 \\
46.5 \\
52.8 \\
67.1 \\
91.2 \\
96.0 \\
107.2 \\
381.7 \\
382.8 \\
388.5\end{array}$ & $\begin{array}{r}2.8 \\
68.9 \\
0.1 \\
4.5 \\
1.2 \\
3.7 \\
0.9 \\
0.0 \\
0.1 \\
1.9 \\
0.4 \\
0.0 \\
0.0 \\
3.8 \\
3.1 \\
0.4 \\
0.7 \\
2.1 \\
3.5 \\
0.6 \\
0.1 \\
0.2 \\
0.6 \\
0.0 \\
0.0 \\
0.0 \\
99.6\end{array}$ \\
\hline
\end{tabular}




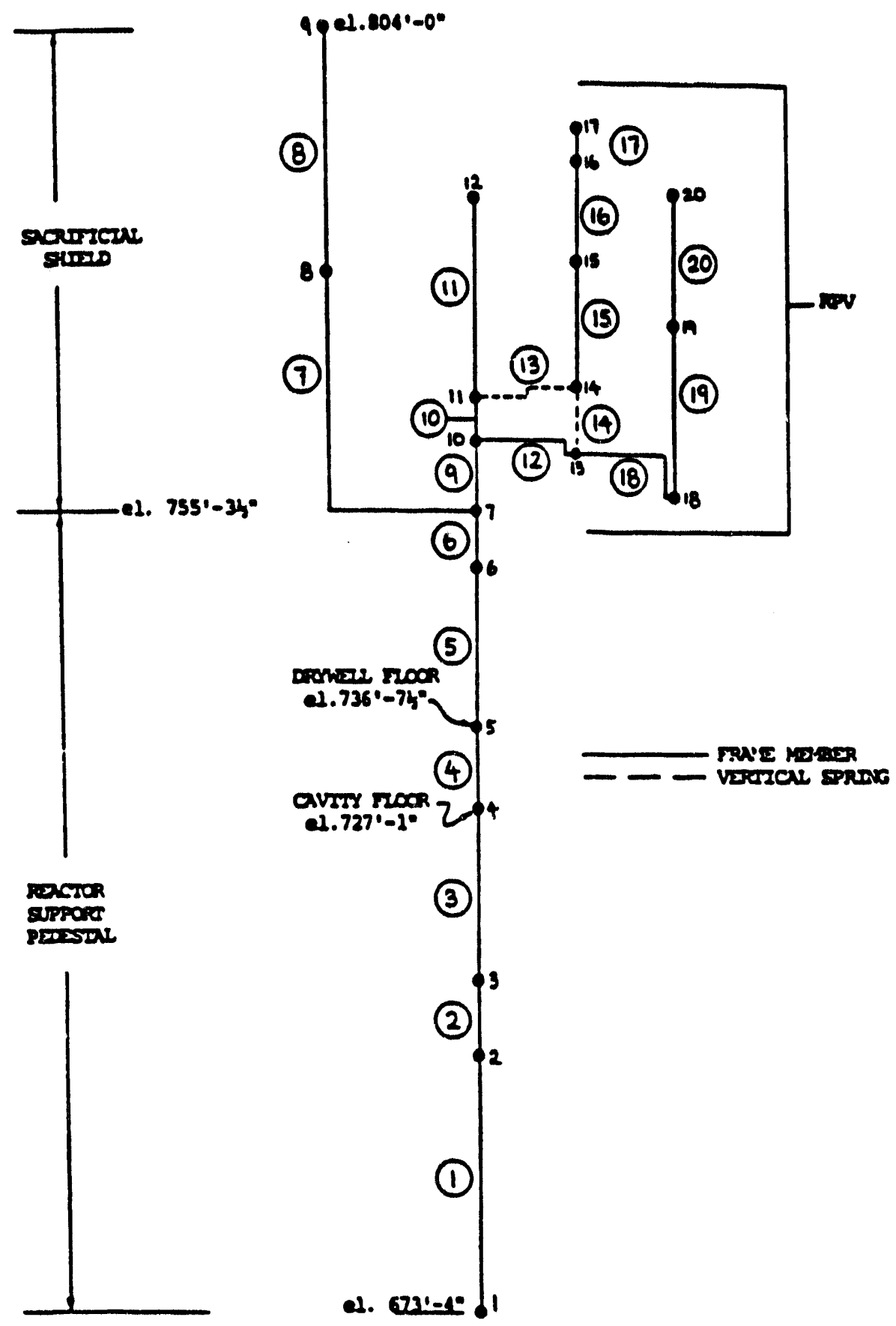

Figure 5.18. Vertical seismic model of RPV, sacrificial shield, and reactor pedestal. 
Table 5.8. Dynamic characteristics of the vertical model of the RPV, sacrificial shield and reactor pedestal.

\begin{tabular}{ccc}
\hline & \multicolumn{2}{c}{ Mass Participation (pct) } \\
Mode & Frequency (Hz.) & \\
& & Z-Trans \\
1 & 17.1 & 78.8 \\
2 & 31.9 & 0.1 \\
3 & 33.6 & 6.4 \\
4 & 50.0 & 0.5 \\
5 & 60.8 & 8.6 \\
6 & 70.3 & 0.0 \\
7 & 79.6 & 0.0 \\
8 & 99.1 & 4.1 \\
9 & 108.2 & 0.4 \\
10 & 125.3 & 0.3 \\
11 & 134.3 & 0.0 \\
12 & 144.3 & 0.1 \\
13 & 175.9 & 0.0 \\
14 & 218.5 & 0.1 \\
15 & 254.5 & 0.0 \\
16 & 311.3 & 0.0 \\
17 & 328.7 & 0.0 \\
18 & 362.7 & 0.0 \\
19 & 863.4 & 0.0 \\
& & 99.5
\end{tabular}




\subsection{Probabilistic Responses}

SMACS analyses were performed for two intervals of the seismic hazard curve. SMACS performs repeated deterministic analyses, each analysis simulating an earthquake occurrence. Variability in each aspect of the process is treated explicitly. In the seismic input, variability is introduced by considering an ensemble of earthquake motions. For LaSalle, the ensemble includes local site amplification, described previously. Variability in SSI is introduced through modeling soil shear modulus and material damping as random variables with lognormal distributions--medians corresponding to the nominal values of Table 5.1 and coefficients of variation of 0.4 and 0.5 , respectively. Variability in structure dynamic behavior is modeled by treating structure frequency and modal damping as random variables. Median values of structure frequency are those discussed in Sec. 5.3. Median values of structure damping were 10 percent of critical. Coefficients of variation of 0.25 and 0.35 were assumed for structure frequency and damping, respectively. In all cases, variability introduced here represents random uncertainty and is consistent with values used in previous analyses..$^{11.31}$ These discrete input parameters were sampled for each earthquake simulation. The samples were combined according to a Latin hypercube experimental design.

Two types of in-structure response were of interest--structure forces and moments for structure element fragility assessment and in-structure response spectra for equipment and component fragility assessment. Only median values of response were of interest. Variability and correlation of responses are to be assigned based on previous studies and recorded data. ${ }^{[1.3)} \mathrm{A}$ brief discussion of each type of result is presented. One hundred-and-forty-five structure forces and moments were calculated for structure element fragility assessment. For each earthquake simulation, several types of forces and moments were determined.

- Maximum shear in individual walls, i.e., maximum over the time history.

- Maximum shear transferred to slabs by major wall groups. The shear transferred to a slab is given by the difference in shear forces in those wall springs of the group that terminate in the slab. In the SMACS analyses, the procedure is equivalent to calculating the modal shear transfer time histories followed by modal superposition in the time domain. The maximum is over the time history.

- Maximum moment at a given section of major wall groups. For major walls which exterid continuously through several slabs, the moment used to determine flexural stresses at a section should be calculated by integrating the shear diagram of the walls immediately above the section of interest. Integrating the shear diagram to determine the moment is not consistent with the assumptions used in formulating the stiffness of the slab-wall portion of the dynamic model but this procedure will give a conservative check on the flexural stresses in the shear walls. The moment for each mode was calculated by integrating the corresponding shear diagram at each time point to yield the complete time histories of modal response. This is followed by mode superposition in the time domain. Again the maximum recorded is the maximum over the time history.

- Maximum vector sum shears and moments in the reactor containment portion of the complex. The vector sum shears and moments were calculated time step-by-time step over the duration of the earthquake simulation and the maximum recorded. 
Having obtained a set of thirty values of each response for the acceleration range of interest, a median response was calculated assuming a lognormal distribution. These median values are presented in Table 5.9 for acceleration ranges 1 and 2. A discussion of selected responses is contained in Sec. 7. However, one point of interest is that the increase in amplitude from acceleration range 1 to acceleration range 2 is small which is due, principally, to the very soft soil profile and its effect on the free-field ground motion and on the dominant frequencies of the soilstructure system.

Forty-eight in-structure response spectra comprised the second type of response calculated here. In some instances, the LaSalle SSE design response spectra ${ }^{[5.41}$ are overplotted for comparison purposes. Those cases are shown in Fig. 5.19 for horizontal response and in Fig. 5.20 for vertical response in the reactor and auxiliary buildings. One difficulty in interpreting the comparisons of median range 1 and 2 response spectra and the design spectra is a lack of correlation of the design free-field ground motion and that of acceleration range 1 and/or 2 . Recall, the LaSalle seismic hazard for this study was defined on a hypothetical rock outcrop and local site amplification was treated explicitly. The LaSalle seismic design criteria specified the design ground motion at foundation level in the free-field ( $44 \mathrm{ft}$. below the soil free surface). Broad-band response spectra anchored to a $0.2 \mathrm{~g}$ horizontal peak ground acceleration (PGA) comprised the specification. For seismic analysis purposes, this motion was assumed to act within the soil column at foundation level. During Sargent \& Lundy's SSI analysis or prior to it, using a 1-D wave propagation analysis, response spectra on the soil free surface could have been calculated. We then could have compared our free surface median response spectra for acceleration ranges 1 and 2 with those generated by Sargent \& Lundy and make definitive statements concerning the comparison. However, free surface motions corresponding to the utility's design specification were not calculated by Sargent \& Lundy and not easily generated. Hence, no direct comparison of free-field ground motion can easily be made. The median horizontal PGA of acceleration ranges 1 and 2 on the soil free surface is approximately $0.22 \mathrm{~g}$ and $0.35 \mathrm{~g}$, respectively. Neither of these correspond to the PGA expected on the soil free surface for the design specification. Acceleration range 1 likely comes the closest. Frequency content of the free surface motion for this study and the design are likely to differ also. Hence, a direct comparison is difficult. However, one can still make observations concerning horizontal and vertical response as follows. For horizontal response, one observes that the design spectra are generally conservative compared to median response spectra for acceleration range 1 and, in some frequency ranges, notably above $1 \mathrm{~Hz}$, very conservative. Comparing the design spectra with those of acceleration range 2 , the latter frequently exceeds the former at low frequencies but the design spectra are generally very conservative in the high frequency range. For the vertical direction, the design spectra are generally conservative compared to median response spectra for acceleration range 1 and in the frequency range above $2 \mathrm{~Hz}$. very conservative. Comparing the design spectra with those of acceleration range 2 , the latter exceeds the former at low frequencies (below $3 \mathrm{~Hz}$.), but the design spectra are generally very conservative for higher frequencies. These response spectra provide the basis for the seismic responses in the seismic PRA for equipment and component fragility assessment.

For the pipe moments and valve accelerations, we employed the response factor approach developed in the SSMRP.[1.1] The response factor approach may be simply represented by one pair of parameters, i.e., a mean response factor, $F_{R}$, and the associated $\mathrm{COV}$ (coefficient of variation). In general, $\mathrm{F}_{\mathrm{R}}$ may be represented by a reference response factor, $\mathrm{CF}_{\mathrm{Ref}}$, and four adjusting factors as follows:

$$
\mathrm{F}_{\mathrm{R}}=\mathrm{CF}_{\mathrm{Ref}} \cdot \mathrm{F}_{\mathrm{M}} \cdot \mathrm{F}_{\mathrm{D}} \cdot \mathrm{F}_{\mathrm{I}} \cdot \mathrm{F}_{\mathrm{S}}
$$


Table 5.9. In-structure forces and moments for acceleration ranges 1 and 2 , median values.

response identifier

1 horiz model: flex diaphragm to y-spring 1011006

2 horiz modcl: flex diaphragm to y-spring 1011036

3 horiz model: $x$-spring 1502023 (shear)

4 horiz modcl: $x$-spring 1501025 (shear)

5 horiz modcl: $x$-spring 102027 (shear)

6 horiz model: $x$-spring 203001 (shear)

7 horiz modcl: $x$-spring 210027 (shear)

8 horiz model: $x$-spring 304001 (shear)

9 horiz model: $x$-spring 405001 (shear)

10 horiz model: $y$-spring 1501032 (shear)

11 horiz modcl: $y$-spring 1501044 (shcar)

12 horiz modcl: y-spring 1502082 (shcar)

13 horiz modcl: $y$-spring 210016 (shcar)

14 horiz modcl: $y$-spring 210032 (shcar)

15 horiz modcl: $y$-spring 210038 (shcar)

16 horiz modcl: $y$-spring 1011036 (shcar)

17 horiz modcl: $y$-spring 304008 (shcar)

18 horiz model: $y$-spring 1104018 (shear)

19 horiz modcl: y-spring 1114020 (shear)

20 horiz model: $y$-spring 414006 (shcar)

21 horiz model: $y$-spring 507014 (shear)

22 horiz model: grp 1x slab 7 (moment)

23 horiz modcl: grp $1 \times$ slab 5 (moment)

24 horiz modcl: grp 1x slab 4 (mumcnl)

25 horiz modcl: grp Ix slab 3 (moment)

26 horiz modcl: grp 1x slab 2 (moment)

27 horiz model: grp $1 \times$ slab 1 (moment)

28 horiz modcl: grp 1x slab 15 (moment)

29 horiz model: grp $2 x$ slab 7 (moment)

30 horiz model: grp 2x slab 6 (moment)

31 horiz model: grp $2 \times$ slab 5 (moment)

32 horiz model: grp $2 x$ slab 14 (moment)

33 horiz model: grp $2 x$ slab 4 (moment)

34 horiz model: grp $2 x$ slab 11 (moment)

35 horiz model: grp $2 x$ slab 3 (moment)

36 horiz modcl: grp $2 \times$ slab 10 (moment)

37 horiz modcl: grp $2 x$ slab 2 (moment)

38 horiz modcl: grp $2 x$ slab 1 (moment)

39 horiz modcl: grp $2 x$ slab 15 (moment)

40 horiz moddl: grp 24x slab 10 (moment)

41 horiz model: grp $24 \times$ slab 2 (moment)

42 horiz model: grp 24x slab 15 (momcnt)

43 horiz model: grp $32 x$ slab 7 (moment)

44 horiz model: grp $32 \times$ slab 5 (moment)

45 horiz model: grp $32 \times$ slab 14 (moment) median response (lognormal)

$\begin{array}{ll}\text { range } 1 & \text { range 2 } \\ & \\ 3.896 e+02 & 5.840 e+02 \\ 3.929 e+02 & 5.836 e+02 \\ 1.821 e+03 & 2.630 e+03 \\ 8.852 e+03 & 1.281 e+04 \\ 2.185 e+03 & 3.154 e+03 \\ 1.090 e+04 & 1.545 e+04 \\ 6.511 e+03 & 9.303 e+03 \\ 9.000 e+03 & 1.262 e+04 \\ 7.318 e+03 & 1.019 e+04 \\ 4.998 e+03 & 7.971 e+03 \\ 4.512 e+03 & 7.190 e+03 \\ 3.468 e+03 & 5.491 e+03 \\ 1.964 c+03 & 3.084 e+03 \\ 5.688 c+03 & 8.898 e+03 \\ 5.558 c+03 & 8.667 e+03 \\ 1.901 e+03 & 2.920 e+03 \\ 5.779 e+03 & 8.821 e+03 \\ 4.286 e+03 & 6.494 e+03 \\ 1.310 e+03 & 1.980 e+03 \\ 7.153 e+03 & 1.088 e+04 \\ 1.951 e+02 & 2.791 e+02 \\ 1.225 e+04 & 1.687 e+04 \\ 1.729 e+05 & 2.409 e+05 \\ 3.594 e+05 & 5.007 e+05 \\ 5.483 e+05 & 7.657 e+05 \\ 8.696 e+05 & 1.221 e+06 \\ 1.021 e+06 & 1.439 e+06 \\ 1.181 e+06 & 1.670 e+06 \\ 2.113 e+04 & 2.931 e+04 \\ 4.926 e+04 & 6.835 e+04 \\ 1.906 e+05 & 2.659 e+05 \\ 2.702 e+05 & 3.773 e+05 \\ 3.073 e+05 & 4.292 e+05 \\ 3.435 e+05 & 4.800 e+05 \\ 3.727 e+05 & 5.210 e+05 \\ 4.053 e+05 & 5.669 e+05 \\ 6.352 c+05 & 8.953 e+05 \\ 8.175 c+05 & 1.159 e+06 \\ 1.004 c+06 & 1.429 e+06 \\ 1.417 c+04 & 1.998 e+04 \\ 2.298 c+04 & 3.254 e+04 \\ 9.060 e+04 & 1.302 e+05 \\ 1.366 e+04 & 1.861 e+04 \\ 1.273 e+05 & 1.766 e+05 \\ 2.024 e+05 & 2.819 e+05\end{array}$


Table 5.9. In-structure forces and moments for acceleration ranges 1 and 2 , median values. (cont.)

response identifier

median response (lognormal)

horiz model: grp 32x slab 11 (moment) horiz model: grp $32 \times$ slab 10 (moment) horiz model: grp $32 x$ slab 2 (moment) horiz modcl: grp 32x slab 1 (moment) horiz model: grp 8y slab 7 (moment) horiz model: grp 8y slab 6 (moment) horiz model: grp 8y slab 5 (moment) horiz model: grp 8y slab 4 (moment) horiz model: grp 8y slab 11 (moment) horiz model: grp 8y slab 3 (moment) horiz modcl: grp 8 y slab 10 (moment) horiz model: grp 8y slab 2 (moment) horiz model: grp 8 y slab 1 (moment) horiz model: grp 8y slab 15 (moment) horiz model: grp 10y slab 1 (moment) horiz model: grp 10y slab 15 (moment) horiz model: grp 14y slab 7 (moment) horiz model: grp 14y slab 6 (moment) horiz model: grp 14 y slab 5 (moment) horiz model: grp 14 y slab 4 (moment) horiz model: grp 14y slab 3 (moment) horiz model: grp 14y slab 2 (moment) horiz model: grp 14y slab 1 (moment) horiz model: grp 14y slab 15 (moment) horiz model: grp 25y slab 11 (momen!) horiz model: grp 25y slab 10 (moment) horiz modcl: grp 25y slab 2 (mencnt) horiz model: grp $28 y$ slab 7 (noment) horiz model: grp $28 y$ slab 5 (moment) horiz model: grp 28y slab 14 (moment) horiz model: grp 28 y slab 11 (moment) horiz model: grp 28y slab 10 (moment) horiz model: grp 28 y slab 2 (moment) horiz model: grp 28y slab 15 (moment) horiz modcl: grp 37y slab 13 (momcnt) horiz model: grp 37y slab 10 (moment) horiz model: grp $37 y$ slab 2 (moment) horiz modcl: grp $1 \times$ slab 7 (shcar) horiz modcl: grp $2 x$ slab 2 (shear) horiz model: grp $2 x$ slab 6 (shear) horiz model: grp $28 x$ slab 7 (shear) horiz modcl: grp 29x slab 7 (shear) horiz model: grp 32x slab 7 (shcar) horiz model: grp $7 y$ slab 6 (shcar)

range $1 \quad$ range 2

$\begin{array}{ll}3.379 c+05 & 4.729 e+05 \\ 5.391 c+05 & 7.582 e+05 \\ 8.057 e+05 & 1.139 e+06 \\ 8.854 e+05 & 1.254 e+06 \\ 1.940 e+04 & 2.767 e+04 \\ 7.590 e+04 & 1.096 e+05 \\ 1.790 e+05 & 2.581 e+05 \\ 3.331 e+05 & 4.898 e+05 \\ 3.840 e+05 & 5.675 e+05 \\ 4.298 e+05 & 6.381 e+05 \\ 4.877 e+05 & 7.260 e+05 \\ 6.010 e+05 & 9.064 e+05 \\ 7.163 e+05 & 1.093 e+06 \\ 8.564 e+05 & 1.320 e+06 \\ 1.099 e+05 & 1.745 e+05 \\ 2.157 e+05 & 3.432 e+05 \\ 9.956 e+03 & 1.467 e+04 \\ 1.330 e+04 & 1.990 e+04 \\ 6.551 e+04 & 9.552 e+04 \\ 2.170 e+05 & 3.239 e+05 \\ 3.377 e+05 & 5.086 e+05 \\ 5.670 e+05 & 8.664 e+05 \\ 6.394 e+05 & 9.825 e+05 \\ 7.324 e+04 & 1.133 e+06 \\ 3.261 e+04 & 4.979 e+04 \\ 5.993 c+04 & 9.148 e+04 \\ 9.999 c+04 & 1.545 e+05 \\ 4.468 c+03 & 6.397 e+03 \\ 1.072 e+04 & 1.533 e+04 \\ 1.077 e+04 & 1.540 e+04 \\ 3.542 e+04 & 5.291 e+04 \\ 6.944 e+04 & 1.053 e+05 \\ 1.003 e+05 & 1.531 e+05 \\ 2.273 e+05 & 3.561 e+05 \\ 1.728 e+04 & 2.533 e+04 \\ 3.665 e+04 & 5.563 e+04 \\ 1.501 e+05 & 2.332 e+05 \\ 4.552 e+03 & 6.351 e+03 \\ 7.177 e+02 & 9.230 e+02 \\ 4.571 e+03 & 6.307 e+03 \\ 1.117 e+03 & 1.557 e+03 \\ 1.117 e+03 & 1.557 e+03 \\ 3.025 e+03 & 4.211 e+03 \\ 3.386 e+01 & 4.862 e+01\end{array}$




\section{Table 5.9. In-structure forces and moments for acceleration ranges 1 and 2 , median values. (cont.)}

\section{response identifier}

\begin{tabular}{|c|}
\hline 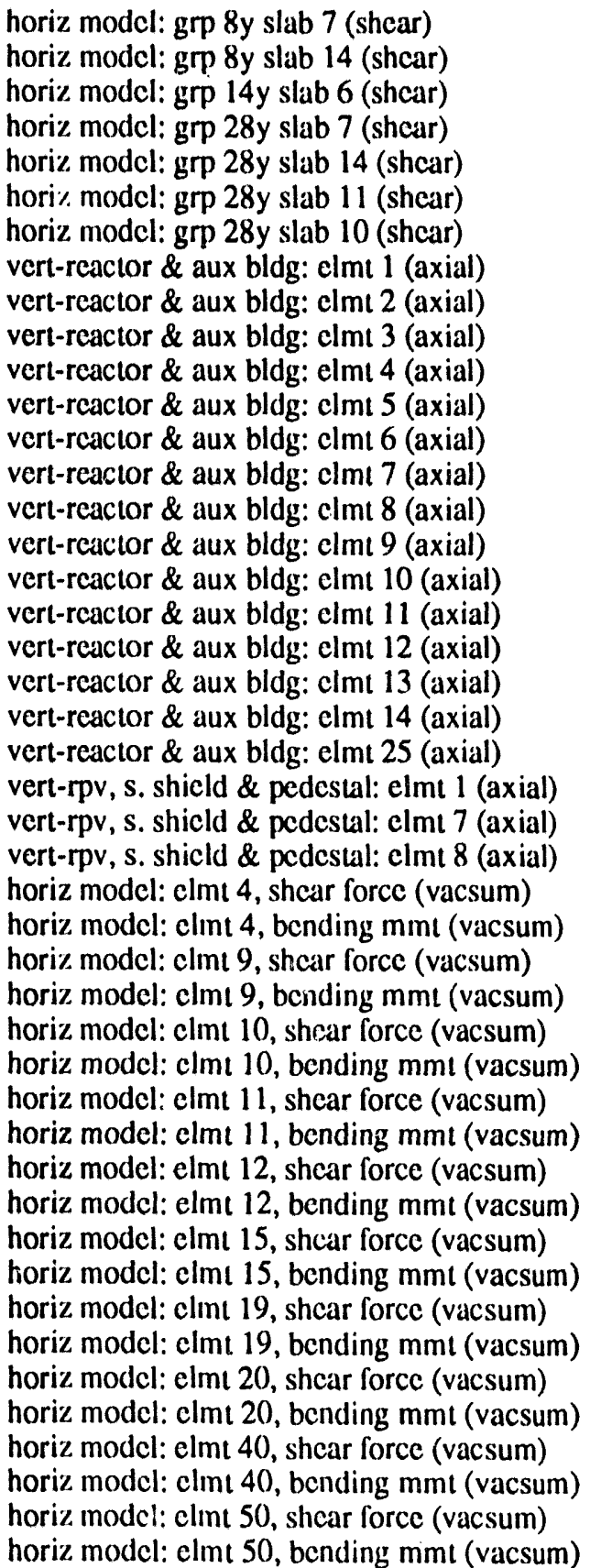 \\
\hline
\end{tabular}

median response (lognormal)

\begin{tabular}{|c|c|}
\hline range 1 & range 2 \\
\hline $4.311 c+03$ & $6.160 e+03$ \\
\hline $1.761 c+03$ & $2.609 e+03$ \\
\hline $2.281 c+03$ & $3.258 e+03$ \\
\hline $1.661 c+01$ & $2.371 e+01$ \\
\hline $1.308 c+03$ & $1.976 c+03$ \\
\hline $6.089 e+02$ & $9.551 e+02$ \\
\hline $3.944 e+02$ & $5.859 e+02$ \\
\hline $2.464 \mathrm{e}+04$ & $4.681 e+04$ \\
\hline $2.169 e+04$ & $4.106 e+04$ \\
\hline $2.187 e+03$ & $4.150 \mathrm{e}+03$ \\
\hline $1.330 c+04$ & $2.505 e+04$ \\
\hline $6.414 c+03$ & $1.207 e+04$ \\
\hline $2.922 \mathrm{e}+03$ & $5.477 e+03$ \\
\hline $8.197 e+03$ & $1.536 e+04$ \\
\hline $2.508 \mathrm{e}+03$ & $4.697 e+03$ \\
\hline $5.373 c+03$ & $1.004 e+04$ \\
\hline $1.979 c+03$ & $3.693 e+03$ \\
\hline $7.037 e+03$ & $1.313 e+04$ \\
\hline $2.593 \mathrm{e}+03$ & $4.833 e+03$ \\
\hline $3.400 \mathfrak{c}+03$ & $6.329 e+03$ \\
\hline $7.586 \mathrm{e}+03$ & $1.411 \mathrm{e}+04$ \\
\hline $1.078 \mathrm{e}+04$ & $2.034 e+04$ \\
\hline $1.010 e+03$ & $1.892 e+03$ \\
\hline $1.332 e+02$ & $2.471 e+02$ \\
\hline $4.451 e+01$ & $8.244 e+01$ \\
\hline $7.953 e+03$ & $1.249 c+04$ \\
\hline $6.075 c+05$ & $9.419 e+05$ \\
\hline 4.54()$c+03$ & $6.854 e+03$ \\
\hline $3.506 c+04$ & $4.970 e+04$ \\
\hline $4.540 c+03$ & $6.854 c+03$ \\
\hline $1.140 c+05$ & $1.756 e+05$ \\
\hline $3.966 \mathrm{e}+03$ & $5.956 e+03$ \\
\hline $1.893 e+05$ & $2.890 e+05$ \\
\hline 2. $103 e+03$ & $3.110 c+03$ \\
\hline $2.019 e+05$ & $3.080 c+03$ \\
\hline $7.151 e+02$ & $1.068 c+03$ \\
\hline $5.942 c+04$ & $9.345 c+04$ \\
\hline $8.107 c+01$ & $6.710 c+01$ \\
\hline $3.921 \mathrm{e}+03$ & $3.225 c+03$ \\
\hline $8.107 c+01$ & $6.710 c+01$ \\
\hline $1.384 c+03$ & $1.136 c+03$ \\
\hline $1.536 c+02$ & $1.869 e+02$ \\
\hline $4.759 c+03$ & $5.003 c+03$ \\
\hline $2.095 c+02$ & $2.657 c+02$ \\
\hline $5.941 \mathrm{e}+03$ & $6.423 e+03$ \\
\hline
\end{tabular}


Table 5.9. In-structure forces and moments for acceleration ranges 1 and 2 , median values. (cont.)

response identifier

136

137

138

139

140

141

142

143

144

145 horiz modcl: clint 51, shear force (vacsum) horiz model: elmt 51, bending mmt (vacsum) horiz modcl: elmt 53, shcar force (vacsum) horiz model: elmt 53, bending $\mathrm{mmt}$ (vacsum) horiz modcl: input elmt 21, shear force (vacsum) horiz model: input elmt 21 , bending $\mathrm{mmt}$ (vacsum) horiz modcl: input elmt 42, shcar force (vacsum) horiz model: input clmt 42, bending $\mathrm{mmt}$ (vacsum) horiz modcl: input clmt 52, shear forcc (vacsum) horiz model: input clmt 52, bending mmt (vacsum) median response (lognormal)

range $1 \quad$ range 2

$1.447 \mathrm{e}+02$

$2.228 \mathrm{e}+02$

$1.733 \mathrm{e}+03$

$1.171 e+02$

$1.757 e+03$

$8.372 \mathrm{e}+02$

$1.133 \mathrm{e}+02$

$7.388 \mathrm{e}+02$

$1.161 \mathrm{e}+02$

$6.355 \mathrm{e}+02$

$1.546 c+00$
$2.292 e+03$

$1.209 \mathrm{e}+02$

$1.818 \mathrm{e}+03$

$1.172 \mathrm{e}+03$

$1.463 \mathrm{e}+02$

$1.037 \mathrm{e}+03$

$1.495 \mathrm{e}+02$

$8.577 e+02$

$2.009 \mathrm{e}+00$ 

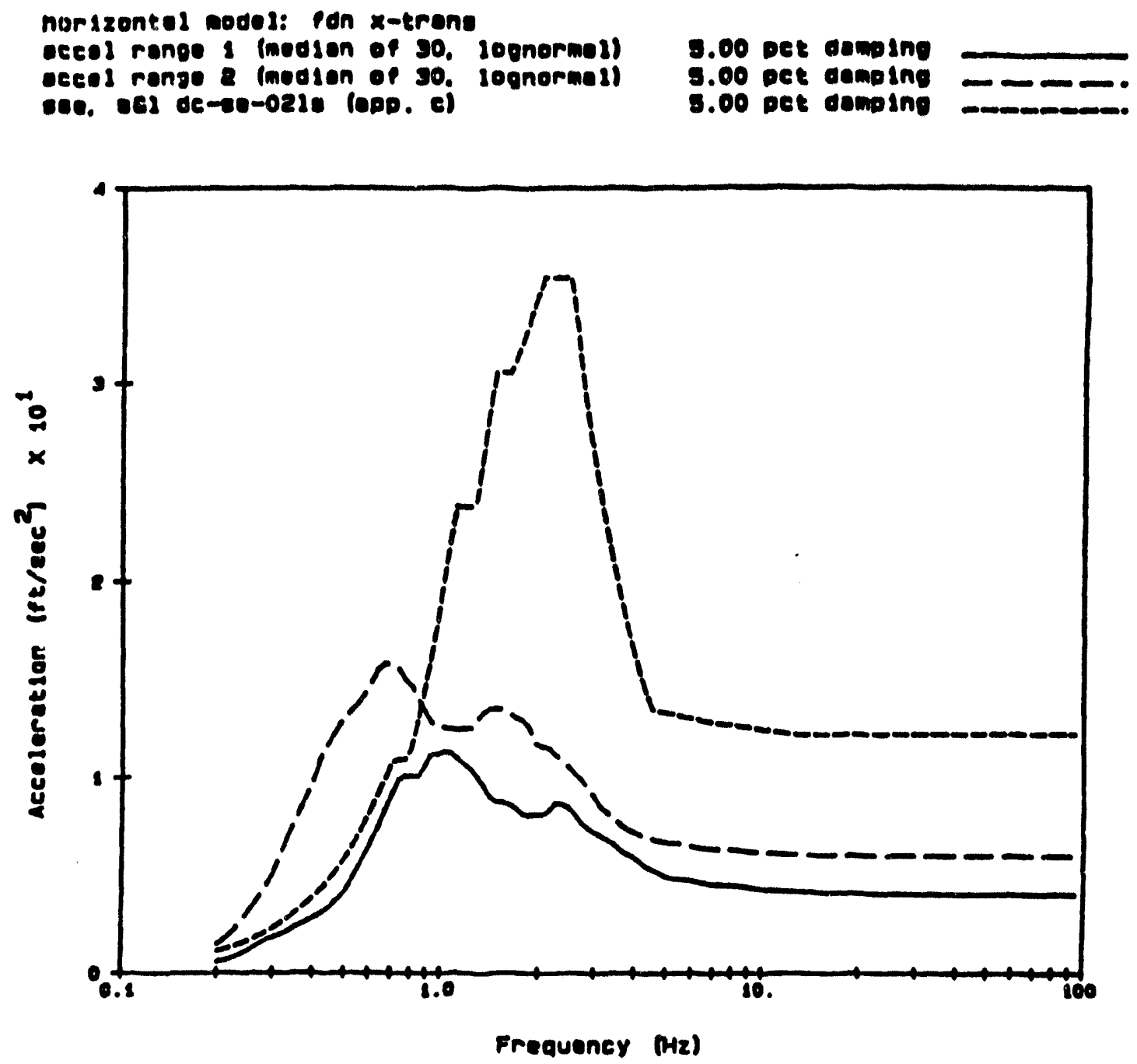

Figure 5.19. Horizontal in-structure response spectra, median acceleration ranges 1 and 2, and design spectra: a) Foundation X-direction (N-S) 

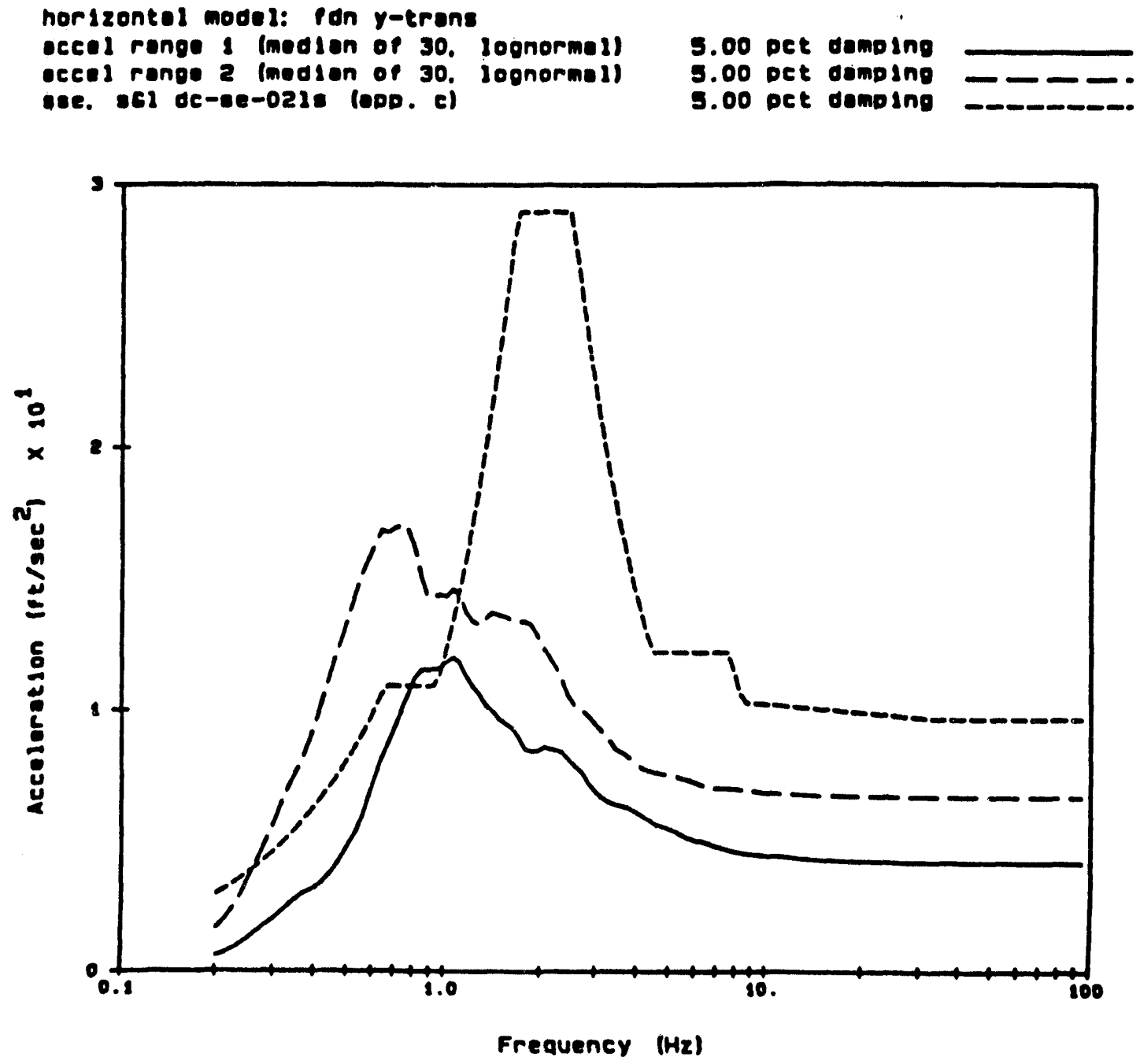

Figure 5.19. Horizontal in-structure response spectra, median acceleration ranges 1 and 2, and design spectra: b) Foundation Y-direction (E-W) 
horszontes coded: node a x-erone

ceced ronge \& (modian of 30 . Ioenormal)

oceed runge 2 (modion of so. Jognornes)

vee. esz de-ce-0220 (0pp. el
9.00 pet domino

9.00 pet denping -

5.00 pet doming.

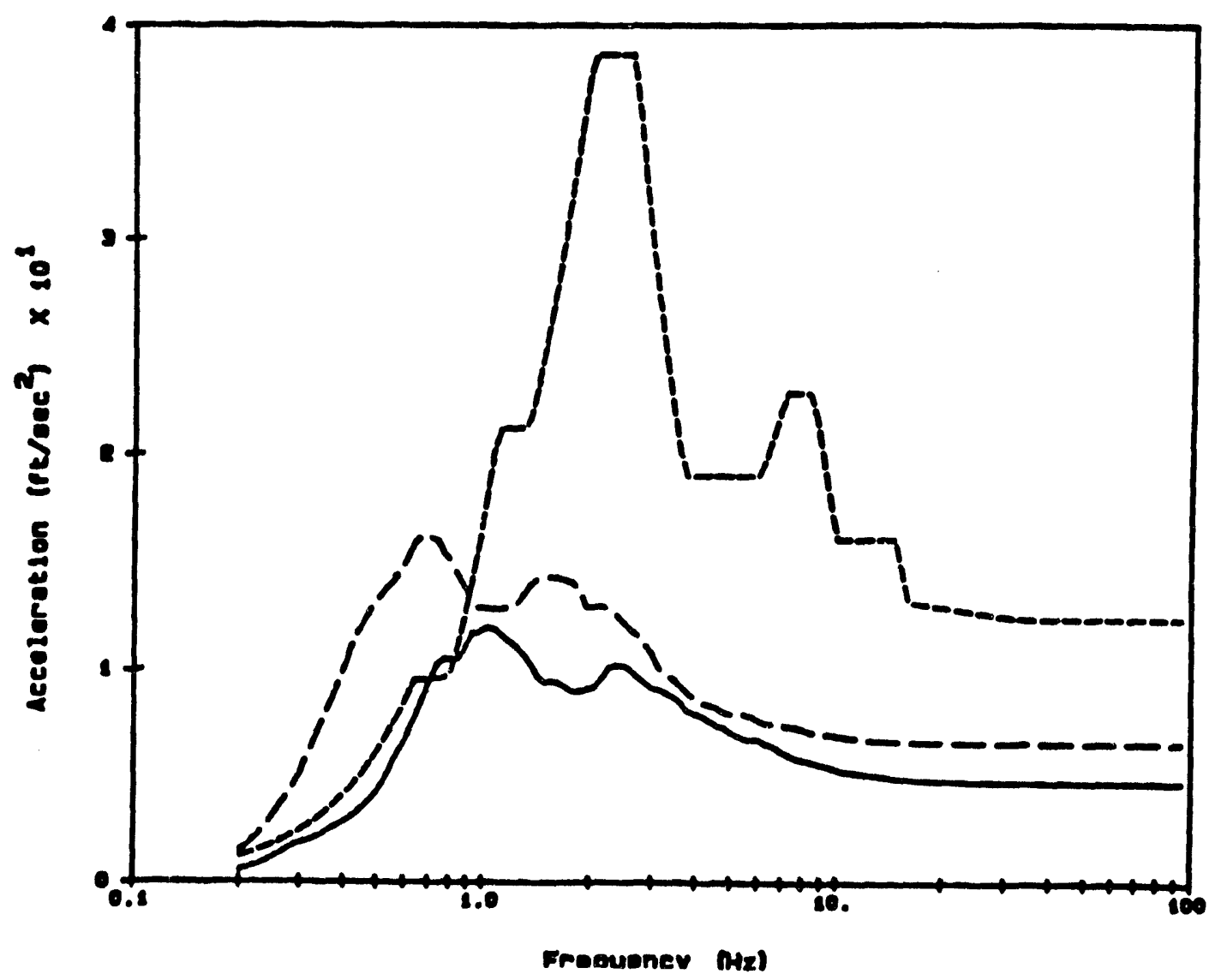

Figure 5.19. Horizontal in-structure response spectra, median acceleration ranges 1 and 2, and design spectra: c) Node 8, X-direction (N-S). 

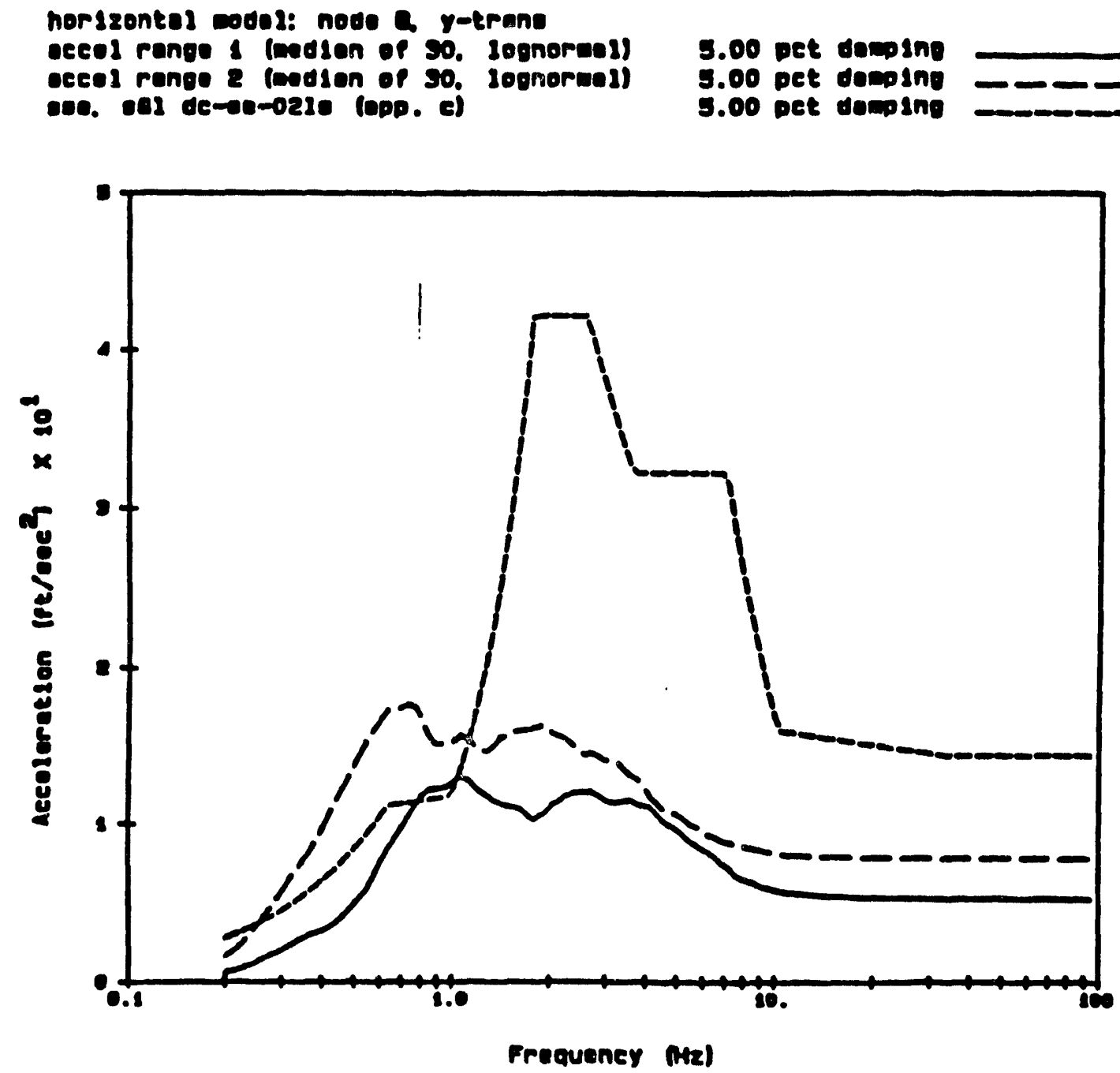

Figure 5.19. Horizontal in-structure response spectra, median acceleration ranges 1 and 2, and design spectra: d) Node 8, Y-direction (E-W). 
hordzontel model: nodes4, n-trone

oces pange \& (modson of 30, Ioenormas)

eceel renos 2 (oodsen of 30. Ioonormol)

veo. de-ae-oede (0pp. el
8.00 pee domino

8.00 pet domine

g.00 pet domine

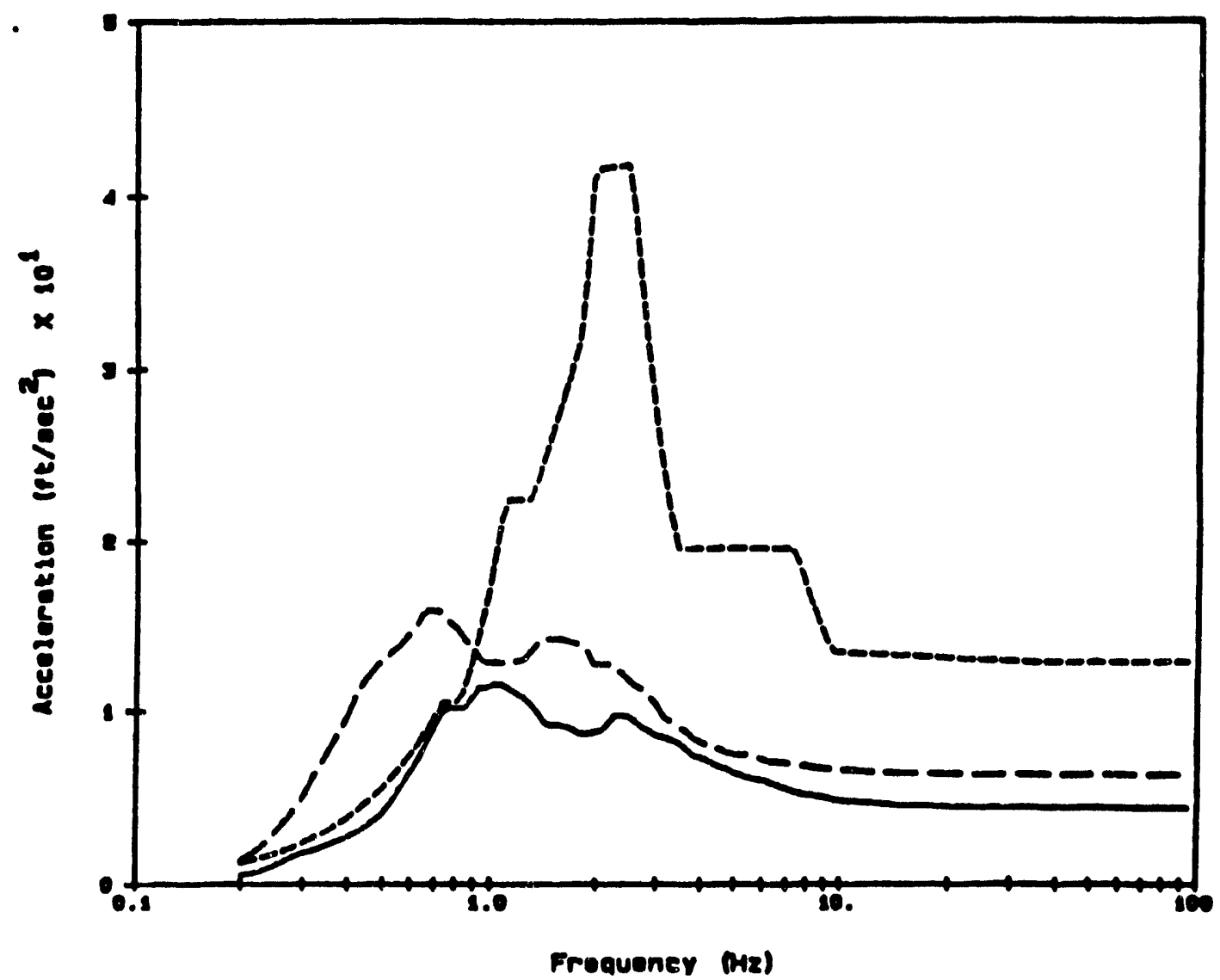

Figure 5.19. Horizontal in-structure response spectra, median acceleration ranges 1 and 2, and design spectra: e) Node 14, C-direction (N-S). 
horlzontel codes: nodos4, y-erons

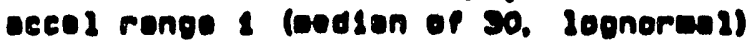
ocepl ronoo 2 (modson of 30. Jognormad)

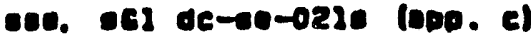

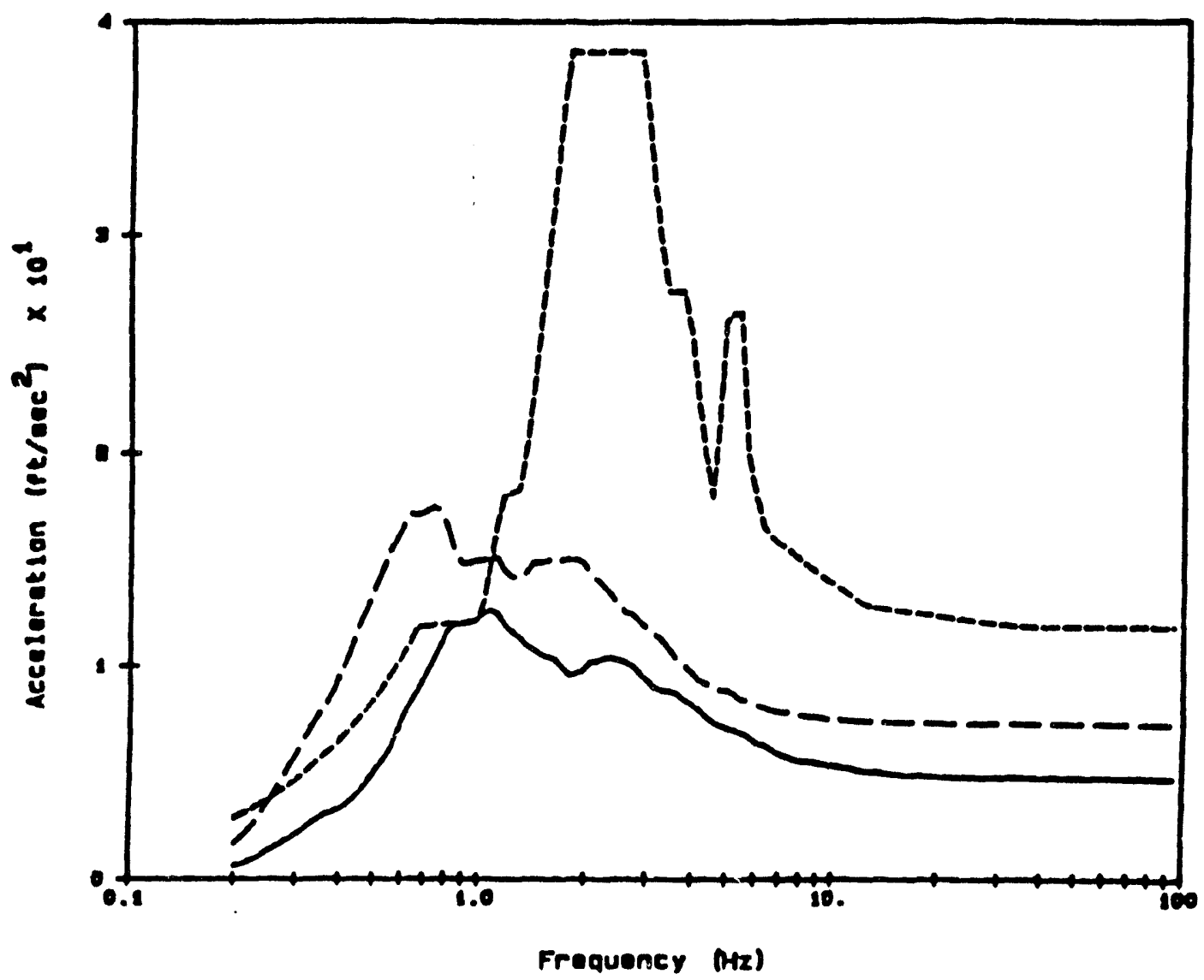

Figure 5.19. Horizontal in-structure response spectra, median acceleration ranges 1 and 2, and design spectra: f) Node 14, Y-direction (E-W). 

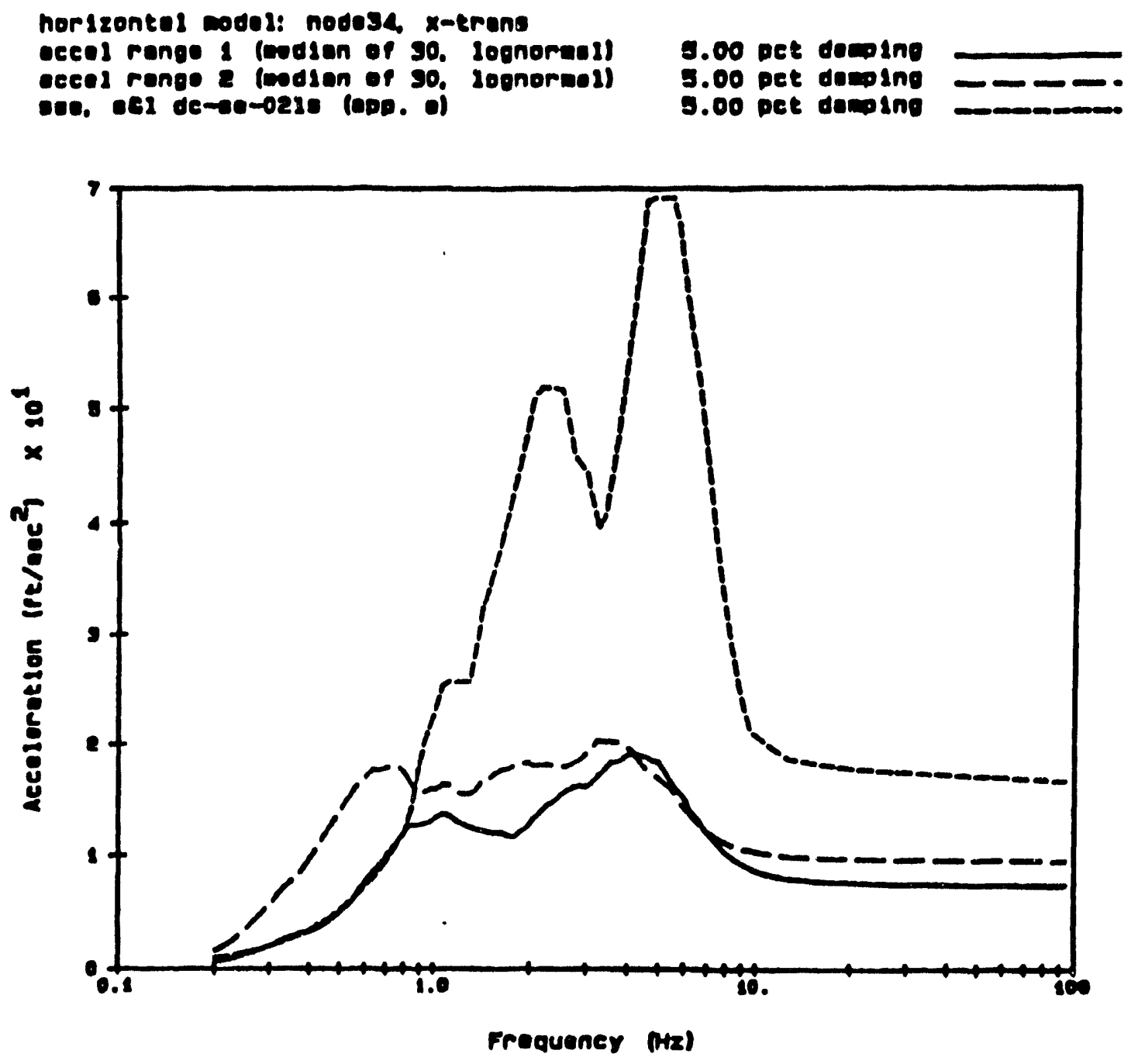

Figure 5.19. Horizontal in-structure response spectra, median acceleration ranges 1 and 2 and design spectra: g) Node 34, X-direction (N-S). 
horlzontel codel: nodesh y-trene

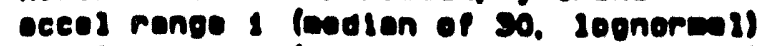

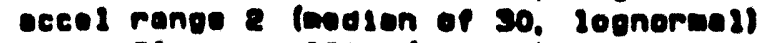
ver. aes de-co-0ese (OPp. o)

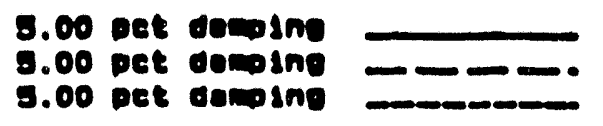

8.00 pet donino g.00 pet doping

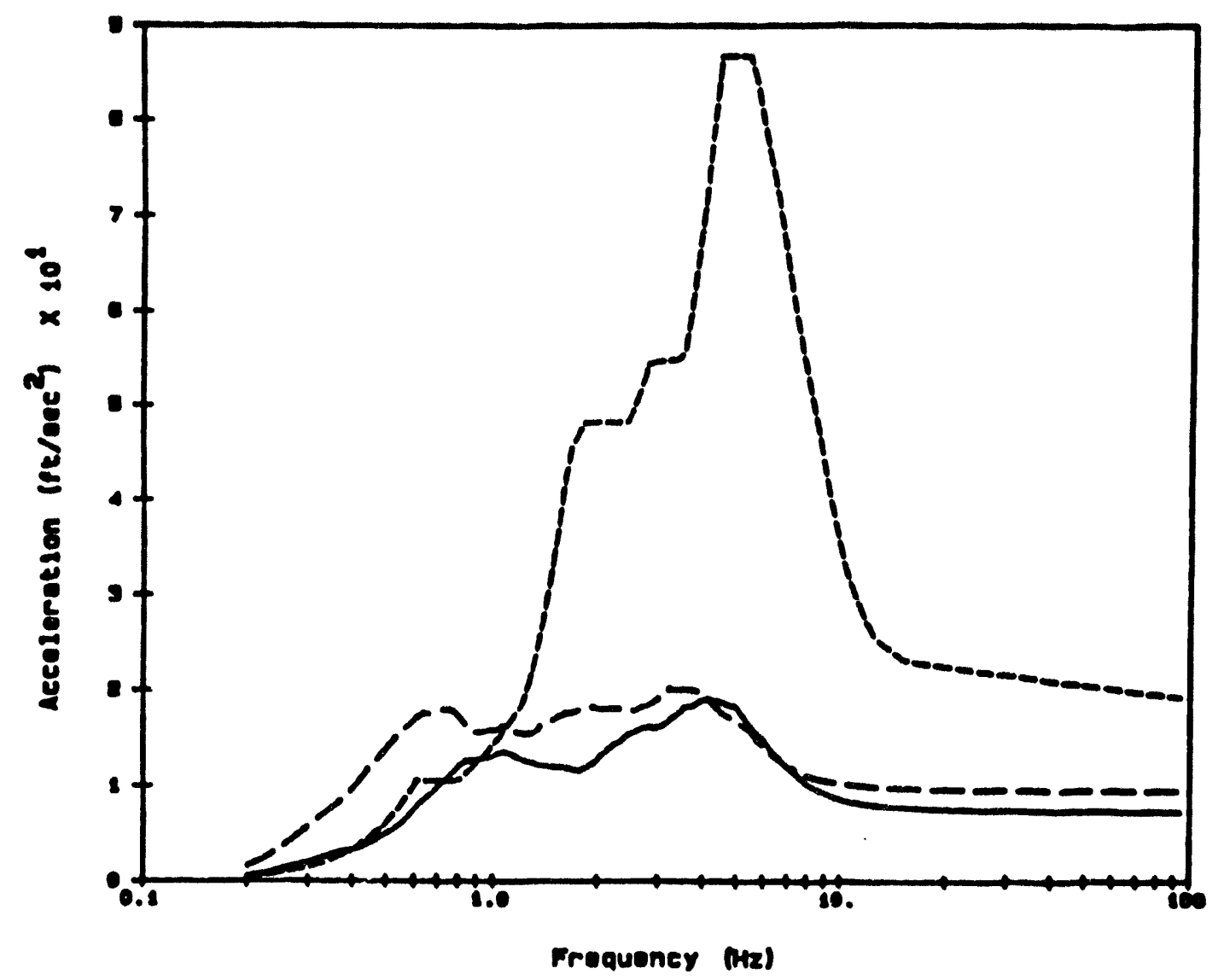

Figure 5.19. Horizontal in-structure response spectra, median acceleration ranges 1 and 2, and design spectra: h) Node 34, Y-direction (E-W). 
horlzontel modes: Pon, z-trane eccel ronge 1 (modson of 30, Jognomall) occel ronos 2 (medion of 30 . loonormal) ese. scl de-0e-021s (ODP. c)
9.00 pet dompino

5.00 pet denping

g.00 pet damping

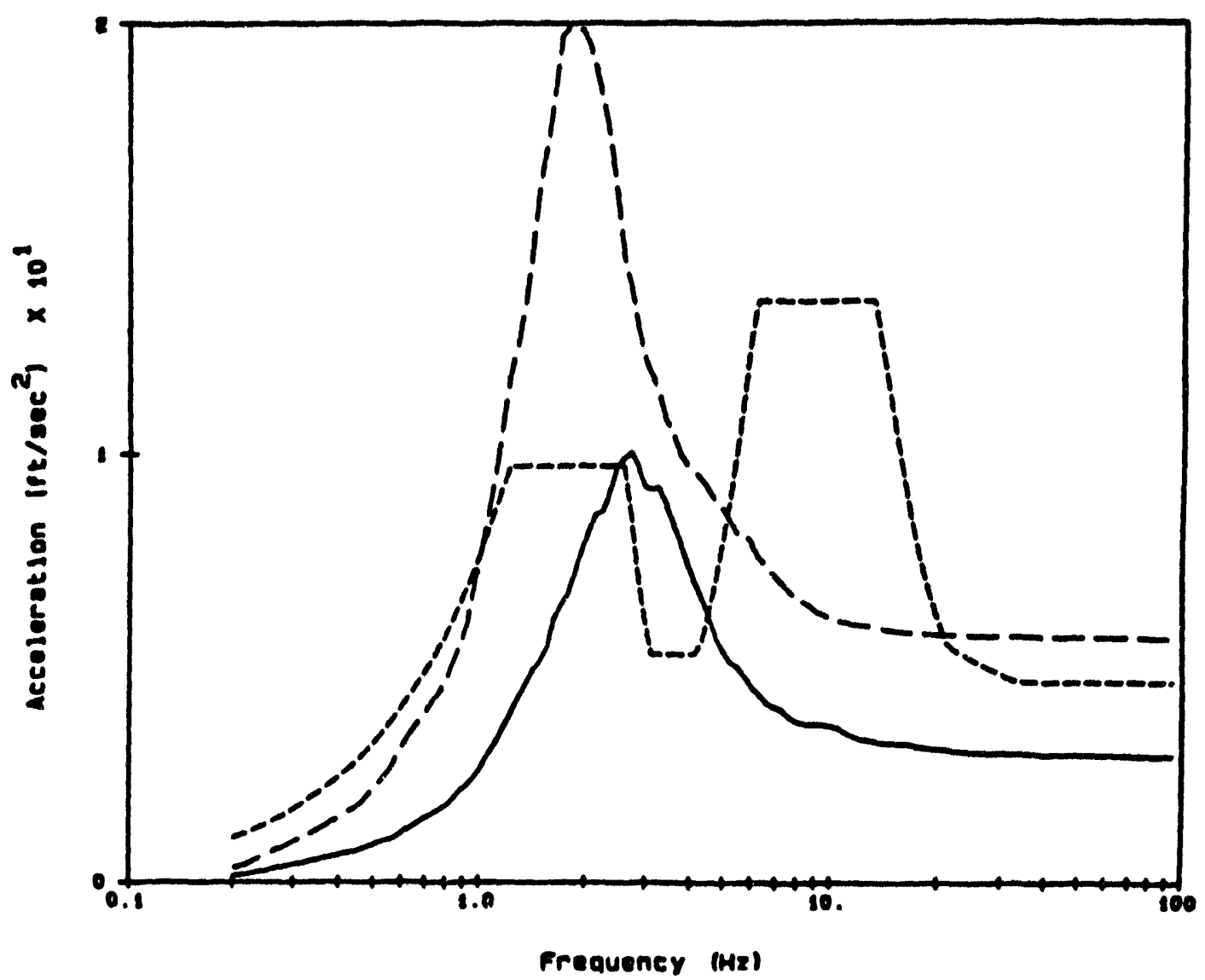

Figure 5.20. Vertical in-structure response spectra, median acceleration ranges 1 and 2, and design spectra: a) Foundation. 

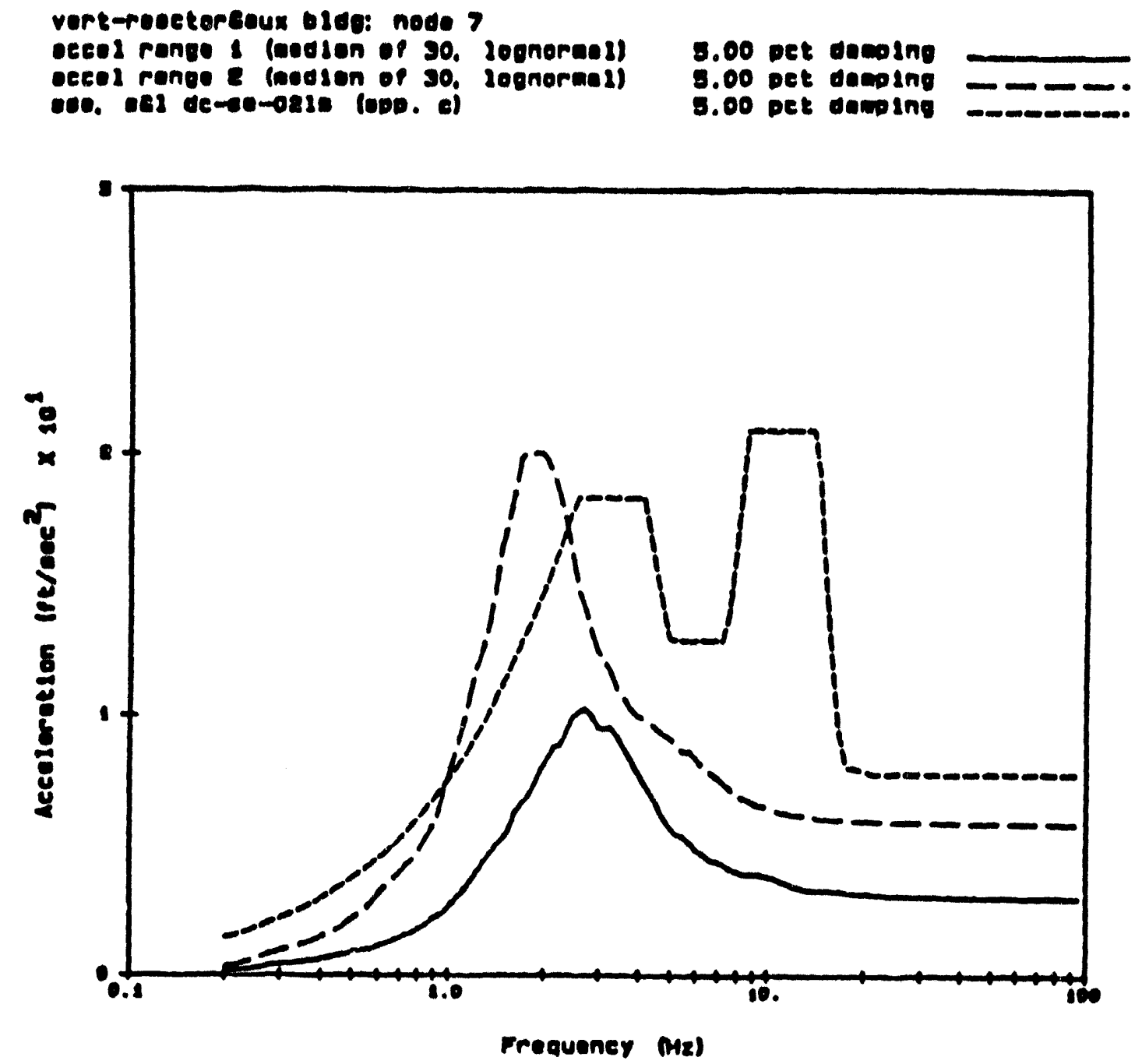

Figure 5.20. Vertical in-structure response spectra, median acceleration ranges 1 and 2, and design spectra: b) Reactor and auxiliary building, node 7. 
vert-rectorsoux blog: noweed

ocesl ronge 1 (meosion of 30. Leonermed)

ecesi renge fo (madion of 30. legnormos)

cee. ec2 de-ce-021s (000. e)

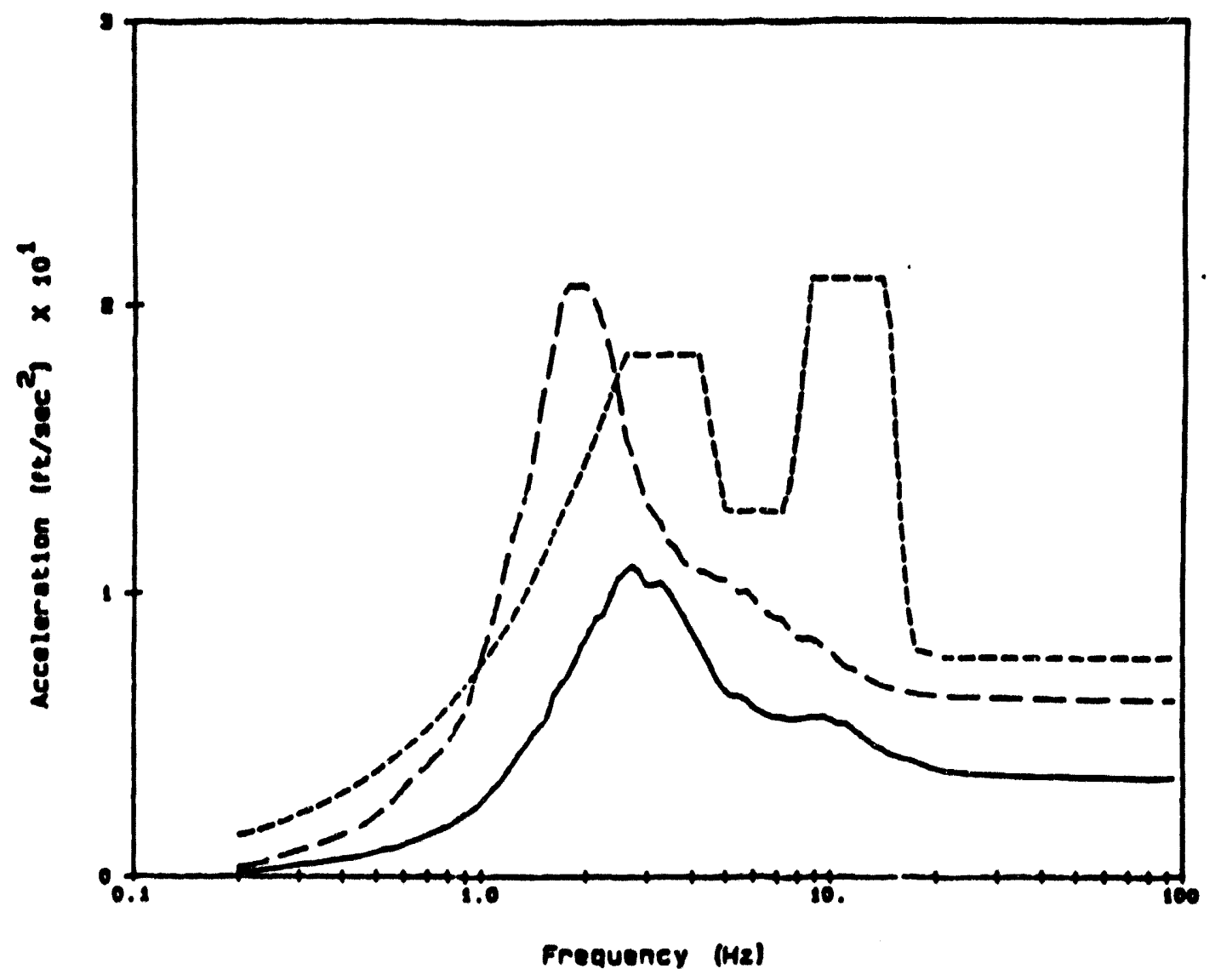

Figure 5.20. Vertical in-structure response spectra, median acceleration ranges 1 and 2, and design spectra: c) Reactor and auxiliary building, node 22. 

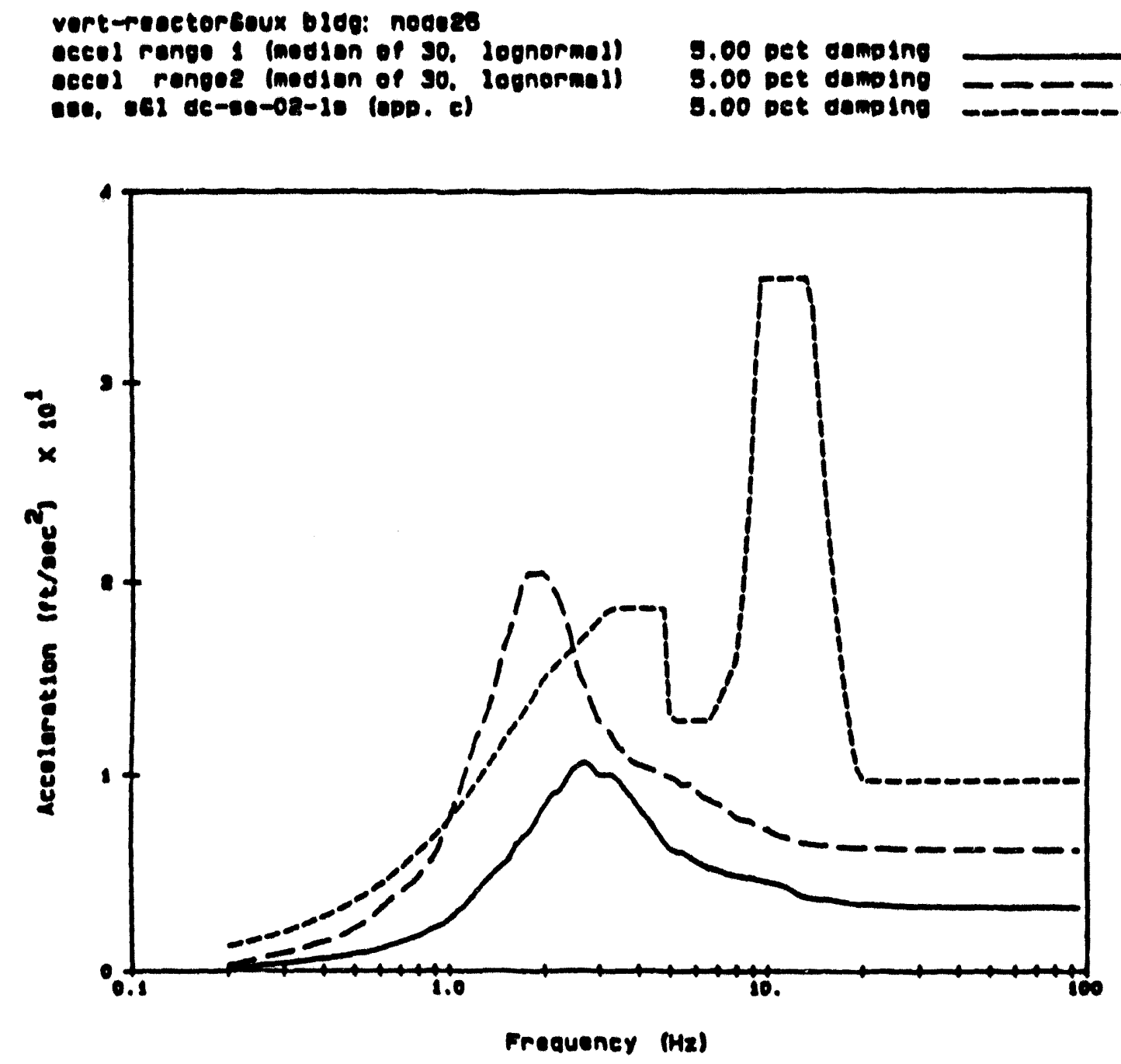

Figure 5.20. Vertical in-structure response spectra, median acceleration ranges 1 and 2 , and design spectra: d) Reactor and auxiliary building, node 26 . 
vert-rectopcoux osde: nodess

oceed roneo \& (modson of 30. Ioenormad)

oceed ronge 2 (moasion of 30, Iognormad)

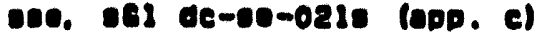

g.00 pet domping

g.00 pet dempsing

g.00 pet damping

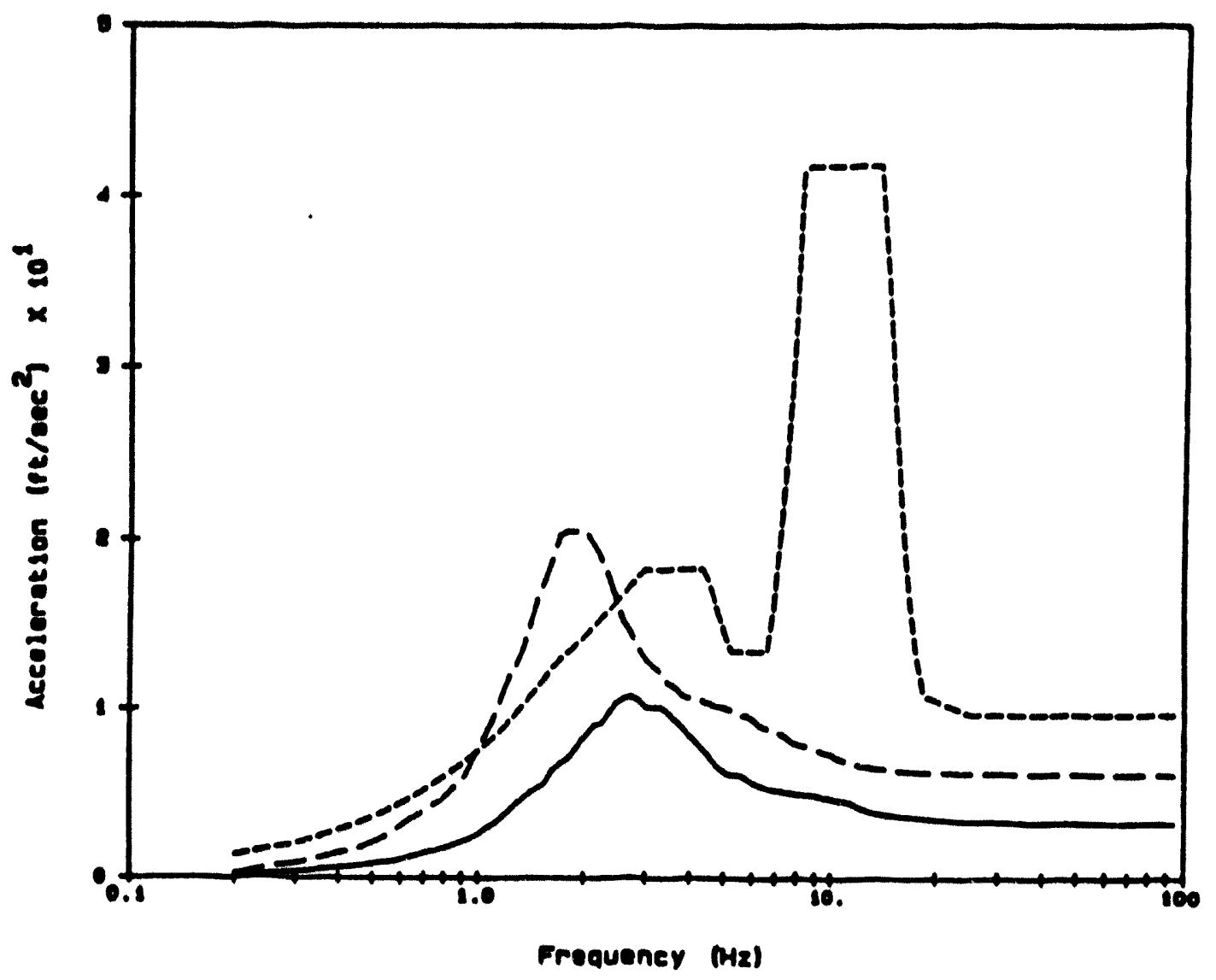

Figure 5.20. Vertical in-structure response spectra, median acceleration ranges 1 and 2, and design spectra: e) Reactor and auxiliary building, node 33. 
The reference response factor, $\mathrm{CF}_{\mathrm{Ref}}$, represents the mean ratio between the seismic response based on a response spectrum method with analysis method and the best estimate seismic response. The adjusting factors, $F_{M}, F_{D}$ and $F_{I}$, are required to account for the deviations in analysis methods, damping, and specification of input pipe support spectrum, respectively, from those used in computing the reference response factor, etc. The fourth adjusting factor, FS, is intended to account for the effect of the uncertainty in soil-structure interaction analysis methodology on the seismic response of the piping. Such adjustment may be necessary because, in a nuclear plant, the in-structure response spectra may be generated based on a soil-structure interaction analysis method that is not best estimate.

We assumed all pipe segments subjected to the maximum code allowable stress for service level D given in the FSAR Sec. 3.9, i.e., 66720 psi, and all valves subjected to the maximum BOP valve qualification limit i.e., $10 \mathrm{~g}$. The adjusting factors $F_{M}, F_{D}, F_{I}$, and $F_{S}$ and reference response factor CFRef for LaSalle County Station are as follows:

$$
\begin{aligned}
F_{M} & =1.0 \\
F_{D} & =1.49 \text { (pipe moment) } \\
F_{D} & =1.41 \text { (valve acceleration) } \\
F_{I} & =1.0 \\
F_{S} & =2.4 \\
C_{\text {Ref }} & =6.6 \text { (pipe moment) } \\
\text { CF }_{\text {Ref }} & =11.3 \text { (valve acceleration) }
\end{aligned}
$$

Therefore, the response factor for pipe moments is

$$
F_{R}=23.64 \text { (pipe moment) }
$$

and the response factor for valve acceleration is

$$
\mathrm{F}_{\mathrm{R}}=37.97 \text { (valve acceleration). }
$$

Using the response factor approach as delineated in Ref. 1.2, the median responses for the acceleration range 1 for the pipe moment and valve acceleration are:

$$
\begin{array}{ll}
\text { Median pipe moment } & =(66720 / 23.64) \times 17.81 \\
& =50260 \mathrm{in}-\mathrm{lbs} \\
\text { Median valve acceleration } & =10 / 37.97=.263 \mathrm{~g}
\end{array}
$$

where $17.81 \mathrm{in}^{3}$ is the section modulus of the pipe (6 in. Sch. 160) for the master fragility. 
The median pipe moment and vaive acceleration for the other acceleration ranges is based on the median response for acceleration with the average slopes of structural responses between acceleration levels 1 and 2 .

Table 5.10 presents the equipment responses used for all earthquake levels. The first column is the response number associated with that response. It is used in the quantification phase. The second and third columns represent the associated node and frequency respectively. The remaining six columns list the median responses for each of the six earthquake levels. All values are in g's except response 81. This response (piping) is in in-lbs. 
Table 5.10. Equipment responses for all earthquake levels.

\begin{tabular}{|c|c|c|c|c|c|c|c|c|}
\hline $\begin{array}{c}\text { NR\# } \\
=====\end{array}$ & $\begin{array}{l}\text { Node\# } \\
====\end{array}$ & $\begin{array}{c}\text { Freq } \\
====\end{array}$ & $\begin{array}{c}\mathrm{Eq \# 1} \\
=====\end{array}$ & $\underset{=====}{\mathrm{Eq \# 2}}$ & $\begin{array}{c}\mathrm{Eq \# 3} \\
=====\end{array}$ & $\underset{====}{\text { Eq\#4 }}$ & Eq\#5 & $\mathrm{Eq \# 6}$ \\
\hline 1 & 1 & $2-5$ & .21 & .23 & .24 & .26 & .28 & .31 \\
\hline 2 & 1 & $5-10$ & .16 & .17 & .19 & .20 & .22 & .24 \\
\hline 3 & 1 & $10-20$ & .17 & .19 & .20 & .22 & .24 & .27 \\
\hline 4 & 1 & $20-30$ & .18 & .19 & .21 & .22 & .24 & .26 \\
\hline 5 & $i$ & $30-50$ & .16 & .18 & .19 & .21 & .23 & .26 \\
\hline 6 & 1 & 15.0 & .18 & .19 & .21 & .22 & .24 & .26 \\
\hline 7 & 1 & Rigid & .14 & .16 & .17 & .19 & .21 & .24 \\
\hline 8 & 2 & $2-5$ & .22 & .24 & .25 & .27 & .29 & .32 \\
\hline 9 & 2 & $5-10$ & .15 & .17 & .18 & .20 & .22 & .25 \\
\hline 10 & 2 & $10-20$ & .17 & .18 & .20 & .21 & .23 & .25 \\
\hline 11 & 2 & $20-30$ & .16 & .18 & .19 & .21 & .23 & .26 \\
\hline 12 & 2 & $30-50$ & .15 & .17 & .18 & .20 & .22 & .25 \\
\hline 13 & 2 & 10.0 & .16 & .18 & .19 & .21 & .23 & .26 \\
\hline 14 & 2 & Rigid & .13 & .15 & .17 & .19 & .21 & .24 \\
\hline 15 & 3 & $2-5$ & .24 & .26 & .27 & .29 & .31 & .34 \\
\hline 16 & 3 & $5-10$ & .17 & .18 & .20 & .21 & .23 & .25 \\
\hline 17 & 3 & $10-20$ & .16 & .18 & .19 & .21 & .23 & .26 \\
\hline 18 & 3 & $20-30$ & .16 & .18 & .19 & .21 & .23 & .26 \\
\hline 19 & 3 & $30-50$ & .15 & .17 & .19 & .21 & .23 & .26 \\
\hline 20 & 4 & $2-5$ & .26 & .28 & .29 & .31 & .33 & .36 \\
\hline 21 & 4 & $5-10$ & .17 & .19 & .20 & .22 & .24 & .27 \\
\hline 22 & 4 & $10-20$ & .17 & .19 & .20 & .22 & .24 & .27 \\
\hline 23 & 4 & $20-30$ & .17 & .19 & .20 & .22 & .24 & .27 \\
\hline 24 & 4 & $30-50$ & .15 & .17 & .19 & .21 & .23 & .26 \\
\hline 25 & 4 & 9.0 & .16 & .18 & .19 & .21 & .23 & .26 \\
\hline 26 & 4 & 13.0 & .17 & .19 & .20 & .22 & .24 & .27 \\
\hline 27 & 5 & $2-5$ & .28 & .30 & .31 & .33 & .35 & .38 \\
\hline 28 & 5 & $5-10$ & .18 & .20 & .21 & .23 & .25 & .28 \\
\hline 29 & 5 & $10-20$ & .16 & .18 & .20 & .22 & .24 & .27 \\
\hline 30 & 5 & 20.30 & .17 & .19 & .20 & .22 & .24 & .27 \\
\hline 31 & 5 & $30-50$ & .16 & .18 & .19 & .21 & .23 & .26 \\
\hline 32 & 6 & $2-5$ & .34 & .35 & .37 & .38 & .40 & .42 \\
\hline 33 & 6 & $5-10$ & .22 & .23 & .25 & .26 & .28 & .30 \\
\hline 34 & 6 & $10-20$ & .17 & .19 & .21 & .23 & .25 & .28 \\
\hline 35 & 6 & $20-30$ & .16 & .18 & .20 & .22 & .24 & .27 \\
\hline 36 & 6 & $30-50$ & .16 & .18 & .20 & .22 & .24 & .27 \\
\hline 37 & 7 & $2-5$ & .34 & .36 & .37 & .39 & .41 & .44 \\
\hline 38 & 7 & $5-10$ & .22 & .23 & .25 & .26 & .28 & .30 \\
\hline 39 & 7 & $10-20$ & .17 & .19 & .21 & .23 & .25 & .28 \\
\hline 40 & 7 & $20-30$ & .16 & .18 & .20 & .22 & .24 & .27 \\
\hline 41 & 7 & $30-50$ & .16 & .18 & .20 & .22 & .24 & .27 \\
\hline 42 & 7 & 50.0 & .16 & .18 & .20 & .22 & .24 & .27 \\
\hline 43 & 7 & Rigid & .16 & .18 & .20 & .22 & .24 & .27 \\
\hline 44 & 8 & $2-5$ & .35 & .36 & .38 & .39 & .41 & .43 \\
\hline \multirow[t]{2}{*}{45} & 8 & 5-10 & .22 & .23 & .25 & .26 & .28 & .30 \\
\hline & & & & -120 & & & & \\
\hline
\end{tabular}


Table 5.10. Equipment responses for all earthquake levels (cont.)

\begin{tabular}{|c|c|c|c|c|c|c|c|c|}
\hline $\begin{array}{l}\text { NR\# } \\
====\end{array}$ & $\begin{array}{l}\text { Node } \\
:====\end{array}$ & Freq & $\begin{array}{c}E q \# 1 \\
:====\end{array}$ & $\begin{array}{c}\mathrm{Eq \# 2} \\
=====\end{array}$ & Eq\#3 & $\begin{array}{r}\mathrm{Eq} \# 4 \\
=====\end{array}$ & $\begin{array}{c}\mathrm{Eq \# 5} \\
=====\end{array}$ & $\begin{array}{c}E q \# 6 \\
====\end{array}$ \\
\hline 46 & 8 & $10-20$ & .17 & .19 & .21 & .23 & .25 & .28 \\
\hline 47 & 8 & $20-30$ & .17 & .19 & .20 & .22 & .24 & .27 \\
\hline 48 & 8 & $30-50$ & .16 & .18 & .20 & .22 & .24 & .27 \\
\hline 49 & 10 & $2-5$ & .24 & .26 & .27 & .29 & 31 & .34 \\
\hline 50 & 10 & $5-10$ & .16 & .18 & .19 & .21 & .23 & .26 \\
\hline 51 & 10 & $10-20$ & .16 & .18 & .19 & .21 & .23 & .26 \\
\hline 52 & 10 & $20-30$ & .16 & .17 & .19 & .20 & .22 & .24 \\
\hline 53 & 10 & $30-50$ & .15 & .17 & .18 & .20 & .22 & .25 \\
\hline 54 & 10 & 8.0 & .15 & .16 & .18 & .19 & .21 & .23 \\
\hline 55 & 10 & 10.0 & .16 & .18 & .19 & .21 & .23 & .26 \\
\hline 56 & 14 & $2-5$ & .27 & .29 & .30 & .32 & .34 & .37 \\
\hline 57 & 14 & $5-10$ & .18 & .19 & .21 & .22 & .24 & .26 \\
\hline 58 & 14 & $10-20$ & .17 & .19 & .20 & .22 & .24 & .27 \\
\hline 59 & 14 & $20-30$ & .17 & .19 & .20 & .22 & .24 & .27 \\
\hline 60 & 14 & $30-50$ & .15 & .17 & .19 & .21 & .23 & .26 \\
\hline 61 & 14 & 14.0 & .17 & .19 & .20 & .22 & .24 & .27 \\
\hline 62 & 14 & 20.0 & .17 & .19 & .20 & .22 & .24 & .27 \\
\hline 63 & 14 & Rigid & .14 & .16 & .18 & .20 & .22 & .25 \\
\hline 64 & 15 & $2-5$ & .20 & .22 & .24 & .26 & .28 & .31 \\
\hline 65 & 15 & $5-10$ & .15 & .17 & .18 & .20 & 22 & .25 \\
\hline 66 & 15 & $10-20$ & .15 & .17 & .18 & .20 & .22 & .25 \\
\hline 67 & 15 & $20-30$ & .19 & .20 & .22 & .23 & .25 & .27 \\
\hline 68 & 15 & $30-50$ & .18 & .20 & .21 & .23 & .25 & .28 \\
\hline 69 & 15 & 7.8 & .15 & .17 & .18 & .20 & .22 & .25 \\
\hline 70 & 15 & 16.0 & .16 & .18 & .19 & .21 & .23 & .26 \\
\hline 71 & 15 & 19.0 & .16 & .18 & .19 & .21 & .23 & .26 \\
\hline 72 & 15 & 28.0 & .20 & .21 & .23 & .24 & .26 & .28 \\
\hline 73 & 15 & Rigid & .14 & .16 & .17 & .19 & .21 & .24 \\
\hline 74 & 56 & $2-5$ & .36 & .38 & .39 & .41 & .43 & .46 \\
\hline 75 & 56 & $5-10$ & .23 & .24 & .26 & .27 & .29 & .31 \\
\hline 76 & 56 & $10-20$ & .20 & .22 & .23 & .25 & .27 & .30 \\
\hline 77 & 56 & $20-30$ & .18 & .20 & .22 & .24 & .26 & .29 \\
\hline 78 & 56 & $30-50$ & .17 & .19 & .21 & .23 & .25 & .28 \\
\hline 79 & 56 & 6.5 & .25 & .26 & .27 & .29 & .30 & .32 \\
\hline 80 & 56 & 20.0 & .20 & .22 & .23 & .25 & .27 & .30 \\
\hline 81 & & All & 50260 & 55914 & 61569 & 67851 & 75390 & 85442 \\
\hline 82 & & All & .26 & .29 & .32 & .36 & .39 & .45 \\
\hline 83 & & Free Field & .22 & .25 & .28 & .31 & .35 & .40 \\
\hline
\end{tabular}




\section{Section 6. Hydrodynamic Load Investigation}

\subsection{Significance of Different Hydrodynamic Loads}

\subsubsection{Approach}

In evaluating the probability of failure of a particular structure or equipment item due to earthquake occurrence, it is necessary to include the effect of any loads which may occur concurrently with the earthquake. In order to identify these loads and evaluate those that are significant, all possible load event combinations are enumerated as shown in the load event tree (Fig. 6.1). When an earthquake occurs, it may or may not trigger any one of several types of SRV discharge. Similarly, pipe breaks may or may not occur when an earthquake occurs. All such combinations are depicted as paths in the load event tree (Fig. 6.1). Note that there are several earthquake paths, representing the range of possible earthquake intensities.

In order to void plant damage, plant safety systems must be available to mitigate the various load event combinations (event tree paths) postulated. In the seismic risk analysis, the probability of occurrence of the various load combinations and the probability of failure of the safety systems under the load combination is required. The probability of failure of a specific piece of safety equipment, given that an earthquake initiating event occurs, obviously depends on the particular load combination being considered. For example, consider the load event tree path (or load combination) of earthquake (of, say, intensity E), with single valve SRV actuation, and intermediate pipe break. In this case, evaluation of the failure probability of the equipment item must include the effects of seismic loads, the single valve SRV discharge load, and the loads caused by the intermediate pipe break. In the same light, when the load combination being considered does not involve, say, any pipe break event, pipe break need not be included in the failure probability evaluation. With all the possible events and load combinations enumerated, there are several factors which must be considered before deciding whether or not the effects of certain loads actually need to be considered in the seismic failure probability evaluation of a particular component. That is, while a particular load event scenario may include many loads, certain loads may not need to be combined with the seismic loads. These factors are:

1. Magnitude of the response caused by the loads

2. Time-phasing of the loads

3. Probability of occurrence (given earthquake occurrence) of the loads.

If the magnitude of the hydrodynamic response is significantly lower than the seismic response (for the particular earthquake event being considered), then it is clear that no load combination is necessary and the probability of safety system failure need only consider the earthquake loads.

The time-phasing of the loads must also be considered. Due to the relatively short duration of strong earthquake motion (generally, less than 10 to $15 \mathrm{sec}$ ), certain loads on the load event tree path may occur late in time with respect to the earthquake, making combination of the two loads unlikely (e.g., chugging, see Sec. 6.1.2). Finally, the occurrence probability of the loads (given 

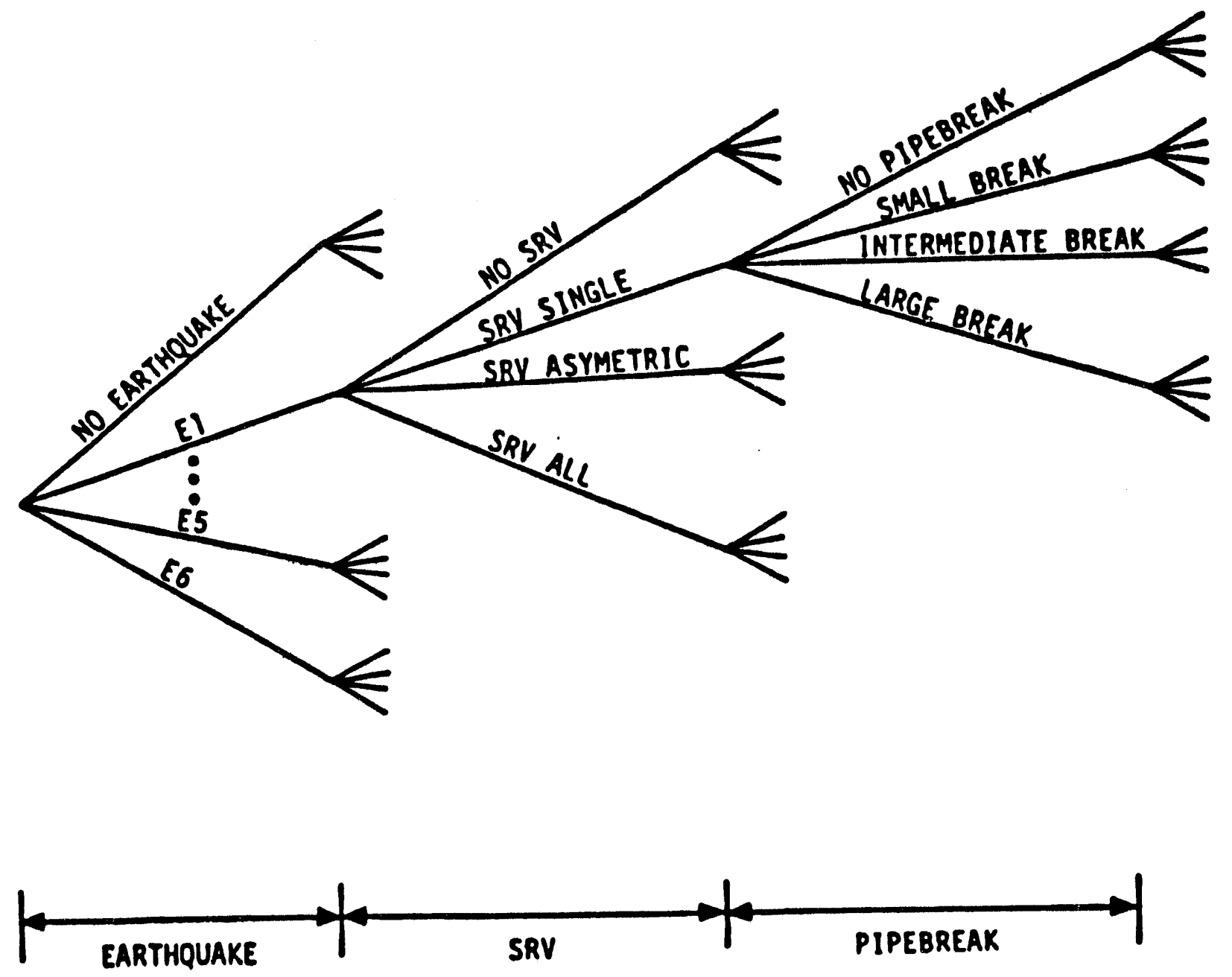

Figure 6.1. Load event tree. 
earthquake occurrence) must be considered. For an earthquake initiating event of a given intensity, the conditional occurrence probability of the SRV and LOCA events can be obtained from curves such as those shown in Fig. 6.2. If the occurrence probability of an event is relatively small, for an earthquake of some given intensity, then the path (i.e., the particular load combination) can be removed from the load event tree. Further, if the conditional occurrence probability of a particular event (e.g., large LOCA) can be shown to be small for even large earthquakes, then all the earthquake initiated load event tree paths which include this event can be removed from the tree. The loads caused by this event, therefore, need not be considered in the load combination problem. The occurrence frequency of plant damage by a load event tree path that includes this event would be small compared to the occurrence frequency of other paths to plant damage.

The following sections examine the various hydrodynamic loads which may occur in a Mark II nuclear power plant with regard to the afore mentioned criteria. The final objective is to discover those loads which may act concurrently with seismic loads and can significantly affect the probability of safety system failure due to earthquake initiated events.

\subsubsection{Description of Hydrodynamic Loads}

General. This section describes the hydrodynamic loads which could be induced by an earthquake initiated accident condition in a Mark II nuclear power plant. ${ }^{[6.1]}$ Specifically, induced hydrodynamic loads in the LaSalle County Station are examined to determine their probable magnitudes and load effects, their occurrence times, and the likelihood of a seismic event inducing such loads. The objective is to identify the hydrodynamic loads which can combine with seismic loads and affect the safety of plant structures and equipment.

Accident-induced hydrodynamic loads which are considered in this study fall into two major categories; dynamic loads due to safety relief valve (SRV) discharge, and dynamic loads resulting from LOCA. The LOCA induced loads are further broken down into the specific phenomena: jet forces, pool swell, condensation-oscillation, and chugging.

Safety/Relief Valve Discharge Loads. SRVs provide a means of pressure relief in a Mark II pressure suppression system during certain transients. In this process, steam is discharged through the valves into the suppression pool for condensation.

Each SRV line in the suppression pool of LaSalle is fitted with a quencher device which enhances the heat transfer during SRV actuation. SRV actuations may occur because of the following events:

1. Reactor system transient pressure increase.

2. Planned operation action.

3. Failure or error affecting one SRV.

4. Automatic depressurization system (ADS) activation.

An SRV discharge initiated by operator action or due to activation of he ADS is not expected to occur until after the seismic event has terminated. Also, the probability of simultaneous occurrence of SRV loads due to a random error with seismic loads is expected to be negligible. Hence, loads 


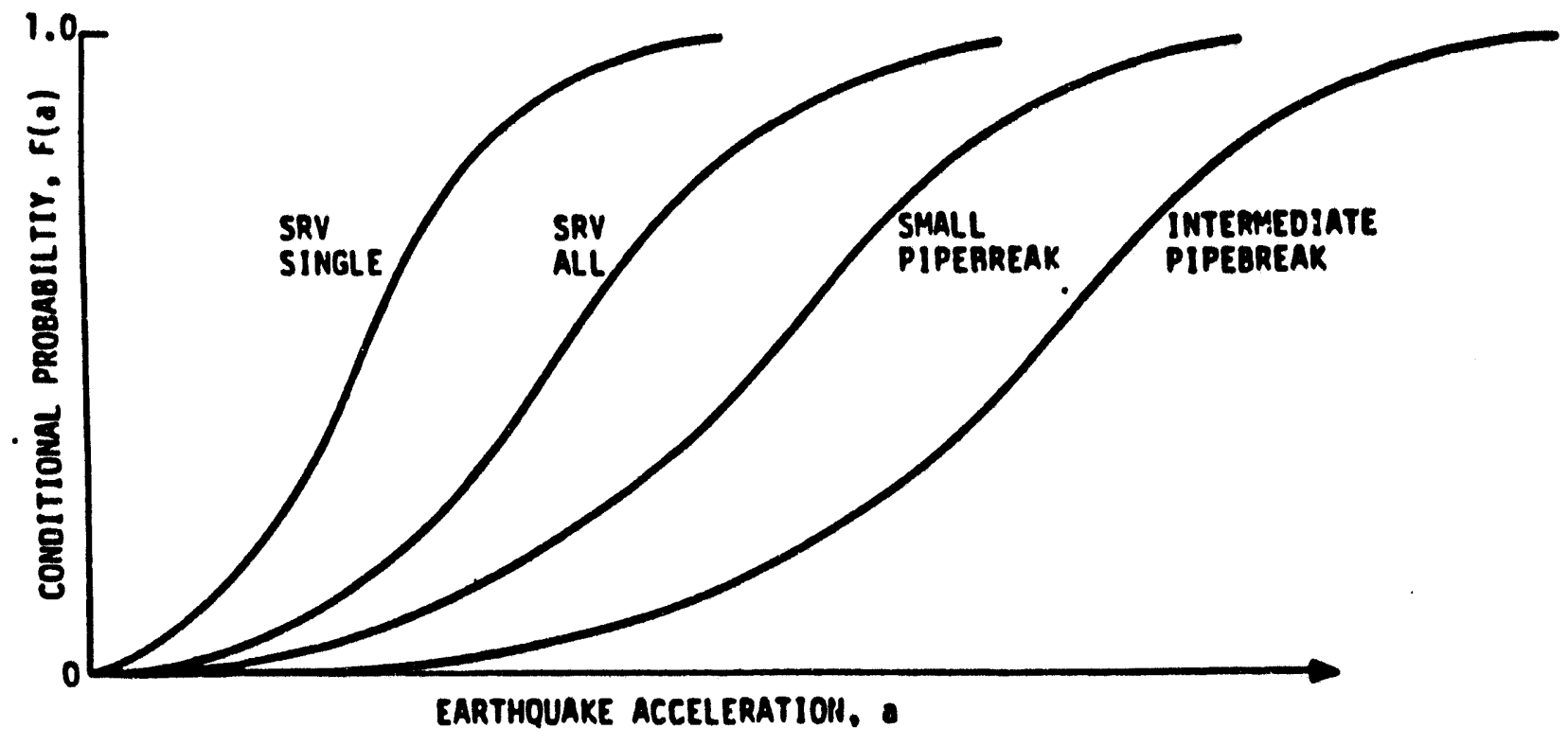

Figure 6.2. Example hydrodynamic event conditional probability curves. 
due to this type of SRV discharge need not be considered in this study. It is, however, likely, that earthquakes will induce a system transient pressure increase. SRV actuation in a seismic-initiated load event tree path would most likely be caused by a reactor system transient pressure increase. Since even small earthquakes can cause loss-of-offsite power to the plant, a transient and a turbine or reactor trip, therefore, SRV actuation is likely for all earthquake levels.

The SRV discharge lines which are submerged in the suppression pool contain ambient air and a column of water whose tree surface is initially the same as the water level in the suppression pool. Upon actuation of the SRV lines, steam pressure compresses the air column and forces the water column through the quencher thus resulting in a "water clearing" phase of the SRV discharge. Following the water clearing, air is forced into the suppression pool in the form of high pressure bubbles. Once in the pool, the air bubbles expand and rise to the surface which induces an oscillatory and decaying pressure field. Following the air clearing phase, steady-steam discharge flow is established and continues until the SRV closes. Steam condensation at the quencher exit results in oscillatory loads which induce pressures and thus accelerations on the wetwell walls below the pool surface. The induced loads are then transferred through the wetwell walls and floor to the sacrificial shield, containment wall and other parts of the reactor building.

For multiple SRV discharge conditions, the loads on the suppression pool are effectively the combination of SRV discharge loads at a number of locations in the suppression pool. There are three SRV cases of concern here; SRV-single valve, SRV-asymmetric valves, and SRV-all valves. SRV-single valve refers to the loads induced when only a single valve actuates, SRV-asymmetric valves refers to the loads induced when multiple SRVs discharge simultaneously and the valves that are discharging are not in symmetric positions around the reactor vessel, and SRV-all valves refers to the loads induced when all the valves discharge.

LOCA-Induced Loads. Three LOCA accident conditions are used in the design of the Mark II containment system. These are the Design Basis Accident (DBA), the Intermediate Break Accident (IBA), and the Small Break Accident (SBA). These accidents cover the spectrum of conditions which can cause LOCA-induced hydrodynamic loads. The DBA is defined as a double-ended break of either a recirculation line or a main steamline, an IBA is defined as a liquid line break of approximately $0.1 \mathrm{ft}$. in area, and a typical SBA is a $0.01 \mathrm{ft}$ steam line break.

Jet Forces. Following a DBA and depressurization of the reactor, the drywell pressure increases as a result of the mass and energy release from the break. Consequently, the water initially in the vent system is accelerated out from the vents. During this vent clearing process, the water exits the vents will form submerged jets in the suppression pool which can produce loads on structures near vent exits and on the suppression pool basement. These jet forces are less severe for an IBA because the intermediate line break will increase drywell pressure and temperature at a more moderate rate. Since the jet forces, which are induced by a DBA, mainly affect the structures near the vent exits (which are conservatively designed to withstand these forces), they are not further considered in this study.

Pool Swell. Pool swell is one of the hydrodynamic loads induced in a Mark II containment as a result of a DBA. As discussed in the previous section, a vent-clearing process occurs following a DBA which results in jet loads in the suppression pool. Immediately following vent water clearing, bubbles containing air and steam form at the vent exits. As the flow of air and steam from the drywell becomes established in the vent system, the initial vent-exit bubbles expand with the bubble pressure nearly equal to the drywell pressure. The steam fraction of the flow into the pool will be condensed, but continued injection of drywell air and the resultant expansion of the air 
bubbles will produce a rapid rise of the suppression pool surface (pool swell). The expanding bubble will create a pressure field in the suppression pool which causes loads on submerged structures and the pool boundaries.

During the early stages of pool swell, a slug of water is accelerated upward by the expanding air bubble. Structures and equipment close to the pool surface will experience impact loads as the rising pool surface contacts the lower surface of the structures. In addition to these inertial drag loads, dissipative drag loads will develop as water flows past structures and equipment at elevations above the vent exit and below the maximum swell height. The rising slug of water will be stopped by the combined effect of gravity and compression of the wetwell airspace. This compression produces an upward force on the diaphragm floor. Subsequently, the air bubble breaks through the surface with essentially no further swell. This begins the fallback process in which the water settles back in the suppression pool. Concurrently, air flow through the main vents rapidly decreases and steam flow is established.

For an IBA, the drywell pressurization rate is less than for a DBA. Consequently, the bubble pressure in the suppression pool is less and the moderate rate of drywell pressurization does not result in significant pool swell.

The pool swell loads, as for the let forces, mainly affect the structures submerged in the suppression pool. Also, the effect of pool swell on the suppression pool boundary walls is such that it does not significantly affect structures and equipment outside of the wetwell boundary. Therefore, the pool swell loads need not be considered further in this study.

Condensation-(Oscillation. The condensation-oscillation (CO) loads in a Mark II plant follow the jet forces and pool swell as a result of a design basis accident. During this period, the steam condenses as it exits from the vents. For initial high flow rates, the steam-water interface at the vent exit oscillates as the steam is condensed. The condensation process induces pressure loads on the wetwell, vent, submerged structures and pool boundaries. Like the SRV loads, the main effect of $\mathrm{CO}$ loads is to induce pressures and accelerations in the wetwell below the water surface level which are then transferred to other parts of the sacrificial shield wall and the containment through the wetwell walls and slab.

It should be noted that $\mathrm{CO}$ loads do not occur as a result of an IBA or a SBA. During the IBA, a high drywell pressure signal scrams the reactor and leads to closure of the main steamline isolation valves (MSIVs). The closure of MSIVs can result in an increase in RPV pressure which is relieved by opening of the SRVs. Therefore, for intermediate site breaks, the steam flow rate through the vents may be insufficient to cause CO loads. Although MSIVs would not close immediately in the event of a small break accident, the steam flow rate is, again, insufficient to cause CO loads.

Chugging. The high steam flow rate through the main vent system during a DBA is followed by a decreasing flow rate as the blowdown progresses. At lower flow rates, the steam bubble at the vent exit alternately grows and collapses in a condensation process referred to as chugging, i.e., the steam-water interface is no longer sustained outside the vent and a process of unsteady condensation begins. The structures that are affected by chugging are the vents and the suppression pool wetted perimeter. As for $\mathrm{CO}$, the main effect of chugging is to induce accelerations on the wetwell below the water surface which would then be transferred to other parts of the reactor building through the wetwell walls and basemat.

For intermediate size breaks, the steam flow rate through the vents may be insufficient to cause $\mathrm{CO}$ 
loads, but chugging loads will be experienced until the reactor vessel blowdown is reduced to a flow rate where chugging becomes insignificant. Also, for small break accidents, there may be sufficient steam flow to cause chugging.

\subsubsection{Hydrodynamic Loads Screening}

Based on the criteria given in Sec. 6.1.1, the hydrodynamic loads described in Sec. 6.1.2 can be screened to discern which of these are significant and must be considered in the bad combination analysis.

SRV Loads. For the LaSalle seismic risk assessment, it should be conservatively assumed that all earthquake levels induce SRV actuation as discussed in Sec. 6.1.2. Thus, evaluation of the seismic fragility of the safety systems should consider seismic load combined with SRV load if the response due to the SRV bad is significant compared with that of the earthquake.

Examination of the SRV design response spectra for LaSalle shows that for horizontal accelerations, SRV-asymmetric is the critical case and for vertical accelerations, SRV-all is the critical case. Although the risk assessment should consider all possible SRV cases and the occurrence probability of each case, it will suffice for the present purpose of screening significant SRV loads to look only at the most critical cases. This is a conservative approach since the more significant loads could have lower occurrence probabilities.

Table 6.1 shows a comparison of horizontal accelerations (maximum floor acceleration, i.e., ZPA, and peak spectral acceleration) due to the SRV-asymmetric loads and the seismic SSE loads at several representative locations within the reactor and containment structures. Figure 6.3 shows the analytical model used in the Sargent \& Lundy hydrodynamic loads analysis, with the locations for the loads comparison circled. Based on the results shown in Table 6.1, it is clear that horizontal SRV accelerations are not significant and need not be combined with seismic loads.

Table 6.2 compares the vertical accelerations due to the SRV-all loads with the vertical accelerations due to the seismic SSE loads at the same locations used for the horizontal accelerations comparison (Fig. 6.3). Note that the SRV-all accelerations are significant (even when compared with twice the SSF accelerations) along the vertical length of the reactor vessel. This results because all SRVs opening induces large vertical pulsive forces on the reactor building basemat, causing the reactor pedestal and reactor vessel to accelerate vertically as a rigid body. It is clear then, that for components located in and around the vertical length of the reactor vessel and pedestal, SRV-all vertical accelerations must be considered with the seismic accelerations for seismic fragility evaluation. A bad combination analysis will be carried out for this case. Of course, the actual impact of the SRV bad will depend the frequency of the affected component.

As a final note, it is pointed out that the loads comparison was based on design spectral accelerations and not the median-centered spectral accelerations. The design spectra were used herein, since they were generally available for locations throughout the structure. For purposes of load magnitude comparisons, it is felt that the use of design spectra is acceptable. This assumes that the degree of conservatism in the design SRV and the design seismic spectra is equivalent. 
Table 6.1. Horizontal accelerations for SRV-asymmetric and seismic (SSE) loads.

\begin{tabular}{|c|c|c|c|c|c|}
\hline & & \multicolumn{2}{|c|}{ sav-assmetnic } & \multicolumn{2}{|c|}{ stismic (sse) } \\
\hline & Locallon & ${ }_{(g)}^{\text {Maseel. }}$ & 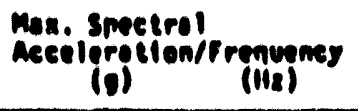 & ${ }^{\text {Mox }}$ (g) $)^{\text {neell. }}$ & 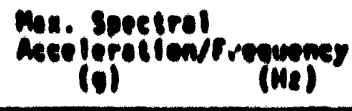 \\
\hline 11 & $\begin{array}{l}\text { Contalmone Noll } \\
\left(694^{\prime}-6^{\prime \prime}\right)\end{array}$ & 0.26 & $0.00 / 80$ & - & - \\
\hline 8) & $\begin{array}{l}\text { Confolmment Noll } \\
\left(740^{\circ} .0^{\circ}\right)\end{array}$ & 0.068 & $0.26 / 17$ & 0.92 & $1.0 / 8.8$ \\
\hline 31 & $\begin{array}{l}\text { Reactor Sunport } \\
\left(15 i^{-} \cdot 3^{\circ}\right)^{-1}\end{array}$ & 0.058 & $0.34 / 18$ & 0.50 & $8.4 / 2.2$ \\
\hline a) & 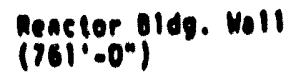 & 0.07 & $0.28 / 10$ & 0.30 & 1.012 .8 \\
\hline s) & $\begin{array}{l}\text { Confelompal Moll } \\
\left(604^{-0.04}\right)\end{array}$ & 0.05 & $0.11 / 20$ & 0.08 & 1.1/8.8 \\
\hline 6) & $\begin{array}{l}\text { Apector glds. Moll } \\
(00) \cdot 00 \%)\end{array}$ & 0.08 & 0.08 & 0.04 & 1.02 .8 \\
\hline 11 & $\begin{array}{l}\text { Recetor Yesse' } \\
\text { (Pop-e24'.4p) }\end{array}$ & 0.18 & $1.0 / 10$ & 1.10 & $1.0 / 4.0$ \\
\hline 81 & 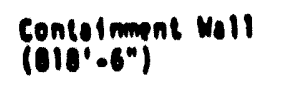 & $\cdot 0.05$ & - 0.05 & - & - \\
\hline
\end{tabular}

- Spectral eccelerattons ore for 28 domplng

- Spectra nol ovolleble

- See rigure 6.3 for location of points 
Table 6.2. Vertical accelerations for SRV-all and seismic (SSE) loads.

\begin{tabular}{|c|c|c|c|c|c|}
\hline \multirow{2}{*}{\multicolumn{2}{|c|}{ Lecelten }} & \multicolumn{2}{|r|}{ servatel } & \multicolumn{2}{|c|}{ stisnc (see) } \\
\hline & & $\frac{\text { Max. Aceel. }}{(0)^{-}}$ & $\begin{array}{l}\text { Mon. Smetrol } \\
\text { Aceilgreltion/fropupmey } \\
\text { (s) }\end{array}$ & Mon, Aecel. & 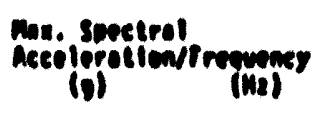 \\
\hline 1) & $\begin{array}{l}\text { Contolnmene Moll } \\
\left(010^{\circ} \cdot 0^{\circ}\right)\end{array}$ & 0.11 & $0.70 / 11$ & $\bullet$ & - \\
\hline 2) & $\begin{array}{l}\text { Contolmment Moll } \\
\left(740^{\circ} .0 \%\right)\end{array}$ & 0.16 & $0.61 / 17$ & 0.32 & $2.1 / 12$ \\
\hline 31 & 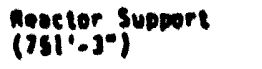 & 0.21 & $2.4 / 4.3$ & 0.24 & $1.2 / 12$ \\
\hline 4 & $\begin{array}{l}\text { Aesetergldg. Wall } \\
\left(1610^{\circ}\right)\end{array}$ & 0.11 & $0.41 / 1.6$ & 0.30 & $1.1 / 12$ \\
\hline 31 & $\begin{array}{l}\text { Coned lnmene Woll } \\
\left(600^{\circ} \cdot 0^{\circ}\right)\end{array}$ & 0.10 & $0.45 / 11$ & 0.32 & $2.8 / 12$ \\
\hline 6$)$ & $\begin{array}{l}\text { Receter } 1 \text { idg. Mall } \\
\left.(80)^{\circ} .0^{\circ}\right)\end{array}$ & 0.085 & $0.48 / 4.5$ & 0.30 & $2.0 / 12$ \\
\hline$\pi$ & $\begin{array}{l}\text { Aeoctor Yessp) } \\
\text { (Pop-020'-4.) }\end{array}$ & 0.20 & $2.4 / 4.5$ & 0.24 & 1.2118 \\
\hline 11 & 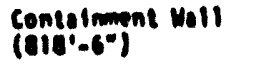 & 0.08 & $0.38 / 4.5$ & 0.33 & $2.8 / 12$ \\
\hline
\end{tabular}

- Spectrol eceelerations ore for 28 domping

- Spectra mol ovalloble

- See ilgure 6.3 for location of polnts 


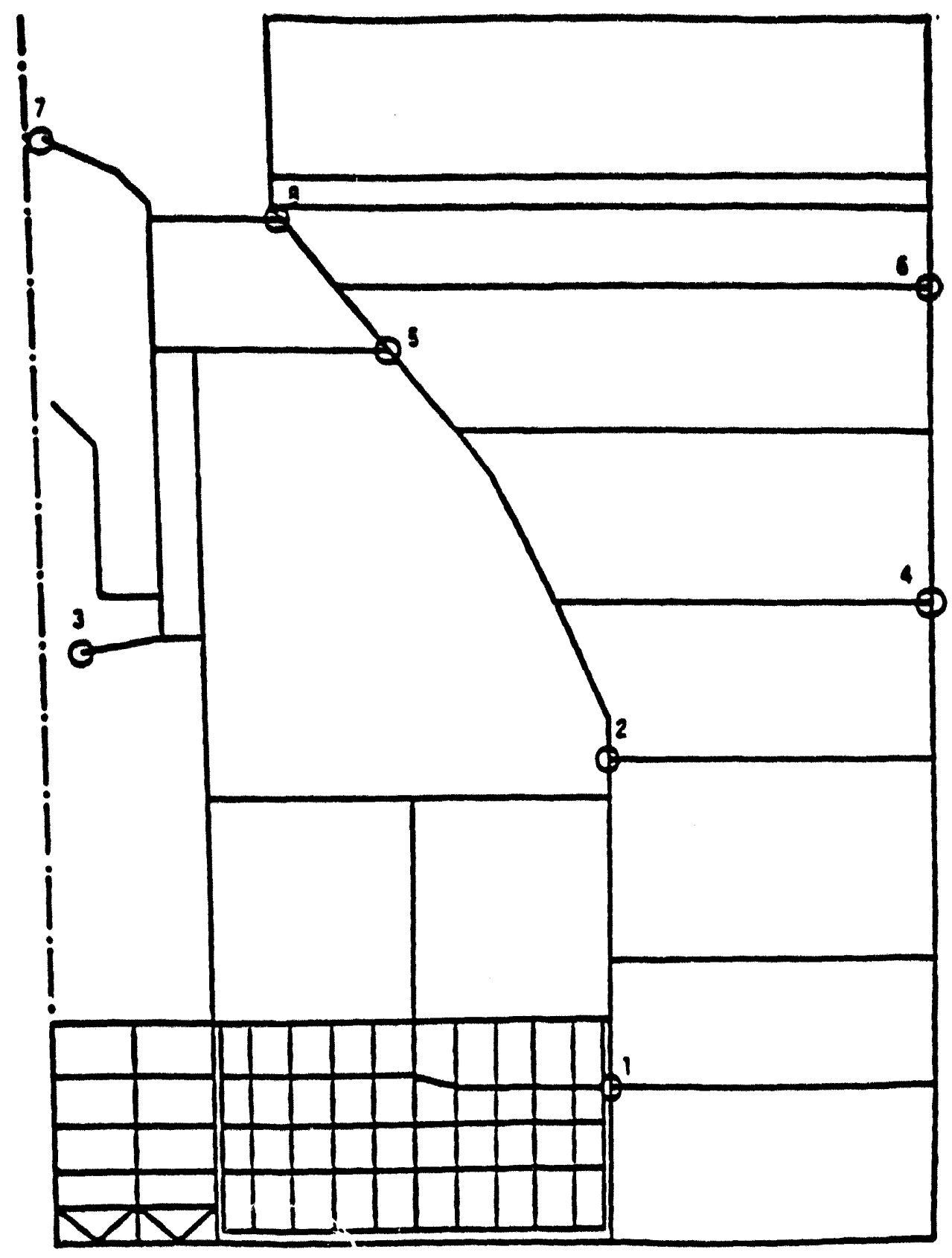

Figure 6.3. LaSalle County Station reactor and containment building model. 
Although a review of the available median entered spectra indicates that the degrees of conservatism vary, the conclusion that SRV accelerations are primarily significant for vertical accelerations along the length of the reactor vessel and pedestal still holds.

LOCA-Induced Loads. The LOCA-induced hydrodynamic loads have been broken down by the specific phenomena of jet forces, pool swell, condensation-oscillation (CO), and chugging. As discussed earlier, the jet force and pool swell loads tend to be highly localized, mainly affecting the structures submerged in the suppression pool. In addition, jet forces do not induce significant stresses in these structures and the effect of pool swell on the suppression pool boundary walls is such that it does not significantly affect the structures and equipment outside the wetwell boundary. For these reasons, jet forces and pool swell loads would not significantly affect the LaSalle seismic risk analysis results and are not considered further in this study.

The loads induced by chugging tend to occur late in time with respect to the earthquake. If there is a seismic-induced LOCA, it is reasonable to assume that it will occur sometime after the onset of strong motion, and chugging generally occurs more than 15 seconds after pipe break. Earthquake strong motion typically lasts for less than 10 to 15 seconds; hence, it is unlikely that significant chugging loads will occur concurrently with the significant seismic loads. Thus, chugging loads need not be considered further.

The remaining hydrodynamic bad is condensation-oscillation (CO). $\mathrm{CO}$ loads will occur early in time after a pipe break and can coincide with the strong motion of the earthquake. Table 6.3 shows the horizontal and vertical design accelerations for CO loads in the same manner as the SRV loads were shown. From the table, it is seen that $\mathrm{CO}$ loads can induce significant vertical accelerations.

These CO loads occur as a result of the design basis accident (DBA). As discussed in the preceding section, the small and intermediate break accidents do not generate sufficient steam flow to cause $\mathrm{CU}$ loads. Thus, only those seismic-initiated load event tree paths which involve a LOCA greater than the IBA (0.1 ft. 4-1/4" diameter) need consider CO loads combined with seismic loads, for occurrence frequency evaluation. This leads to consideration of the occurrence probability of seismic-induced LOCAs. Several studies are presently underway to evaluate the probability of direct and indirect DEGB failures of the main steam, recirculation and feedwater piping in BWRs ${ }^{(6.2)}$. The probability of seismic failure of any smaller lines which can cause CO must also be considered, although the $\mathrm{CO}$ luads would not be as severe as for the DBA. If the occurrence probability of these seismic-induced LOCAs can be shown to be small, the CO loads need not be combined with the seismic loads for seismic risk evaluation. The load combination method (presented in the following section) can be used to combine the $\mathrm{CO}$ and seismic loads if it is determined that $\mathrm{CO}$ loads need to be combined with seismic loads. The method will be illustrated and applied only for the case of SRV loads.

\subsubsection{Evaluation of Median and Variability of SRV Loading}

For our study, median-centered SRV spectra are required. From the Sargent \& Lundy in-plant SRV test results at LaSalle, realistic SRV-all time histories and response spectra were derived ${ }^{16.3]}$ (note that an SRV-all test case was not actually performed). Comparison of the design and realistic spectra for the critical case of SRV-all vertical showed that the spectral peaks (which occur at 3-5 $\mathrm{Hz}$ ) matched fairly well but the design spectra were conservative in the high-frequency region. In 
general, the design floor spectra may be scaled down by a factor of 1.7 for frequencies in the 10$30 \mathrm{~Hz}$ range. For other frequencies, the design spectra may be taken as being median-centered. For horizontal SRV loading, the case of SRV-asymmetric is dominant. The results of the preceding sections have shown that these loads are small when compared with the horizontal earthquake loads and may be neglected. For the sake of completeness, however, it was determined that the horizontal design SRV-asymmetric spectra may be scaled down by a factor of 2.2 to obtain median-centered spectra. This is based on a comparison of design versus measured suppression pool pressures ${ }^{[6.4]}$. Also, for these test results, the logarithmic standard deviation of the SRV loading, $\beta$ SRV is estimated as 0.3 .

As an illustration, Table 6.4 shows the median-centered, worst case horizontal SRV spectral accelerations obtained using the scale factors discussed above. The locations of the nodal points are shown in Fig. 6.4, the LaSalle structural model used for the dynamic analysis. Table 6.5 shows these same results at discrete frequencies, corresponding to the frequencies of critical equipment items quantified in this study.

\subsubsection{Summary}

In the foregoing, the various hydrodynamic loads that can occur in a Mark II BWR as the result of an earthquake were reviewed. It was found that only vertical accelerations induced by $\mathrm{CO}$ loads and SRV actuation could both occur concuriently with the earthquake strong motion and could be of large enough magnitude to warrant further attention. The vertical accelerations can be combined with the seismic vertical accelerations based on the results of the load combination described in the following sections.

The method will be illustrated, and conclusions drawn, only for the case of SRV luads, as discussed earlier, although the method could be applied to the case of CO loads if necessary. 
Table 6.3. Horizontal and vertical accelerations for condensation-oscillation loads.

\begin{tabular}{|c|c|c|c|c|c|}
\hline \multirow{2}{*}{\multicolumn{2}{|c|}{ Locetton }} & \multicolumn{2}{|c|}{ cO - InOAIzominaL } & \multicolumn{2}{|c|}{ CO - VeriICal } \\
\hline & & $\operatorname{Max}_{(\boldsymbol{g})}{ }^{\text {Accel. }}$ & $\begin{array}{l}\text { Mos. Snectrol } \\
\text { Acceleratlon/frenwency } \\
\text { (g) (III) }\end{array}$ & Mox, Accel. & $\begin{array}{l}\text { Mar. Smectrol } \\
\text { Accelerellon/frequemey } \\
\text { (ọ) (III) }\end{array}$ \\
\hline 1) & 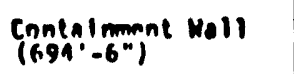 & 0.32 & $1.8 / 30$ & 0.12 & 1.213 .5 \\
\hline 2) & $\begin{array}{l}\text { Conenesmmene Holl } \\
\left(790^{\circ}-0^{\circ}\right)\end{array}$ & 0.03 & $0.30 / 50$ & 0.12 & $1.2 / 3.5$ \\
\hline 3) & $\begin{array}{l}\text { Reoctor Supdort } \\
\left.(75)^{\prime}-3^{\prime \prime}\right)^{-1}\end{array}$ & 0.05 & $0.06 / 50$ & 0.32 & $4.5 / 4.5$ \\
\hline a) & $\begin{array}{l}\text { Reoelor Ridg. Mall } \\
\left.(16)^{\circ}=0^{-1}\right)^{-}\end{array}$ & 0.05 & $0.08 / 30$ & - & - \\
\hline 5) & $\begin{array}{l}\text { Contelnment } \mathrm{Kall} \\
\left(00 \mathrm{~N}^{\circ}-0^{\circ}\right)^{-}\end{array}$ & 0.05 & $0.14 / 50$ & 0.10 & 1.213 .5 \\
\hline 6) & $\begin{array}{l}\text { Reactor gidg. Moll } \\
\text { (B0)-0.1) }\end{array}$ & $<0.05$ & 0.05 & 0.085 & $1.2 / 3.5$ \\
\hline$\pi$ & $\begin{array}{l}\text { Repctor Vessel } \\
\left.\text { (100 }-824^{-1}-A^{\prime \prime}\right)\end{array}$ & $=0.05$ & 0.05 & 0.37 & 4.514 .5 \\
\hline 8) & $\begin{array}{l}\text { Contermment Moll } \\
\left(018^{\circ}-6^{\circ}\right)^{-}\end{array}$ & 0.05 & 0.05 & 0.085 & 1.213 .5 \\
\hline
\end{tabular}

- Spectral scceleratlons are for 28 damping

- Spectra not avallable

- See figure 6.3 for location of points 
Table 6.4. Median horizontal SRV spectral accelerations (SRV-asymmetric).

(*) See Figure 6.4 for nodal point locations

\begin{tabular}{|c|c|c|}
\hline Node \# & Frcquency Range & Median Spectral Acceleration (g) \\
\hline 1 & $\begin{array}{r}2 \cdot 5 \\
5-10 \\
10 \div 20 \\
20 \div-30 \\
30 \div 50\end{array}$ & $\begin{array}{l}0.02 \\
0.04 \\
0.11 \\
0.12 \\
0.09\end{array}$ \\
\hline 2 & $\begin{array}{r}2-5 \\
5 \div 10 \\
10 \div 20 \\
20-30 \\
30-50\end{array}$ & $\begin{array}{l}0.02 \\
0.03 \\
0.09 \\
0.10 \\
0.07\end{array}$ \\
\hline 3 & $\begin{array}{r}2: 5 \\
5 \div 10 \\
10 \div 20 \\
20 \div 30 \\
30 \div 50\end{array}$ & $\begin{array}{l}0.02 \\
0.03 \\
0.08 \\
0.08 \\
0.05\end{array}$ \\
\hline 4 & $\begin{array}{r}2: 5 \\
5 \div 10 \\
10 \div 20 \\
20 \div 30 \\
30 \div 50\end{array}$ & $\begin{array}{l}0.02 \\
0.03 \\
0.09 \\
0.09 \\
0.05\end{array}$ \\
\hline 5 & $\begin{array}{r}2 \div 5 \\
5 \div 10 \\
10 \div 20 \\
20 \div 30 \\
30 \div 50\end{array}$ & $\begin{array}{l}0.02 \\
0.02 \\
0.06 \\
0.07 \\
0.04\end{array}$ \\
\hline 6 & $\begin{array}{r}2: 5 \\
5 \div 10 \\
10 \div 20 \\
20 \div 30 \\
30 \div 50\end{array}$ & $\begin{array}{l}0.00 \\
0.00 \\
0.00 \\
0.00 \\
0.00\end{array}$ \\
\hline 7 & $\begin{array}{r}2: 5 \\
5 \div 10 \\
10 \div 20 \\
20 \div 30 \\
30 \div 50\end{array}$ & $\begin{array}{l}0.00 \\
0.00 \\
0.00 \\
0.00 \\
0.00\end{array}$ \\
\hline 8 & $\begin{array}{r}2 \div 5 \\
5 \div 10 \\
10 \div 20 \\
20 \div 30 \\
30 \div 50\end{array}$ & $\begin{array}{l}0.00 \\
0.00 \\
0.00 \\
0.00 \\
0.00\end{array}$ \\
\hline
\end{tabular}




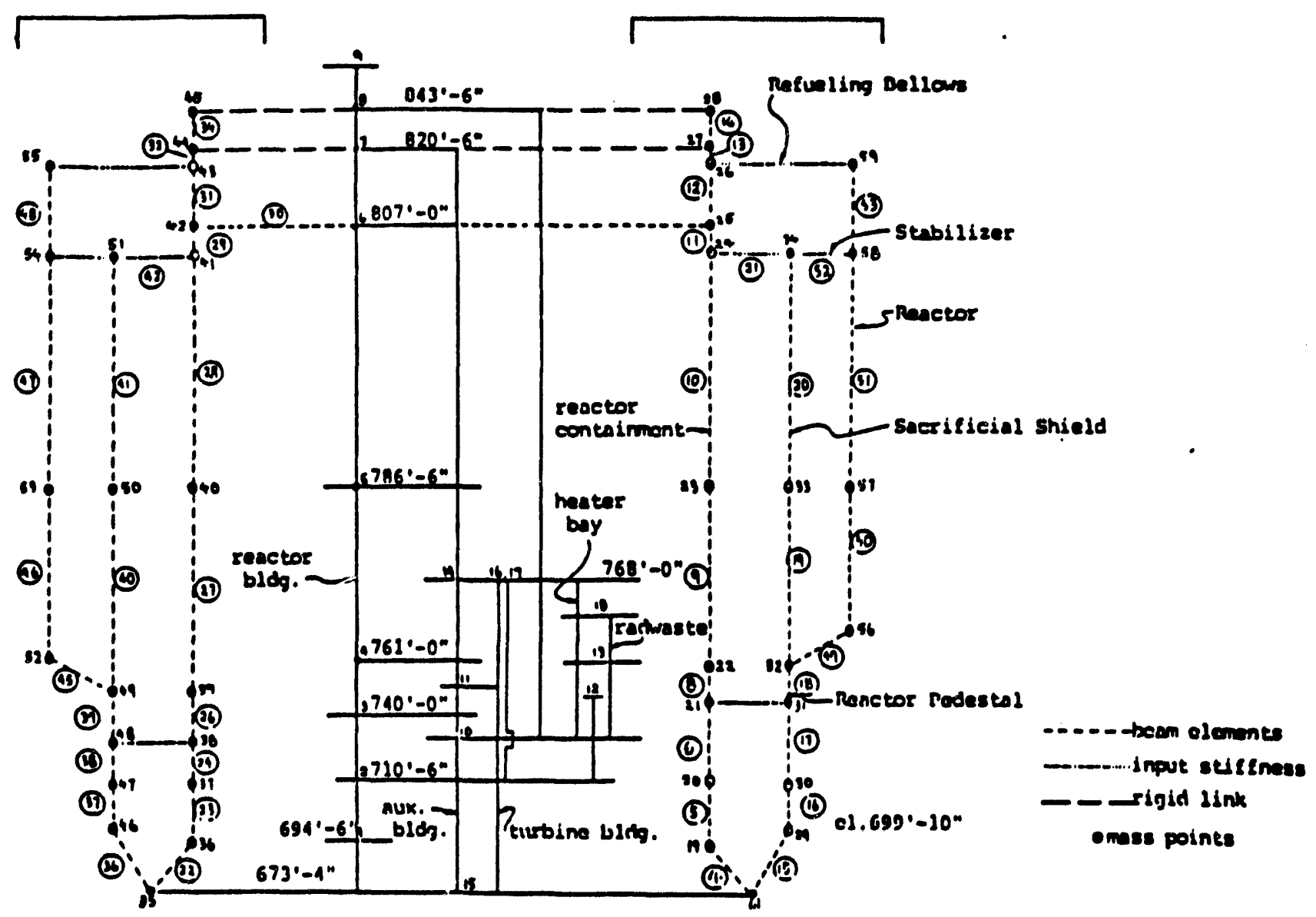

Figure 6.4. Schematic of the LaSalle County Station complex horizontal structure model. 
Table 6.5. Median horizontal SRV spectral accelerations at discrete frequencies.

\begin{tabular}{c|c|c}
\hline Nodc\# & Frequency Range & Mcdian Spectral Acceleration (g) \\
\hline 1 & 15.0 & 0.12 \\
2 & Rigid & 0.04 \\
& 10.0 & 0.05 \\
4 & Rigid & 0.03 \\
& 9.0 & 0.04 \\
7 & 13.0 & 0.09 \\
& 50.0 & 0.00 \\
10 & Rigid & 0.00 \\
& 8.0 & 0.03 \\
14 & 10.0 & 0.05 \\
& 14.0 & 0.10 \\
& 20.0 & 0.10 \\
15 & Rigid & 0.03 \\
(fdn) & 7.8 & 0.04 \\
& 16.0 & $0.09 *$ \\
& 19.0 & $0.10^{*}$ \\
56 & 28.0 & $0.04^{*}$ \\
& Rigid & 0.04 \\
& 6.5 & 0.11 \\
\hline
\end{tabular}

* From SRV-Symmetric load case. All others are from SRV-Asymmetric load case.

** See Figure 6.4 for nodal point locations 


\subsection{Load Combination Approach}

\subsubsection{Introduction}

The SRV loads can be significant and are likely to occur simultaneously with the earthquake loads; thus, the maximum of the combined responses must be determined. Due to the randomness of the SRV and earthquake time histories, the maximum response due to the SRV Loading, the maximum response due to the earthquake loading and the maximum of the combined response are random variables. The objective here is to evaluate descriptors of the combined maximum response random variable (e.g., median, standard deviation, etc.). Such information will be necessary for evaluating the probability of component failure due to the simultaneous occurrence of SRV and earthquake loads.

Obviously, it would be highly unlikely for the maximum response due to the SRV loading and due to the earthquake loading to occur simultaneously. Hence, the absolute sum of the SRV and earthquake median maximum responses would be a very conservative estimate of the median maximum combined response. For this reason, a load combination analysis must be carried out.

Two approaches are possible. The first is a simulation approach in which a large number of earthquake and SRV time histories are randomly generated and used to calculate response time histories. Each earthquake response time history is added to an SRV response time history and the maximum response calculated. In this manner, a large sample of combined maximum response values are obtained allowing statistical description of the combined maximum response. The second approach is based on the theory of stochastic processes and random vibrations. This approach is significantly more efficient than the simulation approach and was therefore selected to be used in this study. The details of the methods employed are described in the following sections.

For practical applications, when the combined maximum response must be calculated for many locations in a structure and for many different components, use of the random vibration approach, although relatively efficient when compared with simulation, is cumbersome. For this reason, simple rules for combining the statistics of the individual responses to obtain the statistics of the combined response will be developed based on the results of the load combination applied for several selected cases.

\subsubsection{Load Combination Methodology}

General. The method used in this study was developed by Winterstein and Cornell[65] as an extension of work by Larrabee and Cornell.166.6] The problem of calculating the combined maximum response due to coincidentally acting earthquake and SRV random loadings is illustrated in Fig. 6.5. As depicted, the duration of the response due to the SRV loading $\left(T_{2}\right)$ is much shorter than that due to the earthquake $\left(T_{1}\right)$. This is based on the results of Sargent \& Lundy's in-plant SRV tests at LaSalle. ${ }^{[6.4]}$ In addition, since we are particularly concerned with SRV loads "triggered" by earthquake, the time lag variable, $\theta$, is introduced. This is the length of time from the initiation of the earthquake motion to the beginning of the SRV motion. Due to the large uncertainty associated with predicting the value of $\theta$, it is modeled as a random variable as will be discussed in Sec. 6.2.2. 


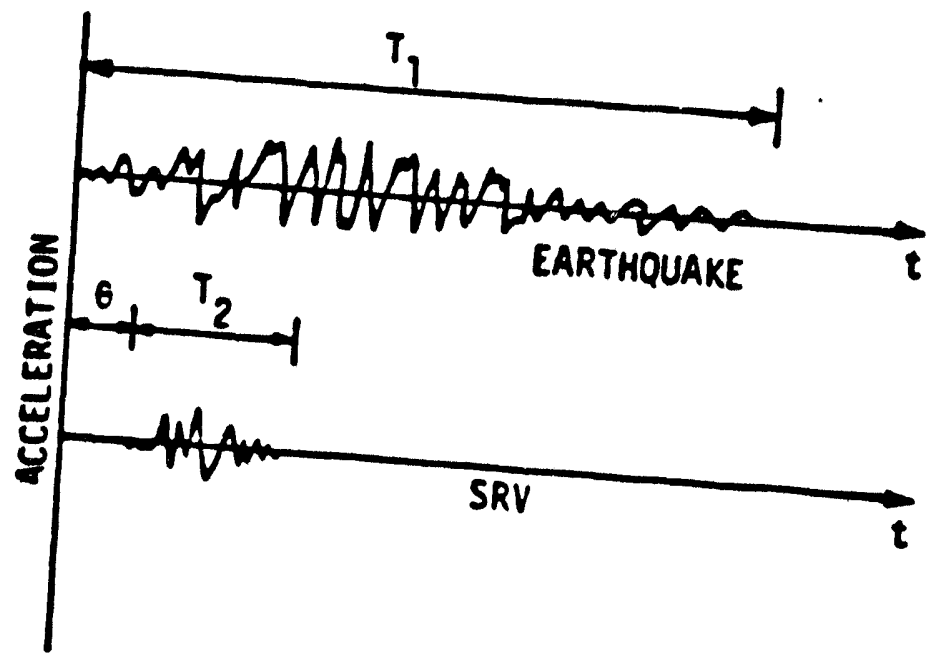

Figure 6.5. Coincidentally acting earthquake and SRV responses. 
The load combination methodology is conceptually depicted by the flow diagram in Fig. 6.6. The response of a particular component due to earthquake or SRV loading is a random process (since the loadings are random processes) and is characterized by its mean upcrossing rate and probability density functions. The upcrossing rate function is the rate at which the response crosses particular response levels (e.g., number of crossings of say, $0.1 \mathrm{~g}$, per second) and the probability density function gives the probability that the response will take on certain values (e.g., the probability that the response will be between $0.09 \mathrm{~g}$ and $0.11 \mathrm{~g}$ ). Using the extended point crossing formula of Winterstein and Cornell, 16.5$]$ the mean upcrossing rate function of the combined response is calculated using the upcrossing rate and probability density functions of the earthquake-alone response, and the SRV-alone response. With the upcrossing rate function of the combined response, the distribution of the maximum combined response can be estimated.

Note that the distribution of the maximum response due to the SRV-alone and due to the earthquake can also be estimated using the individual upcrossing rate functions. Thus, the response maximum distributions can be compared with the combined response maximum distribution for the evaluation of simple load combination rules.

Evaluation of Earthquake Response Upcrossing Rate and Probability Density Function. As discussed earlier, the mean upcrossing rate function defines the number of times the response is expected to cross a particular response level and the probability density function defines the probability that the response will take on certain values. For LaSalle, 30 floor response time histories (for earthquake) were generated at all locations at which there is critical safety equipment. ${ }^{66.7]}$ Hence, the upcrossing rate and density functions for the response of a particular component could be obtained in a straightforward manner. The response time history due to each of the input floor time histories is first calculated and the upcrossing rate and probability density functions then evaluated as shown in Fig. 6.7, and as described below.

For evaluation of the probability density function, the range of response levels is broken down into bands of width $\Delta y$. The density function is then evaluated (approximately) as:

$$
f\left(y_{i}\right)=\frac{1}{\Delta y} \frac{n\left(y_{i}, y_{i}+\Delta y\right)}{N}
$$

where

$$
\begin{aligned}
& n\left(y_{i}, y_{i}+\Delta y\right)=\begin{array}{r}
\text { number of response time history points (digitized at equal time } \\
\text { intervals) that fall in the band } \Delta y
\end{array} \\
& N=\text { total number of points in the response time history. } \\
& \Delta y=\text { bandwidth }
\end{aligned}
$$




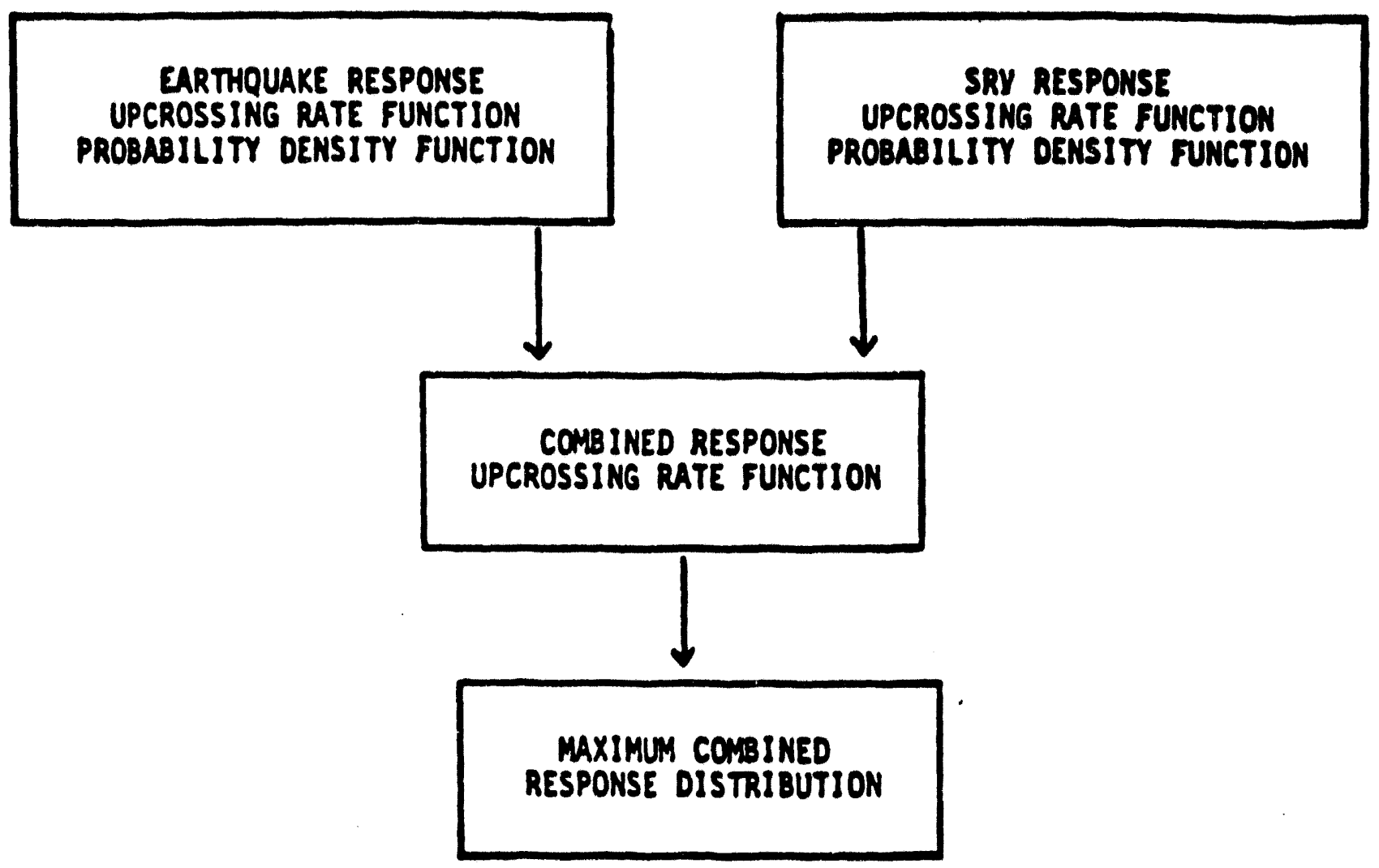

Figure 6.6. Load combination methodology. 
An exact value of $f\left(y_{i}\right)$ would be obtained if the band width $\Delta_{y}$, was infinitesimally small. For purposes herein, $\Delta_{y}$ was chosen small enough such that the values of any $f\left(y_{i+1}\right)$ and $f\left(y_{i}\right)$ did not vary significantly.

For definition of the upcrossing rate function, the number of times the response crosses up through various response levels is counted and divided by the time duration. That is, for evaluation of the upcrossing rate, $v\left(y_{1}\right)$ the number of upcrossings of $y_{i}$ is counted ( 2 in Fig. 6.7b) and divided by the time duration ( $T_{d}$ in Fig. 6.7b).

Since the earthquake response is nonstationary (i.e., the intensity of the response is expected to vary with time), the upcrossing rate and density functions calculated would be different if different portions of the response time history were used. In order to preserve the nonstationary character of the earthquake response, the upcrossing rate and density functions were calculated for specific time segments of two-second lengths. That is, the functions were calculated using the digitized response data from all 30 time histories for the first 2 seconds of response (i.e., 0-2 seconds), for the next two seconds of response (2-4 seconds), etc. Such an approach was deemed necessary since the intensity of the earthquake response at the time at which the SRV load acts can significantly affect the combined maximum response.

Figures 6.8 and 6.9 show sample upcrossing rate functions obtained as described above. Figure 6.8 illustrates how the rate at which the response crosses the $0.25 \mathrm{~g}$ response acceleration level varies with time. Initially, the upcrossing rate increases as the intensity of the earthquake builds up, then exhibits a relatively constant phase, corresponding to the strong motion phase of the earthquake, and then finally decreases as the earthquake motion subsides. Figure 6.9 shows the rates of crossing various acceleration levels at time $=4$ seconds. Obviously, the upcrossing rates decrease with increasing response level. The probability density functions exhibit similar behavior, that is, the function will decrease with increasing response level, and the temporal variation of the function will be similar to that shown for the upcrossing rate function.

Evaluation of SRV Response Upcrossing Rate and Probability Density Function. As mentioned earlier, the duration of the SRV loading is relatively short when compared with that of the earthquake. This implies that the intensity of the earthquake response (and, therefore, the values of the upcrossing rate and probability density functions) will not vary significantly during the time at which the SR ' response is coincidentally acting. For this reason, it is not necessary to preserve the non-stationary characteristics of the SRV response. This allows modeling of the SRV response as a stationary random process. With this simplification, application of random vibration theory to directly evaluate the upcrossing rate and density functions (i.e., without recourse to time history analysis) is straightforward.

From Sargent \& Lundy's in-plant SRV test program ${ }^{(6.4)}$ derived realistic SRV time histories were available at several locations within the reactor building. Although their time histories are quite useful in determining realistic SRV loads and for evaluating the conservatism in SRV design spectra, a single test response does not provide sufficient data to develop a model of the randomness inherent in SRV time histories. In order to maintain some generality in the study, the design response spectrum was used to represent the characteristics of the SRV load. 


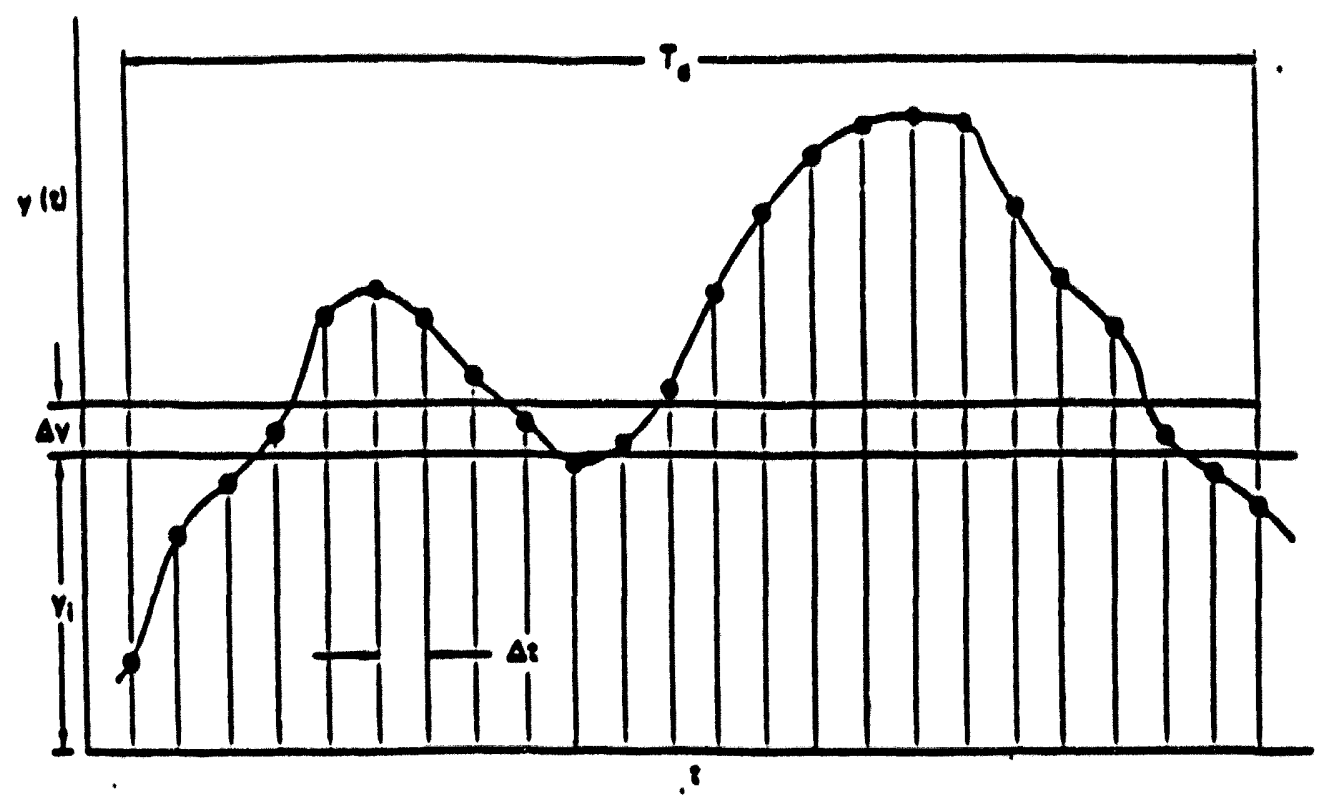

a) Calculation of Probability Ounsity Function

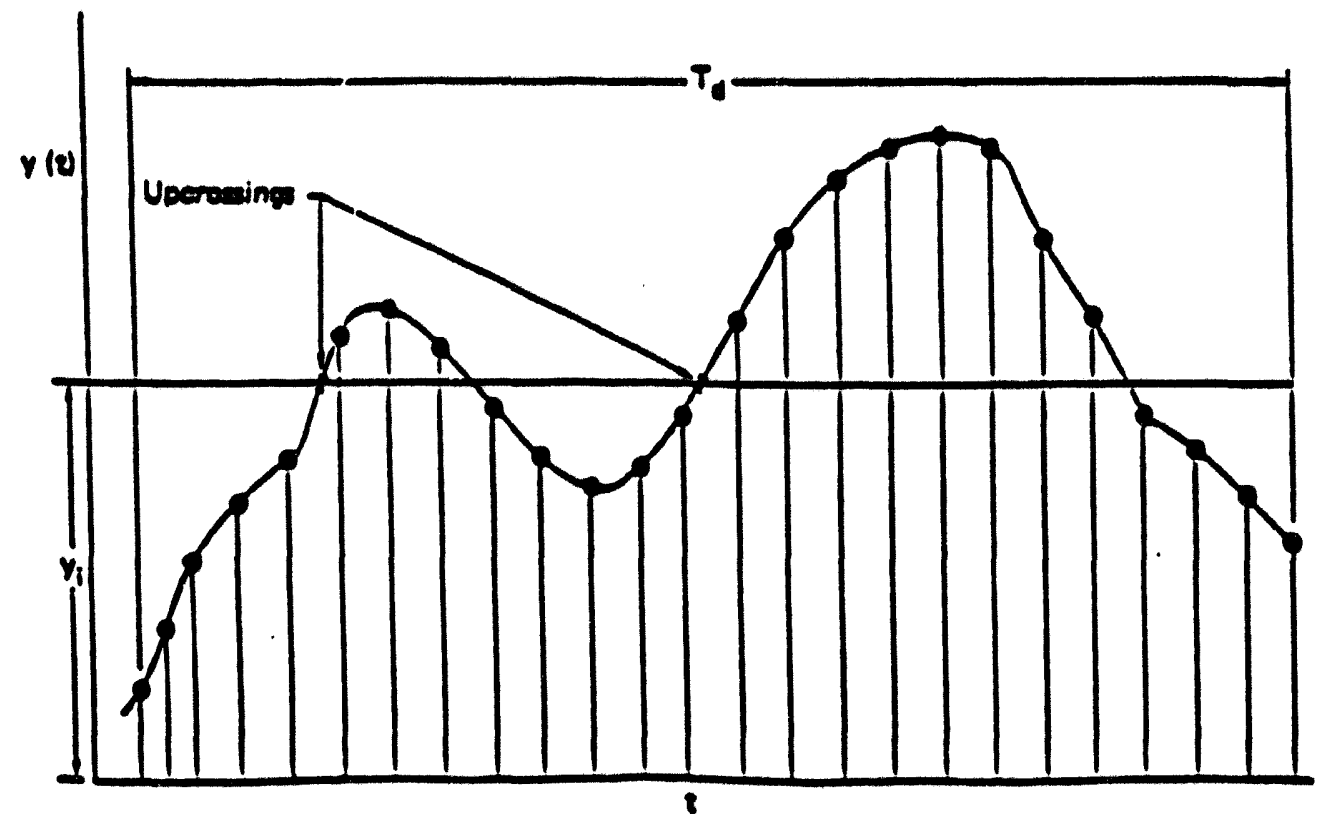

b) Calculation of Uperossing Rate Function

Figure 6.7. Calculation of probability density and upcrossing rate functions. 


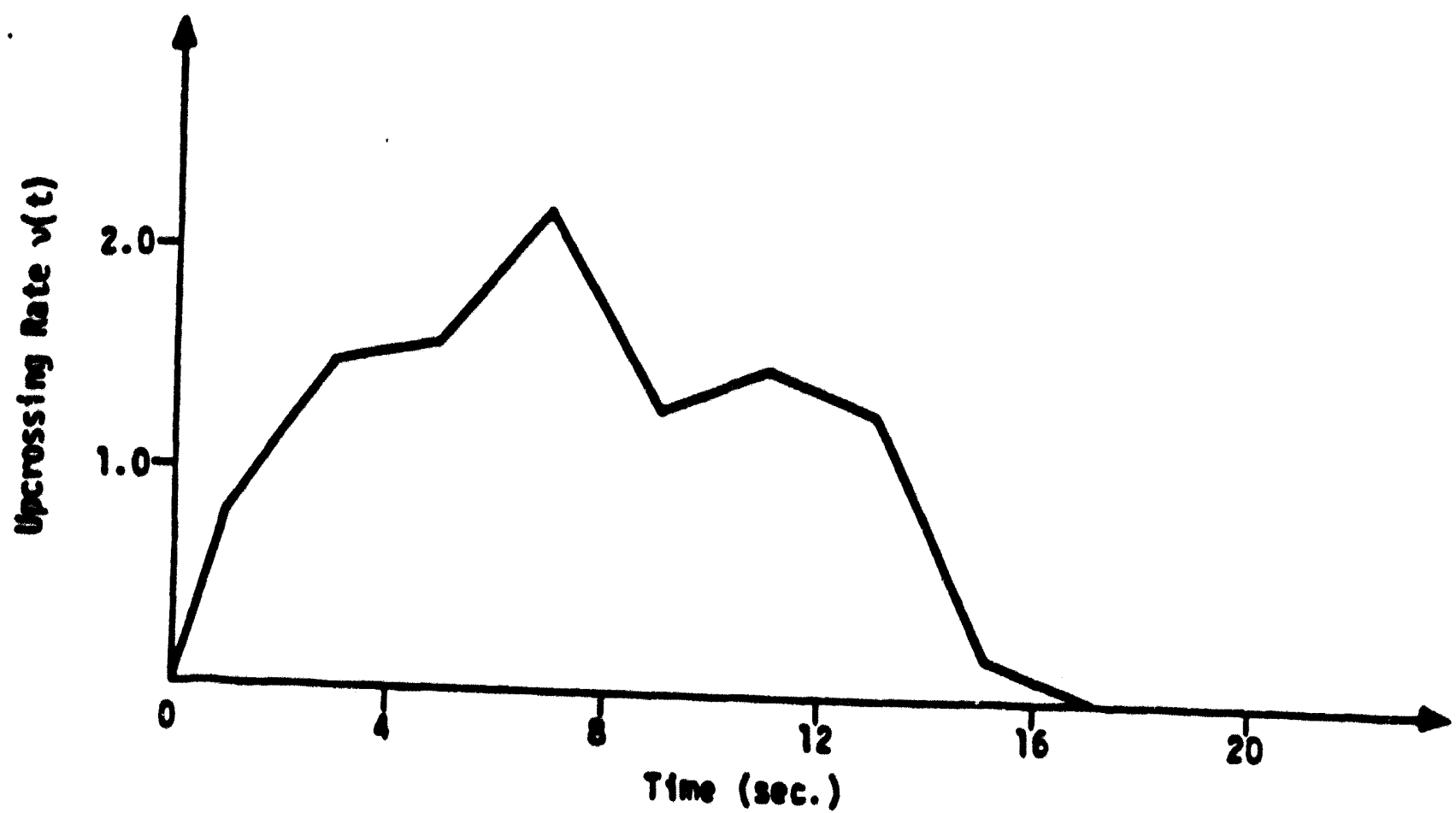

Figure 6.8. Variation of upcrossing rate (of level $0.25 \mathrm{~g}$ ) with time.

$-144-$ 


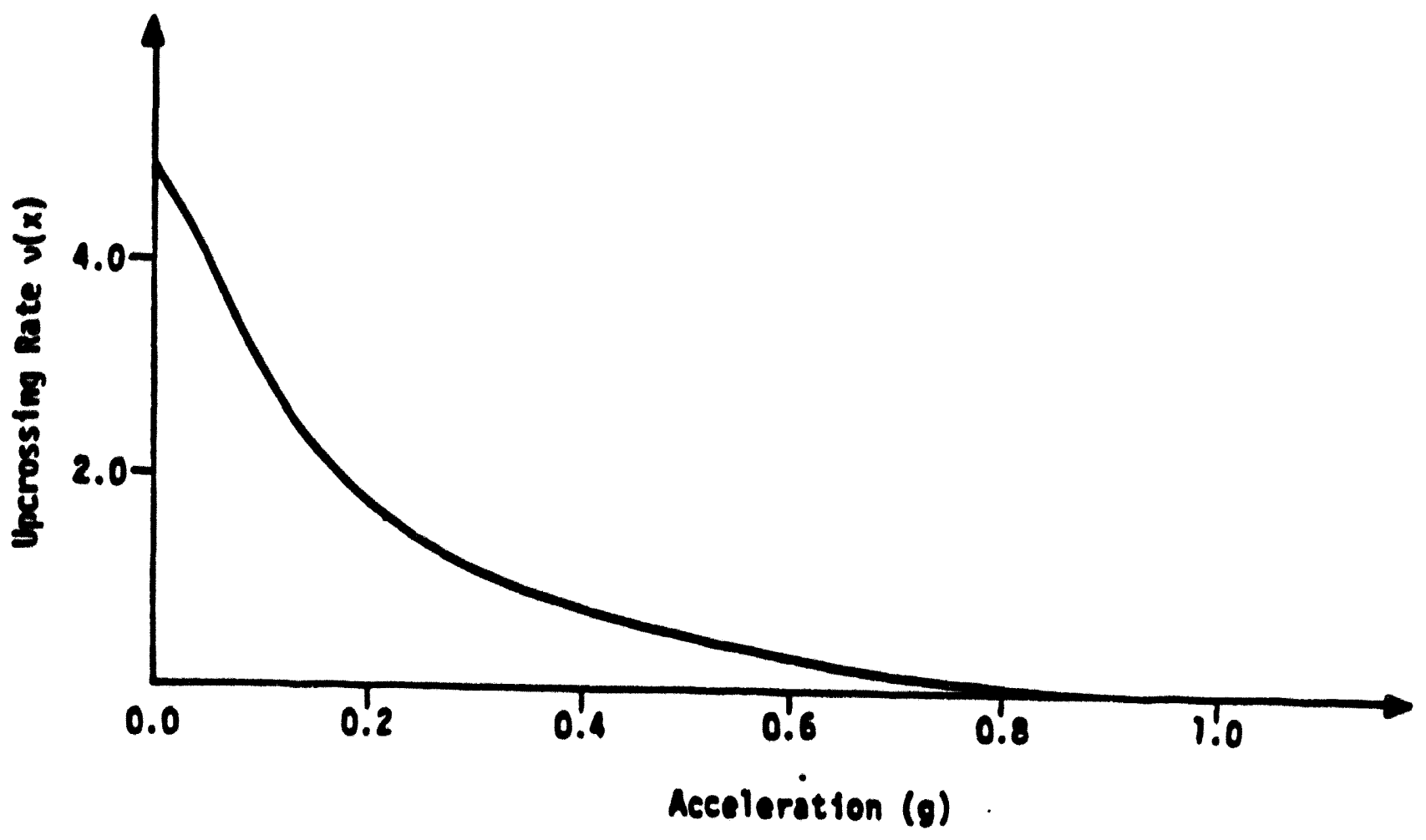

Figure 6.9. Variation of upcrossing rate (at time $=4 \mathrm{sec}$ ) with acceleration level. 
Using the program SIMQKE, ${ }^{|6 .|} \mid$ a power spectral density function for the SRV floor acceleration, $S_{a a}(\omega)$ is generated, based on the input design response spectrum. For a component (modeled as a single degree-of-freedom system) with natural frequency, $\omega_{0}$ and damping ratio, $\zeta_{0}$ the relative displacement response power spectral density function, $S_{\mathbf{x x}}(\omega)$, is evaluated as:

$$
S_{x x}(\omega)=|H(\omega)|^{2} S_{a x}(\omega)
$$

where $H(\omega)$ is the frequency transfer function for the component, i.e.,

$$
|H(\omega)|^{2}=\frac{1}{\left(\omega^{2}-\omega_{0}^{2}\right)^{2}+4 \zeta_{0}^{2} \omega^{2} \omega_{0}^{2}}
$$

With the relative displacement response power spectral density function, $S_{x x}(\omega)$, evaluated, the mean square relative displacement and mean square relative velocity of the response are calculated, respectively, as:

$$
\sigma_{x}^{2}=\int_{0}^{\infty} S_{x x}(\omega) d \omega
$$

and

$$
\sigma_{x}^{2}=\int_{0}^{\infty} W^{2} S_{x x}(\omega) d \omega
$$

Using the definition of spectral acceleration, the root-mean-square, pseudo-absolute acceleration can be evaluated as:

$$
\sigma_{\mathrm{a}}=\omega_{\mathrm{u}}^{2} \sigma_{\mathrm{x}}
$$

Note that the appropriate transfer function could be used to obtain absolute acceleration directly; however, the purpose of this study is to develop simple rules for combining spectral accelerations; hence, the above approach.

If the SRV input and, therefore, the response is assumed to be a zero-mean Gaussian process, then 
the mean upcrossing rate, $v$, and probability density function, $f$, may be evaluated directly as:

$$
v(a)=\frac{1}{2 \pi} \frac{\sigma_{x}}{\sigma_{x}} \exp \left[-\frac{1}{2}\left(\frac{a}{\sigma_{a}}\right)\right]^{2}
$$

and

$$
f(a)=\frac{1}{\sigma_{a} \sqrt{2 \pi}} \exp \left[-\frac{1}{2}\left(\frac{a}{\sigma_{a}}\right)\right]^{2}
$$

respectively.

With this approach, the upcrossing rate and density functions are calculated in an efficient and straight forward manner without recourse to time history analysis. Note that a similar approach could have been used for the earthquake response analysis. However, this would have required a more complex nonstationary random vibration analysis. For practical expediency, this approach was not implemented and the time history analysis, as described in the previous section, was employed.

Evaluation of SRV Response Time Lag. The time at which the SRV load initiates with respect to the onset of earthquake motions, is an important parameter in this study. Obviously, ine combined maximum response will be greater if the SRV load acts during the strong motion portion of the earthquake time history than if it acts during the build-up or decay phases. In Fig. 6.5, the time lag is shown as the variable, $\theta$.

The event sequence which is most likely to cause simultaneous earthquake and SRV loading is that of seismic-induced loss-of-offsite power causing a plant transient and actuation of the SRVS. This is due to the fact that even small earthquakes can cause a loss-of-offsite power. Also, nuclear power plant safety-related equipment generally have seismic capacities significantly greater than that of the offsite power system, thereby precluding other sequence initiators from being more dominant. Based on this assumed event sequence, the time lag may be calculated as the time at which the earthquake causes a loss-of-offsite power (the additional time lag until the SRV loads actually build-up in the structure and equipment begins to respond, is neglected and would have a trivial impact due to the uncertainty associated with predicting the time at which offsite power is lost, as is discussed below).

The time at which offsite power will be lost is a function of the intensity of the earthquake motion and the seismic capacity of the offsite power system. Both are random quantities with large uncertainty; hence, the time at which SRV response initiates with respect to the initiation of earthquake motion, is modeled as a random variable, $\theta$ 
Given that the ground acceleration capacity of the offsite power system is known, the probability distribution of $\theta$ can be determined by examining simulated earthquake ground motion time histories and determining at what time each ground motion exceeds the given capacity. These are 60 such time histories available for two ranges of earthquake intensity. The first set of time histories, Range 1, corresponds to a median rock outcrop peak acceleration of $0.2 \mathrm{~g}$. The median peak free surface acceleration for this intensity range is $0.22 \mathrm{~g}$. The second set of time histories, Range 2, corresponds to a median rock outcrop peak acceleration of $0.6 \mathrm{~g}$. The median peak free surface acceleration for this intensity range however, is only $0.35 \mathrm{~g}$ as a result of soil deamplification. The load combination studies to be carried ot:, herein, will be performed using both earthquake intensity ranges.

Distributions for $\theta$ were obtained for the earthquake Range 1 and Range 2 time histories assuming that offsite power is lost at a ground acceleration of $0.1 \mathrm{~g}$. This is a lower bound on offsite power capacity, based on earthquake experience data. ${ }^{16.91}$ Rigorous treatment of the load combination problem would require evaluation of the probability distribution of $\theta$ for all possible values of offsite power capacity and consideration of the probability distribution of offsite power capacity. For the purposes of the present study, i.e., to examine the applicability of simple load combination rules, the use of a single deterministic value for offsite power capacity was deemed adequate.

Figure 6-10 shows the $\theta$ distributions obtained for the earthquake Range 1 and Range 2 events. Due to the large variability in the data and the physical bounds on the values of $\theta$, a uniform distribution was fit to the data. Note that the probability distribution of $\theta$ was also evaluated for an SSE event with offsite power capacity assumed to be $0.3 \mathrm{~g}$. This distribution will be used to study the sensitivity of the results to the assumed offsite power capacity.

As is expected, the time to initiation of SRV loading is much less for the Range 2 event than the Range 1 event. For the case in which offsite power capacity is increased to $0.3 \mathrm{~g}$, the time to initiation of SRV loading generally increases, as expected. The data showed a very wide variability, however, and the power bound is taken as zero.

Combined Response Upcrossing Rate Function. As depicted in the flow diagram of Fig. 6.6, the mean upcrossing rate function of the combined response can be evaluated using the upcrossing rate and probability density function of the SRV-alone response and the earthquakealone response, calculated as described in the previous sections. Using the extended point crossing formula ${ }^{[6.5]}$ the combined upcrossing rate function is:

$$
\begin{gathered}
v(z)=\frac{1}{T_{1}+T_{2}} \int_{\theta} f_{\theta}(\theta)\left\{\int_{0}^{\theta} v_{E Q}(z, t) d t+\int_{\theta}^{\theta+T_{2}}\left[v_{E Q}(y, t) * f_{S R V}(z-y, t-\theta)\right.\right. \\
\left.\left.+v_{S R V}(y, t-\theta) * f_{E Q}(q-y, t)\right] d t+\int_{\theta+T_{2}}^{T_{1}} v_{E Q}(z, t)\right\} d \theta
\end{gathered}
$$




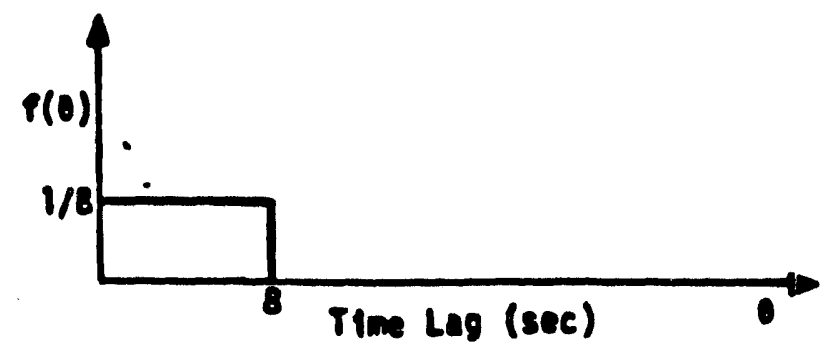

1) Range 1 Earthquake, Offsite Power Capacity $=0.19$

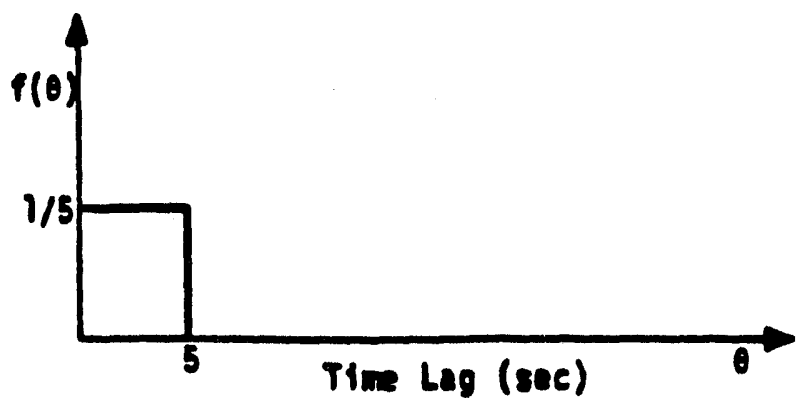

b) Range 2 Earthquake, Offsite Power Capactty $=0.19$

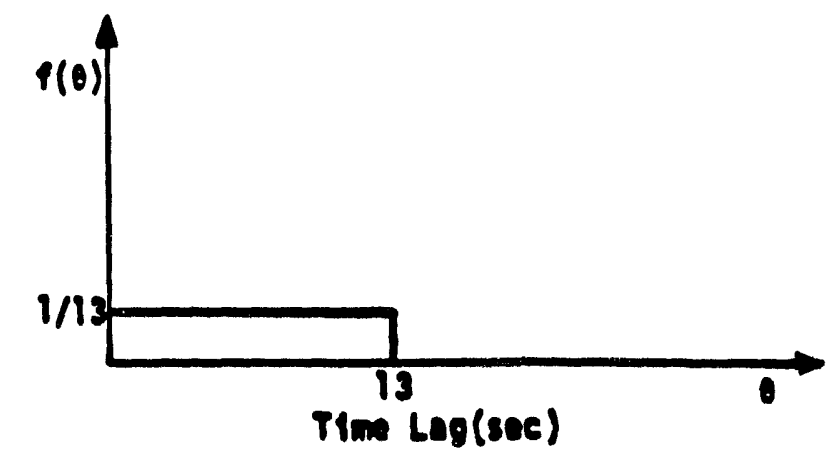

c) Range 2 Earthquake, Offsite Power Capacity $=0.39$

Figure 6.10. Distribution of the SRV time lag random variable, $\theta$. 
where

$$
\begin{aligned}
v\left(y, t_{1}\right) * f\left(z-y, t_{2}\right) & =\int_{-\infty}^{\infty} v\left(y, t_{1}\right) f\left(z-y, t_{2}\right) d y=\int_{\infty}^{\infty} v\left(y, t_{1}\right) f\left(z-y, t_{2}\right) d y \\
T_{1} & =\text { earthquake duration } \\
T_{2} & =\text { SRV duration }
\end{aligned}
$$

and

$$
\theta=\text { SRV time lag random variable }
$$

as illustrated in Fig. 6.5. The probability density function of the SRV time lag random variable, $f_{\theta}(\theta)$, is obtained as described in the preceding section.

Note that the combined upcrossing rate, given by Eq. 6-9, is a time-averaged upcrossing rate. Since the earthquake response is modeled as a nonstationary random process, the combined response is also nonstationary and the upcrossing rate is not constant in time. For the purposes of approximating the maximum combined response distribution and verification of simple load combination rules, the time averaged function given by Eq. 6-9, is sufficient. The first and last integrals, within the braces, thus give the number of upcrossings of the level $z$ during the times at which only the earthquake response acts. The first integral sums up the number of upcrossings from $t=0$ to $t=\theta$, the time prior to SRV actuation (Fig. 6.5), and the last sums up the number of upcrossings from $t=\theta+T_{2}$ to $t=T_{1}$, the time after SRV response has ceased (Fig. 6.5). The middle integral terms (the point crossing formula of Ref. 6.8 evaluates the number of crossings that occur during the time at which the earthquake and SRV responses coincide. The total number of upcrossings from all three terms are summed together and the calculation repeated for all possible values of the time $\log$ variable $\theta$. A weighted-average of these results, governed by the probability distribution of $\theta$, gives the final number of upcrossings of the level $z$ due to the combined response. The number of upcrossings is then divided by the total duration, $T_{D}=T_{1}+$ $\mathrm{T}_{2}$, to give the mean combined upcrossing rate.

Physical meaning can be ascribed to the point crossing formula (the middle integral term). The mean rate at which the earthquake response crosses level $y,\left(v_{E Q}(y)\right)$, is multiplied by the probability that the SRV response is at a value, $(\operatorname{SRV}(z-y))$, that makes the total combined response equal to $z$. All such products are summed over all the values of $y$ for the earthquake response (as denoted by the convolution ' ${ }^{\prime}$ ). A similar explanation holds for the second term within the integral. Here the mean rate at which the SRV response crosses the level y is multiplied by the probability that the earthquake response is at the value $z$ - y; again, all such products are summed over all the values of $y$ for the SRV response.

Maximum Response Disíribution. As described in the preceding sections, the mean upcrossing rate functions for the earthquake response, the SRV response, and the combined response are calculated. From these upcrossing rate functions, the probability distribution of the 
maximum response can be estimated as described below. Note that since upcrossings of positive and negative response levels are equally important, the upcrossing rates used for calculating the maximum response distribution are the rates at which the positive level plus the rates at which the negative level are crossed.

For high response levels, the events of upcrossing tend to be infrequent and can be assumed to be independent. The number of upcrossings in a given time interval, then, can be modeled by a non homogeneous Poisson process distribution with occurrence rate given by the mean upcrossing rate function, $v(x, t)$. The cumulative distribution function of the maximum response is thus given by the probability of no occurrences (crossings) in the given time duration. That is,

$$
F_{x_{m}}(x)=P[N=O]=\exp \left[-\int_{0}^{T_{D}} v(x, t) d t\right]
$$

where $T_{D}$ is the total duration of the response. If the upcrossing rate function is constant in time (as for the SRV response) or we have the time-averaged upcrossing rate function (as for the combined response), the integral term simplifies to $v(x) \cdot T_{D}$.

The median maximum response, $\breve{\mathrm{xm}}$ is obtained by finding the response level for which $F_{x_{m}}(x)=0.5$. That is, solving

$$
0.5=\exp \left[-v\left(\breve{x}_{m}\right) \cdot T_{D}\right]
$$

for $\breve{x}_{m}$, where $v(x)$ in Eq. 6-11, is the time-averaged upcrossing rate function. Note that the Poisson process assumption tends to give a conservative estimate of the maximum response distribution (i.e., the cumulative distribution function values are underestimated) and will result in a conservative estimate of the median maximum response, $\breve{x}_{m}$. This approximation is used consistently, however, to predict the earthquake, SRV, and combined median maximum responses in what follows and is expected not to bias the development of simplified load combination rules, which is the primary purpose of study.

\subsection{Probabilistic Load Combination Case Studies}

\subsubsection{Introduction}

As was shown in Sec. 6.1, only the vertical accelerations due to the SRV-all case need to be combined with seismic loads in the LaSalle seismic risk assessment. Further, the SRV-all vertical accelerations are primarily significant along the reactor vessel and pedestal. For this reason, the 
load combination case studies will be carried out using the SRV-ail vertical and seismic vertical accelerations at the reactor support (Location 3 in Fig. 6.3).

The objective here is to determine a simple rule for combining SRV and earthquake responses based on the results of the load combination case studies. As discussed in the preceding section, the upcrossing rate and probability density functions of the response of a single-degree-of-freedom oscillator of a given frequency and damping ratio, can be used to obtain the combined response upcrossing rate function and estimate the probability distribution of the maximum combined response. The load combination will be carried out using the Range 1 and Range 2 for time histories (see Sec. 6.2.2). Also, several different component frequencies will be selected for the response combination. This will ensure that a range of SRV and earthquake response levels will be covered. These cases should be sufficient to test the applicability of any simple load combination rule. 


\subsubsection{SSE Case Study}

Three frequencies were selected for study for the Range 1 case; $2 \mathrm{~Hz}, 4 \mathrm{~Hz}$, and $25 \mathrm{~Hz}$. These were selected to cover the amplified portion of the SRV and earthquake response spectra, and the rigid region of the earthquake spectrum. The response upcrossing rate functions for single-degreeof-freedom oscillators with each of the selected frequencies were calculated as discussed in the preceding section. The combined up-crossing rate function was then calculated for each case Eq. (6-9) using the probability distribution for the time lag variable, $\theta$, shown in Fig. 6-10a. The results for selected response levels are shown in Tables 6.6 through 6.8 and plotted in Figs. 6.11 through 6.13. Note that these are time averaged upcrossing rate functions, and have been normalized to a duration of 24.0 seconds (the mean duration of the earthquake time histories, 20.5 sec., plus the mean duration of the SRV load, $3.5 \mathrm{sec}$.). Thus, for example, the stationary upcrossing rate function calculated for the SRV response is multiplied by 3.5 and then divided by 24.0. This gives the time-averaged SRV response upcrossing rate function which can be taken to act over the entire 24.0 second combined response duration used for the load combination analysis (see Eq. 6-9). Such a normalization is necessary for comparison of earthquake and SRV upcrossing rate functions since the SRV response acts for only 3.5 seconds. Note also, that the upcrossing rates include both plus $(+)$ and minus $(-)$ crossings of the given response level (i.e., the rates shown are the rates at which the positive level plus the rates at which the negative level are crossed, see Sec. 6.2.2).

For the $2 \mathrm{~Hz}$ oscillator, the earthquake response dominates since $2 \mathrm{~Hz}$ is near the peak of the earthquake response spectrum and in the "soft," deamplified region of the SRV spectrum. At $4 \mathrm{~Hz}$, however, we are near the peak of the SRV spectra and the SRV response dominates. At $25 \mathrm{~Hz}$, SRV response is again dominant since $25 \mathrm{~Hz}$ is in the rigid region of the earthquake spectrum and the SRV response is still somewhat amplified. 
Table 6.6. Upcrossing rates for earthquake range 1, SRV, and combined response (oscillator frequency $=2 \mathrm{H} \%$, damping $=5 \%$ ).

\begin{tabular}{|c|c|c|c|}
\hline \multirow{2}{*}{$\begin{array}{c}\text { RESPONSE } \\
\begin{array}{c}\text { ACELERATION } \\
\text { LEVEL } \\
(G)\end{array}\end{array}$} & $\begin{array}{c}\text { TARTHQUAKE } \\
\text { ALONE } \\
\left(\text { SEC }^{-1}\right)\end{array}$ & $\begin{array}{c}\text { SRV } \\
\text { ALONE } \\
\left(\text { SEC }^{-1}\right)\end{array}$ & $\begin{array}{c}\text { COMBINED } \\
\text { RESPONSE } \\
\left(\text { SEC }^{-1}\right)\end{array}$ \\
\hline 0.0 & 3.4 & 0.72 & 3.7 \\
0.15 & 0.74 & 0.35 & 1.1 \\
0.30 & 0.18 & 0.039 & 0.32 \\
0.44 & 0.061 & 0.0 & 0.091 \\
0.56 & 0.019 & 0.0 & 0.029 \\
\hline
\end{tabular}


Table 6.7. Upcrossing rates for earthquake range $1, \mathrm{SRV}$, and combined response (oscillator frequency $=4 \mathrm{H} \mathrm{t}$, damping $=5 \%$ ).

\begin{tabular}{|c|c|c|c|}
\hline \multirow{2}{*}{$\begin{array}{c}\text { RESPONSE } \\
\text { ACCELERATION } \\
\text { LEVEL } \\
(\mathrm{G})\end{array}$} & $\begin{array}{c}\text { TIME A VERAGED ( } \pm \text { UPCROSSING RATES } \\
\text { ALONE } \\
\left(\mathrm{SEC}^{-1}\right)\end{array}$ & $\begin{array}{c}\text { SRV } \\
\text { ALONE } \\
\left(\mathrm{SEC}^{-1}\right)\end{array}$ & $\begin{array}{c}\text { COMBINED } \\
\text { RESPONSE } \\
\left(\mathrm{SEC}^{-1}\right)\end{array}$ \\
\hline 0.0 & 5.7 & 1.2 & 6.0 \\
0.21 & 0.48 & 1.1 & 1.6 \\
0.42 & 0.097 & 0.99 & 1.2 \\
0.74 & 0.031 & 0.72 & 0.81 \\
2.11 & 0.0 & 0.024 & 0.029 \\
\hline
\end{tabular}


Table 6.8. Upcrossing rates for earthquake range $1, \mathrm{SRV}$, and combined response (oscillator frequency $=25 \mathrm{~Hz}$, damping $=5 \%$ ).

\begin{tabular}{|c|c|c|c|}
\hline \multirow{2}{*}{$\begin{array}{c}\text { RESPONSE } \\
\text { ACCELERATION } \\
\text { LEVEL } \\
(G)\end{array}$} & \begin{tabular}{c} 
TIME AVERAGED ( \pm UPCROSSING RATES \\
\cline { 2 - 4 } \\
ALONE \\
$\left(\right.$ SEC $\left.^{-1}\right)$
\end{tabular} & $\begin{array}{c}\text { SRV } \\
\text { ALONE } \\
\left(\mathrm{SEC}^{-1}\right)\end{array}$ & $\begin{array}{c}\text { COMBINED } \\
\text { RESPONSE } \\
\left(\text { (SEC }^{-1}\right)\end{array}$ \\
\hline 0.0 & 10.1 & 5.0 & 13.9 \\
0.10 & 0.19 & 4.5 & 4.7 \\
0.18 & 0.022 & 3.7 & 3.8 \\
0.26 & 0.001 & 2.7 & 2.8 \\
0.76 & 0.0 & 0.025 & 0.029 \\
\hline
\end{tabular}




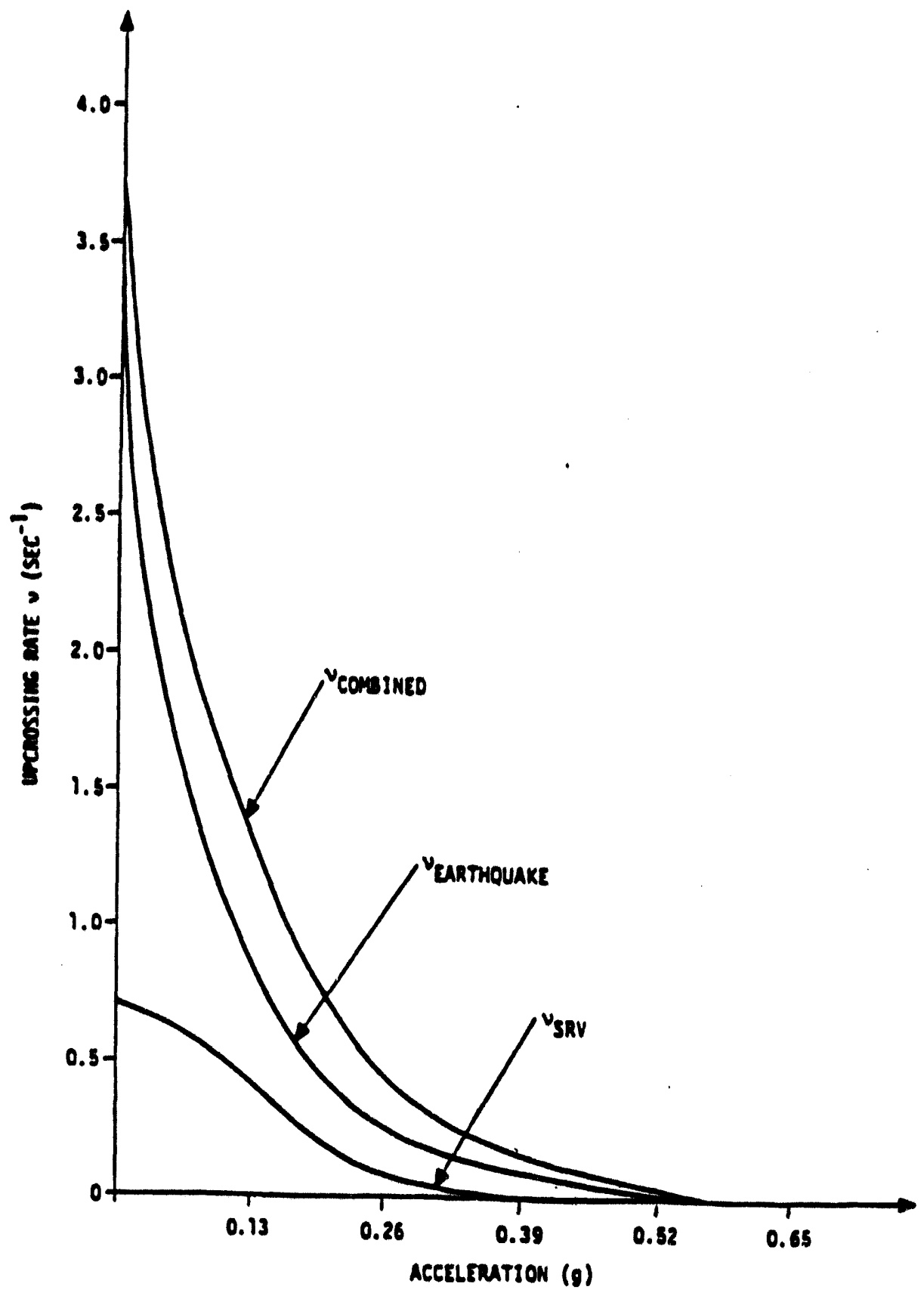

Figure 6.11. Time-averaged upcrossing rates for earthquake range 1, SRV, and combined response (oscillator frequency $=2 \mathrm{H} \%$, damping $=5 \%$ ). 


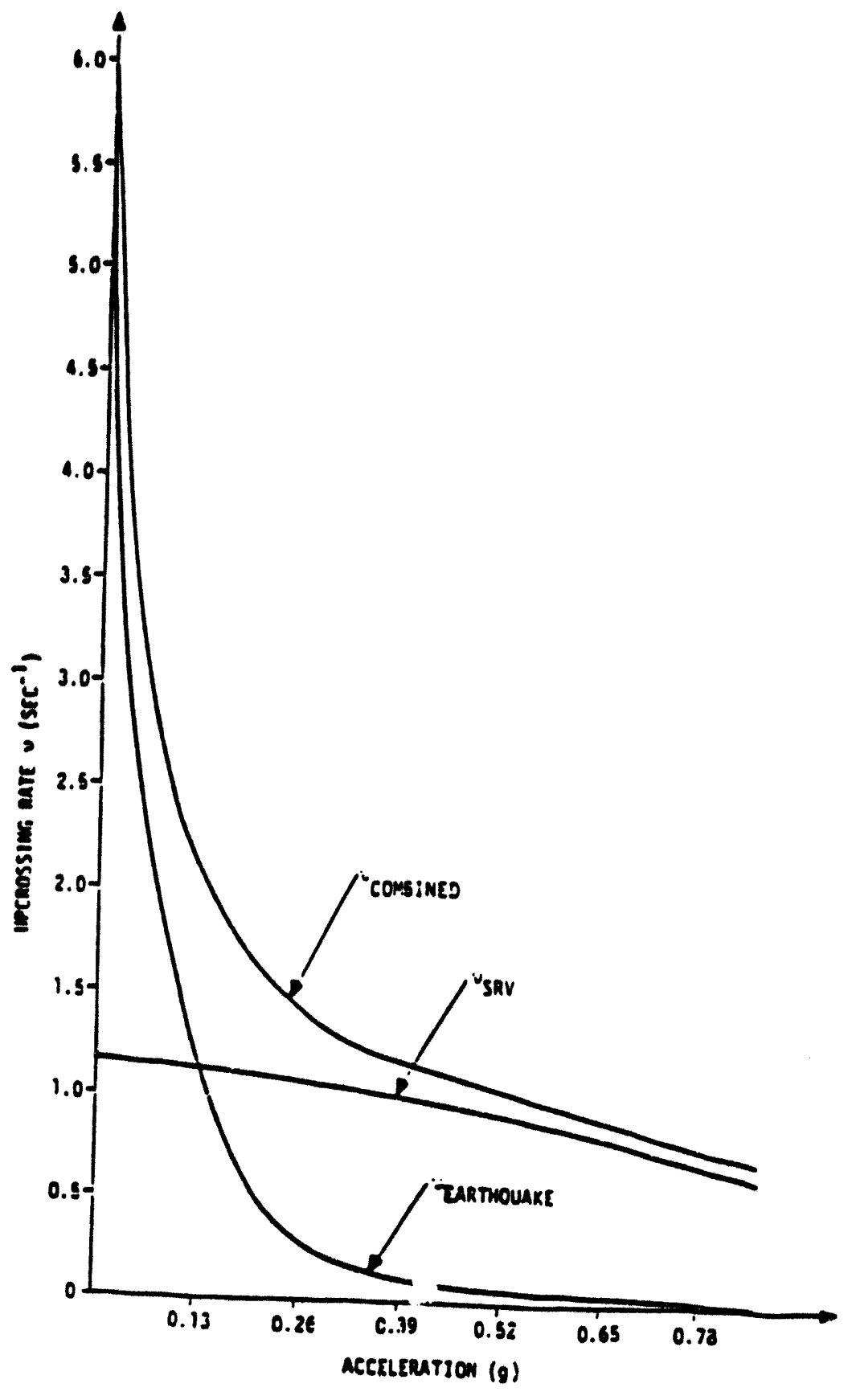

Figure 6.12. Time-averaged upcrossing rates for earthquake range 1, SRV, and combined response (oscillator frequency $=4 \mathrm{H} \%$, damping $=5 \%$ ). 


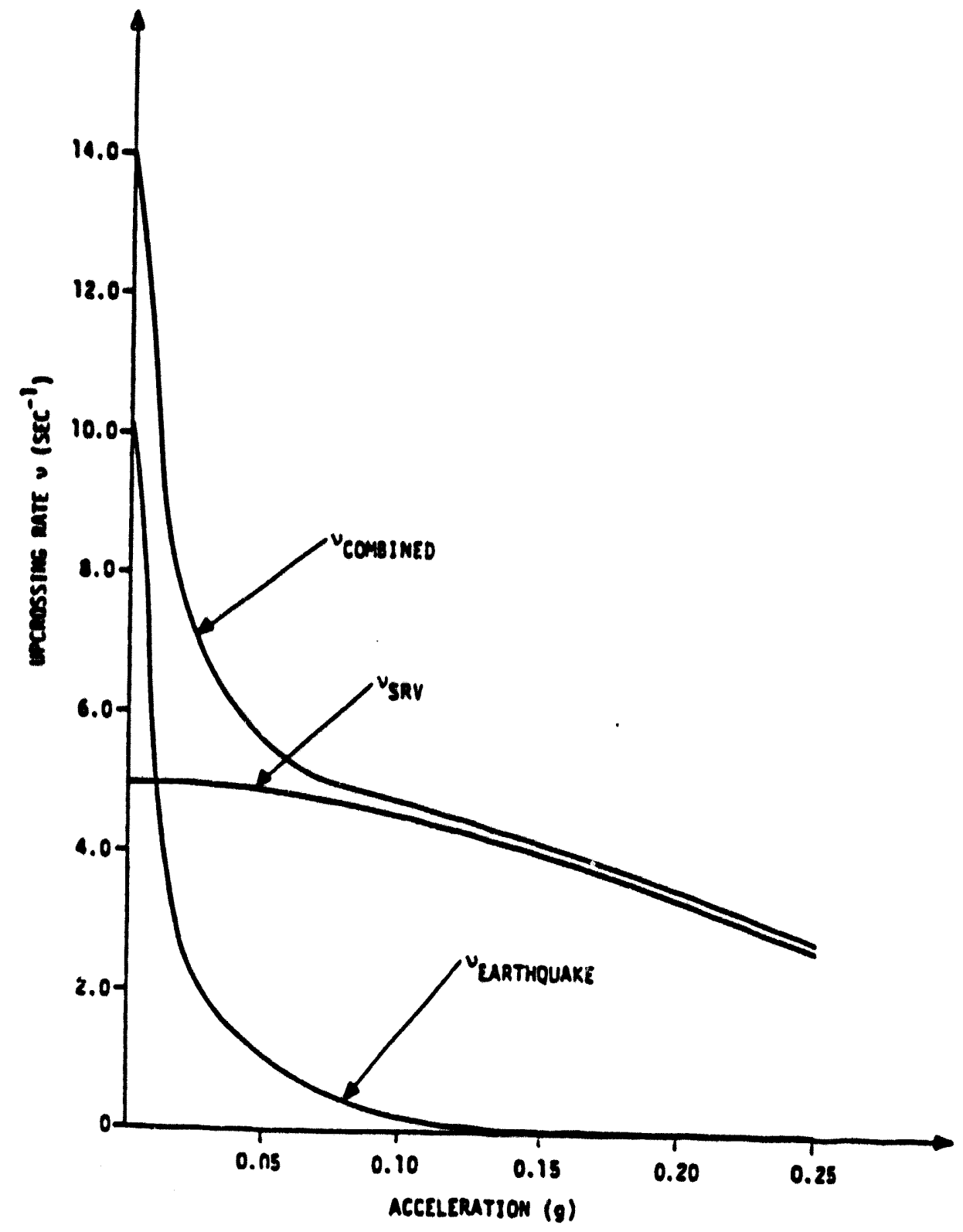

Figure 6.13. Time-averaged upcrossing rates for earthquake range $1, \mathrm{SRV}$, and combined response (oscillator frequency $=25 \mathrm{H} \%$, damping $=5 \%$ ). 
Note that except for the very low response levels, the combined upcrossing rate is greater than the sum of the individual upcrossing rates. This is to be expected, and can be understood by reexamining the physical meaning of the point crossing formula (Sec. 6.2.2). For example, consider the $2.11 \mathrm{~g}$ upcrossing rate for the $4 \mathrm{~Hz}$ oscillator (Tuble 6.7). The rate at which the earthquake crosses $2.11 \mathrm{~g}$ is essentially zero; that is, we do not expect that the oscillator response would exceed $2.11 \mathrm{~g}$ due to earthquake loading alone. The response due to the SRV load, however, is expected to cross this level 0.024 times per second. If the SRV response is at say, $2.10 \mathrm{~g}$, there is a finite probability that the earthquake response is $0.01 \mathrm{~g}$ so that the combined response crosses $2.11 \mathrm{~g}$ with some finite probability. The point crossing formula, as discussed earlier, considers all such combinations. Thus, the earthquake response contributes to the combined upcrossing rate of $2.11 \mathrm{~g}$ even though the range at which the earthquake-alone response would cross this level is essentially zero.

From the upcrossing rate function, the median maximum response may be estimated Eq. (6-11). Note that the estimates will tend to be conservative due to the Poisson process assumption used to obtain the probability distribution of the maximum response as discussed in Sec. 6.2.2. Table 6.9 shows the median maximum responses for the three cases studied, obtained using the earthquake, SRV and combined upcrossing rate functions. Also shown in the table, are the absolute sum and square-root of the sum-of-the-squares (SRSS) combinations of the earthquake and SRV median maximum responses.

As can be seen from the table, the absolute sum combination gives very conservative results as expected. The SRSS combination gives results slightly on the conservative side as compared with the median maximum obtained using the load combination approach. This should be expected since the SRSS rule does not specifically account for the fact that the SRV load is a very short duration load. This greatly reduces the likelihood that the maximum SRV response will combine with the maximum earthquake response. The SRSS rule is most conservative when the maximum responses due to the two individual loads are close together. However, even for the worst case $(f=$ $2 \mathrm{~Hz}$ ), it overestimates by only 5 percent. This will be investigated further in the Range 2 earthquake case studies.

\subsubsection{SSE Case Study}

For the Range 2 earthquake cases, four frequencies were selected for study; $2.0 \mathrm{~Hz}, 2.8 \mathrm{~Hz}, 3.0$ $\mathrm{Hz}$, and $4.0 \mathrm{~Hz}$. This was to ensure that cases in which the response due to the SRV load was significantly larger than that due to the earthquake and vice versa were covered, and that cases in which the two responses were similar were covered (since the Range 1 earthquake studies showed that the SRSS rule could potentially be in error for this latter case). Tables 6.10 though 6.13 show the upcrossing rate results for selected response levels and Figs. 6.14 through 6.17 show plots of these results. These wert 'tained in the same manner as was discussed for the earthquake Range 1 case. Similar observations can be made for these results as for the Range 1 case results. It is again seen, that except at the very low response levels, the combined upcrossing rate is greater than the sum of the individual upcrossing rates, as is expected.

Table 6.14 shows the median maximum response due to the earthquake and SRV loads, estimated from the response upcrossing rate functions Eq. (6-11). Also shown are the median maximum responses for the four cases studied obtained from the combined response upcrossing rate function. Again, the median maximun response estimates for the earthquake, SRV, and combined 
Table 6.9. Comparison of SRSS and absolute sum combination with probabilistic combination (earthquake $=$ ringe 1 ).

\begin{tabular}{|c|c|c|c|c|c|}
\hline $\begin{array}{l}\text { OSCILLATOR } \\
\text { FREQUENCY } \\
\\
\left(\mathrm{Hz}_{\mathrm{z}}\right. \\
\end{array}$ & $\begin{array}{c}\text { EARTHQUAKE } \\
\text { MEDIAN MAXIMUM } \\
\text { RESPONSE } \\
\text { (G) }\end{array}$ & $\begin{array}{l}\text { SRV } \\
\text { MEDLANMUXMUM } \\
\text { RESPONSE } \\
\text { (C) }\end{array}$ & $\begin{array}{l}\text { ABSOLUTE } \\
\text { SUM } \\
\text { (G) } \\
\end{array}$ & $\begin{array}{l}\text { SRSS } \\
\text { (0) }\end{array}$ & $\begin{array}{l}\text { PROBABILISTIC } \\
\text { COMBNATION } \\
\text { MEDLNMAXIMUM } \\
\text { RESPONSE } \\
\text { (O) }\end{array}$ \\
\hline 2.0 & 0.51 & 0.31 & 0.82 & 0.59 & 0.56 \\
\hline 4.0 & 0.75 & 2.05 & 2.80 & 2.18 & 2.11 \\
\hline 25.0 & 0.17 & 0.75 & 0.92 & 0.77 & 0.76 \\
\hline
\end{tabular}


Table 6.10. Upcrossing rates for earthquake range $2, \mathrm{SRV}$, and combined response (oscillator frequency $=2 \mathrm{~Hz}$, damping $=5 \%$ ).

\begin{tabular}{|c|c|c|c|}
\hline \multirow[b]{2}{*}{$\begin{array}{l}\text { RESPONSE } \\
\text { ACCELERATION } \\
\text { LEVEL } \\
\text { (G) }\end{array}$} & \multicolumn{3}{|c|}{ TIME AVERAGED $( \pm)$ UPCROSSING RATES } \\
\hline & $\begin{array}{c}\text { EARTHQUAKE } \\
\text { ALONE } \\
\left(\text { SEC }^{-1}\right)\end{array}$ & $\begin{array}{l}\text { SRV } \\
\text { ALONE } \\
\left(\text { SEC }^{-1}\right)\end{array}$ & $\begin{array}{c}\text { COMBINED } \\
\text { RESPONSE }^{-1} \text { (SEC-1) }\end{array}$ \\
\hline 0.0 & 3.3 & 0.72 & 3.5 \\
\hline 0.15 & 1.6 & 0.32 & 1.9 \\
\hline 0.46 & 0.57 & 0.001 & 0.64 \\
\hline 0.77 & 0.19 & 0.0 & 0.21 \\
\hline 1.24 & 0.026 & 0.0 & 0.031 \\
\hline
\end{tabular}


Table 6.11. Upcrossing rates for earthquake range 2, SRV, and combined response (oscillator frequency $=2.8 \mathrm{~Hz}$, damping $=5 \%$ ).

\begin{tabular}{|c|c|c|c|}
\hline \multirow{2}{*}{$\begin{array}{c}\text { RESPONSE } \\
\text { ACCELERATION } \\
\begin{array}{c}\text { LEVEL } \\
\text { (G) }\end{array}\end{array}$} & $\begin{array}{c}\text { TIME A VERAGED ( } \pm \text { ) UPCROSSING RATES } \\
\text { EAHQUAKE } \\
\text { ALONE } \\
\left(\text { SEC }^{-1}\right)\end{array}$ & $\begin{array}{c}\text { SRV } \\
\text { ALONE } \\
\left(\text { SEC }^{-1}\right)\end{array}$ & $\begin{array}{c}\text { COMBINED } \\
\text { RESPONSE } \\
\left(\text { SEC }^{-1}\right)\end{array}$ \\
\hline 0.0 & 4.0 & 0.92 & 4.4 \\
0.29 & 0.71 & 0.57 & 1.3 \\
0.48 & 0.25 & 0.25 & 0.61 \\
0.76 & 0.038 & 0.031 & 0.13 \\
0.95 & 0.0014 & 0.0046 & 0.0347 \\
\hline
\end{tabular}


Table 6.12. Upcrossing rates for earthquake range 2, SRV, and combined response (oscillator frequency $=3 \mathrm{~Hz}$, damping $=5 \%$ ).

\begin{tabular}{|c|c|c|c|}
\hline \multirow{2}{*}{$\begin{array}{c}\text { RESPONSE } \\
\begin{array}{c}\text { ACCELERATION } \\
\text { LEVEL } \\
(G)\end{array}\end{array}$} & $\begin{array}{c}\text { EARTHQUAKE } \\
\text { ALONE } \\
\left(\text { SEC }^{-1}\right)\end{array}$ & $\begin{array}{c}\text { SRV } \\
\text { ALONE } \\
\left(S^{-1}\right)\end{array}$ & $\begin{array}{c}\text { COMBC A VERAGED ( } \pm \text { ) UPCROSSING RATES } \\
\text { RESPONSE } \\
(\text { SEC }\end{array}$ \\
\hline 0.0 & 4.2 & 0.95 & 4.6 \\
0.28 & 0.64 & 0.73 & 1.4 \\
0.46 & 0.21 & 0.46 & 0.77 \\
0.74 & 0.028 & 0.15 & 0.26 \\
0.92 & 0.0 & 0.054 & 0.10 \\
\hline
\end{tabular}


Table 6.13. Upcrossing rates for earthquake range 2, SR.V, and combined response (oscillator frequency $=4 \mathrm{~Hz}$, damping $=5 \%$ ).

\begin{tabular}{|c|c|c|c|}
\hline \multirow{2}{*}{$\begin{array}{c}\text { RESPONSE } \\
\text { ACCELERATION } \\
\text { LEVEL } \\
\text { (G) }\end{array}$} & $\begin{array}{c}\text { TIME A VERAGED ( } \pm \text { ) UPCROSSING RATES } \\
\text { ALONE } \\
\text { (SEC-1) }\end{array}$ & $\begin{array}{c}\text { SRV } \\
\text { ALONE } \\
\text { (SEC-1) }\end{array}$ & $\begin{array}{c}\text { COMBINED } \\
\text { RESPONSE } \\
\text { (SEC-1) }\end{array}$ \\
\hline 0.0 & 5.1 & 1.2 & 5.5 \\
0.20 & 0.83 & 1.1 & 1.8 \\
0.40 & 0.11 & 1.0 & 1.2 \\
0.53 & 0.022 & 0.91 & 1.0 \\
2.10 & 0.0 & 0.024 & 0.029 \\
\hline
\end{tabular}




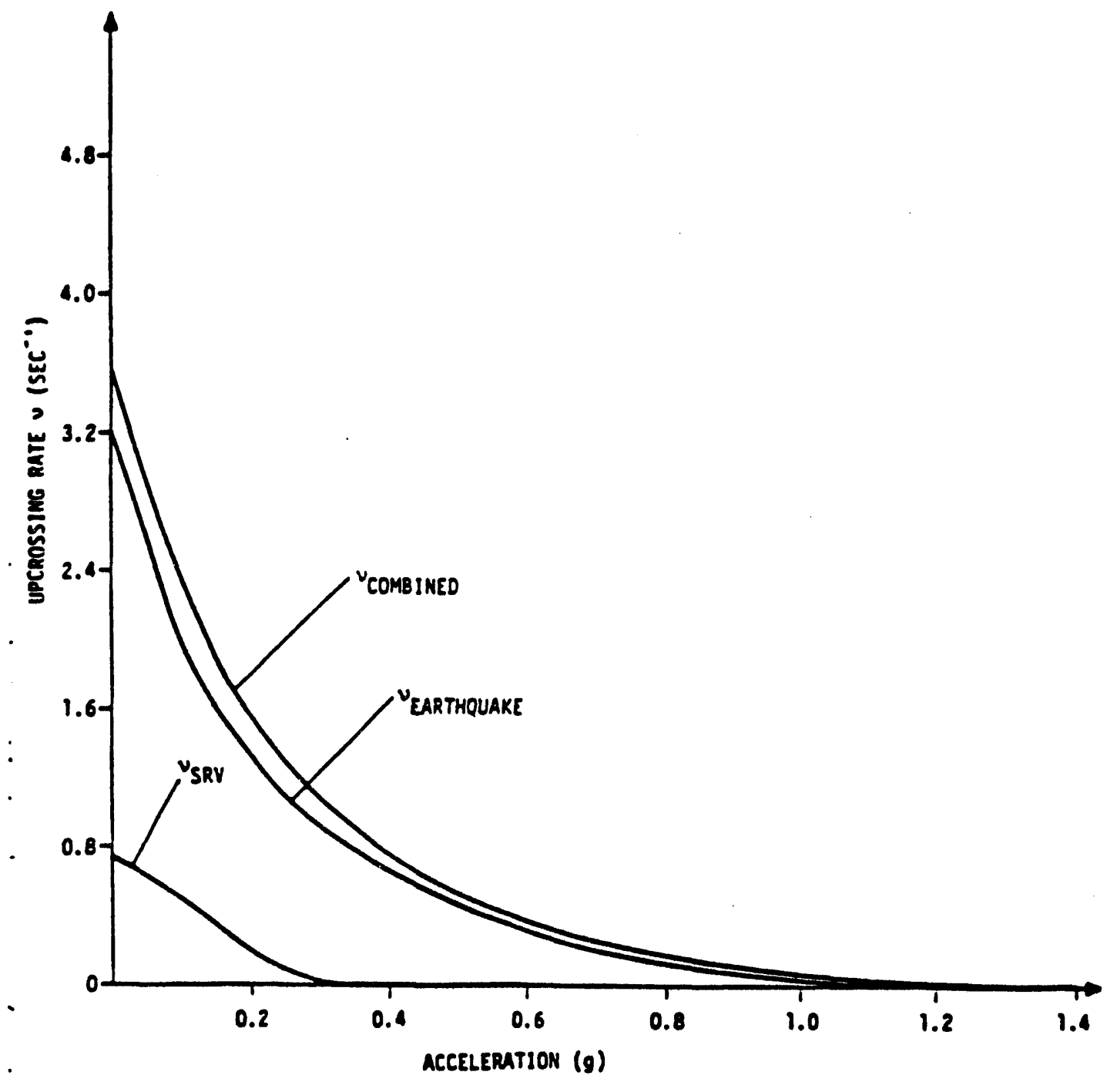

Figure 6.14. Time-averaged upcrossing rates for earthquake range $2, \mathrm{SRV}$, and combined response (oscillator frequency $=2 \mathrm{~Hz}$, damping $=5 \%$ ). 


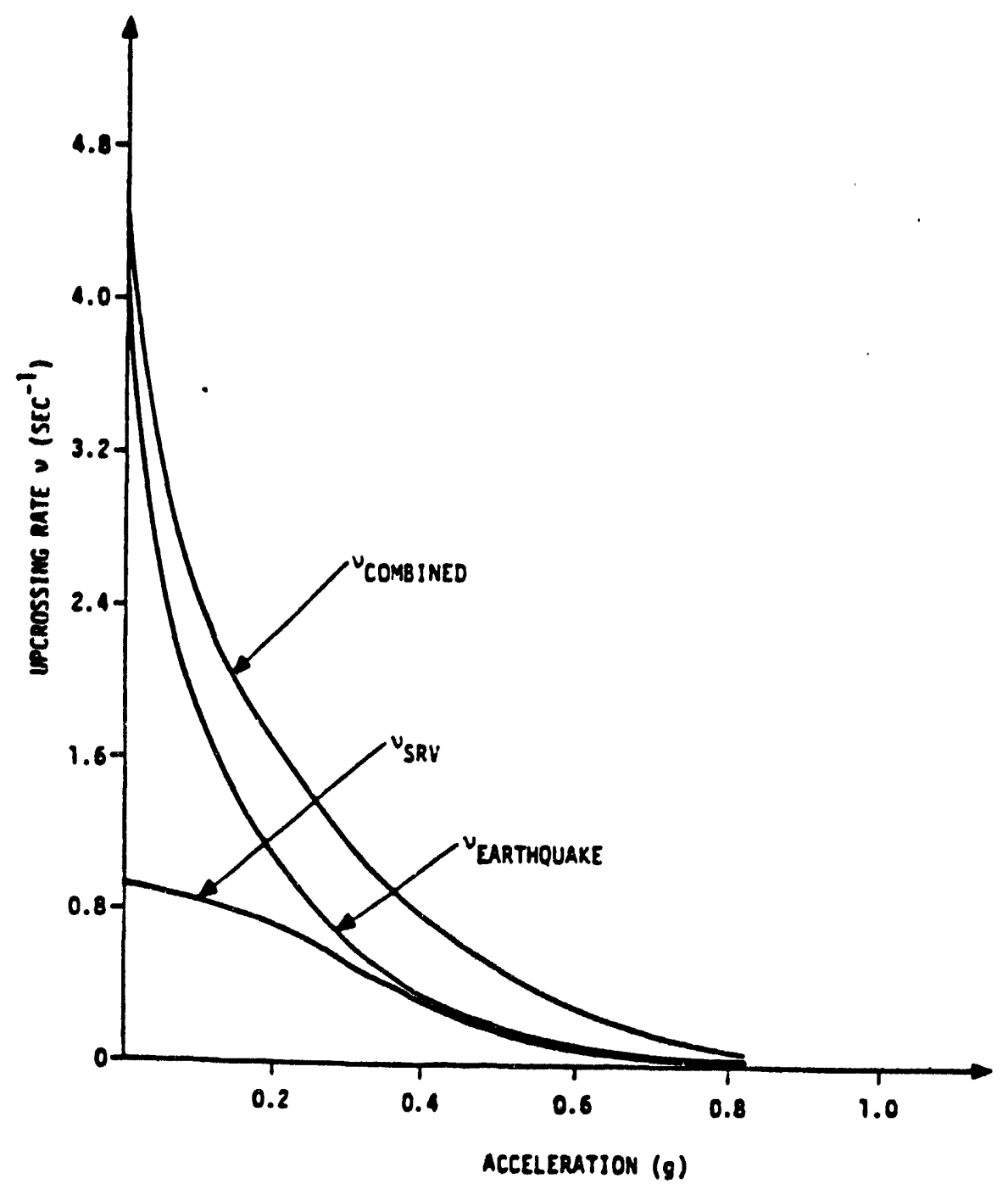

Figure 6.15. Time-averaged upcrossing rates for earthquake range 2, SRV, and combined response (oscillator frequency $=2.8 \mathrm{~Hz}$, damping $=5 \%$ ).

$$
-167-
$$




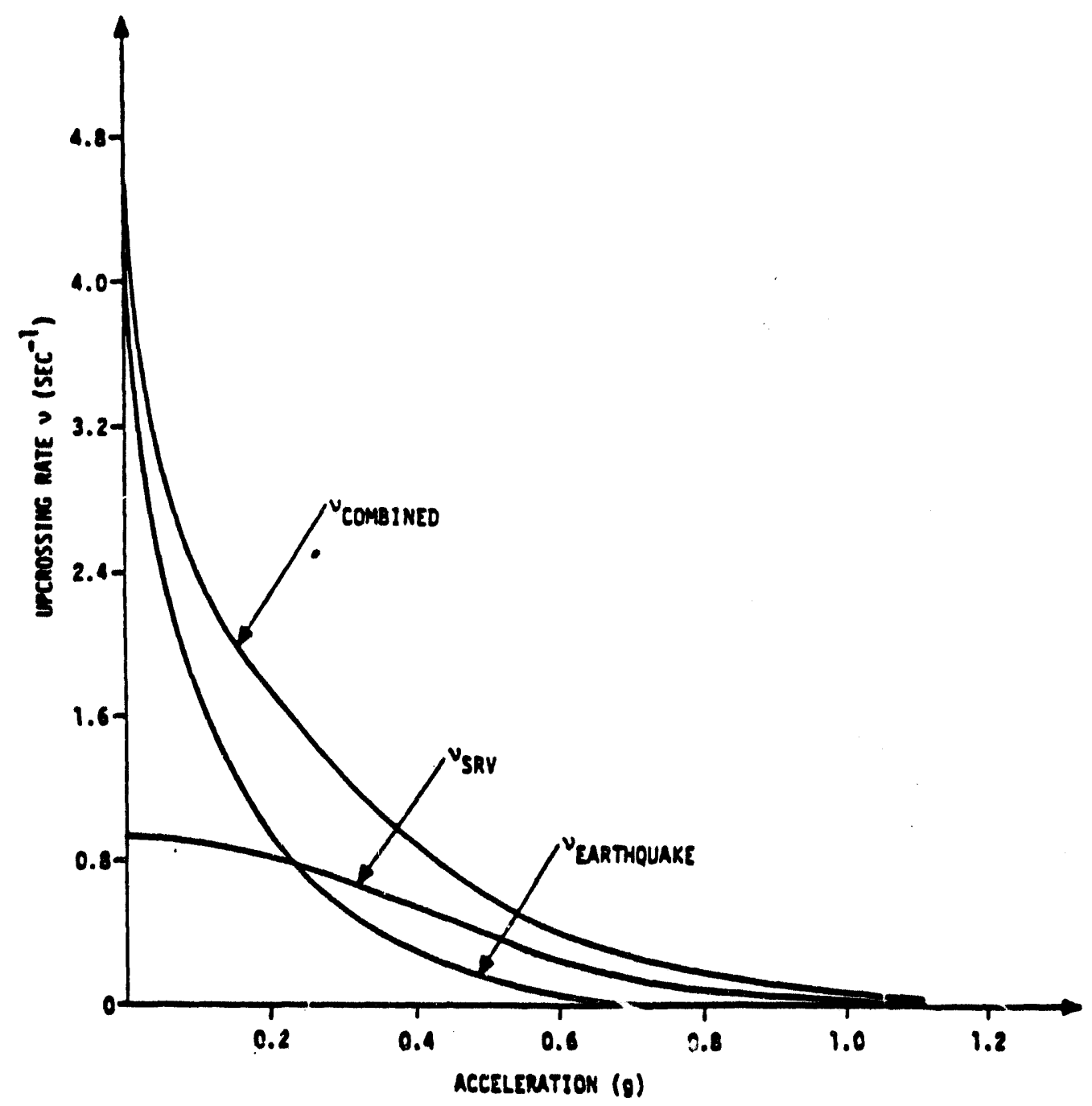

Figure 6.16. Time-averaged upcrossing rates for earthquake range 2, SRV, and combined response (oscillator frequency $=3 \mathrm{H} \%$, damping $=5 \%$ ). 


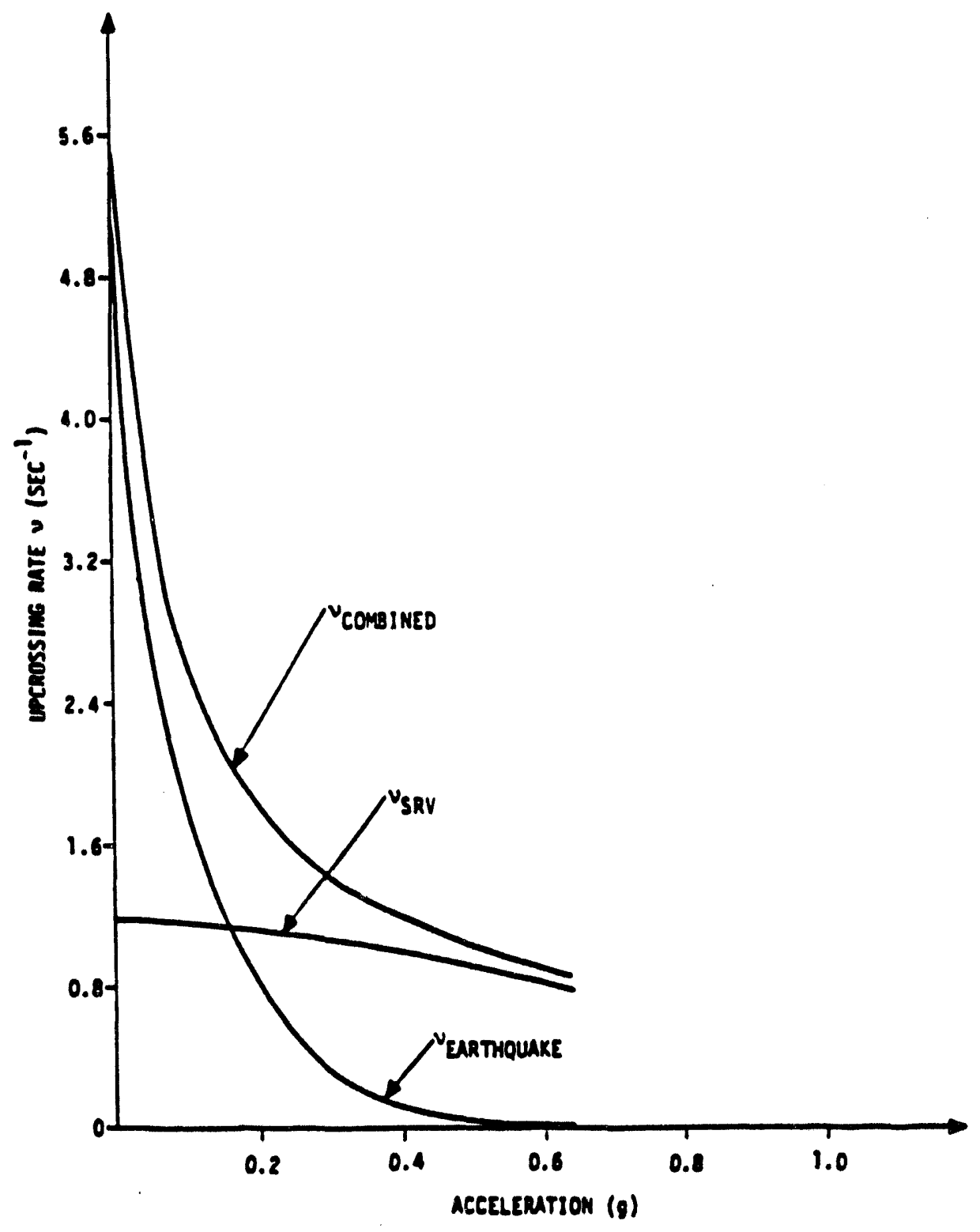

Figure 6.17. Time-averaged upcrossing rates for earthquake range 2, SRV, and combined response (oscillator frequency $=4 \mathrm{~Hz}$, damping $=5 \%$ ). 
Table 6.14. Comparison of SRSS and absolute sum combination with probabilistic combination (earthquake $=$ range 2 ).

\begin{tabular}{|c|c|c|c|c|c|}
\hline $\begin{array}{c}\text { OSCILLATOR } \\
\text { FREQUENCY }\end{array}$ & $\begin{array}{c}\text { EARTHQUAKE } \\
\text { MEDIAN MAXIMUM } \\
\text { RESPONSE }\end{array}$ & $\begin{array}{c}\text { SRV } \\
\text { MEDIAN MAXIMUM } \\
\text { RESPONSE } \\
(\mathrm{Hz})\end{array}$ & $\begin{array}{c}\text { ABSOLUTE } \\
\text { SUM }\end{array}$ & SRSS & $\begin{array}{c}\text { PROBABILISTIC } \\
\text { COMBNATION } \\
\text { MEDIANMAXIMUM } \\
\text { RESPONSE }\end{array}$ \\
\hline 2.0 & 1.23 & 0.31 & 1.54 & 1.27 & $(\mathrm{G})$ \\
2.8 & 0.79 & 0.77 & 1.56 & 1.10 & 1.26 \\
3.0 & 0.73 & 1.02 & 1.75 & 1.25 & 0.98 \\
4.0 & 0.52 & 2.05 & 2.57 & 2.11 & 1.15 \\
& & & & & 2.10 \\
\hline
\end{tabular}


load are conservative due to the Poisson process assumption (see Sec. 6.2.2). As was found in the Range 1 earthquake case study, the absolute sum combination gives very conservative results and the SRSS combination is only slightly conservative as compared with the load combination results. The interesting case here is for $\mathrm{f}=2.8 \mathrm{~Hz}$. Here, the earthquake and SRV median maximum responses are almost the same. Even for this case, the SRSS combination exceeds the load combination result by only 12 percent. Again, for the cases in which the median maximum responses are farther apart, the SRSS rule and the load combination results show little difference.

As mentioned earlier, the load combination analysis was carried out assuming that the distribution of the time lag variable, $\theta$, is that given in Fig. 6.10b. This distribution was obtained by assuming that offsite power is lost and, therefore, a plant transient and SRV actuation occurs, when the ground acceleration is $0.1 \mathrm{~g}$. As discussed in Sec. 6.2.2, rigorous treatment of the load combination problem would require evaluation of the probability distribution of $\theta$ for all possible values of offsite power capacity and consideration of the probability distribution of offsite power capacity.

In order to study the influence of the distribution of $\theta$ and to aid in bringing out any limitations of the present results (obtained assuming that offsite power is lost at $0.1 \mathrm{~g}$ ), a case was studied in which the distribution of $\theta$ was taken as that shown in Fig. 6.10c. That is, $\theta$ was taken to be uniformly distributed between 0 and 13 seconds (rather than 0 and 5 seconds). This distribution was obtained based on the assumption that offsite power is lost at $0.3 \mathrm{~g}$. Table 6.15 shows a comparison of the combined upcrossing rate results obtained with the two different $\theta$ distributions.

As can be seen, the difference is negligible. This can be understood by examining Fig. 6.18 which shows the temporal variation of the earthquake upcrossing rate of $0.20 \mathrm{~g}$. From this plot, it is seen that the earthquake upcrossing rate does not drop off significantly until after 13 seconds. Similar behavior is observed for the upcrossing rates of other levels. For this reason, the results are not particularly sensitive to the distribution of $\theta$. Of course, if the response rise time was longer, the earthquake strong motion shorter, the $\theta$ distribution non-uniform, or the $\theta$ upper bound larger, we would expect to see a greater difference in the upcrossing rates. For the present study, however, the assumption that offsite power is lost at $0.1 \mathrm{~g}$ does not appear to significantly affect the results.

\subsubsection{Recommended Response Combination Rule}

From the foregoing, it is clear that the square-root of the sum-of-the-squares is a reasonable rule for combining the median maximum responses due to SRV and earthquake loads. For the extreme case in which the median maximum responses are nearly identical, the SRSS rule was found to yield a combined response which exceeded that obtained using the load combination approach by only 12 percent. It should be kept in mind that the load combination approach used herein is an approximate method which has been shown to give slightly conservative results. ${ }^{[6.10]}$ Hence, the SRSS rule may be slightly more conservative than indicated here. Considering the large uncertainties in predicting structural capacities, however, this conservatism will have a negligible effect on the seismic risk analysis results. Thus, for our study, 
Table 6.15. Sensitivity of combined upcrossing rate to time lag variable distribution.

\begin{tabular}{|c|c|c|}
\hline \multirow{2}{*}{$\begin{array}{c}\text { RESPONSE } \\
\text { ACCELERATION } \\
\text { LEVEL } \\
\text { (g) }\end{array}$} & $0 \leq \theta \leq 5$ & $0 \leq \theta \leq 13$ \\
\hline 0.0 & 4.43 & 4.42 \\
0.10 & 2.44 & 2.48 \\
0.48 & 0.61 & 0.60 \\
0.95 & 0.035 & 0.034 \\
\hline
\end{tabular}




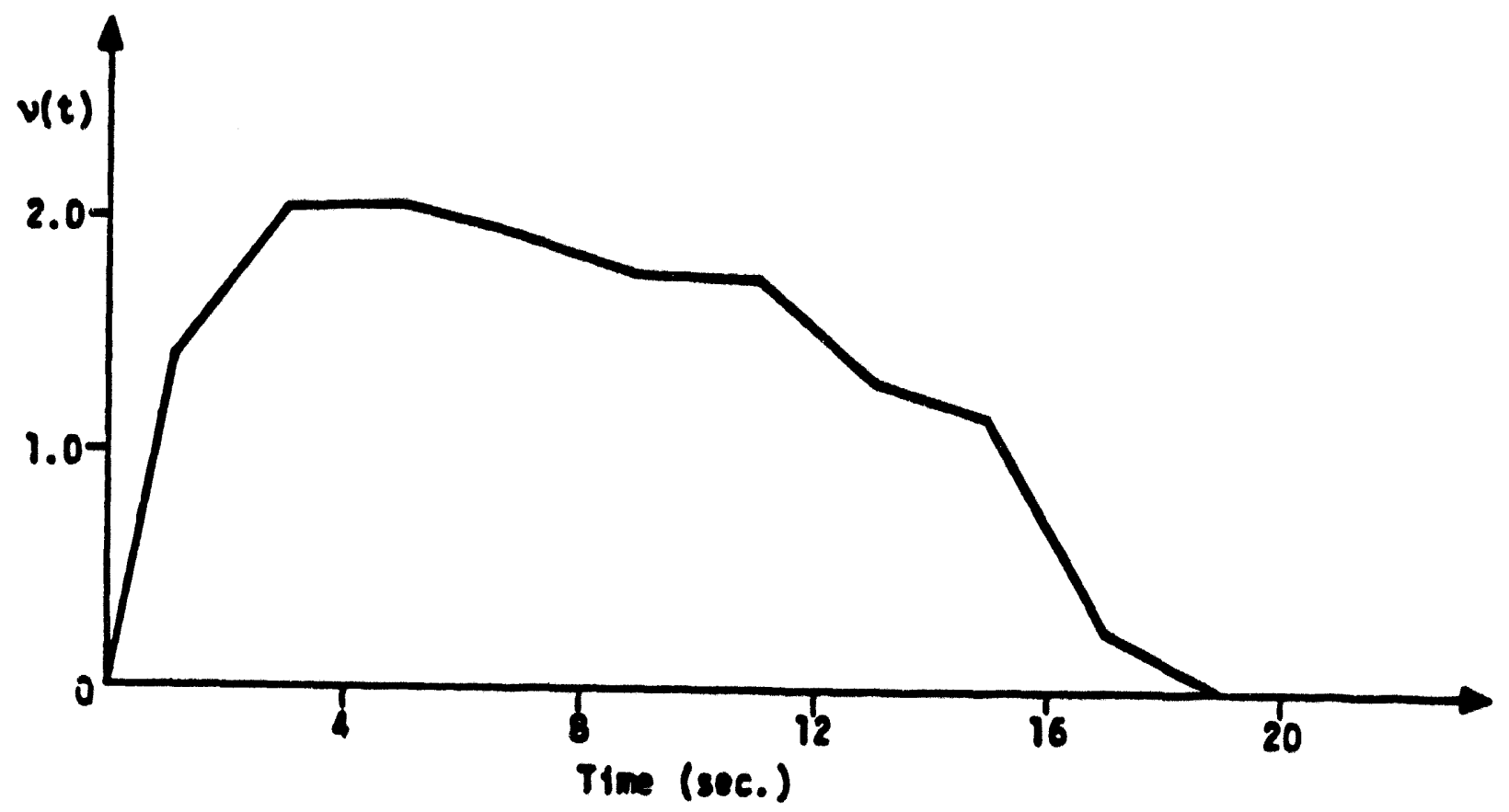

Figure 6.18. Variation of upcrossing rate of $0.20 \mathrm{~g}$ with time (earthquake range $2, f=2.8 \mathrm{H} \%$ ). 


$$
A_{C_{\max }}=\sqrt{\AA_{E Q_{\max }^{2}}^{2}+\AA_{S R V_{\text {max }}^{2}}^{2}}
$$

where

$$
\begin{gathered}
A_{C_{\max }}=\text { median combined maximum response acceleration } \\
A_{\mathrm{EQ}_{\max }}=\text { median earthquake maximumu response acceleration } \\
A_{S R V_{\max }}=\text { median } S R V \text { maximum response acceleration }
\end{gathered}
$$

For seisnic risk analysis, the variability of the combined response in addition to the median, must be known. It is possible to estimate the variability of the combined response based on the distribution of the maximum response Eq. (6-10). This approach, however, is highly dependent on the Poisson process assumption which is known to be conservative (i.e., the probability of no crossings of a given response level is underestimated) particularly, in the lower tail of the distribution. Evaluation of the logarithmic standard deviation is dependent on the entire distribution; hence, this approach is not pursued. While better approximations are availablel [6.11,6.12] they are more complex and would require additional analyses which would not be warranted for the present purposes.

From the result that the SRSS combination is a good approximation for the combination of median maximum responses and since the logarithmic standard deviations of the maximum seismic and SRV response are known, an alternate approach is possible. Assuming that the response maximum random variables combine according to an SRSS rule (say, for some unknown physical reason), then a first-order-second-moment approximation for the combination of logarithmic standard deviations can be obtained and is given by:

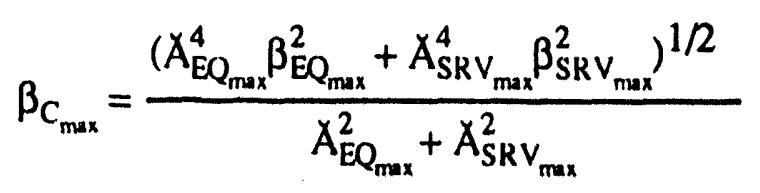

The covariance matrix of the combined response at all locations of interest is also required for the risk assessment. A review of the critical safety equipment item locations and the loadings at these locations, indicates that earthquake will be the doninant loading. Hence, for reasons of simplicity, the covariance matrix of the combined responses can be developed using the correlation matrix of the seismic responses. 


\section{Section 7. Structural Fragilities}

The fragilities of the LaSalle structures were generated using the same basic methodology previously developed for the SSMRP. A description of this methodology is contained in Ref. 7.1 and is not repeated here. Improvements in the methodology and the techniques used to predict the structure capacities were incorporated in this evaluation when possible. These improvements are described in the discussion below.

The fragilities of a number of structural elements were determined in this study. These structural elements were selected for detailed evaluation based upon an initial, preliminary review of the available structure drawings and the original design analysis seismic loads as well as previous experience in the review of the LaSalle structures. The detailed fragilities evaluation was performed for a number of shear walls and diaphragms, the primary containment, and the concrete internals located within the containment.

\subsection{Method of Fragility Evaluation}

In this evaluation, the fragilities for the individual structural elements were expressed in terms of their equivalent elastic capacities. As in the SSMRP, the structure fragilities were assumed to be lognormally distributed. For example, the equivalent elastic capacity for shear strength of a shear wall, $V_{\text {ue, }}$ can be represented by:

$$
V_{\text {ue }}=\hat{V}_{\text {ue }} \varepsilon_{R} \varepsilon_{U}
$$

where $V_{w}$ is the median equivalent elastic shear capacity and $\varepsilon_{R}$ and $\boldsymbol{E}_{U}$ are random variables with unit median, representing the inherent randomness about the median and the uncertainty in the median value. The variables $\boldsymbol{E}_{R}$ and $\boldsymbol{E}_{U}$ were assumed to be lognormally distributed with logarithmic standard deviations $\beta_{R}$ and $\beta_{U}$ respectively. The properties of the lognormal distribution are presented in Ref. 7.1.

The equivalent elastic capacity for an individual structural e:ement was defined as the product of two terms:

1. The ultimate strength of the structural element.

2. The inelastic energy absorption factor, $F_{\mu}$ that accounts for the ability of the structure to withstand seismic levels in excess of those corresponding to yield through ductile, nonlinear response.

The median equivalent elastic capacity is the product of the median values of these two terms. As an example, the median equivalent elastic shear capacity of a shear wall, $\hat{V}_{u e}$, is given by: 


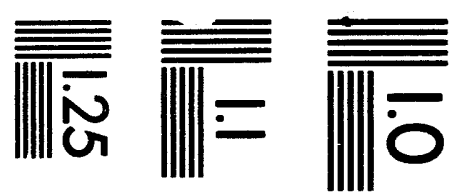

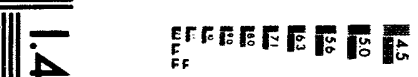

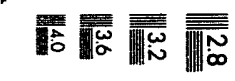

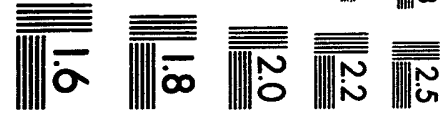




$$
\begin{aligned}
& \boldsymbol{\omega} \\
& \stackrel{\mathrm{o}}{\boldsymbol{\omega}}
\end{aligned}
$$




$$
\hat{V}_{u e}=\hat{F}_{\mu} \cdot \hat{V}_{u}
$$

where $\hat{V}_{u}$ is the median ultimate shear strength. Using the properties of the lognormal distribution, the logarithmic standard deviation associated with the equivalent elastic shear capacity is given by:

$$
\beta_{\mathrm{ue}}=\sqrt{\beta_{\mathrm{us}}^{2}+\beta_{\mu}^{2}}
$$

where $\beta_{u s}$ and $\beta_{\mu}$ are the logarithmic standard deviations associated with the element ultimate strength and the inelastic energy absorption. These individual variabilities were in tum composed of the individual contributions from randomness and uncertainty.

In this study, the guidelines used to estimate the values of $\beta_{R}$ and $\beta_{U}$ were based on considering the inherent randomness, $\beta_{R}$, to be associated with the earthquake characteristics themselves, and $\beta_{\cup}$ to be associated with other lack of knowledge. In general, it is not considered possible to significantly reduce randomness by additional analysis or test based on current state-of-the-art techniques. Thus, the only source of randomness in the structural fragilities was the effect of certain earthquake characteristics on the structure inelastic energy absorption capability. Uncertainty, on the other hand, is considered to result primarily from analytical modeling assumptions and other lack of knowledge concerning variables such as material strength, member strength, etc., which could in many cases be reduced by additional study or test. These earthquake characteristics include response spectra shape and amplification, duration, energy content, numbers and phasing of peak excitation cycles, etc.

\subsection{Development of Structural Element Capacities}

As previously noted, the LaSalle structural fragilities were expressed in terms of the equivalent elastic capacities for a number of structural elements. The equivalent elastic capacity for each element is the product of its ultimate strength and a factor accounting for the structure's inelastic energy absorption capability. The basic techniques used to determine the median values and associated variabilities of the terms were essentially those developed for the SSMRP as described in Ref. 7.1. More recent research, as well as review of the fragilities development approach, has led to the possibility of improvement in these techniques. These improvements were incorporated in the generation of LaSalle structure fragilities where possible.

\subsubsection{Structural Element Ultimate Strengths}

Two major considerations are involved in the determination of the ultimate strengths of the individual structural elements. One is the definition of the strengths of the materials composing the members. For the fragilities evaluation, the median material strengths and variabilities were determined from the plant-specific test data, when available. The other is the determination of the 
evaluation were based upon research results available in the literature.

The median material strengths and variabilities used in the fragilities evaluation are listed in Table 7.1. The values for the $4000 \mathrm{psi}$ and $4500 \mathrm{psi}$ concrete and the reinforcement were bar upon summaries of available plant-specific strength test results presented in Ref. 7.2. The effect or aging on the concrete compressive strength was included in the same manner as for the SSMRP. ${ }^{[7.1]}$ Test results for the 3500 pi concrete and the postensioning tendons were not available from Ref. 7.2. Consequently, the median compressive strength and variability of the $3500 \mathrm{psi}$ concrete were estimated from the values for the other two concretes. The median yield strength and variability for the postensioning tendons were estimated from the results of testing conducted for the tendons used in the construction of the Zion containments.

The median ultimate strength capacities of the various structural elements were found using the known median material strengths and member configurations (i.e., geometry, reinforcement, etc.) in conjunction with available predictive formulations or approaches. The approaches and formulations used were those appropriate for the type of element (i.e., shear wall, cylindrical containment, etc.) and loading (i.e., shear, flexure, etc.). They were typically found to provide essentially median-centc ed capacities when compared to the results of available experimental testing. For example, the predictive equations used to determine the median ultimate strengths of the LaSalle shear walls subjected to in-plane shear and flexure were reported in Ref. 7.1. Approaches used to determine the median ultimate strengths of other members are briefly discussed in the following sections.

Variability of the structural element ultimate strengths was considered to be highly uncertain since it is associated with a lack of knowledge. Uncertainty attributed to material strength was based upon the variability in the available test data. Comparisons of the predicted strength capacities to the available test results provided estimates of the uncertainty in the predictive strength formulations. Additional uncertainty attributable to variabilities associated with other sources, such as member geometry, reinforcement spacing, openings, workmanship, differences between field and laboratory conditions, accuracy of the predicted load distributions, etc., were also included.

\subsubsection{Structure Inelastic Energy Absorption}

The ability of the structure to withstand seismic levels, in excess of those corresponding to $y^{\prime}$ eld through ductile, nonlinear response was accounted for by the inelastic energy absorption fau iur: $F_{\mu}$. Similar to the SSMRP, the inelastic energy absorption factor was based upon the Rid id $\mathrm{Si}$. Newmark response deamplification accession factor presented in Ref. 7.3. Using this aps rocts

the median inelastic energy absorption factor, $\hat{F}_{\mu}$, corresponding to some ductility ratio, $w_{\text {, }}$ given by:

$$
\hat{F}_{\mu}=\left[p_{\mu}-q\right]^{r}
$$


Table 7.1. Median material strengths and variabilities.

Concrete Design Strength

$4000 \mathrm{psi}$

$4500 \mathrm{psi}$

$3500 \mathrm{psi}$

Reinforcing Steel

Bar sizes \#2 to \#11

Bar sizes \#14 to \#18

Prestress Tendons

All $\underline{f^{\prime} c(p s i)}$

B

5350

6150

4700)

0.12

0.12

0.14

fy $(k s i)$

B

66.9

57.0

0.06

fpy (ksi)

B

224

0.05 
where

$$
\begin{aligned}
p & =q+1 \\
q & =3.0 \delta^{-0.30} \text { in the amplified acceleration region. } \\
& =2.7 \delta^{-0.40} \text { in the amplified velocity region. } \\
r & =0.48 \delta^{-0.08} \text { in the amplified acceleration region. } \\
& =0.66 \delta^{-0.04} \text { in the amplified acceleration region. } \\
\delta & =\text { percent of critical damping. }
\end{aligned}
$$

Inspection of the in-structure response spectra indicated that the overall response of the structure was most strongly influenced by horizontal modes having frequencies less than about $2.5 \mathrm{~Hz}$. Consequently, the Riddell-Newmark parameters appropriate for the amplified velocity region were typically used.

The Riddell-Newmark response deamplification factor was based upon a series of nonlinear analyses utilizing single-degree-of-freedom (SDOF), fixed-base models subjected to time histories of large magnitude, long duration earthquakes. As these characteristics are not entirely representative of the LaSalle site and structures, modifications to the Riddell-Newmalk approach were implemented. These modifications were made through the use of an effective, system cuctility, $\mu *$, accounting for these effects in place of the element or story drift ductility, $\mu_{\mathrm{S}}$.

The system ductility, $\mu$, for use in conjunction with the Riddell-Newmark response deamplification factor is a measure of the ductility throughout the structure. For fixed-base SDOF structures, the system ductility is equal to the element or story drift ductility, $\mu_{\mathrm{S}}$. However, for multi-degree-offreedom (MDOF) structures, the system ductility may be less than the element ductility if the ratio of the story demand to story capacity is not uniform through the structure height. The system ductility, $\mu$, can be related to the story drift ductility, $\mu_{\mathrm{s}}$ by a factor $\mathrm{M}$ :

$$
\mu=\frac{\mu_{\mathrm{s}}-1}{M}
$$

The factor $M$ is unity for fixed-base MDOF structures with uniform demand to capacity ratios. However, Ref. 7.4 estimated $M$ to be 3.33 for the fixed-base structure evaluated in Ref. 7.5 which had a highly localized nonlinearity. The factor $M$ may be even greater for structures for which soilstructure interaction (SSI) effects are significant. For example, Ref. 7.4 estimated $M$ to be 10 for the structure evaluated in Ref. 7.5 including SSI. This reduction in effective system ductility for structures having significant SSI effects occurs because the major effects of structure nonlinear response (frequency shift and increased damping) are not as important to the overall response of a 
system which is strongly influenced by the soil flexibility and radiation damping.

For the structural fragilities, the effective system ductility assigned to the structural element was based upon appropriate estimates of the element or story drift ductility, $\mu_{s}$, and $M$. In the determination of these parameters, consideration was given to the degree of nonlinearity associated with the structure as a whole when failure of a particular element occurs. This approach is more appropriate than treatment of the failure modes as being entirely independent of each other since the different failure modes typically exhibit a strong degree of correlation. For example, an element ductility of 5 was judged to be appropriate for the major shear walls. The major SSI effects were considered in the analyses performed to generate the elastic structure loads. These effects are significant since the fundamental structure frequencies are fairly low. The significance of SSI along with a reasonable, although not exactly uniform, distribution of nonlinearity through the structure at failure led to the selection of a value of 4.5 for $M$ for failure of the major shear walls.

An additional drawback of the Riddell-Newmark response deamplification factor is that it does not reflect the relationship between earthquake magnitude and ductility. It is well known that lower magnitude earthquakes are not as damaging to structures and equipment as higher magnitude earthquakes with the same peak ground acceleration. The reason for this is that the lower magnitude earthquakes have lower energy content and shorter durations which develop fewer strong response cycles. Structures are able to withstand larger deformations (i.e., higher ductility) for a few cycles compared to the larger number of cycles resulting from longer duration events.

The method used in the structural fragilities evaluation to account for this effect was based on the use of an effective ductility, $\mu^{*}$, in conjunction with the Riddell-Newmark response deamplification factor approach. The following formulation was developed to calculate the effective ductility.

$$
\mu^{*}=1.0+C_{D}(\mu-1.0)
$$

where the duration correction factor, $C_{D}$, is a function of the earthquake magnitude and $\mu$ is the previously defined system ductility.

The results of the analyses performed in Ref. 7.5 were used to provide estimates of the duration coefficient, $C_{D}$, as a function of earthquake magnitude. For earthquakes having magnitudes ranging from 4.5 to 6.0 , a duration coefficient of 1.4 was determined to be appropriate by correlating the inelastic eniargy absorption factor from the Riddell-Newmark formulation to the results of Ref. 7.5. Similarly, a duration coefficient of 0.7 was estimated for earthquake magnitudes in the 6.5 to 7.5 range. Based upon information supplied showing the LaSalle seismic hazard as a function of earthquake magnitude, most of the seismic risk was estimated to result from earthquakes having magnitudes ranging from about 5.3 to 6.3 and centered on 5.8. A duration coefficient of 1.3 was considered to be appropriate for the LaSalle structures.

It should be noted that, for purposes of this study, structures are considered to fail functionally when inelastic deformations of the structure under seismic load are estimated to be sufficient to potentially interfere with the operability of safety-related equipment attached to the structure. The element and system ductility limits chosen for structures are estimated to correspond to the onset of 
significant structural damage. For many potential modes of failure, this is believed to represent a conservative bound on the level of inelastic structural deformation which might interfere with the operability of components housed within the structure. It is important to note that considerably greater margins of safety against structural collapse are believed to exist for these structures than many cases reported within this study. Thus, the structural element capacities reported herein should not be inferred as corresponding to structure collapse.

\subsection{Structural Element Fragilities}

Fragilities for the various structural elements selected for detailed evaluation were determined using the approach described in the preceding sections. The results of the structural fragilities evaluation for these elements are presented below. A few other structural elements were initially selected for detailed review based upon a preliminary investigation. This includes certain shear walls and diaphragms, the vent stack, and the masonry walls. However, additional approximate calculations indicated that these structural elements would not dominate. The approximate capacities for these elements are consequently not reported herein.

The structural fragilities developed in this evaluation will be compared to seismic loads predicted by elastic dynamic analyses. Many of the structural elements considered are ductile and can withstand deformations in excess of those corresponding to yield. As these elements yield, the seismic had will be redistributed to other members. Prediction of structural failure from a comparison of individual element capacities with elastic seismic loads is therefore somewhat conservative. In a few cases, dynamic model modifications were incorporated to account for the load redistribution. However, the development of had redistribution analyses is fairly complicated and, in general, was considered to be beyond the scope of this study.

\subsubsection{Shear Walls}

A number of shear walls located throughout the main LaSalle structure were selected for evaluation. The in-plane shear and flexural ultimate strengths were determined using the equations previously developed for the SSMRP. ${ }^{[7.1]}$ Shear was found to be controlling for all of the walls investigated. The shear wall ultimate strengths, equivalent elastic capacities, and variabilities are listed in Table 7.2. Variabilities were determined only for those walls expected to be controlling. 
Table 7.2. Summary of shear wall fragilities.

\begin{tabular}{|c|c|c|c|c|c|c|}
\hline $\begin{array}{c}\text { Column } \\
\text { Line }\end{array}$ & Story & $\begin{array}{c}\text { Spring } \\
\text { No. }\end{array}$ & $\begin{array}{r}V_{u} \\
(k)\end{array}$ & $\begin{array}{l}V_{\text {ue }} \\
(\mathrm{k})\end{array}$ & $\beta R$ & $\beta u$ \\
\hline A & El. $710^{\circ}-6 "$ to El. $740^{\circ}-0^{\prime \prime}$ & 203001 & 79,790 & 154,000 & 0.23 & 0.27 \\
\hline A & El. $740^{\prime}-0 "$ to El. $761^{\prime}-()^{\prime \prime}$ & 304001 & 74,060 & 143,000 & 0.23 & 0.27 \\
\hline A & El. $761^{\circ}-0 "$ to El. $786^{\prime}-6^{\prime \prime}$ & 405001 & 68,610 & 132,000 & -- & .- \\
\hline H & El. $673^{\circ}-4^{\prime \prime}$ to El. $694^{\prime}-6^{\prime \prime}$ & 1502023 & 24,640 & 47,600 & .- & -- \\
\hline $\mathrm{j}$ & El. $673^{\prime}-4^{\prime \prime}$ (1) El. $694^{\prime}-6^{\prime \prime}$ & 1501025 & 120,600 & 233,000 & -- & .- \\
\hline $\mathbf{R}$ & El. $710^{\circ}-6^{\prime \prime}$ to El. $731^{\prime}-\left(0^{\prime \prime}\right.$ & 210027 & 46,510 & 89,800 & 0.23 & 0.29 \\
\hline 13 & El. $673^{\circ}-4^{\prime \prime}$ to El. $694^{\prime}-6^{\prime \prime}$ & 1501032 & 41.950 & 81,000 & 0.23 & 0.27 \\
\hline 15 & El. $673^{\prime}-4^{\prime \prime}$ to El. $694^{\prime}-6^{\prime \prime}$ & 1501044 & 54,360 & 105,000 & -. & -- \\
\hline 15 & El. $740^{\circ}-0^{\prime \prime}$ เo El. $761^{\circ}-0^{\prime \prime}$ & 304008 & 63,070 & 122,000 & . & .. \\
\hline 21.1 & El. $710^{\prime}-6^{\prime \prime}$ to El. $731^{\circ}-0^{\prime \prime}$ & 210032 & 46,950 & 90,600 & 0.23 & 0.27 \\
\hline 21.1 & El. 749'-(1)" to El. 761'-0" & 1104018 & 52,200 & 101,000 & - & $\ldots$ \\
\hline 21.1 & El. $761^{\prime}-0^{\prime \prime}$ 10 El. $768^{\circ}-0^{\prime \prime}$ & 414006 & 54,800 & 106,000 & 0.23 & 0.28 \\
\hline 24 & El. $673^{\prime}-4^{\prime \prime}$ to El. $710^{\prime}-6^{\prime \prime}$ & 1502082 & 33,370 & 64,400 & -. & -. \\
\hline 24 & El. $731^{\prime}-0^{\prime \prime}$ to El. $749^{\prime}-0^{\prime \prime}$ & 1011036 & 19,580 & 37,800 & -. & -- \\
\hline 24 & El. $749^{\prime}-()^{\prime \prime}$ to El. $768^{\prime}-()^{\prime \prime}$ & 1114020 & 14,890 & 28,700 & -- & .. \\
\hline 26 & El. $710^{\prime}-6 "$ to El. $731^{\prime}-()^{\prime \prime}$ & 210038 & 36,080 & 69,600 & 0.23 & 0.29 \\
\hline
\end{tabular}

$\mathrm{V}_{\mathrm{u}}=$ Ultimate strength

$\mathrm{V}_{\mathrm{ue}}=$ Equivalent elastic capacity 
Elastic loads on the structural elements generated as a part of this program for acceleration ranges 1 and 2 are tabulated in Table 5.9. The median elastic loads for each range are compared to the shear wall equivalent elastic capacities in Tables 7.3 and 7.4. A measure of which walls are more likely to fail can be gained by comparing the ratio of median capacity to median load, also shown in Tables 7.3 and 7.4.

Column Line 26, El. 710'-6" to El. 731'-0". Inspection of Tables 7.3 and 7.4 indicates that the wall with the lowest capacity is the wall on Column Line 26 from El. 710'-6" to El. 731'$0 "$. This wall is located in the non-seismic Category I turbine building. Failure of this wall may lead to damage of equipment located in the turbine building immediately adjacent to the wall. However, since the turbine building was originally non-seismic Category I, safety-related equipment should not be affected by failure of this wall. The structure should have sufficient redundancy such that failure at this wall will not result in gross failure of the entire structure.

Walls Above El. 710'-6". Inspection of Tables 7.3 and 7.4 indicates that many of the walls exhibiting the lower ratios of capacity to load are located above El. 710'-6" in the reactor, auxiliary, and diesel generator buildings. There are several major walls that have capacity to acceleration range 1 load ratios varying from 14. to 16. Because these are major elements of the lateral load resisting system, failure of any of these walls should be considered to essentially correspond to gross structural failure and to cause damage to equipment located throughout the entire main LaSalle structure above El. 710'-6". This interpretation may introduce some conservatism since the prediction of equipment damage is based upon a comparison of wall capacities with elastic loads.

Walls Below El. 710'-6". The walls below El. 710'-6" exhibiting the lowest capacities are the walls along Column Lines 13 and 24 from El. 673'-4" to El. 694'-6". Because there are several other large walls at this story, the redundancy of the structure should prevent gross structural failure when the capacity of these walls is reached. Failure of the wall should only result in localized damage resulting from loss of anchors to equipment mounted on the wall or on the slabs immediately adjacent to this wall or concrete spalling. Inspection of the capacities of the major walls below El. 710'-6" indicates that they are well in excess of the capacities of the walls along Column Lines 13 and 24. Although, load redistribution analysis was not performed, gross structural failure and resulting equipment damage below El. 710'-6" would be expected to occur at seismic levels somewhat greater than those corresponding to failure of the walls along Column Lines 13 and 24.

\subsubsection{Diaphragms}

Fragilities were developed for a number of diaphragm elements located throughout the main LaSalle structure. The calculated fragilities are presented in Table 7.5. Variabilities were determined only for those diaphragms expected to be controlling. The diaphragms evaluated were typically found to be governed by in-plane shear rather than flexure. In some cases, shear failure is expected to occur in the slabs themselves. The diaphragm slab shear strengths were determined using the same equations developed for the shear walls. In other cases, shear failure is expected to occur due to shear transfer across cracked concrete, steel on concrete, or construction joint 
interfaces. Shear friction capacities and variability were determined from the results of research reported in Refs. 7.6 through 7.9. The capacities of steel studs were based upon the recommendations of Refs. 7.10 and 7.11. The ratios of median capacity to median elasti- load are shown in Tables 7.6 and 7.7. Median elastic loads were obtained from Table 5.9.

Diaphragm failure is usually relatively localized. The consequences of the diaphragm element failures exhibiting the lower capacities are discussed below. 
Table 7.3. Comparison of shear wall equivalent elastic capacities with elastic loads acceleration range 1 .

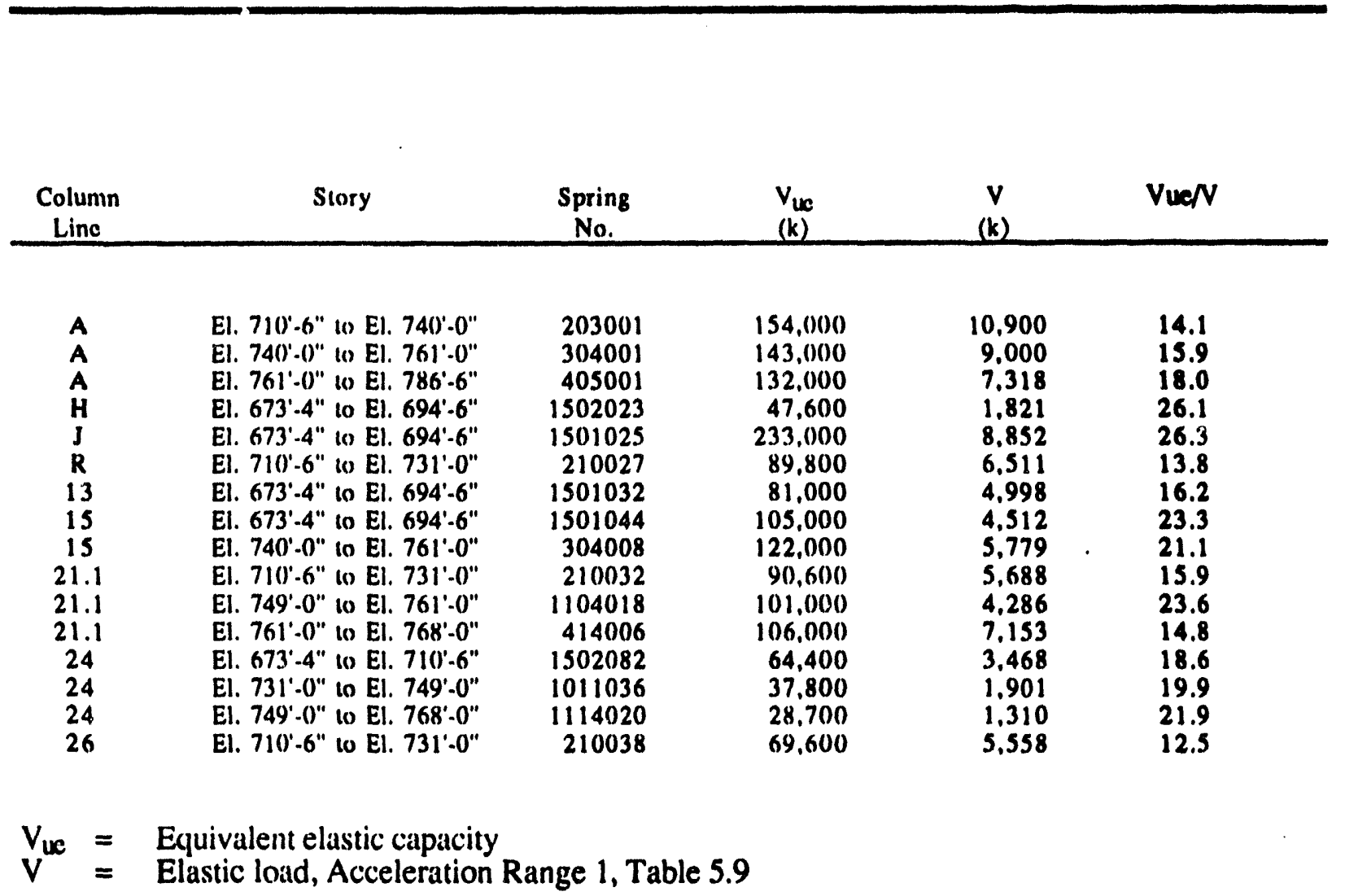


Table 7.4. Comparison of shear wall equivalent elastic capacities with elastic loads acceleration range 2 .

\begin{tabular}{|c|c|c|c|c|c|}
\hline $\begin{array}{c}\text { Column } \\
\text { Line }\end{array}$ & Story & $\begin{array}{c}\text { Spring } \\
\text { No. }\end{array}$ & $\begin{array}{l}v_{u c} \\
(k)\end{array}$ & $\begin{array}{c}V \\
(k)\end{array}$ & Vue/V \\
\hline $\begin{array}{c}\text { A } \\
\text { A } \\
\text { A } \\
H \\
J \\
R \\
13 \\
15 \\
15 \\
21.1 \\
21.1 \\
21.1 \\
24 \\
24 \\
24 \\
26\end{array}$ & 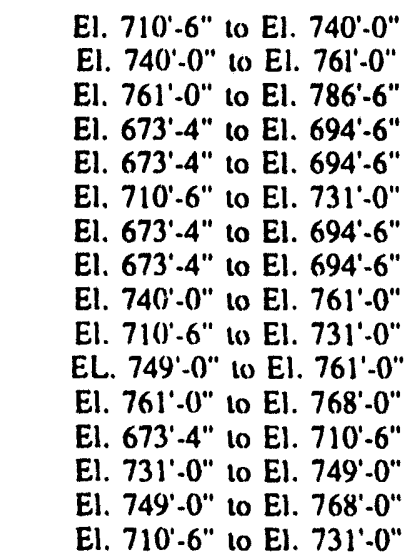 & $\begin{array}{r}203001 \\
304001 \\
405001 \\
1502023 \\
150102 S \\
210027 \\
1501032 \\
1501044 \\
304008 \\
210032 \\
1104018 \\
414006 \\
1502082 \\
1011036 \\
1114020 \\
210038\end{array}$ & $\begin{array}{r}154,000 \\
143,000 \\
132,000 \\
47,600 \\
233,000 \\
89,800 \\
81,000 \\
105,000 \\
122,000 \\
90,600 \\
101,000 \\
106,000 \\
64,400 \\
37,800 \\
28,700 \\
69,600\end{array}$ & $\begin{array}{r}15,450 \\
12,620 \\
10,190 \\
2,630 \\
12,810 \\
9,303 \\
7,971 \\
7,190 \\
8,821 \\
8,898 \\
6,494 \\
10,880 \\
5,491 \\
2,920 \\
1,980 \\
8,667\end{array}$ & $\begin{array}{r}10.0 \\
11.3 \\
13.0 \\
18.1 \\
18.2 \\
9.7 \\
10.2 \\
14.6 \\
13.8 \\
10.2 \\
15.5 \\
9.7 \\
11.7 \\
12.9 \\
14.5 \\
8.0\end{array}$ \\
\hline
\end{tabular}


Table 7.5. Summary of diaphragm fragilities.

\begin{tabular}{|c|c|c|c|c|c|c|}
\hline $\begin{array}{c}\text { Column } \\
\text { Line }\end{array}$ & Floor & $\begin{array}{l}\text { Response } \\
\text { Identifier }\end{array}$ & $\begin{array}{l}V_{u} \\
(k)\end{array}$ & $\begin{array}{l}V_{\text {ue }} \\
(k)\end{array}$ & $\beta_{R}$ & Bu \\
\hline $\mathbf{A}$ & El. $820^{\circ}-6^{\prime \prime}$ & X Group 1, Slab 7 & 36,950 & 71,300 & 0.23 & 0.27 \\
\hline J & El. $807^{\prime}-0^{\prime \prime}$ & X Group 2, Slab 6 & 71,040 & 137,000 & - & 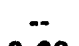 \\
\hline $\mathbf{N}$ & El. $815^{\circ}-0^{\prime \prime}$ & $X$ Group 29, Slab 7 & 7,234 & 12.500 & 0.19 & 0.29 \\
\hline $\mathbf{R}$ & El. $815^{\prime}-0^{\prime \prime}$ & Spring No. 507009 & 65,060 & 126,000 & -- & -- \\
\hline 8.9 & El. $807^{\prime}-0^{\prime}$ & Y Group 7, Slab 6 & 6,448 & 12,400 & - & - \\
\hline 21 & El. $768^{\prime}-0^{\prime \prime}$ & Y Grcisp 8, Slab 14 & 19.870 & 38,400 & $\ddot{10}$ & $\ddot{90}$ \\
\hline 21 & El. 815'-0"/El. 820'-6" & Y Group 8, Slab 7 & 25,300 & 39,200 & 0.15 & 0.26 \\
\hline 24 & El. $731^{\prime}-0^{\prime \prime} / \mathrm{El} .736^{\circ}-6^{\prime \prime}$ & $\begin{array}{l}\text { Flexible Element to } \\
\text { Spring No. } 1011036\end{array}$ & 3,540 & 4,720 & 0 & 0.40 \\
\hline 24 & El. $749^{\circ}-0^{\prime \prime}$ & Spring No. 1011036 & 14.510 & 28,000 & .0 .23 & 0.30 \\
\hline 24 & E]. 815'-0" & Y Group 28, Slab 7 & 1,276 & 2,460 & -- & $-\bullet$ \\
\hline
\end{tabular}

$\mathrm{V}_{\mathrm{u}}=$ Ultimate strength

$V_{\mathrm{ue}}=$ Equivalent elastic capacity 
Table 7.6. Comparison of diaphragm equivalent elastic capacities with elastic loads acceleration range 1 .

\begin{tabular}{|c|c|c|c|c|c|}
\hline $\begin{array}{c}\text { Column } \\
\text { Line }\end{array}$ & Floor & $\begin{array}{l}\text { Response } \\
\text { Identifier }\end{array}$ & $\begin{array}{l}V_{u e} \\
(k)\end{array}$ & $\begin{array}{r}V \\
(k)\end{array}$ & $V_{\text {ud }} \mathbf{V}$ \\
\hline $\begin{array}{l}\text { A } \\
\text { J } \\
N \\
21 \\
21 \\
24\end{array}$ & $\begin{array}{l}\text { El. } 820^{\prime}-6^{\prime \prime} \\
\text { El. } 807^{\prime}-0^{\prime \prime} \\
\text { El. } 815^{\prime}-0^{\prime \prime} \\
\text { El. } 768^{\prime}-0^{\prime \prime} \\
\text { El. } 815^{\prime}-0^{\prime \prime} / \text { El. } 820^{\prime}-6^{\prime \prime} \\
\text { El. } 731^{\prime}-0^{\prime \prime} / \text { El. } 736^{\prime}-6^{\prime \prime}\end{array}$ & $\begin{array}{l}\text { X Group 1, Slab 7 } \\
\text { X Group 2, Slab 6 } \\
\text { X Group 29, Slab } 7 \\
\text { Y Group 8, Slab 14 } \\
\text { Y Group 8, Slab } 7 \\
\text { Flexible element to } \\
\text { Spring No. 1011036 }\end{array}$ & $\begin{array}{r}71,300 \\
137,000 \\
12,500 \\
38,400 \\
39,200 \\
4,720\end{array}$ & $\begin{array}{l}4.552 \\
4.571 \\
1,117 \\
1.761 \\
4,311 \\
392.9\end{array}$ & $\begin{array}{r}15.7 \\
30.0 \\
11.2 \\
21.8 \\
9.1 \\
12.0\end{array}$ \\
\hline
\end{tabular}

$V_{\text {ue }}=$ Equivalent elastic capacity

$\mathrm{V}=$ Elastic load, Acceleration Range 1, Table 5.9 
Table 7.7. Comparison of diaphragm equivalent elastic capacities with elastic loads acceleration range 2 .

\begin{tabular}{|c|c|c|c|c|c|}
\hline $\begin{array}{c}\text { Column } \\
\text { Line }\end{array}$ & Floor & $\begin{array}{l}\text { Response } \\
\text { Identifier }\end{array}$ & $\begin{array}{l}v_{u e} \\
(k)\end{array}$ & $\begin{array}{r}V \\
(k)\end{array}$ & $\mathbf{V}_{\mathbf{u}} \boldsymbol{N} \mathbf{V}$ \\
\hline $\begin{array}{l}A \\
J \\
N \\
21 \\
21 \\
24\end{array}$ & $\begin{array}{l}\text { El. } 820^{\prime}-6^{\prime \prime} \\
\text { El. } 807^{\prime}-0^{\prime \prime} \\
\text { El. } 815^{\prime}-0^{\prime \prime} \\
\text { El. 768'-0" } \\
\text { El. 815'-0"/El. } 820^{\prime}-6^{\prime \prime} \\
\text { El. 731'-0"/El. 736'-6" }\end{array}$ & $\begin{array}{l}\text { X Group 1, Slab 7 } \\
\text { X Group 2, Slab } 6 \\
\text { X Group 29, Slab } 7 \\
\text { Y Group 8, Slab } 14 \\
\text { Y Group 8, Slab } 7 \\
\text { Flexible element to } \\
\text { Spring No. } 1011036\end{array}$ & $\begin{array}{r}71,300 \\
137,000 \\
12,500 \\
38,400 \\
39,200 \\
3,540\end{array}$ & $\begin{array}{l}6,351 \\
6,307 \\
1,557 \\
2,609 \\
6,160 \\
583.6\end{array}$ & $\begin{array}{r}11.2 \\
21.7 \\
8.0 \\
14.7 \\
6.4 \\
6.1\end{array}$ \\
\hline 24 & El. $749^{\prime}-0^{\prime \prime}$ & Spring No. 1011036 & 28,000 & 2,920 & 9.6 \\
\hline
\end{tabular}

$V_{v e}=$ Equivalent elastic capacity

$V^{2}=$ Elastic load, Acceleration Range 2, Table 5.9 
Column Line 24, El. 731'-0"/EI. 736'-6". The capacity of this diaphragm was found to be controlled by shear transfer in the slab across the embedded steel beams. Review of Refs. 7.9 and 7.10 indicates that the ultimate strength of concrete on concrete and concrete on steel interfaces is reached at slip displacements of about 0.02 inches while ultimate slips of up to approximately 0.10 inches can be attained without total loss of load transfer capability. Local yielding of this diaphragm at a slip of 0.02 inches is expected to occur at acceleration levels much lower than those corresponding to yielding of the rest of the structure. However, this slip is considered to be insufficient to result in damage to supported equipment. Instead, equipment damage due to loss of anchorage should be assumed to occur at the onset of significant strength degradation at the ultimate slip of approximately 0.10 inches.

Once the diaphragm yields at a slip of about 0.02 inches, seismic load will be redistributed to the other structural elements. To more accurately account for this load redistribution, this diaphragm was represented in the structure model by a flexible element. This flexible element was assigned the diaphragm's secant stiffness based upon attainment of the ultimate strength at the ultimate slip of approximately 0.10 inches. Comparison of this diaphragm's ultimate strength with the elastic loads predicted by the dynamic analysis indicated that the adjacent major shear walls would be expected to yield prior to development of the ultimate slip. Ductile yielding of the walls can result in diaphragm slips in excess of those predicted by the elastic model for a given acceleration level. To account for this additional nonlinearity, the effective ultimate strength reported in Table 7.5 was developed. This effective strength accounts for the effects of shear wall yielding on the diaphragm slips and provides a more accurate prediction of the seismic levels at which failure of this diaphragm may be expected to occur. It may be somewhat conservative due to certain assumptions and approximations, but the conservatism cannot be eliminated without a more complicated analysis.

As a $m$ nimum, failure of the diaphragm is expected to result in loss of support of cable trays, piping, conduit, etc. that are tied to the underside of the slab. Concrete sapling onto the floor below will be prevented by the metal decking. The diesel generator performance should not be affected unless critical support systems suspended from the diaphragm become dislodged. A site walkdown is necessary to determine the support systems affected if this diaphragm is predicted to fail at earthquake levels of interest. If sufficient diaphragm displacement occurs, the steel floor beams may be torn from their end connections, resulting in potential partial collapse of the floor slab and damage to the diesel generator systems. Evaluation of the displacement at which this occurs can only be determined by more elaborate analysis. This displacement could be taken to be the ultimate diaphragm slip of 0.10 inches, but this assumption may be somewhat conservative. Numerous fans and rack mounted control panels for the fans could lose their anchorage and render ventilation cooling systems inoperable for the following:

HPCS D.G. Fuel Storage Tank Room

HPCS D.G. Room

HPCS Cooling Water Pump Room

HPCS Switchgear and Battery Room

Diesel Generator Room

Diesel Generator Fuel Storage Tank Room 
Table 7.7. Comparison of diaphragm equivalent elastic capacities with elastic loads acceleration range 2 .

\begin{tabular}{|c|c|c|c|c|c|}
\hline $\begin{array}{c}\text { Column } \\
\text { Line }\end{array}$ & Floor & $\begin{array}{l}\text { Response } \\
\text { Identifier } \\
\end{array}$ & $\begin{array}{l}y / x e \\
(k)\end{array}$ & $\begin{array}{r}V \\
(k)\end{array}$ & $v_{w e d v}$ \\
\hline $\begin{array}{l}A \\
J \\
N \\
21 \\
21 \\
24\end{array}$ & $\begin{array}{l}\text { El. 820'-6" } \\
\text { El. 807'-0" } \\
\text { El. } 815^{\prime}-0^{\prime \prime} \\
\text { El. 768'-0" } \\
\text { El. 815'-0"/El. 820'-6" } \\
\text { El. 731'-0"/El. 736'-6" }\end{array}$ & $\begin{array}{l}\text { X Group 1, Slab 7 } \\
\text { X Group 2, Slab } 6 \\
\text { X Group 29, Slab } 7 \\
\text { Y Group 8, Slab } 14 \\
\text { Y Group 8, Slab 7 } \\
\text { Flexible element to } \\
\text { Spring No. } 1011036\end{array}$ & $\begin{array}{r}71,300 \\
137,000 \\
12,500 \\
38,400 \\
39,200 \\
3,540\end{array}$ & $\begin{array}{l}6,351 \\
6,307 \\
1,557 \\
2,609 \\
6,160 \\
583.6\end{array}$ & $\begin{array}{r}11.2 \\
21.7 \\
8.0 \\
14.7 \\
6.4 \\
6.1\end{array}$ \\
\hline 24 & El. $749^{\prime} .0^{\prime \prime}$ & Spring No. 1011036 & 28,000 & 2,920 & 9.6 \\
\hline
\end{tabular}

$V_{v e}=$ Equivalent elastic capacity

$\mathrm{V}=$ Elastic load, Acceleration Range 2, Table 5.9 
Column Line 24, El. 731'-0"/El. 736'-6". The capacity of this diaphragm was found to be controlled by shear transfer in the slab across the embedded steel beams. Review of Refs. 7.9 and 7.10 indicates that the ultimate strength of concrete on concrete and concrete on steel interfaces is reached at slip displacements of about 0.02 inches while ultimate slips of up to approximately 0.10 inches can be attained without total loss of load transfer capability. Local yielding of this diaphragm at a slip of 0.02 inches is expected to occur at acceleration levels much lower than those corresponding to yielding of the rest of the structure. However, this slip is considered to be insufficient to result in damage to supported equipment. Instead, equipment damage due to loss of anchorage should be assumed to occur at the onset of significant strength degradation at the ultimate slip of approximately 0.10 inches.

Once the diaphragm yields at a slip of about 0.02 inches, seismic load will be redistributed to the other structural elements. To more accurately account for this load redistribution, this diaphragm was represented in the structure model by a flexible element. This flexible element was assigned the diaphragm's secant stiffness based upon attainment of the ultimate strength at the ultimate slip of approximately 0.10 inches. Comparison of this diaphragm's ultimate strength with the elastic loads predicted by the dynamic analysis indicated that the adjacent major shear walls would be expected to yield prior to development of the ultimate slip. Ductile yielding of the walls can result in diaphragm slips in excess of those predicted by the elastic model for a given acceleration level. To account for this additional nonlinearity, the effective ultimate strength reported in Table 7.5 was developed. This effective strength accounts for the effects of shear wall yielding on the diaphragm slips and provides a more accurate prediction of the seismic levels at which failure of this diaphragm may be expected to occur. It may be somewhat conservative due to certain assumptions and approximations, but the conservatism cannot be eliminated without a more complicated analysis.

As a minimum, failure of the diaphragm is expected to result in loss of support of cable trays, piping, conduit, etc. that are tied to the underside of the slab. Concrete sapling onto the floor below will be prevented by the metal decking. The diesel generator performance should not be affected unless critical support systems suspended from the diaphragm become dislodged. A site walkdown is necessary to determine the support systems affected if this diaphragm is predicted to fail at earthquake levels of interest. If sufficient diaphragm displacement occurs, the steel floor beams may be torn from their end connections, resulting in potential partial collapse of the floor slab and damage to the diesel generator systems. Evaluation of the displacement at which this occurs can only be determined by more elaborate analysis. This displacement could be taken to be the ultimate diaphragm slip of 0.10 inches, but this assumption may be somewhat conservative. Numerous fans and rack mounted control panels for the fans could lose their anchorage and render ventilation cooling systems inoperable for the following:

HPCS D.G. Fuel Storage Tank Room

HPCS D.G. Room

HPCS Cooling Water Pump Room

HPCS Switchgear and Battery Room

Diesel Generator Room

Diesel Generator Fuel Storage Tank Room 


\section{Service Water Pump Cubicles}

\section{CSCS Equipment Cooling System}

The motor driven reactor feed pump could become dislodged causing a feed water line break.

Column Line 21, El. 815'-0"/El. 820' 6". This failure mode is localized and expected to lead to potential damage to equipment anchored to the portion of slab immediately adjacent to the wall on Column Line 21. No equipment in this location was identified from an inspection of the equipment layout drawings. A site walkdown would be necessary to verify this assumption. Failure of the diaphragm to the wall on Column Lines 9 and 21 would be expected to result in a redistribution of the floor inertial load to the other walls supporting this floor. Because a load redistribution analysis was considered to be beyond the scope of this study and was not performed, the impact of this load redistribution is not exactly known. It is noted from a comparison of Tables 7.3 and 7.4 with Tables 7.6 and 5.7 that this diaphragm would be expected to fail at seismic levels somewhat lower than those for the major shear walls (see Sac. 7.3.1). It is also noted, however, that the capacities of the major walls affected by the load redistribution (i.e., Column Line 15, primary containment) have capacity well in excess of the major walls expected to control. It is therefore expected that failure of this diaphragm would not result in a significant reduction in the capacities of the major walls whose failures could lead to gross structural damage.

Column Line N, EI. 815'-0". The embedded steel beam adjacent to the wall on Column Line $\mathrm{N}$ at El. 815'-0" prevents the continuity of the bottom slab reinforcement into the wall. Diaphragm failure is expected to be localized immediately adjacent to this wall. Only equipment, piping, conduit, etc. anchored to the slab at this localized region would be expected to suffer a loss of anchorage. Review of the equipment layout drawings indicates that the $480 \mathrm{~V}$ switchgear located at the floor below at El. 794'-0" may be damaged due to concrete spalling as a consequence of failure of this diaphragm. However, it is not known if this switchgear is safety-related. Review of the structure indicates that sufficient redundancy exists such that failure of this diaphragm would not be expected to result in a significant reduction in capacity of other structural elements.

Column Line 24, EI. 749'-0". The details of the diaphragm are similar to those at the floor below (see Sec. 7.3.2). The capacity of this diaphragm was based upon a comparison of its median equivalent elastic capacity with its elastic load predicted by the dynamic analysis. A flexible element similar to the one included in the structure model at the floor below was not used. The fragility for this diaphragm may consequently be somewhat conservative. As a result of failure of this diaphragm, cable trays, conduits, piping etc. suspended from the roof of the diesel generator room and anchored into concrete could become dislodged and fail. A site walkdown is necessary to identify the systems supported from the roof if failure of this diaphragm is predicted at earthquake levels of interest.

Column Line A, El. 820'-6". The capacity of this diaphragm reported in Tables 7.5 to 7.7 corresponds to failure of the slab itself between Column Lines A and C. Failure of this diaphragm is expected to result in a loss of anchorage for the SBLC pumps, SBLC storage tanks, and any cable trays, conduit, piping, etc. anchored into the underside of the slab. Equipment mounted on the slab below may be damaged due to concrete spalling. A site walkdown is necessary to identify 
the systems supported below the slab if failure of this diaphragm is predicted for earthquake levels of interest.

\subsubsection{Primary Containment and Internal Structures}

Fragilities were developed for the primary containment and the important components of the internal structures, including the reactor support, sacrificial shield wall, and the stabilizer truss. The capacities of these structural elements were determined using different techniques appropriate for each particular configuration. In general, the resistances against both horizontal shear and moment were determined. Fragilities for the primary containment and the internal structures are presented in Tables 7.8. The median equivalent elastic capacities are compared to the median elastic loads from the appropriate elements of the analysis for acceleration ranges 1 and 2 and presented in Tables 7.9 and 7.10 . 
Table 7.8. Summary of primary containment fragilities.

\begin{tabular}{|c|c|c|c|c|c|c|}
\hline Structure & Load & $\begin{array}{c}\text { Element } \\
\text { No. }\end{array}$ & $\begin{array}{l}\text { Ultimate } \\
\text { Strength } \\
\end{array}$ & $\begin{array}{l}\text { Equivalent } \\
\text { Elastic } \\
\text { Capacity } \\
\end{array}$ & $\mathrm{B}$ & $\beta u$ \\
\hline Containment & Shear & 4 & $121,200 k$ & $234,000 \mathrm{k}$ & 0.23 & 0.30 \\
\hline $\begin{array}{l}\text { Containment } \\
\text { Reactor Support }\end{array}$ & $\begin{array}{l}\text { Moment } \\
\text { Shear }\end{array}$ & $\begin{array}{l}4 \\
15\end{array}$ & $\begin{array}{c}7.94 \times 10^{6} k-f t \\
10,650 k\end{array}$ & $\begin{array}{c}1.53 \times 10^{7} k-f t \\
20,600 k\end{array}$ & $\begin{array}{l}0.23 \\
0.23\end{array}$ & $\begin{array}{l}0.29 \\
0.29\end{array}$ \\
\hline $\begin{array}{l}\text { Reactor Support } \\
\text { Sacrificial Shield Wall } \\
\text { Sacrificial Shield Wall } \\
\text { Stabilizer Truss }\end{array}$ & $\begin{array}{l}\text { Moment } \\
\text { Shear } \\
\text { Moment } \\
\text { Shear }\end{array}$ & $\begin{array}{l}15 \\
40 \\
40 \\
21\end{array}$ & $\begin{array}{c}612,400 k-f t \\
18,560 k \\
278,000 k-f t \\
8,516 k\end{array}$ & $\begin{array}{c}1.18 \times 10^{6} k-f t \\
35,300 k \\
528,000 k-f t \\
16,200 k\end{array}$ & $\begin{array}{c}0.23 \\
-- \\
-- \\
0.22\end{array}$ & $\begin{array}{c}0.27 \\
- \\
0.32\end{array}$ \\
\hline
\end{tabular}


Table 7.9. Comparison of primary containment equivalent elastic capacities with elastic loads acceleration range 1 .

\begin{tabular}{|c|c|c|c|c|c|}
\hline Suucture & Load & $\begin{array}{c}\text { Element } \\
\text { No. } \\
\end{array}$ & $\begin{array}{c}\text { Equivalent } \\
\text { Elastic } \\
\text { Capacity } \\
\end{array}$ & $\begin{array}{l}\text { Elastic } \\
\text { Load }\end{array}$ & $\begin{array}{l}\text { Capacity } \\
\text { Load }\end{array}$ \\
\hline Containment & Shear & 4 & $234,000 \mathrm{k}$ & $7,953 \mathrm{k}$ & 29.4 \\
\hline $\begin{array}{l}\text { Containment } \\
\text { Reactor Support }\end{array}$ & $\begin{array}{l}\text { Moment } \\
\text { Shear }\end{array}$ & $\begin{array}{l}4 \\
15\end{array}$ & $\begin{array}{l}1.53 \times 10^{7} \mathrm{k}-\mathrm{ft} \\
20,600 \mathrm{k}\end{array}$ & $\begin{array}{c}607,500 \mathrm{k}-\mathrm{ft} \\
715.1 \mathrm{k}\end{array}$ & $\begin{array}{l}25.2 \\
28.8\end{array}$ \\
\hline $\begin{array}{l}\text { Reactor Support } \\
\text { Sacrificial Shield Wall } \\
\text { Sacrificial Shield Wall } \\
\text { Stabilizer Truss }\end{array}$ & $\begin{array}{l}\text { Moment } \\
\text { Shear } \\
\text { Moment } \\
\text { Shear }\end{array}$ & $\begin{array}{l}15 \\
40 \\
40 \\
21\end{array}$ & $\begin{array}{c}1.18 \times 10^{6} k-f t \\
35,300 k \\
528,000 k-f t \\
16,200 k\end{array}$ & $\begin{array}{c}59.420 \mathrm{k}-\mathrm{ft} \\
153.6 \mathrm{k} \\
4.759 \mathrm{k}-\mathrm{ft} \\
837.2 \mathrm{k}\end{array}$ & $\begin{array}{l}19.9 \\
>30 \\
>30 \\
19.4\end{array}$ \\
\hline
\end{tabular}


Table 7.10. Comparison of primary containment equivalent elastic capacities with elastic loads acceleration range 2.

\begin{tabular}{lccccc} 
Structure & Load & $\begin{array}{c}\text { Element } \\
\text { No. }\end{array}$ & $\begin{array}{c}\text { Equivalent } \\
\text { Elastic } \\
\text { Capacity }\end{array}$ & $\begin{array}{c}\text { Elastic } \\
\text { Load }\end{array}$ & $\begin{array}{c}\text { Capraity } \\
\text { Load }\end{array}$ \\
\hline Containment & Shear & 4 & $234,000 \mathrm{k}$ & $12,490 \mathrm{k}$ & 18.7 \\
Containment & Moment & 4 & $1.53 \times 10^{7} \mathrm{k}-\mathrm{ft}$ & $941.900 \mathrm{k}-\mathrm{ft}$ & 16.2 \\
Reactor Support & Shear & 15 & $20,600 \mathrm{k}$ & $1,068 \mathrm{k}$ & 19.3 \\
Reactor Support & Moment & 15 & $1.18 \times 10^{6} \mathrm{k}-\mathrm{ft}$ & $93,450 \mathrm{k}-\mathrm{ft}$ & 12.6 \\
Sacrificial Shield Wall & Shear & 40 & $35,300 \mathrm{k}$ & $186.9 \mathrm{k}$ & $>30$ \\
Sacrificial Shield Wall & Moment & 40 & $528,000 \mathrm{k}-\mathrm{ft}$ & $5,003 \mathrm{k}-\mathrm{ft}$ & $>30$ \\
Stabilizer Truss & Shear & 21 & $16,200 \mathrm{k}$ & $1,172 \mathrm{k}$ & 13.8
\end{tabular}


Primary Containment. Considerable amount of testing has been conducted in Japan on scale models of reinforced and prestressed concrete containment structures. The purpose of this testing was to determine the resistances of containment structures against seismic loads with and without internal pressure. The shear strength of the primary containment was based upon the results of testing summarized in Ref. 7.12. The use of these test results is appropriate since they directly incorporate many of the parameters influencing the containment shear strength. The resistance against the moment due to horizontal seismic response was determined by applying the basic flexural strength design principles of $\mathrm{ACI} 349-80^{[7.13]}$ to the primary containment structure as a whole. The validity of this approach was confirmed by comparison to the results of scale model testing reported in Ref. 7.14.

Failure of the primary containment is expected to result in a breech of the containment boundary. This failure is expected to occur at the lower elevations of the containment. The ratios between median capacity and median load for acceleration ranges 1 and 2 are shown in Tables 7.9 and 7.10.

Internal Structures. The major components of the internal structures for which fragilities were developed were the reactor support, the sacrificial shield wall, and the stabilizer truss. The resistances of these various structures to seismic load were determined using techniques appropriate for each particular structural configuration. The reactor support is a thick-walled reinforced concrete cylinder. The horizontal shear strength was based upon the results of testing conducted on these types of structures reported in Ref. 7.15. The flexural strength of the reactor support was determined using the same approach developed for the primary containment. The details between the reactor support and the base mat were found not to be controlling.

Shear failure of the sacrificial shield wall was found to be controlled by yielding of the steel shell that provides the lateral load resistance. The flexural strength of the sacrificial shield wall was found to be controlled by the ability of the connection to the reactor support to transmit uplift force resulting from the seismic moment. It should be noted that the large ratios of capacity to load for the sacrificial shield wall can be attributed to the relatively low seismic loads acting on this structure. The stabilizer truss between the sacrificial shield wall and the primary containment was found to fail due to yielding of the pipe braces. A compilation of test results presented in Ref. 7.16 shows that short, stubby steel members, such as these braces, are capable of developing compressive stresses in excess of yield. Reference 7.16 postulates that this may be due to strain hardening and a compressive yield stress greater than the tensile yield stress. Reference 7.17 confirms the high local buckling stress for steel tubes and indicates that large strain ductilities are possible. Failure of the internal structures is expected to result in RPV damage and simultaneous rupture of the steam, feedwater, and recirculation lines.

\subsection{Condensate Storage Tank}

The condensate storage tank is a field erected water tank with approximately 350,000 gallon capacity. The tank is approximately $24 \mathrm{ft}$. high with a $25^{\prime}-3^{\prime \prime}$ radius. The tank is fabricated from 6061-T6 aluminum plate with thicknesses of $1 / 4$ and 3/8 inches. No anchor bolts are provided. Due to the lack of anchor bolts, uplift forces in the side wall due to overturning moment are resisted by fluid pressure on the base plate. Out-of-plane flexural yielding of the base plate is followed by compressive buckling of the side wall. Failure of the plate weld with subsequent loss of the tank contents is assumed to occur upon buckling. The median ground acceleration capacity for this mode of failure is expected to be aproximately $0.34 \mathrm{~g}$. 


\section{Section 8. Equipment Fragility}

Fragilities for selected LaSalle components were derived by extrapolating design information. The equipment for which fragilities were derived constitute the important components of the safe shutdown and accident mitigation systems. Fragilities are defined as a conditional probability of failure vs. a structural response parameter. For the LaSalle components, the response parameter is spectral acceleration defined at the equipment fundamental frequency and at a stated damping value. This definition is identical to that of the SSMRP fragility study..$^{(8.1)}$ Failure is defined as the response level at which the component will no longer perform its intended function. This might be trip of a circuit breaker, failure of equipment anchorage or pressure boundary failure. In some cases, permanent structural deformation will take place at levels substantially below the failure threshold.

Design and seismic qualitication information was provided by Sargent and Lundy, the plant $\mathrm{A} E$, in the form of Seismic Qualification Review Team (SQRT) summaries. Microfiche cards containing mnre detailed information on equipment qualitication were also provided and were used where necessary to supplement the information contained in SQRT summaries.

Fragilities are assumed to fit a lognormal distribution and are defined by a median spectral acceleration capacity $S_{a}$ and two variables $\beta_{R}$ and $\beta_{U}$ defined as the logarithmic standard deviations representing randomness and uncertainty about the median. A complete description of the lognormal representations of fragility curves is contained in Ref. 8.2 and is not repeated here.

BWRs have suppression pool generated hydrodynamic loading events that affect the dynamic input environment for components mounted in affected areas of the containment and reactor building. The effects ot these hydrodynamic loads have not been incorporated into the fragility descriptions contained here.

\subsection{Methodology}

The general methodology applied in development of fragility descriptions was to define the overall factor of satety, F, between the best estimate of equipment response to a reference earthquake, usually the safe shutdown earthquake (SSE), and the best estimate of the actual capacity. This factor of safety multiplied times the spectral accelerations for the SSE at the equipment mounting location defines the overall capacity or median value of the fragility.

$$
\hat{S}_{\mathrm{a}}=\hat{F} \cdot S_{a_{\text {SSE }}}
$$

where

$$
\hat{S}_{\mathrm{a}}=\text { the median spectral acceleration capacity }
$$




$$
\begin{aligned}
& S_{\text {2SE }}=\text { the spectral acceleration at the SSE level } \\
& \hat{F}=\text { the median factor of safety }
\end{aligned}
$$

\subsubsection{Equipment Qualified by Analysis}

For equipment qualified by analysis the following methodology was applied. The factor of safety, $F$, is composed of three factors. $F_{c}$ represents the margin on strength between design and actual capacity. $F_{\mu}$ is a factor accounting for inelastic energy absorption. $F_{R E}$ is a factor that defines the conservatism in the computed design response of the component. Each factor is a random variable and assumed to be lognormally distributed.

$$
\hat{F}=\hat{F}_{c} \cdot \hat{F}_{\mu} \cdot \hat{F}_{\mathrm{RE}}
$$

\section{Capacity Factor}

The capacity factor $\left(F_{c}\right)$ is computed from

$$
F_{c}=\frac{P_{c}-P_{N}}{P_{S S E}}
$$

where

$$
\begin{aligned}
& P_{c}=\text { the limit load or stress which results in gross yielding } \\
& P_{N}=\text { the normally applied load or stress } \\
& P_{S S E}=\text { the design seismic load or stress for the SSE }
\end{aligned}
$$

Note that the hydrodynamic loading could be applied in equation 8-3 and assumed to reduce the capacity of the component. In the LaSalle analysis, the effect of hydrodynamic loads is applied to the response and is discussed in Sec. 6.

Inelastic Energy Absorption Factor. The inelastic energy absorption (ductility) factor $\left(F_{\mu}\right)$ is applicable to ductile equipment that fails in a structural mode. Section 7.2.2 describes the 
methodology behind the inelastic energy absorption factor and the governing equations. For equipment, the equations presented for structures are assumed to be reasonably applicable. Equipment frequencies are typically higher than for the supporting structures and are in the amplified acceleration or rigid range. For brittle failure modes, a ductility factor is not applicable. The capacity, $P_{c}$, in Eq. 8-3 is based on the ultimate strength of the element instead of the limit or yield strength. For functional failure modes evaluated by analysis, the inelastic energy absorption factor is also not applicable. The capacity, $\mathrm{P}_{\mathrm{c}}$, represents the functional limit of the component.

Equipment Response Factor. The equipment response factor ( $F_{R E}$ ) is comprised of the product of several factors representing important variables in the equipment response analyses. Each factor is a random variable.

$$
F_{R E}=F_{Q M} F_{M E} F_{D} F_{M C} F_{E C C}
$$

These factors and their variability are described in detail in Refs. 8.1 and 8.2 and are only briefly redefined here. FQM is the measure of conservatism or unconservatism and the uncertainty in
the method of qualification.

FME is the measure of conservatism or unconservatism and the uncertainty in the modelling assumptions (modeling error)

$F_{D} \quad$ is the measure of conservatism and uncertainty in design damping

$\mathrm{F}_{\mathrm{MC}} \quad$ is a measure of the deviation from median response due to the method used for combining modal responses

FECC represents the deviation from median response for the method of combining earthquake component responses.

The qualification method factor, $\mathrm{F}_{\mathrm{QM}}$, is only used if analysis methods other than a dynamic analysis were used. If a static coefficient method was used the factor is the ratio of the static coefficient to the design SSE spectral acceleration at the equipment fundamental frequency. The uncertainty in FQM reflects the fact that the factor derivation is not exact due to multimode response or other parameters that reflect the uncertainty in static analysis. The modeling error factor, $\mathrm{F}_{\mathrm{ME}}$, is usually assumed to be unity if the analyst has conducted a median centered analysis. The uncertainty in the factor reflects the estimated error in response that could occur from imprecise boundary condition modeling, approximate modeling of the structural elements, weight and elastic modulus error, etc. A complex component is assigned a greater coefficient of variation (approximate logarithmic standard deviation) on response than a simple component. The 
uncertainty, $\beta \mathrm{U}$ is typically 0.15 for a moderately complex component and 0.1 or less for a simple component.

The damping factor, $\mathrm{FD}_{\mathrm{D}}$ is taken as unity regardless of the design damping values since the reference spectral acceleration, $S_{\text {ase }}$ in Eq. (8-1) is the spectral acceleration input for design. The response spectra to be compared to $S_{a}$ in Eq. (8-1) are based on median damping for the component and the variability due to damping uncertainty is contained within the variability on spectral acceleration generated from the structural analysis.

The mode combination factor, FMC. is in all cases taken as unity as the method of combining modal responses for LaSalle components was considered median centered. The random variability, $\beta_{R}$ was estimated to be about 0.15 for multimode response. For single degree-of-freedom systems, the random variability due to mode combination is zero by definition.

The earthquake component combinations factor, FECC reflects the fact that the median spectral acceleration is referenced to a single direction of earthquake input whereas the component failure mode could be influenced by more than one input direction. In the design of LaSalle components, the response to the worst horizontal direction component was combined by absolute sum with the response for the vertical component. This criterion was compared to median response phasing developed in previous generic studies for idealized structural shapes with and without cross coupling of response. Table 8.1 lists the factors and their random and uncertainty variability representing random response due to earthquake phasing and uncertainty in response due to coupling.

Since the fragility descriptions are referenced to a single horizontal component, they correspond to original design criteria and the factors developed for the design case are applicable to the derived fragility descriptions.

\subsubsection{Equipment Qualified by Test}

Qualification test data cannot directly be extrapolated to equipment capacity. Therefore, for equipment qualified by test, the fragility analyst must draw upon generic data to estimate the threshold of failure and use the test level to establish the tails of the fragility curves. In instances where equipment is tested to a high level, it can conservatively be assumed without compromising risk analysis results that the functional failure capacity is some modest level (about 25 percent) above the achieved test level with the test level defining the lower tail of the fragility curves.

Conservative strength for ductile failure modes may also be estimated from achieved test levels. If the test report indicates no evidence of gross yielding, the actual capacity may be estimated to be equal to or greater than the test level times an appropriate inelastic energv absorption factor, $F_{\mu}$. Alternately, the capacity may be estimated as equal to or greater than the achieved test level times the ratio of ultimate strength to yield strength. This is often done for anchor bolt or attachment bolt 
Table 8.1. Earthquake component combination factors.

\begin{tabular}{|c|c|c|c|c|}
\hline & Case & Description & $\beta_{\mathbf{R}}$ & $\beta_{U}$ \\
\hline 1 & $3 \mathrm{D}$ & 3 directional components contribute to failure ......1.09 & 0.12 & 0.01 \\
\hline 2 & 2D & $\begin{array}{l}\text { Median coupling: both horizontal components } \\
\text { contribute to failure }\end{array}$ & 0.10 & 0.04 \\
\hline 3 & 2D & $\begin{array}{l}\text { No coupling: both horizontal components } \\
\text { contribute to failure }\end{array}$ & 0.06 & 0.00 \\
\hline 4 & $2 \mathrm{D}$ & $\begin{array}{l}\text { Median coupling: } 1 \text { horizontal and the vertical } \\
\text { components contribute to failure .....................1.12 }\end{array}$ & 0.09 & 0.03 \\
\hline 5 & 2D & $\begin{array}{l}\text { No coupling: } 1 \text { horizontal and the vertical } \\
\text { components contribute to failure }\end{array}$ & 0.03 & 0.00 \\
\hline 6 & ID & $\begin{array}{l}\text { Any one of the directional components } \\
\text { alone is responsible for the failure .... }\end{array}$ & 0.00 & 0.00 \\
\hline
\end{tabular}


failure modes. Again, the test levels are considered to define the lower tail of the fragility curves. The achieved test level is estimated to represent an acceleration level where there is 95 percent confidence that there is less than 5 percent probability of failure.

The estimation of median capacities from test results is almost always conservative, but in the absence of fragility data or analysis, it is the only reasonable method to establish fragility descriptions.

Recent effort by the Seismic Qualification Utility Group (SQUG) has documented earthquake experience data for several major classes of equipment. These data are very useful in establishing the lower bounds on failure. The data are referenced to peak ground acceleration and to use the data for the SSMRP approach, they must be converted to estimates of spectral acceleration at the component level. Since the commercial facilities from which the data were obtained did not have a seismic analysis, the estimates contain a great deal of uncertainty but are useful to establish a range at which seismic failures are not expected to occur.

The treatment of response factors and uncertainties for qualification testing is covered in detail in Refs. 8.1 and 8.2 and are not repeated here.

\subsubsection{Hydrodynamic Loads}

Hydrodynamic loading from discharge of steam into the suppression pool is transmitted into the containment and reactor buildings. Loading cases are defined for several combinations of safety relief valve discharge and for condensate oscillation and chugging. The condensate oscillation and chugging loads result from pipe breaks whereas the SRV discharge loading may occur from pressure transients resulting from a loss of off-site power with a resulting turbine trip.

In the design process, hydrodynamic loads are combined with seismic, normal operating and other transient loading conditions and compared to code allowables. For purposes of developing component fragilities it was assumed that simultaneous random pipe break induced loading and earthquake loading was of such low probability that consideration of the combination was not credible in risk results. On the other hand, SRV discharge loads are assumed to occur simultaneously with a strong motion earthquake. The phasing of seismic and SRV responses is random and for the LaSalle design both absolute sum and SRSS response combinations were examined. The load combination methodology was examined based upon SRV test data from LaSalle. See Sec. 6 for details.

\subsection{Results}

Table 8.2 lists our results. The first column, "Cat," gives the category number assigned to that type of component. A "--" in that column indicates that is another mode of failure for that component. They were not used in the quantitative process. The component description, location, and appropriate node number from the structural model are also listed. The component fundamental frequency as determined from the qualification reports are listed. The spectral acceleration from the response analysis should be compared to the median spectral acceleration capacity, $S_{a}$, at the fundamental frequency listed. 
It is estimated that 5 percent damping is median although design damping varied from 1 percent to 4 percent. The median spectral acceleration from the response analysis in-structure response spectra used for comparison to median capacity should be the 5 percent damped spectral acceleration.

The governing failure mode for the component is listed. For electrical equipment containing relays, two values are given. One value is for relay chatter which is a recoverable failure mode and one is for a nonrecoverable failure mode. Consequences of relay chatter are not known. The chatter fragility is provided for the systems analysts to consider in their treatment of operator error, etc. The median spectral acceleration capacity, $S_{a}$, and the logarithmic standard deviations, $\beta_{R}$ and,$\beta U$ representing randomness and uncertainty are provided.

The notes identified by letters $a, b$, c denote the earthquake component direction for which the fragility is referenced. Numerically numbered notes offer additional information regarding the failure which the systems analyst may wish to consider.

When determining the appropriate fragility category to use for a specific component on the fault trees, the analyst first received the components listed in Table 8.2. If the component of interest was not contained in Table 8.2 (LaSalle specific components), then a generic database was used to determine the appropriate fragility. That generic database is provided in Table 8.3. 
Table 8.2. LaSalle specific equipment fragilities

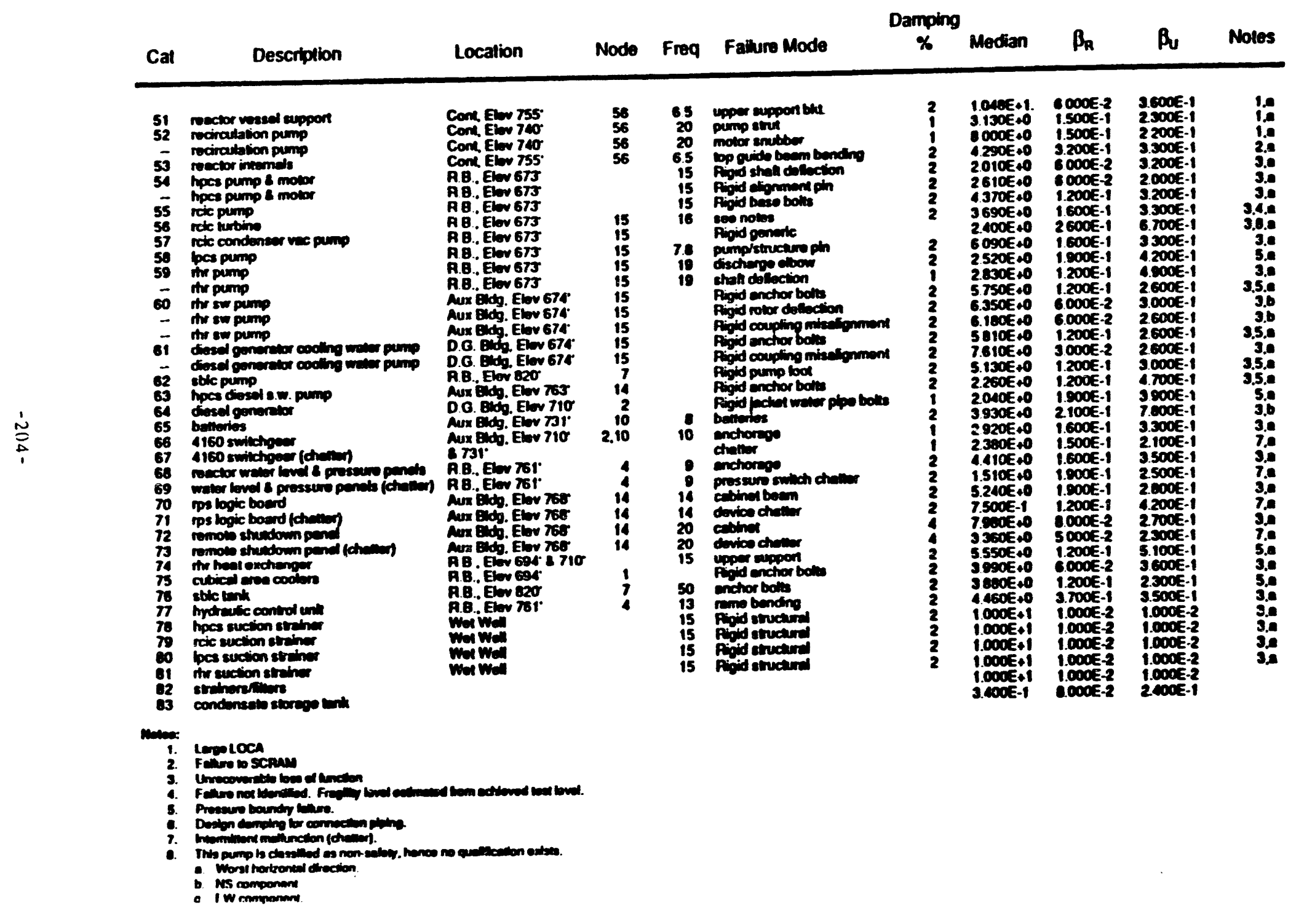


Table 8.3. Generic seismic equipment fragilities.

\begin{tabular}{|c|c|c|c|c|c|}
\hline Case & Description & Freq & Median & $\beta_{R}$ & $\beta_{U}$ \\
\hline $\begin{array}{l}1 \\
2 \\
3 \\
4 \\
5 \\
6 \\
7 \\
8 \\
9 \\
10 \\
11 \\
12 \\
13 \\
14 \\
15 \\
16 \\
17 \\
18 \\
20 \\
22 \\
23 \\
24 \\
25 \\
26 \\
27 \\
28 \\
29 \\
30 \\
31 \\
32 \\
33 \\
34 \\
35 \\
36 \\
37 \\
38 \\
39 \\
40 \\
41 \\
42 \\
49 \\
50\end{array}$ & 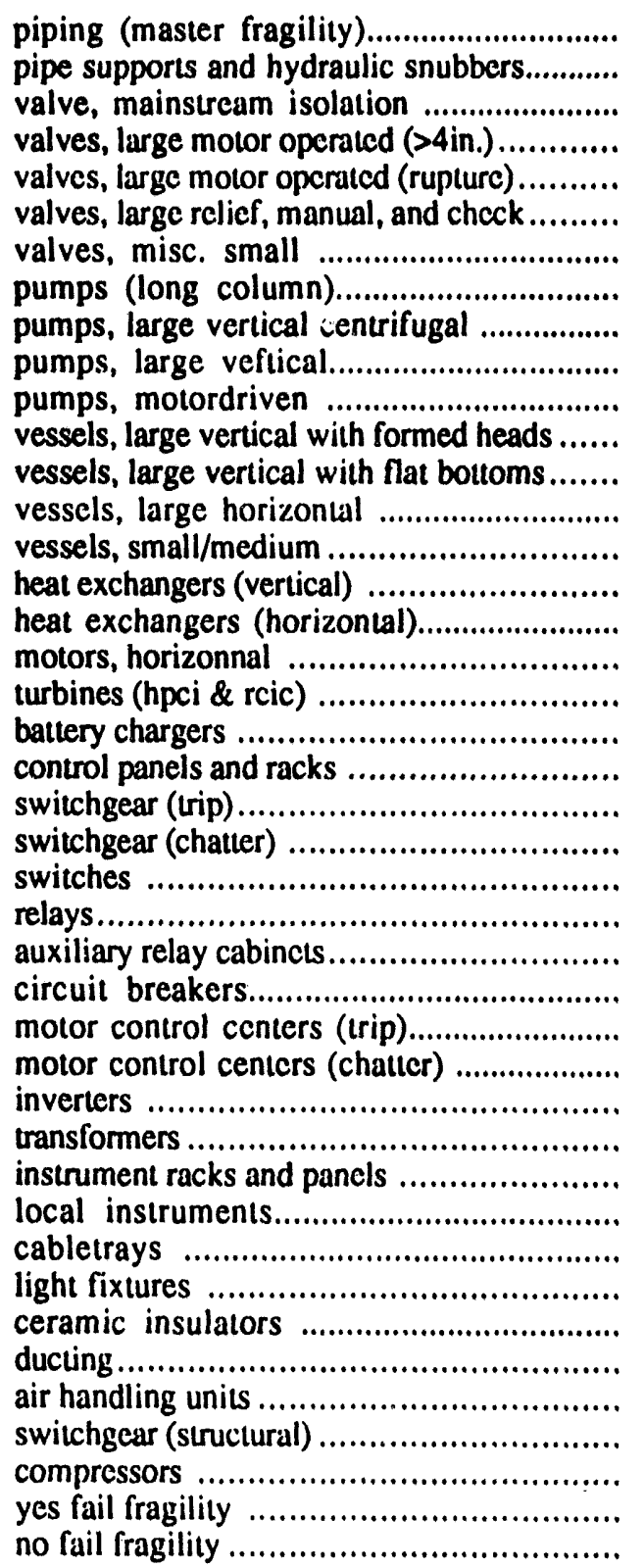 & $\begin{array}{l}\text { 5-20 } \\
\text { 5-20p } \\
\text { 5-20p } \\
5-20 \mathrm{p} \\
\text { 7-Rig } \\
\text { 7-Rig } \\
\text { 7-Rig } \\
\text { 3-Rig } \\
\text { 5-15 } \\
\text { 8-Rig } \\
22-\text { Rig } \\
\text { 6-Rig } \\
14-\text { Rig } \\
\\
\text { 15-Rig } \\
\text { 5-20 } \\
\\
\text { 4-10 } \\
4-10 \\
13-20 \\
2-20 \\
2-20 \\
2-20 \\
4-10 \\
4-18 \\
8-14 \\
5-20 \\
\text { Rigid }\end{array}$ & $\begin{array}{l}2.44000 \mathrm{E}+6 \\
1.46000 \mathrm{E}+0 \\
4.41741 \mathrm{E}+0 \\
6.69610 \mathrm{E}+0 \\
1.44000 \mathrm{E}+1 \\
7.63683 \mathrm{E}+0 \\
2.81032 \mathrm{E}+0 \\
1.45658 \mathrm{E}+0 \\
2.21000 \mathrm{E}+0 \\
1.89376 \mathrm{E}+0 \\
1.85158 \mathrm{E}+0 \\
1.92759 \mathrm{E}+0 \\
2.86933 \mathrm{E}-1 \\
1.92564 \mathrm{E}+0 \\
1.17977 \mathrm{E}+1 \\
1.50152 \mathrm{E}+0 \\
1.98124 \mathrm{E}+0 \\
1.21000 \mathrm{E}+1 \\
4.05212 \mathrm{E}+0 \\
2.15108 \mathrm{E}+0 \\
1.15000 \mathrm{E}+1 \\
4.81133 \mathrm{E}+0 \\
3.30862 \mathrm{E}+0 \\
1.61864 \mathrm{E}+0 \\
2.54136 \mathrm{E}+0 \\
4.72920 \mathrm{E}+0 \\
6.24489 \mathrm{E}+0 \\
6.84663 \mathrm{E}+0 \\
2.74608 \mathrm{E}+0 \\
1.51913 \mathrm{E}+0 \\
6.18011 \mathrm{E}+0 \\
1.15000 \mathrm{E}+0 \\
7.64216 \mathrm{E}+0 \\
2.23000 \mathrm{E}+0 \\
9.20000 \mathrm{E}+0 \\
2.07220 \mathrm{E}-1 \\
3.97000 \mathrm{E}+0 \\
3.74912 \mathrm{E}+0 \\
2.39431 \mathrm{E}+0 \\
2.21625 \mathrm{E}+0 \\
0.00000 \mathrm{E}+0 \\
5.00000 \mathrm{E}+1\end{array}$ & $\begin{array}{l}1.80000 \mathrm{E}-1 \\
2.20000 \mathrm{E}-1 \\
2.40000 \mathrm{E}-1 \\
2.10000 \mathrm{E}-1 \\
2.80000 \mathrm{E}-1 \\
2.70000 \mathrm{E}-1 \\
1.90000 \mathrm{E}-1 \\
1.70000 \mathrm{E}-1 \\
2.20000 \mathrm{E}-1 \\
2.10000 \mathrm{E}-1 \\
1.50000 \mathrm{E}-1 \\
1.50000 \mathrm{E}-1 \\
2.60000 \mathrm{E}-1 \\
1.90000 \mathrm{E}-1 \\
1.90000 \mathrm{E}-1 \\
2.10000 \mathrm{E}-1 \\
1.6000 \mathrm{E}-1 \\
2.70000 \mathrm{E}-1 \\
1.90000 \mathrm{E}-1 \\
2.40000 \mathrm{E}-1 \\
4.80000 \mathrm{E}-1 \\
2.10000 \mathrm{E}-1 \\
1.70000 \mathrm{E}-1 \\
2.50000 \mathrm{E}-1 \\
1.90000 \mathrm{E}-1 \\
2.10000 \mathrm{E}-1 \\
2.50000 \mathrm{E}-1 \\
2.10000 \mathrm{E}-1 \\
1.60000 \mathrm{E}-1 \\
1.40000 \mathrm{E}-1 \\
2.60000 \mathrm{E}-1 \\
4.80000 \mathrm{E}-1 \\
3.40000 \mathrm{E}-1 \\
3.40000 \mathrm{E}-1 \\
1.40000 \mathrm{E}-1 \\
3.00000 \mathrm{E}-1 \\
2.90000 \mathrm{E}-1 \\
2.50000 \mathrm{E}-1 \\
2.40000 \mathrm{E}-1 \\
1.20000 \mathrm{E}-1 \\
0.00000 \mathrm{E}+0 \\
1.0000 \mathrm{E}-1\end{array}$ & $\begin{array}{l}3.30000 \mathrm{E}-1 \\
4.90000 \mathrm{E}-1 \\
4.00000 \mathrm{E}-1 \\
5.00000 \mathrm{E}-1 \\
5.60000 \mathrm{E}-1 \\
1.00000 \mathrm{E}-1 \\
5.10000 \mathrm{E}-1 \\
5.60000 \mathrm{E}-1 \\
3.20000 \mathrm{E}-1 \\
4.50000 \mathrm{E}-1 \\
4.10000 \mathrm{E}-1 \\
5.10000 \mathrm{E}-1 \\
4.50000 \mathrm{E}-1 \\
4.50000 \mathrm{E}-1 \\
3.70000 \mathrm{E}-1 \\
4.00000 \mathrm{E}-1 \\
6.3000 \mathrm{E}-1 \\
3.10000 \mathrm{E}-1 \\
2.30000 \mathrm{E}-1 \\
4.40000 \mathrm{E}-1 \\
7.40000 \mathrm{E}-1 \\
2.90000 \mathrm{E}-1 \\
3.70000 \mathrm{E}-1 \\
5.90000 \mathrm{E}-1 \\
5.00000 \mathrm{E}-1 \\
6.00000 \mathrm{E}-2 \\
4.30000 \mathrm{E}-1 \\
6.00000 \mathrm{E}-2 \\
4.60000 \mathrm{E}-1 \\
2.80000 \mathrm{E}-1 \\
3.50000 \mathrm{E}-1 \\
6.60000 \mathrm{E}-1 \\
3.30000 \mathrm{E}-1 \\
1.90000 \mathrm{E}-1 \\
1.40000 \mathrm{E}-1 \\
3.70000 \mathrm{E}-1 \\
4.60000 \mathrm{E}-1 \\
6.10000 \mathrm{E}-1 \\
7.10000 \mathrm{E}-1 \\
1.90000 \mathrm{E}-1 \\
0.00000 \mathrm{E}+0 \\
1.00000 \mathrm{E}-1\end{array}$ \\
\hline
\end{tabular}




\section{Section 9. Plant Logic Models}

\subsection{Initiating Events}

The classes of seismic initiating events which were considered for LaSalle are essentially the same as those in the RMII:P|l al analysis. These classes are:

(1) Reactor Vessel Rupture (RVR) and equivalent events

(2) Large l oss-of-Conlant Accident (Large-1.OCA)

(3) Medium Loss-t Coolant Accident (Medium-LOCA)

(4) Small Loss-ol-Coolant Accident (Small-LOCA)

(5) Transients (1.OSP)

The fault tree molels of the safiely systems included both random and seismically induced failures. However, only saismically induced litilures are included in the definition of the initiating events. This ensures that the cilculitied core damage frepuency is a full measure of, and only of, the seismic increment to risk. In the following sections there will be a discussion of each initiating event class. Table 9.1 lists the number of cut sets used in the analysis for each initiating event and Table 9.2 lists the occurrence probibilities over the range of earthquakes considered for each initiating event.

\subsubsection{Reactor Vessel Rupture}

An RVR is any loss-of-coolant accidem which is too large to be mitigated by the emergency core cooling systems. Such events as a rupture of the reactor pressure vessel or multiple primary system pipe ruptures can produce a coolant leak with a greater rate than the ECCS has capacity to overcome. In addition, other events cinn occur (RVR equivalent events) which lead directly to core damage.

The events which were considered to be in the RVR class of initiators are:

1) reactor pressure vessel rupture,

2) multiple pipe ruptures eciuivalent to vessel rupture conlant loss,

3) interfacing LOCAs, and

4) total loss of reactor water level indication.

The most probable meins of occurrence of item 1, vessel rupture, was seismic failure of the vessel supports. Calculations indicated, however, that this failure has a negligible probability occurrence. Sinilarly, item 2 was modeled as failure of the support struts to both of the recirculation pumps 
and was found to have a small probability of occurrence.

Item 3, interfacing LOCAs, occur when the high pressure piping communicates with the low pressure piping (outside containment) due to check valve and/or MOV failures. It was assumed in the Reactor Safety Study ${ }^{(9.1)}$ that interfacing LOCAs cause core melt. ECCS is not designed to mitigate the effects of LOCAs outside containment. Since, for LaSalle, this event requires the failure of two or more check valves and/or MOVs, interfacing LOCAs were found to have a negligible occurrence probability in the seismic analysis.

Finally, our analysis showed that seismically induced total loss of all reactor water level indication had a small probability of occurrence. Thus, all four mechanisms for experiencing an RVR or equivalent event had insignificant probabilities of occurrence. Consequently, no significant seismic risk is attributed to LaSalle from this class of initiating events.

\subsubsection{Large-LOCA}

The Large-LOCA event is defined as any reactor coolant loop rupture, either steam or liquid, with area equal to or greater than .3 square feet. The Large-LOCA initiator was modeled as failure of either of the recirculation pump support struts, failure of 3 or more Safety Relief 'Valves (SRVs) to reclose or failure of a large piping segment. Table 9.3 lists all the piping segments which, if ruptured, would contribute to a Large-LOCA.

\subsubsection{Medium-LOCA}

The Medium-LOCA event is defined as a reactor coolant loop liquid piping rupture with area between .005 and .3 square feet or reactor coolant loop steam piping rupture with area between .1 and 3 square feet. A Medium-LOCA may also be caused by failure of two Safety Relief Valves to reclose. Table 9.4 lists all the piping segments which, if ruptured, would contribute is a MediumLOCA.

\subsubsection{Small-LOCA}

A Small-LOCA is a reactor coolant loop liquid piping rupture of .005 square feet or less, or a steam piping rupture of .1 square feet or less. A Small-LOCA may also be caused by the failure of one Safety Relief Valve to reclose. Table 9.5 lists all the piping segments which, if ruptured, would contribute to a Small-LOCA.

\subsubsection{Transient}

A transient is an abnormal condition at the plant which forces the shutdown of the nuclear reactor. There are several events, including loss-of-offsite power, turbine trip, loss-of-condenser vacuum, etc., which qualify as transient initiating events. Like LOCAs, transient events are planned for in the design of the power plant. However, because of failures in the safety systems to respond as designed, even such highly anticipated events as a loss-of-offsite power have the potential to result in reactor core damage. 
Table 9.1. Number of initiating event cut sets.

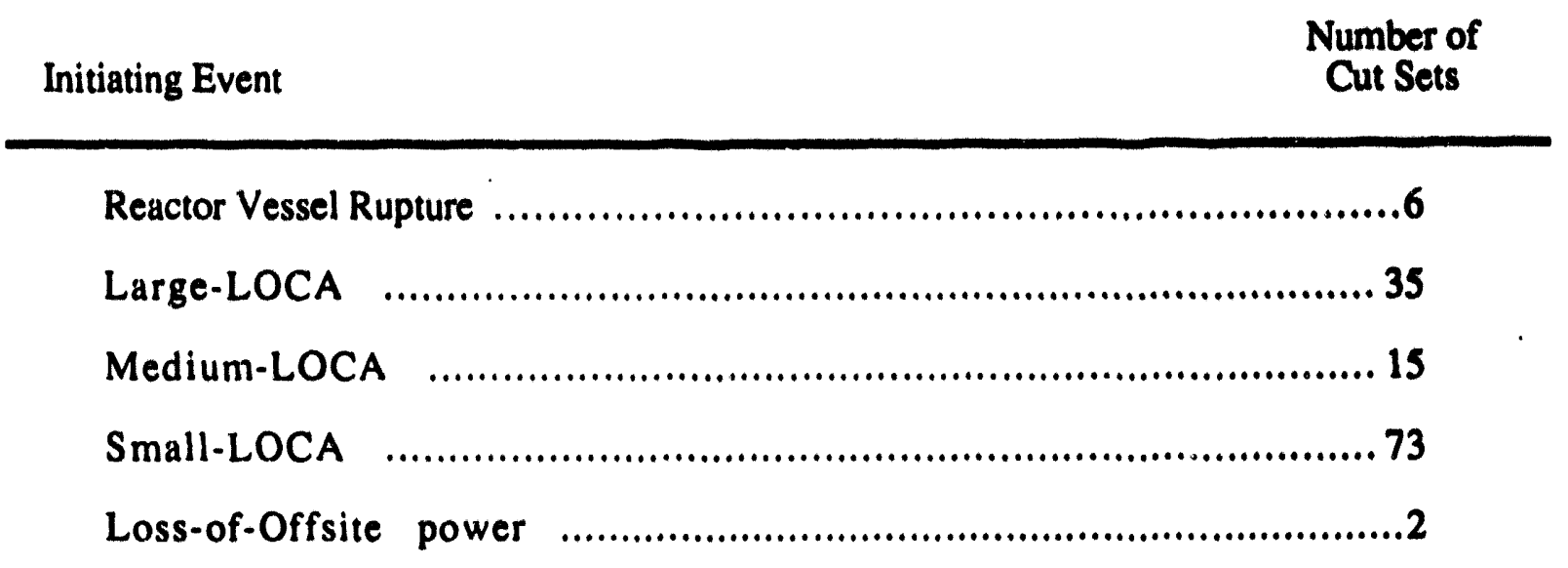


Table 9.2. Probability of initiating events.

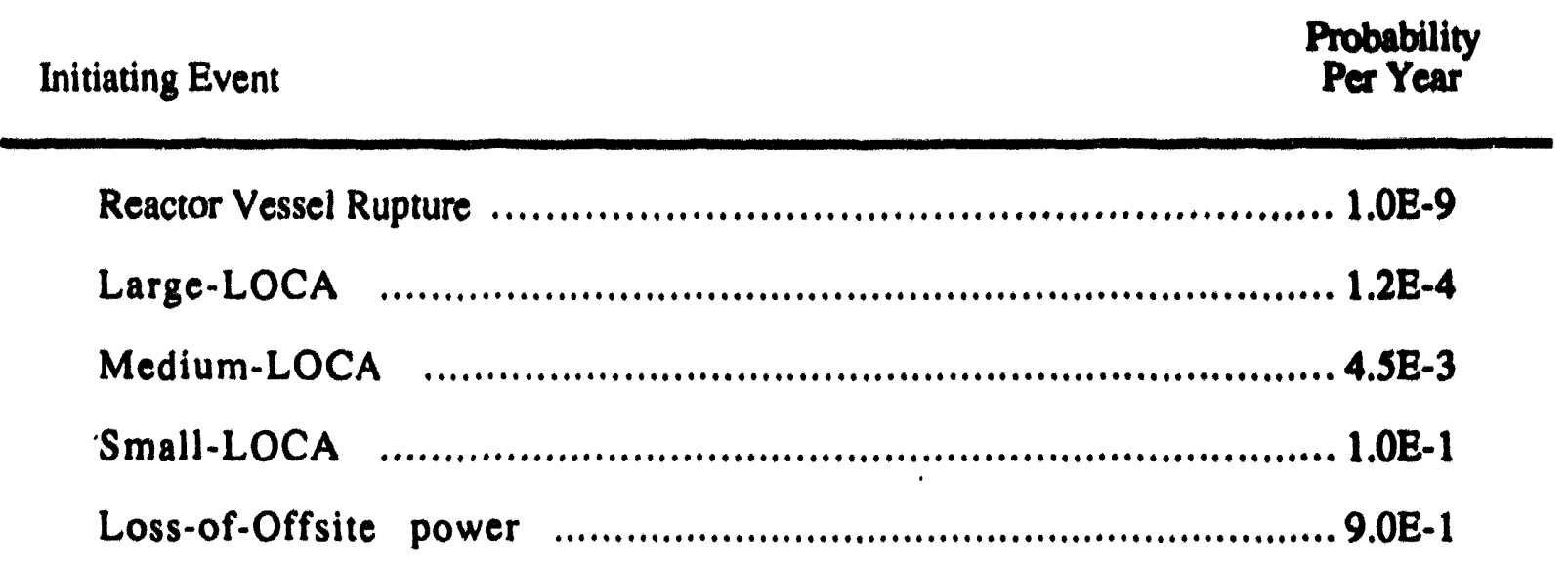


The assumption made during the seismic analysis of LaSalle is that, during a seismic event in the range we are considering, there will be a loss-of-offsite power. This assumption has been made both because of the uncertainty in maintaining an offsite power supply at the plant during an earthquake and because this assumption results in several simplifications in our analysis, which is, after all, a demonstration of a simplified seismic risk methodology. Offsite power is dependent on a series of transmission lines, towers, insulators, etc. over which the utility may have limited control or concern and for which we have very little available data. Further, a relatively low intensity earthquake at the plant site may be due to an earthquake whose epicentral ground motion is of a much higher intensity (i.e., the plant is not near the epicenter). Consequently, even at low plant site accelerations, the probability of offsite power loss may be high because the power grid near the epicenter is experiencing much higher accelerations than the plant.

Since a loss-of-offsite power transient subsumes the other transient initiating events, one need only examine the loss-of-offsite power event in our transient analysis. However, note that, although a number of component failures have been ruled out as transient initiators, we continue to consider them as enabling events for accident progression.

The assumption of a loss-of-offsite power, and the further assumption that offsite power will not be restored for the duration of the reactor shutdown period, has a significant impact on the results. It would be desirable to make a more detailed examination of the likelihood of offsite power loss during earthquakes at the LaSalle site. Such an investigation is outside the scope of our current analysis.

\subsubsection{LOSP as LOCA Inltlator}

It should be emphasized here that the loss-of-offsite power is the only initiating event at LaSalle which can be attributed to the seismic event. As discussed for the LOCA events, all classes of LOCAs have the failure of the SRVS to reclose as contributors to initiator probability. From our calculations, it is clear that the SRV failures are the only significant contributors to the LOCA probabilities.

The random failure data obtained from RMIEP indicates a 10 percent probability of failure to reclose each SRV. Because the failures of other valves and equipment at LaSalle are dominated by the random failure mode, we feel that the SRVs are no exception and have not significantly attributed any additional probability of failure due to a seismic failure mode.

Thus, the seismic event is not likely to directly cause the LOCAs but causes them indirectly by first initiating a loss-of-offsite power. The LOSP event then leads to opening and random failure to reclose the safety relief valves. The dominant contribution to risk at LaSalle comes mostly from Transient-LOSP sequences. Thus the issue of LOCA origination takes on less importance. Nevertheless, for strict accuracy in understanding, it should be remembered that the only initiating event directly caused by the seismic event is the loss-of-offsite power. All of the LOCAs then result as a consequence of random failure of the SRVs to reclose on demand following the LOSP. 
Table 9.3. Pipe breaks resulting in large-LOCA.

\begin{tabular}{|c|c|c|c|c|}
\hline System & Pipe Segment & $\begin{array}{l}\text { Drawing } \\
\text { Number }\end{array}$ & $\begin{array}{c}\text { Type of } \\
\text { Break }\end{array}$ & $\begin{array}{c}\text { Pipe } \\
\text { Diameter }\end{array}$ \\
\hline Main Steam & $2 \mathrm{M} 501$ & M-116 Sht 1,2 & Steam & $20 "$ \\
\hline Main Steam & 2M502 & M-116 Sht 1,2 & Steam & $20 "$ \\
\hline Main Steam & $2 \mathrm{M} 503$ & M-116 Sht 1,2 & Steam & $20 "$ \\
\hline Feedwater & 2M504 & M-116 Sht 1,2 & Steam & $20 "$ \\
\hline Feedwater & 2FW01 & M-118 & Liquid & $12^{\prime \prime}-24 "$ \\
\hline Feedwater & 2FW02 & M-118 & Liquid & $12 "-24 "$ \\
\hline Nuclear Boiler & 2RH01 & M-139 Sht 1 & Liquid & $12 "-24 "$ \\
\hline Nuclear Boiler & 2RH02 & M-139 Sht 2 & Liquid. & $12 "-24^{\prime \prime}$ \\
\hline LPCS & 2LP01 & M-140 & Liquid & $10 "-12 "$ \\
\hline HPCS & 2HP01 & $M-141$ & Liquid & $10^{\prime \prime}-12^{\prime \prime}$ \\
\hline RHRS & $2 \mathrm{RH}-4$ & M-142 Sht 1 & Liquid & $12^{\prime \prime}$ \\
\hline RHRS & 2RH05 & M-142 Sht 2 & Liquid & $12^{\prime \prime}$ \\
\hline RHRS & 2RHK06 & M-142 Sht 3 & Liquid & $12^{\prime \prime}$ \\
\hline RHRS & $2 \mathrm{RH} 768$ & M-142 Sht 3 & Liquid & $12^{\prime \prime}$ \\
\hline $\mathrm{RCIC}$ & $2 \mathrm{M} 502$ & M-147 Sht 1 & Steam & $10^{\prime \prime}$ \\
\hline
\end{tabular}


Table 9.4. Pipe breaks resulting in medium-LOCA

\begin{tabular}{|c|c|c|c|c|}
\hline System & Pipe Segment & $\begin{array}{l}\text { Drawing } \\
\text { Number }\end{array}$ & $\begin{array}{c}\text { Type of } \\
\text { Break }\end{array}$ & $\begin{array}{c}\text { Pipe } \\
\text { Diameter }\end{array}$ \\
\hline Reactor water clean-up & 2RR01 & M-143 & Liquid & $2 "-6 "$ \\
\hline SLC & $25 \mathrm{CO} 2$ & $M-145$ & Liquid & $11 / 2^{\prime \prime}$ \\
\hline Control Rod Drive & 2RD99 & M-146 Sht 1 & Liquid & 1" \\
\hline $\mathrm{RCIC}$ & 2R104 & M-147 Sht 2 & Steam & 6" \\
\hline Nuclear Boiler & $2 \mathrm{NBC} 2$ & M-139 Sht 4 & Liquid & 2" \\
\hline Nuclear Boiler & 2NBB4 & M-139 Sht 4 & Liquid & 2" \\
\hline Nuclear Boiler & 2NBB2 & M-139 Sht 5 & Liquid & $2^{\prime \prime}$ \\
\hline
\end{tabular}


Table 9.5. Pipe breaks resulting in small-LOCA

$\begin{array}{llccc}\text { System } & \text { Pipe Segment } & \text { Drawing } & \text { Type of } & \text { Pipe } \\ \text { Number } & \text { Break } & \text { Diameter }\end{array}$

\begin{tabular}{|c|c|c|c|c|}
\hline RPV VENT & 2RH77K & M-116 & steam & $2 "$ \\
\hline Nuclear & $2 \mathrm{NBH} 4$ & M-139 Sht 3 & liquid/steam & $3 / 4^{\prime \prime}$ \\
\hline Boiler \& & $2 \mathrm{NBH} 2$ & M-239 Sht 3 & liquid/steam & $3 / 4^{\prime \prime}$ \\
\hline Reactor & $2 \mathrm{NBH} 5$ & M-139 Sht 3 & liquid/steam & $3 / 4 "$ \\
\hline Circulating & 2NBD1 & M-139 Sht 3 & liquid/steam & $3 / 4^{\prime \prime}$ \\
\hline$"$ & $2 \mathrm{NBH} 3$ & M-139 Sht 3 & liquid/steam & 3/4" \\
\hline$"$ & $2 \mathrm{NBJ} 2$ & M-139 Sht 3 & liquid/steam & $3 / 4 "$ \\
\hline$"$ & 2NBH6 & M-139 Sht 3 & liquid/steam & $3 / 4^{\prime \prime}$ \\
\hline$"$ & $2 \mathrm{NBJ} 1$ & M-139 Sht 3 & liquid/steam & $3 / 4 "$ \\
\hline$"$ & $2 \mathrm{NBJ} 3$ & M-139 Sht 3 & liquid/steam & $3 / 4^{\prime \prime}$ \\
\hline$"$ & $2 \mathrm{NBJ} 4$ & M-139 Sht 3 & liquid/steam & $3 / 4^{\prime \prime}$ \\
\hline$"$ & 2NBD3 & M-139 Sht 3 & liquid/steam & $3 / 4 "$ \\
\hline$"$ & 2NBJ5 & M-139 Sht 3 & liquid/steam & $3 / 4 "$ \\
\hline$"$ & 2NBF3 & M-139 Sht 3 & liquid/steam & $3 / 4 "$ \\
\hline$"$ & 2NBF6 & M-139 Sht 3 & liquid/steam & $3 / 4 "$ \\
\hline$"$ & $2 \mathrm{NBF} 2$ & M-139 Sht 3 & liquid/steam & $3 / 4 "$ \\
\hline$"$ & 2NBF5 & M-139 Sht 3 & liquid/steam & $3 / 4 "$ \\
\hline$"$ & $2 \mathrm{NBF} 7$ & M-139 Sht 3 & liquid/steam & $3 / 4 "$ \\
\hline$"$ & 2NBD8 & M-139 Sht 3 & liquid/steam & $3 / 4^{\prime \prime}$ \\
\hline$"$ & $2 \mathrm{NBF} 4$ & M-139 Sht 3 & liquid/steam & $3 / 4^{\prime \prime}$ \\
\hline ". & 2NBD6 & M-139 Sht 3 & liquid/steam & $3 / 4^{\prime \prime}$ \\
\hline$"$ & 2NBD2 & M-139 Sht 3 & liquid/steam & $3 / 4 "$ \\
\hline$"$ & 2NBD7 & M-139 Sht 3 & liquid/steam & $3 / 4^{\prime \prime}$ \\
\hline$"$ & 2NBD4 & M-139 Sht 3 & liquid/steam & $3 / 4 "$ \\
\hline " & 2NBD5 & M-139 Sht 3 & liquid/steam & $3 / 4^{\prime \prime}$ \\
\hline$"$ & $2 \mathrm{NBH} 1$ & M-139 Sht 3 & liquid/steam & $3 / 4^{\prime \prime}$ \\
\hline$"$ & $2 \mathrm{NBF} 1$ & M-139 Sht 3 & liquid & $3 / 4^{\prime \prime}$ \\
\hline$"$ & $2 \mathrm{NBG} 1$ & M-139 Sht 3 & liquid & $3 / 4 "$ \\
\hline
\end{tabular}


Table 9.5. Pipe breaks resulting in small-LOCA (cont.)

\begin{tabular}{|c|c|c|c|c|}
\hline System & Pipe Segment & $\begin{array}{l}\text { Drawing } \\
\text { Number }\end{array}$ & $\begin{array}{c}\text { Type of } \\
\text { Break }\end{array}$ & $\begin{array}{c}\text { Pipe } \\
\text { Diameter }\end{array}$ \\
\hline$"$ & 2NB01 & M-139 Sht 4 & steam & 4" \\
\hline$"$ & 2NBA3 & M-139 Sht 4 & steam & 1" \\
\hline$"$ & 2NBA4 & M-139 Sht 4 & steam & $1 "$ \\
\hline \multirow[t]{2}{*}{$"$} & 2NBB3 & M-139 Sht 4 & steam & 2" \\
\hline & 2NBA1 & M-139 Sht 5 & steam & $2^{\prime \prime}$ \\
\hline$"$ & 2NBA2 & M-139 Sht 5 & steam & $2 "$ \\
\hline$"$ & 2NBB 1 & M-139 Sht 5 & steam & 2" \\
\hline$"$ & $2 \mathrm{NBCl}$ & M-139 Sht 5 & steam & $2^{\prime \prime}$ \\
\hline
\end{tabular}




\subsection{Event Trees/Accident Sequences}

The evaluation of the seismically induced core damage frequency at the LaSalle Unit 2 nuclear power plant requires calculation of the frequency of the accidents leading to core damage. To do this, the sequences of events which lead to core damage must be delineated. This delineation is provided in the form of event trees (to define the sequence of events on the system level) and supplemented by system fault trees (to allow for accident definition at the component level).

In the previous section, five classes of initiating events were defined. These included the Reactor Vessel Rupture (RVR); Large Loss-of-Coolant Accident (Large-LOCA); Medium Loss-of-Coolant Accident (Medium-LOCA); Small Loss-of-Coolant Accident (Small-LOCA); and Transients (LOSP). In this section, we discuss the set of event trees that were used during the our analyses. However, before the specifics of the models are discussed, a general discussion of the plant response to a LOCA is appropriate.

\subsubsection{Pla t response to LOCA}

The LOCA event trees represent the plant response to a LOCA. To mitigate a LOCA, it is necessary to shut down the nuclear reaction, protect the containment from early overpressurization by condensing steam released from the vessel, keep the fuel covered with water, remove decay heat, and protect the containment from late overpressurization by transferring heat from the containment to the ultimate heat sink.

Reactor Shutdown. Shutting down the nuclear reaction is normally accomplished by inserting the control rods into the core. Failure to shut down the nuclear reaction would result in large amounts of the remaining water in the core boiling off. The loss of coolant would result in a power reduction. However, the coolant makeup systems would reintroduce coolant into the core for decay heat removal. The reactor power would increase again and boil off the coolant. This cycle could continue until the operator took manual control of the plant in order to reduce core power and stabilize the plant. Failure to stabilize the plant can result in core damage and/or containment failure.

Safety Relief Valves. Failure to reduce core power following an LOSP can result in boiling off of the core coolant. Since the turbine bypass capability is only 25 percent of full power, the normal heat removal system, if available, would not be capable of removing all the generated steam. Excess pressure would be relieved to the containment through safety relief valves (SRVs).

Given a successful shutdown, the decay heat in the core will continue to produce steam. Even with successful turbine bypass the pressure in the core may increase above normal operating levels. The RCS integrity function allows the reactor coolant system pressure to be relieved by opening the safety relief valves and transferring the steam to the suppression pool. Multiple openings of the relief valves will occur if turbine bypass is not available.

Failure of the relief valves to open will result in overpressurization and possible rupture of the reactor vessel. If overpressure protection succeeds, the pressure in the vessel is reduced but coolant is lost from the vessel to the vapor suppression pool. It thus becomes necessary to provide coolant to the vessel to keep the core covered. 
Once the pressure in the vessel is relieved, the safety/relief valves should reclose to minimize coolant loss. If one or more of the valves fail to reclose, a continuous flow of steam from the vessel to the suppression pool will occur. Such an occurrence would require that the suppression pool remain intact, that makeup water be supplied to the vessel, and that the heat transferred to the suppression pool be eventually transferred to the environment. Successful reclosure of the safety/relief valves must be followed by decay heat removal from the vessel.

Early Containment Overpressure Protection. Once the reactor is shutdown, it is still necessary to remove heat from the core and replace lost coolant. During a LOCA, the normal heat removal path may be disrupted by a pipe break while coolant is released to the containment atmosphere as steam. The steam generited in the drywell of the containment is forced by its own pressure to flow through downcomers into the wetwell. The wetwell contains a pool of water for condensing the steam and thus reducing the temperature and pressure of the drywell. This vapor suppression pool has sufficient heat capacity for several hours after a LOCA before it becomes necessary to transfer heat from the containment to the ultimate heat sink.

If the steam released during a LOCA is not condensed by the vapor suppression pool, pressure will build up in the primary containment and the containment will rupture. The containment overpressurization may result in failure of the coolant injection systems and containment heat removal equipment. If the early containment overpressure protection function is available, it is still necessary to replace lost coolant in the core and eventually remove the heat transported to the suppression pool.

Core Coolant Makeup. A LOCA, by definition, results in loss-of-coolant from the reactor core that must be replaced in order to prevent core damage. The emergency core cooling (ECC) systems are designed to initially provide cooling water to the core from an external source or from the suppression pool following initiation of a LOCA. This cooling water passes through the core removing heat and transferring $\mathrm{H}$ to the vapor suppression pool. Later, some ECC systems are realigned to take suction from the suppression pool to form a continuous circulation bop for cooling the core. Eventually, the stored heat in the suppression pool must be transferred to the ultimate heat sink.

Non-emergency related systems are also capable of injecting water from external sources into the vessel during a LOCA. However, these systems are not capable of recirculating water from the suppression pool. Failure of the coolant makeup function will result in loss of core cooling and core damage. Success of this function must be followed by removal of heat stored in the suppression pool.

Containment Heat Removal. In the later stages of a LOCA, the heat buildup in the suppression pool can reach the pool storage capacity. If this storage capacity is exceeded, the suppression pool can boil and then evolved steam can cause overpressurization and rupture of the containment.

There are systems to transfer heat to the ultimate heat sink from the suppression pool via heat exchangers. During a LOCA, these systems are aligned to take suction from the suppression pool, pass the water through heat exchangers, and inject it into the core or into the containment.

If the containment heat removal function is successful, the plant is stabilized and core damage is averted. The LOCA is thus mitigated and no other functions are required. 


\subsubsection{Event Trees/Accident Sequences}

The event trees used for the analysis of LaSalle are presented in Figs. 9.1 through 9.4. These event trees are taken directly from the RMIEP event trees, with two simplifying modifications. First, since we have assumed a loss-of-offsite power will occur during a seismic event, we have eliminated from the event trees those systems which are dependent upon offsite power. Those systems are feedwater, condensate, control rod drive, and power conversion. Where branches occur for these systems in the RMIEP trees, we assumed failure of the system and followed the failure path in the development of our tree sequences.

The second simplification is related to the fact that we are concerned only with core damage in this study. Consequently, we do not develop the accident sequences past the point where core damage, or lack thereof, is guaranteed. This results in the elimination of some systems from some event trees since they have effect only on the consequence analysis, not on the probability of sustaining core damage. Specifically, we have eliminated the suppression pool cooling and containment spray systems from the Large and Medium LOCA event trees. Also, we have eliminated the venting system from all of the event trees.

The removal of the venting system involves an important assumption on our part. The RMIEP event trees include the venting system in the accident mitigation (as opposed to consequence mitigation) portion of the event trees out of concern over what is known as Containment Thermal Vulnerability (CTV). In a CTV situation, the containment becomes highly over-pressurized and over-heated. This leads to a potential failure of the equipment hatch seals which results in the escape of steam from the containment into the reactor building. In the event that this steam should travel into the adjoining cubicles which house the various safety system pumps and other equipment, a common cause failure of that safety equipment could occur. This could result in a core damage state. For example, RMIEP has defined the containment failure pressure as 150 psig. This implies that the HPCS may be susceptible to failure due to pumping appropriately $370^{\circ} \mathrm{F}$ suppression pool water. When the ADS values reclose at 100 psig some high pressure injection is needed until the containment ruptures.

We have not included the CTV scenario for several reasons. First, the mechanism leading to core damage is not well understood. We did not evaluate the CTV and we believe that more investigation is needed before including the scenario in our seismic risk estimate. However, an estimate of the effect of considering CTV would be possible since our results are driven largely by random failures and the CTV scenario does not appear to be affected by a seismic event. Secondly, from our plant visits, we feel that such a scenario has a low probability of occurrence. Therefore, even if the CTV scenario was included, it is likely that the scenario would not survive probabilistic culling. 


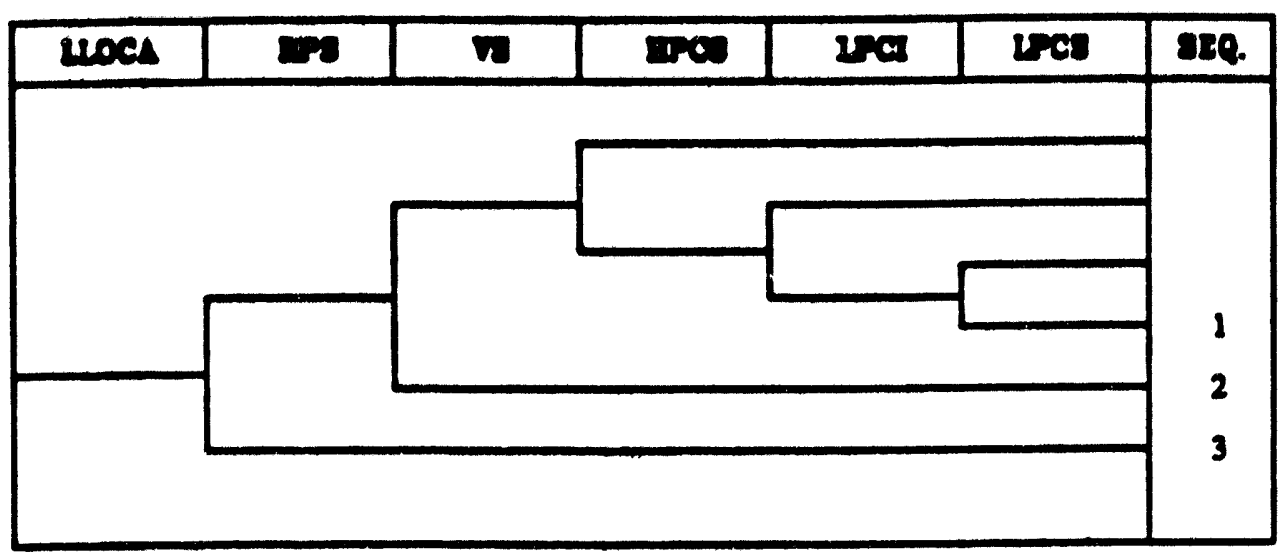

Figure 9.1. Large-LOCA event tree.

$-218-$ 


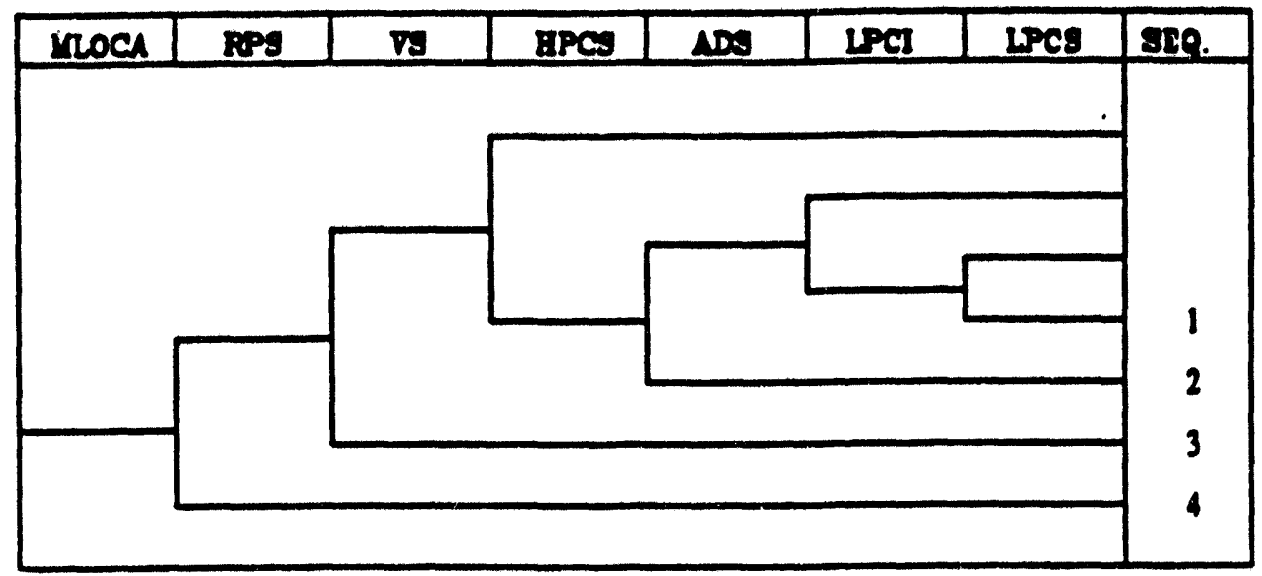

Figure 9.2. Medium-LOCA event tree. 


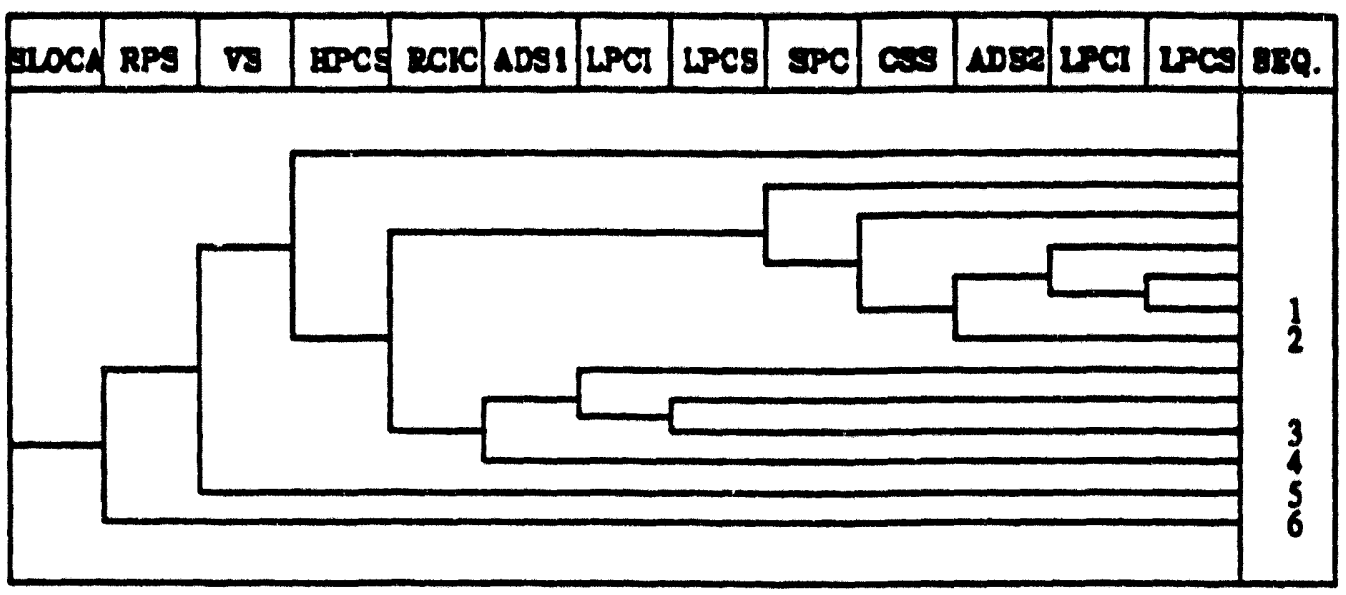

Figure 9.3. Small-LOCA event tree. 


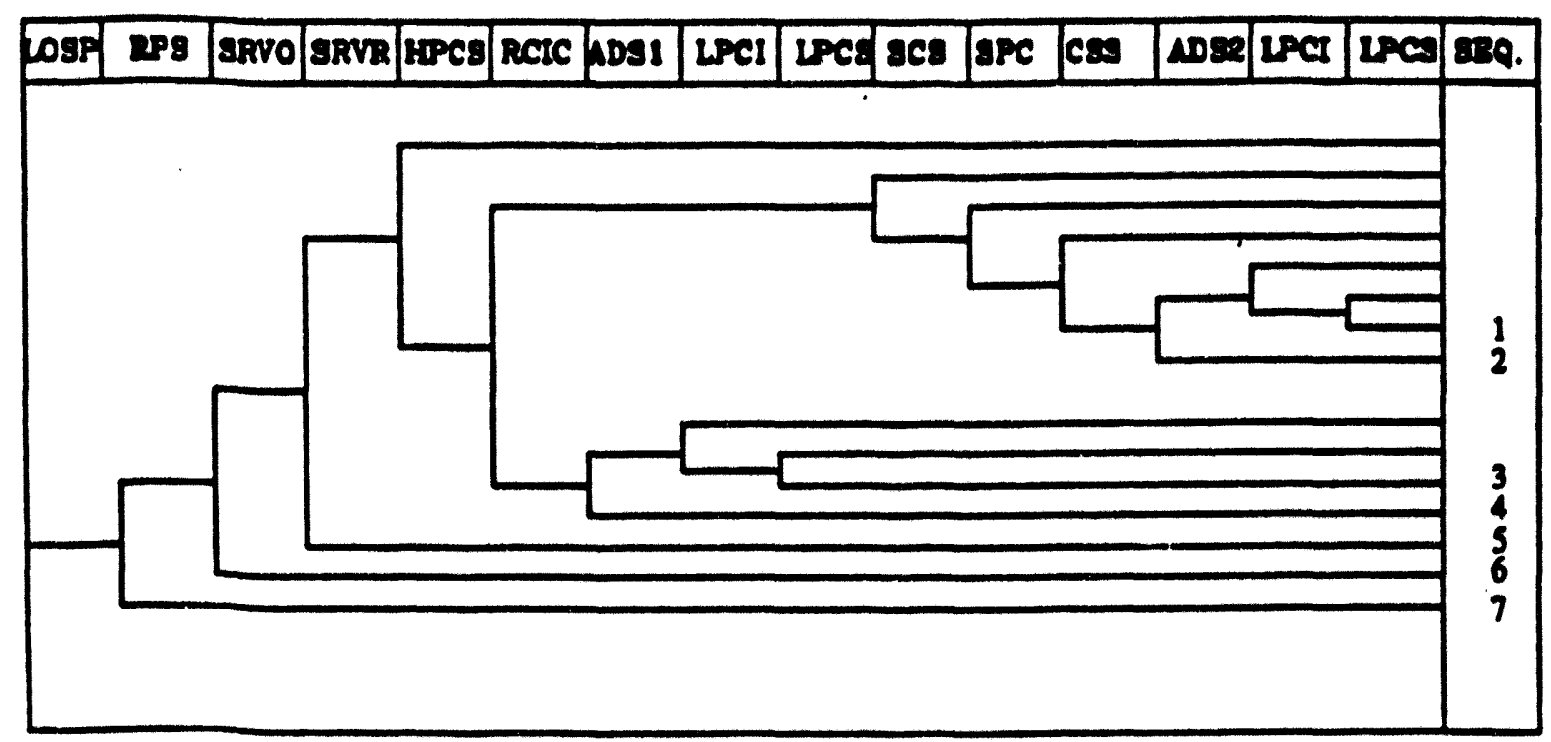

Figure 9.4. l.oss-of-offsite power event tree. 
From Figs. 9.1 through 9.4, it can be seen that there are three Large-LOCA, four Medium-LOCA, six Small-LOCA, and seven Loss-of-Offsite Power sequences leading to core damage, for a total of 20 accident sequences which we analyzed in our study. Since our study focused only on the core damage frequency we do not extend the sequences beyond the point of core damage nor do we distinguish between various grades of core damage severity. Table 9.6 provides a summary of the accident sequences used. The minus sign indicates system success. Events with no minus sign indicate system failure.

Table 9.6. Summary of accident sequences.

\section{Large-LOCA}

1) -RPS * -VS * HPCS * LPCI * LPCS

2) - RPS * VS

3) RPS

\section{Medium-LOCA}

1) -RPS *-VS * HPCS * -ADS * LPCI * LPCS

2) - RPS * -VS * HPCS * ADS

3) -RPS * VS

4) RPS

Small-LOCA

1) -RPS * -VS * HPCS * -RCIC * SPC * CSS * -ADS * LPCI * LPCS

2) -RPS * -VS * HPCS * -RCIC * SPC * CSS * ADS

3) -RPS * -VS * HPCS * RCIC * -ADS * LPCI * LPCS

4) - RPS * -VS * HPCS * RCIC * ADS

5) -RPS * VS

6) RPS

\section{Loss-of-Offsite Power}

1) $-\mathrm{RPS}$ * -SRVO * -SRVR * HPCS * -RCIC * SCS * SPC * CSS * -ADS * LPCI * LPCS

2) -RPS * -SRVO *-SRVR * HPCS *-RCIC * SCS * SPC * CSS * ADS

3) -RPS * -SRVO * -SRVR * HPCS * RCIC * -ADS * LPCI * LPCS

4) -RPS * -SRVO * -SRVR * HPCS * RCIC * ADS

5) -RPS * -SRVO * SRVR (Leads 10 LOCA Event Tree)

6) -RPS * SRVO

7) RPS (Anticipated Transient Without Scram (ATWS)) 


\subsection{Fault Trees/Safety Systems}

\subsubsection{Syatem Descriptions}

Below, we provide brief descriptions of the safety system functions and the equipment designs. More in-depth discussions of the systems can be found in LaSalle FSAR ${ }^{(3.1)}$ and the RMIEP final report. ${ }^{(1.4)}$

\section{Automatic Depressurization System (ADS)}

Systom Function. The ADS provides a method of automatically depressurizing the reactor vessel following small breaks in the system pressure boundary. This rapid reduction in pressure allows the Low Pressure Coolant Injection (LPCI) mode of RHR and the Low Pressure Core Spray (LPCS) to inject coolant into the reactor vessel to keep the core cooled. ADS acts in conjunction with the low pressure injection systems as a backup to the High Pressure Core Spray system (HPCS).

System Design. Eighteen safety/relief valves, connected to the four main steam lines between the vessel and the inboard main steam isolation valves, protect the reactor vessel from overpressure. Each of these valves serves a dual function as both a safety and a relief valve. The safety function is mechanically actuated by an internal bellows and spring. The relief function is actuated by an external pneumatic cylinder that opens the valve independently of the mechanical actuator. Seven of these valves also serve as ADS valves. The ADS function is actuated by the same pneumatic cylinder that opens the valve for the relief function. Air to the pneumatic cylinder for each valve is applied by any one of three solenoid valves which, when open, admit air to a header connected to the pneumatic cylinder.

For the seven valves designated for ADS, two of the solenoid valves are controlled by ADS signals with the third solenoid valve controlled by relief signals. Actuating air is supplied by drywell pneumatics. An accumulator in the air supply line for each valve provides reserve air for operation of the valve if the air supply is lost. The ADS valves opening is initiated by the simultaneous occurrence of a low reactor vessel water level (Level 1 ) and high drywell pressure.

\section{Condensate System (CDS)}

Systom Function. The purpose of the condensate system is to provide a means of purifying and transferring water from the condenser hotwell to the suction of the reactor feedwater pumps. In addition, the flow of condensate serves as the cooling medium for the steam jet air ejector condensers, the gland exhaust condenser, and the off-gas condensers. For the RMIEP analysis, in terms of the PRA, the condensate system function is to provide condensate to the feedwater pumps or to act as a low pressure injection system. 
Systom Destgn. The condensate system transfers condensate from the condenser hotwell to the feedwater pumps. The system consists of four condensate pumps, two steam let air electors, two gland seal condensers, two off-gas condensers, seven condensate polishers, four condensate booster pumps, and three strings of low-pressure heaters each containing five heaters.

\section{Core Standby Cooling Syatem (CSCS)}

Syrtom Functlon. The Core Standby Cooling Systems (CSCS) equipment cooling water system provides component cooling during operation of the individual core standby cooling systems, (RHR heat exchangers, CSCS area cooling coils, diesel generators, RHR pump sea coolers, and the LPCS pump motor cooling coils). The system also provides a source of emergency make-up water for fuel pol cooling and can provide containment flooding for post accident recovery.

Systom Design. The CSCS system consists of three divisions which independently supply filtered cooling water to their respective loads. Each division utilizes a common water supply header and consists of one or more subsystems. There are eight cooling water pumps for LaSalle Unit 2. One Unit 1 pump, the ODO pump, is designated as a swing pump supplying either unit.

The three divisions of CSCS consist of the following:

Division 1/Three pumps supplying:

RHR Service Water Subsystem (Train A)

-RHR heat exchanger " $A$ "

-RHR pump "A" seal cooler

ODG Cooling Water Subsystem

-Diesel generator "O" cooler

-Unit 1 and Unit 2 LPCS pump motor coolers

-Unit 1 and Unit 2 N.E. area coolers (RCIC and LPCS comer room)

Fuel Pool Make-up Subsystem

Fuel pool emergency make-up supply " $A$ "

Note: The ODG cooling water subsystem and fuel make-up subsystem loads c-f above are supplied by the swing pump (D/G cooling water pump "OA") located on Unit 1 side.

Division 2/Four pumps supplying:

RHR Service Water Subsystem (Train B)

-RHR heat exchanger " $B$ "

-RHR pump "B" seal cooler

-RHR pump "C" seal cooler 
DC2a Cooling Water Subsystem

- Diesel generator "A" cooler

-South east area cooler (RHR pump "B" and "C" comer room

Fuel Pool Makeup Subsystem

-Fuel pool emergency make-up supply "B"

-Containinent flooding supply

Division 3/One pump supplying:

HPCS DO Cooling Water Subsystem

-Diesel generator "B" cooler (HPCS D/G)

-South west area cooler (HPCS comer room)

\section{Containment Spray System (CSS)}

Systom Function. The Containment Spray System (CSS) is a subsystem of the RHR system and a subfunction of the containment cooling function. The containment cooling mode consists of suppression pool cooling and containment spray. Containment spray is used to depressurize the drywell. The drywell spray removes energy from the drywell atmosphere by condensing the water vapor.

System Design. The containment spray subsystem consists of two independent fluid trains that draw water from the suppression pool, pass it through the RHR heat exchangers, and deliver it to the drywell spray headers. The CSS trains are configured from RHR trains A and B and uso the RHR pumps, heat exchangers, and the drywell spray lines.

\section{High Preasure Core Spray (HPCS) System}

System Function. The purpose of the HPCS system is to maintain the reactor vessel water level above the top of the core, without reliance upon other ECCS systems, following LOCAs which do not result in rapid depressurization of the reactor vessel. However, the HPCS system is capable of maintaining the reactor vessel water level throughout the full range of breaks up to and including a Design Basis Accident (DBA). The HPCS system also serves as a back up to the Reactor Core Isolation Cooling (RCIC) system should the RCIC system fail to maintain the reactor vessel water level following a reactor vessel isolation.

System Design. The HPCS system injects water from either the suppression pool or condensate storage tank into the reactor vessel through a single injection line. The system consists of one motor driven pump, a flow distribution sparger inside the reactor vessel and associated piping, valves, instrumentation and controls. 


\section{Low Preasure Coolant Injection (LPCI) System}

System Function. The Low-Pressure Coolant Injection (LPCI) system is one of the independent operating subsystems of the RHR system. It operates to restore and, if necessary, maintain the coolant inventory in the reactor vessel after a LOCA. LPCI operation provides protection to the core for a large break in the reactor coolant pressure boundary and also extends protection to cover a small break in which the ADS or HPCS have reduced the reactor vessel pressure to the LPCI operating range. LPCI is a low-head, high-flow function that delivers flow to the reactor vessel when the differential pressure between the vessel and drywell is less than 225 psid. The LPCI is designed to reflood the reactor vessel to at least two-thirds core height and to maintain this level.

System Design. The LPCI subsystem consists of three independent fluid trains that carry water from the suppression pool and inject it into the reactor vessel via three separate nozzles. Two of the LPCI trains are configured from RHR pumps, valves and piping that may also be used in other RHR modes. The third train is used only in LPCI mode and contains no components common to other subsystems.

\section{Low Pressure Core Spray System (LPCS).}

System Function. The Low Pressure Core Spray system (LPCS) is a loop similar to, but independent of, the HPCS. The low pressure system is designed to provide core protection in case of larger breaks which would rapidly depressurize the reactor vessel. The LPCS limits the maximum fuel cladding temperature and cools it to saturation upon flooding the core. Such protection also extends to a small break in which the ADS or HPCS has operated to lower the reactor vessel pressure to the operating range of the LPCS. The LPCS has no containment heat removal capability.

System Design. The LPCS system is a single independent loop consisting of a centrifugal pump that can be powered by normal auxiliary power or by the standby a-c power system; a spray sparger in the reactor vessel above the core (separate from the HPCS sparger); piping and valves to convey water from the suppression pool to the sparger; and associated controls and instrumentation.

\section{Reactor Core Isolation Cooling System (RCIC)}

System Function. The RCIC system cools the core and reduces reactor vessel pressure following a reactor shutdown when it is isolated from its normal coolant source. Reactor vessel water is maintained or supplemented by RCIC:

- when the reactor vessel is isolated and maintained in the hot standby condition,

- when the reactor vessel is isolated and accompanied by a loss-of-normal coolant flow from the reactor feedwater system, and 
- when a complete plant shutdown following a loss-of-normal feedwater is started before the reactor is depressurized enough to allow operation of the reactor shutdown cooling mode of RHR.

The RCIC system may also be used to backup the HPCS system or operated in conjunction with other ECCS systems, if desired.

System Design. The RCIC turbine driven pump operates on steam generated by decay heat, injecting water from either the suppression pool or CST into the reactor vessel through a single injection line. The pump can also be aligned to take a suction on the RHR heat exchanger for shutdown cooling. The system consists of one turbine driven pump discharging to the head spray nozzle inside the reactor vessel, and associated piping, valves, instrumentation and controls.

\section{Shutdown Cooling System (SCS)}

System Function. The shutdown cooling mode is an integral part of the RHR system. It is operated during a normal shutdown and cooldown and may also be used following LPCI core reflood. The initial phase of nuclear system cooldown is accomplished by dumping steam from the reactor vessel to the main condenser. When nuclear system temperature has decreased to where the steam supply pressure is not sufficient to maintain the turbine shaft gland seals, vicuum in the main condenser cannot be maintained and the RHR system is placed in the shutdown cooling mode of operation. The shutdown cooling subsystem is able to complete cooldown to $125^{\circ} \mathrm{F}$ for reactor refueling and servicing.

During the initial phase of cooling the reactor, only a portion of RHR system heat exchanger capacity is required for SCS operation. This leaves the remaining portion of the RHR system with its heat exchanger, associated pumps, and valving available for the LPCI mode. The LPCI mode is shifted to shutdown cooling mode after the reactor is depressurized so the proper cooling rate may be achieved with the lower reactor water inlet temperature.

System Desigr. The Shutdown Cooling System consists of two independent fluid trains that take suction from one of the reactor recirculation loops (A) through a common suction line. The water is pumped through the RHR heat exchangers and returned to the reactor vessel via " $A$ " or "B" recirculation loops. The SCS trains are configured from RHR trains A and B, and use the RHR pumps, heat exchangers, and test return lines.

\section{Suppression Pool Cooling System (SPC)}

System Function. Suppression Pool Cooling (SPC) is a subsystem of the RHR system and a subpart of the containment cooling mode. The containment cooling mode consists of suppression pool cooling and containment spray.

In the event of a LOCA, the short-term energy release from the reactor primary system will be dumped to the suppression pool. Subsequent to the accident, fission product decay heat will result in a continuing energy dump to the pool. If this energy is not removed from the primary 
containment, it will eventually result in unacceptable suppression pool temperatures and containment pressures. The containment cooling mode of the RHR system is used to limit the temperature of the water in the suppression pool such that, immediately after the design-basis LOCA, pool temperature does not exceed $170^{\circ} \mathrm{F}$. Tests show that at $170^{\circ} \mathrm{F}$, complete condensation of blowdown steam from this LOCA can be expected. Complete condensation is also likely at higher pool temperatures, but no test data is available.

System Design. The suppression pool cooling subsystem consists of two independent fluid trains that draw water from the suppression pool, pass it through the RHR heat exchangers, and return it to the suppression pool. The SPC trains are configured from RHR trains $A$ and $B$ and use the RHR pumps, heat exchangers, and test retum lines.

\subsubsection{Fault Trees/Safety Systems}

Of the twelve safety systems/functions shown in the event trees, we received large, detailed fault trees from RMIEP for all but four. These were the reactor protection (RPS)/alternate rod insertion (ARI) system, the vapor suppression system, and the Safety Relief Valve Opening (SRVO) and Reclosing (SRVR) functions. The RPS/ARI is a single point failure leading to core damage in all four of the event trees. We used the RMIEP estimate of 1.E-05/yr as the random failure frequency for the RPS/ARI system. The Vapor Suppression (VS) system, like the RPS/ARI, is also a single point failure leading to core damage. However, since the VS system is passive, and since our calculations showed low probabilities for pipe failures in the system, we assumed success of the vapor suppression system throughout our analysis.

The Safety Relief Valve (SRV) functions were modeled as follows. First, based on the fact that, in general, the plant equipment failures were highly dominated by the random mode rather than by the seismically induced mode, it was appropriate to treat the SRVs as random failures. Consequently, it was assumed that success for the SRVO function and the SRVR function was assessed based on an individual SRV reclose failure rate of .1 per demand. A more detailed discussion is given in Sec. 9.1.6.

The remaining systems, for which we received detailed fault trees, were modified to include seismic failure modes. Any event in the fault tree which could be the result of either a random failure or a seismically induced failure was modified as follows. The event was converted into an OR-gate with two basic event inputs, one representing the random failure mode and one representing the seismic failure mode. Of the original 3414 events in the fault trees, 2562 were modified in this way. The remaining 852 basic events, therefore, represent events for which there is no equivalent seismic failure mode, e.g., maintenance errors, which are committed prior to the earthquake, or equipment items which are passive and considered to be relatively impervious to seismic motion, e.g., electrical fuses.

We considered the modification of the fault trees through the addition of new failure paths which were not considered during the development of the internal event fault trees. However, our preliminary calculations showed probabilities of structural failure at LaSalle to be small; this being the type of failure which can lead to common mode failure of independent safety systems trains, redundant systems, etc., and the dominant class of seismic failures found in the Zion study. We feel further scrutiny of the plant equipment diagrams and operating procedures to determine more subtle means of failing the safety systems would not have yielded anything significant. 


\subsection{Solution Process and Results}

Once the event trees and fault trees have been developed, the process of solving the systems and accident sequences begins. Figure 9.5 illustrates the procedure. A detailed database, providing the basic event name, associated response fragility, and random probability data, was needed. After developing this database and the appropriate SEISIM[9.2] input files, the basic event list was used as input into SEISIM and marginal probabilities generated. These marginal probabilities were generated for all six earthquake levels (see Sec. 4.4 for earthquake level definitions). Having completed the SEISIM runs to generate marginal probabilities at all six levels of earthquakes we then developed an "average" probability for each basic event. These "average" probabilities are called culling values and were used in our culling process. ${ }^{[9.31}$ As indicated earlier, each basic event on the fault tree was evaluated and determined to be either a random failure, a fragility-related failure, or capable of failing in either mode. The culling values generated using SEISIM were the fragility-related values. The random failure probabilities were taken from the database.

The culling values described in the previous paragraph were then used in solving the safety systems. The computer code used to solve the systems was FTAP (Fault Tree Analysis Program). ${ }^{[9.4]}$ All runs were made on an LLNL VAX-750. The system solutions were then reviewed for correctness and to determine the effect due to an earthquake.

At this point, the fault tree solutions for the systems were input back into FTAP along with the event trees. Accident sequence solutions were then generated. Note, the same culling values that were used for culling the systems were also used here. However, the culling procedure differed slightly in that after reviewing the system solutions, it was found that there were no two dominant seismic events that appeared in the same cut set. Therefore, while the systems solutions were culled using a minimum criteria, i.e., culled based on the lowest probability basic event appearing in a cut set, the accident sequence culling was performed based on cut set probability.

The detailed fault trees received from the RMIEP were large. This situation was worsened when 2562 gates and basic events were added to the numerous gates and events already found in the trees. Consequently, it was assumed from the beginning that there would be a need to make use of probabilistic culling techniques ${ }^{[9.3]}$ in order to solve the fault and event trees. 


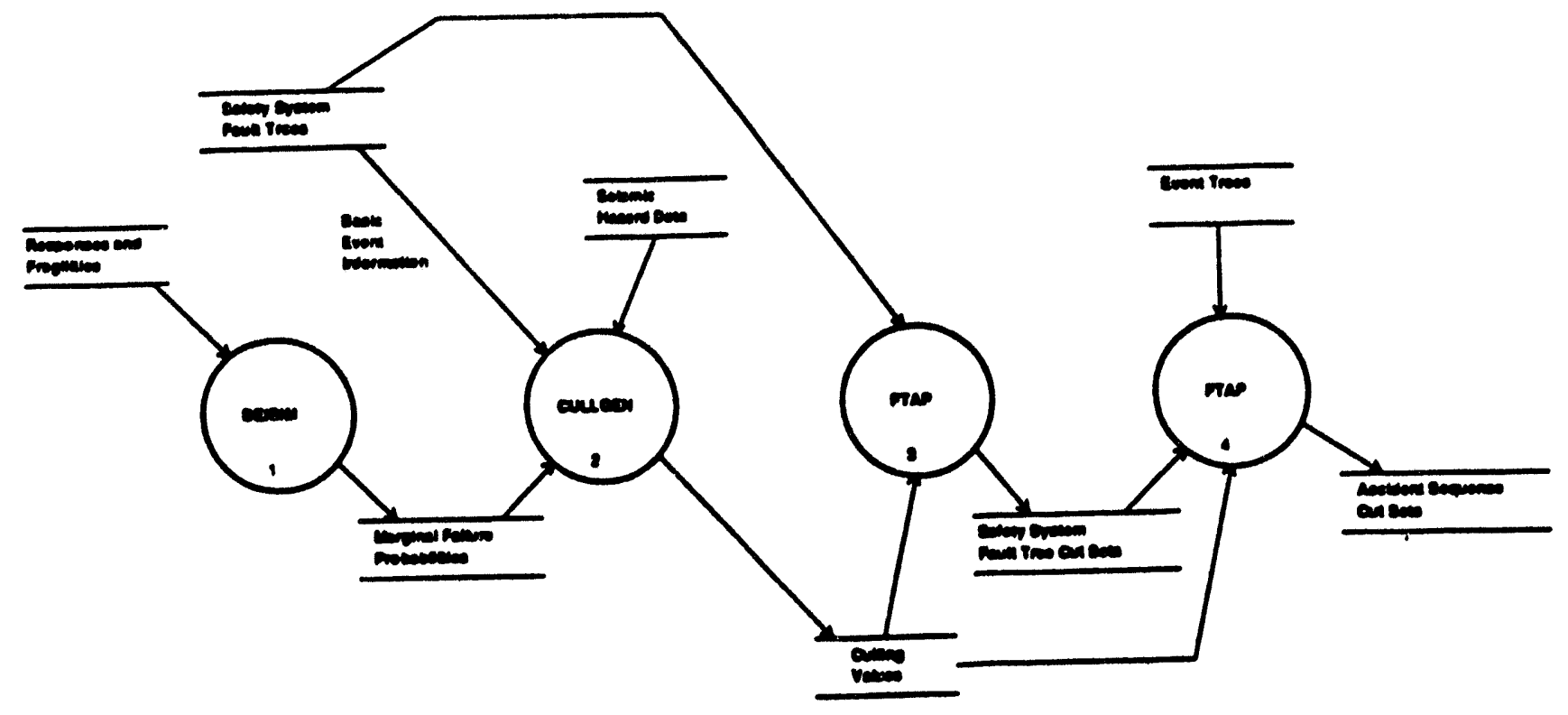

Figure 9.5. System and accident sequence solution process. 
A probability of 1.0E-04 was the cutoff used in the culling process. In earlier studies, ${ }^{11.3)}$ we made use of a culling technique based upon the minimum marginal failure probability of the elements of a cut set (minimal element approach). This was necessary in order to account for correlation between cut set elements, which can be significant for seismic analyses.

In the LaSalle study, we had no basic event culling values which exceeded $1.0 \mathrm{E}-05$, with the exception of the condensate storage tank. Thus, at a culling cutoff of 1.0E-04, there was no chance of survival, even under the minimum element approach. Therefore, the product of cut set elements estimator was used.

Table 9.7 contains a list of all the accident sequence cut set that survived the culling process. Those accident sequences not listed did not survive the culling process. Note that a hyphen in front of a basic event indicates a success, no hyphen indicates a failure.

In reviewing Table 9.7, it can be found that only 18 failure events survived the culling process. The 18 are listed in Table 9.8. 
Table 9.7. Culled accident sequence cut set numbers.

Accident Sequence

Number of

Cut Sets

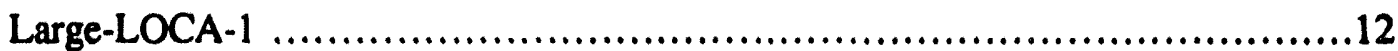

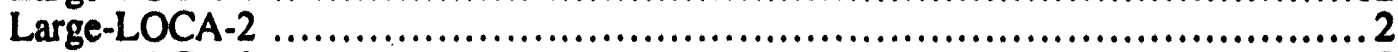

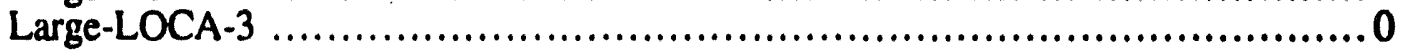

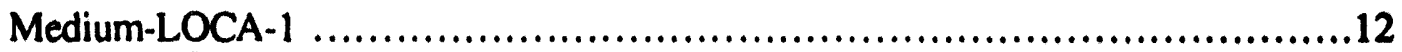

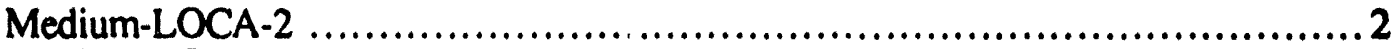

Medium-LOCA-3 …........................................................ 0

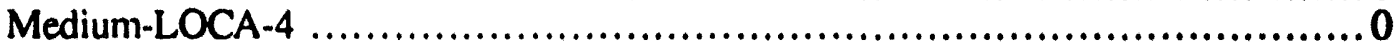

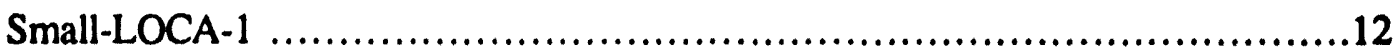

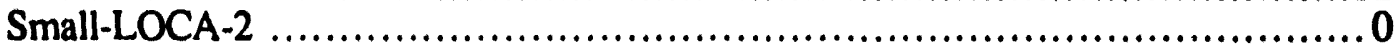

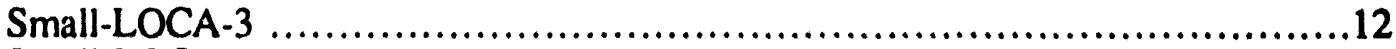

Small-LOCA-4 …........................................................................ 2

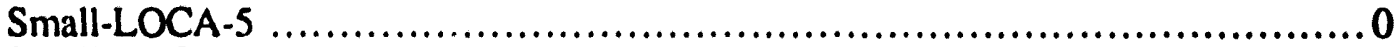

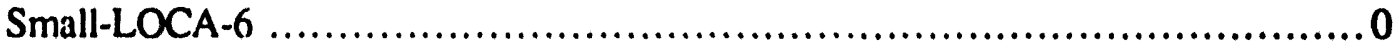

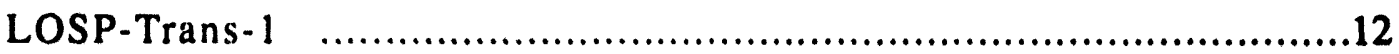

LOSP-Trans-2

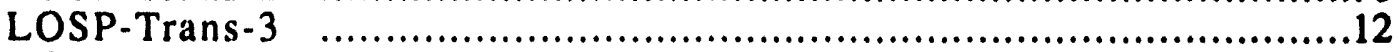

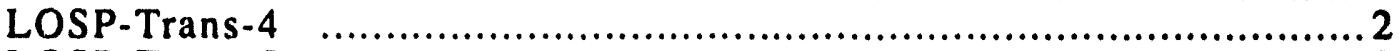

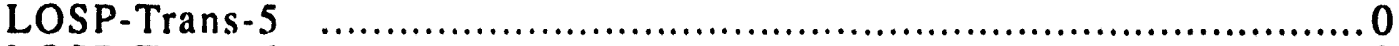

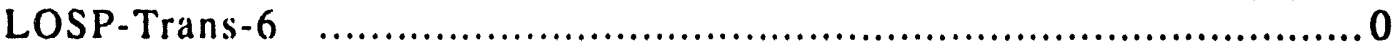

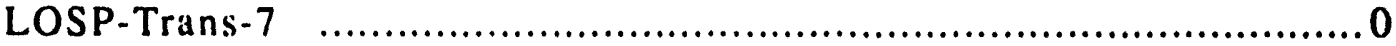


Table 9.8. Basic events in accident sequence cut sets.

Basic Event Name Description

adsminit-qoo-oe-

ap037x3-roo-Ifo0

ap039 2 -roo-Ifo0

ap040x3-roo-Ifo0

cst-tnk-...-.....

d0vb101x-bco-Ifu

d0vb102x-bco-Ifo

d0vb201x-bco-If0

d0vb202x-bco-Ifo

dg-cm--.-.-..-

dg0-gen-1f-.-.--0

dg2a-gen-1f-.---0

lak10rca-roo-lfo

losp-ie-------.-

rcicrmcool-flag-

t14-ie--.-.-....-
ADS manual initiating operator error

Local fault of relay apo $037 \times 3$ fails open

Local fault of relay apo039 2 fails open

Local fault of relay apo040 3 fails open

Failure of the condensate storage tank

Local fault if circuit breaker $d$-vb101x

Local fault if circuit breaker $d$-vbl $02 x$

Local fault if circuit breaker $d-v b 201 x$

Local fault if circuit breaker d-vb202x

Diesel generators not available

Local fault of diesel dg0

Local fault of diesel dg2a

Fault in relay coil lak10rca-00 in RHR signal path 11

Probability of having an offsite power initiating event

Flag used for RCIC room cooling

Loss of water level instrumentation 


\section{Section 10. Risk Quantification}

The procedure used to quantify risk in this study is essentially unchanged from that of the SSMRP seismic risk methodology. For that reason, we shall provide only a brief discussion.

Once logic models (fault trees and event trees) have been developed and solved to determine the equipment failure combinations which result in the various accident sequences, it becomes necessary to attach probabilistic estimates to those failures in order to quantify the frequency of occurrence of the accident sequences and, thus, core damage. In the SSMRP Simplified Methodology, the computer program SEISIM ${ }^{(9.2)}$ is used to quantify the accident sequence frequencies. This program takes account of the correlation between seismic failure modes for equipment. These correlations arise from equipment items being simultaneously subjected to the same earthquake responses.

The data requirements of SEISIM are briefly described in Sec. 10.1 and the actual quantification process is discussed in Sec. 10.2.

\subsection{Data Requirements}

As shown in Fig. 10.1, SEISIM requires 6 types of information in order to quantify seismic risk. They are:

- seismicity data (discretized seismic hazard curve)

- initiating event definitions

- plant structure and equipment seismic responses

- plant structure and equipment fragilities

- structure and equipment random failure probabilities

- logic model solution results (safety system/accident sequence cut sets)

SEISIM uses the seismic response, fragility data, and random failure data to quantify the accident sequence cut sets. The result is a core damage frequency estimate conditional on both earthquake and initiating event. SEISIM then factors in the initiating event probability, producing a core damage frequency estimate conditional only on earthquake. Lastly, SEISIM combines this estimate with the seismicity information to produce an unconditional estimate of the core damage frequency. In the results presented in Sec. 11, each of these three different estimates are presented; each one providing valuable information regarding the seismic risk at the LaSalle nuclear power plant. 


\section{Fragilities}
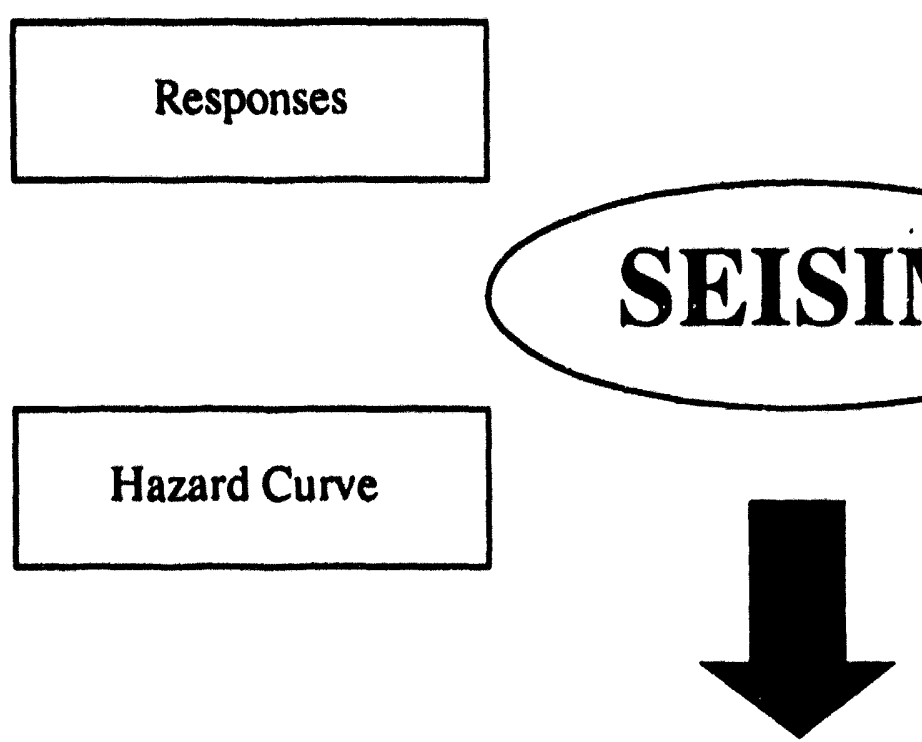

\section{Event/Fault}

Tree Information

\section{OUTPUTS:}

- Core Damage Frequency

- Accident Sequence Probabilities

- Marginal Component Failure Probabilities

- System Failure Probabilities

Figure 10.1. Quantification of seismic risk. 


\subsection{Quantification Process}

\subsubsection{Input Files}

The data used for the SEISIM calculations comes from several sources. First, the data used to control the SEISIM calculations (INFILE) were based on those used in earlier SSMRP runs of SEISIM. This data consists of SEISIM control information and the use of earlier values is based on the similarity in the types of numerical calculations being performed in this analysis of LaSalle and the earlier analyses of the SSMRP. One notable exception to this is the discretized seismic hazard data which is input to SEISIM.

These probabilities for the LaSalle hazard curve are given in Table 4.1. The analysis which provided this data was described in Sec. 4.

The fragility data (FRFILE) had two primary sources. These included plant-specific fragilities, which were developed for the LaSalle study, and generic fragility data, which is not specific to LaSalle. A more complete discussion was given in Sec. 8. By contrast, the response data (REFILE and REFILN) used in our analysis were all plant-specific. The methods used to obtain the response data was described earlier in this report. The initiating event definitions (IEFILE) were developed specifically for LaSalle.

The remaining SEISIM data files (ASFILE, LCFILE, CRFILE, and LUFILE) were developed for the RMilEP event and fault trte models. The data in these files was generated by the event and fault trees used by the analyses, as well as the logic model solution process employed.

Table 10.1 provides a list of these files and summarizes their contents.

\subsubsection{SEISIM Computational Procedure Summary}

In the analysis, SEISIM was run on a CDC-7600. One run was made at each of the defined six earthquake levels. Then, the six sets of results were combined using the seismicity data. The computational time used by SEISIM was small because of the number of accident sequence cut sets and the presence of only a few seismic failures. Modifications to the existing versions of SEISIM were necessary to provide a capability for handling basic event descriptors with 16 characters (RMIEP fault trees), to handle a larger number of random failure events, and to allow responses means, standard deviations, and correlations to be input into SEISIM (Simple Methodology). 
Table 10.1. SEISIM Input miles.

Filename

Contents

INFILE Run control information: run name and description, integration error control, Hunter's bound control, integration partition counter, integration time limit, earthquake probability, etc.

LUFILE Basic event look-up tile: numbers associated with basic events.

FRFILE Fragility function and random event data: fragility function indicators, means, standard deviations, and correlations, and random event names, probability estimates, and probability estimate standard deviations.

CRFILE Cross reference file: acceleration dependent basic event names, response numbers, and fragility function numbers.

REFILE Response file: response distribution function indicators.

REFILN Response file: response means, standard deviations, and correlations for a specified earthquake level.

IEFILE Initiating event file: initiating event names, cut set counters and cut sets.

LCFILE Logical component tile: system names, cut set counters, and cut sets.

ASFILE Accident sequence file: initiating event name, accident sequence number, containment failure modes and associated probabilities and release categories, cut set counter, and cut sets. Data in this file also indicates accident sequences that can be initiated by other accident sequences. 


\section{Section 11. Results}

\subsection{Point Eotimates of Core Damage Frequency}

Our analysis resulted in a seismically induced core damage frequency point estimate of 6.0E-7/yr for LaSalle Unit-2. Table 11.1 lists the breakdown of contributions to this total seismic risk estimate from the nine significant accident sequences and six earthquake levels which we considered. A number of interesting observations can be made from this table.

First, and perhaps most significantly, the risk contribution is seen to monotonically decrease as one moves from the lowest level earthquake (Level 1) to the highest level (Level 6). This decrease is significant because it implies that the greatest seismic risk is contributed by the smaller earthquakes. A large percent of the risk (89.9) fell into the lower three earthquake levels. More importantly, it also implies that the greatest risk at LaSalle may be from low intensity earthquakes. By contrast, in the Zion study, ${ }^{(1.3)}$ it was found that the greatest contribution to risk came in the 2 to 4 times SSE level. The fact that a large percent of the risk $(89.9 \%)$ fell into the lower three earthquake levels indicates that the core damage frequency would be lower than the estimated 6.0B$7 / y r$ for LaSalle Unit 2 if the final instead of the preliminary seismic hazard curves were used. The core damage frequency would be lower because, as shown in Figure 4.7, the final seismic hazard curves fall completely below the preliminary curves for these three earthquake levels.

It is believed that this dominance by the lower level earthquakes can be traced directly to our assumption of a loss-of-offsite power during an earthquake. To facilitate understanding this conclusion, examine Table 11.2, in which the contributions to core damage frequency, conditional on earthquake frequency are presented. In this table, it can be seen that the conditional core damage frequency increases with increasing earthquake level. In fact, the total risk increases by a factor of 4 from level 1 to level 6 . However, from Table 4.1, we see that a level 1 earthquake is 100 times more frequent than a level 6 earthquake. Thus, we would expect the unconditional risk at level 1 to be roughly 25 times greater than at level 6, which is in fact what we see in Table 11.1 (i.e., 3.4E-7 as compared to 1.2E-8). In other words, the core damage frequency does not increase with earthquake intensity as rapidly as the falloff in frequency of having a higher intensity earthquake.

The reason core damage frequency increases only by a factor of 4 from level 1 to level 6 is twofold. First, because of the relatively low responses at LaSalle, we find that the equipment failure is dominated by the random mode, as opposed to the seismically induced mode. The random failure mode is not subject to increasing probability with increasing earthquake level; thus, one would not expect to see the probability of accident sequences from random failure increase with earthquake level. In Table 11.3, we present the contribution to core damage frequency from the various accident sequences conditional on both earthquake and initiating event frequency. Clearly, the increase from level 1 to level 6 is only about the factor of 4 described above. This increase is due entirely to the condensate storage tank failure, which is the only seismically induced failure which survived probabilistic culling. The CST failure probability increased from a level 1 failure probability of 1.6E-3 to a level 6 failure probability of $1.2 \mathrm{E}-1$. It should be noted that after our analysis of the RMIEP fault trees was completed, RMIEP dici make a change to the fault trees that would reduce the significance of the CST.

Second, we experience little increase in the initiating event probabilities with earthquake level 
because, as explained in Sec. 9.1, we assume a loss-of-offsite power to occur at all carthquake levels. This transient, together with the random failure of the SRVs to reclose, was the primary initiating event in our analysis. Thus, we find that the assumption of experiencing a loss-of-offsite power at all eurthquake levels resuits in the dominance of risk by the lower earthquake levels. Although the result is a direct consecjuence of our assumption, we believe the assumption to be appropriate.

One last important note involves $A C$ power recovery, either through repair of the diesel generators or associated equipment or through reestablishment of offsite power. We have not taken into account the possibility of $A C$ power recovery in our analysis. We have done so primarily because the logic models from RMIEP included no provision for recovery action, but also because we feel that there are large uncertuinties attached to the chance for early recovery of random loss of emergency AC or seismic loss-of-offsite power. Recovery actions will be evaluated in the RMIEP analysis.

\subsection{Duminant Accident Sequences}

The breakdown, by initiating events, of the core damage frequency is given in Table 11.4. The dominance of the transients is expected given that, as can be seen in Table 11.3, the accident sequences do not have widely varying probabilities of occurrence, conditional on the initiating event and earthquake level. Thus, we would expect the risk to be dominated according to the relative probability of the different initiator classes. When the initiating event probabilities are folded in, as in Table 11.2, their effect is clearly seen in the relative contribution of the different accident sequences.

In the right-hand column of Table 11.1 , it can be seen that the three most dominant accident sequences are the three loss-of-offsite power transient sequences, contributing roughly 90 percent of the total core damage frequency. The most dominant sequence is the LOSP-Trans-3, responsible for 2.5E-7/yr (42 percent of Total). The next most probable non-LOSP accident sequence is SmallLOCA-3 (3.1E-8/yr), which is nearly an order of magnitude less probable than the LOSP-Trans-3 sequence.

Interestingly, there is some variation of dominance with earthquake level. From Table 11.1, it can be seen that LOSP-Trans-4 dominates at the first earthquake level, but that LOSP-Trans-3 dominates at levels 2 through 6 . This is due to the fact that LOSP-Trans-3 has the Condendsate Storage Tank as one of its dominant contributors. As earthquake level increases, the probability of tank failure increases as well, causing the sequence probability to increase. LOSP-Trans-4 has only a small contribution from the Condensate Storage Tank and, therefore, does not increase as rapidly with earthquake level. A full discussion of the dominant equipment failures is given in Sec. 11.3.

\subsection{Cominant Component Failures}

We now examine the basic equipment procedural failures which dominate the seismically induced core damage risk at LaSalle. This information is perhaps the most useful, since it points to specific equipment procedures which can be studied further to assess their likely performance during and 
Table 11.1. Unconditional accident sequence frequencies.

Earthquake Level

Accident

$\begin{array}{lllllll}\text { Sequence Level } 1 & \text { Level } 2 & \text { Level } 3 & \text { Level } 4 & \text { Level } 5 & \text { Level } 6 & \text { Total }\end{array}$

\begin{tabular}{|c|c|c|c|c|c|c|c|}
\hline $\begin{array}{l}\text { Large-LOCA-1 } \\
\text { Large-LOCA-2 } \\
\text { Largc-LOCA-3 } \\
\text { Subtolal }\end{array}$ & $\begin{array}{l}1.8 \mathrm{E}-11 \\
0.0 \mathrm{E}+0 \\
0.0 \mathrm{E}+0 \\
1.8 \mathrm{E}-11\end{array}$ & $\begin{array}{l}8.4 \mathrm{E}-12 \\
0.0 \mathrm{E}+0 \\
0.0 \mathrm{E}+0 \\
8.4 \mathrm{E}-12\end{array}$ & $\begin{array}{l}4.9 \mathrm{E}-12 \\
0.0 \mathrm{E}+0 \\
0.0 \mathrm{E}+0 \\
4.9 \mathrm{E} \cdot 12\end{array}$ & $\begin{array}{l}3.0 \mathrm{E}-12 \\
0.0 \mathrm{E}+0 \\
0.0 \mathrm{E}+0 \\
3 .(\mathrm{OE}-12\end{array}$ & $\begin{array}{l}1.8 \mathrm{E}-12 \\
0.0 \mathrm{E}+0 \\
0.0 \mathrm{E}+0 \\
1.8 \mathrm{E}-12\end{array}$ & $\begin{array}{l}1.1 \mathrm{E}-12 \\
0.0 \mathrm{E}+0 \\
0.0 \mathrm{E}+0 \\
1.1 \mathrm{E}-12\end{array}$ & $\begin{array}{l}3.8 \mathrm{E}-11 \\
0.0 \mathrm{E}+0 \\
0.0 \mathrm{E}+0 \\
3.8 \mathrm{E}-11\end{array}$ \\
\hline $\begin{array}{l}\text { Medium-LOCA-1 } \\
\text { Medium-LOCA-2 } \\
\text { Medium-LOCA-3 } \\
\text { Medium-LOCA-4 } \\
\text { Sublotal }\end{array}$ & $\begin{array}{l}\text { 6.9E-10 } \\
7.8 \mathrm{E}-10 \\
0 .(\mathrm{E}+0 \\
(0 .(\mathrm{DE}+() \\
1.5 \mathrm{E}-9\end{array}$ & $\begin{array}{l}3.1 \mathrm{E}-10 \\
2.2 \mathrm{E}-10 \\
0 .(0 \mathrm{E}+() \\
0.0 \mathrm{E}+() \\
5.3 \mathrm{E}-10\end{array}$ & $\begin{array}{l}1.8 \mathrm{E}-10 \\
9.2 \mathrm{E}-11 \\
0.0 \mathrm{E}+0 \\
0.0 \mathrm{E}+0 \\
2.7 \mathrm{E}-10\end{array}$ & $\begin{array}{l}1.1 \mathrm{E}-10 \\
4.4 \mathrm{E}-11 \\
0.0 \mathrm{E}+0 \\
0.0 \mathrm{E}+0 \\
1.5 \mathrm{E}-10\end{array}$ & $\begin{array}{l}6.9 \mathrm{E}-11 \\
2.2 \mathrm{E}-11 \\
0.0 \mathrm{E}+0 \\
0.0 \mathrm{E}+0 \\
9.1 \mathrm{E}-11\end{array}$ & $\begin{array}{l}4.3 \mathrm{E}-11 \\
1.2 \mathrm{E}-11 \\
0.0 \mathrm{E}+0 \\
0.0 \mathrm{E}+0 \\
4.5 \mathrm{E}-11\end{array}$ & $\begin{array}{l}1.4 \mathrm{E}-9 \\
1.2 \mathrm{E}-9 \\
0.0 \mathrm{E}+0 \\
0.0 \mathrm{E}+0 \\
2.6 \mathrm{E}-9\end{array}$ \\
\hline $\begin{array}{c}\text { Small-LOCA-1 } \\
\text { Small-LOCA-2 } \\
\text { Small-LOCA-3 } \\
\text { Small-LOCA-4 } \\
\text { Small-LOCA-5 } \\
\text { Small-LOCA-6 } \\
\text { Sublotal }\end{array}$ & $\begin{array}{l}4.6 E-9 \\
0 .(1 E+0 \\
1.5 E-8 \\
1.7 E-8 \\
0 .(1 E+0 \\
0 .(0 E+0 \\
3.7 E-8\end{array}$ & $\begin{array}{l}1.9 \mathrm{E}-9 \\
0 .(0 \mathrm{E}+0 \\
6.9 \mathrm{E}-9 \\
4.9 \mathrm{E}-9 \\
0.0 \mathrm{E}+(0 \\
0.0 \mathrm{E}+0 \\
1.4 \mathrm{E}-8\end{array}$ & $\begin{array}{l}9.6 \mathrm{E}-10 \\
0.0 \mathrm{E}+0 \\
4.0 \mathrm{E}-9 \\
2.0 \mathrm{E}-9 \\
0.0 \mathrm{E}+() \\
0 .(0 \mathrm{E}+0 \\
7.0 \mathrm{E}-9\end{array}$ & $\begin{array}{l}4.9 \mathrm{E}-10 \\
0.0 \mathrm{E}+0 \\
2.5 \mathrm{E}-9 \\
9.7 \mathrm{E}-10 \\
0.0 \mathrm{E}+0 \\
0.0 \mathrm{E}+0 \\
4.0 \mathrm{E}-9\end{array}$ & $\begin{array}{l}2.3 \mathrm{E}-10 \\
0.0 \mathrm{E}+0 \\
1.5 \mathrm{E}-9 \\
4.9 \mathrm{E}-10 \\
0.0 \mathrm{E}+0 \\
0.0 \mathrm{E}+0 \\
2.2 \mathrm{E}-9\end{array}$ & $\begin{array}{l}9.0 \mathrm{E}-11 \\
0.0 \mathrm{E}+0 \\
9.4 \mathrm{E}-1 \\
2.6 \mathrm{E}-10 \\
0.0 \mathrm{E}+0 \\
0.0 \mathrm{E}+0 \\
1.3 \mathrm{E}-9\end{array}$ & $\begin{array}{l}8.2 \mathrm{E}-9 \\
0.0 \mathrm{E}+0 \\
3.1 \mathrm{E}-8 \\
2.6 \mathrm{E}-8 \\
0.0 \mathrm{E}+0 \\
0.0 \mathrm{E}+0 \\
6.5 \mathrm{E}-8\end{array}$ \\
\hline $\begin{array}{c}\text { LOSP-Trans-1 } \\
\text { LOSP-Trans-2 } \\
\text { LOSP-Trans-3 } \\
\text { LOSP.Trans-4 } \\
\text { LOSP-Trans-5 } \\
\text { LOSP.Trans-6 } \\
\text { LOSP-Trans-7 } \\
\text { Subtotal }\end{array}$ & $\begin{array}{l}3.7 \mathrm{E}-8 \\
0 .(0 \mathrm{E}+() \\
1.2 \mathrm{E}-7 \\
1.4 \mathrm{E}-7 \\
0.0 \mathrm{E}+0 \\
0 .(\mathrm{EE}+() \\
0 .(0 \mathrm{E}+() \\
3 .(0 \mathrm{E}-7\end{array}$ & $\begin{array}{l}1.5 \mathrm{E}-8 \\
0 .(\mathrm{E}+0 \\
5.6 \mathrm{E}-8 \\
4.0 \mathrm{E}-8 \\
0 .(0 \mathrm{E}+() \\
0.0 \mathrm{E}+(1) \\
0 .(\mathrm{E}+0 \\
1.1 \mathrm{E}-7\end{array}$ & $\begin{array}{l}7.8 \mathrm{E}-9 \\
0.0 \mathrm{E}+0 \\
3.3 \mathrm{E}-8 \\
1.6 \mathrm{E}-8 \\
0.0 \mathrm{E}+0 \\
0.0 \mathrm{E}+0 \\
0.0 \mathrm{E}+0 \\
5.7 \mathrm{E}-8\end{array}$ & $\begin{array}{l}4.0 \mathrm{E}-9 \\
0.0 \mathrm{E}+0 \\
2.0 \mathrm{E}-8 \\
7.9 \mathrm{E}-9 \\
0.0 \mathrm{E}+0 \\
0.0 \mathrm{E}+0 \\
0.0 \mathrm{E}+0 \\
3.2 \mathrm{E}-8\end{array}$ & $\begin{array}{l}1.8 \mathrm{E}-9 \\
0.0 \mathrm{E}+0 \\
1.2 \mathrm{E}-8 \\
3.9 \mathrm{E}-9 \\
0.0 \mathrm{E}+0 \\
0.0 \mathrm{E}+0 \\
0.0 \mathrm{E}+0 \\
1.8 \mathrm{E}-8\end{array}$ & $\begin{array}{l}7.3 \mathrm{E}-10 \\
0.0 \mathrm{E}+0 \\
7.7 \mathrm{E}-9 \\
2.1 \mathrm{E}-9 \\
0.0 \mathrm{E}+0 \\
0.0 \mathrm{E}+0 \\
0.0 \mathrm{E}+0 \\
1.1 \mathrm{E}-8\end{array}$ & $\begin{array}{l}6.7 \mathrm{E}-8 \\
0.0 \mathrm{E}+0 \\
2.5 \mathrm{E}-7 \\
2.1 \mathrm{E}-7 \\
0.0 \mathrm{E}+0 \\
0.0 \mathrm{E}+0 \\
0.0 \mathrm{E}+0 \\
5.3 \mathrm{E}-7\end{array}$ \\
\hline Grand Total & $3.4 \mathrm{E} \cdot 7$ & $1.3 \mathrm{E}-7$ & $6.4 \mathrm{E}-8$ & $3.6 \mathrm{E}-8$ & $2.0 \mathrm{E}-8$ & $1.2 \mathrm{E}-8$ & $6.0 \mathrm{E}-7$ \\
\hline
\end{tabular}

Nole: Estimates listed as $0.0 \mathrm{E}+0$ actually represent insignificant, but finite, values. 
Table 11.2. Accident sequence probabilities conditional on earthquake probability.

Earthquake Level

Accident Sequence Level $1 \quad$ Level 2 Level 3 Level 4 Level 5 Level 6 Total

\begin{tabular}{|c|c|c|c|c|c|c|}
\hline $\begin{array}{l}\text { Large-LOCA-1 } \\
\text { Large-LOCA-1 } \\
\text { Large-LOCA-2 } \\
\text { Subtotal }\end{array}$ & $\begin{array}{l}1.7 \mathrm{E}-7 \\
0.0 \mathrm{E}+0 \\
0.0 \mathrm{E}+0 \\
1.9 \mathrm{E}-7\end{array}$ & $\begin{array}{l}2.9 \mathrm{E}-7 \\
0.0 \mathrm{E}+0 \\
0.0 \mathrm{E}+0 \\
2.9 \mathrm{E}-7\end{array}$ & $\begin{array}{l}4.5 E-7 \\
0.0 \mathrm{E}+0 \\
0.0 \mathrm{E}+0 \\
4.5 \mathrm{E}-7\end{array}$ & $\begin{array}{l}6.3 \mathrm{E}-7 \\
0.0 \mathrm{E}+0 \\
0.0 \mathrm{E}+0 \\
6.3 \mathrm{E}-7\end{array}$ & $\begin{array}{l}\text { 8.7E-7 } \\
0.0 \mathrm{E}+0 \\
0.0 \mathrm{E}+0 \\
8.7 \mathrm{E}-7\end{array}$ & $\begin{array}{l}1.1 \mathrm{E}-6 \\
0.0 \mathrm{E}+0 \\
0.0 \mathrm{E}+0 \\
1.1 \mathrm{E}-6\end{array}$ \\
\hline $\begin{array}{l}\text { Medium-LOCA-1 } \\
\text { Medium-LOCA-2 } \\
\text { Medium-LOCA-3 } \\
\text { Medium-LOCA-4 } \\
\text { Subtotal }\end{array}$ & $\begin{array}{l}6 . S E-6 \\
7.2 \mathrm{E}-6 \\
0.0 \mathrm{E}+0 \\
0.0 \mathrm{E}+0 \\
1.4 \mathrm{E}-5\end{array}$ & $\begin{array}{l}1.1 \mathrm{E}-5 \\
7.8 \mathrm{E}-6 \\
0.0 \mathrm{E}+0 \\
0.0 \mathrm{E}+0 \\
1.8 \mathrm{E}-5\end{array}$ & $\begin{array}{l}1.7 \mathrm{E}-5 \\
8.5 \mathrm{E}-6 \\
0.0 \mathrm{E}+0 \\
0.0 \mathrm{E}+0 \\
2.6 \mathrm{E}-5\end{array}$ & $\begin{array}{l}2.4 E-5 \\
9.3 E-6 \\
0.0 E+0 \\
0.0 E+0 \\
3.3 E-5\end{array}$ & $\begin{array}{l}3.3 \mathrm{E}-5 \\
1.0 \mathrm{E}-5 \\
0.0 \mathrm{E}+0 \\
0.0 \mathrm{E}+0 \\
4.3-5\end{array}$ & $\begin{array}{l}4.2 \mathrm{E}-5 \\
1.2 \mathrm{E}-5 \\
0.0 \mathrm{E}+0 \\
0.0 \mathrm{E}+0 \\
5.4 \mathrm{E}-5\end{array}$ \\
\hline $\begin{array}{c}\text { Small-LOCA-1 } \\
\text { Small-LOCA-2 } \\
\text { Small-LOCA-3 } \\
\text { Small-LOCA-4 } \\
\text { Small-LOCA-5 } \\
\text { Small-LOCA-6 } \\
\text { Subtotal }\end{array}$ & $\begin{array}{l}4.3 \mathrm{E}-5 \\
0.0 \mathrm{E}+0 \\
1.4 \mathrm{E} 4 \\
1.6 \mathrm{E}-4 \\
0.0 \mathrm{E}+0 \\
0.0 \mathrm{E}+0 \\
3.4 \mathrm{E}-4\end{array}$ & $\begin{array}{l}6.6 \mathrm{E}-5 \\
0.0 \mathrm{E}+0 \\
2.4 \mathrm{E}-4 \\
1.7 \mathrm{E}-4 \\
0.0 \mathrm{E}+0 \\
0.0 \mathrm{E}+0 \\
4.8 \mathrm{E}-4\end{array}$ & $\begin{array}{l}8.9 \mathrm{E}-5 \\
0.0 \mathrm{E}+0 \\
3.7 \mathrm{E}-4 \\
1.9 \mathrm{E}-4 \\
0.0 \mathrm{E}+0 \\
0.0 \mathrm{E}+0 \\
6.5 \mathrm{E}-4\end{array}$ & $\begin{array}{l}1.0 \mathrm{E}-4 \\
0.0 \mathrm{E}+0 \\
5.2 \mathrm{E}-4 \\
2.1 \mathrm{E}-4 \\
0.0 \mathrm{E}+0 \\
0.0 \mathrm{E}+0 \\
8.3 \mathrm{E}-4\end{array}$ & $\begin{array}{l}1.1 \mathrm{E}-4 \\
0.0 \mathrm{E}+0 \\
7.2 \mathrm{E}-4 \\
2.3 \mathrm{E}-4 \\
0.0 \mathrm{E}+0 \\
0.0 \mathrm{E}+0 \\
1.1 \mathrm{E}-3\end{array}$ & $\begin{array}{l}8.8 \mathrm{E}-5 \\
0.0 \mathrm{E}+0 \\
9.3 \mathrm{E}-4 \\
2.5 \mathrm{E}-4 \\
0.0 \mathrm{E}+0 \\
0.0 \mathrm{E}+0 \\
1.3 \mathrm{E}-3\end{array}$ \\
\hline $\begin{array}{c}\text { LOSP-Trans-1 } \\
\text { LOSP-Trans-2 } \\
\text { LOSP-Trans-3 } \\
\text { LOSP-Trans-4 } \\
\text { LOSP-Trans-5 } \\
\text { LOSP-Trans-6 } \\
\text { LOSP-Trans-7 } \\
\text { Subtotal }\end{array}$ & $\begin{array}{l}3.5 \mathrm{E}-4 \\
0.0 \mathrm{E}+0 \\
1.2 \mathrm{E}-3 \\
1.3 \mathrm{E}-3 \\
0.0 \mathrm{E}+0 \\
0.0 \mathrm{E}+0 \\
0.0 \mathrm{E}+0 \\
2.9 \mathrm{E}-3\end{array}$ & $\begin{array}{l}5.4 E-4 \\
0.0 E+() \\
2.0 E-3 \\
1.4 E-3 \\
0.0 E+0 \\
0.0 E+0 \\
0.0 E+0 \\
3.9 E-3\end{array}$ & $\begin{array}{l}7.3 E-4 \\
0.0 E+0 \\
3.0 E-3 \\
1.5 E-3 \\
0.0 E+0 \\
0.0 E+0 \\
0.0 E+0 \\
5.2 E-3\end{array}$ & $\begin{array}{l}8.5 E-4 \\
0.0 E+0 \\
4.2 E-3 \\
1.7 E-3 \\
0.0 E+0 \\
0.0 E+0 \\
0.0 E+0 \\
6.8 E-3\end{array}$ & $\begin{array}{l}8.7 E-4 \\
0.0 \mathrm{E}+0 \\
5.8 \mathrm{E}-3 \\
1.9 \mathrm{E}-3 \\
0.0 \mathrm{E}+0 \\
0.0 \mathrm{E}+0 \\
0.0 \mathrm{E}+0 \\
8.6 \mathrm{E}-3\end{array}$ & $\begin{array}{l}7.2 \mathrm{E}-4 \\
0.0 \mathrm{E}+0 \\
7.5 \mathrm{E}-3 \\
2.1 \mathrm{E}-3 \\
0.0 \mathrm{E}+0 \\
0.0 \mathrm{E}+0 \\
0.0 \mathrm{E}+0 \\
1.0 \mathrm{E}-2\end{array}$ \\
\hline GrandTotal & $3.2 \mathrm{E}-3$ & $4.4 \mathrm{E}-3$ & $6.0 \mathrm{E}-3$ & $7.6 \mathrm{E}-3$ & $9.7 \mathrm{E}-3$ & $1.2 \mathrm{E}-2$ \\
\hline
\end{tabular}

Note: Estimates listed as $0.0 \mathrm{E}+0$ actually represent insignificant, but finite, values. 
Table 11.3. Accident sequence probabilities conditional on initiator and earthquake probability.

Earthquake Level

Accident

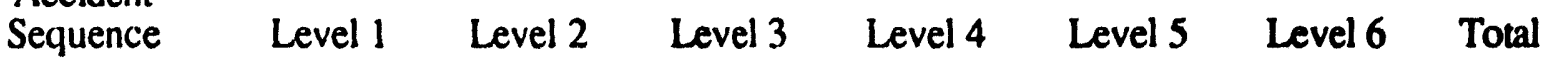

$\begin{array}{lllllll}\text { Large-LOCA-1 } & 1.4 \mathrm{E}-3 & 2.4 \mathrm{E}-3 & 3.8 \mathrm{E}-3 & 5.2 \mathrm{E}-3 & 7.2 \mathrm{E}-3 & 9.3 \mathrm{E}-3 \\ \text { Large-LOCA-1 } & 0.0 \mathrm{E}+0 & 0.0 \mathrm{E}+0 & 0.0 \mathrm{E}+0 & 0.0 \mathrm{E}+0 & 0.0 \mathrm{E}+0 & 0.0 \mathrm{E}+0 \\ \text { Large-LOCA-2 } & 0.0 \mathrm{E}+0 & 0.0 \mathrm{E}+0 & 0.0 \mathrm{E}+0 & 0.0 \mathrm{E}+0 & 0.0 \mathrm{E}+0 & 0.0 \mathrm{E}+0 \\ \text { Subtotal } & 1.4 \mathrm{E}-3 & 2.4 \mathrm{E}-3 & 3.8 \mathrm{E}-3 & 5.2 \mathrm{E}-3 & 7.2 \mathrm{E}-3 & 9.3 \mathrm{E}-3 \\ & & & & & & \\ \text { Medium-LOCA-1 } & 1.4 \mathrm{E}-3 & 2.4 \mathrm{E}-3 & 3.8 \mathrm{E}-3 & 5.2 \mathrm{E}-3 & 7.2 \mathrm{E}-3 & 9.3 \mathrm{E}-3 \\ \text { Mcdium-LOCA-2 } & 1.6 \mathrm{E}-3 & 1.7 \mathrm{E}-3 & 1.9 \mathrm{E}-3 & 2.1 \mathrm{E}-3 & 2.3 \mathrm{E}-3 & 2.6 \mathrm{E}-3 \\ \text { Medium-LOCA-3 } & 0.0 \mathrm{E}+0 & 0.0 \mathrm{E}+0 & 0.0 \mathrm{E}+0 & 0.0 \mathrm{E}+0 & 0.0 \mathrm{E}+0 & 0.0 \mathrm{E}+0 \\ \text { Medium-LOCA-4 } & 0.0 \mathrm{E}+0 & 0.0 \mathrm{E}+0 & 0.0 \mathrm{E}+0 & 0.0 \mathrm{E}+0 & 0.0 \mathrm{E}+0 & 0.0 \mathrm{E}+0 \\ \text { Subtotal } & 3.0 \mathrm{E}-3 & 4.1 \mathrm{E}-3 & 5.7 \mathrm{E}-3 & 7.3 \mathrm{E}-3 & 9.5 \mathrm{E}-3 & 1.2 \mathrm{E}-2 \\ & & & & & & \\ \text { Small-LOCA-1 } & 4.3 \mathrm{E}-4 & 6.6 \mathrm{E}-4 & 8.9 \mathrm{E}-4 & 1.0 \mathrm{E}-3 & 1.1 \mathrm{E}-3 & \mathbf{8 . 8 \mathrm { E } - 4} \\ \text { Small-LOCA-2 } & 0.0 \mathrm{E}+0 & 0.0 \mathrm{E}+0 & 0.0 \mathrm{E}+0 & 0.0 \mathrm{E}+0 & 0.0 \mathrm{E}+0 & 0.0 \mathrm{E}+0 \\ \text { Small-LOCA-3 } & 1.4 \mathrm{E}-3 & 2.4 \mathrm{E}-3 & 3.8 \mathrm{E}-3 & 5.2 \mathrm{E}-3 & 7.2 \mathrm{E}-3 & 9.3 \mathrm{E}-3 \\ \text { Small-LOCA-4 } & 1.6 \mathrm{E}-3 & 1.7 \mathrm{E}-3 & 1.9 \mathrm{E}-3 & 2.1 \mathrm{E}-3 & 2.3 \mathrm{E}-3 & 2.6 \mathrm{E}-3 \\ \text { Small-LOCA-5 } & 0.0 \mathrm{E}+0 & 0.0 \mathrm{E}+0 & 0.0 \mathrm{E}+0 & 0.0 \mathrm{E}+0 & 0.0 \mathrm{E}+0 & 0.0 \mathrm{E}+0 \\ \text { Small-LOCA-6 } & 0.0 \mathrm{E}+0 & 0.0 \mathrm{E}+0 & 0.0 \mathrm{E}+0 & 0.0 \mathrm{E}+0 & 0.0 \mathrm{E}+0 & 0.0 \mathrm{E}+0 \\ \text { Subtotal } & 3.4 \mathrm{E}-3 & 4.8 \mathrm{E}-3 & 6.6 \mathrm{E}-3 & 8.3 \mathrm{E}-3 & 1.1 \mathrm{E}-2 & 1.3 \mathrm{E}-2 \\ & & & & & & \\ & & & & & & \\ \text { LOSP-Trans-1 } & 3.9 \mathrm{E}-4 & 6.0 \mathrm{E}-4 & 8.1 \mathrm{E}-4 & 9.5 \mathrm{E}-4 & 9.7 \mathrm{E}-4 & 8.0 \mathrm{E}-4 \\ \text { LOSP-Trans-2 } & 0.0 \mathrm{E}+0 & 0.0 \mathrm{E}+0 & 0.0 \mathrm{E}+0 & 0.0 \mathrm{E}+0 & 0.0 \mathrm{E}+0 & 0.0 \mathrm{E}+0 \\ \text { LOSP-Trans-3 } & 1.3 \mathrm{E}-3 & 2.2 \mathrm{E}-3 & 3.4 \mathrm{E}-3 & 4.7 \mathrm{E}-3 & 6.5 \mathrm{E}-3 & 8.4 \mathrm{E}-3 \\ \text { LOSP-Trans-4 } & 1.4 \mathrm{E}-3 & 1.6 \mathrm{E}-3 & 1.7 \mathrm{E}-3 & 1.9 \mathrm{E}-3 & 2.1 \mathrm{E}-3 & 2.3 \mathrm{E}-3 \\ \text { LOSP-Trans-5 } & 0.0 \mathrm{E}+0 & 0.0 \mathrm{E}+0 & 0.0 \mathrm{E}+0 & 0.0 \mathrm{E}+0 & 0.0 \mathrm{E}+0 & 0.0 \mathrm{E}+0 \\ \text { LOSP-Trans-6 } & 0.0 \mathrm{E}+0 & 0.0 \mathrm{E}+0 & 0.0 \mathrm{E}+0 & 0.0 \mathrm{E}+0 & 0.0 \mathrm{E}+0 & 0.0 \mathrm{E}+0 \\ \text { LOSP-Trans-7 } & 0.0 \mathrm{E}+0 & 0.0 \mathrm{E}+0 & 0.0 \mathrm{E}+0 & 0.0 \mathrm{E}+0 & 0.0 \mathrm{E}+0 & 0.0 \mathrm{E}+0 \\ \text { Subtotal } & 3.1 \mathrm{E}-3 & 4.4 \mathrm{E}-3 & 5.9 \mathrm{E}-3 & 7.6 \mathrm{E}-3 & 9.6 \mathrm{E}-3 & 1.2 \mathrm{E}-2\end{array}$

Note: Estimates listed as $0.0 \mathrm{E}+0$ actually represent insignificant, but finite, values. 
after an earthquake. We describe the dominant components associated with each of the three most dominant sequences.

\title{
Dominant Sequence \#1--LOSP-3
}

The most dominant sequence in our analysis is the LOSP-Trans-3 sequence. This sequence involves successful operation of the Reactor Protection System (RPS) as well as the Safety Relief Valve (SRV) functions. Following successful reclosure of all the SRVs, both the High Pressure Core Spray (HPCS) and the Reactor Core Isolation Cooling (RCIC) systems fail due to a seismic failure of the condensate storage tank. The Automatic Depressurization System (ADS) successfully depressurizes the reactor vessel, however, both the Low Pressure Coolant Injection (LPCI) and the Low Pressure Core Spray (LPCS) systems fail due to diesel generator and bus failures as well as relay coil and circuit breaker faults, leading to a complete failure to inject coolant and an eventual core damage state.

There were twelve cut sets which survived probabilistic culling for the LOSP-Trans-3 sequence, all with roughly the same contribution. Below, we list the 8 events which combine to form the twelve sequence cut sets. We also include the event probabilities and descriptions of the events.

\section{Condensate Storage Tank Seismic Failure}

\author{
Fault Tree Identifier: \\ Systems Affected: \\ CST-TNK-.-.-.-.-9 \\ HPCS, RCIC
}

Event Description. This event represents the gross failure of the condensate storage tank, with a complete loss of its contents. This is the only seismically induced failure which contributes significantly to the risk of core damage at LaSalle. In fact, it is the only basic event involving a seismic failure mode which survived the probabilistic culling process. The tank has a rather low seismic strength; it is not a Class I equipment item.

The tank acts in this sequence as a singleton for the High Pressure Core Spray (HPCS) system. As modeled, if the tank should suffer a gross failure, the HPCS pumps would burn out from a lack of suction. There is apparently no interlock to prevent this from occurring or else, whatever interlocks do exist are considered to be defeated by the gross failure of the tank. Thus, although the HPCS has an alternate suction supply (the Suppression Pool) a rapid loss of coolant from the CST effectively acts as a singleton for the HPCS system. As indicated earlier in this section, RMIEP analysts have modified the way the CST is handled in the fault trees after our analysis was complete. 
Table 11.4. Core damage frequency contribution by initiating events.

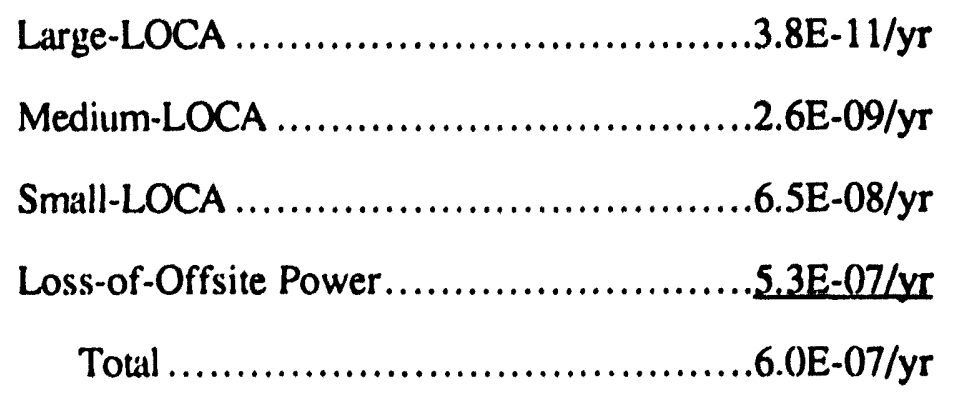




\title{
Diesel Generator Failures
}

\author{
Fault Tree Identifier: $\quad$ DG2A-GEN-LF----0 \\ Systems Affected: $\quad$ CSS,LCI,SCS,SPC \\ Fault Tree Identifier: $\quad$ DG2B-GEN-LF-..-0 \\ Systems Affected: HPCS \\ Fault Tree Identifier: $\quad$ DG0-GEN-LF----0 \\ Systems Affected: $\quad$ CSS,LCI,LCS,RCI,SCS,SPC \\ Fault Tree Identifier: $\quad$ DG-CM-.......... \\ Systems Affected:
}

Event Description. These consist of several types of diesel generator and bus failures which combine to effectively cut off all emergency power to the safety systems which fail in this sequence. These failures are largely common cause failures which contribute to the failure of more than one safety system.

\section{Relay Coil and Circuit Breaker Faults}

$\begin{array}{lll}\text { Fault Tree Identifier: } & \text { AP037X3-ROO-LFO0 } & \text { DOVB101X-BCO-LF0 } \\ & \text { AP039X2-ROO-LFO0 } & \text { DOVB 102X-BCO-LF0 } \\ & \text { AP040X2-ROO-LFO0 } & \text { DOVB201X-BCO-LF0 } \\ & \text { AP040X3-ROO-LFO0 } & \text { D0VB202X-BCO-LF0 } \\ & & \end{array}$

Event Description. Failure of these components leads to loss of the emergency AC power which, together with the assumed loss-of-offsite power, leads to total station blackout and failure of several systems.

\section{Dominant Sequence \#2-LOSP-Trans-4}

The sequence LOSP-4 is nearly as dominant as LOSP-Trans-3. This accident sequence involves successful operation of the Reactor Protection System and the Safety Relief Valves, followed by failure of all high pressure coolant makeup (HPCS and RCIC) due to reactor level instrumentation or the condensate storage tank, and a failure of the Automatic Depressurization System (ADS) due to operator error and reactor water level instrumentation, which prevents use of the low pressure coolant makeup systems.

This sequence has only two cut sets which survived probabilistic culling and, of those, one is highly dominant. The elements of that cut sets are described below: 
Operator Error
Fault Tree Identifier: Systems Affected:
ADSMINIT-QOO-OE- ADS

Event Description. This event represents failure of the reactor operators to manually initiate the Automatic Depressurization System following the loss of the reactor level instrumentation reference legs that are necessary for automatic operation of the ADS.

\section{Reactor Water Level Instrumentation
Fault Tree Identifier: Systems Affected:
T14-IE-..........
ADS, HPCS, RCIC

Event Description. This event represents the failure of the reactor water level instrumentation reference legs. Failure of both legs leads to a complete loss of reactor level water monitoring, which, in turn, leads to a failure to automatically initiate the HPCS and RCIC systems. The random failure probability which was used for this event was significantly lowered by the RMIEP, but not until we had completed our calculations. The revised estimate is 3 orders of magnitude lower than the value we used. Substituting this new estimate into our calculations would have lowered the risk contributed by this sequence by about a factor of 10 , and reduce the total seismic risk by about 25 percent to about $4.5 E-7$.

The other cut set of this sequence involves loss of the HPCS and RCIC systems through the failure of the Condensate Storage Tank and failure of the ADS through a combined failure to manually initiate and failure of reactor level reference leg 15B.

\section{Dominant Sequence \#3--LOSP-Trans-1}

The remaining dominant sequence, LOSP-Trans-1, is a rather involved accident sequence. Following successful operation of the Reactor Protection System and Safety Relief Valves, successful high pressure coolant makeup is accomplished through the Reactor Core Isolation Cooling system, despite failure of the High Pressure Core Spray system. However, failure of the containment heat removal systems (SCS, SPC, and CSS), combined with successful ADS operation and failure of the low pressure coolant makeup systems eventually leads to a core damage state.

Of the three dominant sequences, this sequence is probably the most sensitive to our assumptions about AC power recovery since it is probably has the longest duration before core damage occurrence. The dominating basic events for this sequence are largely the same as those for LOSPTrans-3, which are described earlier in this section. 


\section{Section 12. Conclusions}

This study had several goals. These included performing a seismic risk analysis of a BWR, exercise the simplified methodology developed by the SSMRP, comparing the seismic risks of a BWR and PWR, and comparing the differences resulting from the use of alternate event and fault trees vs the RMIEP event and fault trees.

\subsection{Comparison of BWR Seismic Risk to PWR Seismic Risk}

One of the goals noted above was to provide a basis for making a comparison of the relative seismic susceptibility of boiling water reactors and pressurized water reactors; the Zion study providing the information about PWRs. It is, of course, difficult to make a generalized comparison of two broad reactor classes based upon a limited amount of information. However, there are conclusions which can be drawn from these two studies.

- Hydrodynamic loads, which may be experienced in BWRs during an earthquake, are not significant at LaSalle. Jet forces and pool swell are very localized hydrodynamic loads and their inclusion did not significantly affect the LaSalle seismic risk analysis results. Chugging hydrodynamic loads occur late in time with respect to earthquake strong motion, therefore, chugging load responses need not be combined with the seismic load responses. SRV asymmetric horizontal loadings are small when compared with the horizontal earthquake loads. The hydrodynamic loads that were included in the analysis ranged from $0.0 \mathrm{~g}$ to $.14 \mathrm{~g}$. The average hydrodynamic load (averaged over all responses) was $.04 \mathrm{~g}$. This type of load is absent from PWRs because of the lack a suppression pool and the associated downcomer piping.

- The combination of structure and foundation configuration with the LaSalle soil profile led to considerably lower seismic response as compared to Zion. It is interesting to note that the increase in amplitude from acceleration range 1 to acceleration range 2 is small. This is due, principally, to the soft soil profile and its effect on the free-field ground motion and on the dominant frequencies of the soilstructure system. The Zion study predicted that the lack of a common foundation could lead to relative displacements between buildings and a potential for common mode failure of critical piping which passes from one building to another.

\subsection{General Remarks}

We summarize here with several points which we feel should be emphasized regarding our seismic analysis of LaSalle.

- The only seismic initiating event of significance at LaSalle is the LOSP, which is assumed to occur. The LOCAs examined were dominated by the random failure of the SRVs to reclose following the LOSP transient. This is true because the probability of NSSS piping failure was very small so therefore it did not contribute 
to the probability of a LOCA occuring.

- $\quad$ Our assumption of LOSP proved to be important. The high probability of random failure of the diesel generators leads to a high probability of station blackout following loss-of-offsite power. The assumption of an LOSP, and the further assumption that offsite power will not be restored for the duration of the reactor shutdown period, should be studied in more detail to determine if these assumptions are warranted.

- The major differences between the additional and RMIEP analyses were caused by differing modeling assumptions used in constructing the event trees and fault trees. The event tree differences were dominated by two assumptions. In the additional analysis, it was assumed that RHR was required to prevent core damage. This was not the case for the RMIEP analysis. A second assumption was that for the RMIEP event trees, Small-I.OCA and LOSP-Trans, allowed for the ADS to be used in the event SPC and CSS fail. The additional event trees did not consider this.

The only seismically-induced component failure of significance in the RMIEP analysis is the failure of the condensite storage tank (CST). No seismically-induced component failures were found to be significant in the additional analysis. Random failures dominated. It was assumed in the RMIEP analysis that failure of the CST would cause failure of both the HPCS and RCIC pumps because of loss of suction. This assumption was not made in the additional analysis. As mentioned earlier in this report, after our analysis of the RMIEP faun trees was completed, RMIEP did make a change to the fault trees that would reduce the significance of the CST.

- It is not clear whether greater detail in the event and fault trees leads to higher or lower risk estimates. In our study, we have found that the detailed modeling produced a lower risk estimate. But we do feel that uncertainty associated with these risk estimates will be greater in a more simplified analysis.

- The LaSalle seismic PRA documented in this report was completed in 1987. Since then there were advances in the state-of-the-art in soil-structure interaction analysis, fragility development, and seismic hazard estimates. This report was updated in 1992 to address the effects of these advances on the results.

In SSI analysis, SASSI code based on finite element approach is replacing CLASSI, a continuum mechanics code used in LaSalle, to become a more widely used computer program. CLASSI is linited to flat rigid surface foundations while SASSI does not have these restrictions. We believe the assumption of a flat and rigid foundation is sufficient for the LaSalle seismic PRA. The effect of embedment was inclurled approximately in LaSalle. The appropriateness of this approximation was not assessed in the update because significant amount of additional work using SASSI code is needed. Not addressed in the update for the same reason is the effect of experience data obtained by the Seismic Qualification Utility Group (SQUG) on the fragility development. 
In the area of seismic hazard estimates, the final LaSalle seismic hazard curves developed by LLNL and documented in NUREG/CR-5250 were found to be lower than the preliminary curves used in the LaSalle analysis in the ground accoleration range in which seismic hazard effects are of significance. Since the NUREG/CR5250 work was completed, EPRI developed a similar seismic hazand methodology. See Section 4.2.3. 


\section{Section 13. References}

1.1 Smith, P. D., R. G. Dong, D. L. Bernreuter, M. P. Bohn, T. Y. Chuang, G. E. Cummings, J. J. Johnson, R. W. Mensing, and J. E. Wells, "Seismic Safety Margins Research--Phase I Final Report", Lawrence Livermore National Laboratory, Livermore, California, UCRL-53021, NUREG/CR-2015, Vols. 1-10, 1981.

1.2 Shieh, L. C., J. J. Johnson, J. E. Wells, J. C. Chen, and P. D. Smith, "Simplified Seismic Probabilistic Risk Assessment: Procedures and Limitations", Lawrence Livermore National Laboratory, Livermore, California, UCID-20468, NUREG/CR-4331, 1985.

1.3 Bohn, M. P., L. C. Shieh, J. E. Wells, L. C. Cover, D. L. Bernreuter, J. C. Chen, J. J. Johnson, S. E. Bumpus, R. W. Mensing, W. J. O'Connell, and D. A. Lappa, "Applications of the SSMRP Methodology to the Seismic Risk at the Zion Nuclear Power Plant", Lawrence Livermore National Laboratory, Livermore, California, UCRL-53483, NUREG/CR-3428, 1984.

1.4 Kolb, G. J., et al., "Risk Methods Integration and Evaluation Program Plan", Sandia National Laboratories, Albuquerque, New Mexico, 1984.

2.1 Bernreuter, D. L., et al. (1985), "Seismic Hazard Characterization of the Eastem United States, Volume 1: Methodology and Results for Ten sites and Volume 2: Questionnaires," Lawrence Livermore National Laboratory Report UCID-20421.

2.2 Seismic Qualification Utilities Group (SQUG), "General Implementation Procedure for Seismic Verification of Nuclear Power Plant Equipment", Rev. 2, Palo Alto, CA, September, 1990.

3.1 LaSalle County Station, Final Safety Analysis Report

4.1 D.L. Bernreuter, et al., "Seismic Hazard Characterization of 69 Nuclear Plant Sites East of the Rocky Mountains," Volumes 1 through 8, Lawrence Livermore National Laboratory, UCID-21517; sponsored by the U.S. Nuclear Regulatory Commission, NUREG/CR5250, January, 1989.

5.1 Johnson, J. J., G. L. Goudreau, S. E. Bumpus, and O. R. Maslenikov, "SSMRP Phase I Final Report--SMACS--Seismic Methodology Analysis Chain with Statistics (Project VIII)," Lawrence Livermore National Laboratory, Livermore, CA, UCRL-53021, Vol. 9 , also NUREG/CR-2015, Vol. 9, September, 1981.

5.2 Maslenikov, O. R., J. C. Chen, and J. J. Johnson, "Uncertainty in Soil-Structure Interaction Analysis Arising from Difference in Analytical Techniques," Lawrence Livermore National Laboratory, Livermore, CA, UCRL-53026, also NUREG/CR-2077, July, 1982.

5.3 Sargent \& Lundy, "Seismic Response Spectra Design Criteria--LaSalle County Nuclear Power Station Units 1 and 2," DC-SE-02-LS. 
5.4 J. Lysmer and F. Ostadan, "SASSI - A System for Analysis of Soil-Structure Interaction, User's Manual", University of California, Berkeley, CA; Revised by Bechtel Power Corporation, San Francisco, CA, July 30, 1988.

5.5 H.L. Wong and J.E. Luco, "Soil-Structure Interaction: A Linear Continuum Mechanics Approach (CLASSI)", University of Southern California, Los Angeles, California, 1980.

5.6 "CLASSI/ASD Computer Program for Three-Dimensional Soil/Multiple Foundation Interaction Analysis, User's Manual", Version 2.1, ASD International, Inc., San Francisco, CA, November, 1987.

6.1 General Electric, "Mark II Containment Dynamic Forcing Function Information Report," NEDO- 21061, Revision 4, Class I, November, 1981.

6.2 Hardy, G. S., R. D. Campbell, and M. K. Ravindra, "Load Combination Program Probability of Guillotine Break of the Brunswick GE Mark I Reactor Coolant Piping Indirectly-Induced by Earthquakes", SMA 12211.49, June, 1985.

6.3 Weaver, E. R. (Sargent \& Lundy Engineers), transmittal of realistic all valve SRV acceleration time histories and response spectra to T. Y. Chuang (Lawrence Livermore National Laboratory), Sargent \& Lundy Project No. 7043-51, 28 November 1984.

6.4 Sargent \& Lundy Engineers, "In-Plant SRV Test LaSalle County Station Unit 1," Commonwealth Edison Company, Final Evaluation Report, September, 1983.

6.5 Winterstein, S. R., and C. A. Cornell, "Combined Dynamic Responses: Extremes and Fatigue Damage, MIT, Department of Civil Engineering, R80-46, December, 1980.

6.6 Larrabee, R. D., and C. A. Cornell, "Approximate Stochastic Analysis of Combined Loading," MIT, Department of Civil Engineering, R78-28, September, 1978.

6.7 Johnson, J., R. Campbell, P. Hashimoto, O. Maslenikov, and R. Tiong, "LaSalle Probabilistic Risk Assessment: Response and Fragilities, SMA 12211.21, 1985.

6.8 Gasparini, D., and E. H. Vanmarcke, "Simulated Earthquake Motions Compatible with Prescribed Response Spectra", MIT, Dejartment of Civil Engineering, R76-4, January, 1976.

6.9 Structural Mechanics Associates, "Seismic Fragilities of Structures and Components at the Three Mile Island, Unit 1, Nuclear Power Plant", SMA 12914.01, February, 1985.

6.10 Madsen, H., R. Kilcup, and C. A. Cornell, "Mean Upcrossing Rates for Sums of PulseType Stochastic Loads Processes", Proc. Speciality Conference on Probabilistic Mechanics and Structural Reliability, ASEC, January, 1979.

6.11 Vanmarcke, E. H., "On the Distribution of the First-Passige Time for Normal Stationary Random Processes", Trans. of ASME, Vol. 42, Series E, No. 1, March, 1975. 
6.12 Yang, J. N. and S. C. Liu, "Distribution of Maximum and Statistical Response Spectra," Jour. Eng. Mech. Div., ASCE, Vol. 107, No. EM6, December 1981.

7.1 Wesley, D. A., and P. S. Hashimoto, "Seismic Structural Fragility Investigation for the Zion Nuclear Power Plant," Lawrence Livermore National Laboratory, Livermore, CA, NUREG/CR-2320, October, 1981 .

7.2 Lettet from E. R. Weaver (Sargent and Lundy) to T. Y. Chuang (Lawrence Livermore National Laboratory), "Concrete and Reinforcing Steel-Summary of Test Data," March 29,1984 .

7.3 Riddell, R. and N. M. Newmark, "Statistical Analysis of the Response of Nonlinear Systems Subjected to Earthquakes," Department of Civil Engineering, Report UILU 79. 2016, Urbana, Illinois, August, 1979.

7.4 Kennedy, R. P., R. H. Kinciaid, and S. A. Short, "Effects of Ground Motion Characteristics on Structural Response Considering a Typical PWR Reactor Building with Localized Nonlinearities and Soil-Structure Interaction Effects," Draft, prepared for Woodward-Clyde Consultants by Structural Mechanics Associates, SMA 12702.04-R1-B, June, 1984.

7.5 Kennedy, R. P., et al., "Engineering Characterization of Ground Motion," NUREG/CR3805, May, 1984.

7.6 Hofbeck, J. A., 1. O. Ibrahim, and A. H. Mattock, "Shear Transfer in Reinforced Concrete," ACl Journal, Febnuary, 1969.

7.7 Mattock, A. H., L. Johol, and H. C. Chow, "Shear Transfer in Reinforced Concrete-Recent Research," PCl Joumal, March/April, 1972.

7.8 Mattock, A. H., L. Johol, and H. C. Chow, "Shear Transfer in Reinforced Concrete with Moment or Tension Acting Across the Shear Plane," PCl Journal, July/August, 1975.

7.9 Mattock, A. H., "Cyclic Shear Transfer and Type at Interface," Journal of the Structural Division, ASCE, October, 1981.

7.10 Ollgaard, J. G., R. G. Slutter, and J. W. Fisher, "Shear Strength of Stud Connectors in Lightweight and Normal-Weight Concrete," AlSC Journal, April, 1971.

7.11 McMackin, P. J., R. G. Slutter, and J. W. Fisher, "Headed Steel Anchors under Combined Loading," AISC Journal, Second Quarter, 1973.

7.12 Ogaki, Y., et al., "Shear Strength Tests of Prestressed Concrete Containment Vessels," Paper J4/3, Proceedings Sixth SMiRT Conference, Paris, France, 1981.

7.13 "Code Requirements for Nuclear Safety-Related Concrete Structures (ACI 349-80) and Commentinty- - $\Lambda \mathrm{Cl}$ 349R-80," Americin Concrete Institute, 1980.

7.14 Umemura, H., et al., "Aseismic Characteristics of RC Box and Cylinder Walls," Experimental Studies on Reinforced Concrete Members and Composite Steel and 
Reinforced Concrete Members, Volume 3, Faculty of Engineering, University of Tokyo, December 1977.

7.15 Bader, M., and H. Krawinkler, "Shear Transfer in Thick-Walled Reinforced Concrete Cylinders," Paper J3/7, Proceedings Sixth SMiRT Conference, Paris, France, 1981.

7.16 Hall, D., "Proposed Steel Column Strength Criteria," J. Structural Division, ASCE, April, 1981.

7.17 Kato, B., "Local Buckling of Steel Circular Tubes in Plastic Region," International Colloquium on Structural Stability of Structures under Static and Dynamic Loads, Washington, D.C., May 17-19, 1977.

8.1 Kennedy, R. P., R. D. Campbell, G. Hardy, and H. Banon, "Subsystem Fragility, Seismic Safety Margins Research Program (Phase I)," Lawrence Livermore National Laboratory, Livermore, CA, NUREG/CR-2405, UCRL-15407, February, 1981.

8.2 Kennedy, R. P., R. D. Campbell, D. A. Wesley, H. Kamil, A. Gontayat, and R. Vasudenvan, "Subsystems Response P.eview, Seismic Safety Margins Research Program, Phase I," Lawrence Livermore National Laboratory, Livermore, CA, NUREG/CR-1706, UCRL 15216, October, 1980.

9.1 U.S. Nuclear Regulatory Commission, "Reactor Safety Study: An Assessment of Accident Risks in U.S. Commercial Nuclear Power Plants, WASH-1400," NUREG-75/014, October 1975.

9.2 Wells, J. E., "SEISIM: A Probabilistic Risk Assessment Tool Used in Evaluating Seismic Risk", Proceedings of the Conference on Seisnic Risk and Havy Industrial Facilities, San Francisco, California, 1983.

9.3 Wells, J. E., and D. A. Lappa, "Probabilistic Culling in Fault Tree Evaluation," Proceedings of the 1983 Reliability and Maintainability Symposium, IEEE, New York, NY, 1983.

9.4 Willie, R. R., "Computer-Aided Fault Tree Analysis," Operations Research Center, University of California, Berkeley, CA, ORC 78-14A, August 1978. 


\section{Appendix A. Use of Alternate Event and Fault Trees}

During the study a second set of event trees and fault trees was generated. These trees were generated independently of the RMIEP event and fault trees. The fault trees in the second set were less detailed in that fewer systems were modeled and the systems modeled were modeled in less detail than the RMIEP fault trees. Two sets of trees were generated so that a comparison might be made between them to indicate whether or not highly detailed fault trees are warranted for a simplified seismic risk analysis. In this appendix we will discuss the event trees and fault trees used, the results obtained, and some conclusions which this additional analysis was based on.

\section{A.I Event Trees/f.ccident Sequences Used In Additional Analysis}

There is no event tree for the RVR. For this study we were only interested in core damage and not release, therefore there was no need for an RVR event tree since all RVR failures lead to core damage.

Each of the remaining four event trees follow a basic functional pattern that is the same for each. That is, following an accident initiation, the Reactor Protection System (RPS) must act to quench the nuclear reaction. If this fails, core damage is assumed because the power level is too high to be offset by safety systems. Next, the Vapor Suppression (VS), for the LOCA sequences, must occur. The steam must be carried into the suppression pool and condensed. The steam is carried from the drywell to the suppression pool through downcomers connecting the drywell and suppression pool. If not, the suppression pool will fail from overpressure, which is assumed to result in core damage.

For the LOSP initiator, the vessel must be protected by operation of the Safety Relieve Valves (SRVs). Failure to open (SRVO) is assumed to result in a breech of the reactor pressure boundary and, thus, core damage. Failure of any SRV to reclose (SRVR) is considered a LOCA condition.

If the RPS, SRVs, and VS have succeeded then, for all event trees, the remainder of the sequences are divided into two functional portions: coolant makeup and containment heat removal. Coolant makeup involves High Pressure Core Spray (HPCS), Reactor Core Isolation Cooling (RCIC), Automatic Depressurization System (ADS), Low Pressure Core Spray (LPCS), and Low Pressure Coolant Injection (LPCI), depending on the initiator. Containment heat removal involves Suppression Pool Cooling (SPC) and the Containment Spray (CS), which are both intimately linked to the Residual Heat Removal (RHR) system. For simplification purposes, the RHR system represents the SPC and CS on the event trees.

Both the HPCS and a single train of the LPCI have sufficient capacity for miitgating all sizes of LOCAs. The capacity of the RCIC, however, is insufficient for coolant makeup during a Large or Medium LOCA but is sufficient for a Small LOCA.

The low pressure systems can be used immediately following a large initiator since the reactor vessel would be depressurized. However, for Small and Medium LOCAs, reactor vessel depressurization would be required. Reactor vessel depressurization can be accomplished automatically through the ADS. 
The RHR system, as indicated, can remove containment heat following a LOCA by either suppression pool cooling or containment spray. It was assumed that one RHR heat exchanger is sufficient for maintaining the suppression pool temperature below limiting temperatures. This is assumed to be true in any mode of RHR operation. Flow from two service water pumps is required to remove the heat from one heat exchanger. For a more detailed description of the safety systems needed to mitigate an accident, see Sec. 9.3.

The 'additional' analysis event trees are shown in Figs. A.1 through A.4. A number in the last column on the right of each of these event trees indicates those sequences that lead to core damage. Those not having a number in this column do not lead to core damage. Only those accident sequences leading to core damage were quantified in this study. There are 30 such accident sequences in the event trees that were developed (including the RVR sequence).

Table A.1 provides a summary of the accident sequences used. The minus sign indicates system success. Events with no minus sign indicate system failure.

\section{A.2 Fault Trees/Safety Systems Used In Additional Analysis}

There were six safety systems for which additional fault trees were developed. These included the ADS, HPCS, LPCI, LPCS, RCIC, and RHR. These systems were described earlier in Sec. 9.3. An additional fault tree for the Electrical Power (EP) system was also developed. This system was considered a support system and was used in solving each of the safety systems.

The system failure expressions determined from the fault trees were input into SEISIM ${ }^{[9.2]}$ in the form of minimal cut sets, the smallest sets of basic events that must take place in order for the top event to occur. These fault trees are reduced to unions of intersections of basic events by Boolean algebra.

Table A.2 lists the number of gates and basic events for each of the additional systems. Note that EP is listed separately. The number of gates and basic events for the six safety systems shown do not include the EP system nor are the EP gate transfers included. The number of basic events listed accounts for only one mode of failure. As in the analysis of the RMIEP fault trees, every basic event that was capable of failing in both a random and a seismically-related mode were made into two basic events. Therefore, the number of basic events used in the fault tree solutions was much higher than indicated.

\section{A.3 Solution Process and Results Used in Additional Analysis}

\section{A.3.1 Solution Process}

As with the RMIEP event fault trees, once the event trees and fault trees have been developed, the process of solving the systems and accident sequences begins. Figure 9.5 illustrates the procedure. A detailed database, providing the basic event name, associated response fragility, and random probability data, was needed. After developing this database and the appropriate SEISIM(9.2) input files, the basic event list was used as input into SEISIM and marginal probabilities generated. 


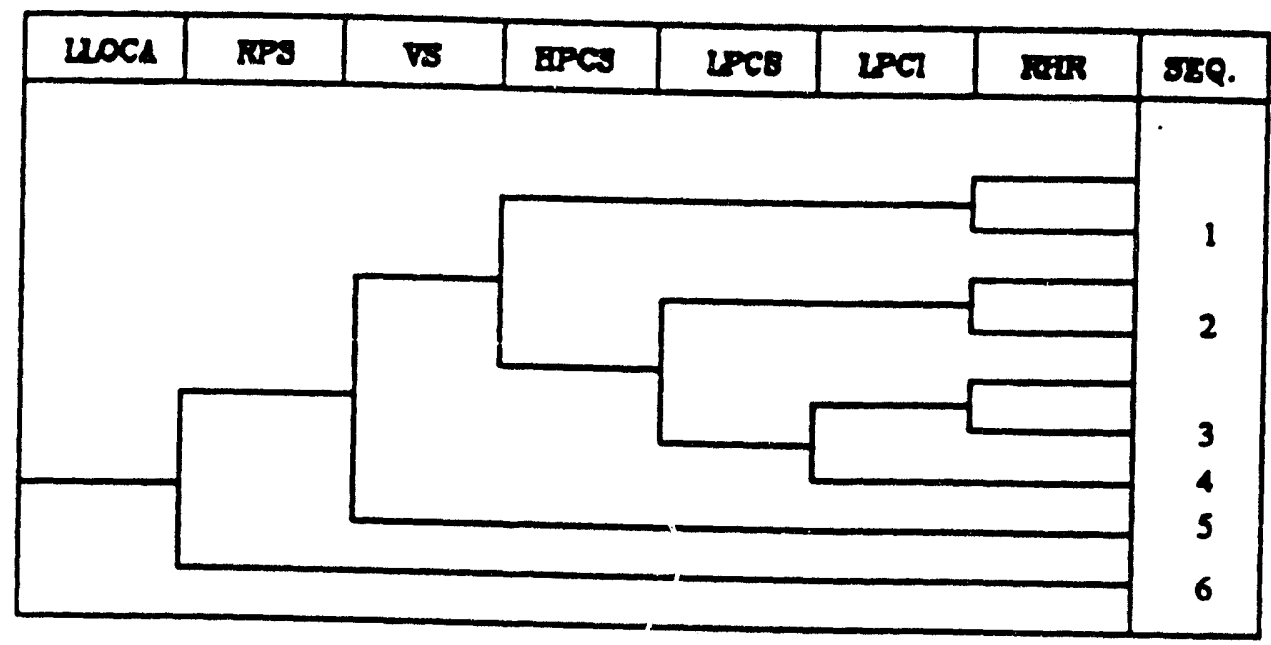

Figure A.1. Large-LOCA event tree (used in additional analysis). 


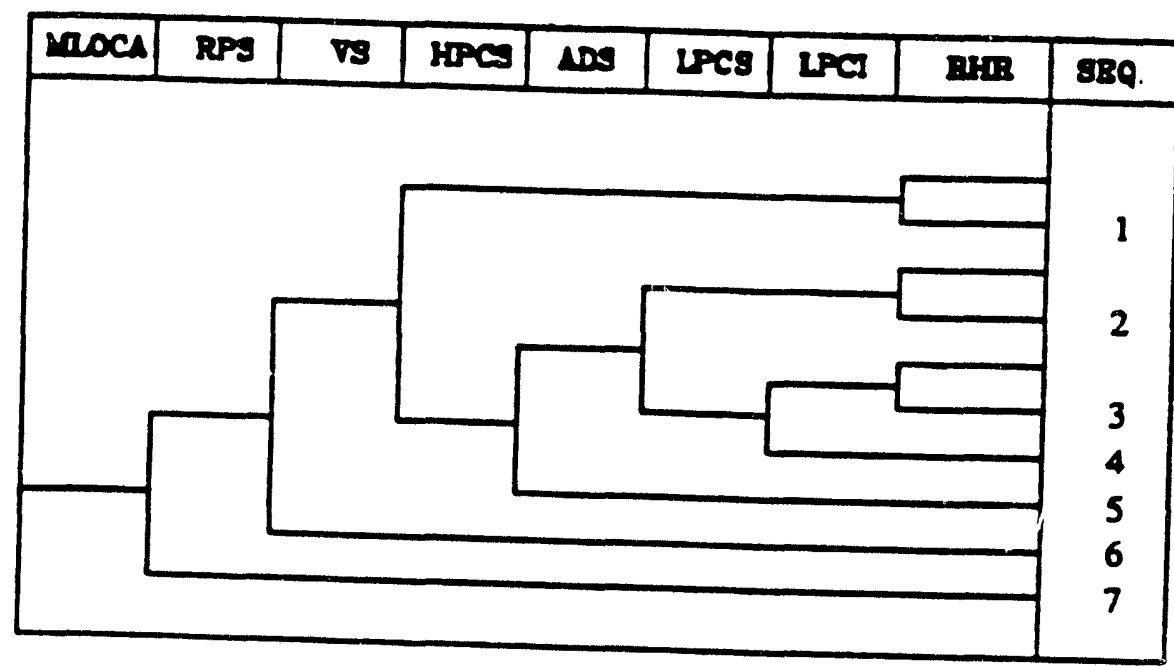

Figure A.2. Medium-LOCA event tree (used in additional analysis). 


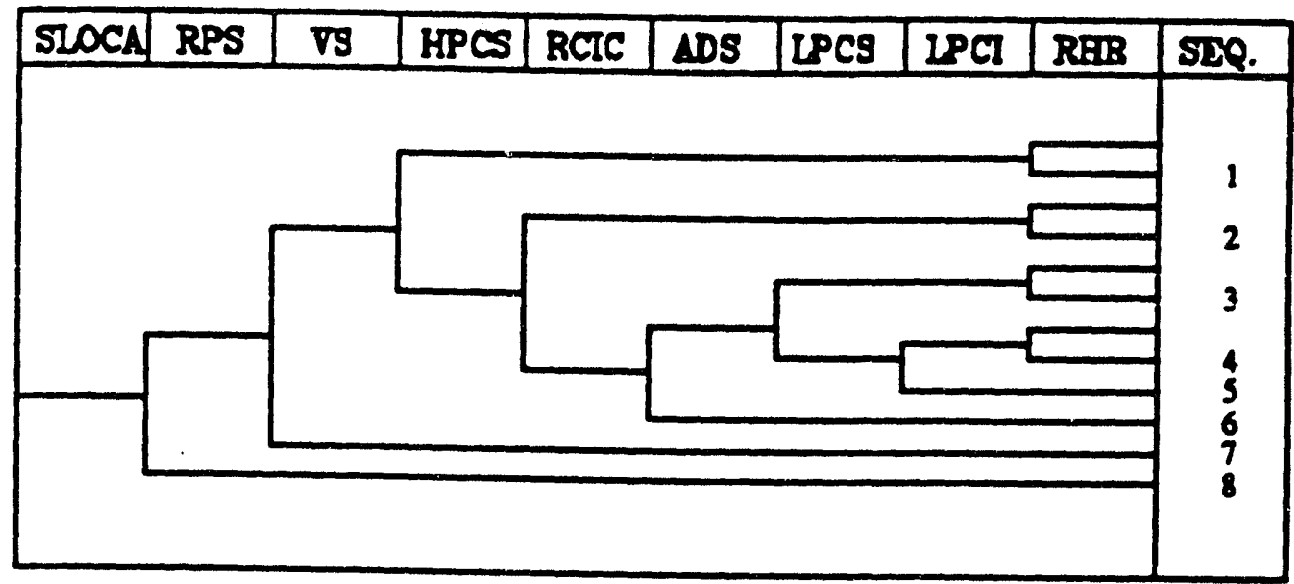

Figure A.3. Small-LOCA event tree (used in additional analysis). 


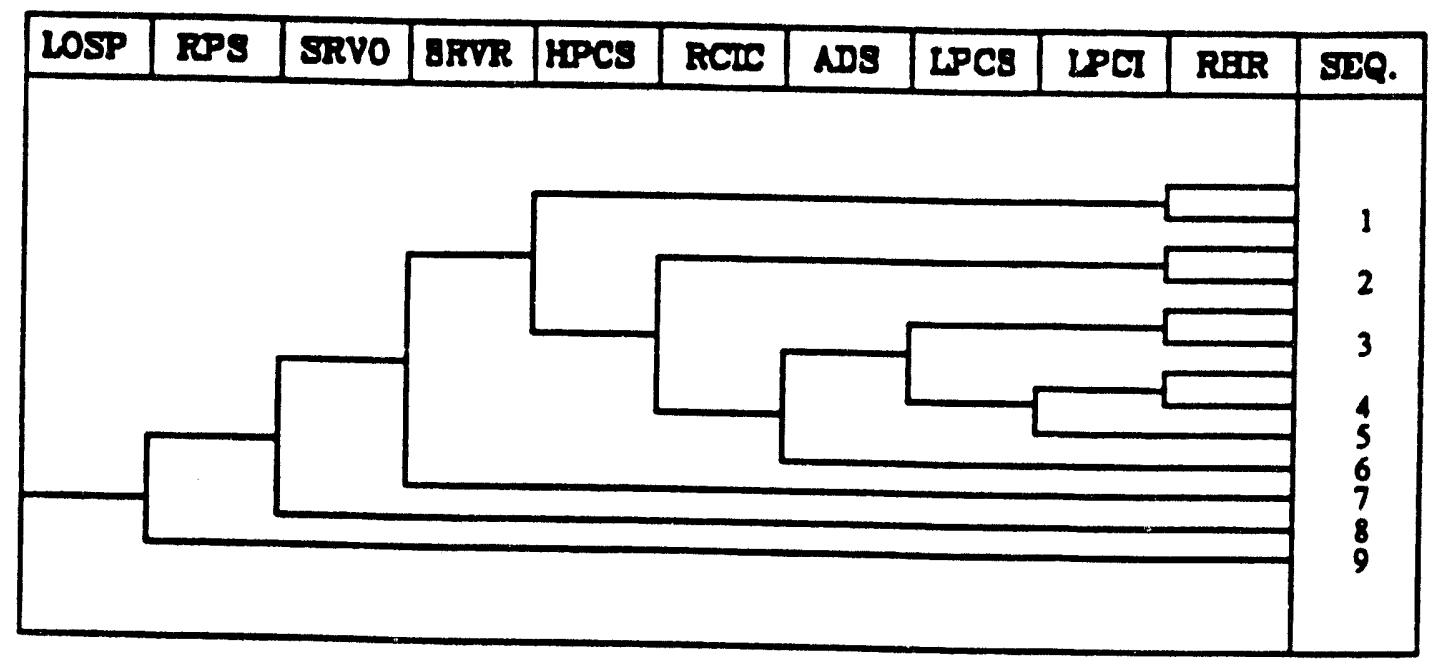

Figurc A.4. Loss-of-offsite power event tree (used in additional analysis).

A-6 
These marginal probabilities were generated for all six earthquake levels (see Sec. 4.4 for earthquake level definitions). Having completed the SEISIM nuns to generate marginal probabilities at all six levels of earthquakes we then developed an "average probability for each basic event. These "average" probabilities are called culling values and were used in our culling process. ${ }^{99.3]}$ As indicated earlier, each basic event on the fault tree was evaluated and determined to be either a random failure, a fragility-related failure, or capable of failing in either mode. The culling values generated using SEISIM were the fragility-related values. The random failure probabilities were taken from the database.

The culling values described in the previous paragraph were then used in solving the safety systems. The computer code used to solve the systems was FTAP (Fault Tree Analysis Program).[9.4] All runs were made on an LLNL VAX-750. The system solutions were then reviewed for correctness and to determine the effect due to an earthquake.At this point, the fault tree solutions for the systems were input back into FTAP along with the event trees.

Accident sequence solutions were then generated. Note, the same culling values that were used for culling the systems were also used here. However, the culling procedure differed slightly in that after reviewing the system solutions, it was found that there were no two dominant seismic events that appeared in the same cut set. Therefore, while the systems solutions were culled using a minimum criteria, i.e., culled based on the lowest probability basic event appearing in a cut set, the accident sequence culling was performed based on cut set probability.

It was assumed from the beginning that there would be a need to make use of probabilistic culling techniques in order to solve the fault and event trees. This was considered necessary since both random and seismically-induced failures were included for those basic events for which it was considered appropriate.

Table A.3 contains a list of the six safety systems considered and the number of cut sets which survived the culling process. Table A.4 contains a list of each of the accident sequences studied and the number of cut sets which survived culling for each sequence.

\section{A.3.2 Point Estimate of Core Damage Frequency}

Tables A.5, A.6 and A.7 contain the accident sequence probabilities based on the additional event and fault trees that were developed for the LaSalle nuclear power plant. Table A.5 lists the unconditional accident sequence probabilities (accident sequence probability $\mathbf{x}$ initiator probability $\mathbf{x}$ earthquake frequency). Table A.6 lists the accident sequence probabilities conditional on earthquake at a specific level (accident sequence probability $\mathbf{x}$ initiator probability). Table A.7 lists the accident sequence probabilities conditional on both earthquake and initiating event.

Table A.5, the unconditional accident sequence frequencies, provides a better look at how the risk is distributed. Looking at the "Grand Total", note the contribution decreases with the level of initiator severity; i.e., the LOSP contribution being the highest and the RVR contribution being the lowest. The core damage frequency psint estimate for LaSalle using the additional event and fault trees is 8.4E-6/yr. Note that 95 percent of the risk falls in the first three earthquake levels with 70 percent of the risk in earthquake level 1 . This result is different from the Zion analysis which showed most of the risk in earthquake level 2,3, and 4 (note that, while the LaSalle earthquake levels are not defined in exactly the same way as the Zion levels, they are similar). This result, combined with the component importance analysis, showed that system failures were dominated by random events. 
Table A.I Summary of accident sequences (additional analysis).

\section{Large-LOCA}
1) -RPS * -VS * -HPCS * RHR
2) -RPS * -VS * HPCS * -LPCS * RHR
3) -RPS * -VS * HPCS * LPCS * -LPCI * RHR
4) -RPS * -VS * HPCS * LPCS * LPCI
5) -RPS *VS
6) RPS

\section{Medium-LOCA}
1) -RPS * -VS * -HPCS * RHR
2) -RPS * -VS * HPCS * -ADS * -LPCS * RHR
3) -RPS * -VS * HPCS * -ADS * LPCS * -LPCI * RHR
4) -RPS * - VS * HPCS * -ADS * LPCS * LPCI
5) $-\mathrm{RPS} *$-VS * HPCS * ADS
6) - RPS *VS
7) RPS

Small-LOCA
1) -RPS * -VS * -HPCS * RHR
2) -RPS * -VS * HPCS * -RCIC * RHR
3) -RPS * -VS * HPCS * RCIC * -ADS * -LPCS * RHR
4) -RPS * - VS * HPCS * RCIC *-ADS * LPCS * -LPCI * RHR
5) $-\mathrm{RPS} *$-VS * HPCS * RCIC * -ADS * LPCS * LPCI
6) -RPS * -VS * HPCS * RCIC* ADS
7) -RPS * VS
8) RPS

\section{Loss-of-offsite power}
1) -RPS * -SRVO * -SRVR * -HPCS * RHR
2) -RPS * -SRVO * -SRVR * HPCS *-RCIC * RHR
3) -RPS * -SRVO * -SRVR * HPCS * RCIC * -ADS * -LPCS * RHR
4) -RPS * -SRVO * -SRVR * HPCS * RCIC* -ADS * LPCS * -LPCI * RHR
5) -RPS * -SRVO * -SRVR * HPCS * RCIC * -ADS * LPCS * LPCI
6) -RPS * -SRVO * -SRVR * HPCS * RCIC * ADS
7) -RPS * -SRVO * SRVR (Leads to LOCA Event Tree)
8) -RPS * SRVO
9) RPS (Anticipated Transient Without Scram (ATWS)) 
Table A.2. Numbers of gates and basic events in safety system fault trees (additional analysis).

\begin{tabular}{lcc}
\hline $\begin{array}{l}\text { Number } \\
\text { System }\end{array}$ & $\begin{array}{c}\text { Number } \\
\text { of Gates }\end{array}$ & Basic Events \\
ADS & 13 & 14 \\
HPCS & 56 & 74 \\
LPCI & 33 & 53 \\
LPCS & 6 & 14 \\
RCIC & 54 & 91 \\
RHR & 45 & 83 \\
EP & 44 & 81
\end{tabular}


Once the initiator probabilities are included (see Table A.6), it is found that the contribution to each of the events decreases with the severity of the initiator. At level 6 the Large-LOCA contributes 8.3E-6, the Medium-LOCA contributes 3.1E-4, Small-LOCA contributes 6.0E-3, and the LOSP. Trans contributes 4.9E-2.

In studying Table A.7 it is interesting to note that the Large-LOCA, Mediuni-LOCA, Small-LOCA, and LOSP-Trans events have roughly the same probability of occurrence. For example, if one examines the level 6 column, the Large-LOCA contribution is 6.9E-2, the Medium-LOCA contribution is 6.9E-2, the Small-LOCA contribution is 6.0E-2, and the LOSP-Trans contribution is $5.4 \mathrm{E}-2$.

\section{A.3.3 Dominant Accident Sequences}

In studying Table A.S it is noted that 93 percent of the core damage frequency is due to only three accident sequences: LOSP-Trans-1, LOSP-Trans-2, and Small-LOCA-1. The Small-LOCA-1 and LOSP-Trans-1 accidents sequence are dominated by the failure of the RHR system. The LOSPTrans-2 event is dominated by failure of the RHR and the HPCS system. These event trees are shown in Figs. A.1 through A.4 and a summary of the accident sequences used are listed in Table A.1. 
Table A.3. Number of safety system cut sets after culling (additional analysis).

\begin{tabular}{|c|c|}
\hline Initiating Event & $\begin{array}{l}\text { Number of } \\
\text { Cut Sets }\end{array}$ \\
\hline ADS.......... & ......339 \\
\hline HPC......... & ........83 \\
\hline LPCI ........ & $\ldots .6600$ \\
\hline LPCS ........ & ........ 32 \\
\hline RCIC........ & .......160 \\
\hline RHR ......... & ..... 1651 \\
\hline
\end{tabular}


Table A.4. Culled accident sequence cut set numbers (additional analysis).

$\begin{array}{cc}\text { Initiating Event } & \begin{array}{c}\text { Number of } \\ \text { Cut Sets }\end{array}\end{array}$

Large-LOCA-1

Large-LOCA-2

Large-LOCA-3

Large-LOCA-4

Large-LOCA-5

Large-LOCA-6

236

473

453

405

0

Medium-LOCA-1

Medium-LOCA-2

Medium-LOCA-3

Medium-LOCA-4

Medium-LOCA-5

Medium-LOCA-6

Medium-LOCA-7

Small-LOCA-1

Small-LOCA-2

Small-L.OCA-3

Small-LOCA-4

Small-LOCA-5

Small-LOCA-6

Small-LOCA-7

Small-LOCA-8

LOSP-Trans-1 LOSP-Trans-2

LOSP-Trans-3

LOSP-Trans-4

LOSP-Trans-5

LOSP-Trans- 6

LOSP-Trans-8

LOSP-Trans-9

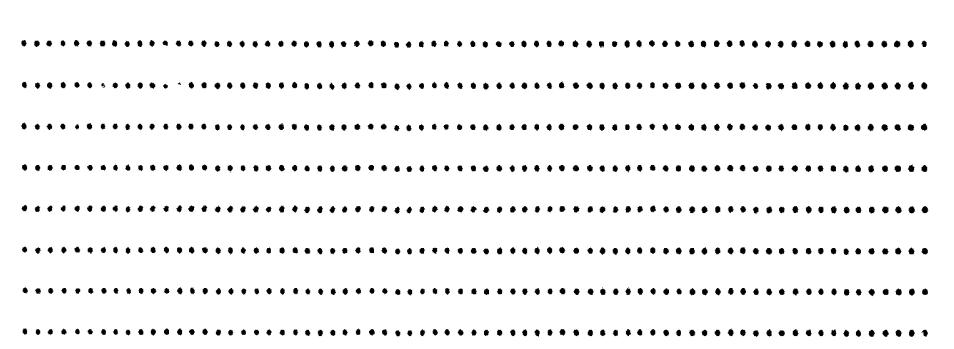


Table A.5. Unconditional accident sequence frequencies (additional analysis).

Earthquake Level

Accident

Sequence

Level 1 Level 2 Level $3 \quad$ Level $4 \quad$ Level 5 Level 6 Total

\begin{tabular}{|c|c|c|c|c|c|c|c|}
\hline $\begin{array}{l}\text { Large-LOCA-1 } \\
\text { Large-LOCA-2 } \\
\text { Large-LOCA-3 } \\
\text { Lar-e-LOCA-4 } \\
\text { Large-LOCA-5 } \\
\text { Large-LOCA-6 } \\
\text { Subtotal }\end{array}$ & $\begin{array}{l}6.3 \mathrm{E}-10 \\
4.3 \mathrm{E}-11 \\
9.7 \mathrm{E}-11 \\
9.1 \mathrm{E}-11 \\
0.0 \mathrm{E}+0 \\
1.3 \mathrm{E}-13 \\
8.6 \mathrm{E}-10\end{array}$ & $\begin{array}{l}1.7 \mathrm{E}-10 \\
1.2 \mathrm{E}-11 \\
2.7 \mathrm{E}-11 \\
2.5 \mathrm{E}-11 \\
0.0 \mathrm{E}+0 \\
3.4 \mathrm{E}-14 \\
2.3 \mathrm{E}-10\end{array}$ & $\begin{array}{l}6.3 \mathrm{E}-11 \\
4.6 \mathrm{E}-12 \\
1.0 \mathrm{E}-11 \\
9.7 \mathrm{E}-12 \\
0.0 \mathrm{E}+0 \\
1.3 \mathrm{E} \cdot 14 \\
8.8 \mathrm{E}-11\end{array}$ & $\begin{array}{l}2.7 \mathrm{E}-11 \\
2.1 \mathrm{E} \cdot 12 \\
4.7 \mathrm{E}-12 \\
4.4 \mathrm{E}-12 \\
0.0 \mathrm{E}+0 \\
5.7 \mathrm{E}-15 \\
3.8 \mathrm{E}-11\end{array}$ & $\begin{array}{l}1.2 \mathrm{E}-11 \\
9.8 \mathrm{E}-13 \\
2.2 \mathrm{E}-13 \\
2.0 \mathrm{E}-12 \\
0.0 \mathrm{E}+0 \\
2.5 \mathrm{E}-15 \\
1.7 \mathrm{E}-11\end{array}$ & $\begin{array}{l}5.8 \mathrm{E}-12 \\
5.0 \mathrm{E}-13 \\
1.1 \mathrm{E}-13 \\
1.0 \mathrm{E}-12 \\
0.0 \mathrm{E}+0 \\
1.2 \mathrm{E}-15 \\
8.5 \mathrm{E}-12\end{array}$ & $\begin{array}{l}9.1 \mathrm{E}-10 \\
6.4 \mathrm{E}-11 \\
1.4 \mathrm{E}-10 \\
1.3 \mathrm{E}-10 \\
0.0 \mathrm{E}+0 \\
1.9 \mathrm{E}-13 \\
1.2 \mathrm{E}-9\end{array}$ \\
\hline $\begin{array}{c}\text { Medium-LOCA-I } \\
\text { Medium-LOCA-2 } \\
\text { Medium-LOCA-3 } \\
\text { Medium-LOCA-4 } \\
\text { Medium-LOCA-5 } \\
\text { Medium-LOCA-6 } \\
\text { Medium-LOCA-7 } \\
\text { Subtotal }\end{array}$ & $\begin{array}{l}2.4 \mathrm{E}-8 \\
1.6 \mathrm{E}-9 \\
3.7 \mathrm{E}-9 \\
3.4 \mathrm{E}-9 \\
0.0 \mathrm{E}+0 \\
0.0 \mathrm{E}+0 \\
4.8 \mathrm{E}-12 \\
3.2 \mathrm{E}-8\end{array}$ & $\begin{array}{l}6.4 \mathrm{E}-9 \\
4.5 \mathrm{E}-10 \\
1.0 \mathrm{E}-9 \\
9.4 \mathrm{E}-10 \\
0.0 \mathrm{E}+0 \\
0.0 \mathrm{E}+0 \\
1.3 \mathrm{E}-12 \\
8.7 \mathrm{E}-9\end{array}$ & $\begin{array}{l}2.4 \mathrm{E}-9 \\
1.7 \mathrm{E}-10 \\
3.9 \mathrm{E}-10 \\
3.6 \mathrm{E}-10 \\
0.0 \mathrm{E}+0 \\
0.0 \mathrm{E}+0 \\
4.9 \mathrm{E}-13 \\
3.3 \mathrm{E}-9\end{array}$ & $\begin{array}{l}1.0 \mathrm{E}-9 \\
7.8 \mathrm{E}-11 \\
1.8 \mathrm{E}-10 \\
1.6 \mathrm{E}-10 \\
0.0 \mathrm{E}+0 \\
0.0 \mathrm{E}+0 \\
2.1 \mathrm{E}-13 \\
1.4 \mathrm{E}-9\end{array}$ & $\begin{array}{l}4.6 \mathrm{E}-10 \\
3.7 \mathrm{E}-11 \\
8.2 \mathrm{E}-11 \\
7.7 \mathrm{E}-11 \\
0.0 \mathrm{E}+0 \\
0.0 \mathrm{E}+0 \\
9.5 \mathrm{E}-14 \\
6.5 \mathrm{E}-10\end{array}$ & $\begin{array}{l}2.2 \mathrm{E}-10 \\
1.9 \mathrm{E}-11 \\
4.2 \mathrm{E}-11 \\
3.9 \mathrm{E}-11 \\
0.0 \mathrm{E}+0 \\
0.0 \mathrm{E}+0 \\
4.6 \mathrm{E}-14 \\
3.2 \mathrm{E}-10\end{array}$ & $\begin{array}{l}3.4 \mathrm{E}-8 \\
2.4 \mathrm{E}-9 \\
5.4 \mathrm{E}-9 \\
5.0 \mathrm{E}-9 \\
0.0 \mathrm{E}+0 \\
0.0 \mathrm{E}+0 \\
6.9 \mathrm{E}-12 \\
4.7 \mathrm{E}-8\end{array}$ \\
\hline $\begin{array}{c}\text { Small-LOCA-1 } \\
\text { Small-LOCA-2 } \\
\text { Small-LOCA-3 } \\
\text { Small-LOCA-4 } \\
\text { Small-LOCA-5 } \\
\text { Small-LOCA-6 } \\
\text { Small-LOCA-7 } \\
\text { Small-LOCA-8 } \\
\text { Subtotal }\end{array}$ & $\begin{array}{l}5.3 \mathrm{E}-7 \\
7.8 \mathrm{E}-8 \\
5.1 \mathrm{E}-9 \\
1.1 \mathrm{E}-8 \\
1.1 \mathrm{E}-8 \\
0.0 \mathrm{E}+0 \\
0.0 \mathrm{E}+0 \\
1.1 \mathrm{E}-10 \\
6.3 \mathrm{E}-7\end{array}$ & $\begin{array}{l}1.4 \mathrm{E}-7 \\
2.1 \mathrm{E}-8 \\
1.5 \mathrm{E}-9 \\
3.3 \mathrm{E}-9 \\
3.3 \mathrm{E}-9 \\
0.0 \mathrm{E}+0 \\
0.0 \mathrm{E}+0 \\
2.9 \mathrm{E}-11 \\
1.7 \mathrm{E}-7\end{array}$ & $\begin{array}{l}5.3 \mathrm{E}-8 \\
8.3 \mathrm{E}-9 \\
6.3 \mathrm{E}-10 \\
1.4 \mathrm{E}-9 \\
1.3 \mathrm{E}-9 \\
0.0 \mathrm{E}+0 \\
0.0 \mathrm{E}+0 \\
1.1 \mathrm{E}-11 \\
6.4 \mathrm{E}-8\end{array}$ & $\begin{array}{l}2.3 \mathrm{E}-8 \\
3.7 \mathrm{E}-9 \\
3.1 \mathrm{E}-10 \\
6.5 \mathrm{E}-10 \\
6.4 \mathrm{E}-10 \\
0.0 \mathrm{E}+0 \\
0.0 \mathrm{E}+0 \\
4.7 \mathrm{E}-12 \\
2.8 \mathrm{E}-8\end{array}$ & $\begin{array}{l}1.0 \mathrm{E}-8 \\
1.7 \mathrm{E}-9 \\
1.6 \mathrm{E}-10 \\
3.2 \mathrm{E}-10 \\
3.2 \mathrm{E}-10 \\
0.0 \mathrm{E}+0 \\
0.0 \mathrm{E}+0 \\
2.1 \mathrm{E}-12 \\
1.3 \mathrm{E}-8\end{array}$ & $\begin{array}{l}4.8 \mathrm{E}-9 \\
8.9 \mathrm{E}-10 \\
8.7 \mathrm{E}-11 \\
1.8 \mathrm{E}-10 \\
1.7 \mathrm{E}-10 \\
0.0 \mathrm{E}+0 \\
0.0 \mathrm{E}+0 \\
1.0 \mathrm{E}-12 \\
6.2 \mathrm{E}-9\end{array}$ & $\begin{array}{l}7.6 \mathrm{E}-7 \\
1.1 \mathrm{E}-7 \\
7.7 \mathrm{E}-9 \\
1.7 \mathrm{E}-8 \\
1.7 \mathrm{E}-8 \\
0.0 \mathrm{E}+0 \\
0.0 \mathrm{E}+0 \\
1.5 \mathrm{E}-10 \\
9.1 \mathrm{E}-7\end{array}$ \\
\hline $\begin{array}{c}\text { LOSP-Trans-1 } \\
\text { LOSP-Trans-2 } \\
\text { LOSP-Trans-3 } \\
\text { LOSP-Trans-4 } \\
\text { LOSP-Trans-5 } \\
\text { LOSP-Trans-6 } \\
\text { LOSP-Trans-8 } \\
\text { LOSP-Trans-9 } \\
\text { Subtolal }\end{array}$ & $\begin{array}{l}4.3 \mathrm{E}-6 \\
6.3 \mathrm{E}-7 \\
4.1 \mathrm{E}-8 \\
9.2 \mathrm{E}-8 \\
9.2 \mathrm{E}-8 \\
0.0 \mathrm{E}+0 \\
0.0 \mathrm{E}+0 \\
9.6 \mathrm{E}-10 \\
5.1 \mathrm{E}-6\end{array}$ & $\begin{array}{l}1.1 \mathrm{E}-6 \\
1.7 \mathrm{E}-7 \\
1.2 \mathrm{E}-8 \\
2.7 \mathrm{E}-8 \\
2.7 \mathrm{E}-8 \\
0.0 \mathrm{E}+0 \\
0.0 \mathrm{E}+0 \\
2.6 \mathrm{E}-10 \\
1.4 \mathrm{E}-6\end{array}$ & $\begin{array}{l}4.3 \mathrm{E}-7 \\
6.7 \mathrm{i}-8 \\
5.1 \mathrm{E}-9 \\
1.1 \mathrm{E}-8 \\
1.1 \mathrm{E}-8 \\
0.0 \mathrm{E}+0 \\
0.0 \mathrm{E}+() \\
9.7 \mathrm{E}-11 \\
5.2 \mathrm{E}-7\end{array}$ & $\begin{array}{l}1.8 \mathrm{E}-7 \\
3.0 \mathrm{E}-8 \\
2.5 \mathrm{E}-9 \\
5.2 \mathrm{E}-9 \\
5.2 \mathrm{E}-9 \\
0.0 \mathrm{E}+0 \\
0.0 \mathrm{E}+0 \\
4.2 \mathrm{E}-11 \\
2.3 \mathrm{E}-7\end{array}$ & $\begin{array}{l}8.3 \mathrm{E}-8 \\
1.4 \mathrm{E}-8 \\
1.3 \mathrm{E}-9 \\
2.6 \mathrm{E}-9 \\
2.6 \mathrm{E}-9 \\
0.0 \mathrm{E}+0 \\
0.0 \mathrm{E}+0 \\
1.9 \mathrm{E}-11 \\
1.0 \mathrm{E}-7\end{array}$ & $\begin{array}{l}3.9 \mathrm{E}-8 \\
7.2 \mathrm{E}-9 \\
7.1 \mathrm{E}-10 \\
1.4 \mathrm{E}-9 \\
1.4 \mathrm{E}-9 \\
0.0 \mathrm{E}+0 \\
0.0 \mathrm{E}+0 \\
9.2 \mathrm{E}-12 \\
5.0 \mathrm{E}-8\end{array}$ & $\begin{array}{l}6.1 \mathrm{E}-6 \\
9.2 \mathrm{E}-7 \\
6.3 \mathrm{E}-8 \\
1.4 \mathrm{E}-7 \\
1.4 \mathrm{E}-7 \\
0.0 \mathrm{E}+0 \\
0.0 \mathrm{E}+0 \\
1.4 \mathrm{E}-9 \\
7.4 \mathrm{E}-6\end{array}$ \\
\hline Grand Total & $5.8 \mathrm{E}-6$ & $1.6 \mathrm{E}-6$ & $5.9 \mathrm{E}-7$ & $2.6 \mathrm{E}-7$ & $1.2 \mathrm{E}-7$ & $5.6 \mathrm{E}-8$ & $8.4 \mathrm{E}-6$ \\
\hline
\end{tabular}

Note: Estimates listed as $0.0 E+0$ actually represent insignificant, but finite values. 
Table A.6. Accident sequence probabilities conditional on earthquake probability (additional analysis).

\author{
Earthquake Level
}

Accident

Sequence Level 1 Level 2 Level 3 Level 4 Level 5 Level 6 Total

\begin{tabular}{|c|c|c|c|c|c|c|}
\hline $\begin{array}{c}\text { Large-LOCA-1 } \\
\text { Large-LOCA-2 } \\
\text { Large-LOCA-3 } \\
\text { Large-LOCA-4 } \\
\text { Large-LOCA-5 } \\
\text { Large-LOCA-6 } \\
\text { Subtotal }\end{array}$ & $\begin{array}{l}5.9 \mathrm{E}-6 \\
4.1 \mathrm{E}-7 \\
9.1 \mathrm{E}-7 \\
8.5 \mathrm{E}-7 \\
0.0 \mathrm{E}+0 \\
1.2 \mathrm{E}-9 \\
8.1 \mathrm{E}-6\end{array}$ & $\begin{array}{l}5.9 \mathrm{E}-6 \\
4.2 \mathrm{E}-7 \\
9.3 \mathrm{E}-7 \\
8.7 \mathrm{E}-7 \\
0.0 \mathrm{E}+0 \\
1.2 \mathrm{E}-9 \\
8.1 \mathrm{E}-6\end{array}$ & $\begin{array}{l}5.8 \mathrm{E}-6 \\
4.3 \mathrm{E}-7 \\
9.6 \mathrm{E}-7 \\
9.0 \mathrm{E}-7 \\
0.0 \mathrm{E}+0 \\
1.2 \mathrm{E}-9 \\
8.1 \mathrm{E}-6\end{array}$ & $\begin{array}{l}5.8 \mathrm{E}-6 \\
4.4 \mathrm{E}-7 \\
. .9 \mathrm{E}-7 \\
9.2 \mathrm{E}-7 \\
0.0 \mathrm{E}+0 \\
1.2 \mathrm{E}-9 \\
8.2 \mathrm{E}-6\end{array}$ & $\begin{array}{l}5.8 \mathrm{E}-6 \\
4.6 \mathrm{E}-7 \\
1.0 \mathrm{E}-7 \\
9.6 \mathrm{E}-7 \\
0.0 \mathrm{E}+0 \\
1.2 \mathrm{E}-9 \\
8.2 \mathrm{E}-6\end{array}$ & $\begin{array}{l}5.7 \mathrm{E}-6 \\
4.9 \mathrm{E}-7 \\
1.1 \mathrm{E}-7 \\
1.0 \mathrm{E}-6 \\
0.0 \mathrm{E}+0 \\
1.2 \mathrm{E}-9 \\
8.3 \mathrm{E}-6\end{array}$ \\
\hline $\begin{array}{c}\text { Medium-LOCA-1 } \\
\text { Medium-LOCA-2 } \\
\text { Medium-LOCA-3 } \\
\text { Medium-LOCA-4 } \\
\text { Medium-LOCA-5 } \\
\text { Medium-LOCA-6 } \\
\text { Medium-LOCA-7 } \\
\text { Subtolal }\end{array}$ & $\begin{array}{l}2.2 E-4 \\
1.5 E-5 \\
3.5 E-5 \\
3.2 E-5 \\
0.0 E+0 \\
0.0 E+0 \\
4.5 E-8 \\
3 .(1 E-4\end{array}$ & $\begin{array}{l}2.2 \mathrm{E}-4 \\
1.6 \mathrm{E}-5 \\
3.5 \mathrm{E}-5 \\
3.3 \mathrm{E}-5 \\
0.0 \mathrm{E}+0 \\
0.0 \mathrm{E}+0 \\
4.5 \mathrm{E}-8 \\
3.0 \mathrm{E}-4\end{array}$ & $\begin{array}{l}2.2 E-4 \\
1.6 \mathrm{E}-5 \\
3.6 \mathrm{E}-5 \\
3.4 \mathrm{E}-\mathrm{S} \\
0.0 \mathrm{E}+0 \\
0.0 \mathrm{E}+0 \\
4.5 \mathrm{E}-8 \\
3.0 \mathrm{E}-4\end{array}$ & $\begin{array}{l}2.2 \mathrm{E}-4 \\
1.7 \mathrm{E}-5 \\
3.7 \mathrm{E}-5 \\
3.5 \mathrm{E}-5 \\
0.0 \mathrm{E}+0 \\
0.0 \mathrm{E}+0 \\
4.5 \mathrm{E}-8 \\
3.1 \mathrm{E}-4\end{array}$ & $\begin{array}{l}2.2 \mathrm{E}-4 \\
1.7 \mathrm{E}-5 \\
3.9 \mathrm{E}-5 \\
3.6 \mathrm{E}-5 \\
0.0 \mathrm{E}+0 \\
0.0 \mathrm{E}+0 \\
4.5 \mathrm{E}-8 \\
3.1 \mathrm{E}-4\end{array}$ & $\begin{array}{l}2.1 E-4 \\
1.8 E-5 \\
4.1 E-5 \\
3.8 E-5 \\
0.0 \mathrm{E}+0 \\
0.0 \mathrm{E}+0 \\
4.5 \mathrm{E}-8 \\
3.1 \mathrm{E}-4\end{array}$ \\
\hline $\begin{array}{c}\text { Small-LOCA-1 } \\
\text { Small-LOCA-2 } \\
\text { Small-LOCA-3 } \\
\text { Small-LOCA-4 } \\
\text { Small-LOCA-5 } \\
\text { Small-LOCA-6 } \\
\text { Small-LOCA-7 } \\
\text { Small-LOCA-8 } \\
\text { Subtotal }\end{array}$ & $\begin{array}{l}4.9 \mathrm{E}-3 \\
7.3 \mathrm{E}-4 \\
4.7 \mathrm{E}-5 \\
1.1 \mathrm{E}-4 \\
1.1 \mathrm{E}-4 \\
0.0 \mathrm{E}+0 \\
0.0 \mathrm{E}+0 \\
1.0 \mathrm{E}-6 \\
5.9 \mathrm{E}-3\end{array}$ & $\begin{array}{l}4.9 \mathrm{E}-3 \\
7.5 \mathrm{E}-4 \\
5.2 \mathrm{E}-5 \\
1.1 \mathrm{E}-4 \\
1.1 \mathrm{E}-4 \\
0.0 \mathrm{E}+0 \\
0.0 \mathrm{E}+0 \\
1.0 \mathrm{E}-6 \\
5.9 \mathrm{E}-3\end{array}$ & $\begin{array}{l}4.9 E-3 \\
7.7 E-4 \\
5.8 E-5 \\
1.3 E-4 \\
1.2 E-4 \\
0.0 E+0 \\
0.0 E+0 \\
1.0 E-6 \\
5.9 E-3\end{array}$ & $\begin{array}{l}4.8 \mathrm{E}-3 \\
7.9 \mathrm{E}-4 \\
6.5 \mathrm{E}-5 \\
1.4 \mathrm{E}-4 \\
1.4 \mathrm{E}-4 \\
0.0 \mathrm{E}+0 \\
0.0 \mathrm{E}+0 \\
1.0 \mathrm{E}-6 \\
6.0 \mathrm{E}-3\end{array}$ & $\begin{array}{l}4.8 \mathrm{E}-3 \\
8.2 \mathrm{E}-4 \\
7.5 \mathrm{E}-5 \\
1.5 \mathrm{E}-4 \\
1.5 \mathrm{E}-4 \\
0.0 \mathrm{E}+0 \\
0.0 \mathrm{E}+0 \\
1.0 \mathrm{E}-6 \\
6.0 \mathrm{E}-3\end{array}$ & $\begin{array}{l}4.7 \mathrm{E}-3 \\
8.7 \mathrm{E}-4 \\
8.6 \mathrm{E}-5 \\
1.7 \mathrm{E}-4 \\
1.7 \mathrm{E}-4 \\
0.0 \mathrm{E}+0 \\
0.0 \mathrm{E}+0 \\
1.0 \mathrm{E}-6 \\
6.0 \mathrm{E}-3\end{array}$ \\
\hline $\begin{array}{c}\text { LOSP-Trans-1 } \\
\text { LOSP-Trans-2 } \\
\text { LOSP-Trans-3 } \\
\text { LOSP-Trans-4 } \\
\text { LOSP-Trans-5 } \\
\text { LOSP-Trans-6 } \\
\text { LOSP-Trans-8 } \\
\text { LOSP-Trans-9 } \\
\text { Subtotal }\end{array}$ & $\begin{array}{l}4.0 \mathrm{E}-2 \\
5.9 \mathrm{E}-3 \\
3.8 \mathrm{E}-4 \\
8.6 \mathrm{E}-4 \\
8.6 \mathrm{E}-4 \\
0 .(\mathrm{E}+0 \\
0.0 \mathrm{E}+0 \\
9.0 \mathrm{E}-6 \\
4.8 \mathrm{E}-2\end{array}$ & $\begin{array}{l}4.0 \mathrm{E}-2 \\
6.0 \mathrm{E}-3 \\
4.2 \mathrm{E}-4 \\
9.3 \mathrm{E}-4 \\
9.3 \mathrm{E}-4 \\
(0.0 \mathrm{E}+() \\
0.0 \mathrm{E}+0 \\
9.0 \mathrm{E}-6 \\
4.8 \mathrm{E}-2\end{array}$ & $\begin{array}{l}4.0 \mathrm{E}-2 \\
6.2 \mathrm{E}-3 \\
4.7 \mathrm{E}-4 \\
1.0 \mathrm{E}-3 \\
1.0 \mathrm{E}-3 \\
0.0 \mathrm{E}+0 \\
0.0 \mathrm{E}+0 \\
9.0 \mathrm{E}-6 \\
4.8 \mathrm{E}-2\end{array}$ & $\begin{array}{l}3.9 E-2 \\
6.4 E-3 \\
5.3 E-4 \\
1.1 E-3 \\
1.1 E-3 \\
0.0 E+0 \\
0.0 E+0 \\
9.0 E-6 \\
4.8 E-2\end{array}$ & $\begin{array}{l}3.9 \mathrm{E}-2 \\
6.7 \mathrm{E}-3 \\
6.0 \mathrm{E}-4 \\
1.2 \mathrm{E}-3 \\
1.2 \mathrm{E}-3 \\
0.0 \mathrm{E}+0 \\
0.0 \mathrm{E}+0 \\
9.0 \mathrm{E}-6 \\
4.9 \mathrm{E}-2\end{array}$ & $\begin{array}{l}3.8 \mathrm{E}-2 \\
7.1 \mathrm{E}-3 \\
6.9 \mathrm{E}-4 \\
1.4 \mathrm{E}-3 \\
1.4 \mathrm{E}-3 \\
0.0 \mathrm{E}+0 \\
0.0 \mathrm{E}+0 \\
9.0 \mathrm{E}-6 \\
4.9 \mathrm{E}-2\end{array}$ \\
\hline Grand Toral & 5.4E-2 & $5.4 E-2$ & $5.4 E-2$ & 5.5E-2 & 5.5E-2 & 5.5E-2 \\
\hline
\end{tabular}

Note: Estimates listed $0.0 k+0$ actually represent insignificant, but finite, values. 


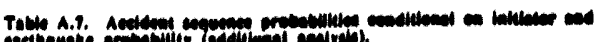

Intapien

\begin{tabular}{|c|c|c|c|c|c|c|}
\hline 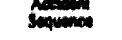 & Lowed I & $\ln 21$ & Lond 3 & Londa & Int & Lende \\
\hline 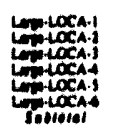 & 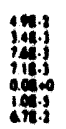 & 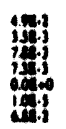 & $\begin{array}{l}4.14 \\
1+1 \\
1+1\end{array}$ & 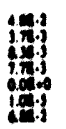 & 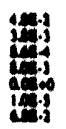 & 造: \\
\hline 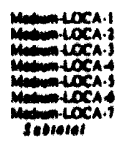 & 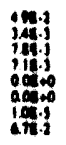 & 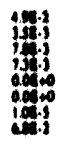 & 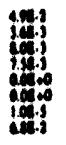 & 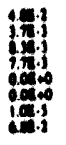 & 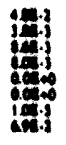 & 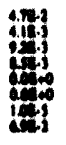 \\
\hline 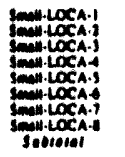 & 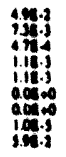 & 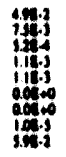 & 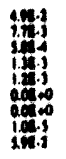 & 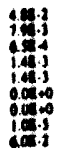 & 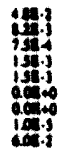 & 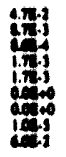 \\
\hline 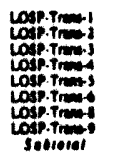 & 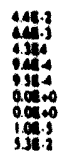 & 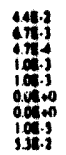 & 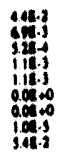 & 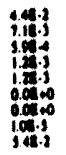 & 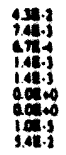 & 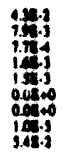 \\
\hline
\end{tabular}

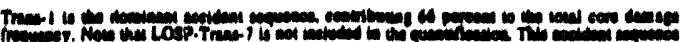

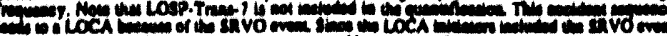

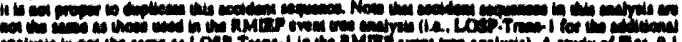

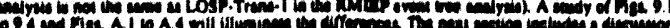

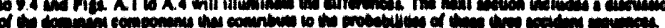

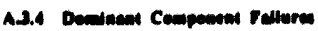

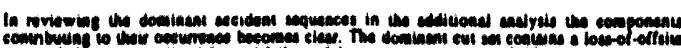

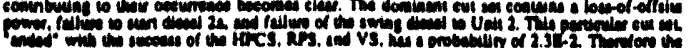

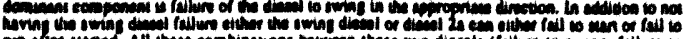

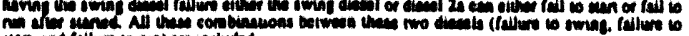

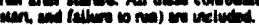

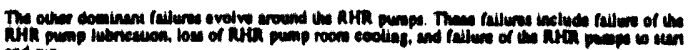
tin nom.

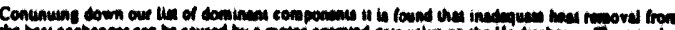

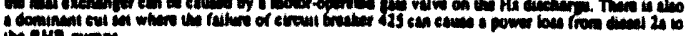
intin poin

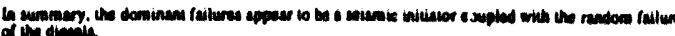
A.4 Compartien of RMILP end Additiona Analyses Reaults

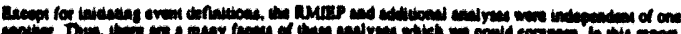

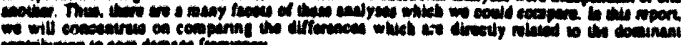

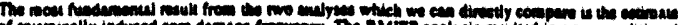

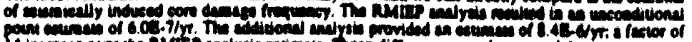

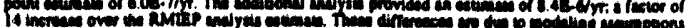

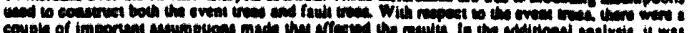

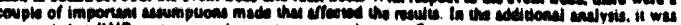

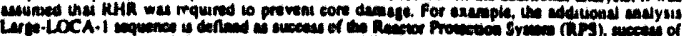

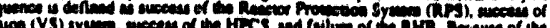

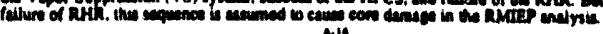

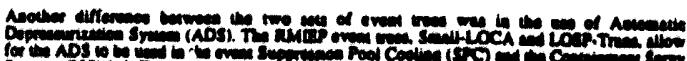

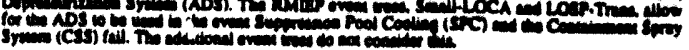

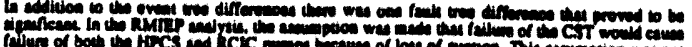

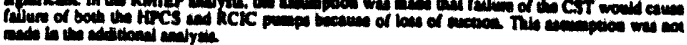

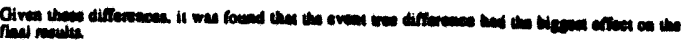

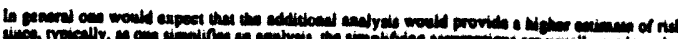

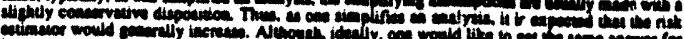

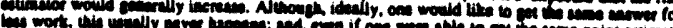

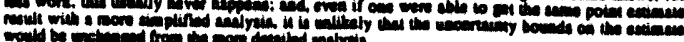

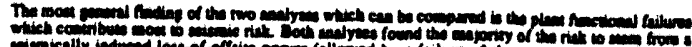

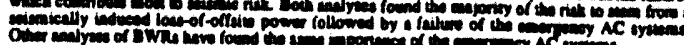

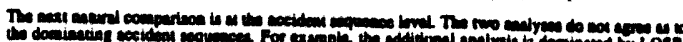

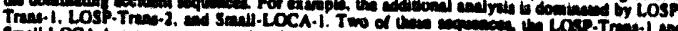

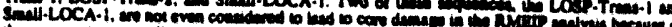

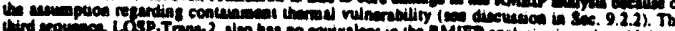

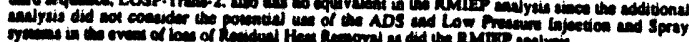

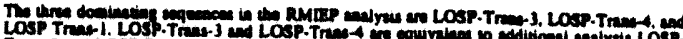

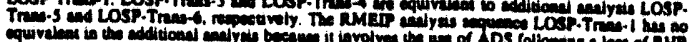

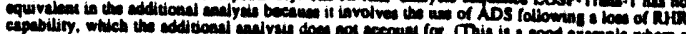

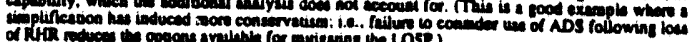

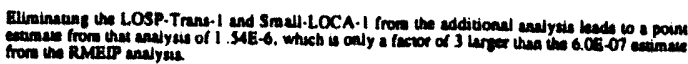

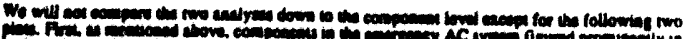

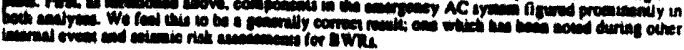

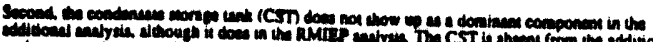

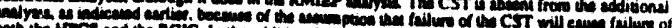
(N)

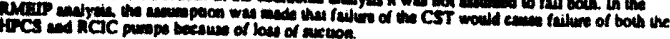

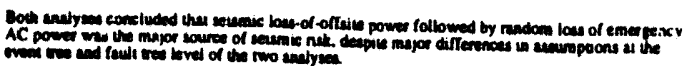

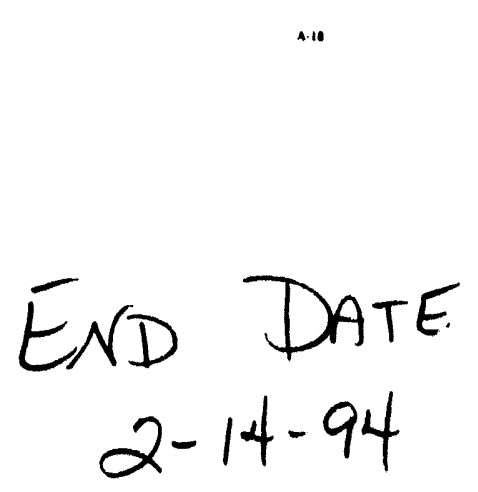

